NIST NCSTAR 1-5A

Federal Building and Fire Safety Investigation of the World Trade Center Disaster

\title{
Visual Evidence, Damage Estimates, And Timeline Analysis
}

\section{(Appendices D-G)}





\section{Appendix D \\ WTC 1 EAST FACE COMBINED FIRE AND WINDOW CONDITION AND WINDOW SMOKE CONDITION}

This appendix provides visual representations of the data contained in data sheets describing observations of fire, smoke. and window condition on a window-by-window basis as a function of time for the east face of WTC 1. The data for window condition and fire observations are combined into single representations, with the data for fire taking precedence. Observations are represented using the key provided in Figure 5-1 as follows: [ - window glass in place. \- window open, - spot fire, - fire visible inside, - external flaming, and - not visible. The data for smoke are shown in separate figures

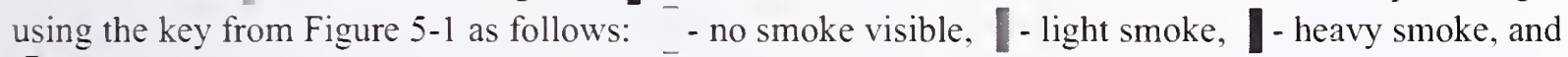
1- not visible. For a given time, the window condition and fire data are shown first. 

WTC 1, East Face
8:48 a.m.

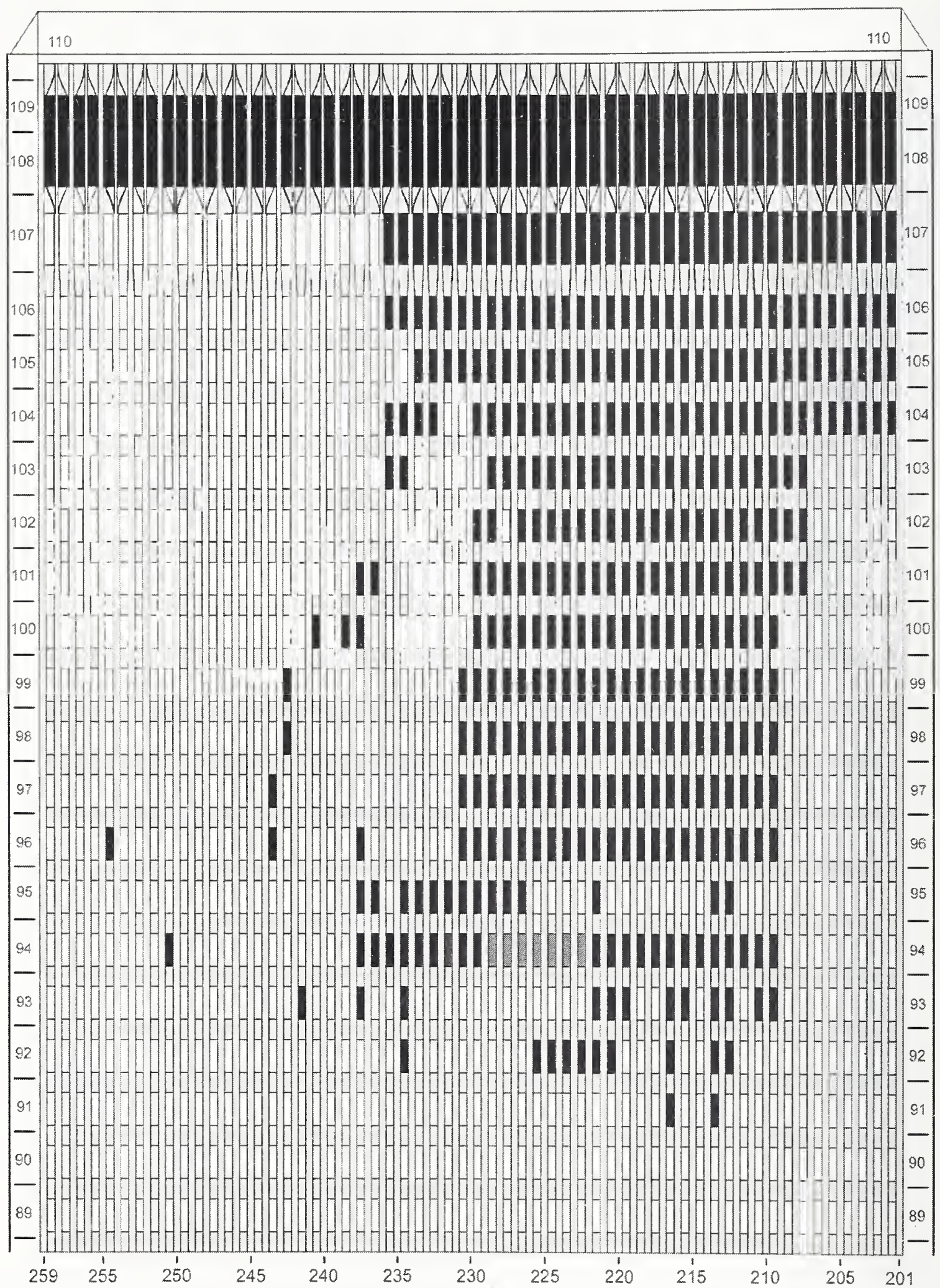

Figure D-1. Diagram of the east face of WTC 1 for floors 89 to 110 at $8: 48$ a.m. showing the condition of windows and locations of fires. 


$$
\text { WTC 1, East Face } \quad \text { 8:48 a.m. }
$$

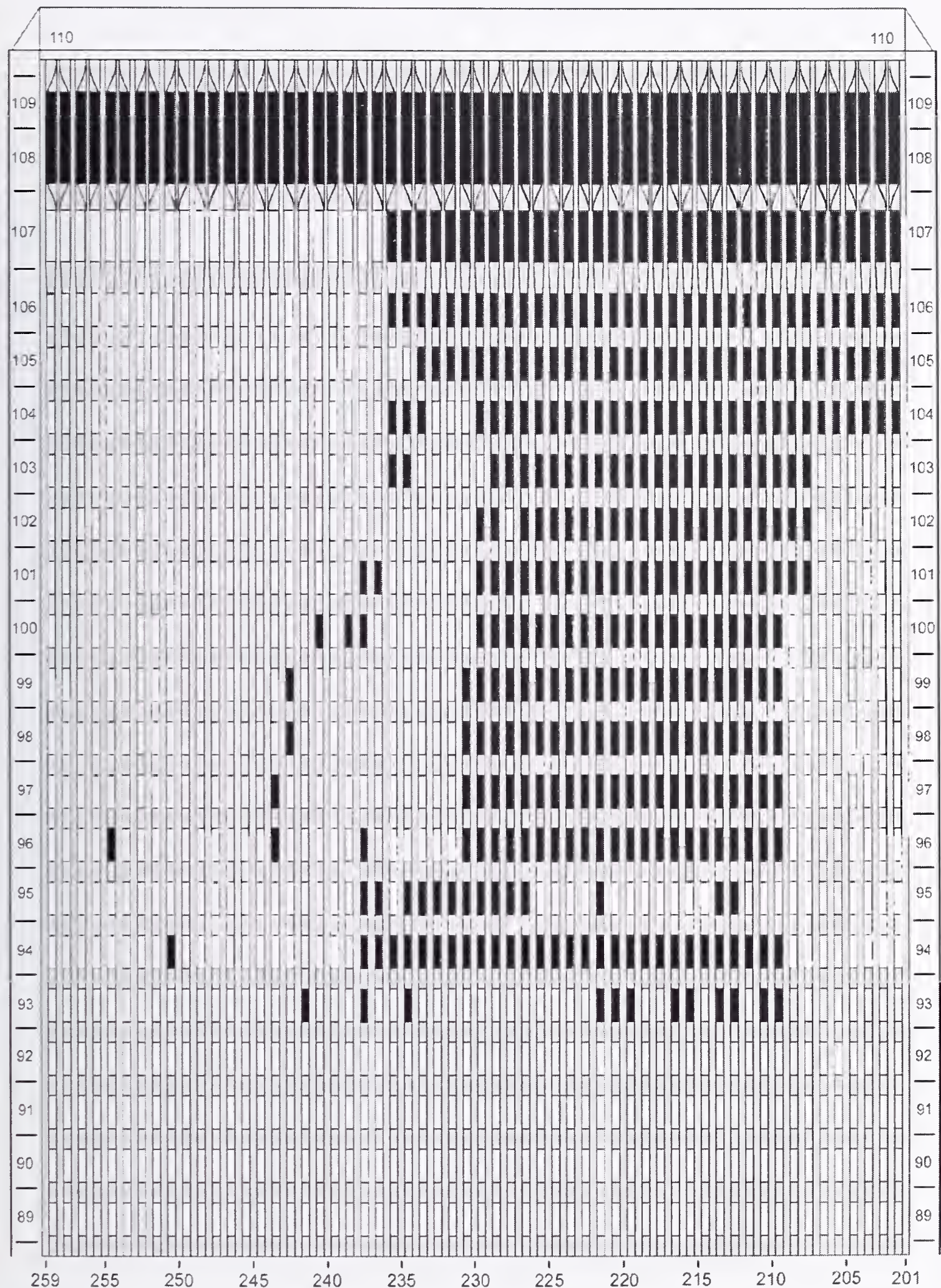

Figure D-2. Diagram of the east face of WTC 1 for floors 89 to 110 at 8:48 a.m. showing windows where smoke was observed and those that were hidden from view. 

WTC 1, East Face
8:52 a.m.

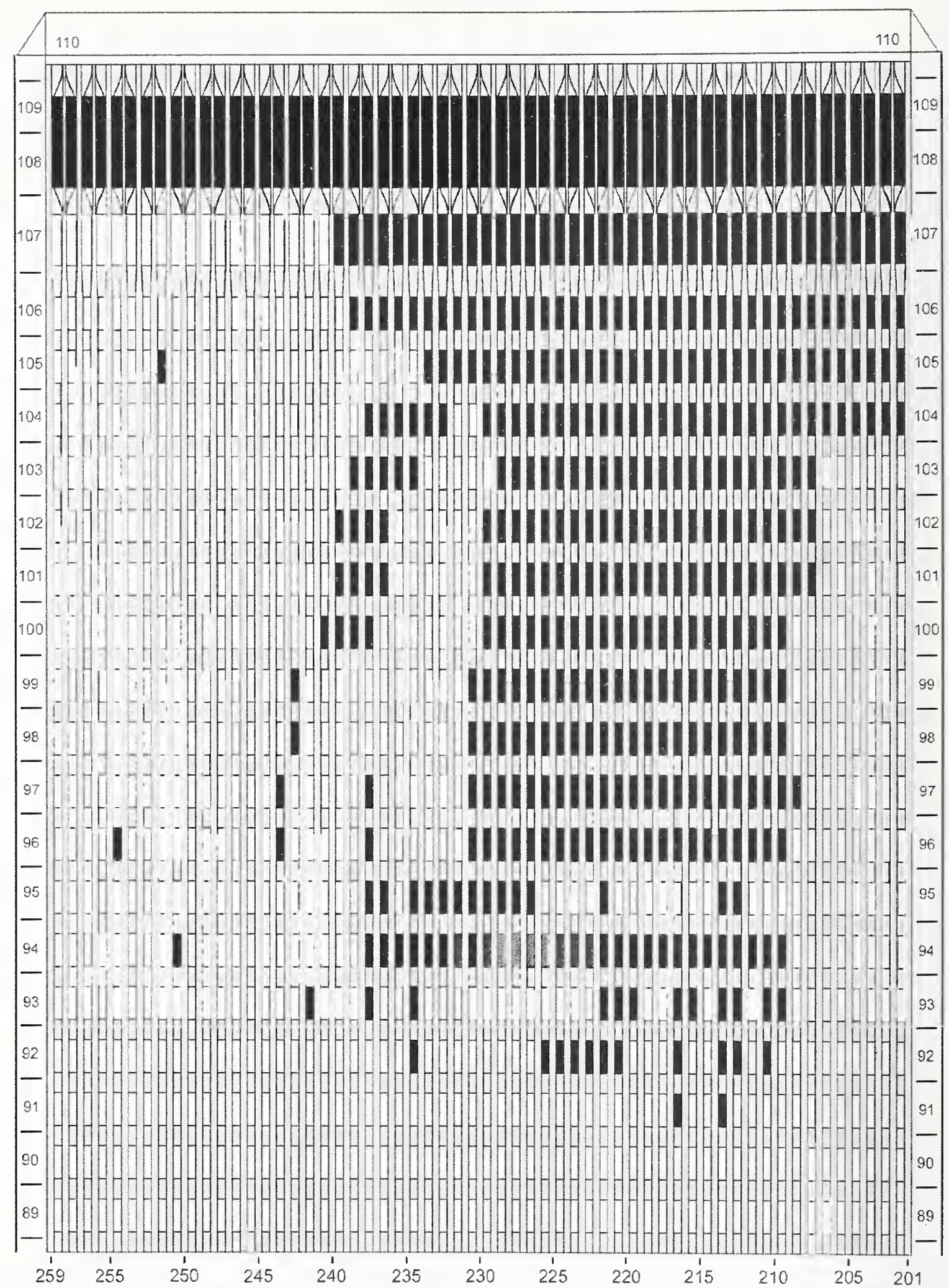

Figure D-3. Diagram of the east face of WTC 1 for floors 89 to 110 at $8: 52$ a.m. showing the condition of windows and locations of fires. 
WTC 1, East Face $\quad 8: 52$ a.m.

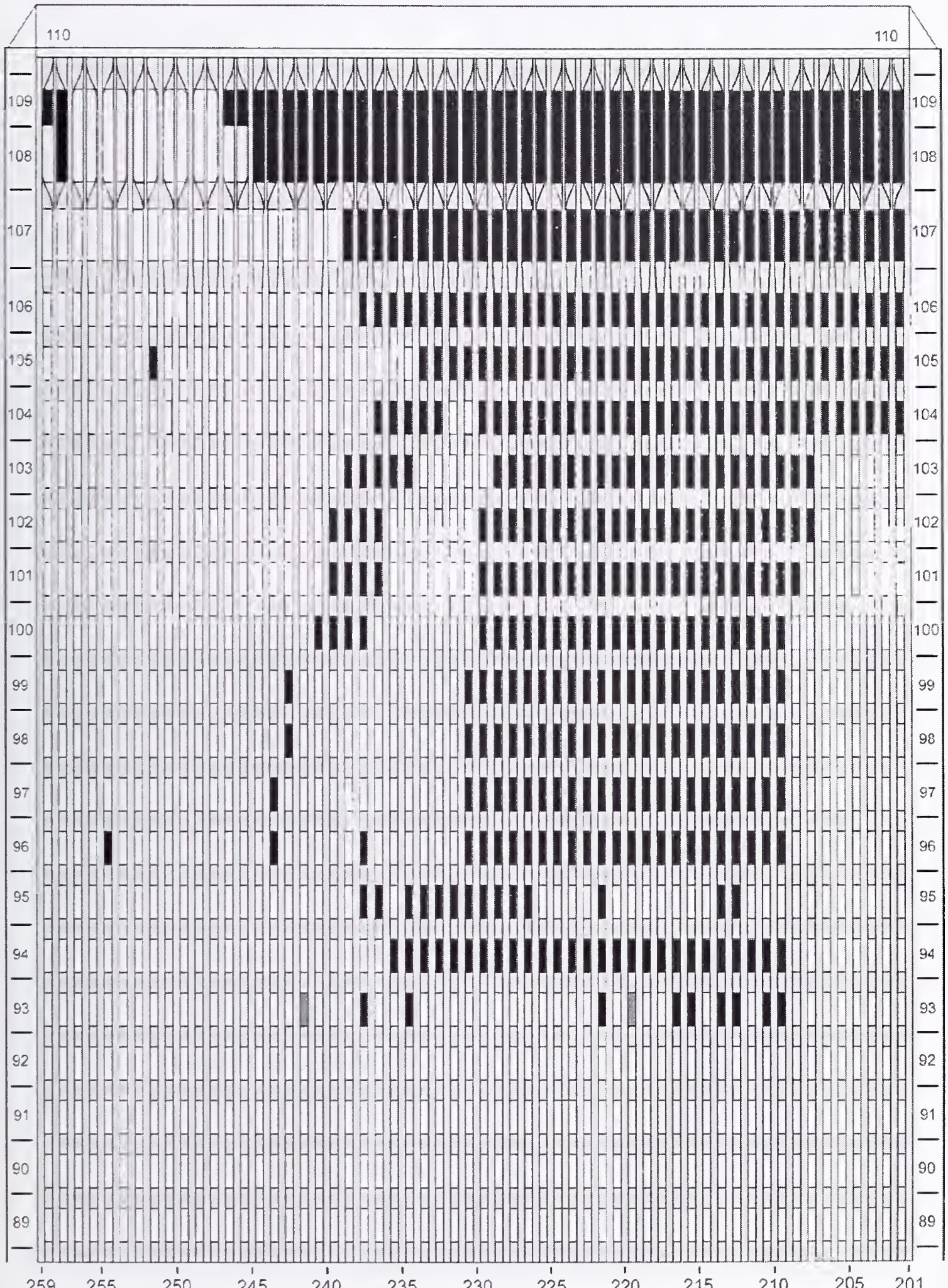

Figure D-4. Diagram of the east face of WTC 1 for floors 89 to 110 at 8:52 a.m. showing windows where smoke was observed and those that were hidden from view. 


\section{WTC 1, East Face $\quad$ 8:54 a.m.}



Figure D-5. Diagram of the east face of WTC 1 for floors 89 to 110 at 8:54 a.m. showing the condition of windows and locations of fires. 


$$
\text { WTC 1, East Face } \quad 8: 54 \text { a.m. }
$$

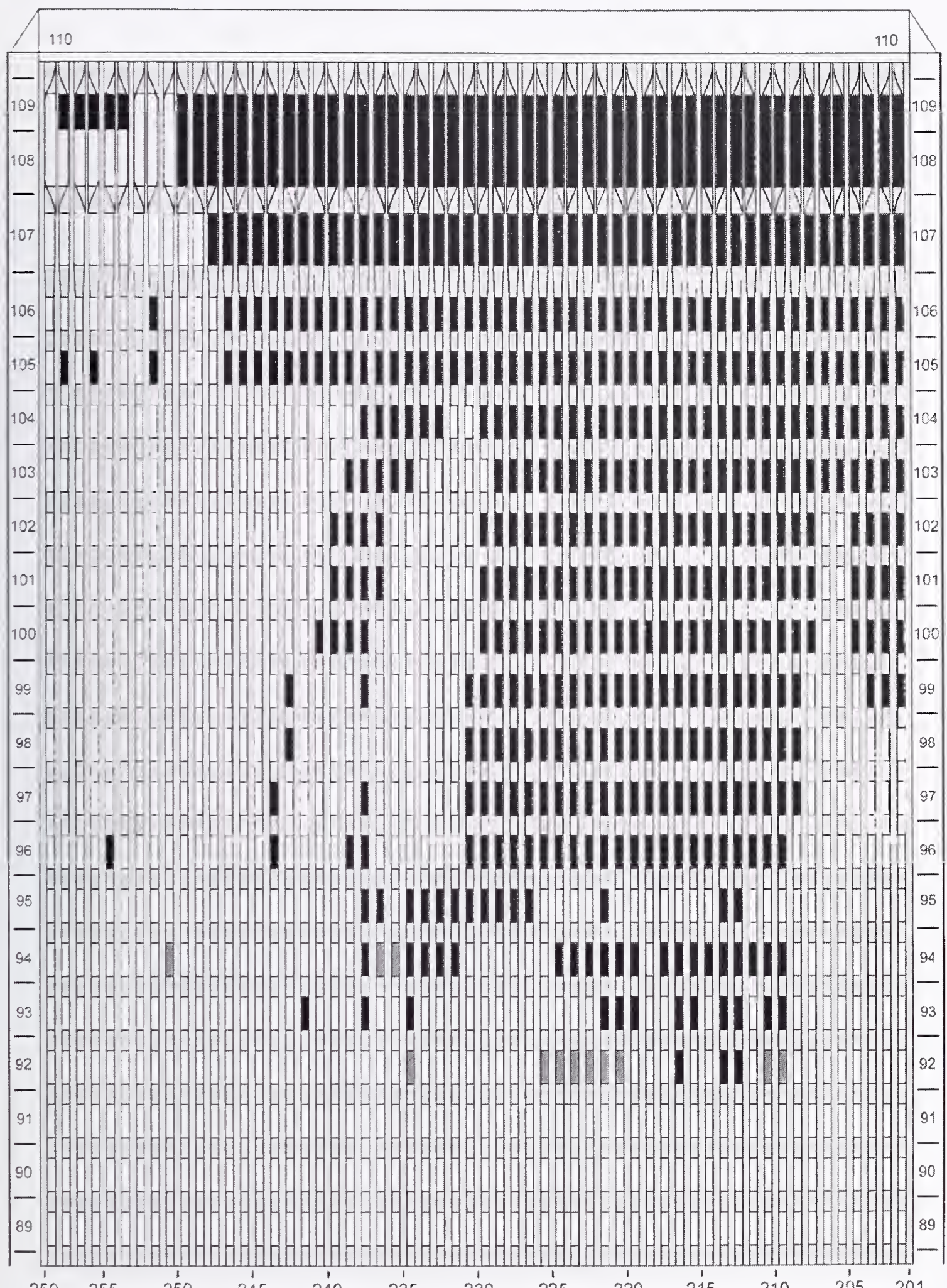

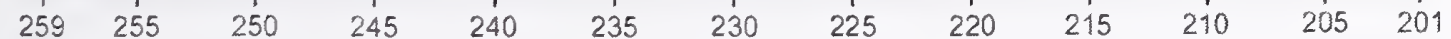

Figure D-6. Diagram of the east face of WTC 1 for floors 89 to 110 at 8:54 a.m. showing windows where smoke was observed and those that were hidden from view. 

WTC 1, East Face
8:58 a.m.

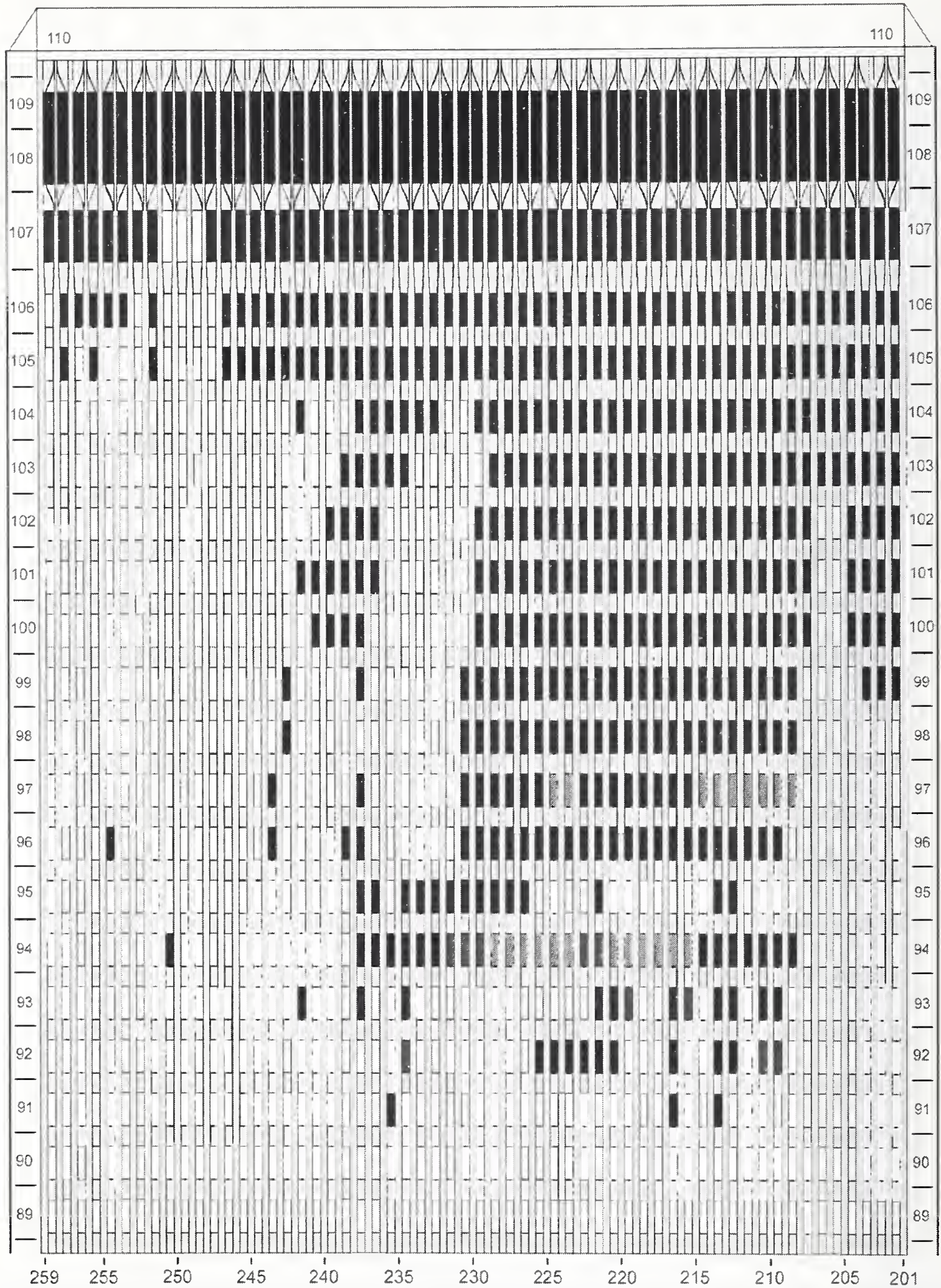

Figure D-7. Diagram of the east face of WTC 1 for floors 89 to 110 at 8:58 a.m. showing the condition of windows and locations of fires. 


$$
\text { WTC 1, East Face } \quad \text { 8:58 a.m. }
$$

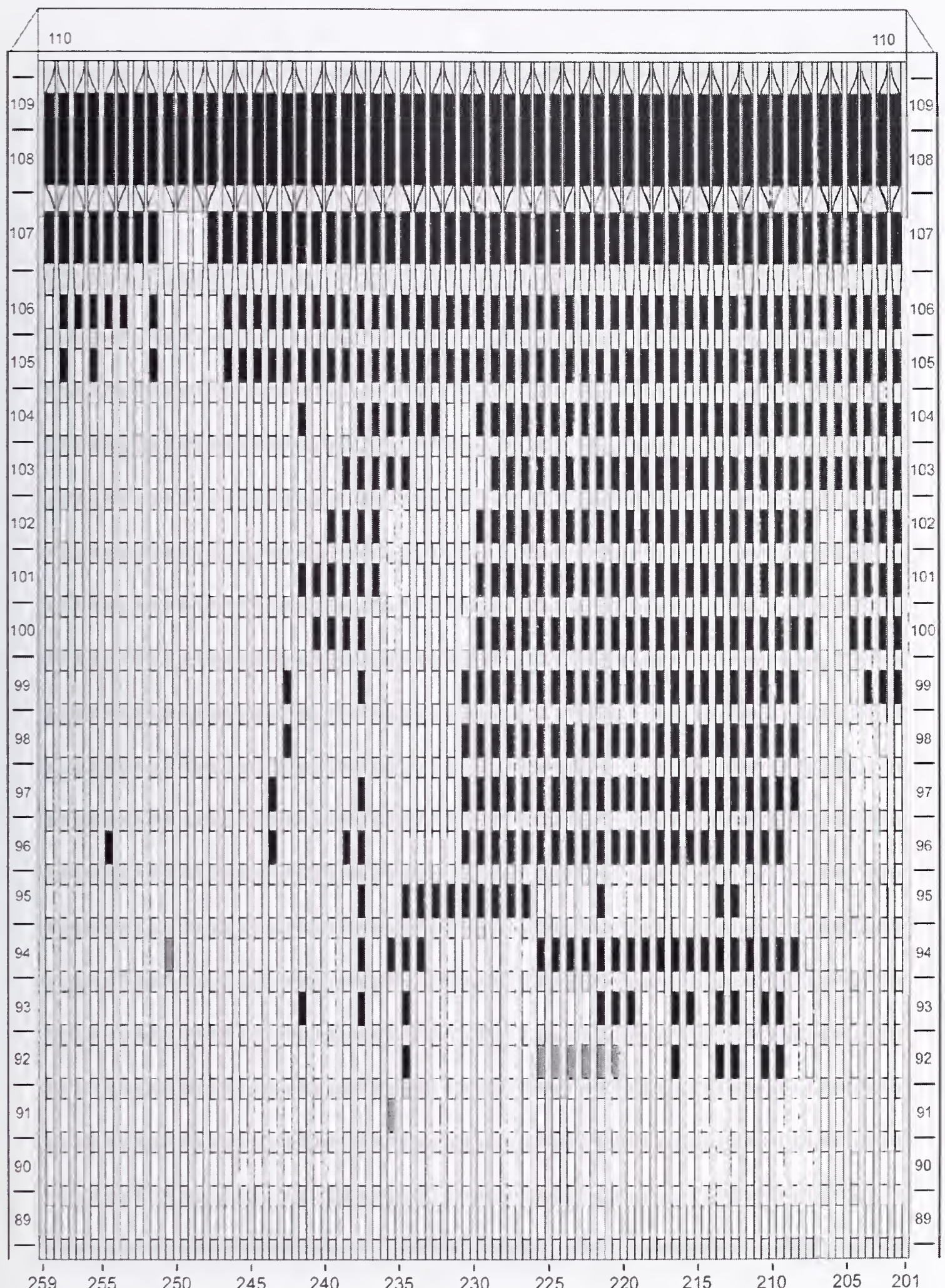

Figure D-8. Diagram of the east face of WTC 1 for floors 89 to 110 at 8:58 a.m. showing windows where smoke was observed and those that were hidden from view. 

WTC 1, East Face
9:00 a.m.

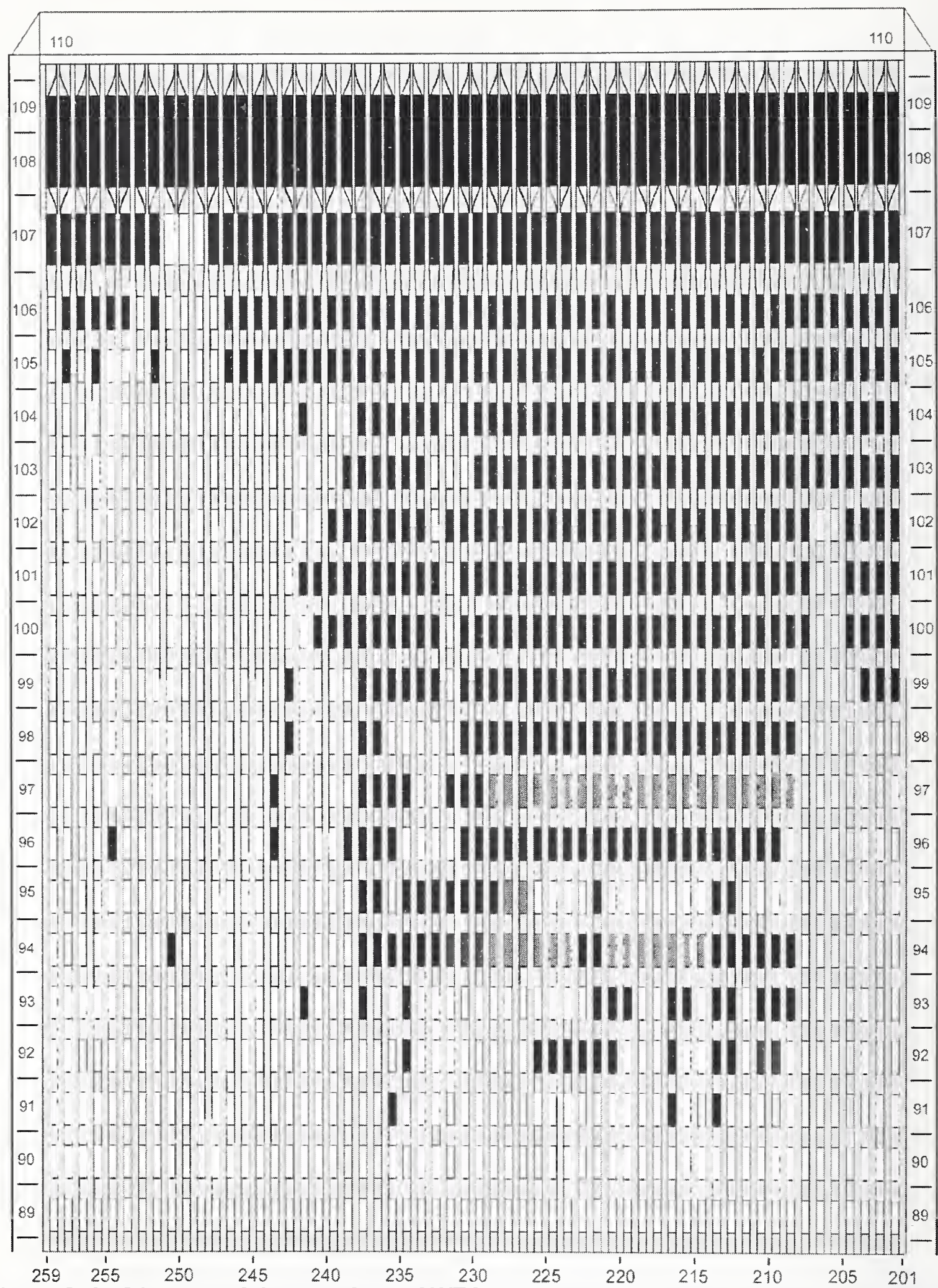

Figure D-9. Diagram of the east face of WTC 1 for floors 89 to 110 at 9:00 a.m. showing the condition of windows and locations of fires. 


$$
\text { WTC 1, East Face 9:00 a.m. }
$$

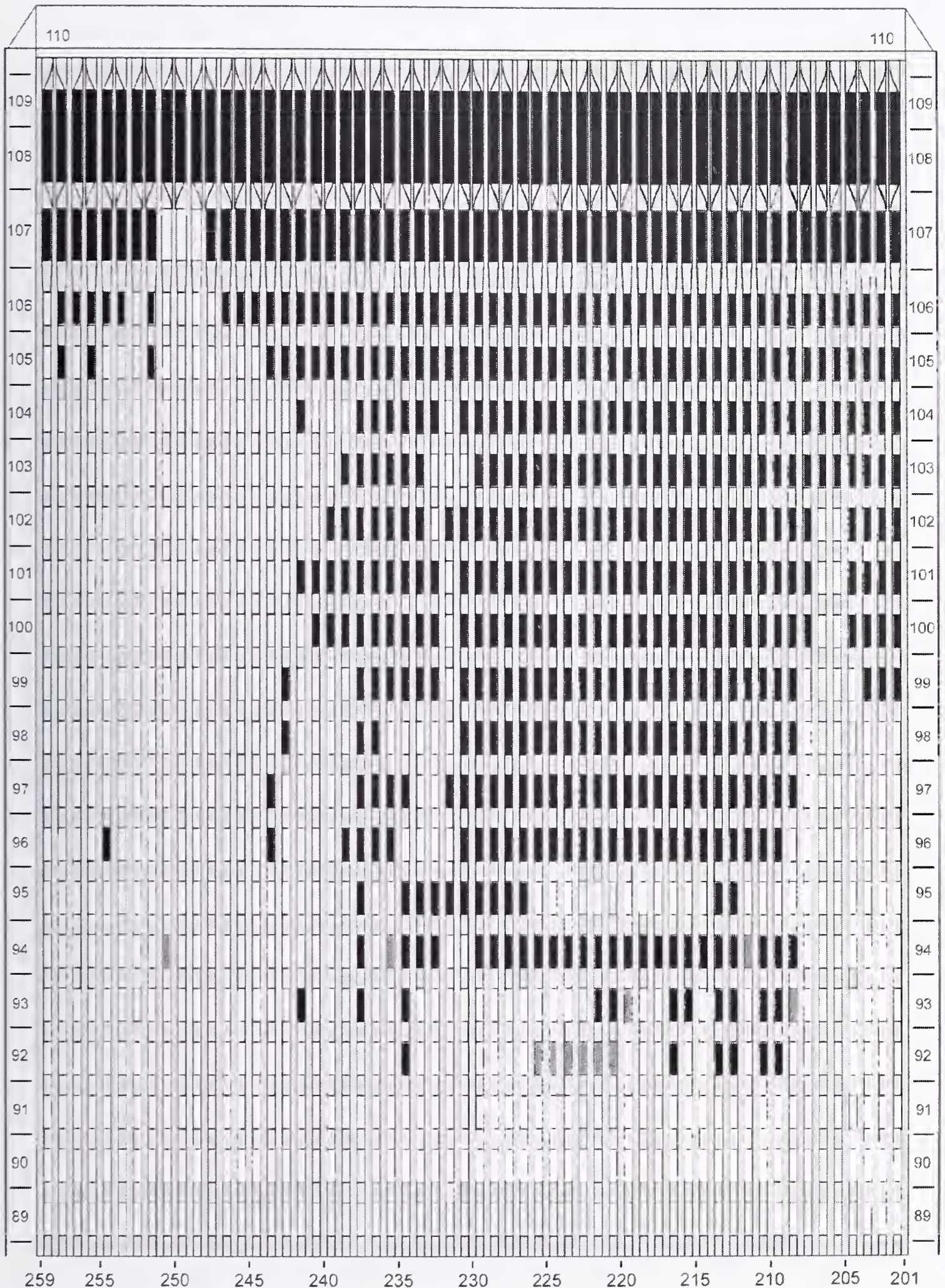

Figure D-10. Diagram of the east face of WTC 1 for floors 89 to 110 at 9:00 a.m. showing windows where smoke was observed and those that were hidden from view. 
WTC 1, East Face

9:02 a.m.

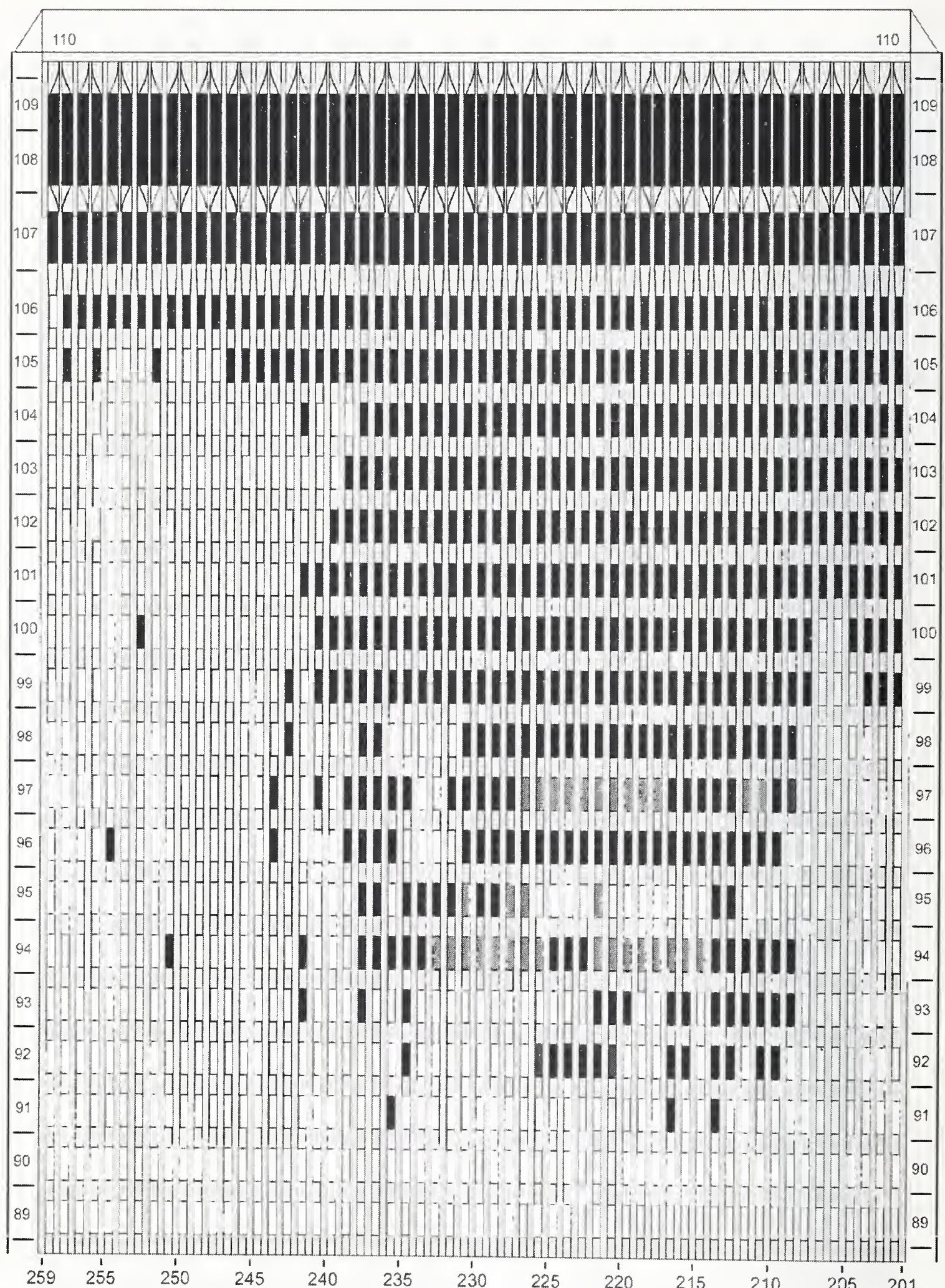

Figure D-11. Diagram of the east face of WTC 1 for floors 89 to 110 at 9:02 a.m. showing the condition of windows and locations of fires. 


$$
\text { WTC 1, East Face 9:02 a.m. }
$$

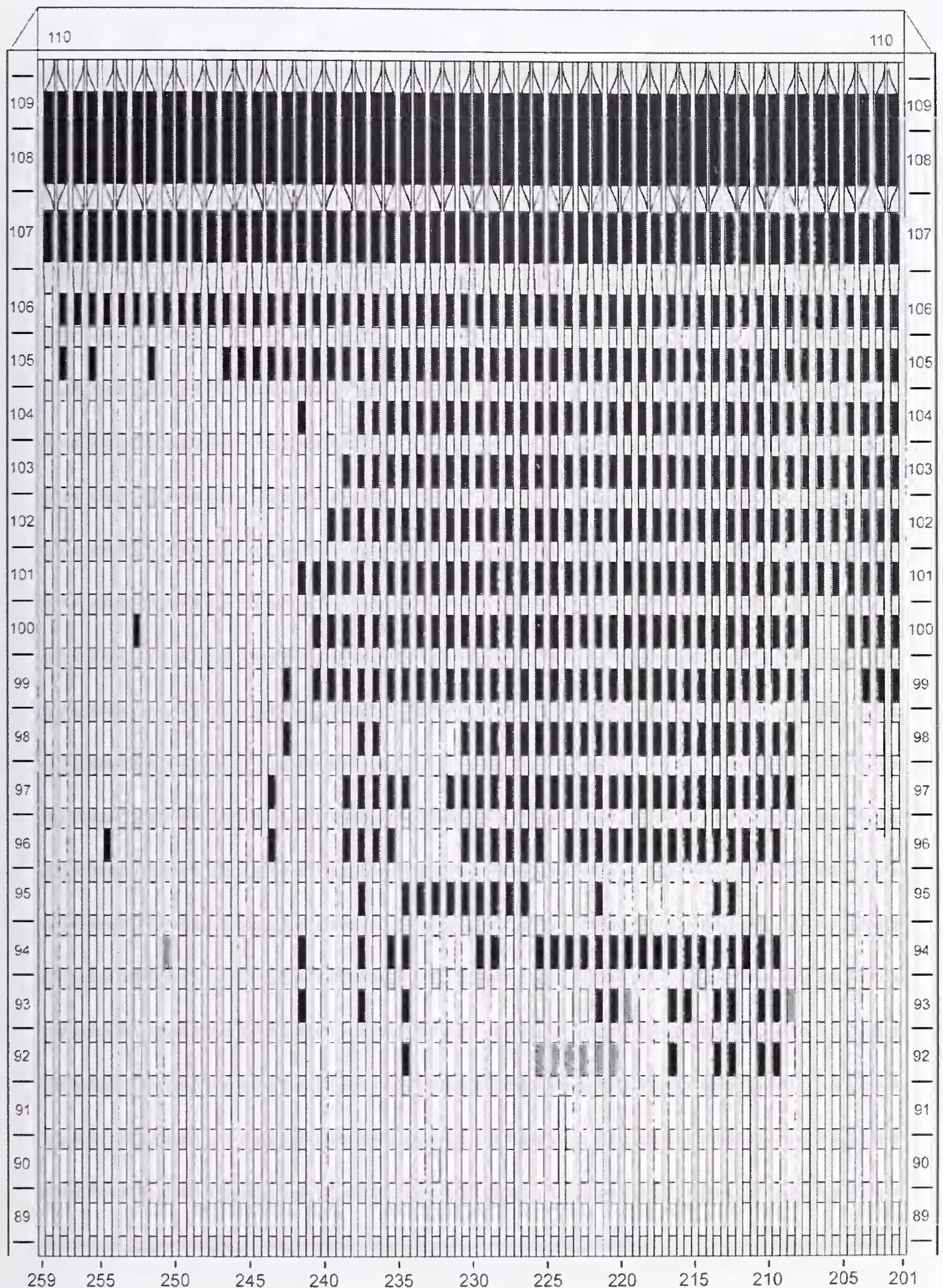

Figure D-12. Diagram of the east face of WTC 1 for floors 89 to 110 at 9:02 a.m. showing windows where smoke was observed and those that were hidden from view. 


\section{WTC 1, East Face 9:08 a.m.}

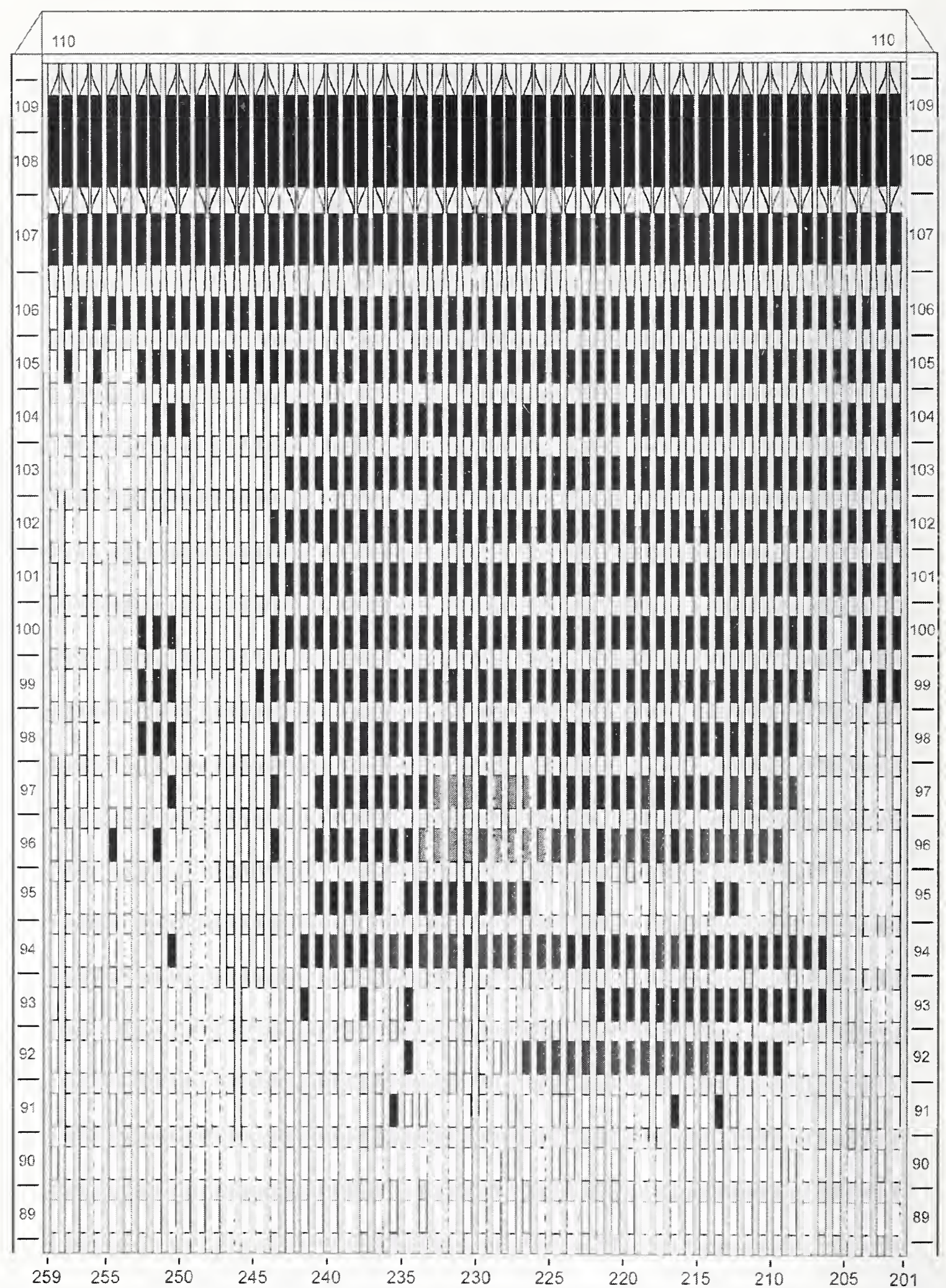

Figure D-13. Diagram of the east face of WTC 1 for floors 89 to 110 at 9:08 a.m. showing the condition of windows and locations of fires. 


$$
\text { WTC 1, East Face 9:08 a.m. }
$$

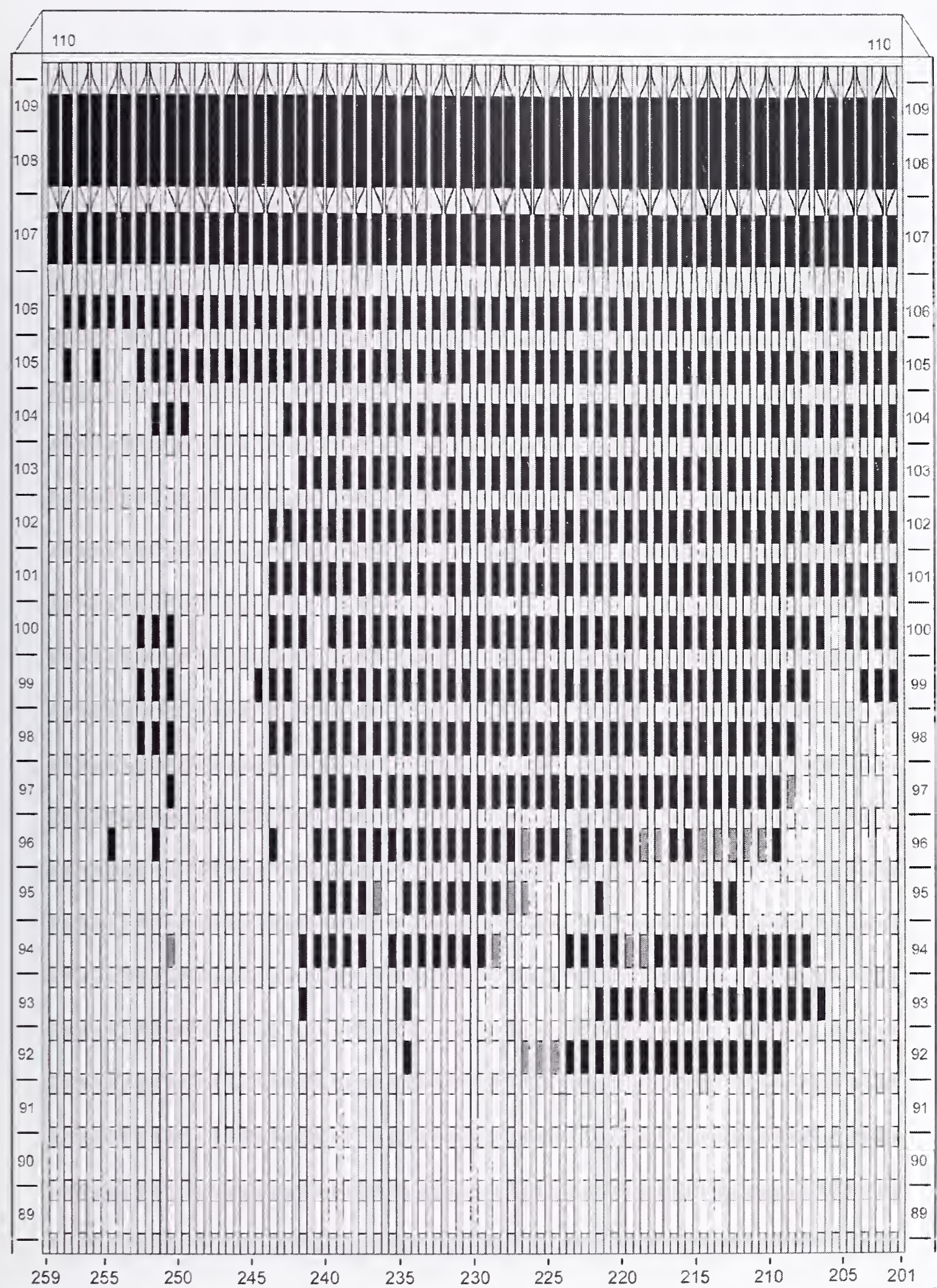

Figure D-14. Diagram of the east face of WTC 1 for floors 89 to 110 at 9:08 a.m. showing windows where smoke was observed and those that were hidden from view. 


$$
\text { WTC 1, East Face } \quad 9: 12 \text { a.m. }
$$

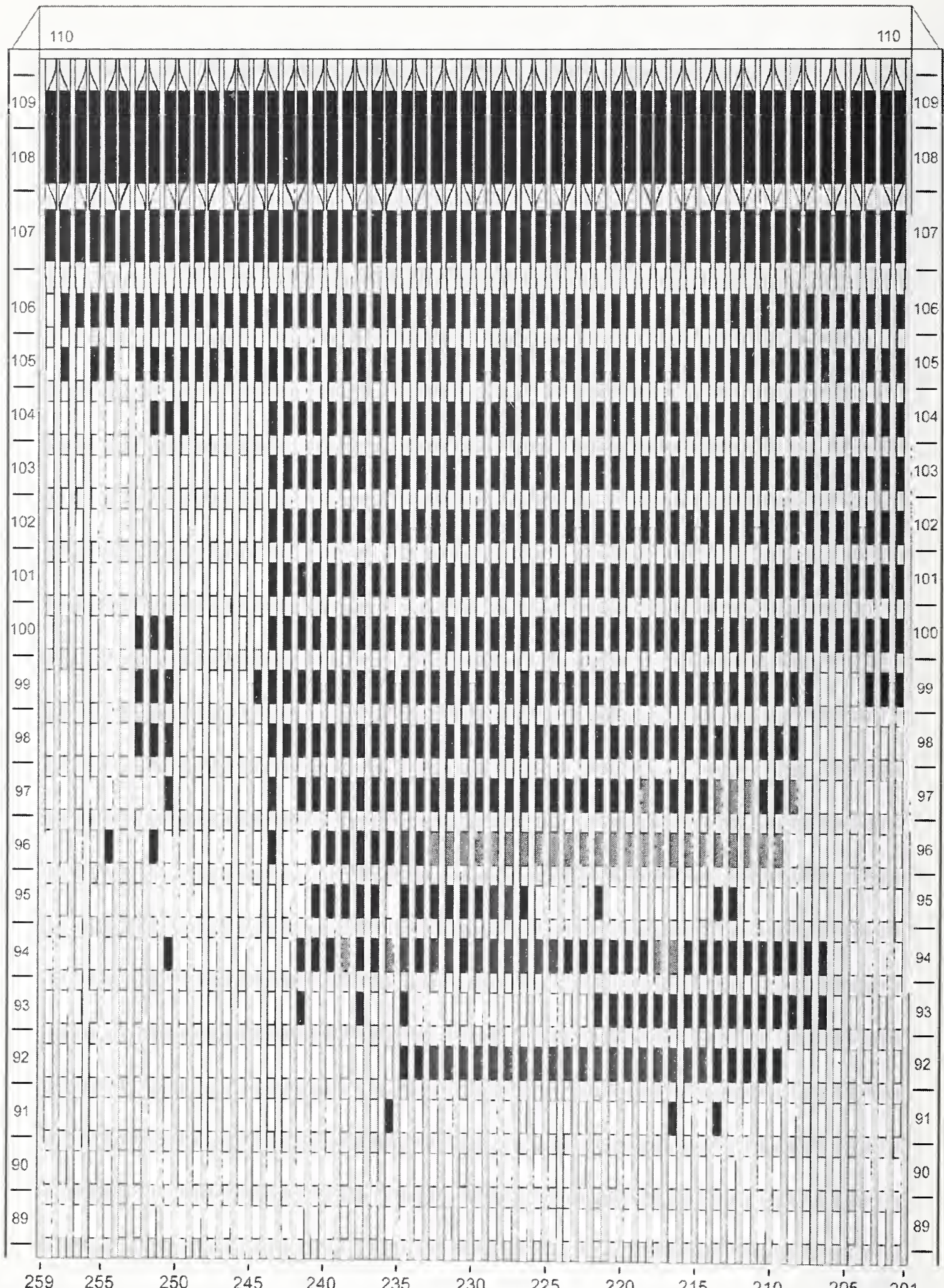

Figure D-15. Diagram of the east face of WTC 1 for floors 89 to 110 at 9:12 a.m. showing the condition of windows and locations of fires. 


$$
\text { WTC 1, East Face 9:12 a.m. }
$$

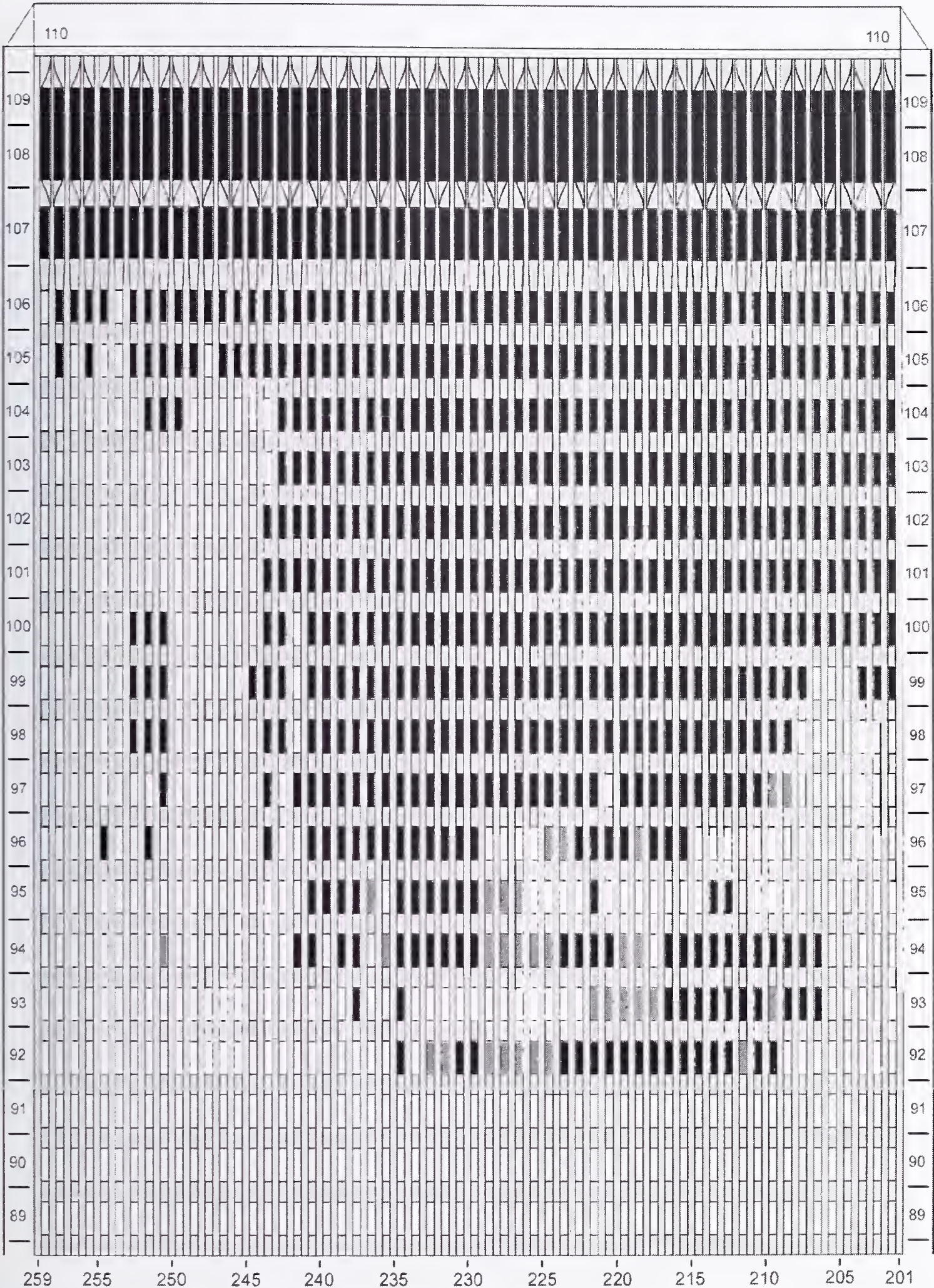

Figure D-16. Diagram of the east face of WTC 1 for floors 89 to 110 at 9:12 a.m. showing windows where smoke was observed and those that were hidden from view. 

WTC 1, East Face
9:14 a.m.

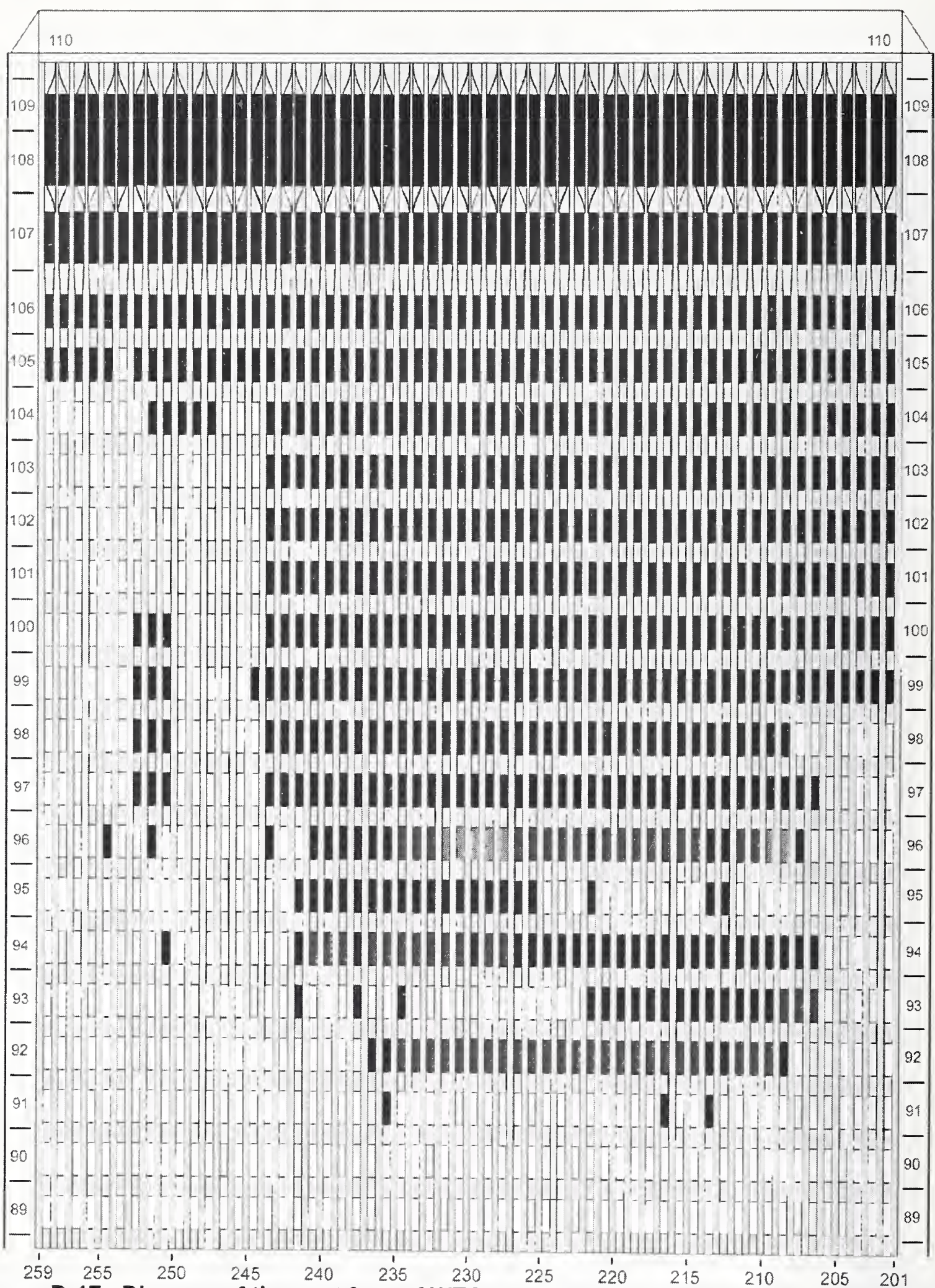

Figure D-17. Diagram of the east face of WTC 1 for floors 89 to 110 at 9:14 a.m. showing the condition of windows and locations of fires. 


$$
\text { WTC 1, East Face } \quad \text { 9:14 a.m. }
$$

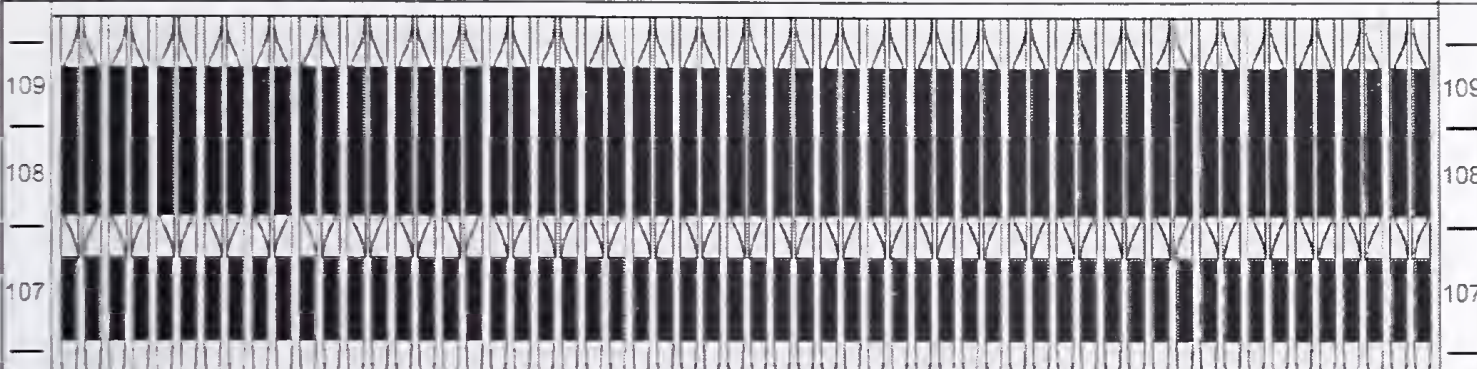

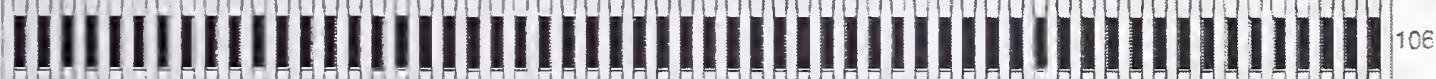

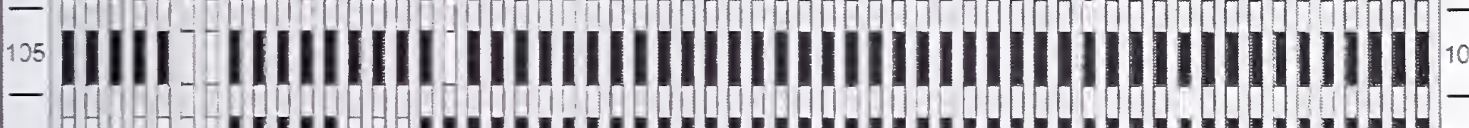

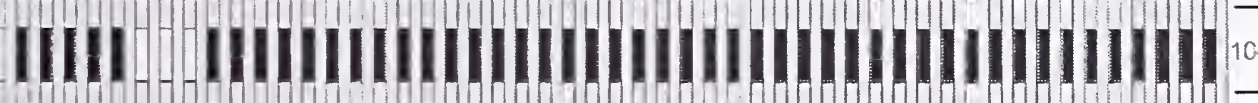

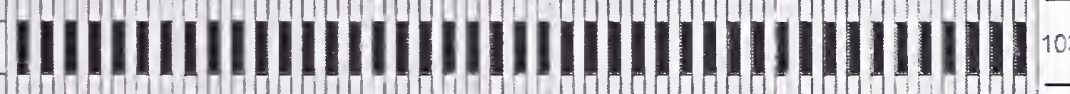
|

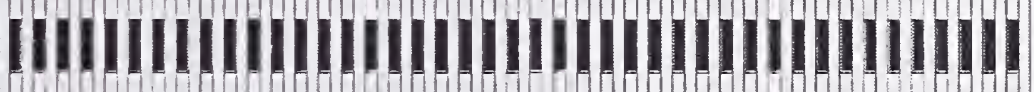

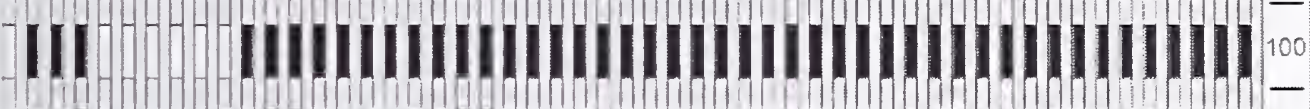

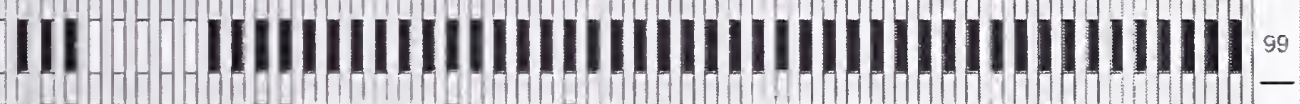
III- III IIIIIIIIIIIII IIIIIIIIIIII

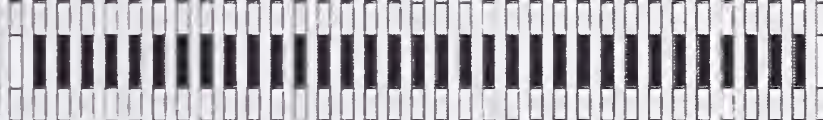

\section{IIIIIII - III I I I}

IIIIIIIIIIIIIIII I- I

IIIII II II [- IIIIIIIIIIIIIII

I. IIIIIIIIIIII

IIIIIIIII II II

Figure D-18. Diagram of the east face of WTC 1 for floors 89 to 110 at 9:14 a.m. showing windows where smoke was observed and those that were hidden from view. 
WTC 1, East Face $\quad$ 9:20 a.m.

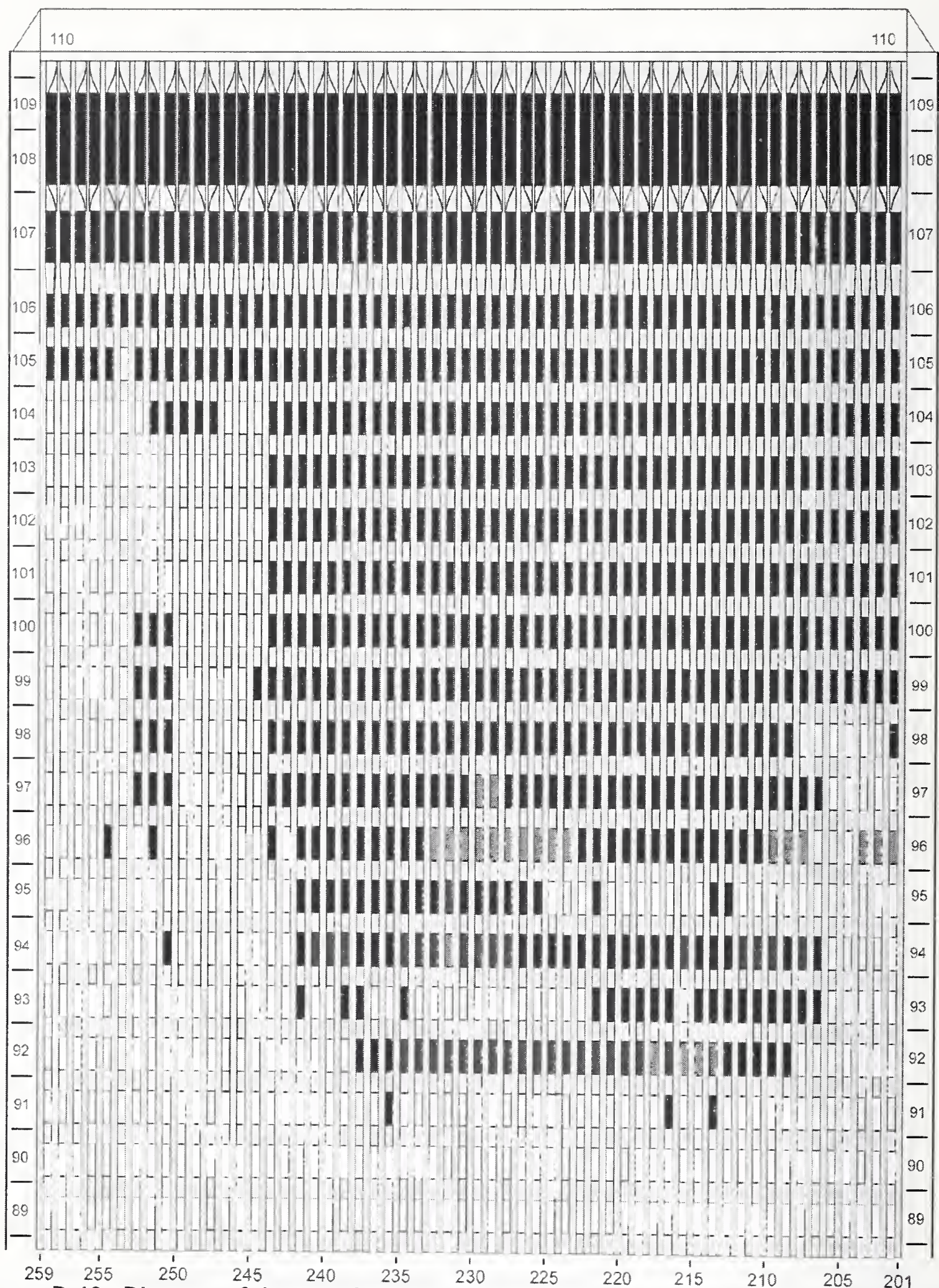

Figure D-19. Diagram of the east face of WTC 1 for floors 89 to 110 at 9:20 a.m. showing the condition of windows and locations of fires. 


$$
\text { WTC 1, East Face 9:20 a.m. }
$$

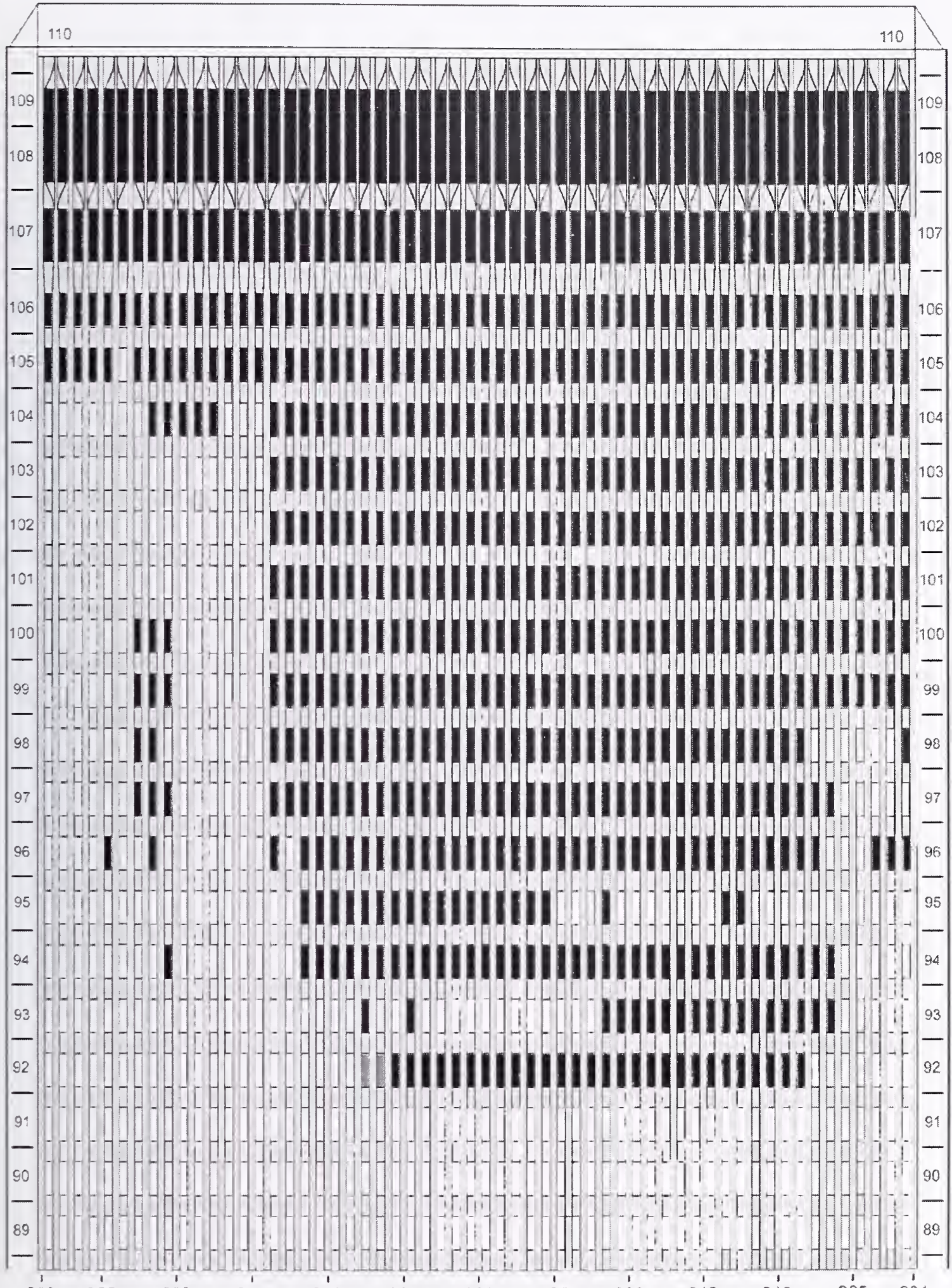

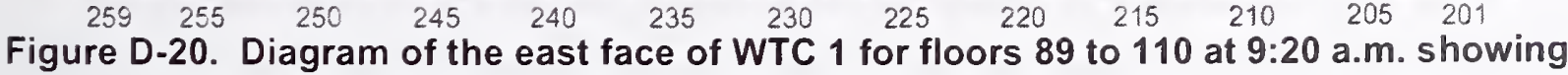
windows where smoke was observed and those that were hidden from view. 


\section{WTC 1, East Face 9:26 a.m.}

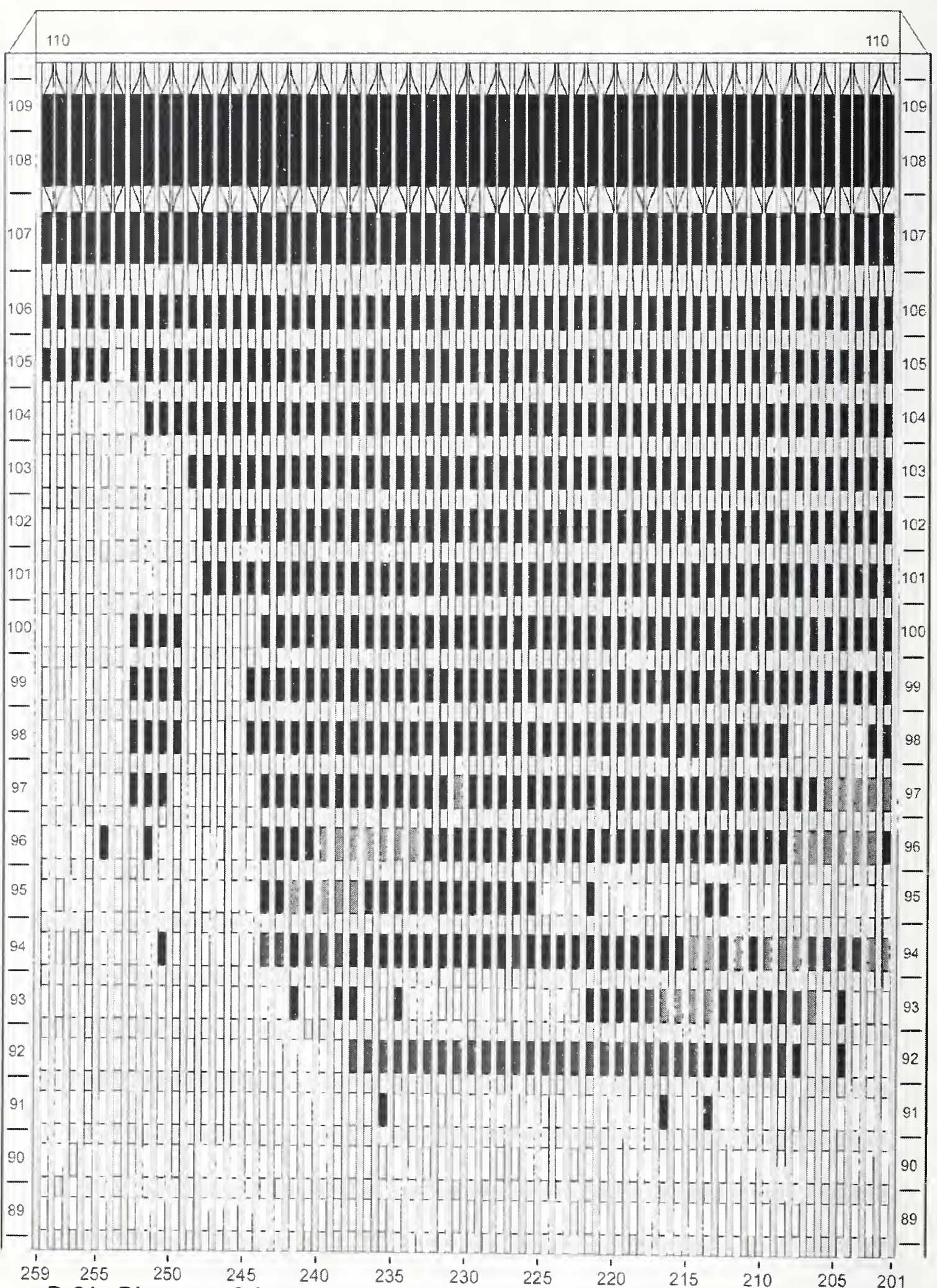

Figure D-21. Diagram of the east face of WTC 1 for floors 89 to 110 at 9:26 a.m. showing the condition of windows and locations of fires. 


$$
\text { WTC 1, East Face } \quad 9: 26 \text { a.m. }
$$

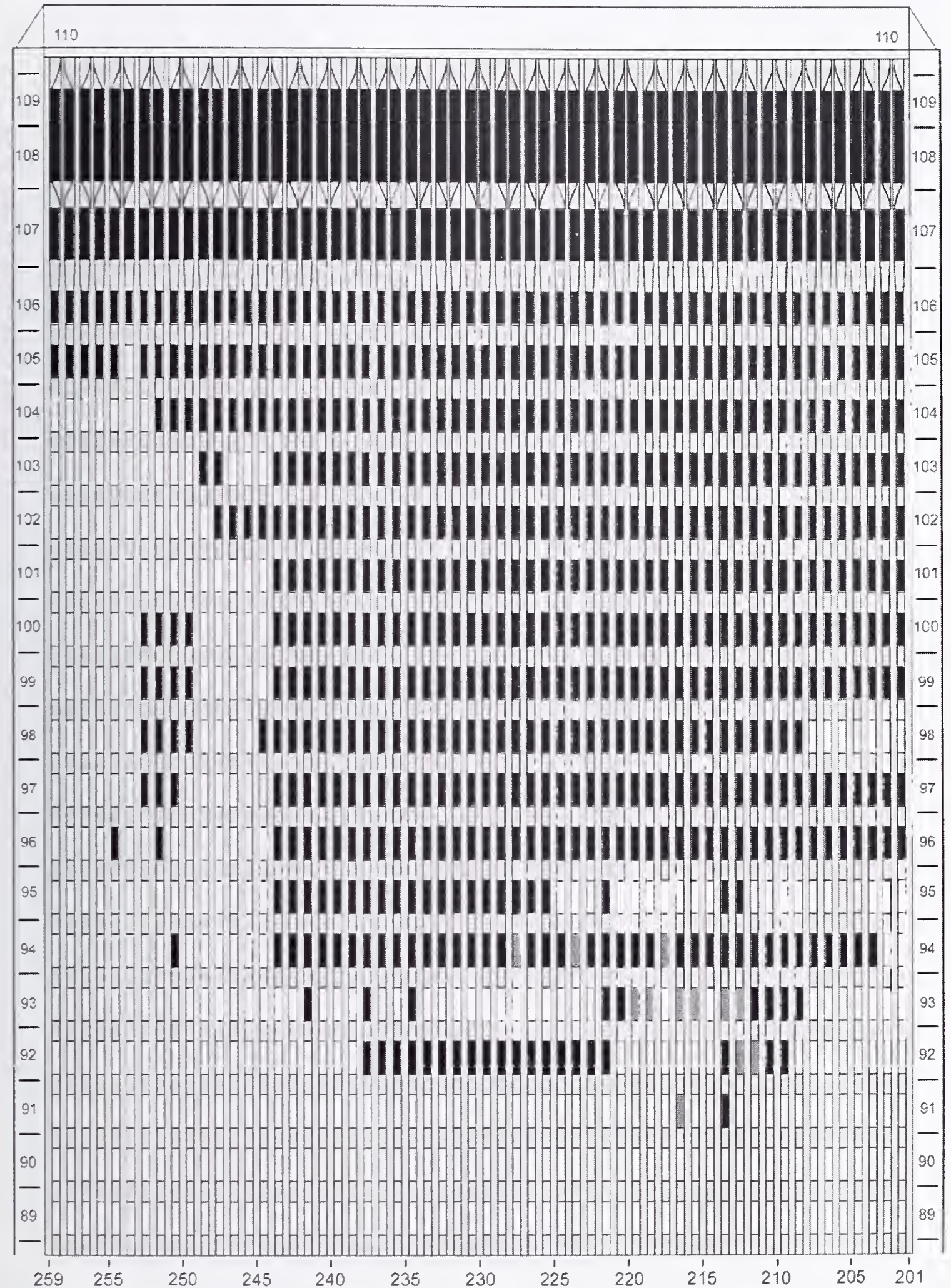

Figure D-22. Diagram of the east face of WTC 1 for floors 89 to 110 at 9:26 a.m. showing windows where smoke was observed and those that were hidden from view. 


$$
\text { WTC 1, East Face } \quad 9: 32 \text { a.m. }
$$

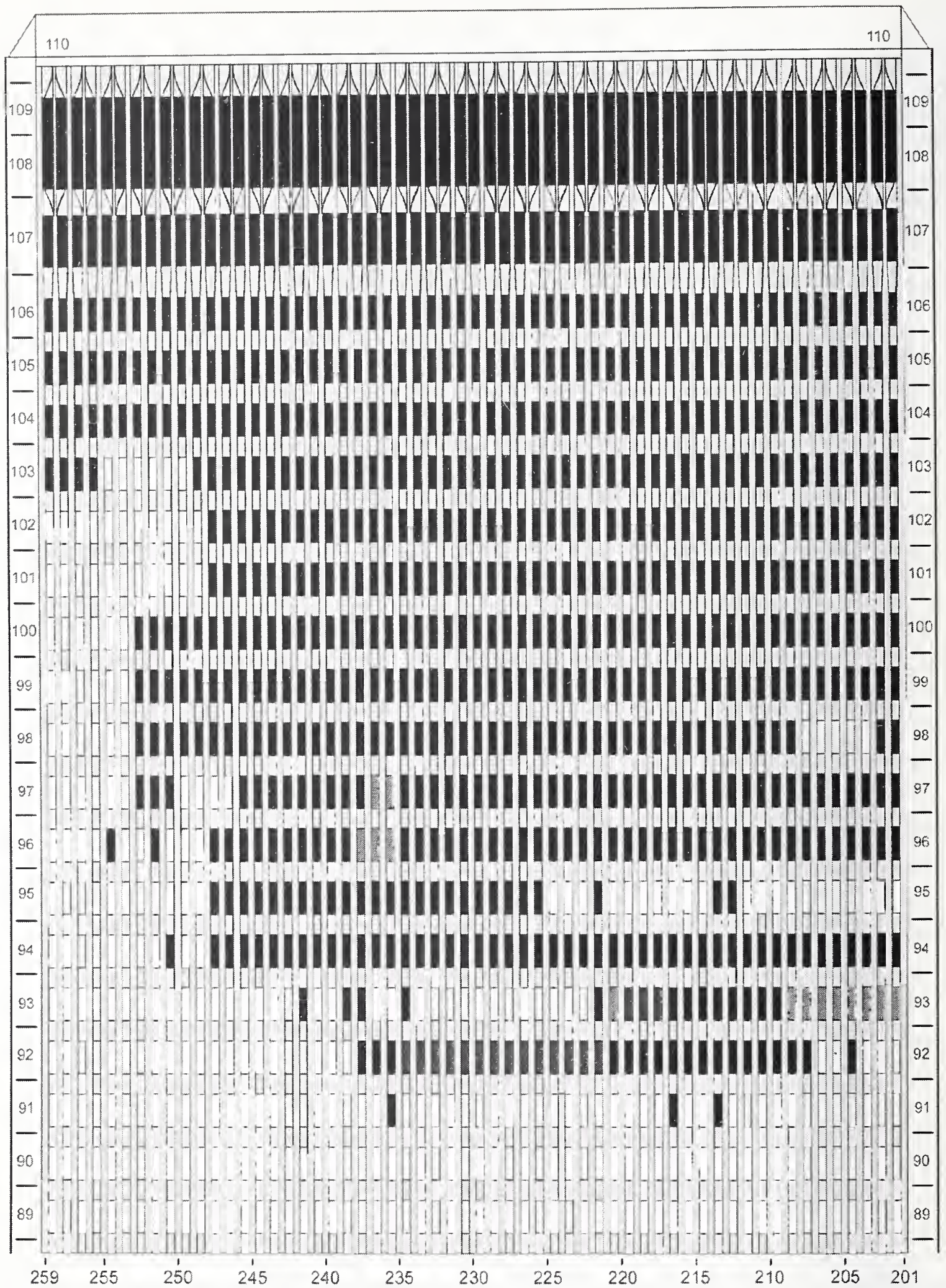

Figure D-23. Diagram of the east face of WTC 1 for floors 89 to 110 at 9:32 a.m. showing the condition of windows and locations of fires. 


$$
\text { WTC 1, East Face 9:32 a.m. }
$$

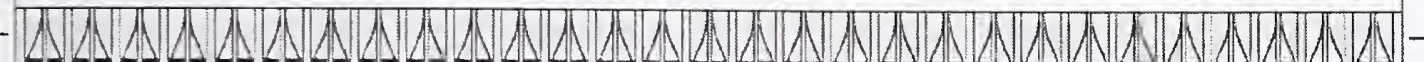
$\frac{109}{108}-\int$

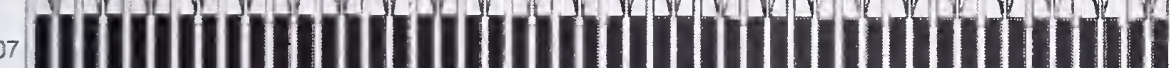

-

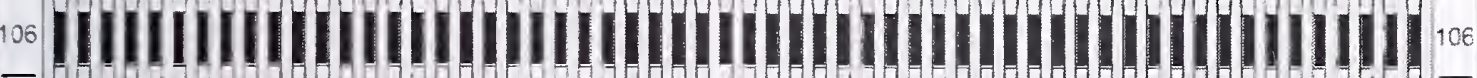

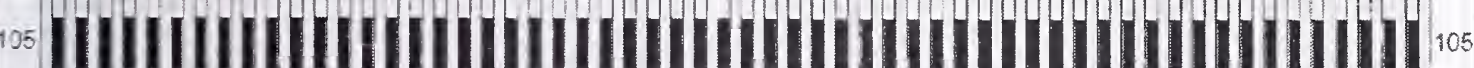

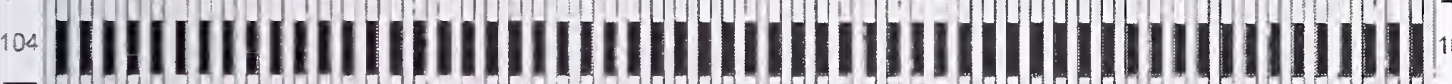

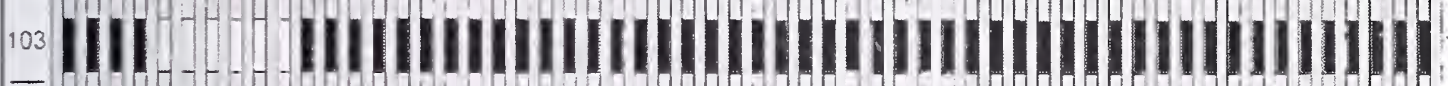
10. 10. |. -

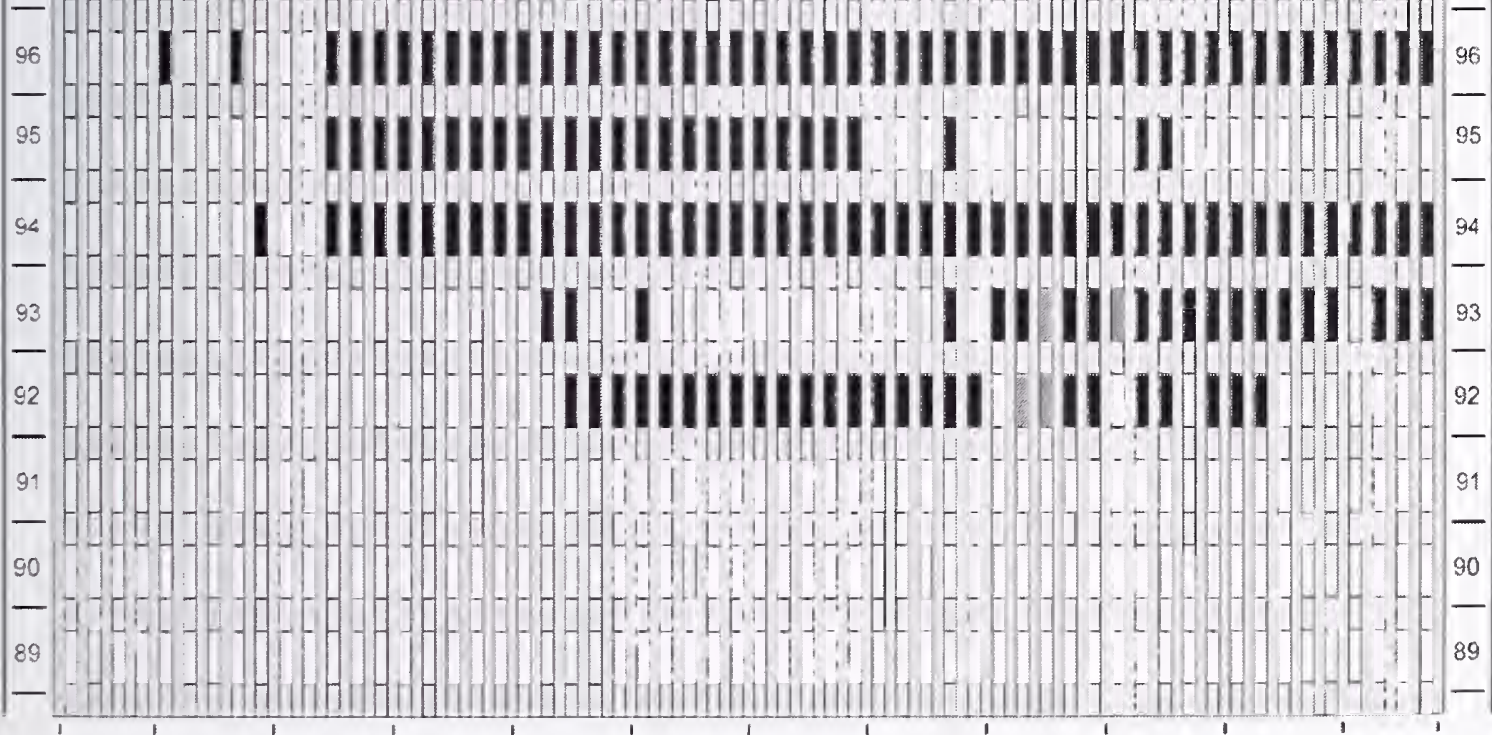

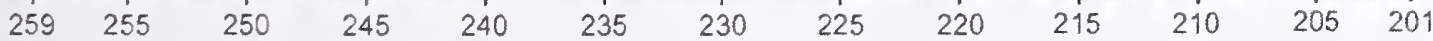

Figure D-24. Diagram of the east face of WTC 1 for floors 89 to 110 at 9:32 a.m. showing windows where smoke was observed and those that were hidden from view. 


$$
\text { WTC 1, East Face } \quad 9: 38 \text { a.m. }
$$

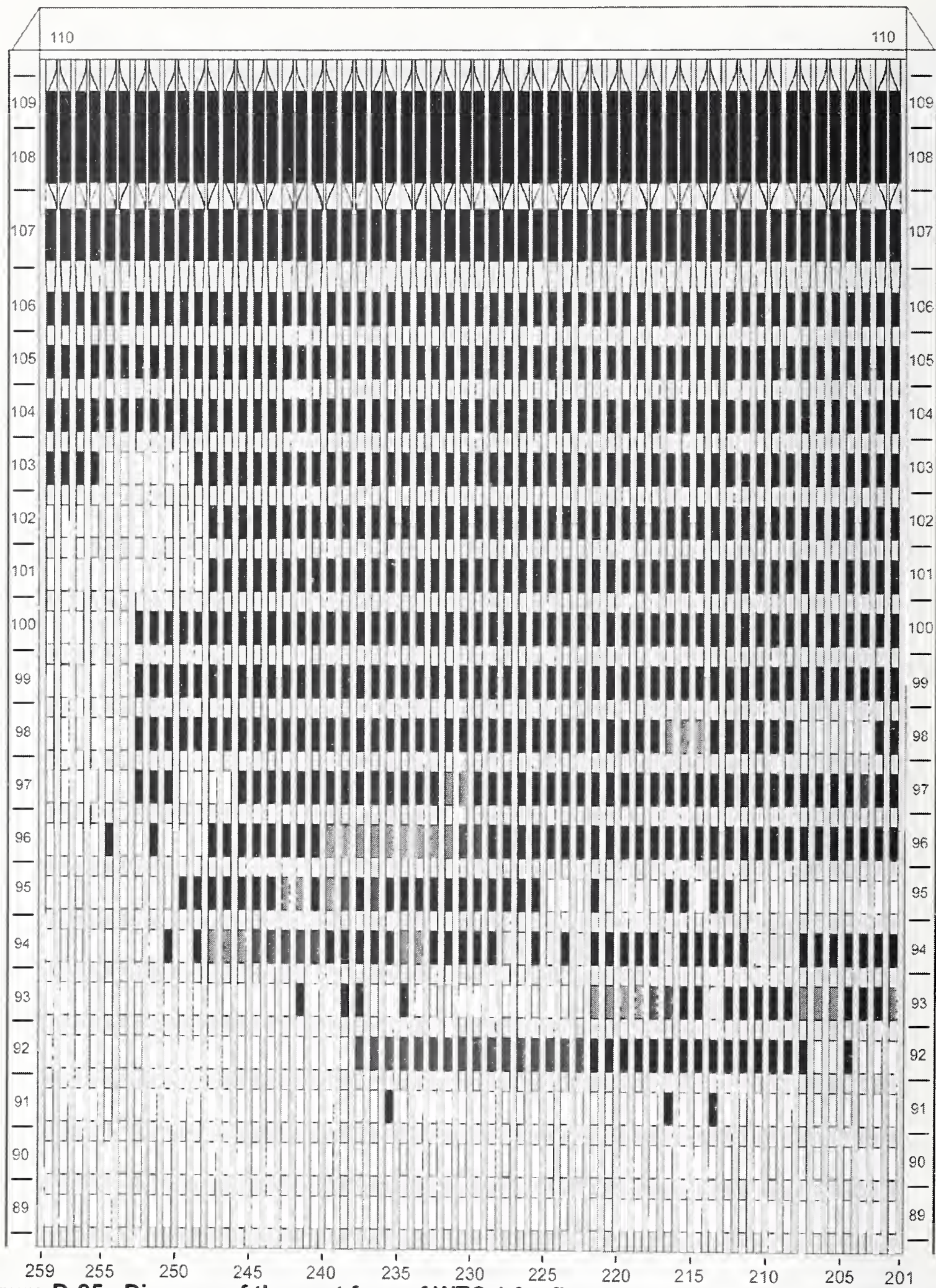

Figure D-25. Diagram of the east face of WTC 1 for floors 89 to 110 at 9:38 a.m. showing the condition of windows and locations of fires. 


$$
\text { WTC 1, East Face 9:38 a.m. }
$$

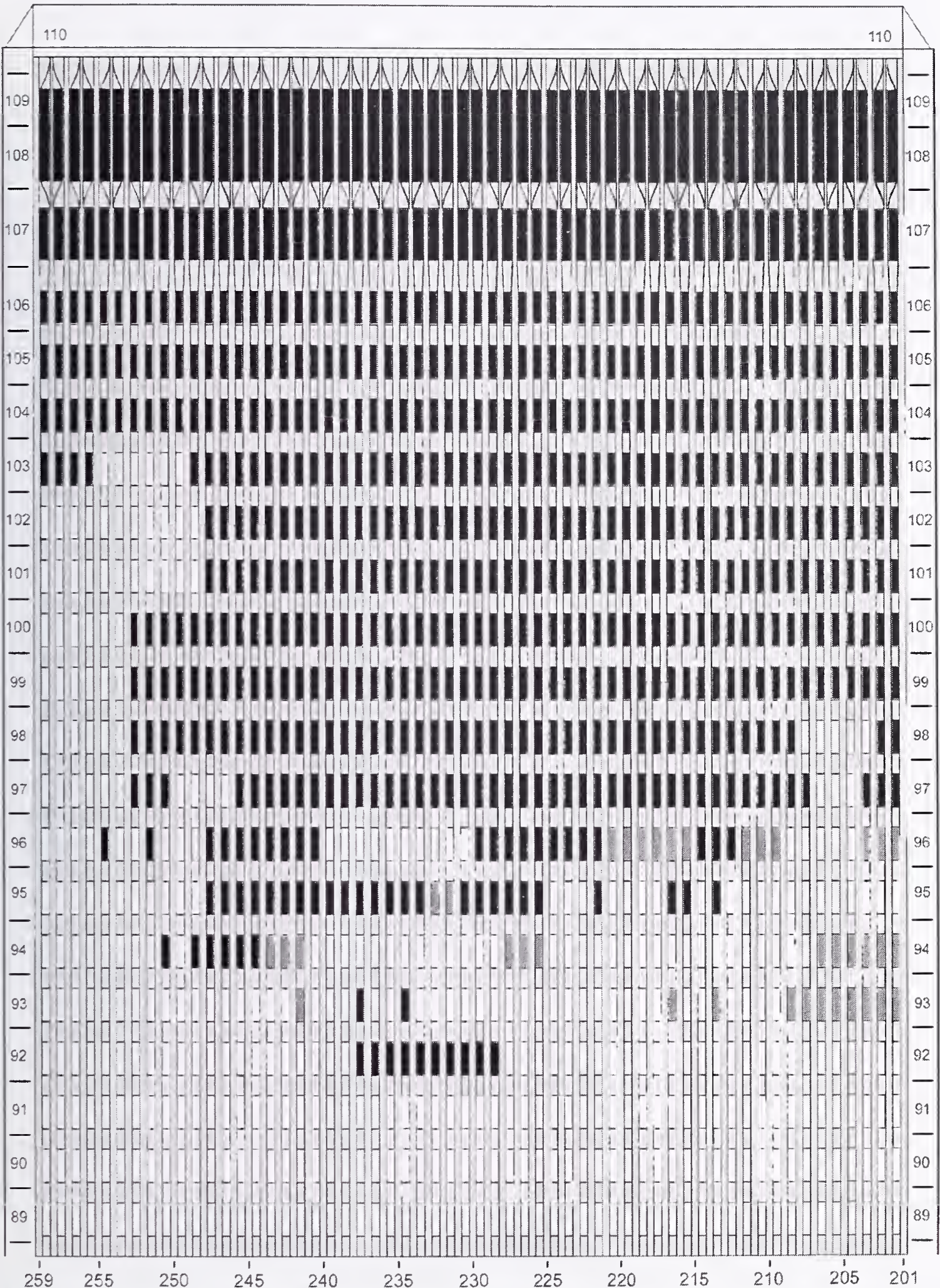

Figure D-26. Diagram of the east face of WTC 1 for floors 89 to 110 at 9:38 a.m. showing windows where smoke was observed and those that were hidden from view. 


$$
\text { WTC 1, East Face 9:42 a.m. }
$$

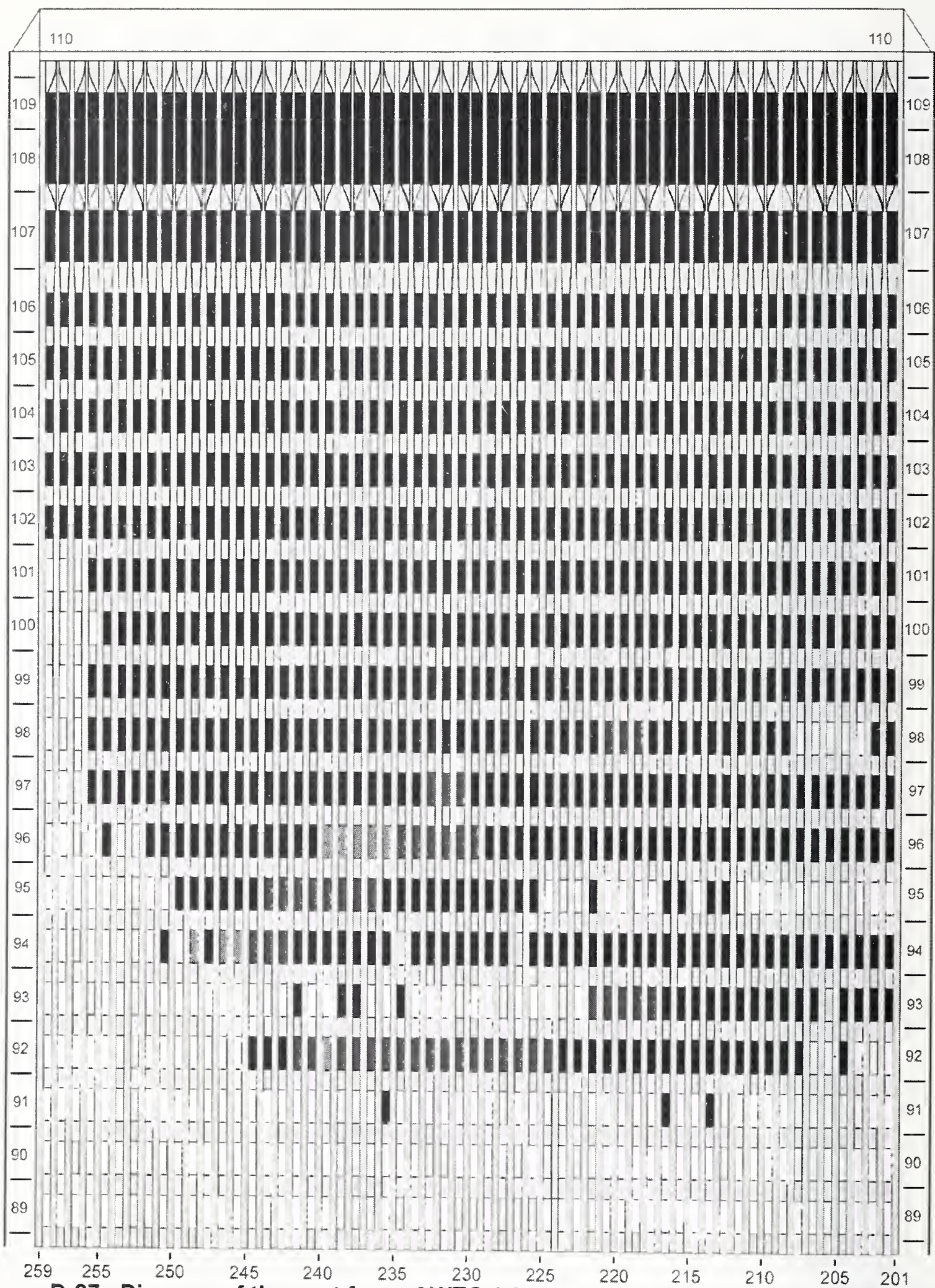

Figure D-27. Diagram of the east face of WTC 1 for floors 89 to 110 at 9:42 a.m. showing the condition of windows and locations of fires. 


$$
\text { WTC 1, East Face 9:42 a.m. }
$$

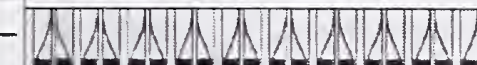

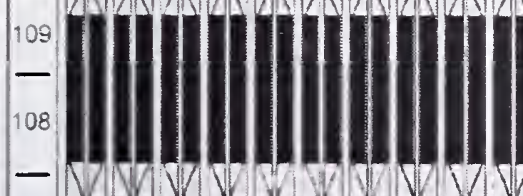

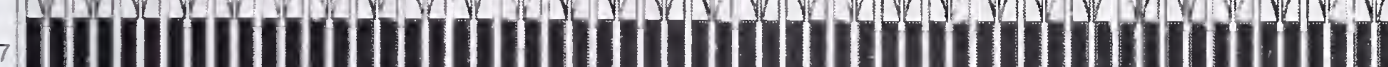
-

|

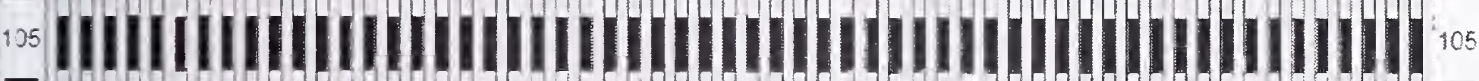

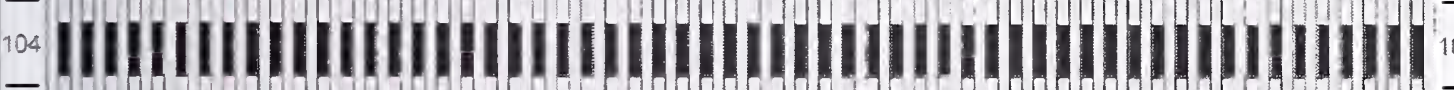

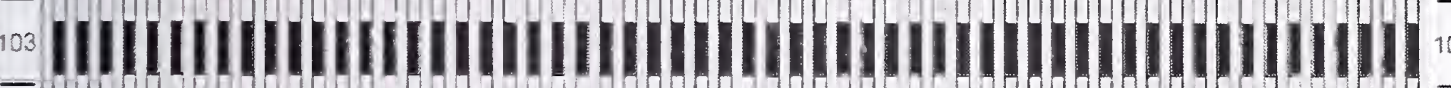

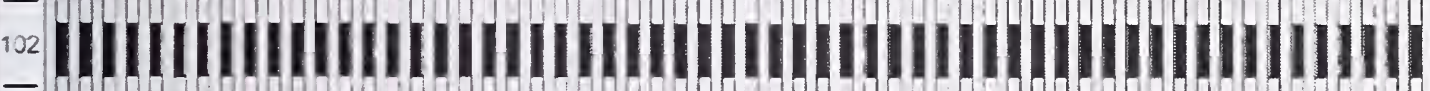
-

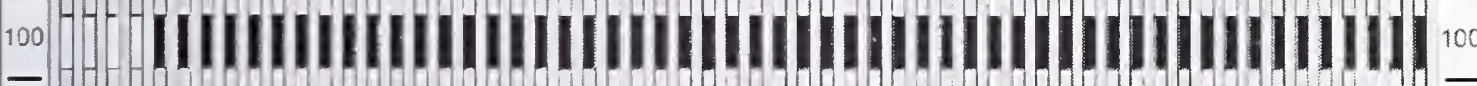

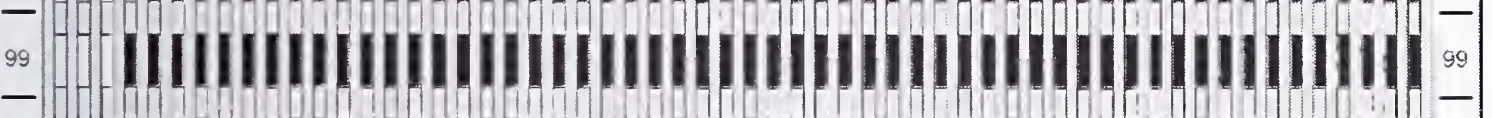

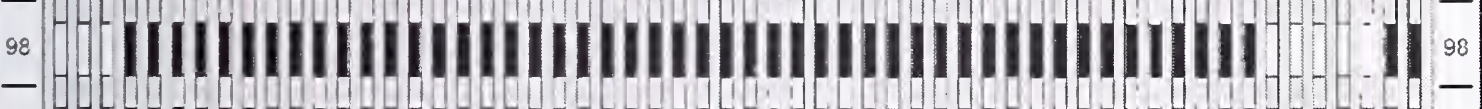

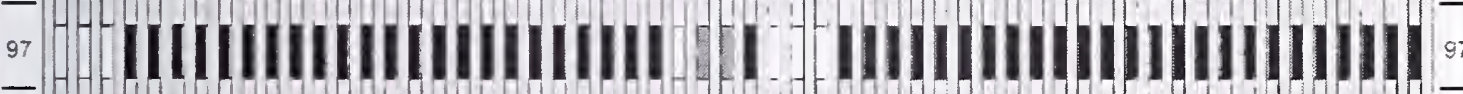

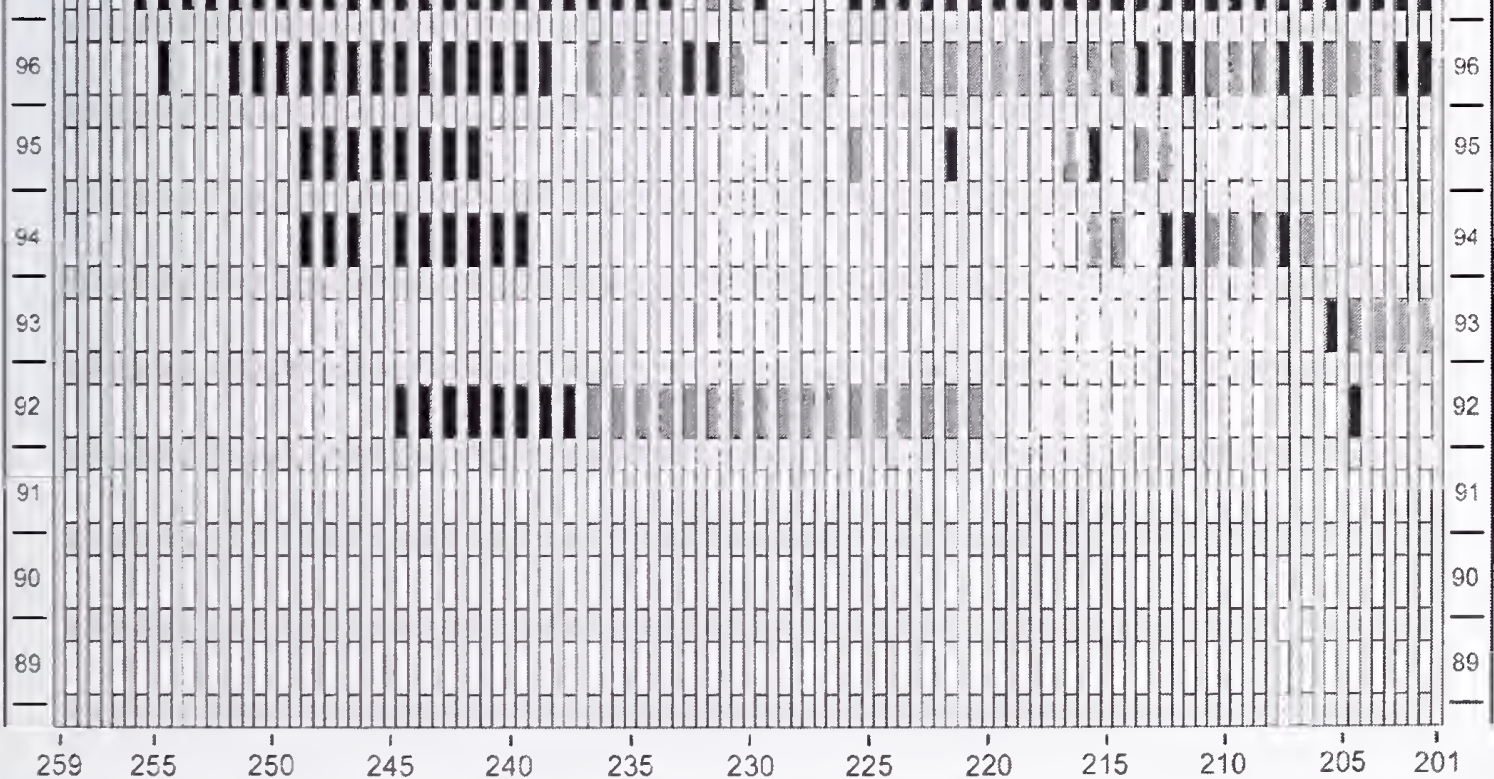

Figure D-28. Diagram of the east face of WTC 1 for floors 89 to 110 at 9:42 a.m. showing windows where smoke was observed and those that were hidden from view. 
WTC 1, East Face $\quad$ 9:46 a.m.

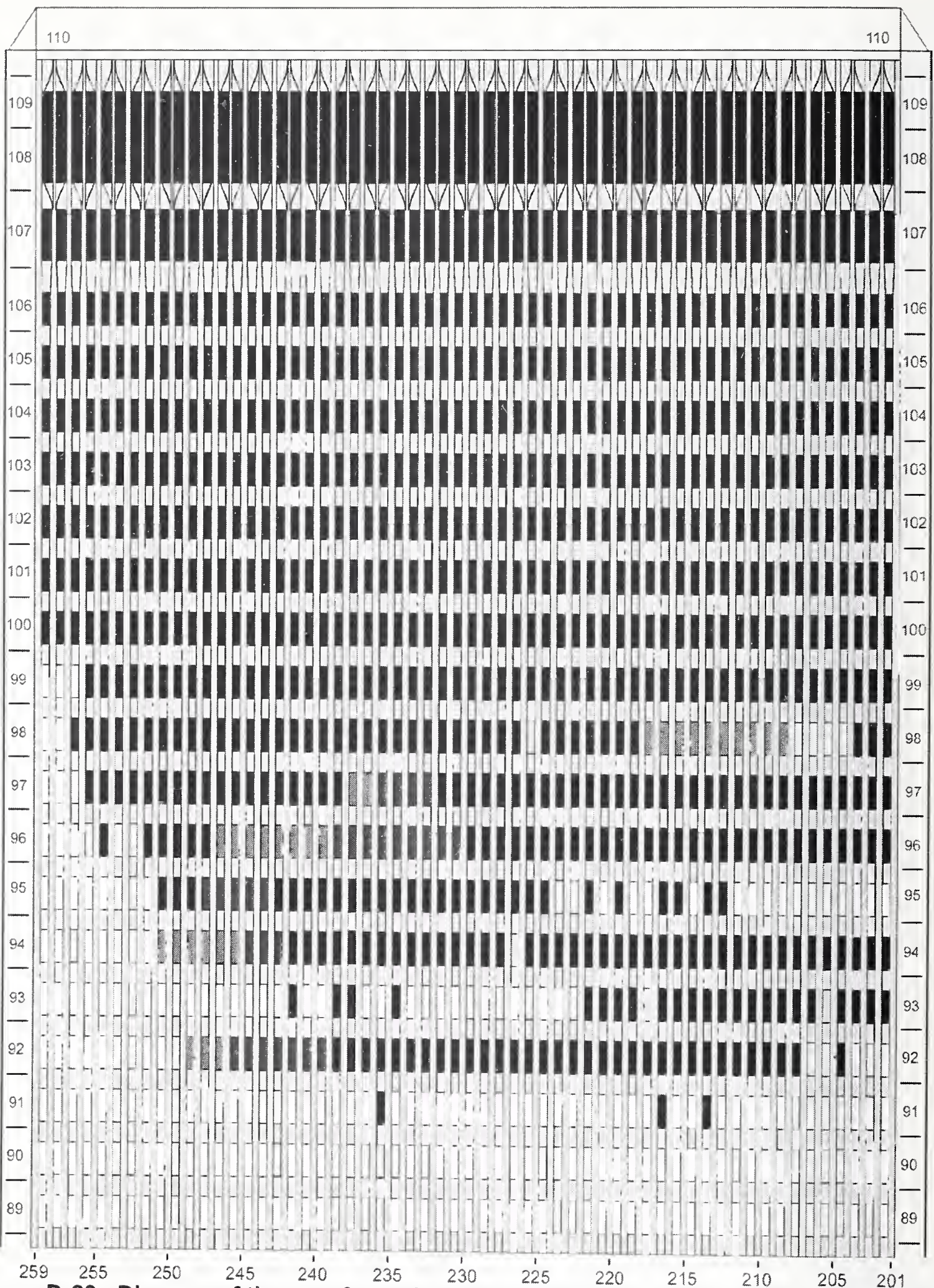

Figure D-29. Diagram of the east face of WTC 1 for floors 89 to 110 at 9:46 a.m. showing the condition of windows and locations of fires. 


$$
\text { WTC 1, East Face 9:46 a.m. }
$$

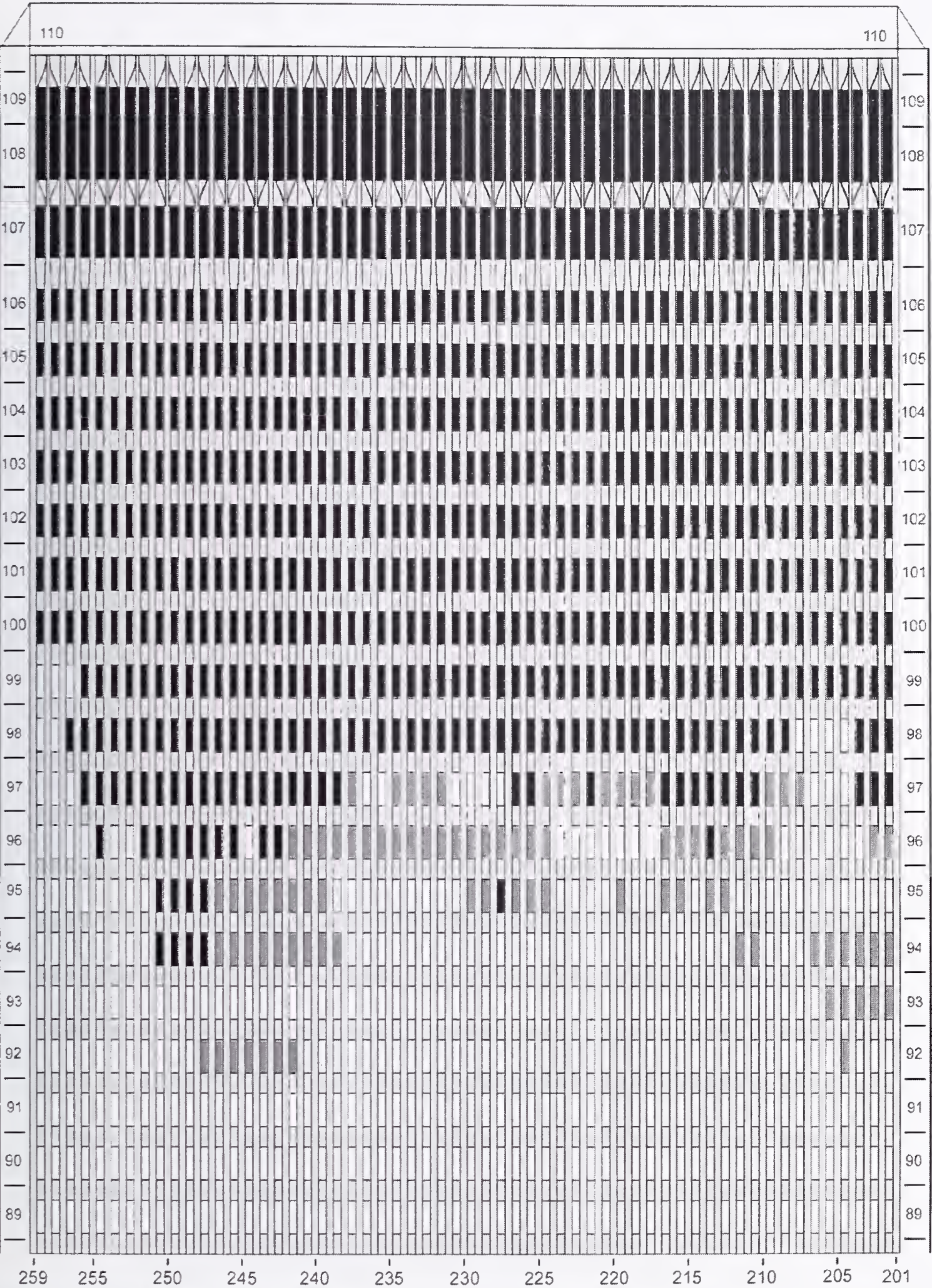

Figure D-30. Diagram of the east face of WTC 1 for floors 89 to 110 at 9:46 a.m. showing windows where smoke was observed and those that were hidden from view. 
WTC 1, East Face

9:50 a.m.

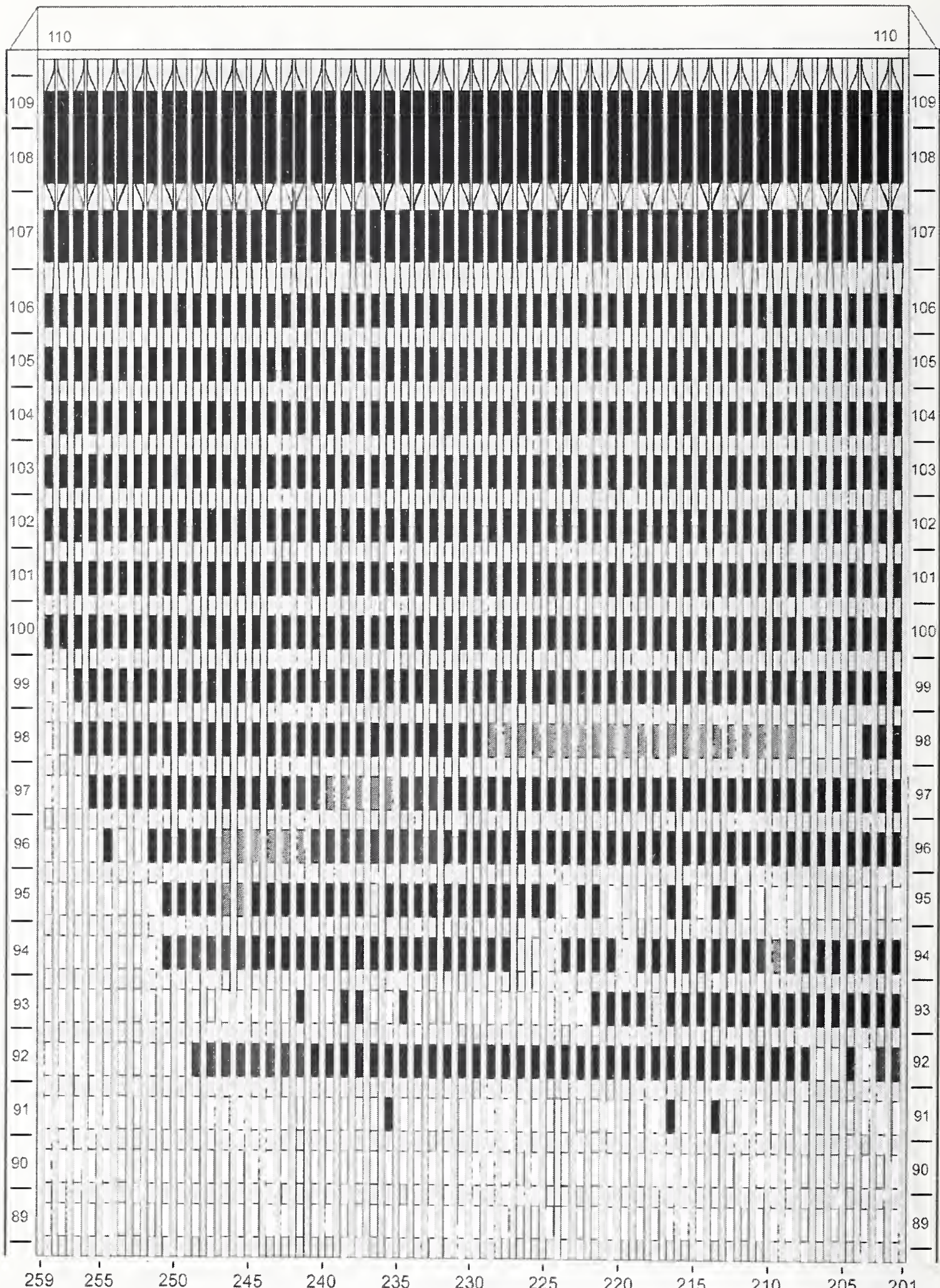

Figure D-31. Diagram of the east face of WTC 1 for floors 89 to 110 at 9:50 a.m. showing the condition of windows and locations of fires. 


$$
\text { WTC 1, East Face } \quad 9: 50 \text { a.m. }
$$

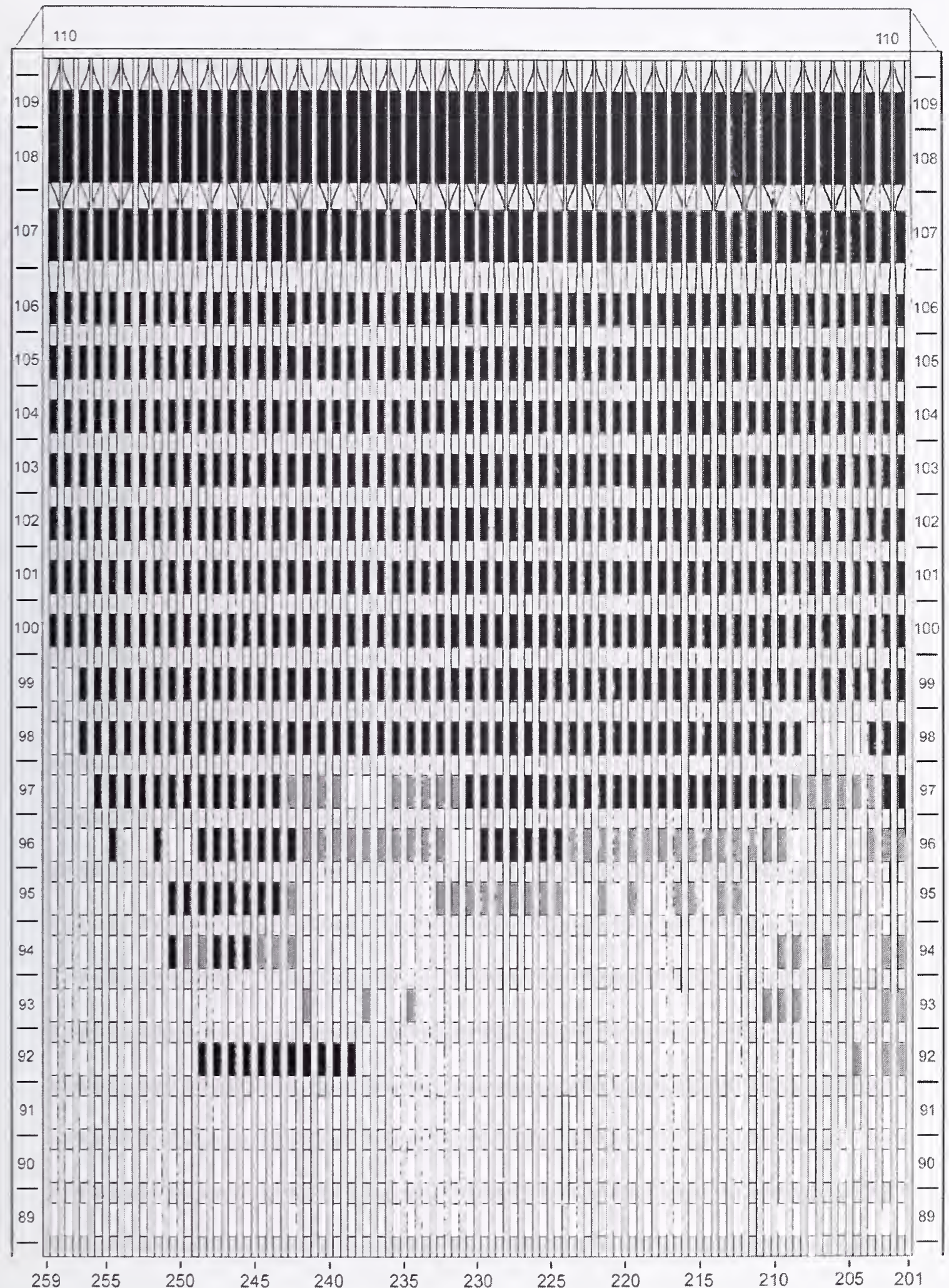

Figure D-32. Diagram of the east face of WTC 1 for floors 89 to 110 at 9:50 a.m. showing windows where smoke was observed and those that were hidden from view. 


$$
\text { WTC 1, East Face 9:54 a.m. }
$$

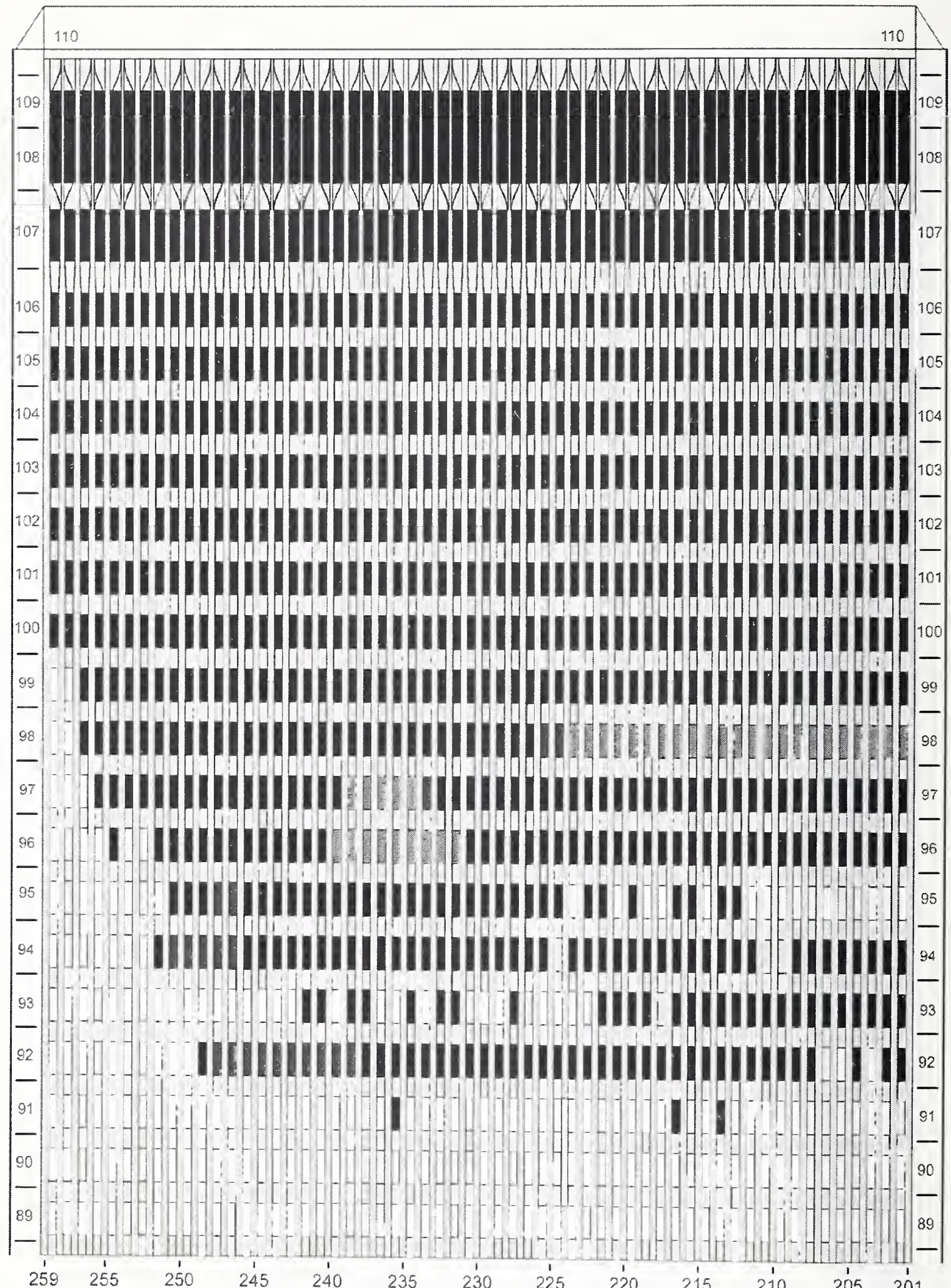

Figure D-33. Diagram of the east face of WTC 1 for floors 89 to 110 at 9:54 a.m. showing the condition of windows and locations of fires. 


$$
\text { WTC 1, East Face 9:54 a.m. }
$$

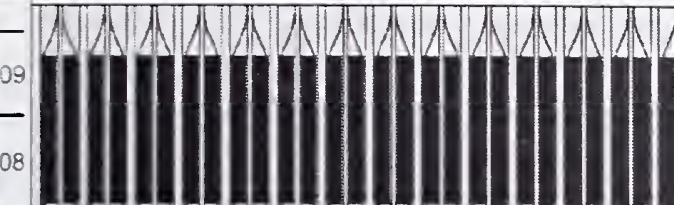

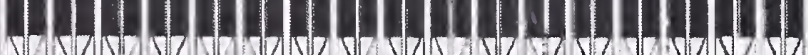
| ||||||||||||||||||||||||||||||||||||||||||||||||||||||||||

-

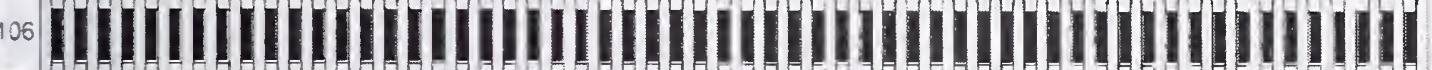

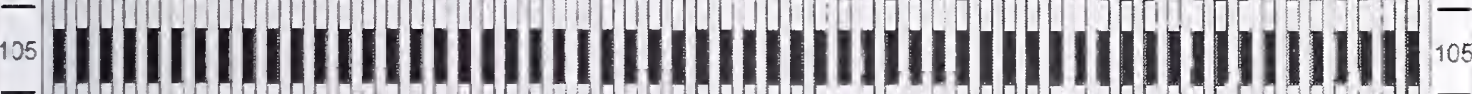

-

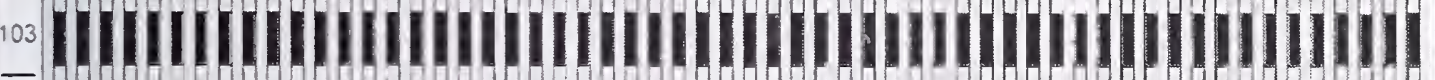

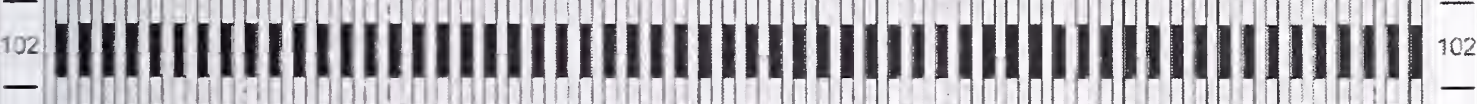

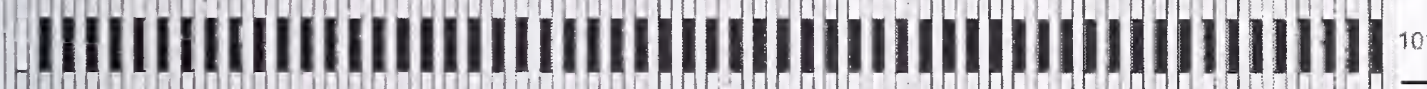

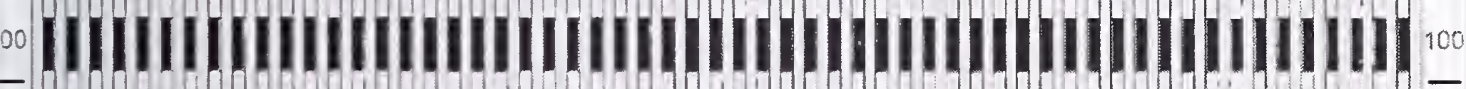

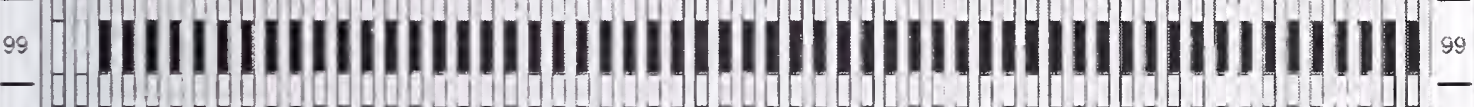

-

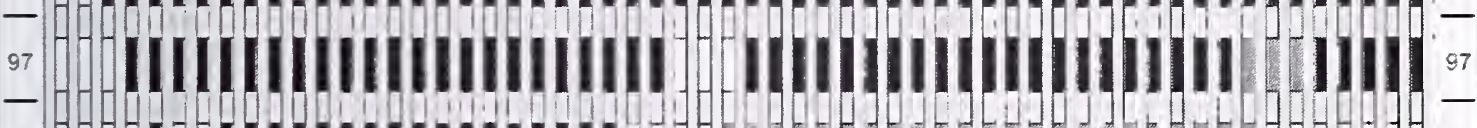

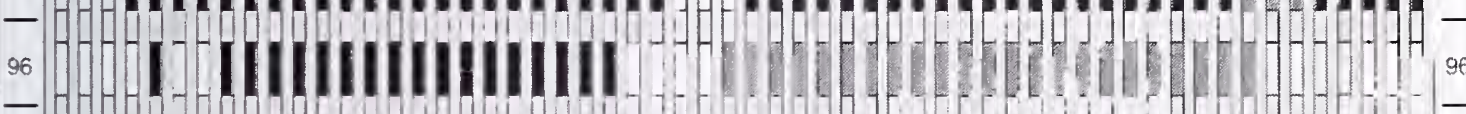

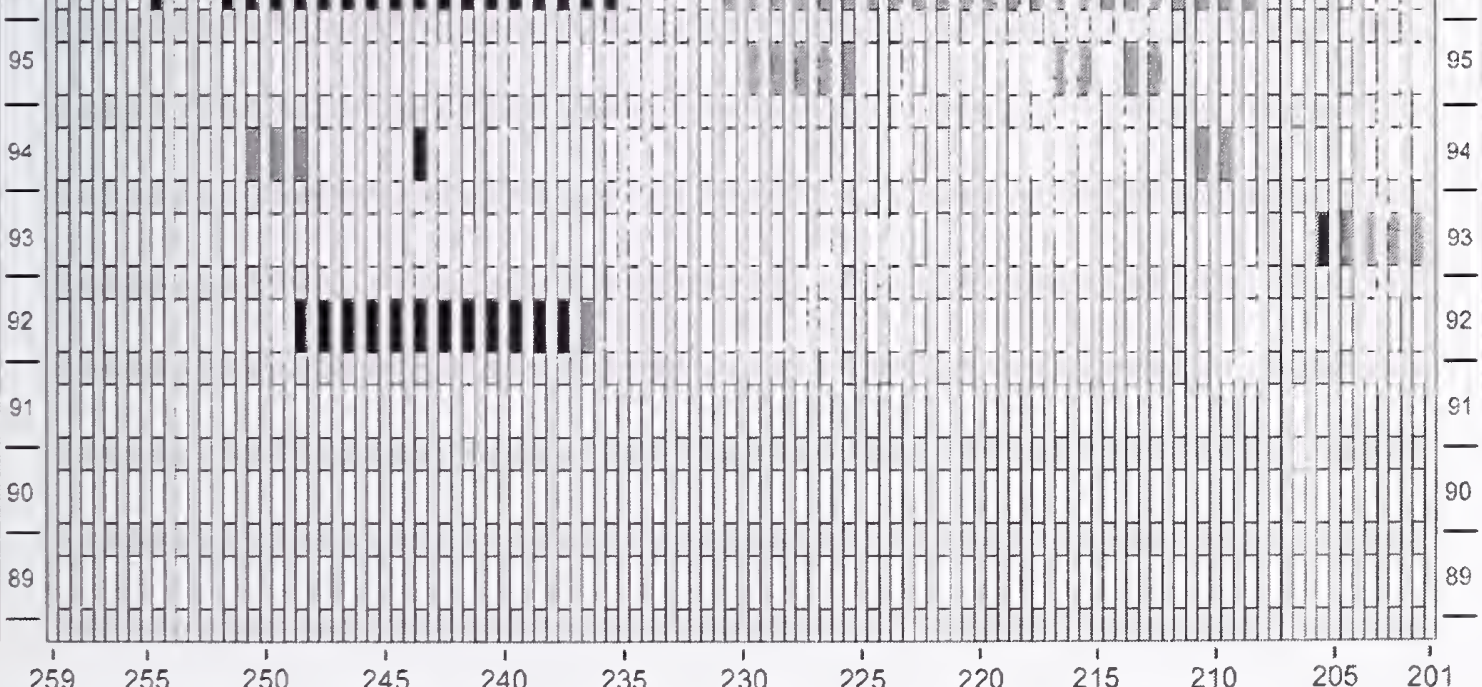

Figure D-34. Diagram of the east face of WTC 1 for floors 89 to 110 at 9:54 a.m. showing windows where smoke was observed and those that were hidden from view. 


$$
\text { WTC 1, East Face } \quad 9: 56 \text { a.m. }
$$



Figure D-35. Diagram of the east face of WTC 1 for floors 89 to 110 at 9:56 a.m. showing the condition of windows and locations of fires. 


$$
\text { WTC 1, East Face 9:56 a.m. }
$$

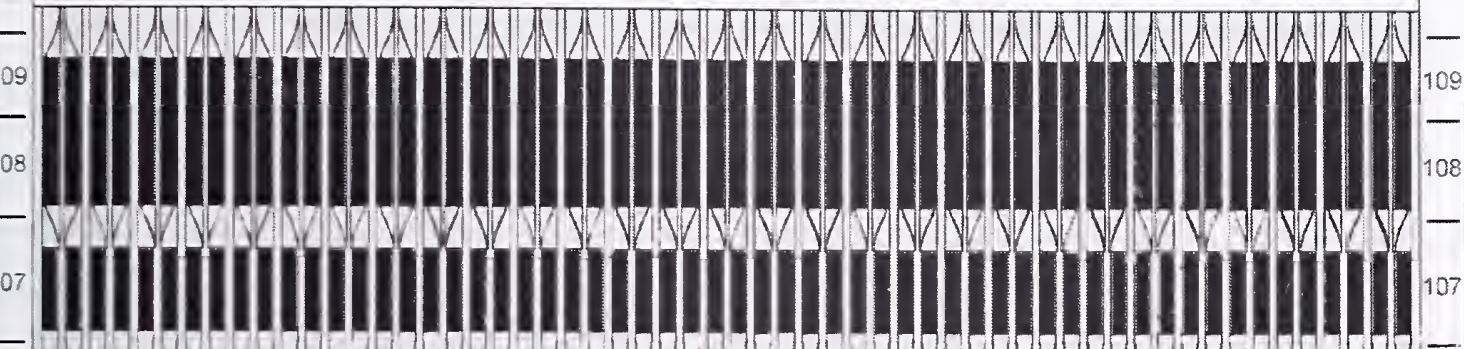

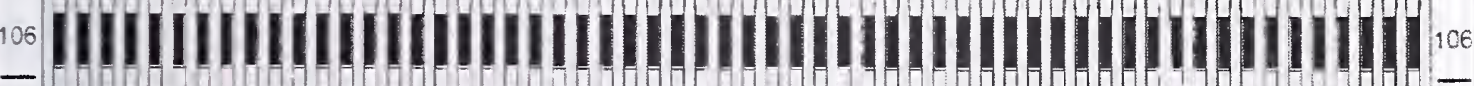

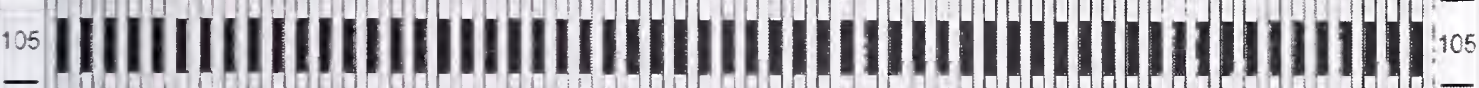

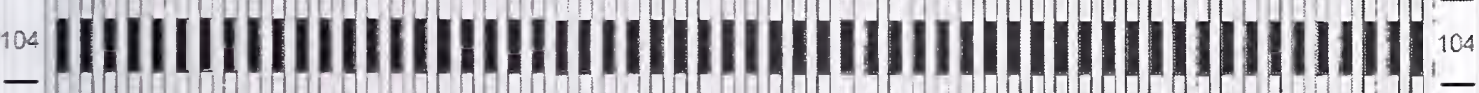

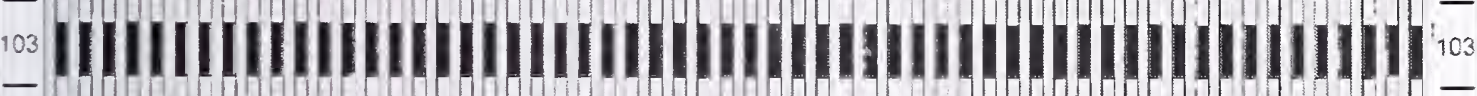

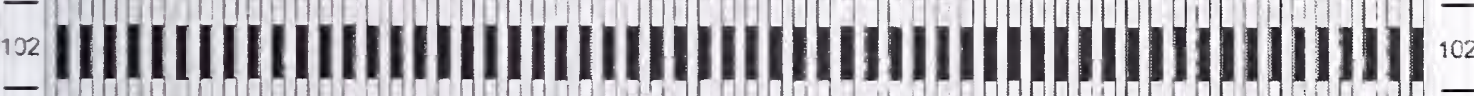

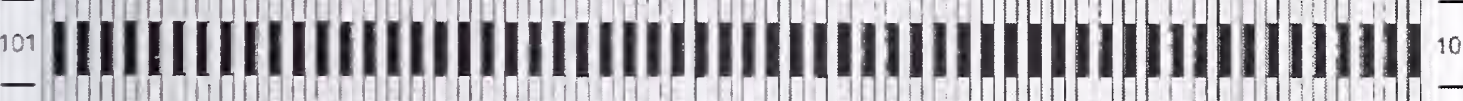

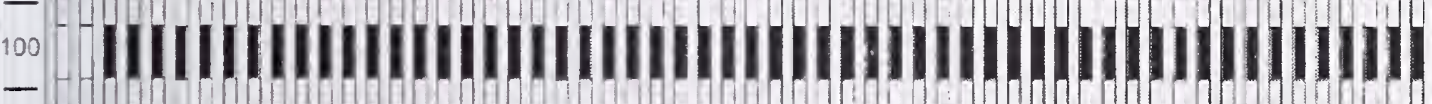

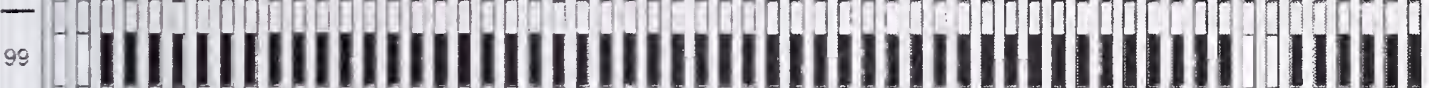

-

|

-

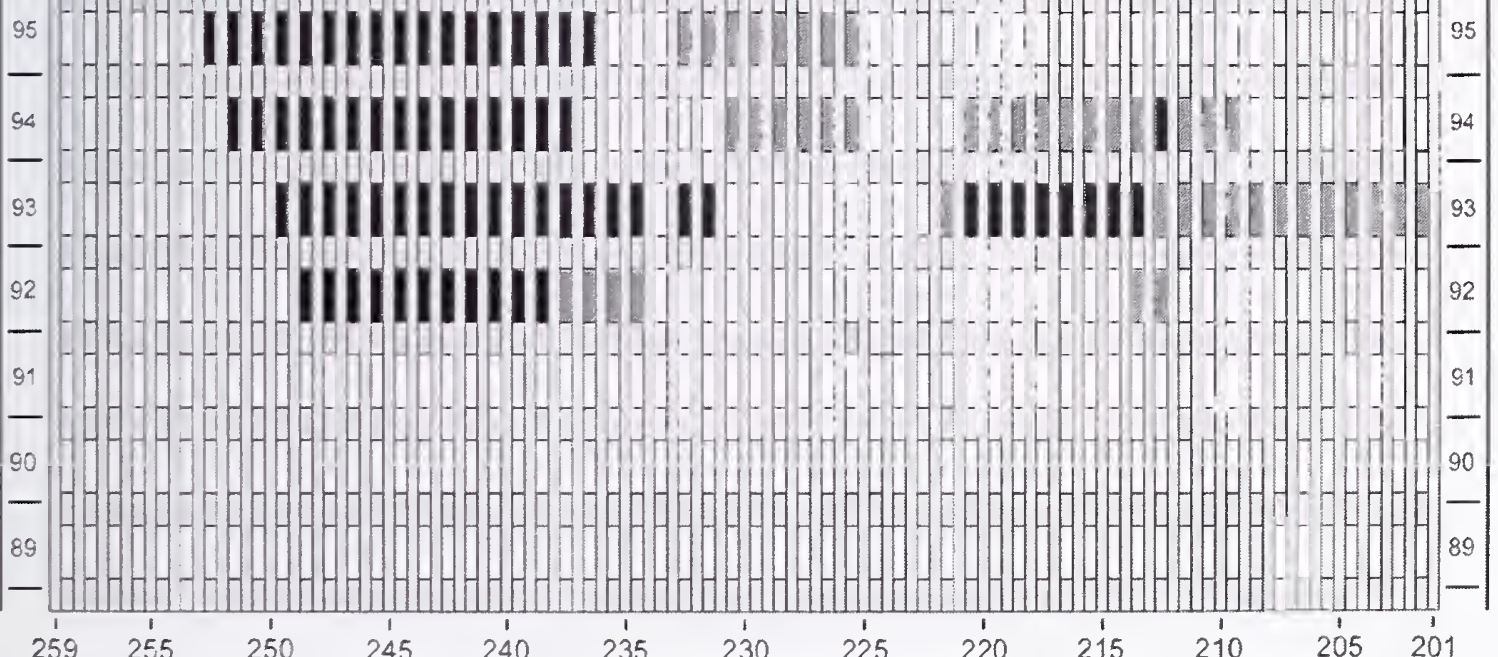

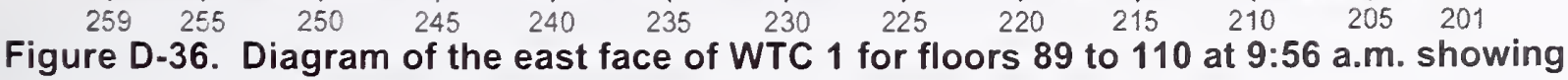
windows where smoke was observed and those that were hidden from view. 


$$
\text { WTC 1, East Face } \quad 9: 58 \text { a.m. }
$$

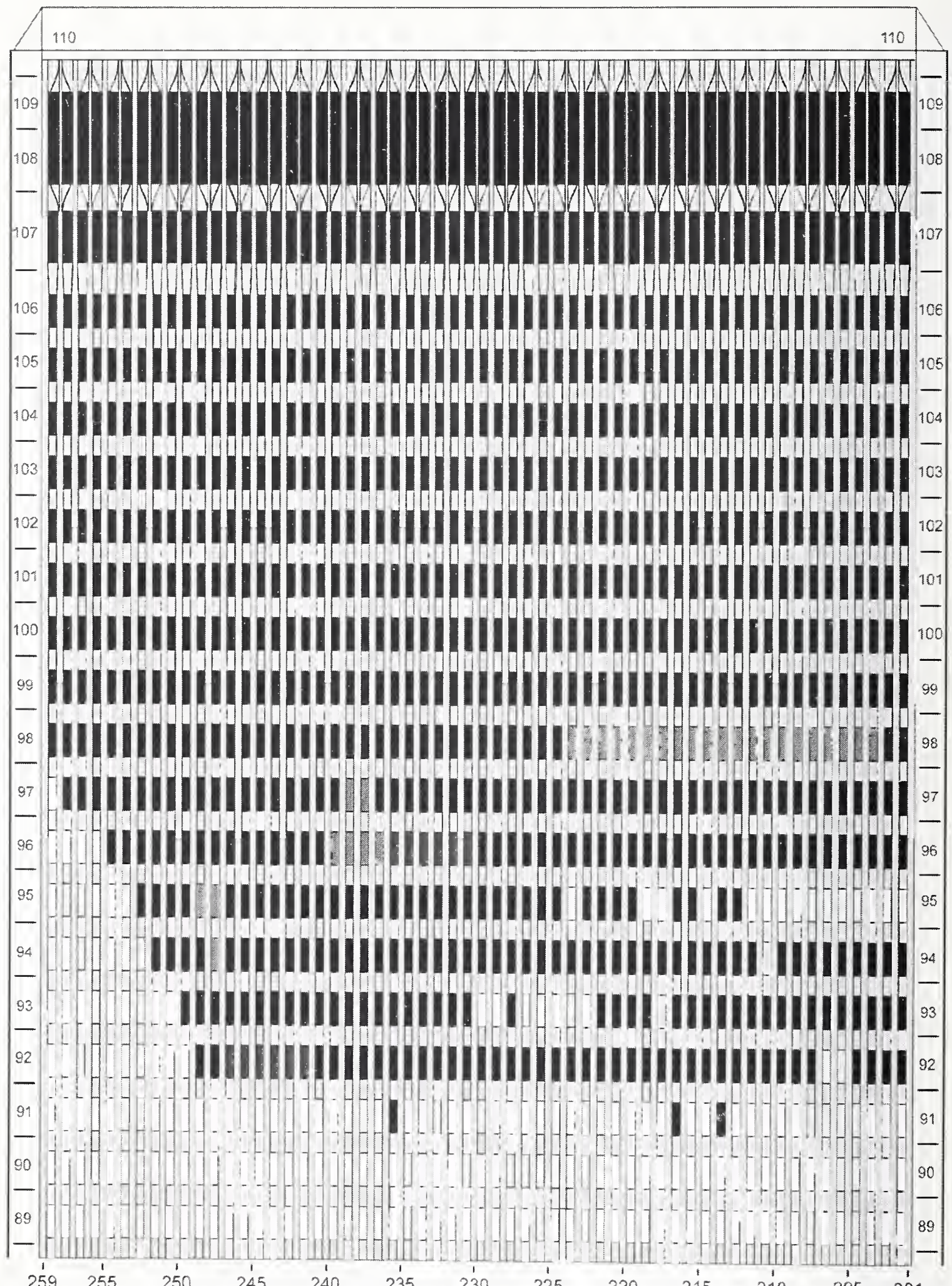

Figure D-37. Diagram of the east face of WTC 1 for floors 89 to 110 at 9:58 a.m. showing the condition of windows and locations of fires. 


$$
\text { WTC 1, East Face 9:58 a.m. }
$$

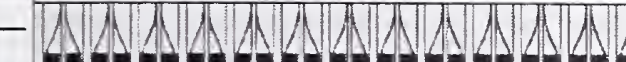

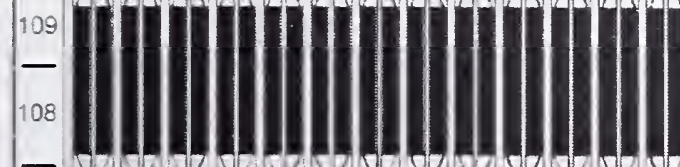

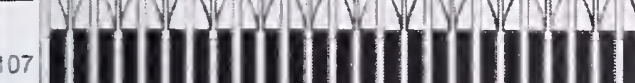

107

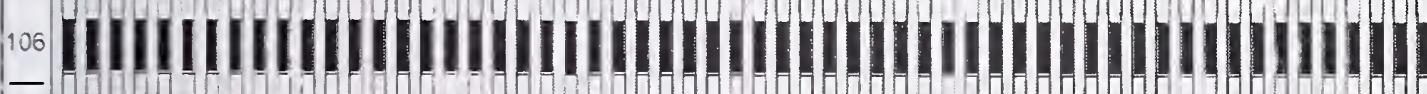

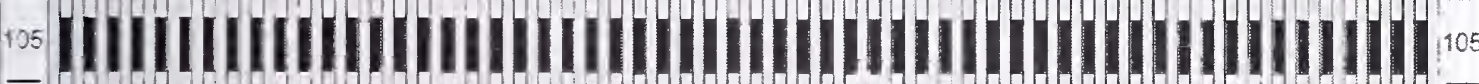

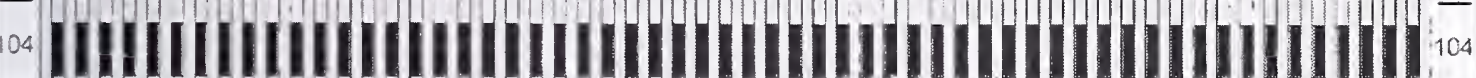

-

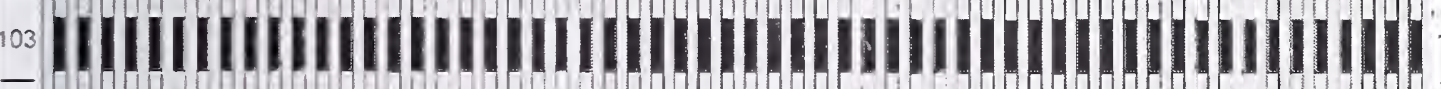

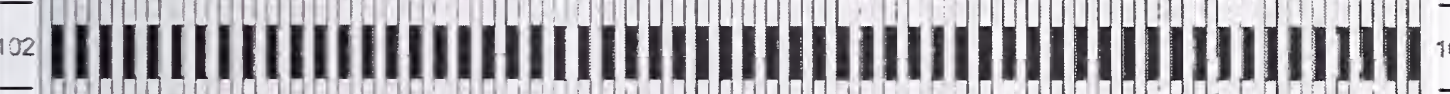

-

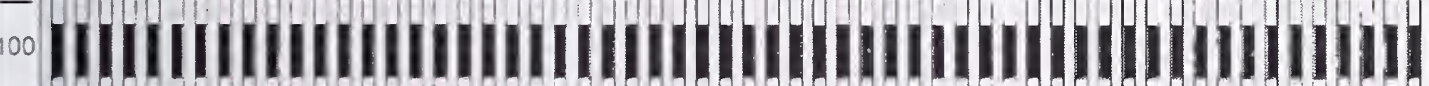

$-$

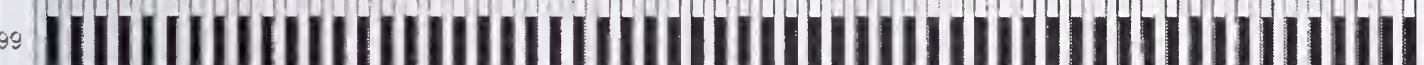

-

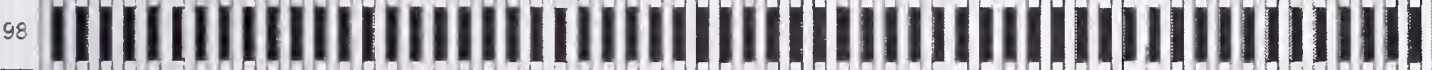

97 IIIIIIIIIIIIIIIIIIIIIIIIIIIIIIIIIIIIIIIIIIIIIIIIIIIIII

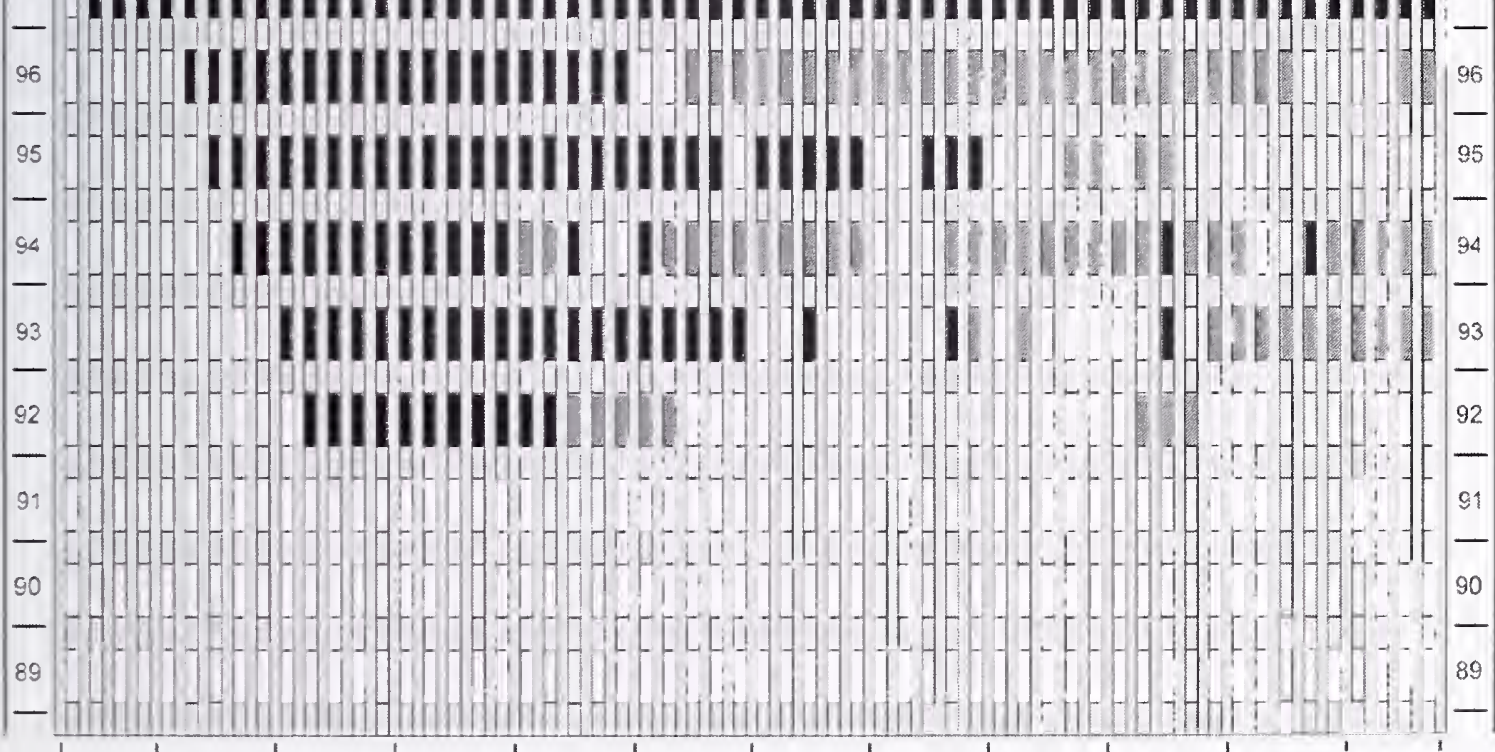

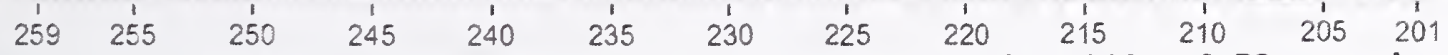

Figure D-38. Diagram of the east face of WTC 1 for floors 89 to 110 at 9:58 a.m. showing windows where smoke was observed and those that were hidden from view. 


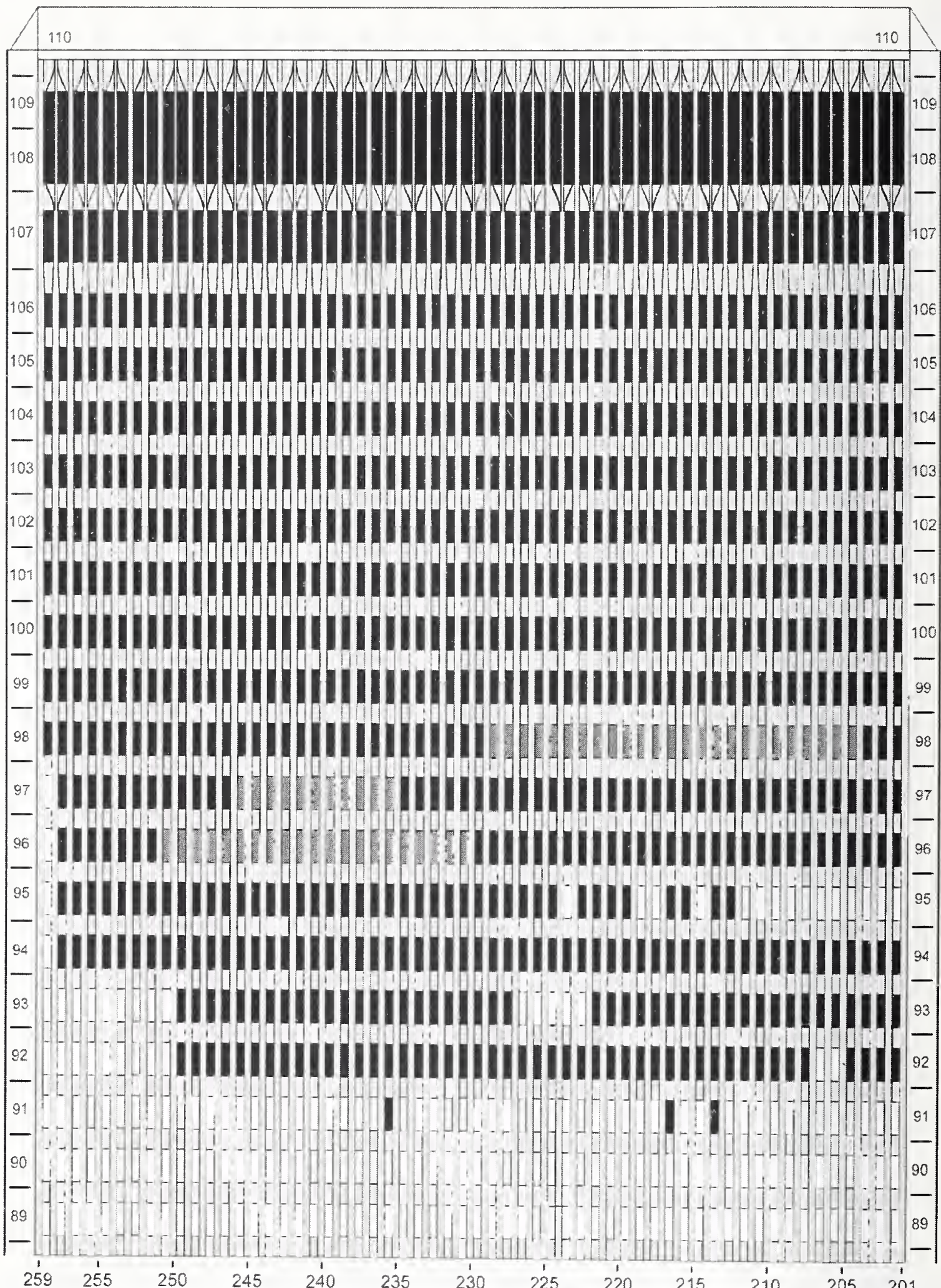

Figure D-39. Diagram of the east face of WTC 1 for floors 89 to 110 at 10:00 a.m. showing the condition of windows and locations of fires. 


$$
\text { WTC 1, East Face } \quad \text { 10:00 a.m. }
$$

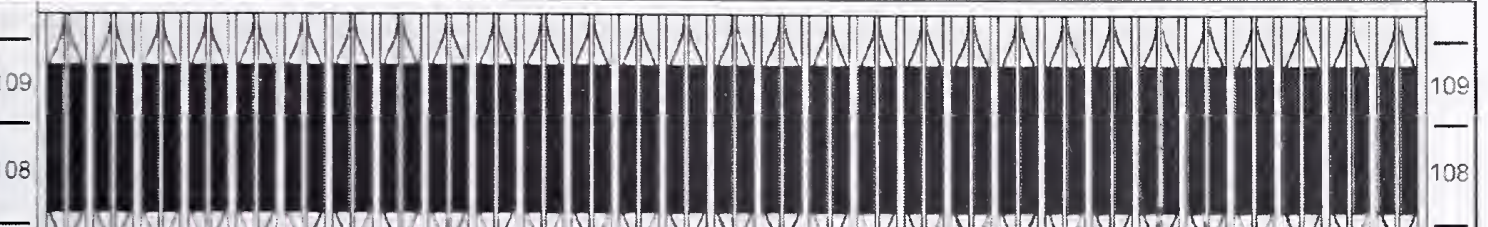

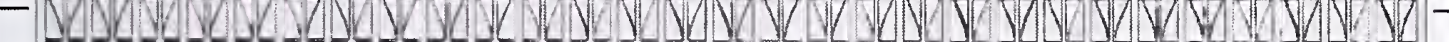

107

- mis

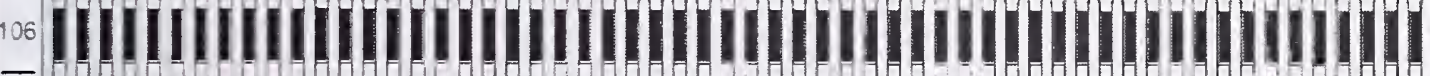

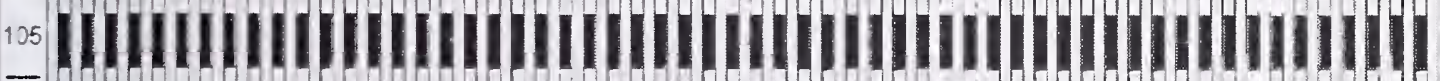

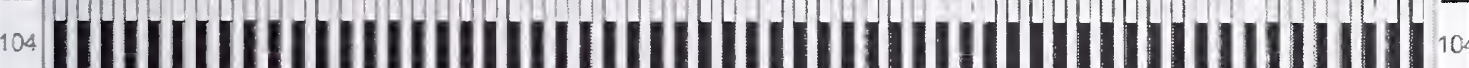
-

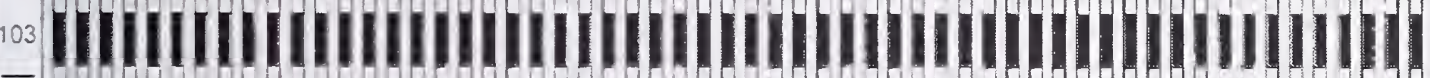

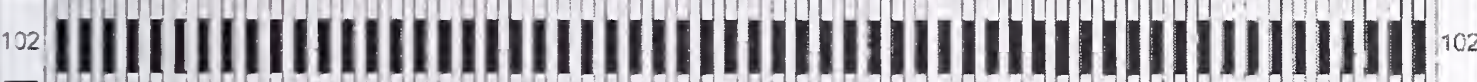

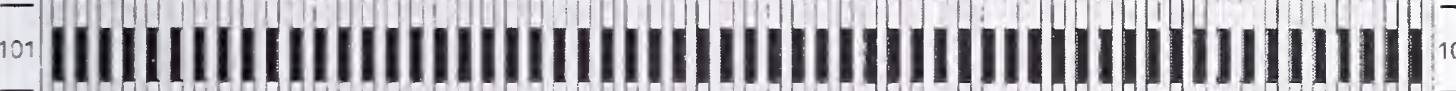

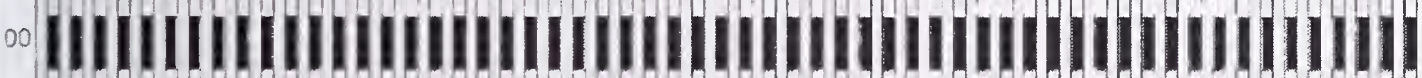

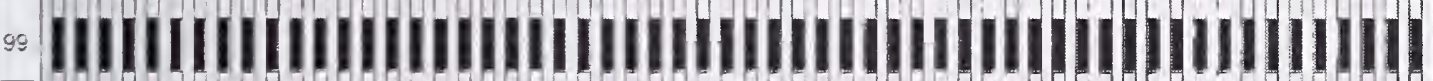

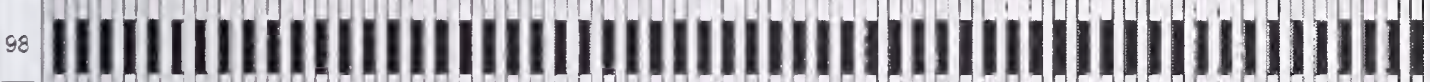

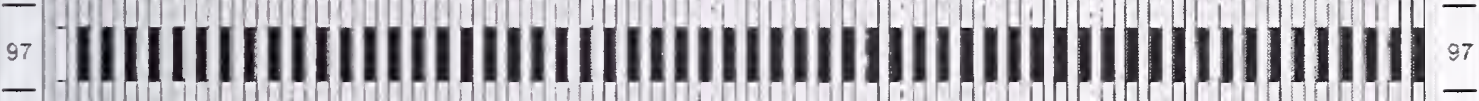

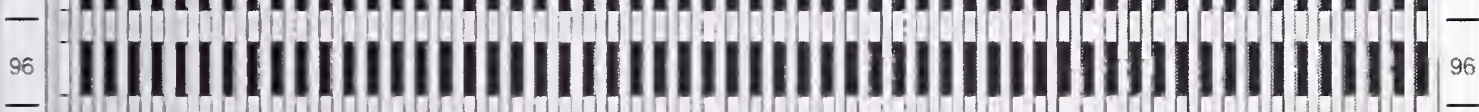

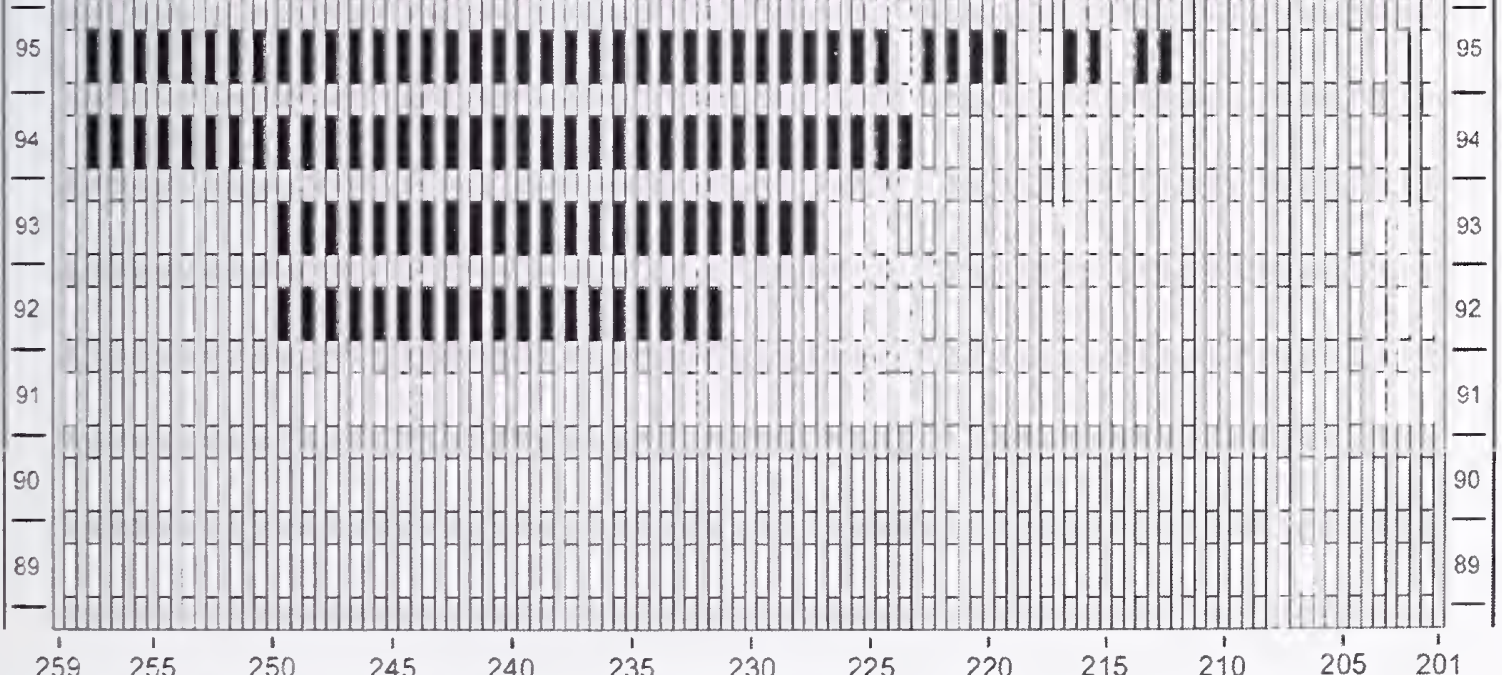

Figure D-40. Diagram of the east face of WTC 1 for floors 89 to 110 at 10:00 a.m. showing windows where smoke was observed and those that were hidden from view. 


$$
\text { WTC 1, East Face } \quad \text { 10:06 a.m. }
$$

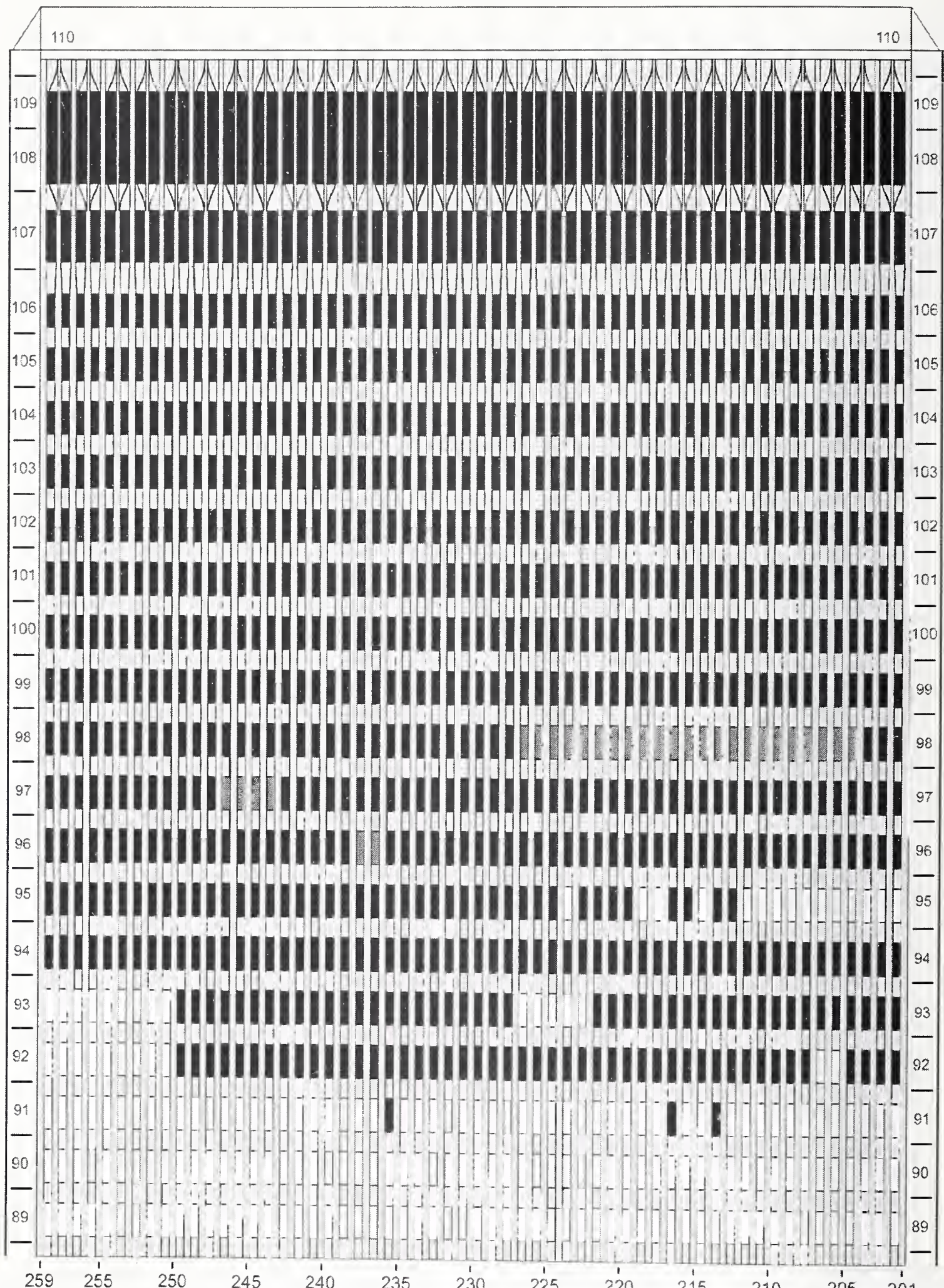

Figure D-41. Diagram of the east face of WTC 1 for floors 89 to 110 at 10:06 a.m. showing the condition of windows and locations of fires. 


$$
\text { WTC 1, East Face 10:06 a.m. }
$$

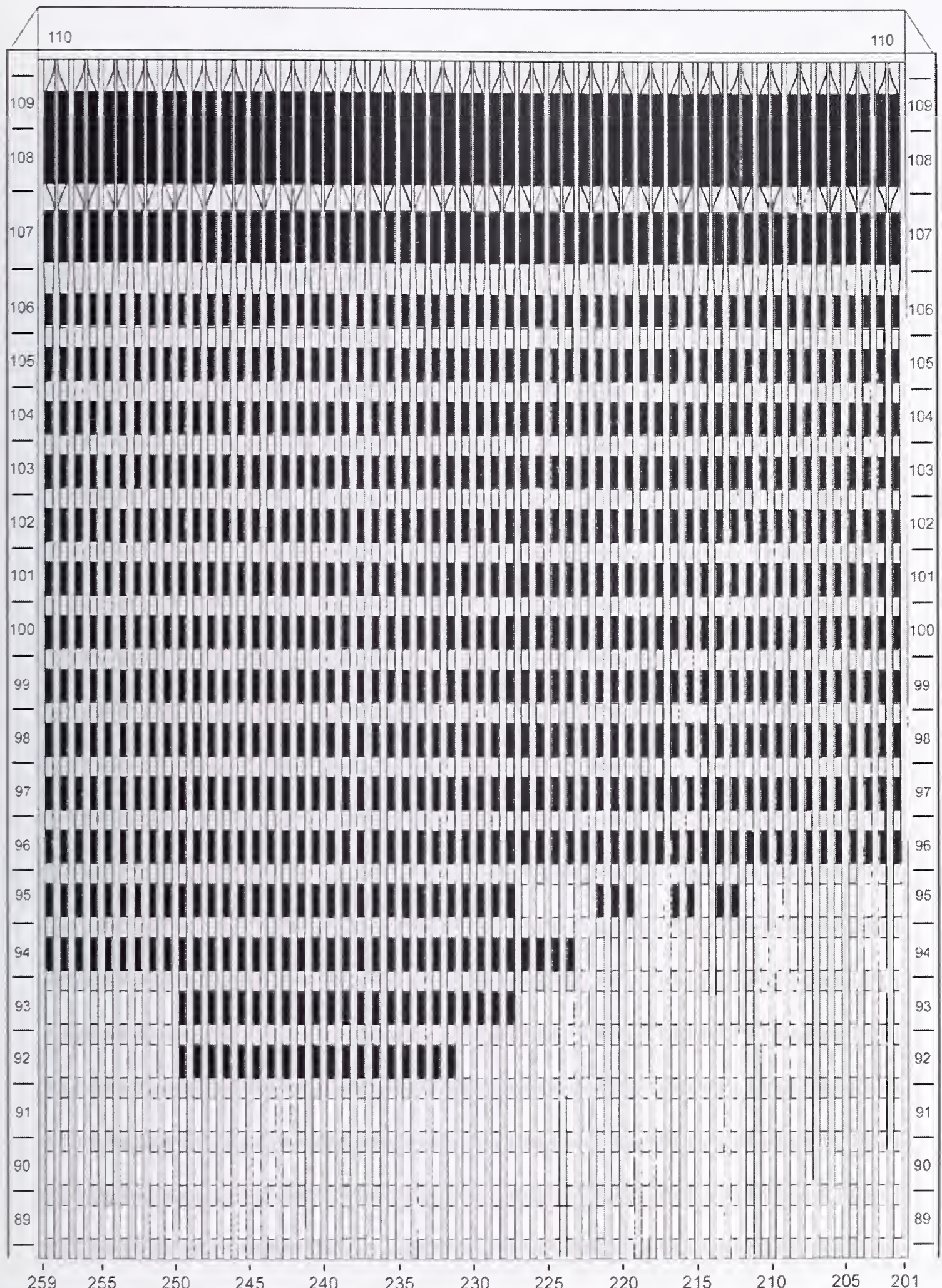

Figure D-42. Diagram of the east face of WTC 1 for floors 89 to 110 at 10:06 a.m. showing windows where smoke was observed and those that were hidden from view. 


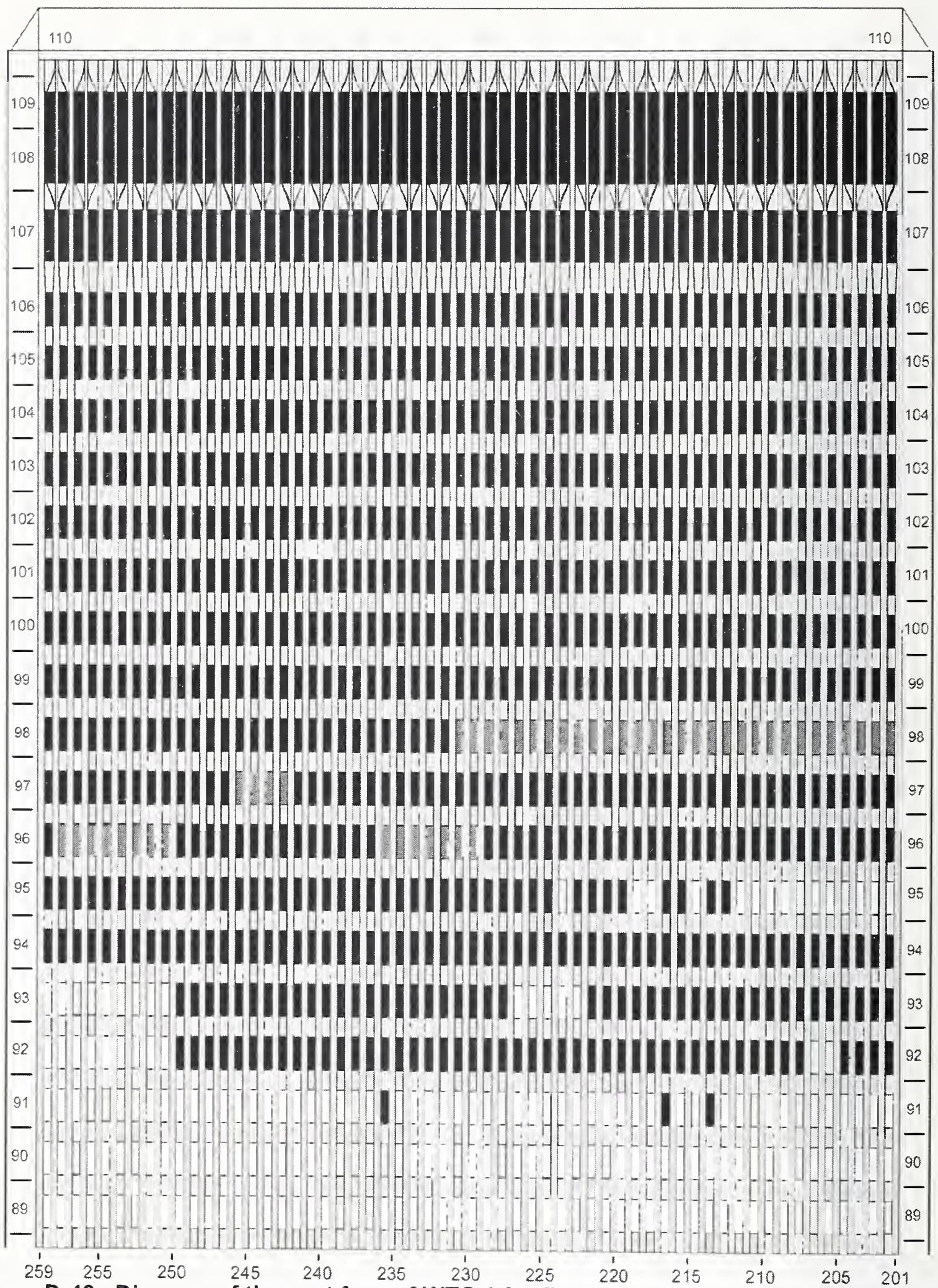

Figure D-43. Diagram of the east face of WTC 1 for floors 89 to 110 at 10:12 a.m. showing the condition of windows and locations of fires. 


$$
\text { WTC 1, East Face 10:12 a.m. }
$$

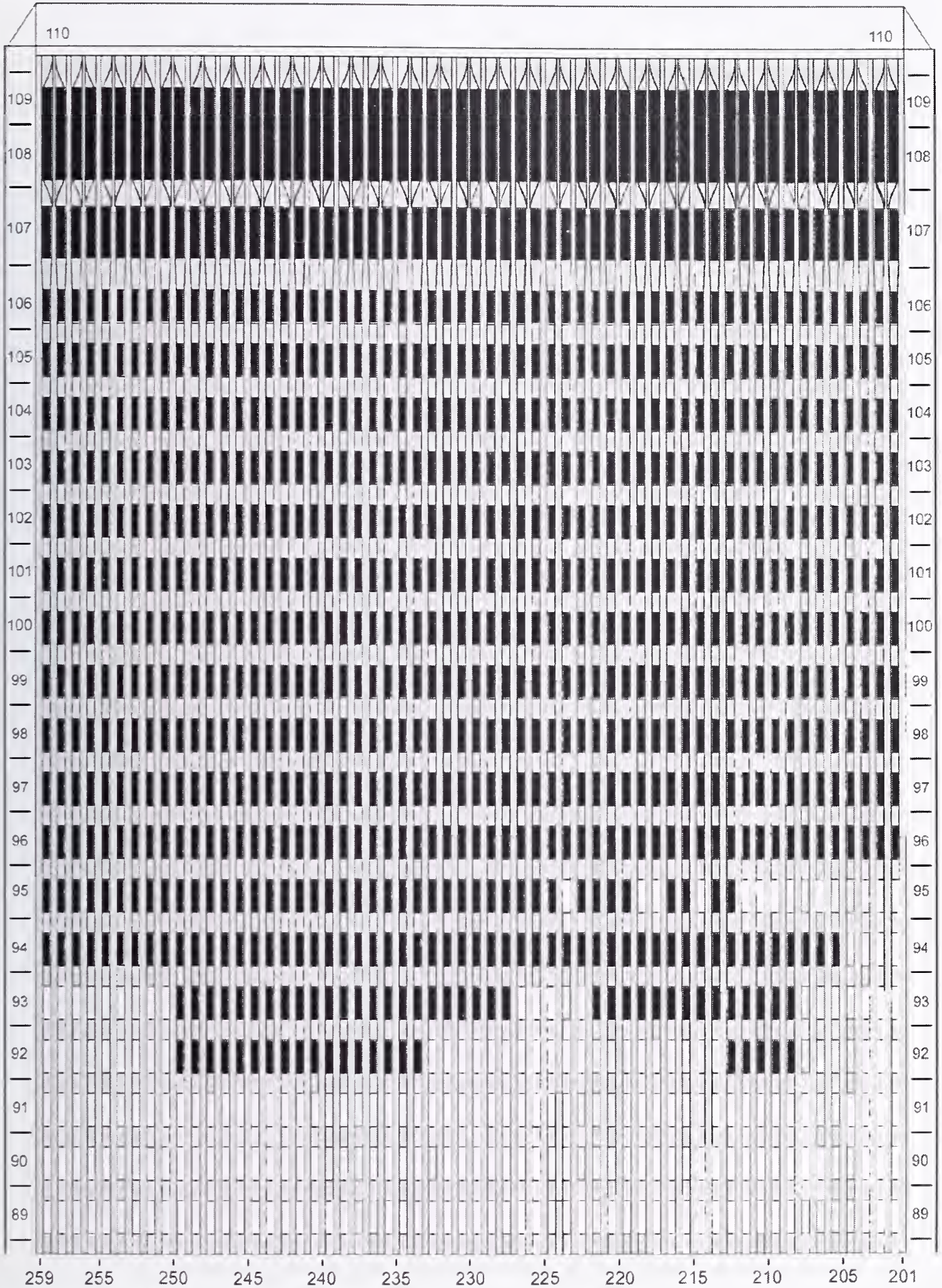

Figure D-44. Diagram of the east face of WTC 1 for floors 89 to 110 at 10:12 a.m. showing windows where smoke was observed and those that were hidden from view. 


$$
\text { WTC 1, East Face } \quad \text { 10:18 a.m. }
$$

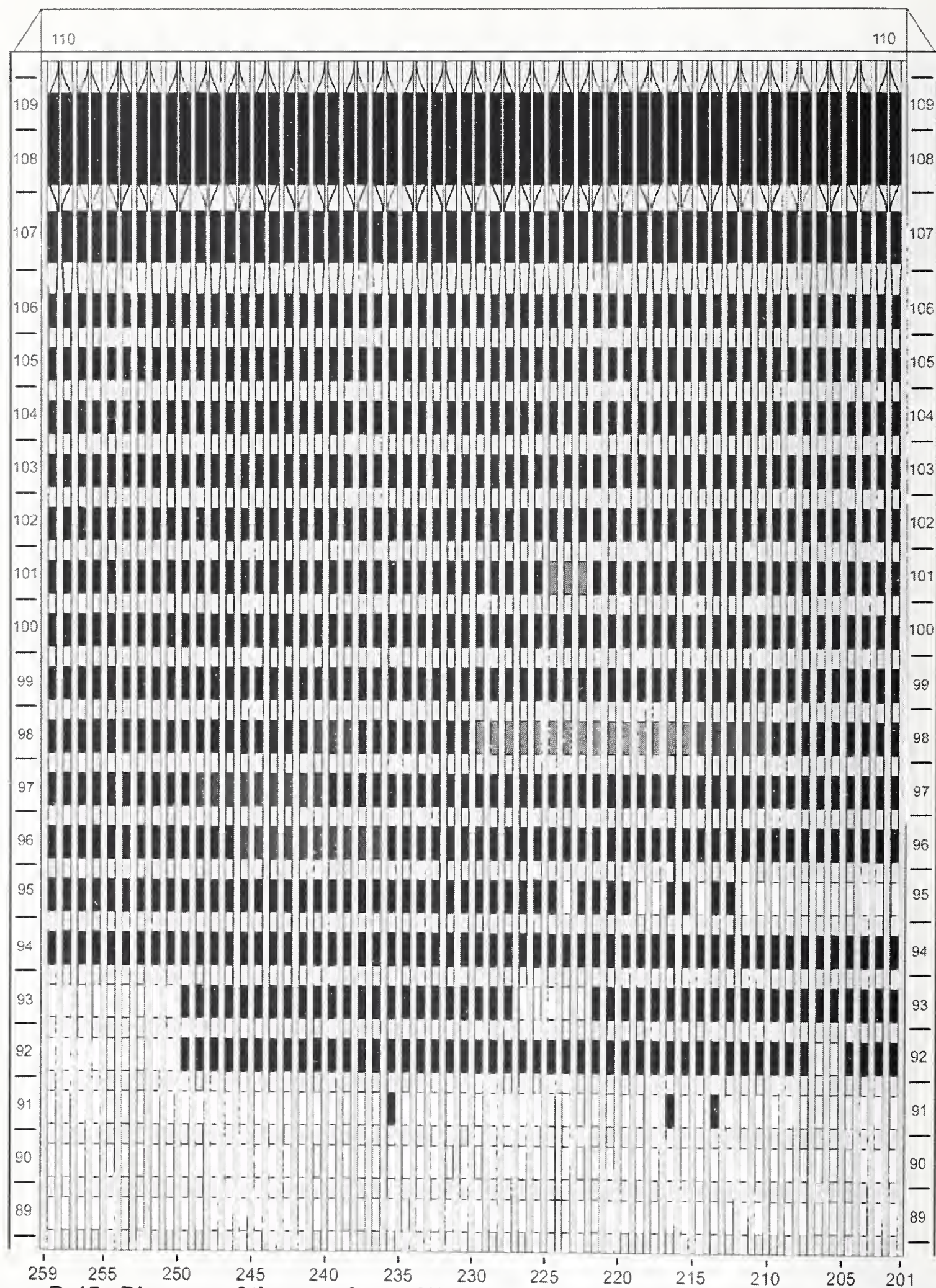

Figure D-45. Diagram of the east face of WTC 1 for floors 89 to 110 at 10:18 a.m. showing the condition of windows and locations of fires. 


$$
\text { WTC 1, East Face 10:18 a.m. }
$$

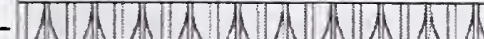

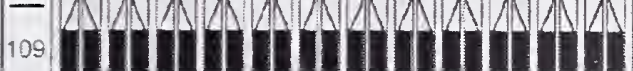

$-$

-

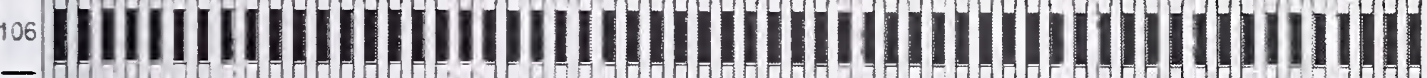



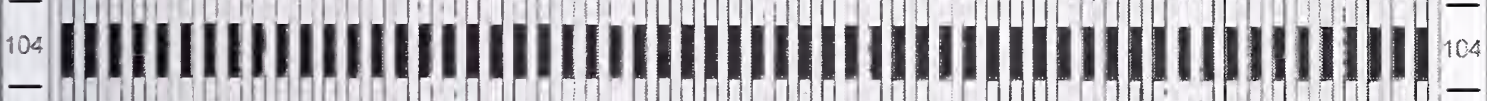

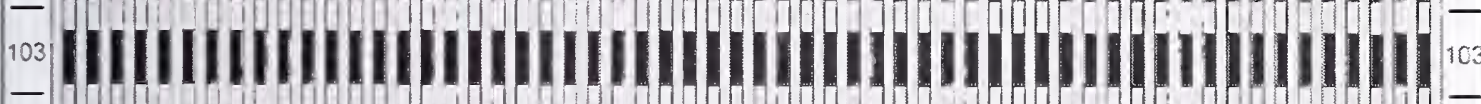

-2 |

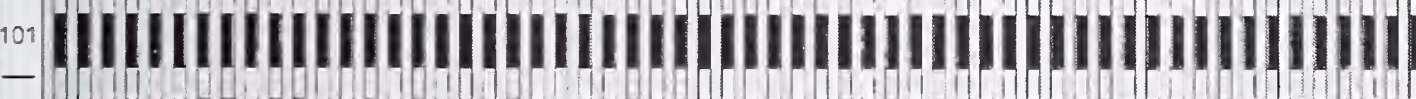

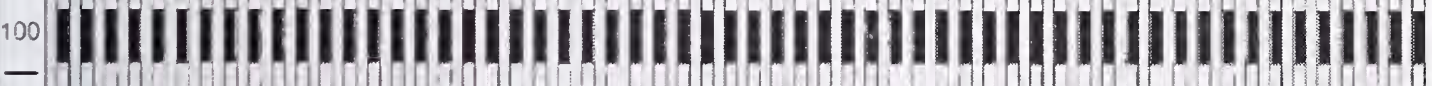

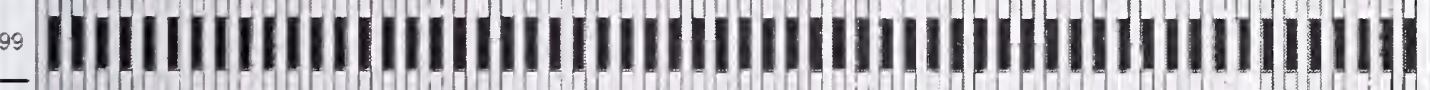

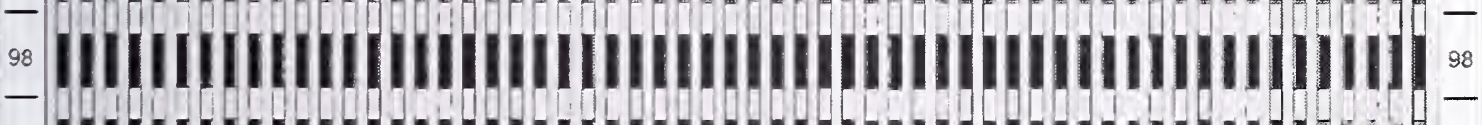

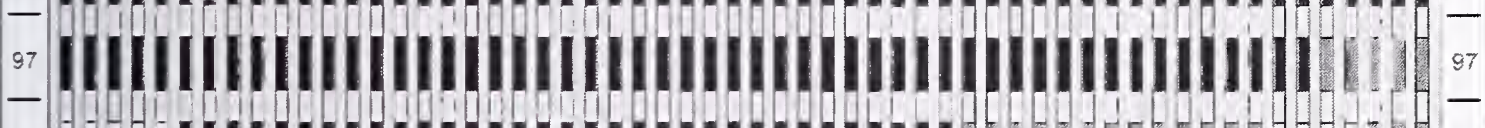

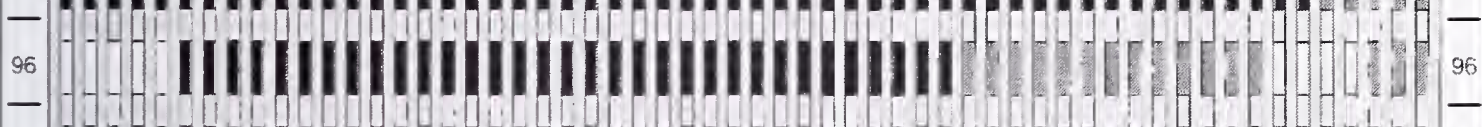

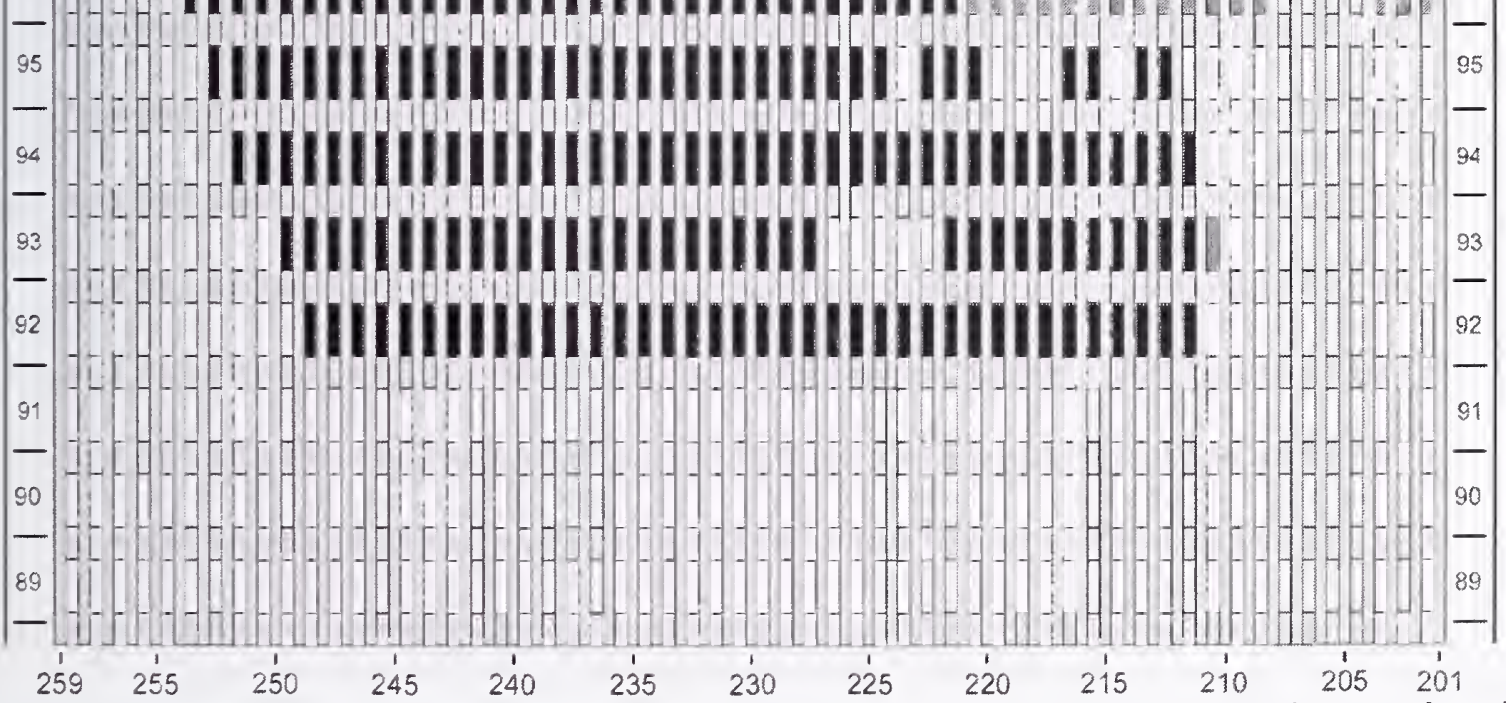

Figure D-46. Diagram of the east face of WTC 1 for floors 89 to 110 at 10:18 a.m. showing windows where smoke was observed and those that were hidden from view. 


$$
\text { WTC 1, East Face } \quad \text { 10:22 a.m. }
$$

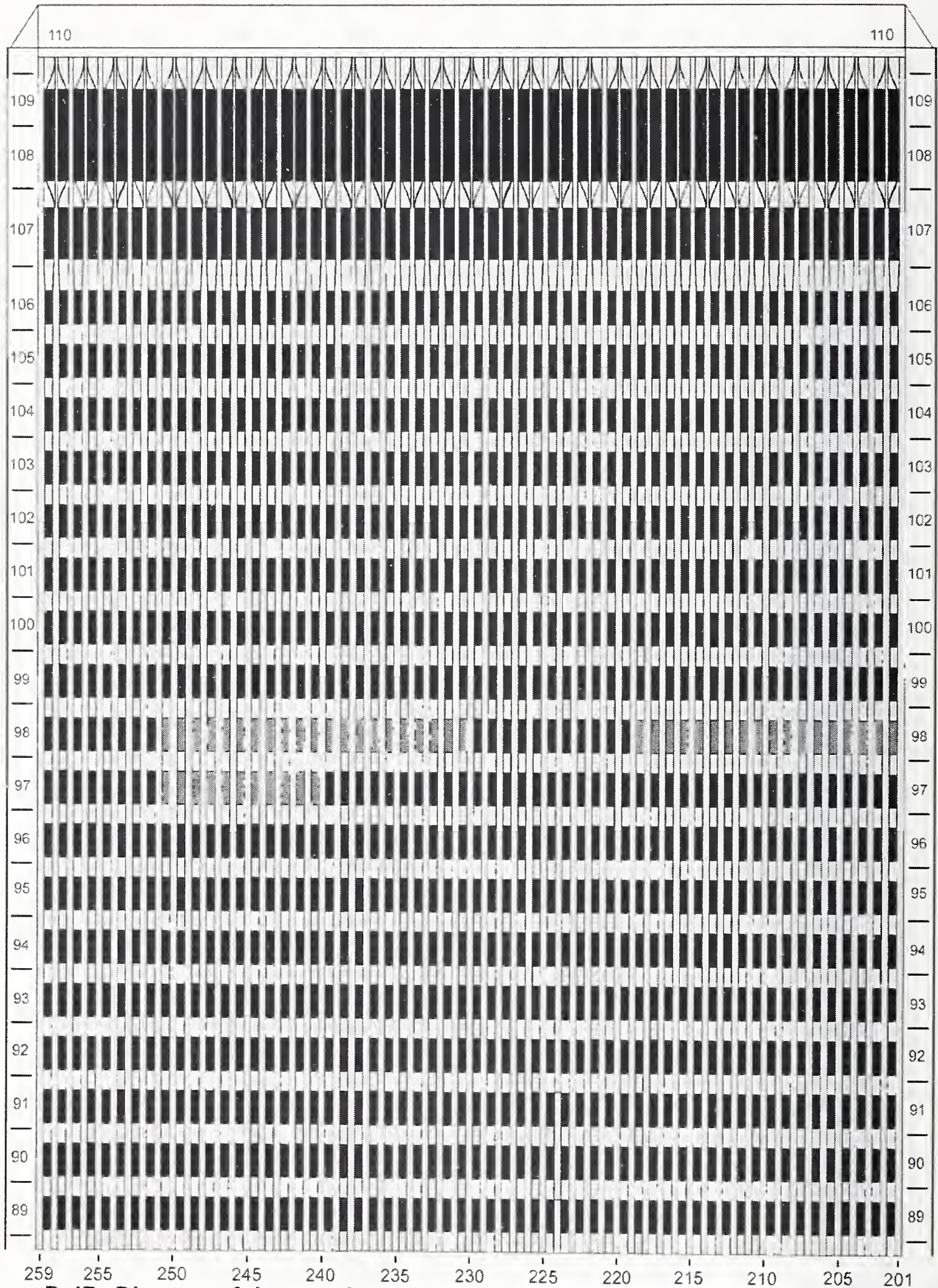

Figure D-47. Diagram of the east face of WTC 1 for floors 89 to 110 at 10:22 a.m. showing the condition of windows and locations of fires. 


$$
\text { WTC 1, East Face 10:22 a.m. }
$$

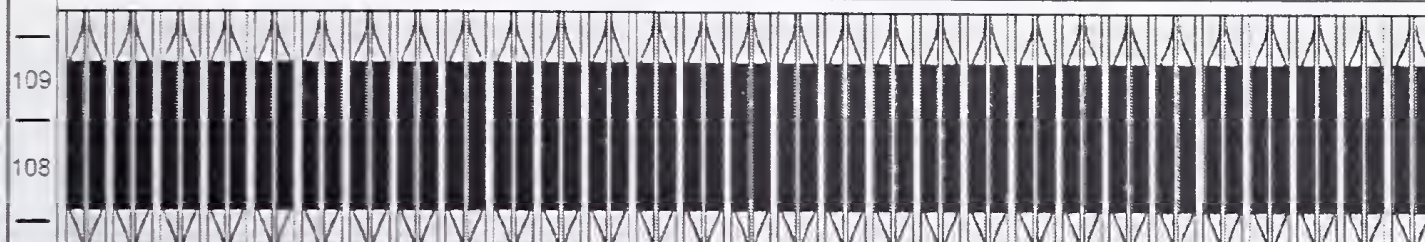

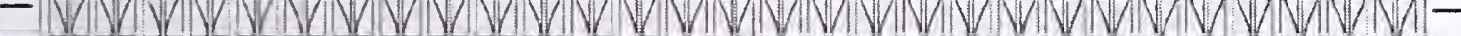
-

-

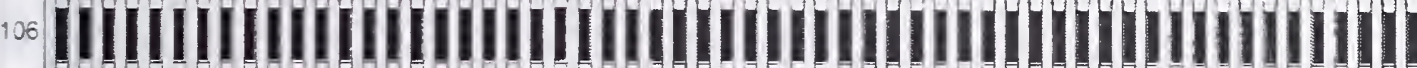

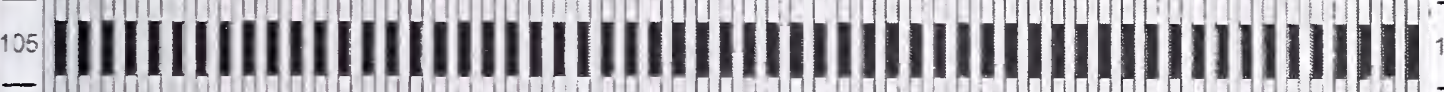

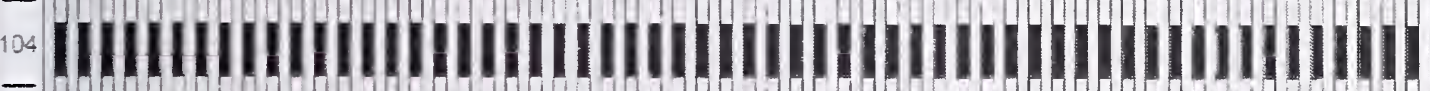

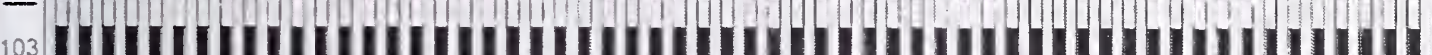

-

102 IIIIIIIIIIIIIIIIIIIIIIIIIIIIIIIIIIIIIIIIIIIIIIIIIIIIIIIII

10:

- mor

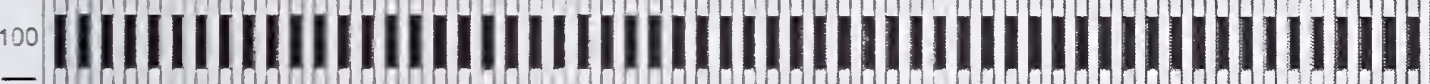

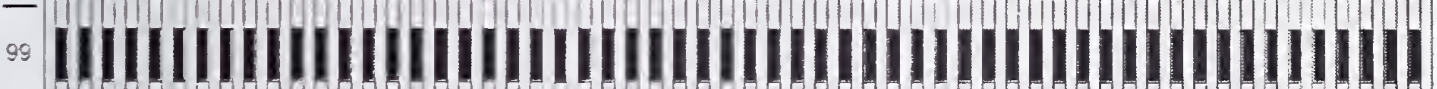

-

"8 [IIIIIIIIIIIIIIIIIIIIIIIIIIIIIIIIIIIIIIIIIIIIIIIIIIIIII

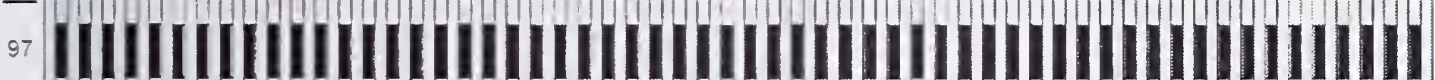

-

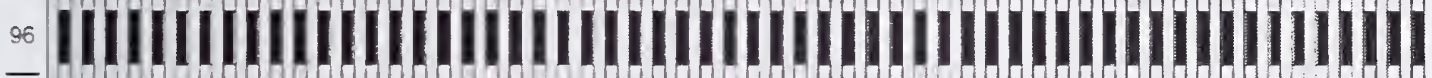

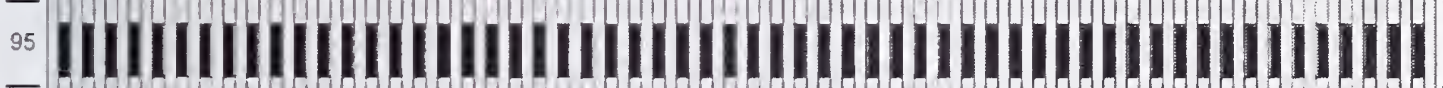

"IIIIIIIIIIIIIIIIIIIIIIIIIIIIIIIIIIIIIIIIIIIIIIIIIIIIIII

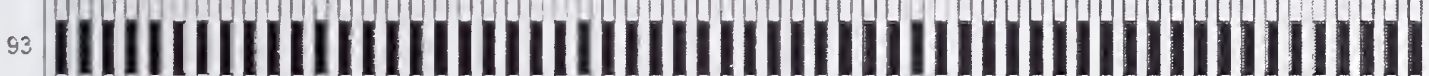

-

s2 IIIIIIIIIIIIIIIIIIIIIIIIIIIIIIIIIIIIIIIIIIIIIIIII

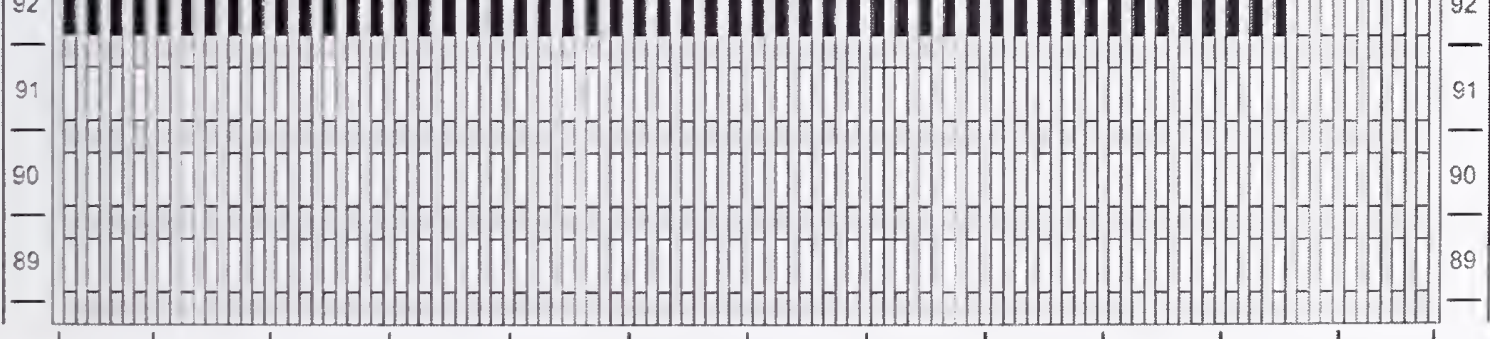

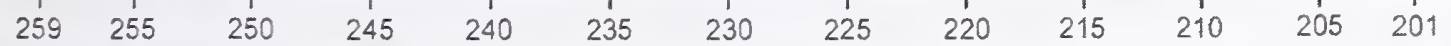

Figure D-48. Diagram of the east face of WTC 1 for floors 89 to 110 at 10:22 a.m. showing windows where smoke was observed and those that were hidden from view. 


$$
\text { WTC 1, East Face } \quad 10: 26 \text { a.m. }
$$

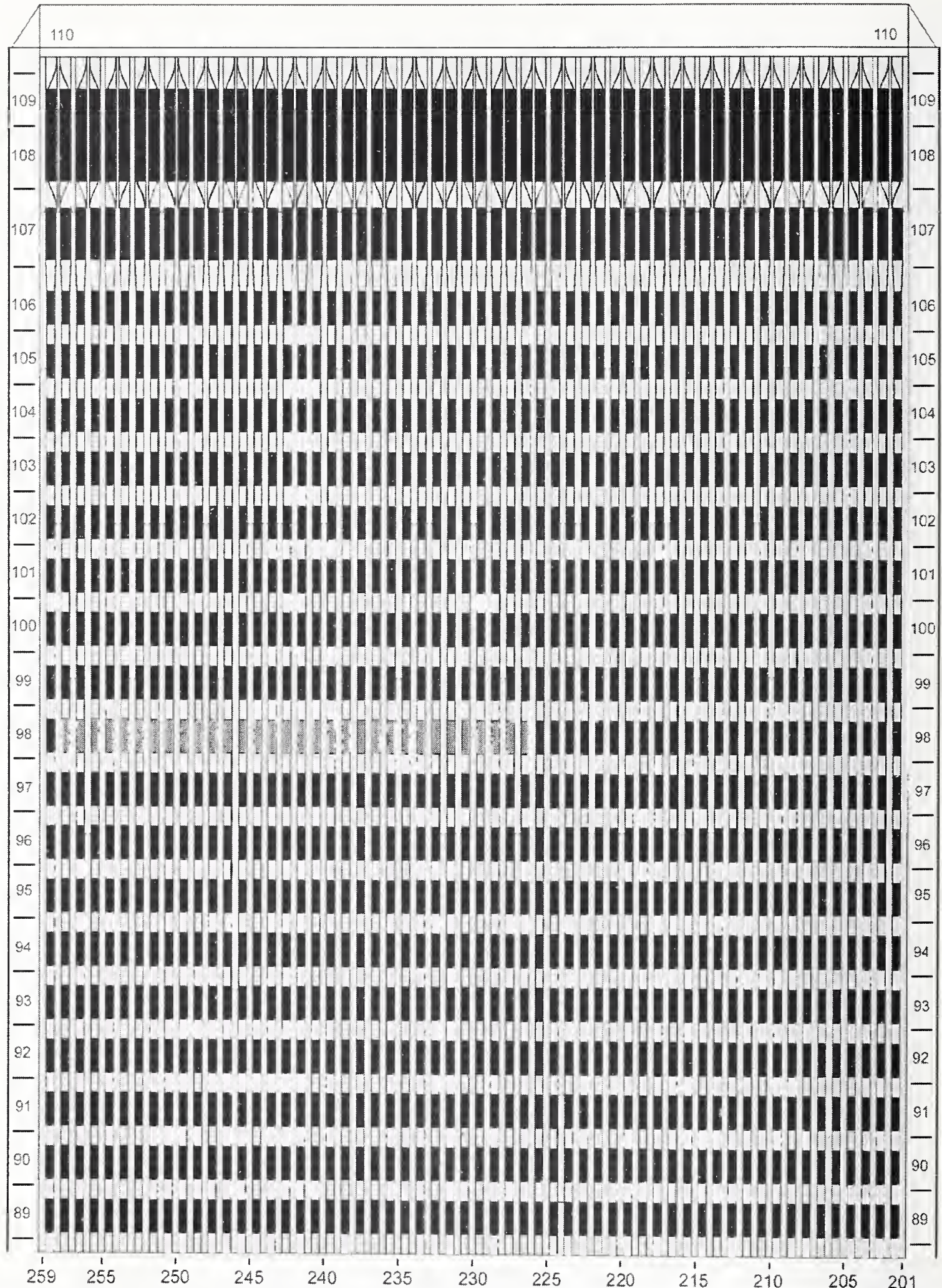

Figure D-49. Diagram of the east face of WTC 1 for floors 89 to 110 at 10:26 a.m. showing the condition of windows and locations of fires. 


$$
\text { WTC 1, East Face 10:26 a.m. }
$$

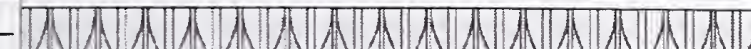

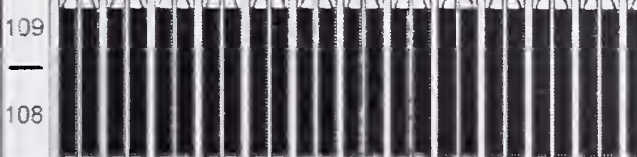

-

107

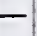

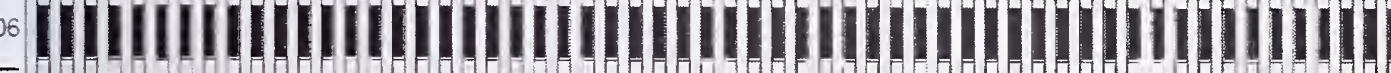

105

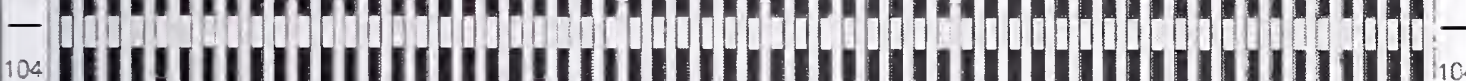

"x

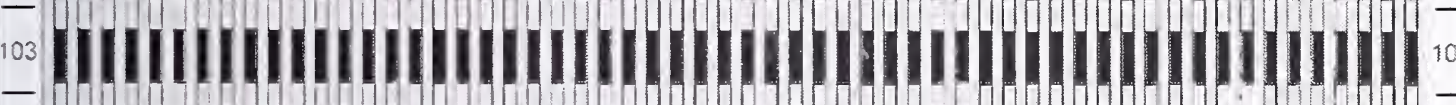

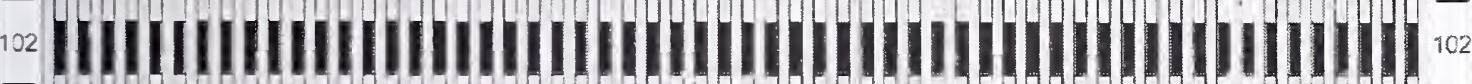

-

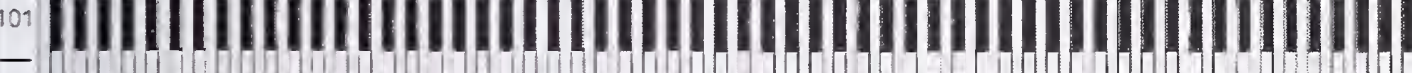

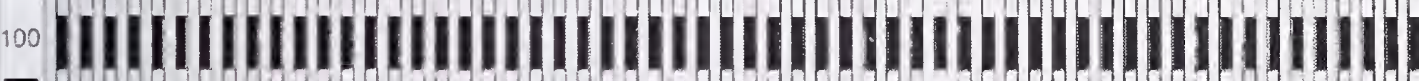

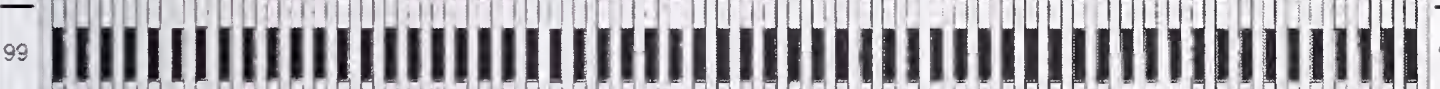

-

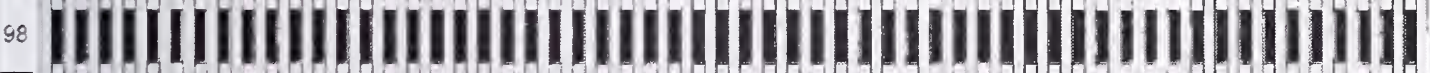

o7 IIIIIIIIIIIIIIIIIIIIIIIIIIIIIIIIIIIIIIIIIIIIIIIIIIIIIIIII

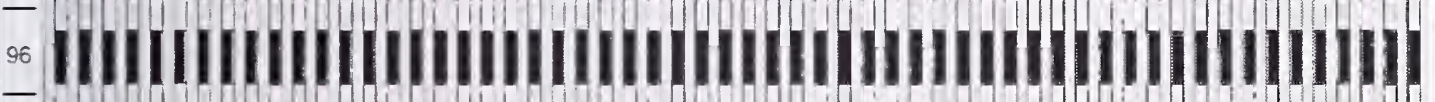

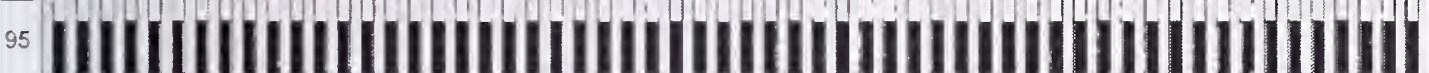

-

= IIIIIIIIIIIIIIIIIIIIIIIIIIIIIIIIIIIIIIIIIIIIIIIIIIIIIIIII

so IIIIIIIIIIIIIIIIIIIIIIIIIIIIIIIIIIIIIIIIIIIIIIIIIIIIIIIIIIII

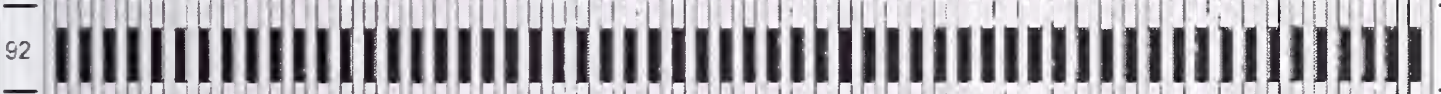

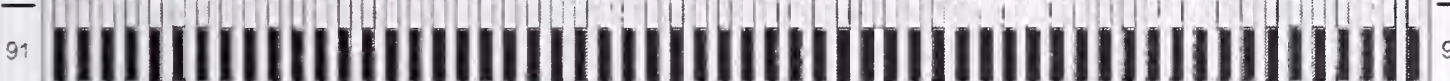

- iny in

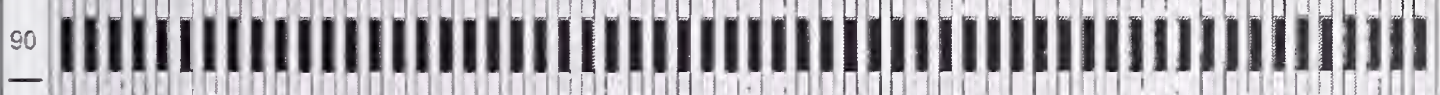

อง |

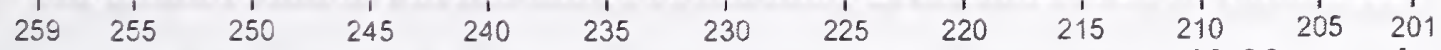

Figure D-50. Diagram of the east face of WTC 1 for floors 89 to 110 at 10:26 a.m. showing windows where smoke was observed and those that were hidden from view. 


$$
\text { WTC 1, East Face } \quad \text { 10:28 a.m. }
$$

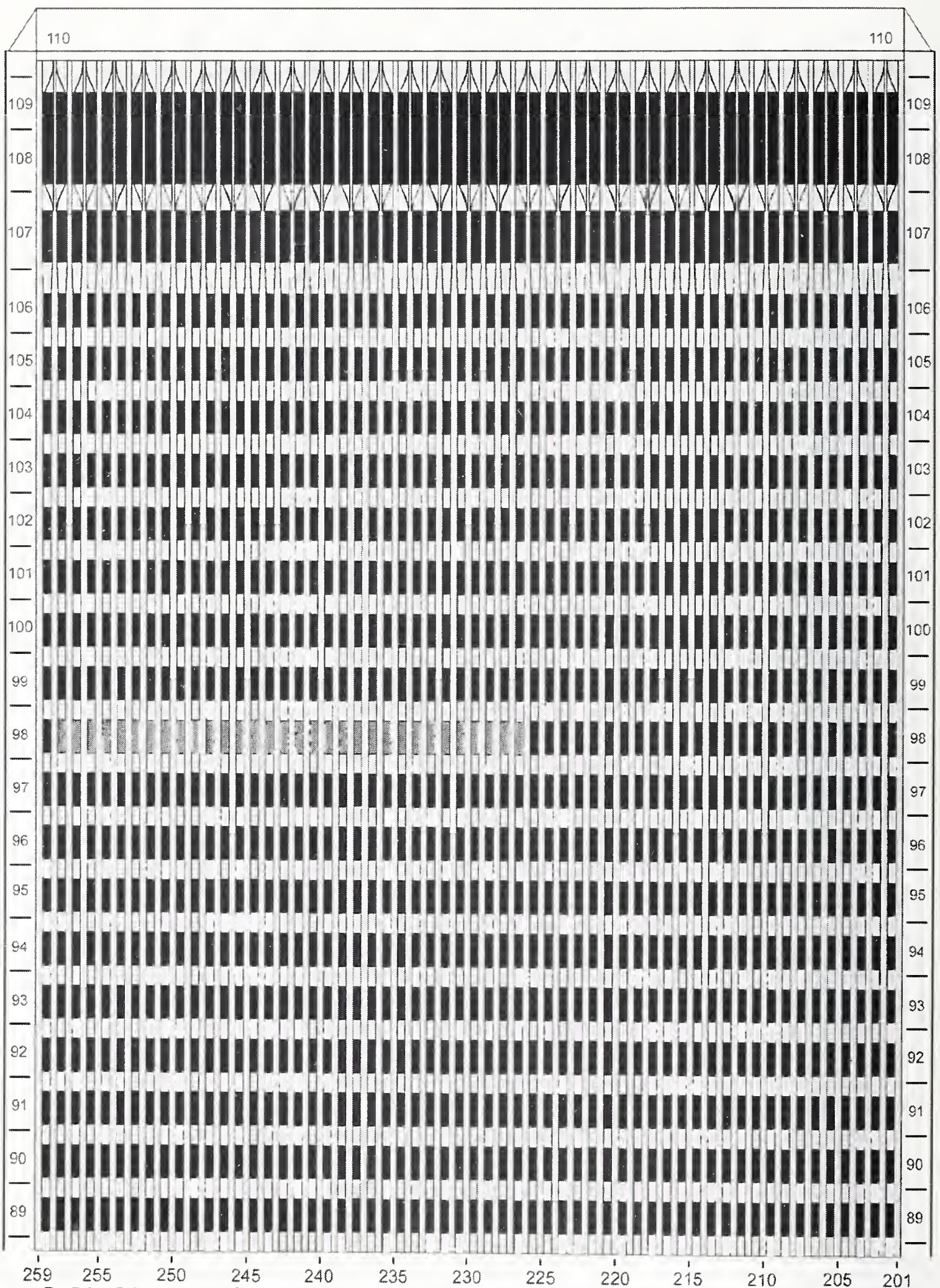

Figure D-51. Diagram of the east face of WTC 1 for floors 89 to 110 at 10:28 a.m. showing the condition of windows and locations of fires. 


$$
\text { WTC 1, East Face 10:28 a.m. }
$$

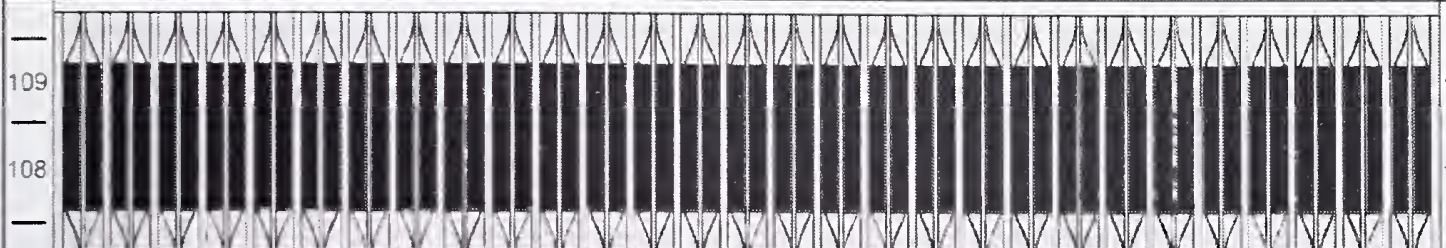

107

2.

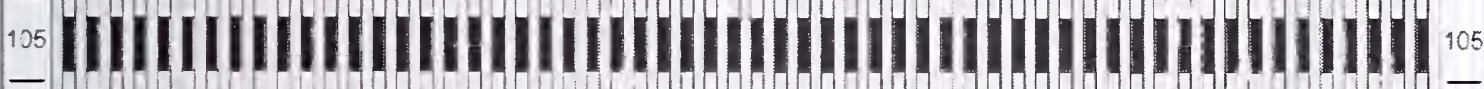

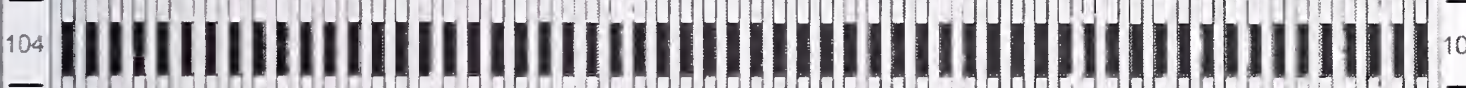

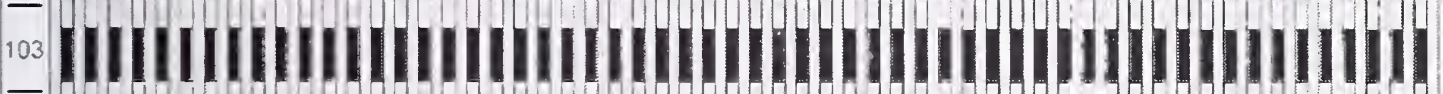

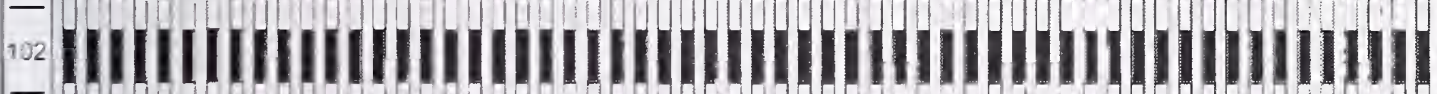

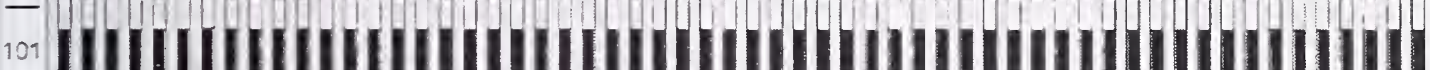
-

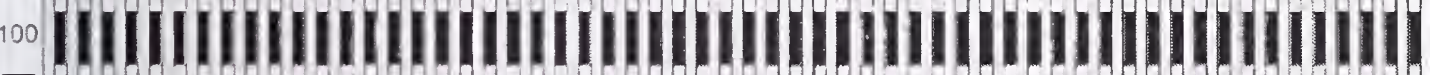

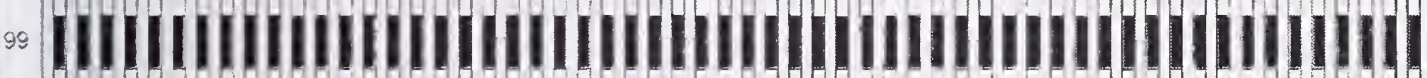

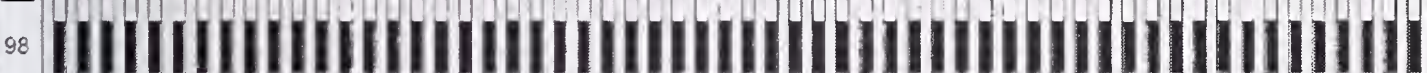

-

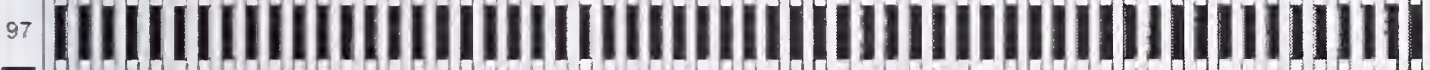

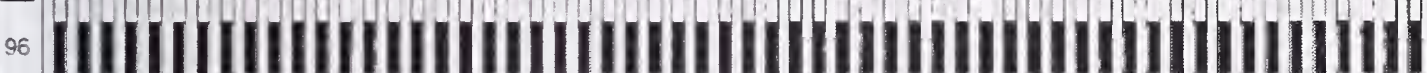
s5 IIIIIIIIIIIIIIIIIIIIIIIIIIIIIIIIIIIIIIIIIIIIIIIIIIIIIIIIIIII s. s: IIIIIIIIIIIIIIIIIIIIIIIIIIIIIIIIIIIIIIIIIIIIIIIIIIIIIIIIII

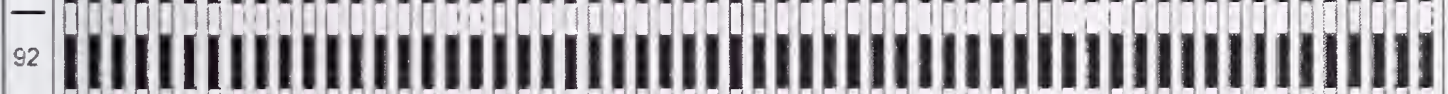

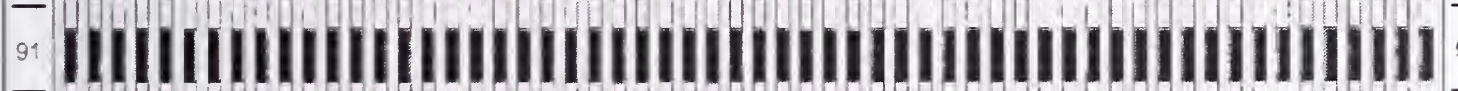
$\left[\begin{array}{c}-0 \\ \text { - }\end{array}\right.$

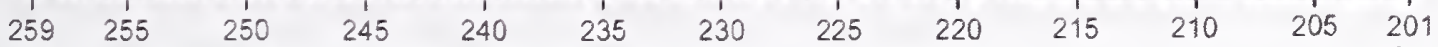

Figure D-52. Diagram of the east face of WTC 1 for floors 89 to 110 at 10:28 a.m. showing windows where smoke was observed and those that were hidden from view. 
This page intentionally left blank. 


\section{Appendix E \\ WTC 1 SOUTH FACE Combined FIRE AND Window Condition AND WINDOW SMOKE CONDITION}

This appendix provides visual representations of the data contained in data sheets describing observations of fire, smoke, and window condition on a window-by-window basis as a function of time for the south face of WTC 1. The data for window condition and fire observations are combined into single representations, with the data for fire taking precedence. Observations are represented using the key provided in Figure 5-1 as follows: - window glass in place, - window open, - spot fire, - fire visible inside, - external flaming, and - not visible. The data for smoke are shown in separate figures using the key from Figure 5-1 as follows: - no smoke visible, - light smoke, - heavy smoke, and - not visible. For a given time, the window condition and fire data are shown first. 


\section{WTC 1, South Face $\quad$ 8:47 a.m.}

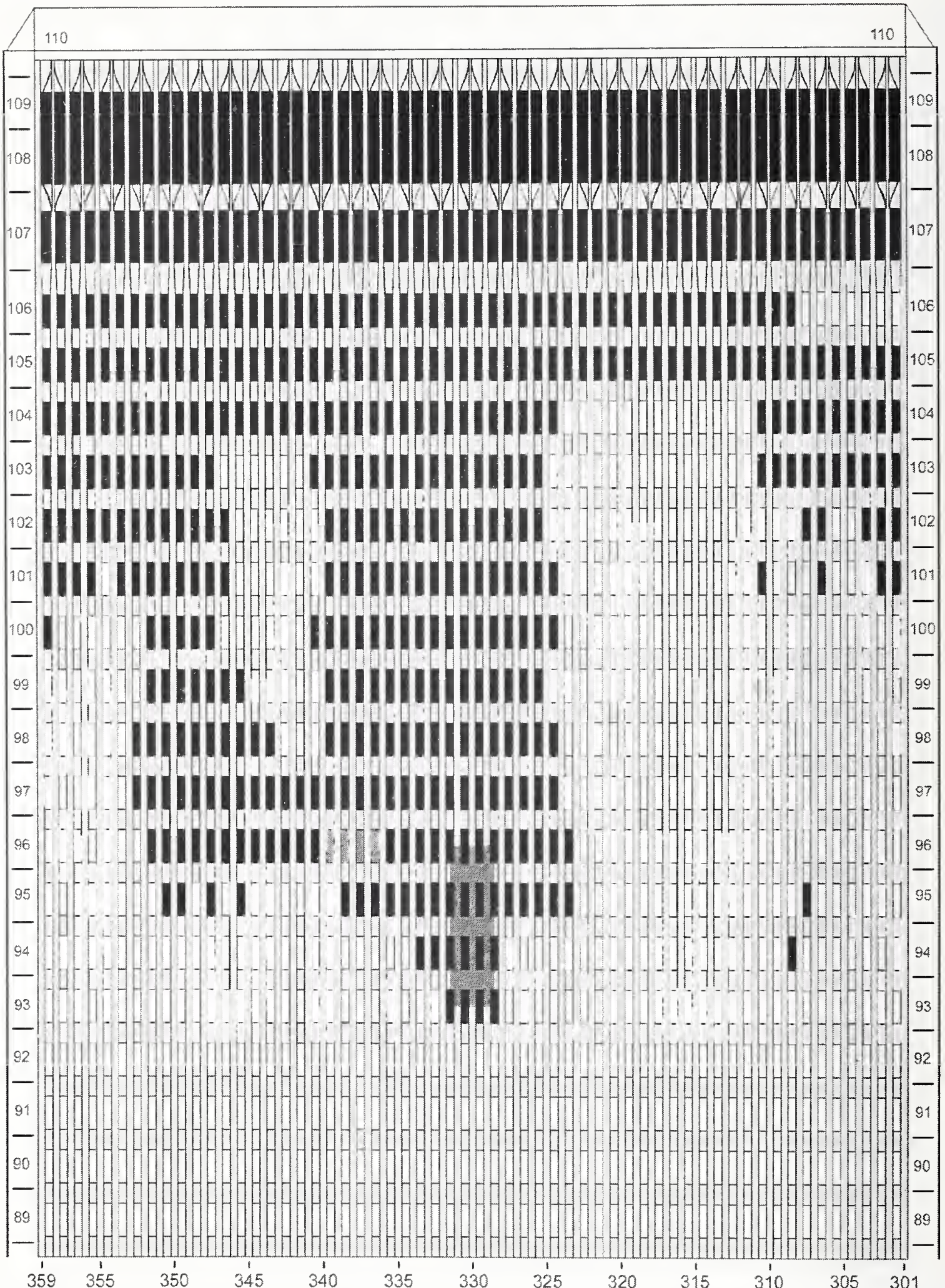

Figure E-1. Diagram of the south face of WTC 1 for floors 89 to 110 at $8: 47$ a.m. showing the condition of windows and locations of fires. 


$$
\text { WTC 1, South Face } \quad \text { 8:47 a.m. }
$$

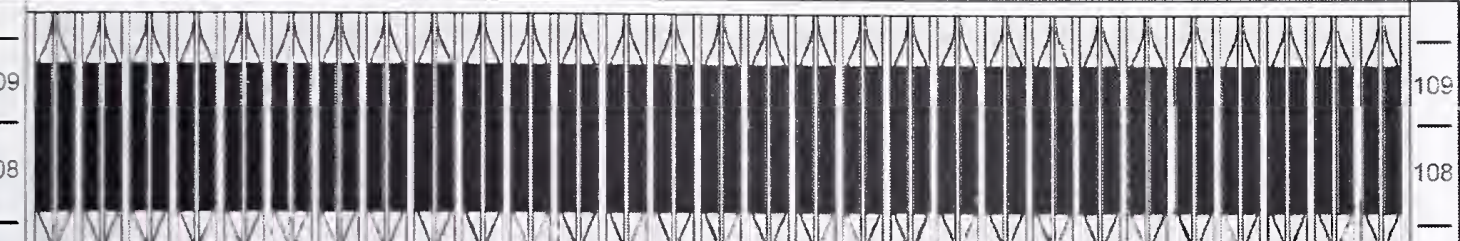

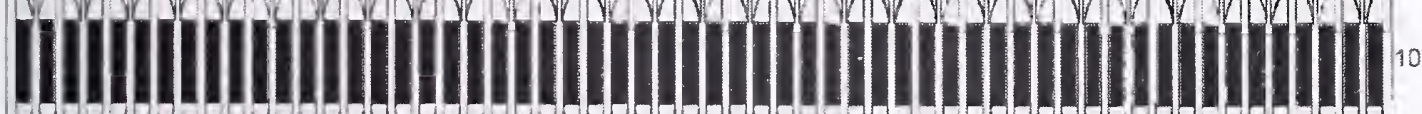

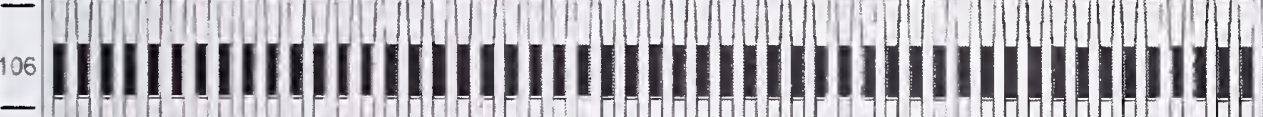

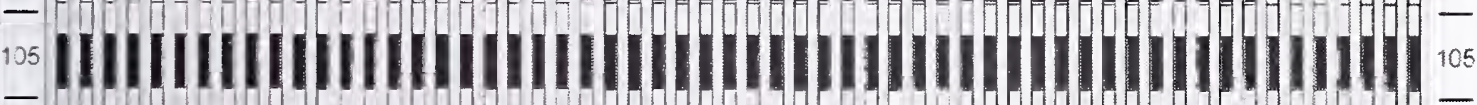

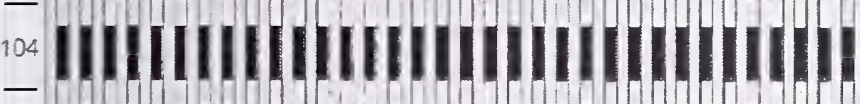

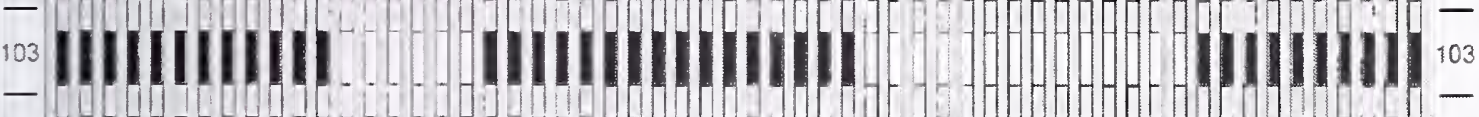

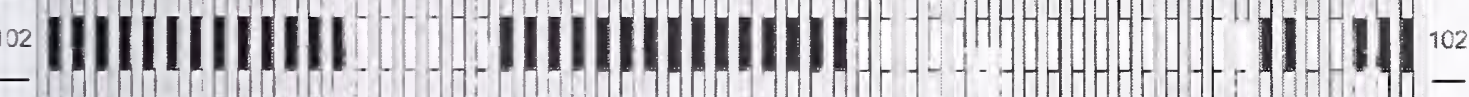

IIIII IIIIIIII III IIIIIIIIIIIIII

T) II IIII IIIIIIIIIIIII

IIIIIIIIII IIIIIIIIIIIIIII

IIIIIIIIIIIIIIIIIIIIIIIIIIIIII

IIIIIIIIIIII IIII!!!I!!

II I I IIIIIIIII- II

IIIII

IIII

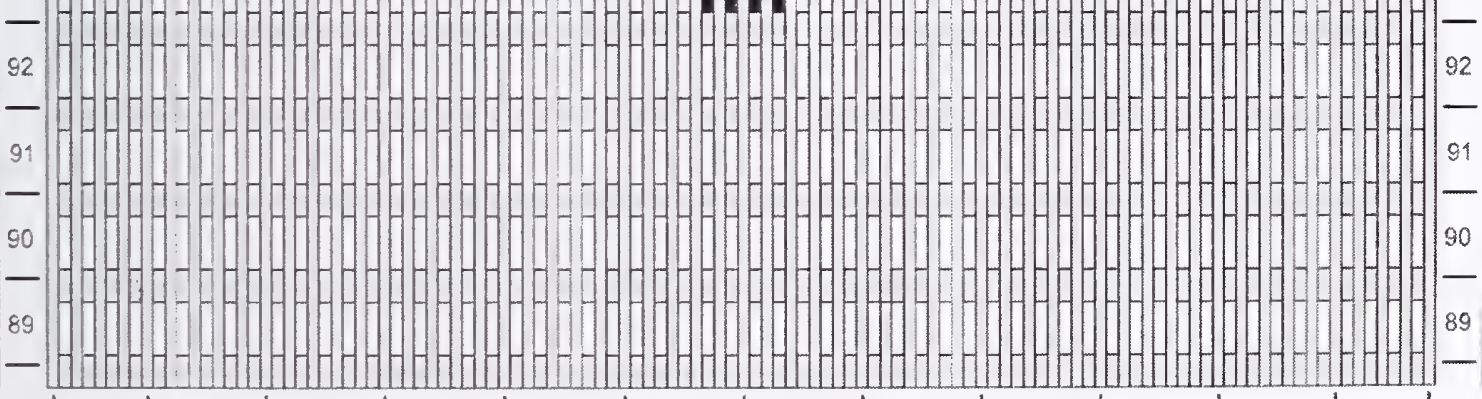

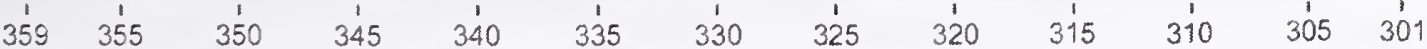

Figure E-2. Diagram of the south face of WTC 1 for floors 89 to 110 at 8:47 a.m. showing windows where smoke was observed and those that were hidden from view. 
WTC 1, South Face $\quad$ 8:52 a.m.

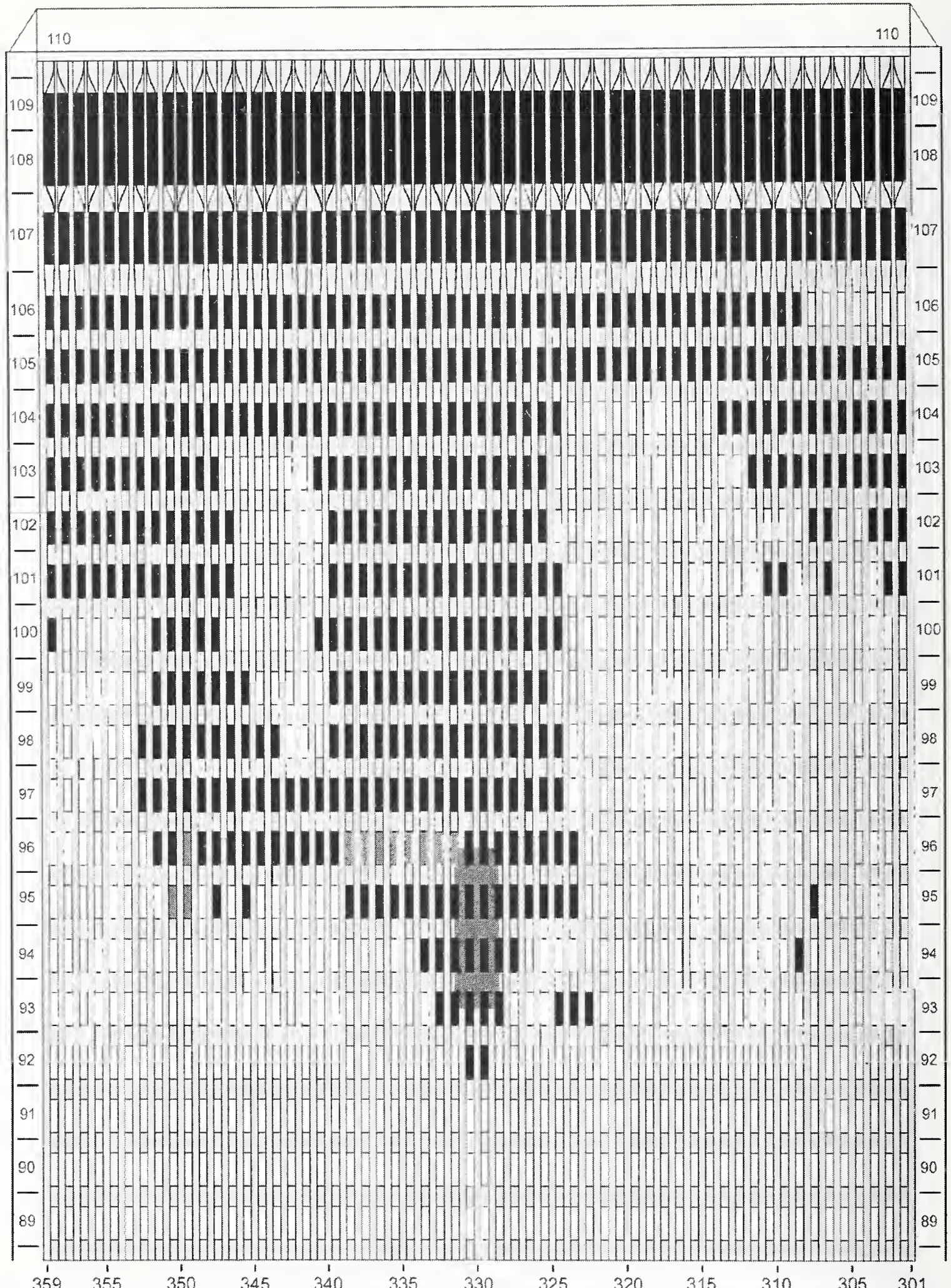

Figure E-3. Diagram of the south face of WTC 1 for floors 89 to 110 at $8: 52$ a.m. showing the condition of windows and locations of fires. 


$$
\text { WTC 1, South Face } \quad \text { 8:52 a.m. }
$$

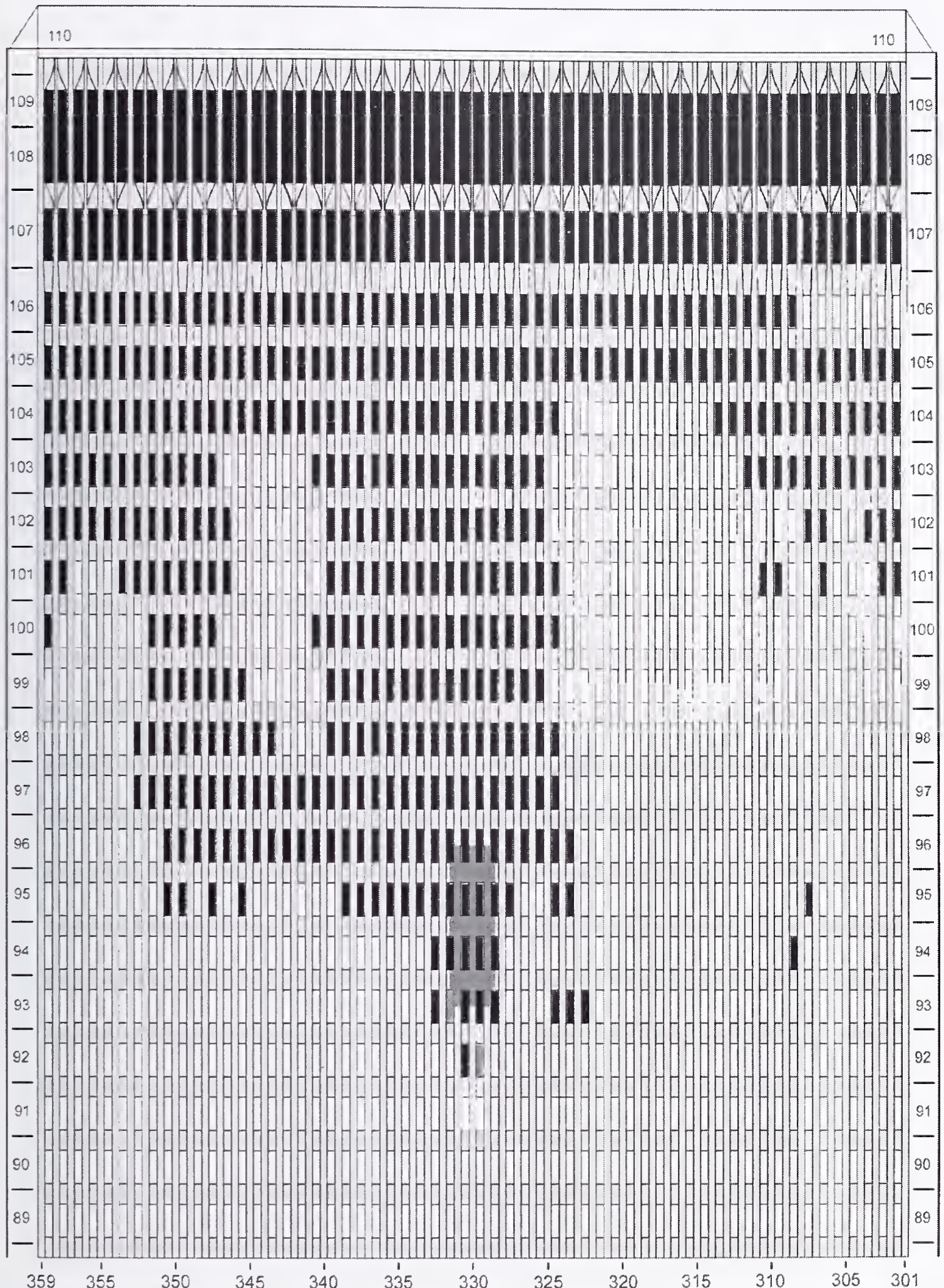

Figure E-4. Diagram of the south face of WTC 1 for floors 89 to 110 at 8:52 a.m. showing windows where smoke was observed and those that were hidden from view. 
WTC 1, South Face $\quad$ 8:56 a.m.

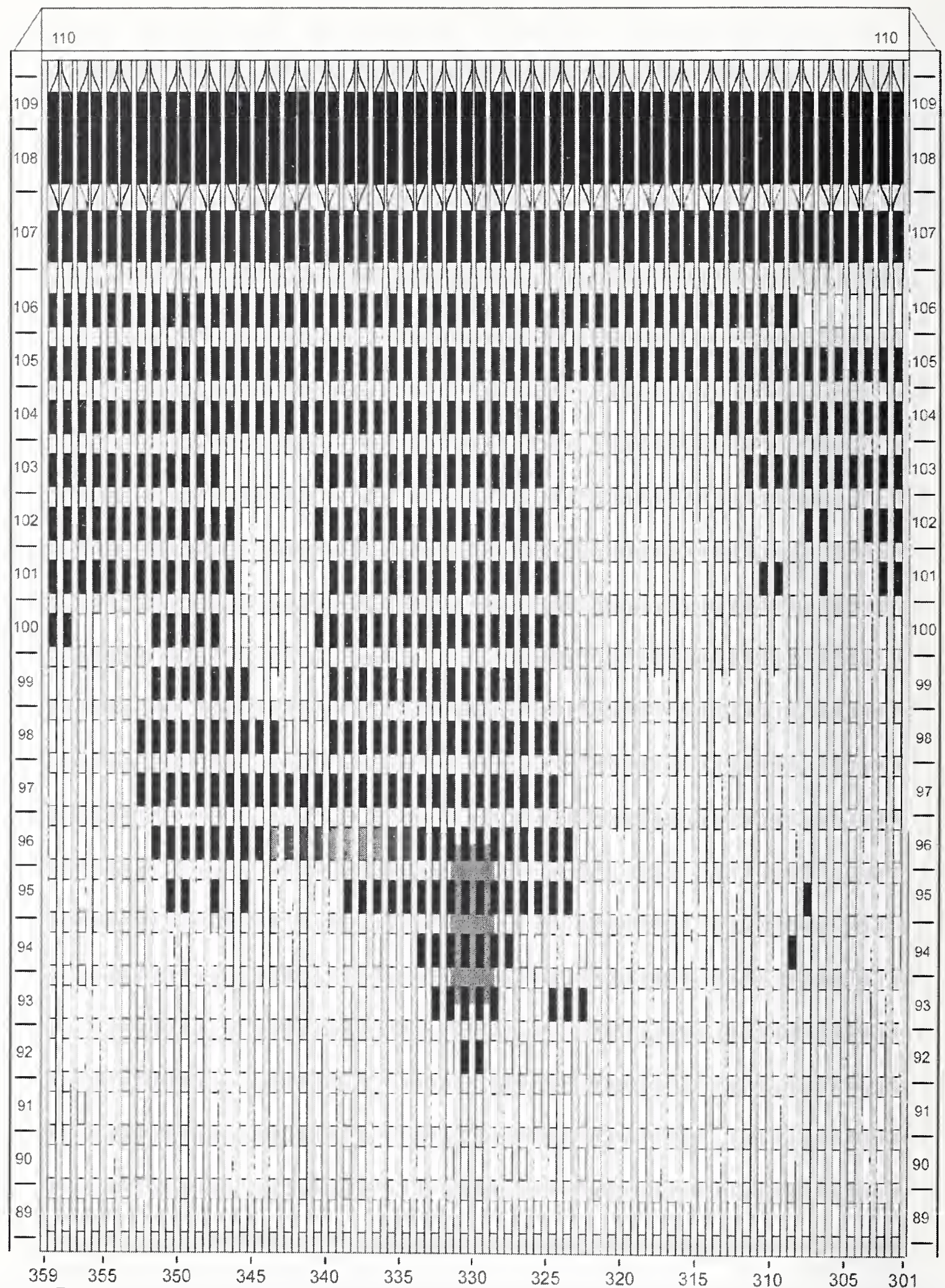

Figure E-5. Diagram of the south face of WTC 1 for floors 89 to 110 at 8:56 a.m. showing the condition of windows and locations of fires. 


$$
\text { WTC 1, South Face } \quad \text { 8:56 a.m. }
$$

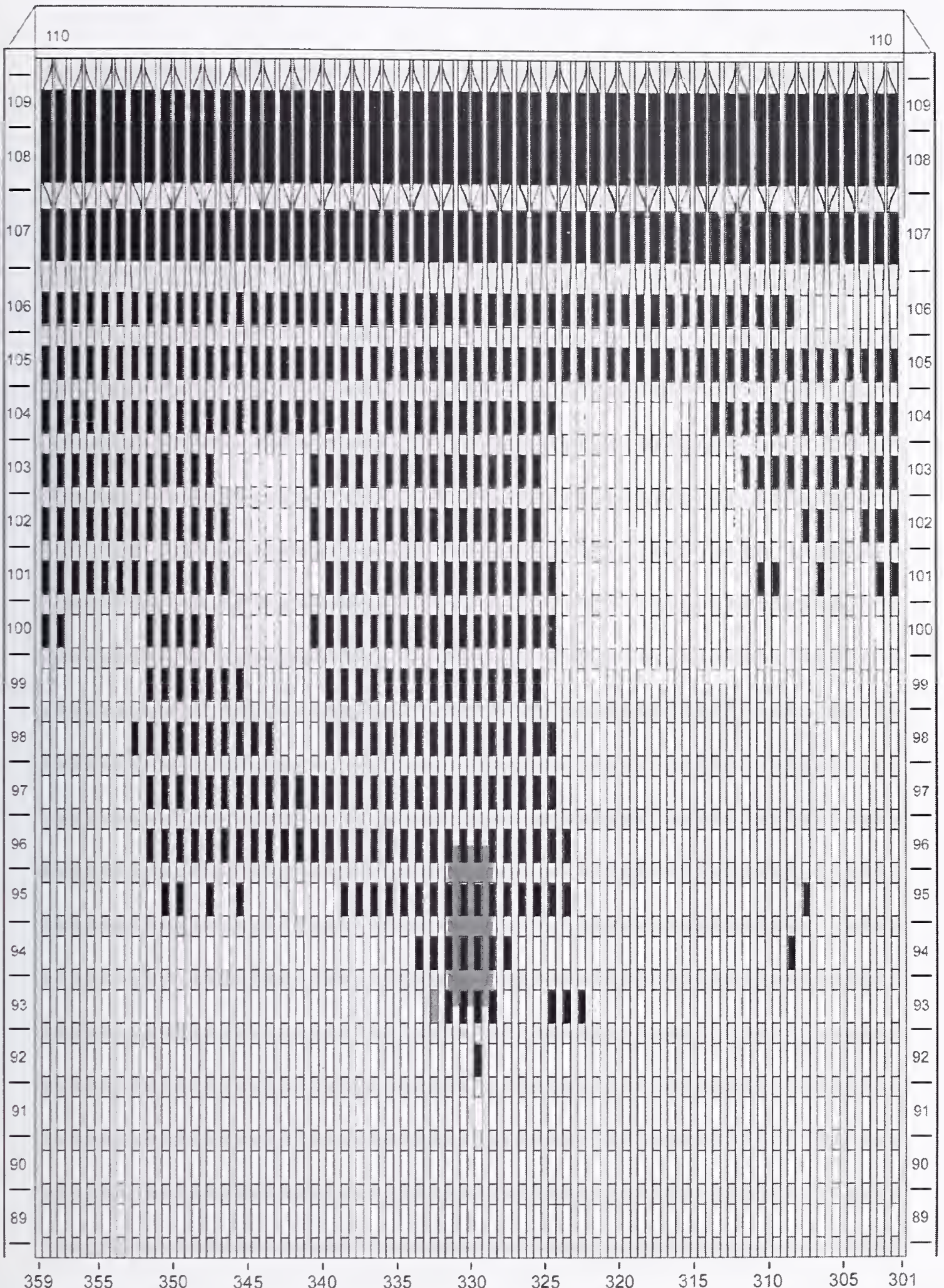

Figure E-6. Diagram of the south face of WTC 1 for floors 89 to 110 at 8:56 a.m. showing windows where smoke was observed and those that were hidden from view. 
WTC 1, South Face $\quad$ 8:58 a.m.

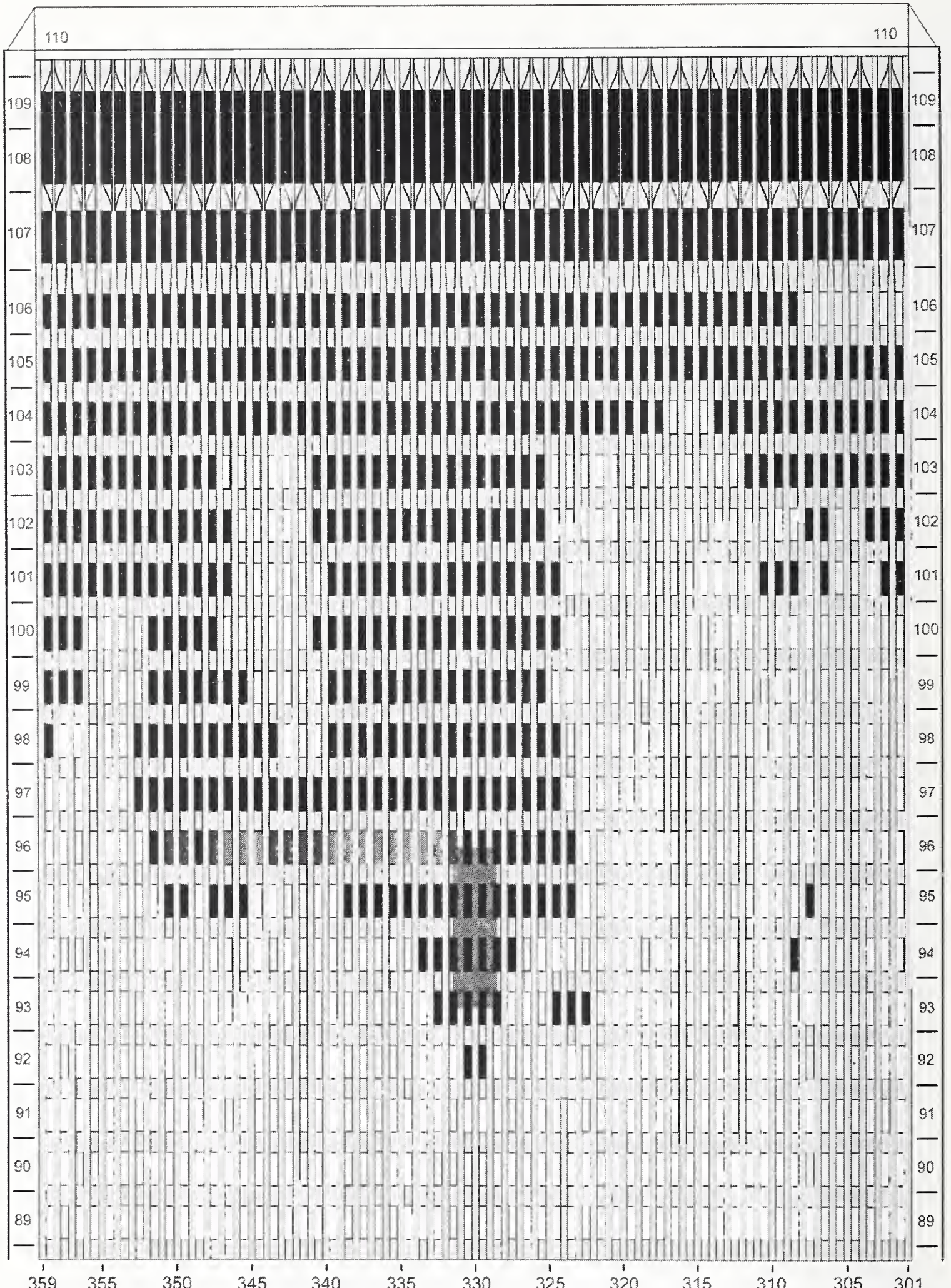

Figure E-7. Diagram of the south face of WTC 1 for floors 89 to 110 at 8:58 a.m. showing the condition of windows and locations of fires. 


$$
\text { WTC 1, South Face } \quad \text { 8:58 a.m. }
$$

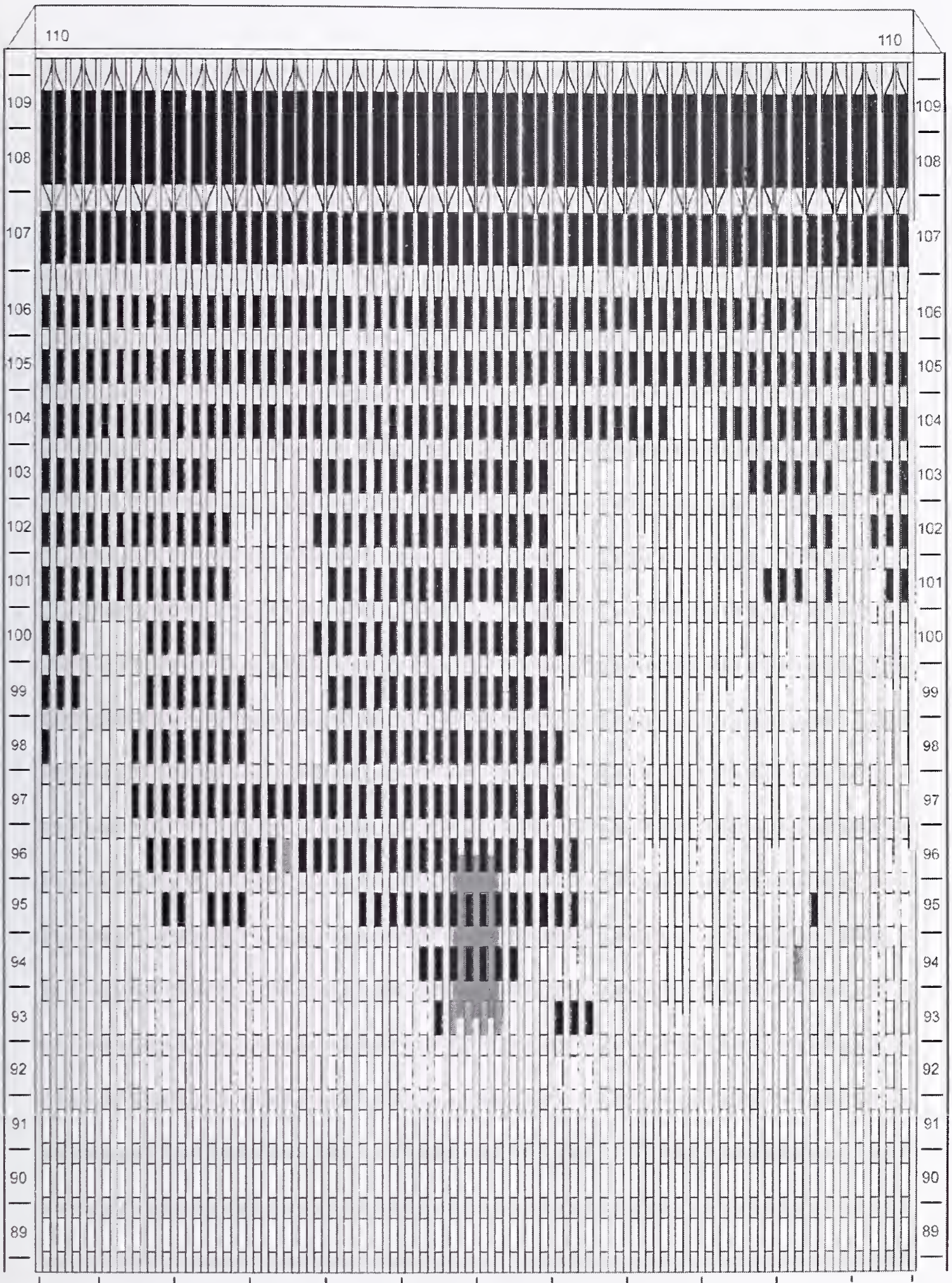

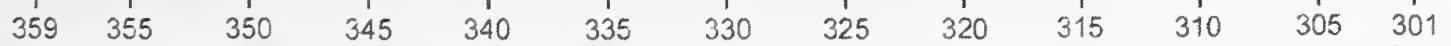

Figure E-8. Diagram of the south face of WTC 1 for floors 89 to 110 at $8: 58$ a.m. showing windows where smoke was observed and those that were hidden from view. 
WTC 1 , South Face

9:02 a.m.

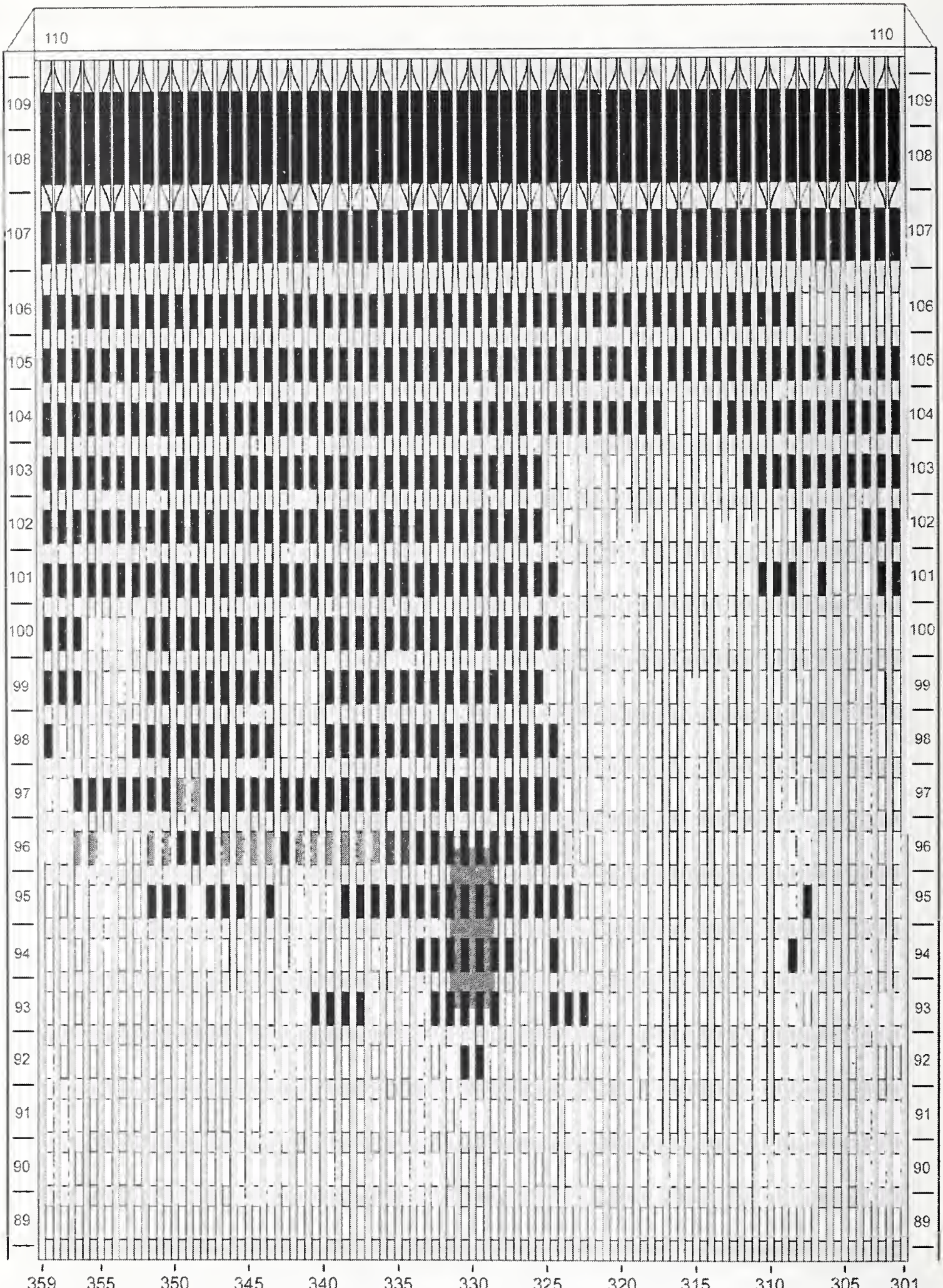

Figure E-9. Diagram of the south face of WTC 1 for floors 89 to 110 at 9:02 a.m. showing the condition of windows and locations of fires. 


$$
\text { WTC 1, South Face 9:02 a.m. }
$$

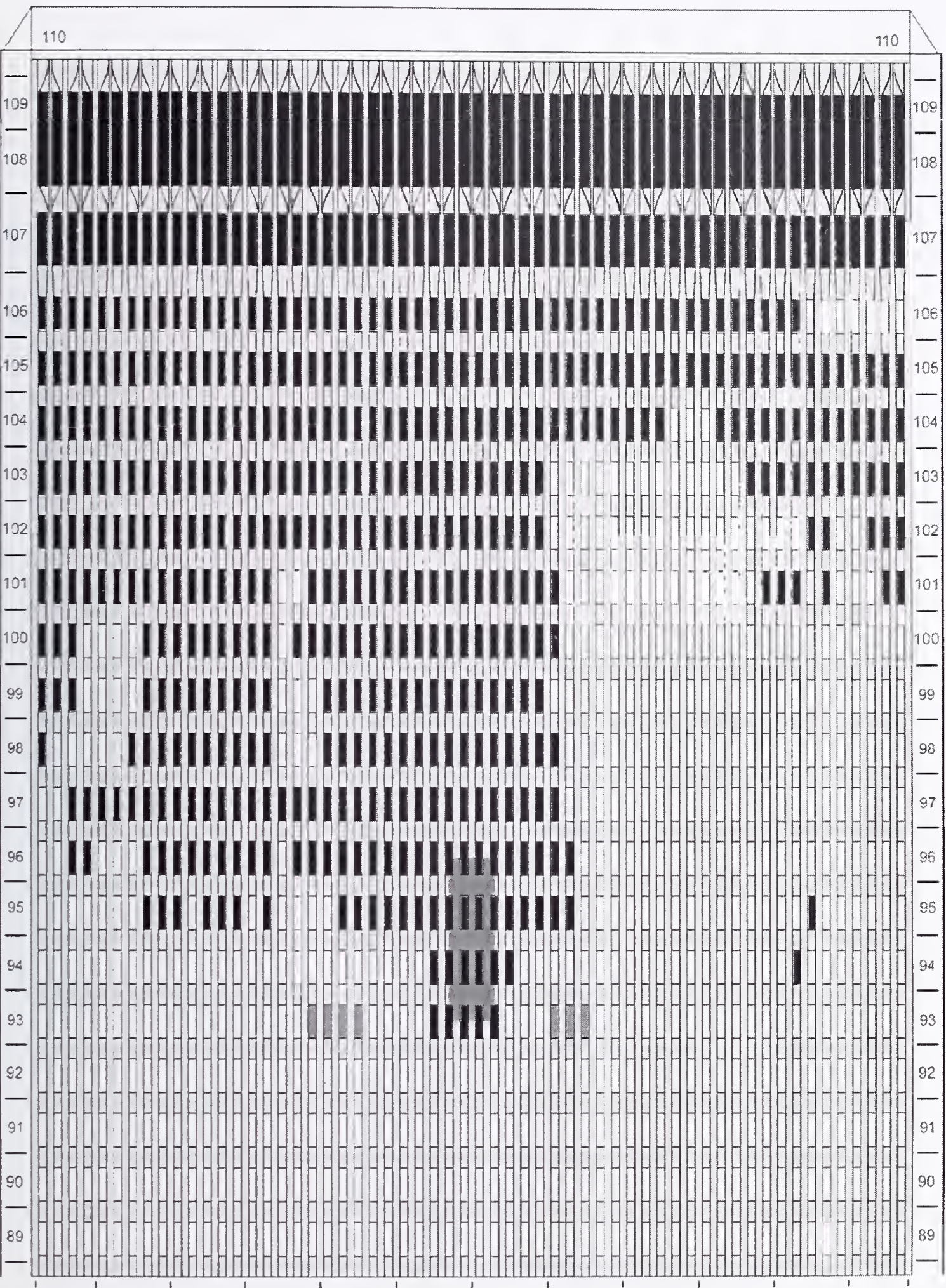

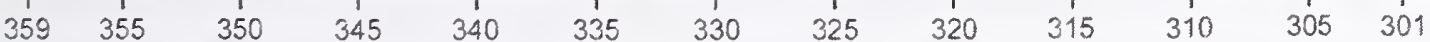

Figure E-10. Diagram of the south face of WTC 1 for floors 89 to 110 at 9:02 a.m. showing windows where smoke was observed and those that were hidden from view. 
WTC 1, South Face $\quad$ 9:08 a.m.

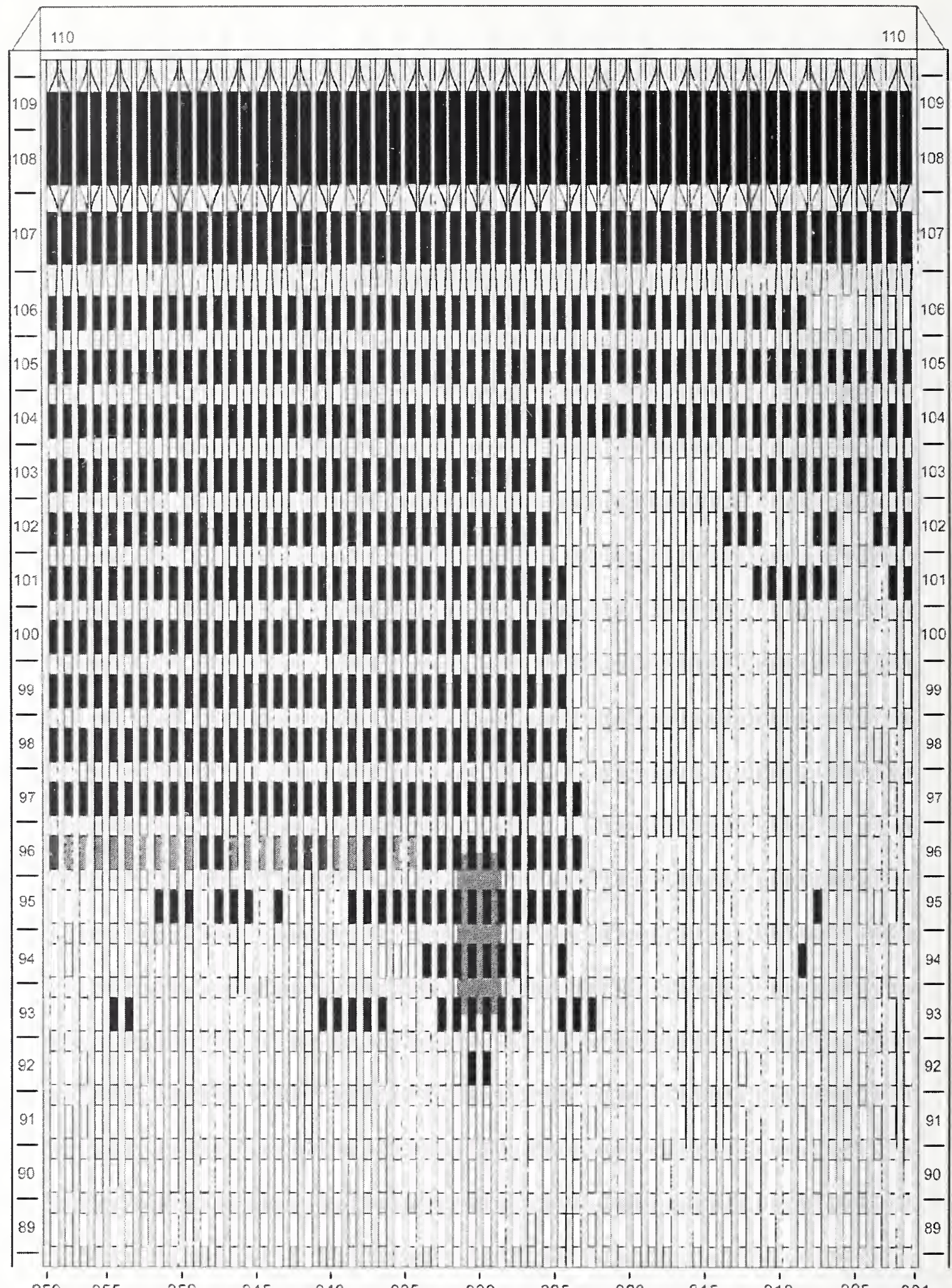

Figure E-11. Diagram of the south face of WTC 1 for floors 89 to 110 at 9:08 a.m. showing the condition of windows and locations of fires. 


$$
\text { WTC 1, South Face 9:08 a.m. }
$$

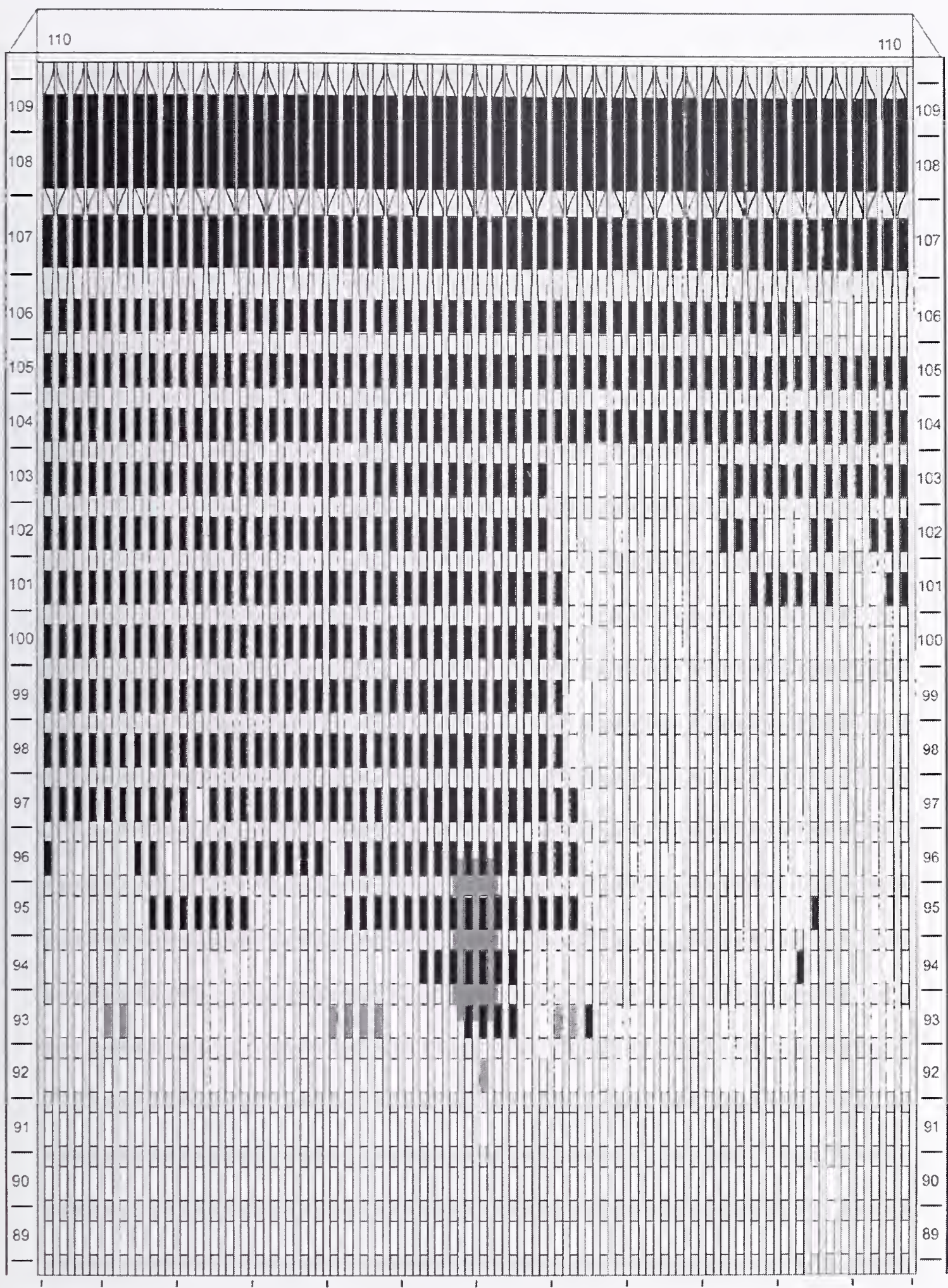

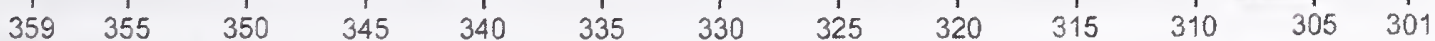

Figure E-12. Diagram of the south face of WTC 1 for floors 89 to 110 at 9:08 a.m. showing windows where smoke was observed and those that were hidden from view. 


$$
\text { WTC 1, South Face } \quad 9: 10 \text { a.m. }
$$

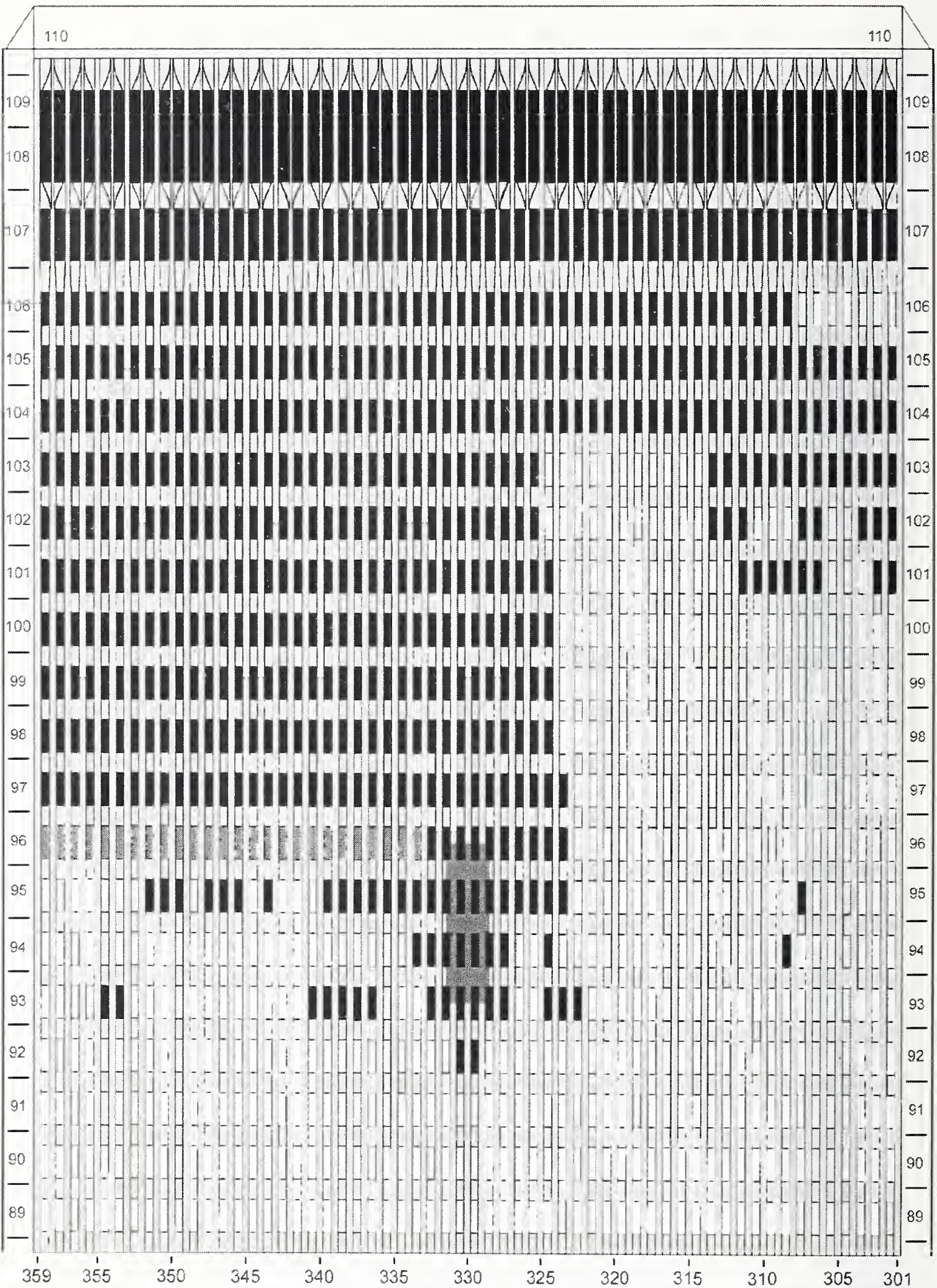

Figure E-13. Diagram of the south face of WTC 1 for floors 89 to 110 at 9:10 a.m. showing the condition of windows and locations of fires. 


$$
\text { WTC 1, South Face 9:10 a.m. }
$$

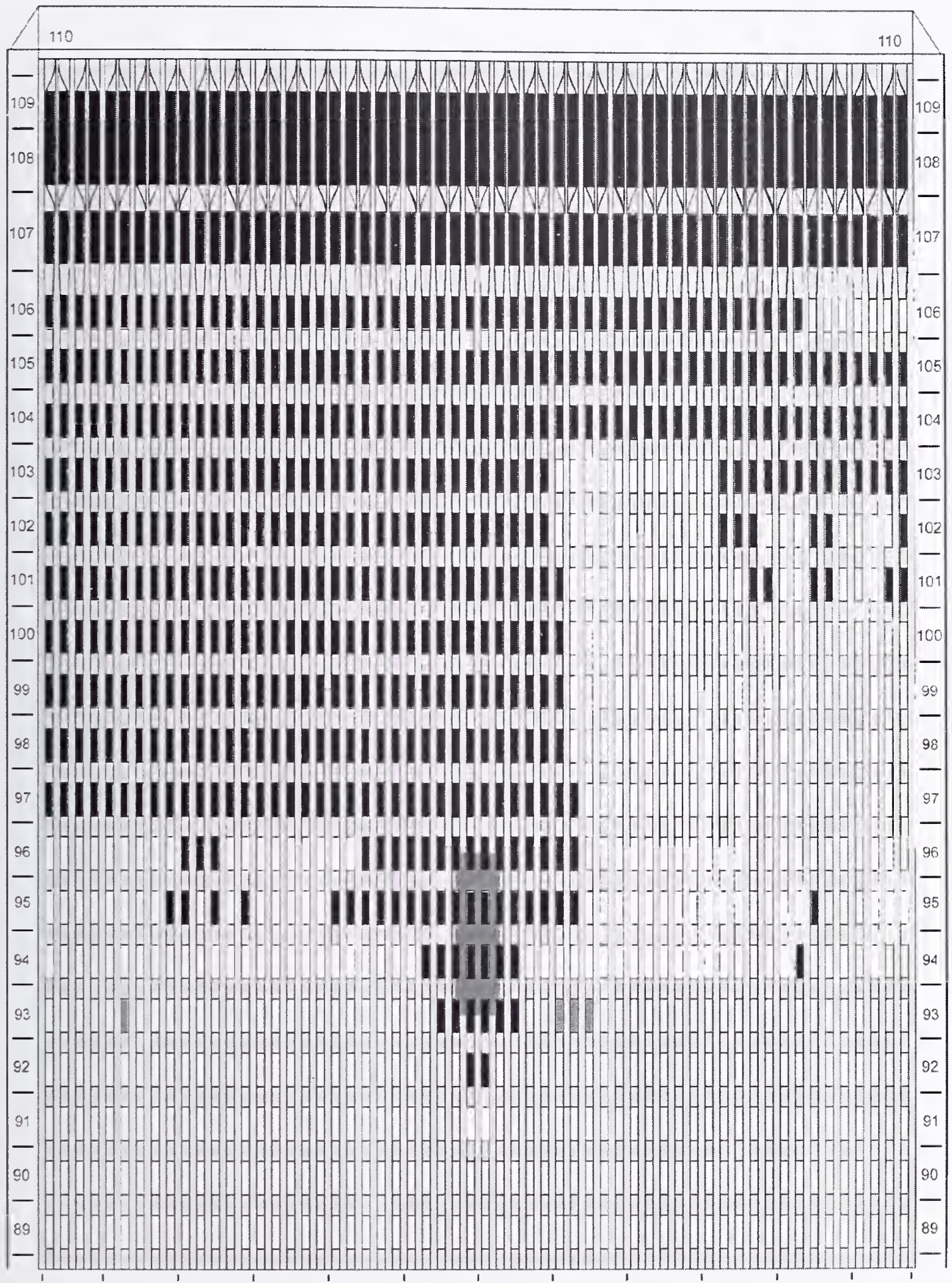

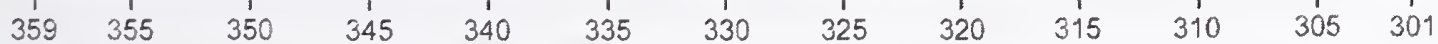

Figure E-14. Diagram of the south face of WTC 1 for floors 89 to 110 at 9:10 a.m. showing windows where smoke was observed and those that were hidden from view. 


$$
\text { WTC 1, South Face } \quad 9: 16 \text { a.m. }
$$

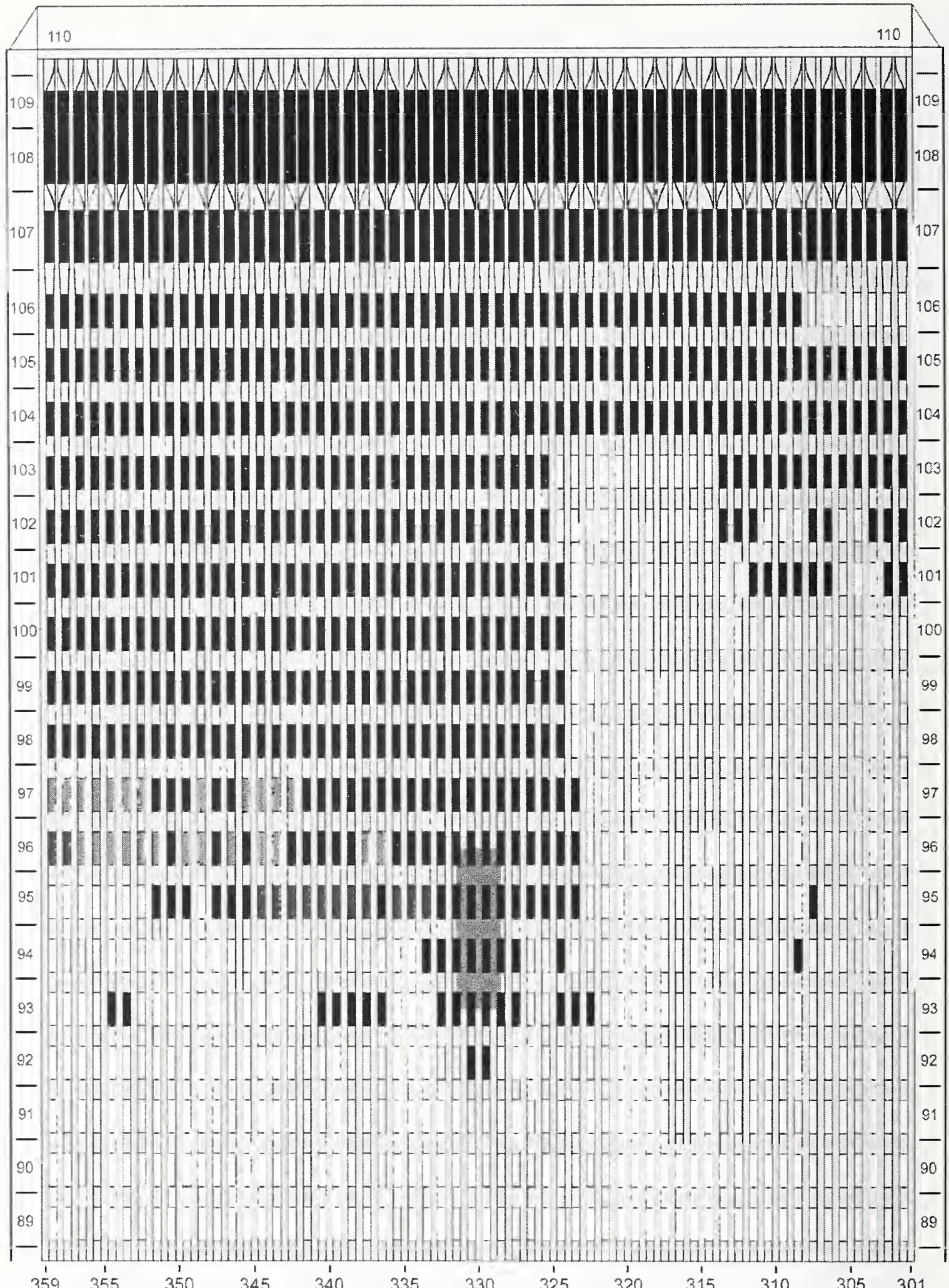

Figure E-15. Diagram of the south face of WTC 1 for floors 89 to 110 at 9:16 a.m. showing the condition of windows and locations of fires. 


$$
\text { WTC 1, South Face 9:16 a.m. }
$$

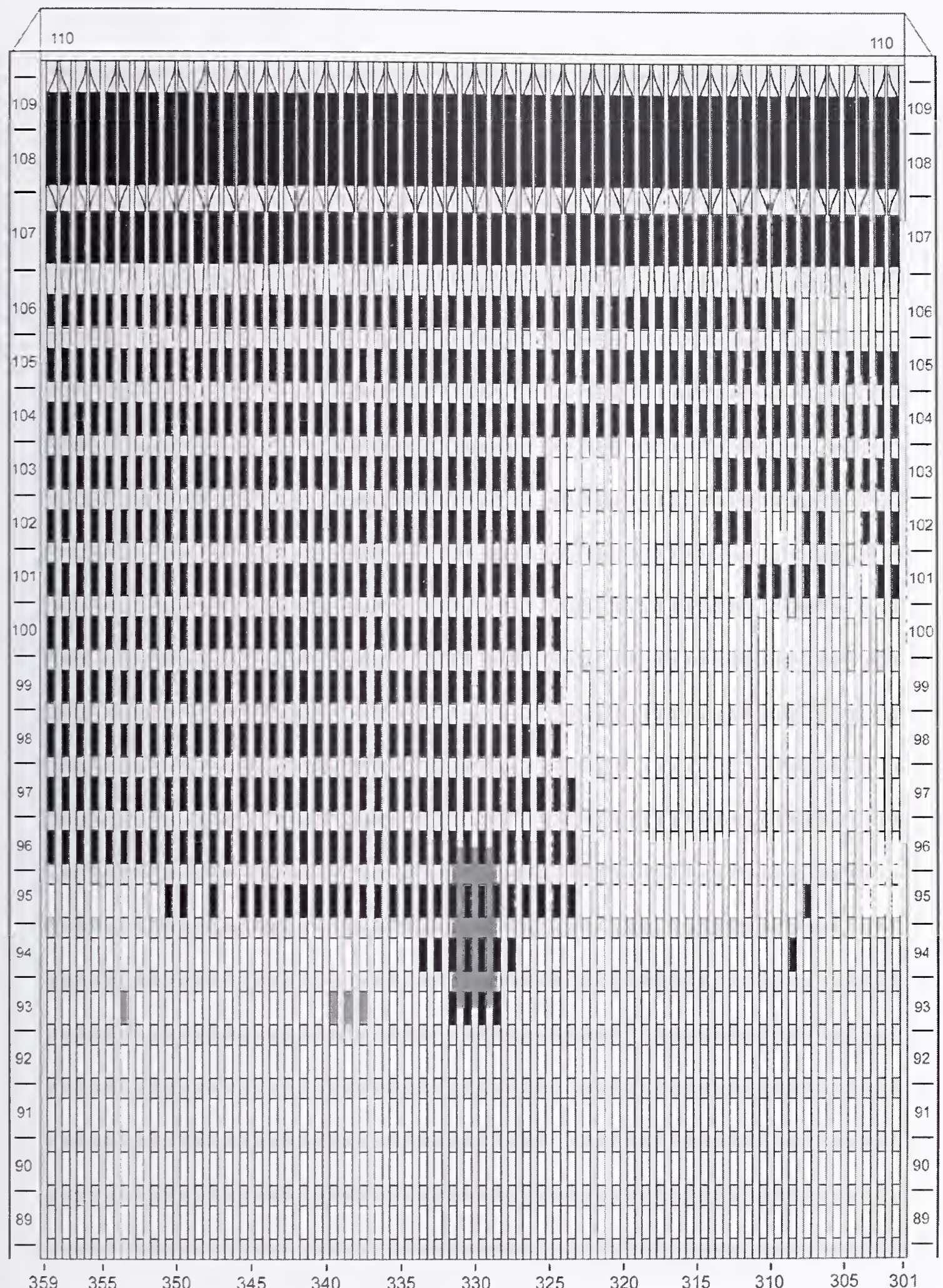

Figure E-16. Diagram of the south face of WTC 1 for floors 89 to 110 at 9:16 a.m. showing windows where smoke was observed and those that were hidden from view. 


$$
\text { WTC 1, South Face } \quad 9: 20 \text { a.m. }
$$

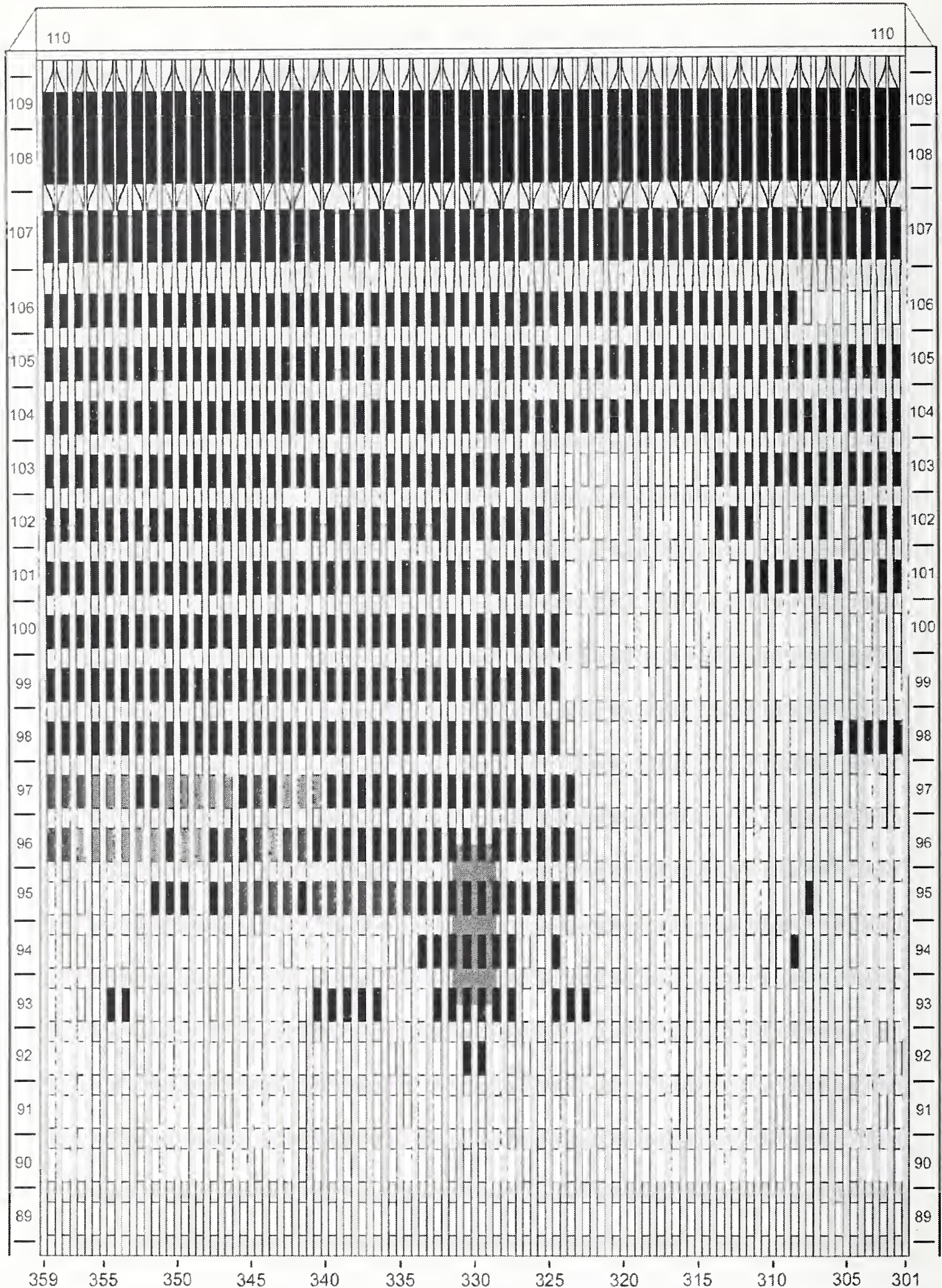

Figure E-17. Diagram of the south face of WTC 1 for floors 89 to 110 at 9:20 a.m. showing the condition of windows and locations of fires. 


$$
\text { WTC 1, South Face } \quad 9: 20 \text { a.m. }
$$

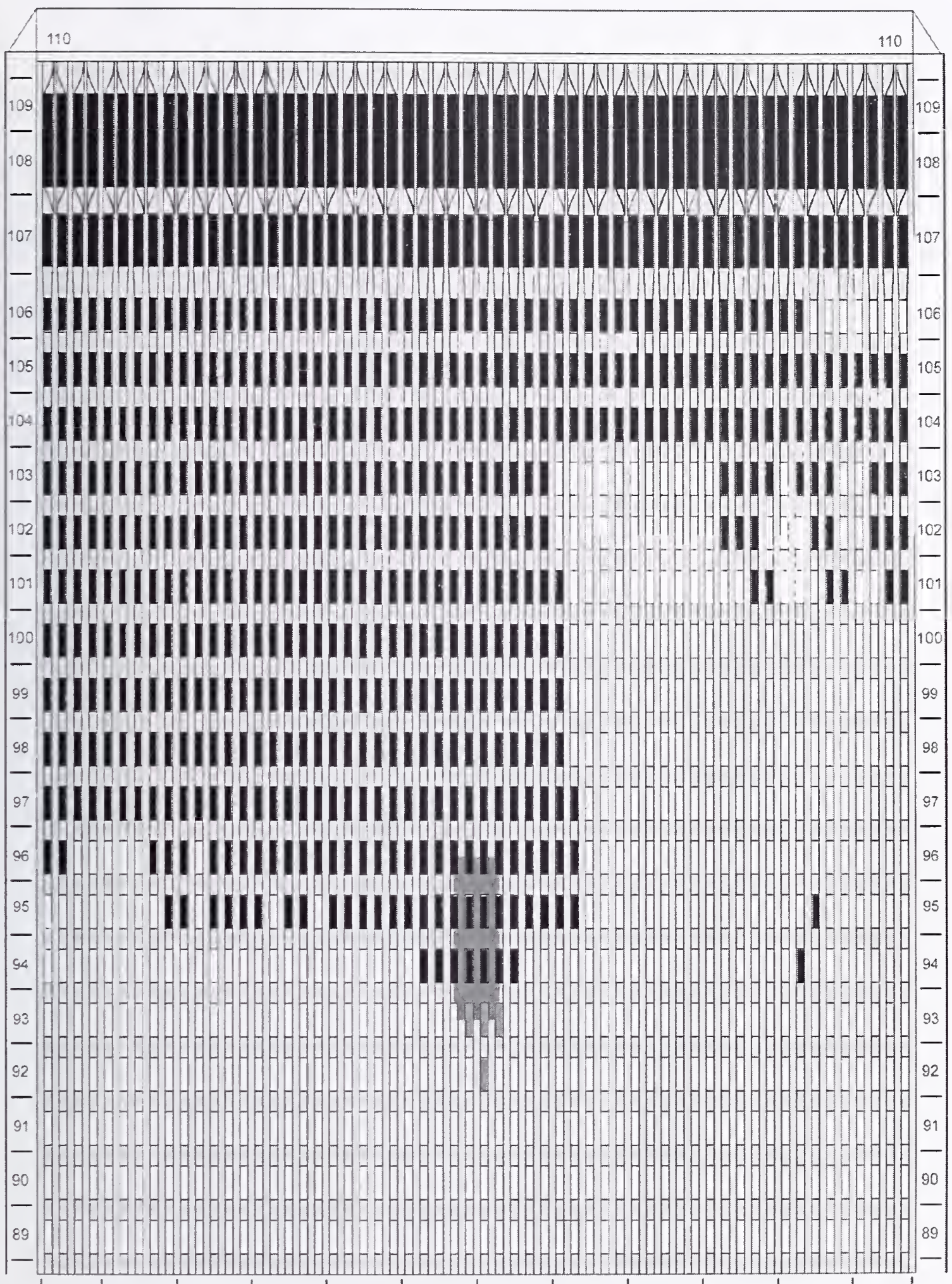

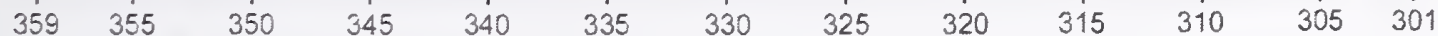

Figure E-18. Diagram of the south face of WTC 1 for floors 89 to 110 at 9:20 a.m. showing windows where smoke was observed and those that were hidden from view. 


$$
\text { WTC 1, South Face } \quad 9: 26 \text { a.m. }
$$

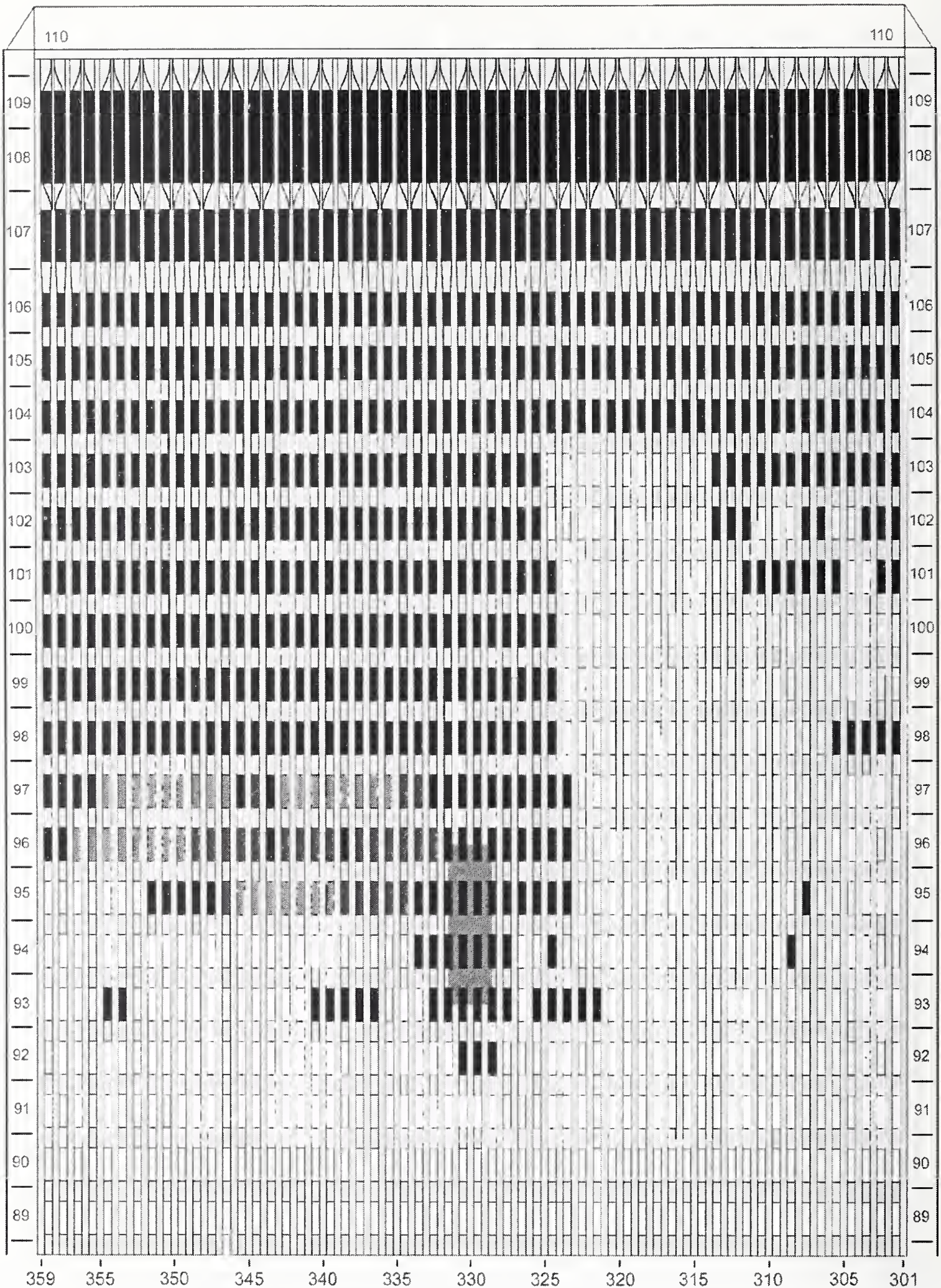

Figure E-19. Diagram of the south face of WTC 1 for floors 89 to 110 at 9:26 a.m. showing the condition of windows and locations of fires. 


$$
\text { WTC 1, South Face 9:26 a.m. }
$$

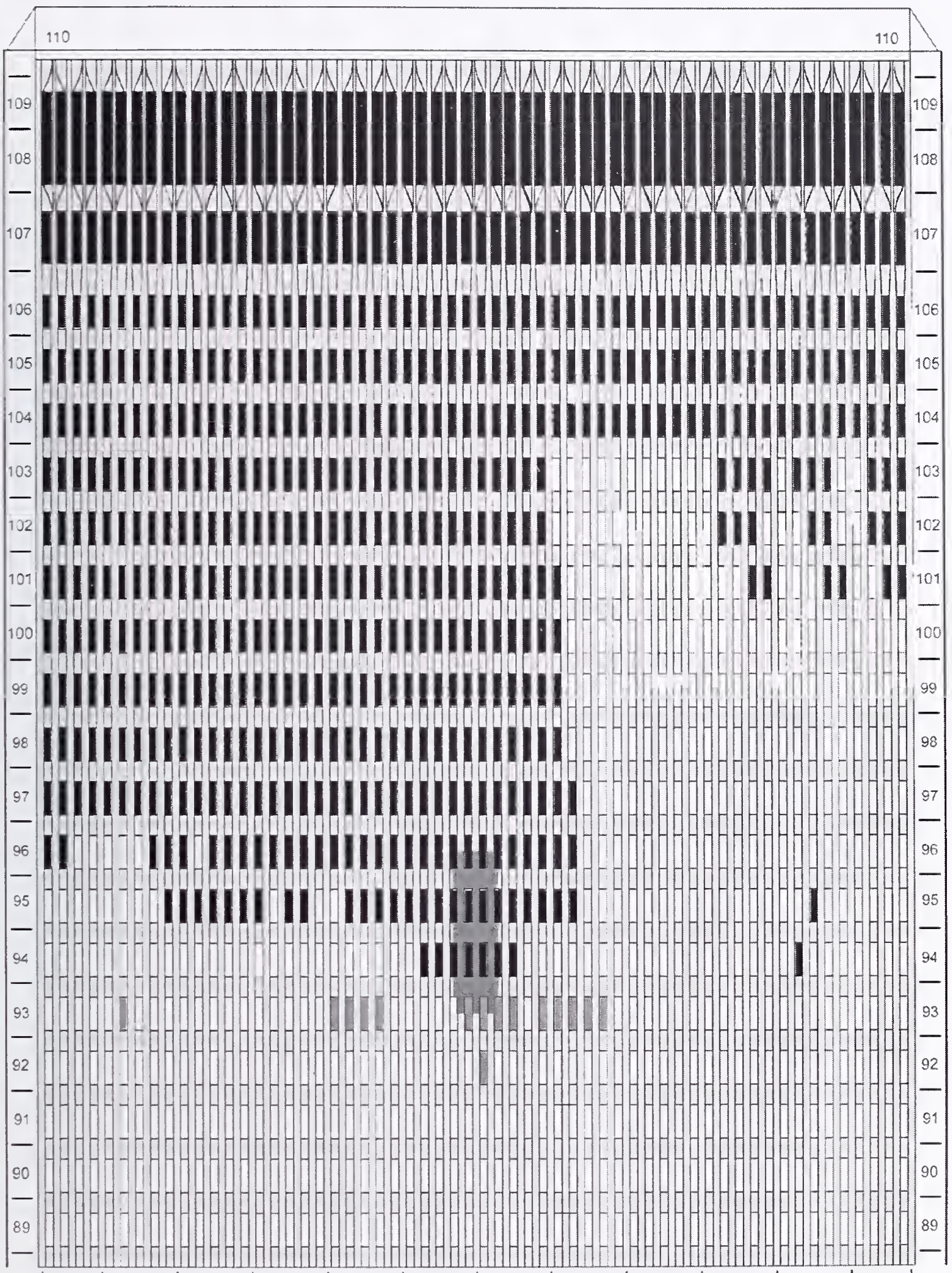

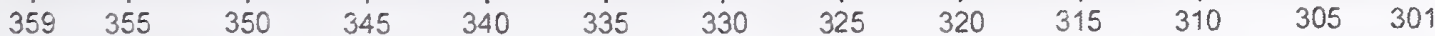

Figure E-20. Diagram of the south face of WTC 1 for floors 89 to 110 at 9:26 a.m. showing windows where smoke was observed and those that were hidden from view. 


\section{WTC 1, South Face $\quad 9: 32$ a.m.}



Figure E-21. Diagram of the south face of WTC 1 for floors 89 to 110 at $9: 32$ a.m. showing the condition of windows and locations of fires. 


$$
\text { WTC 1, South Face } \quad 9: 32 \text { a.m. }
$$

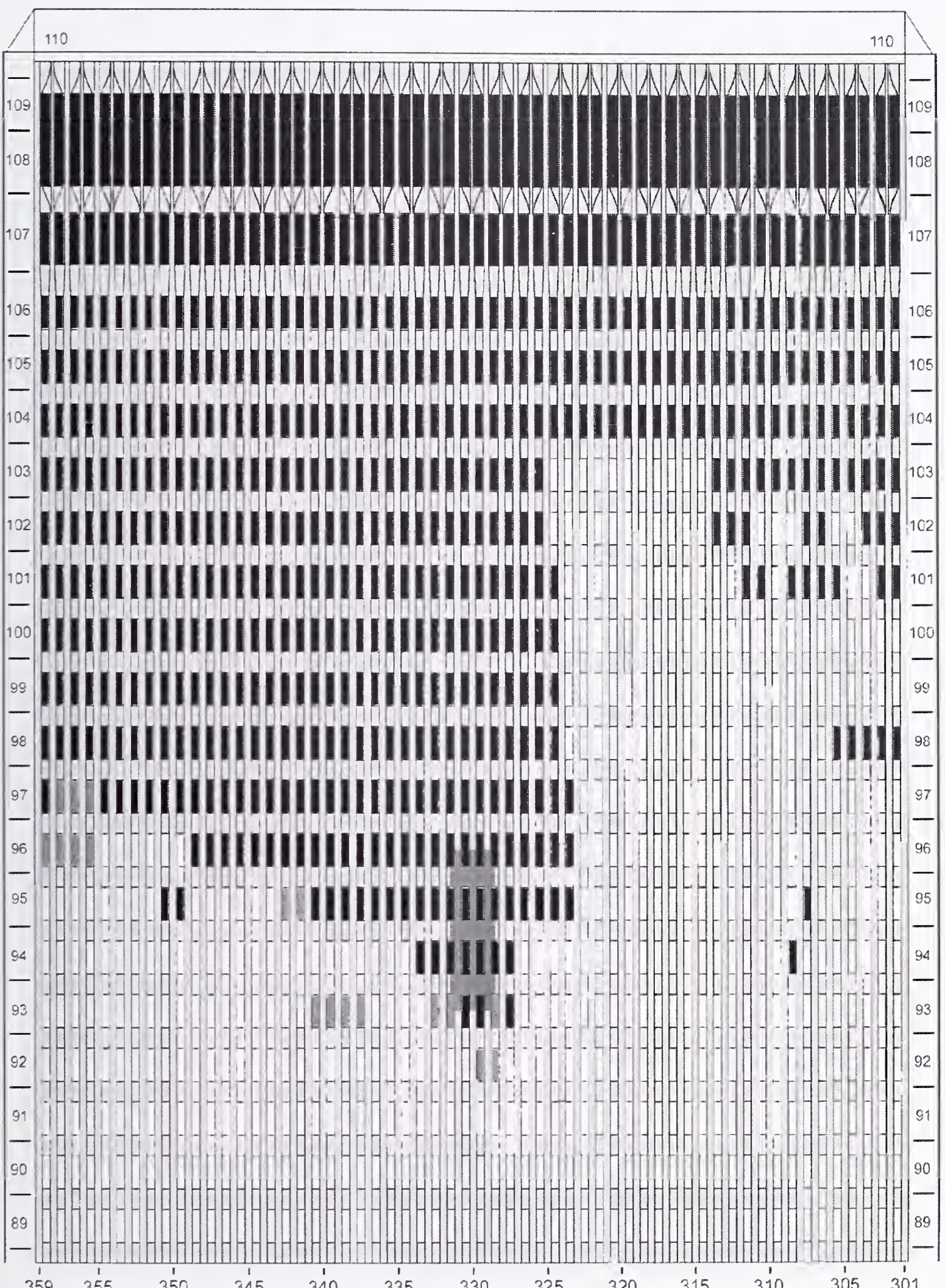

Figure E-22. Diagram of the south face of WTC 1 for floors 89 to 110 at 9:32 a.m. showing windows where smoke was observed and those that were hidden from view. 


\section{WTC 1, South Face $\quad$ 9:34 a.m.}

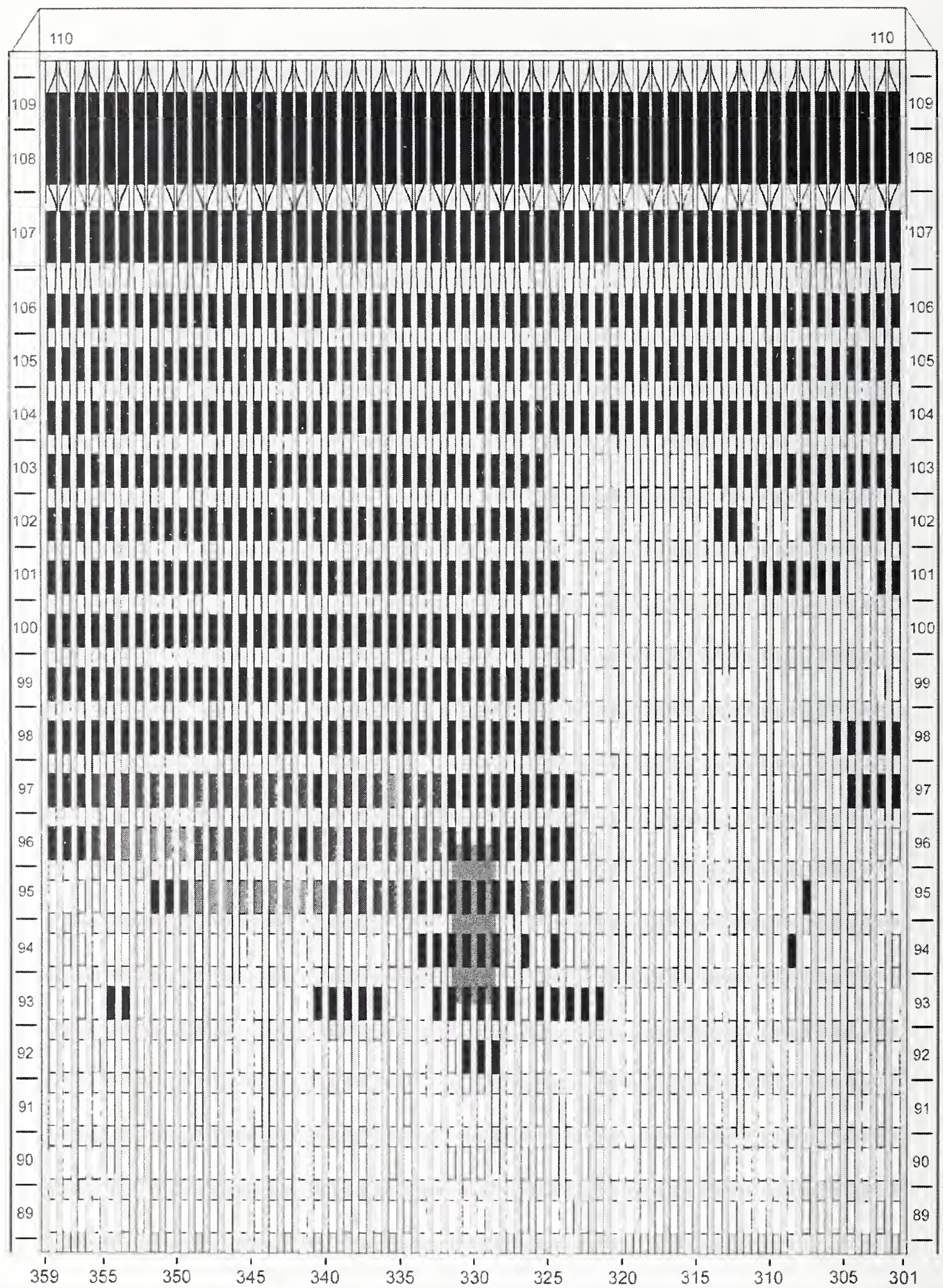

Figure E-23. Diagram of the south face of WTC 1 for floors 89 to 110 at 9:34 a.m. showing the condition of windows and locations of fires. 


\section{WTC 1, South Face $\quad 9: 34$ a.m.}

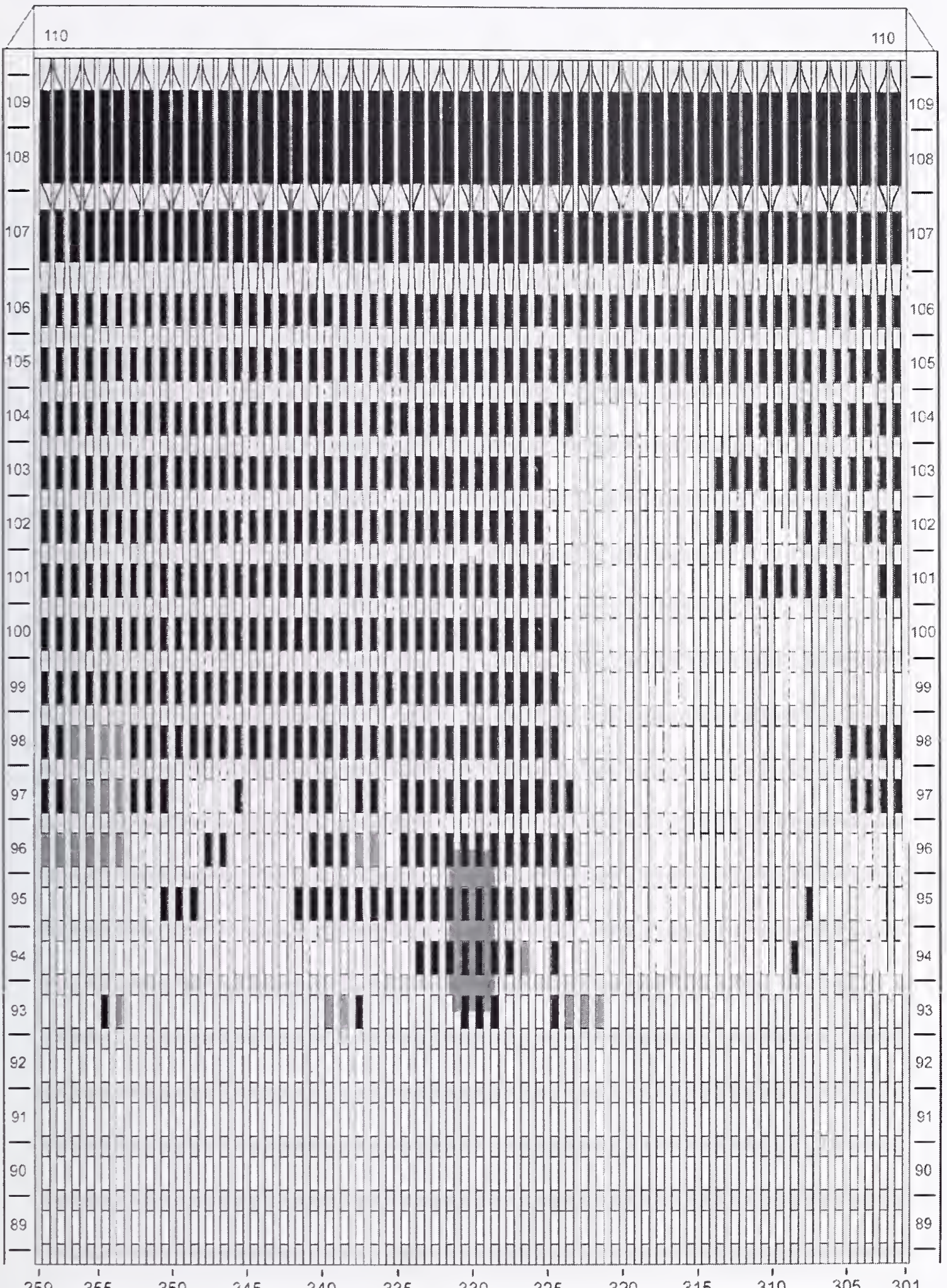

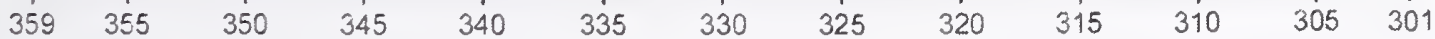

Figure E-24. Diagram of the south face of WTC 1 for floors 89 to 110 at 9:34 a.m. showing windows where smoke was observed and those that were hidden from view. 
WTC 1, South Face $\quad 9: 38$ a.m.

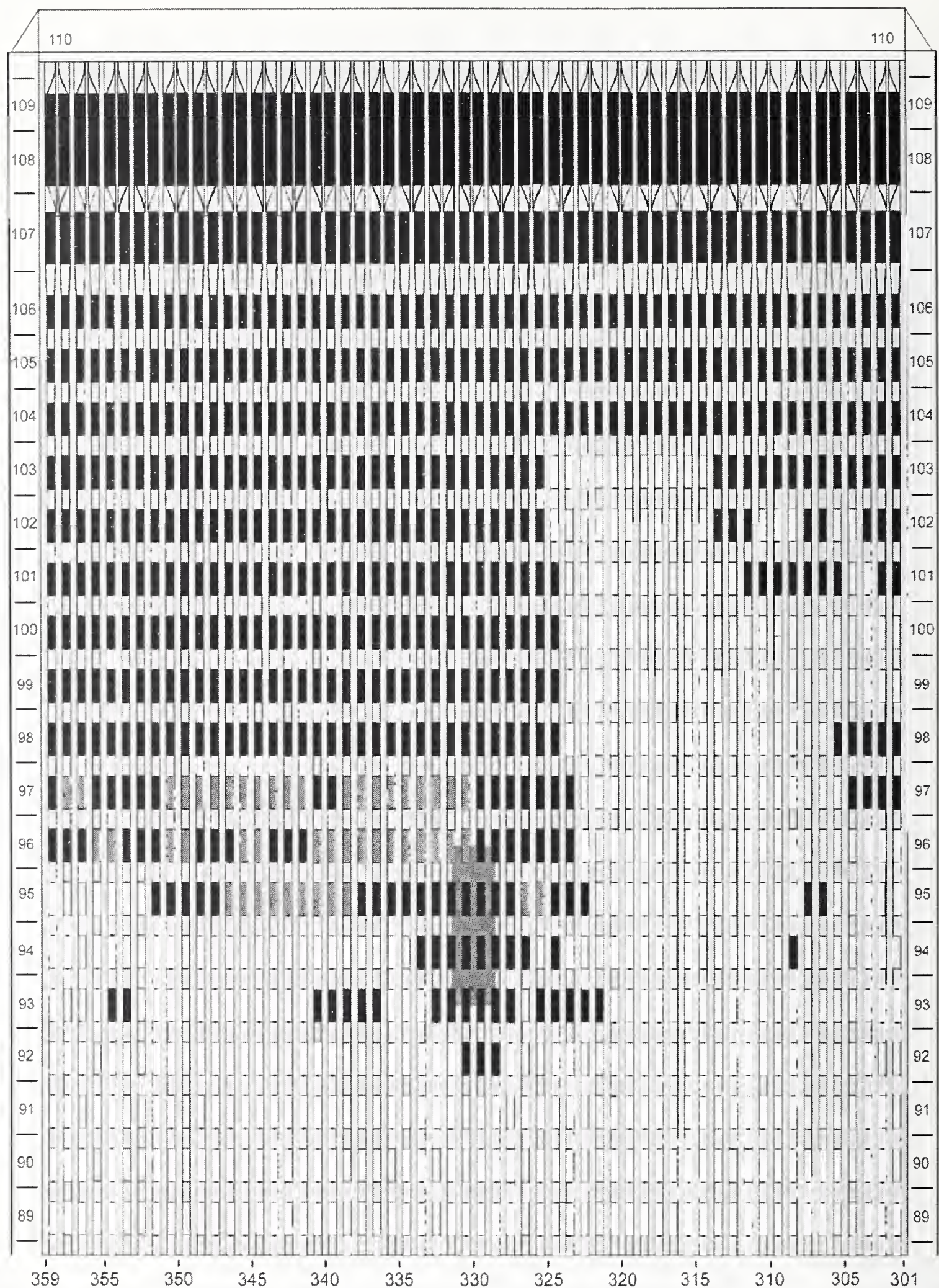

Figure E-25. Diagram of the south face of WTC 1 for floors 89 to 110 at 9:38 a.m. showing the condition of windows and locations of fires. 


\section{WTC 1, South Face $\quad$ 9:38 a.m.}

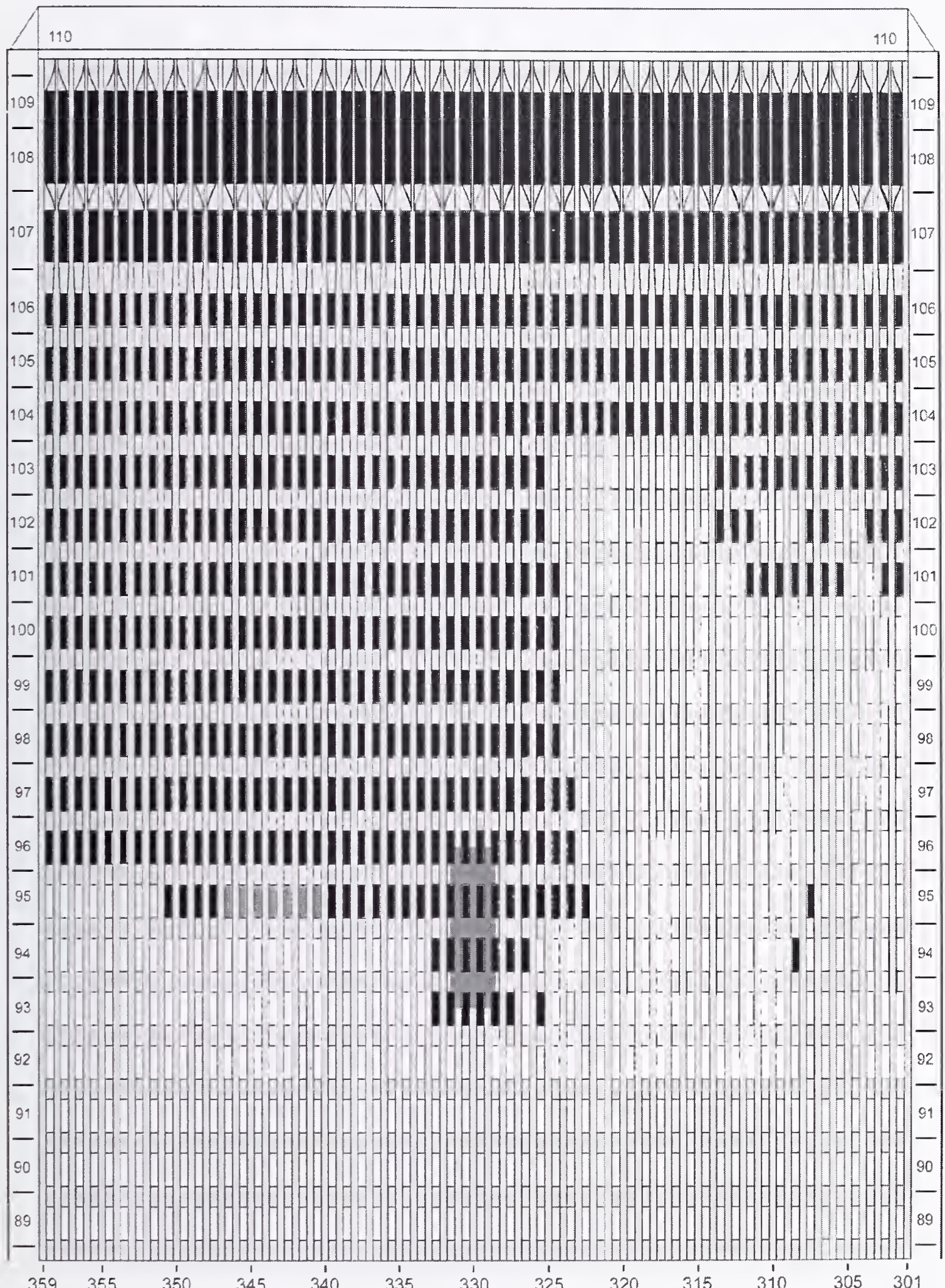

Figure E-26. Diagram of the south face of WTC 1 for floors 89 to 110 at 9:38 a.m. showing windows where smoke was observed and those that were hidden from view. 
WTC 1, South Face $\quad 9: 42$ a.m.

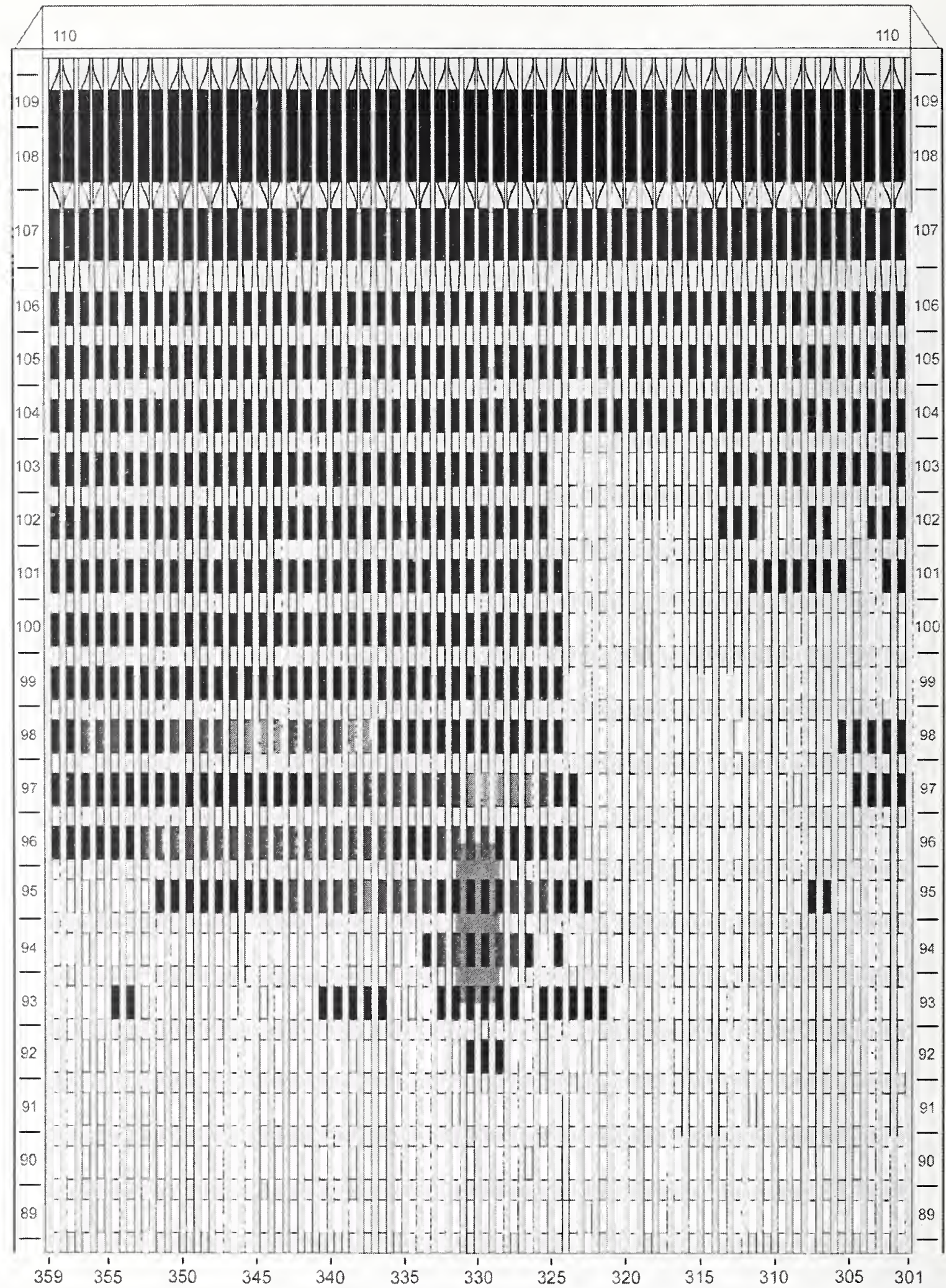

Figure E-27. Diagram of the south face of WTC 1 for floors 89 to 110 at 9:42 a.m. showing the condition of windows and locations of fires. 


$$
\text { WTC 1, South Face 9:42 a.m. }
$$

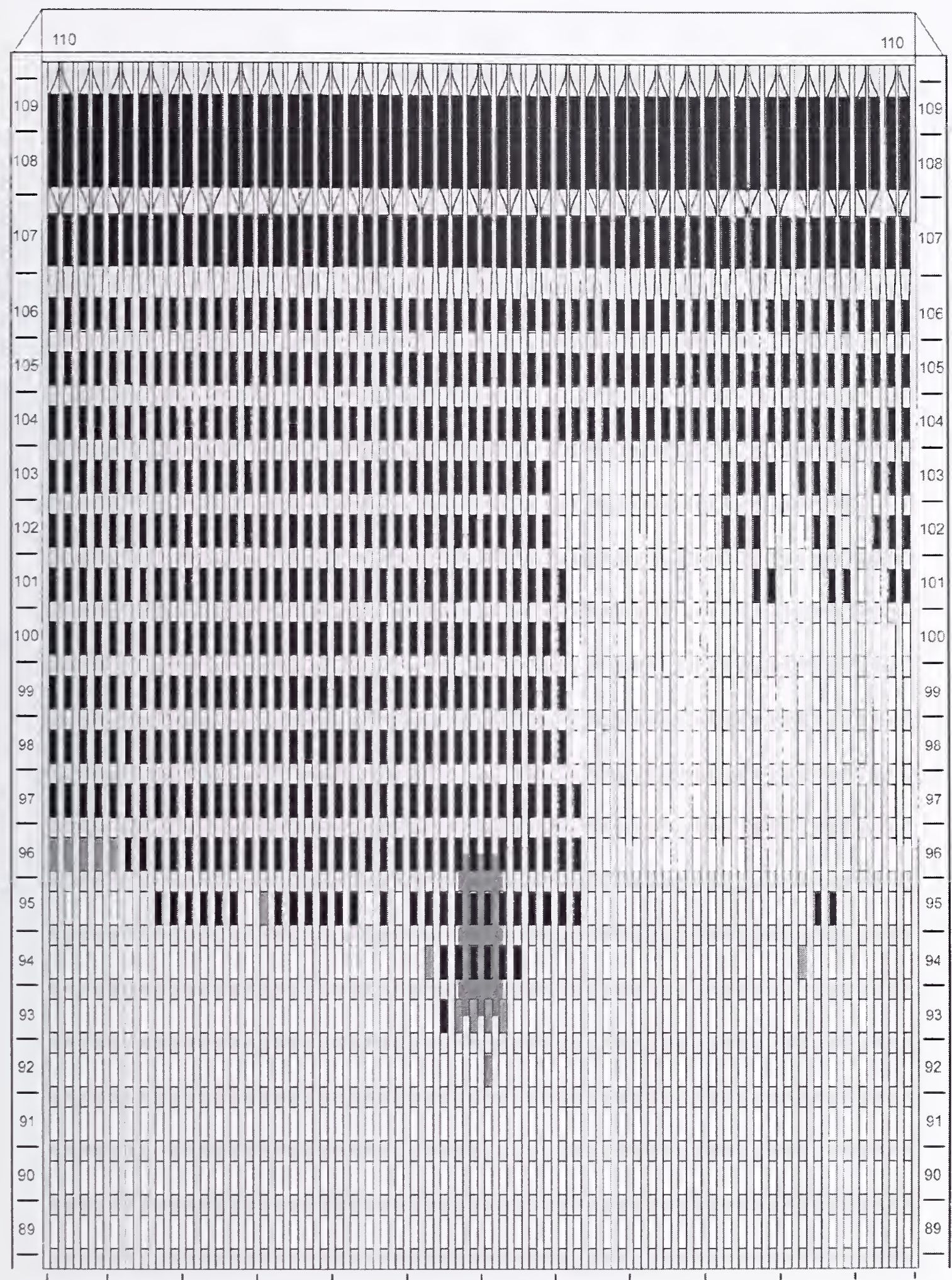

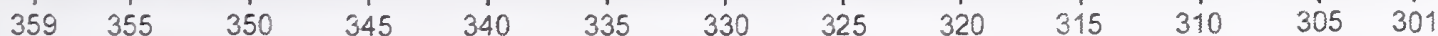

Figure E-28. Diagram of the south face of WTC 1 for floors 89 to 110 at 9:42 a.m. showing windows where smoke was observed and those that were hidden from view. 


$$
\text { WTC 1, South Face 9:44 a.m. }
$$

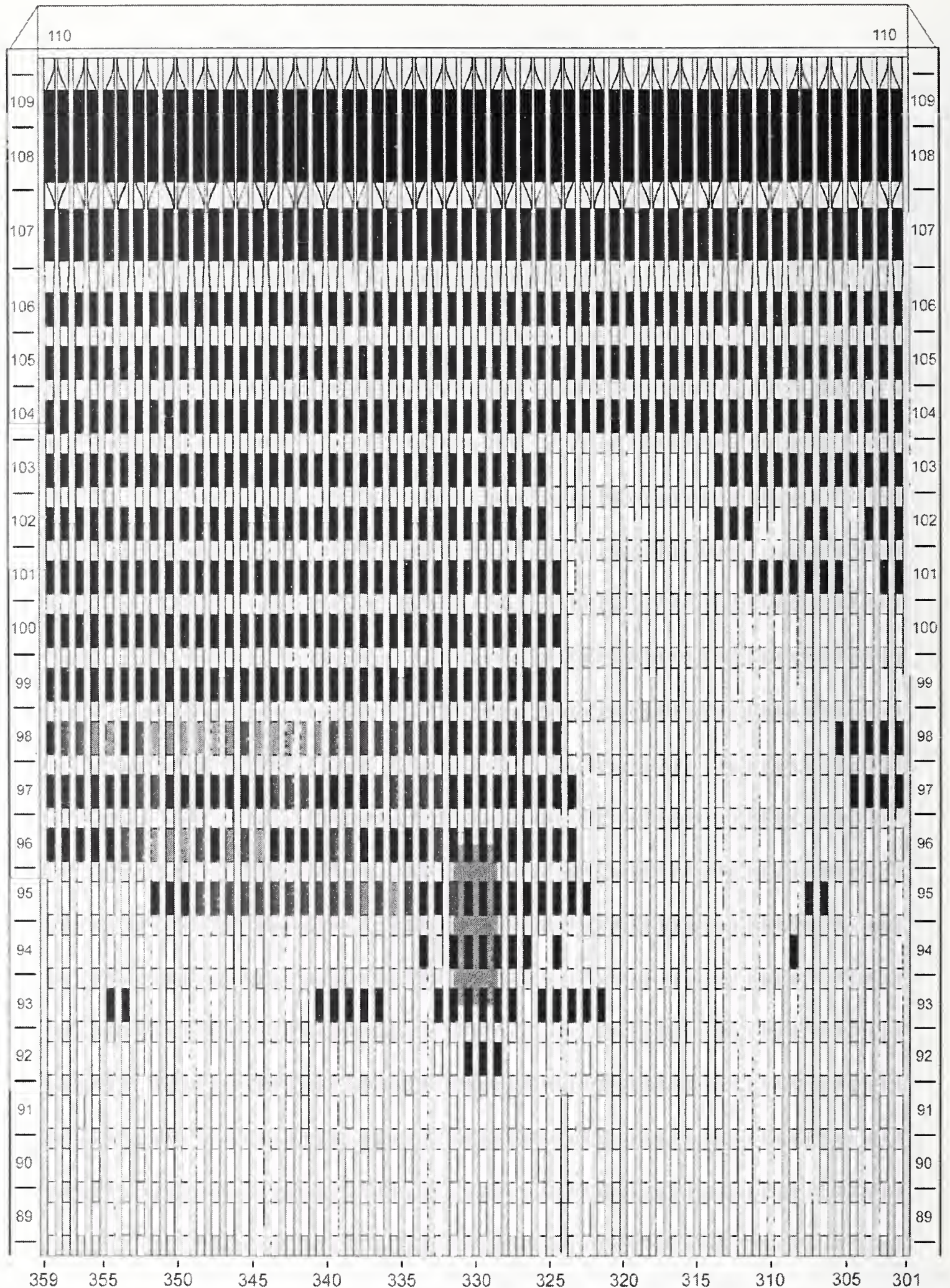

Figure E-29. Diagram of the south face of WTC 1 for floors 89 to 110 at 9:44 a.m. showing the condition of windows and locations of fires. 


$$
\text { WTC 1, South Face 9:44 a.m. }
$$

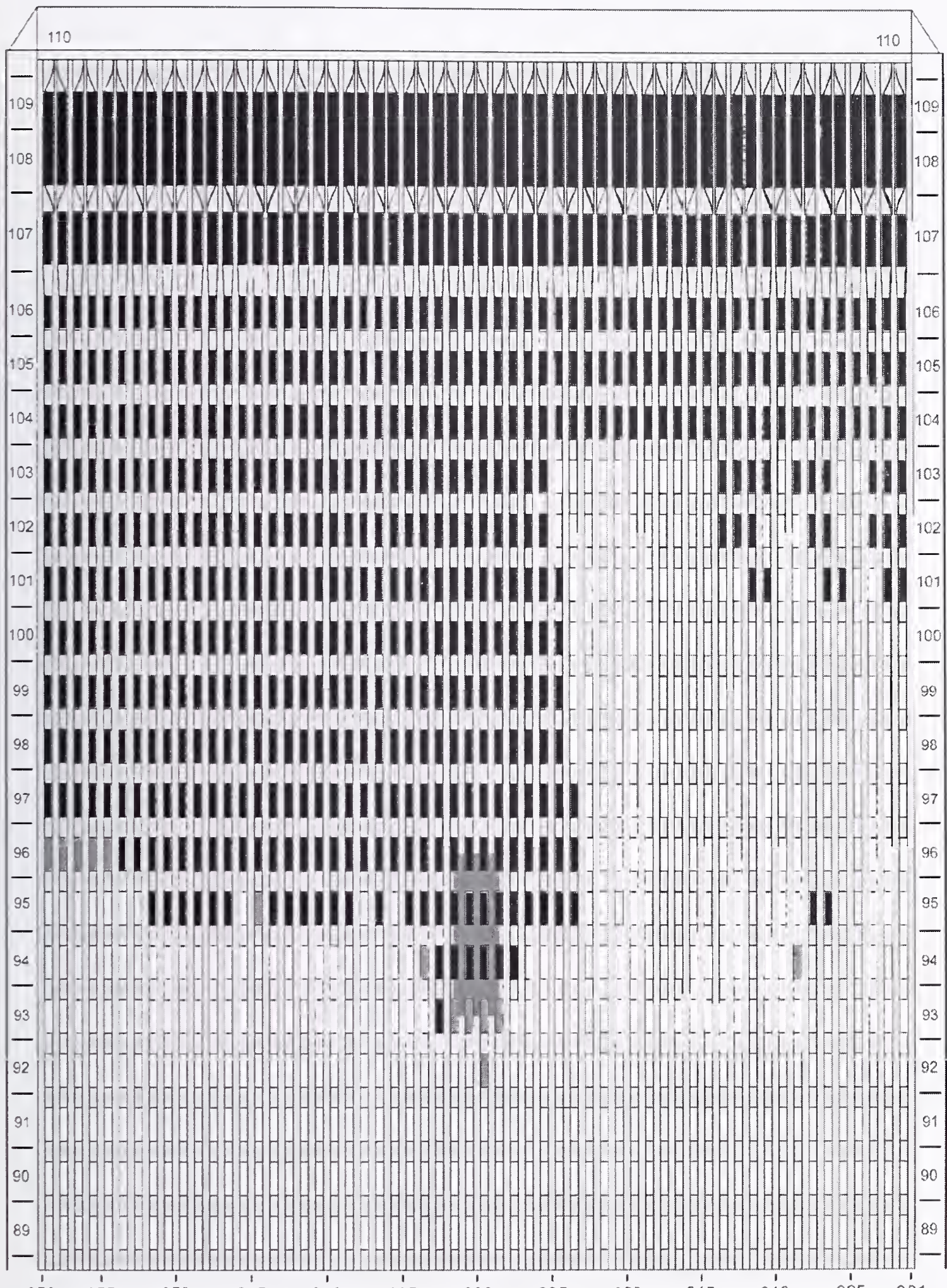

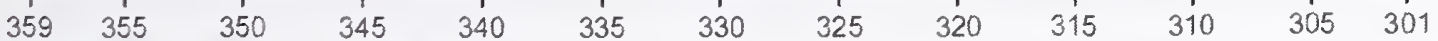

Figure E-30. Diagram of the south face of WTC 1 for floors 89 to 110 at 9:44 a.m. showing windows where smoke was observed and those that were hidden from view. 
WTC 1, South Face

9:46 a.m.

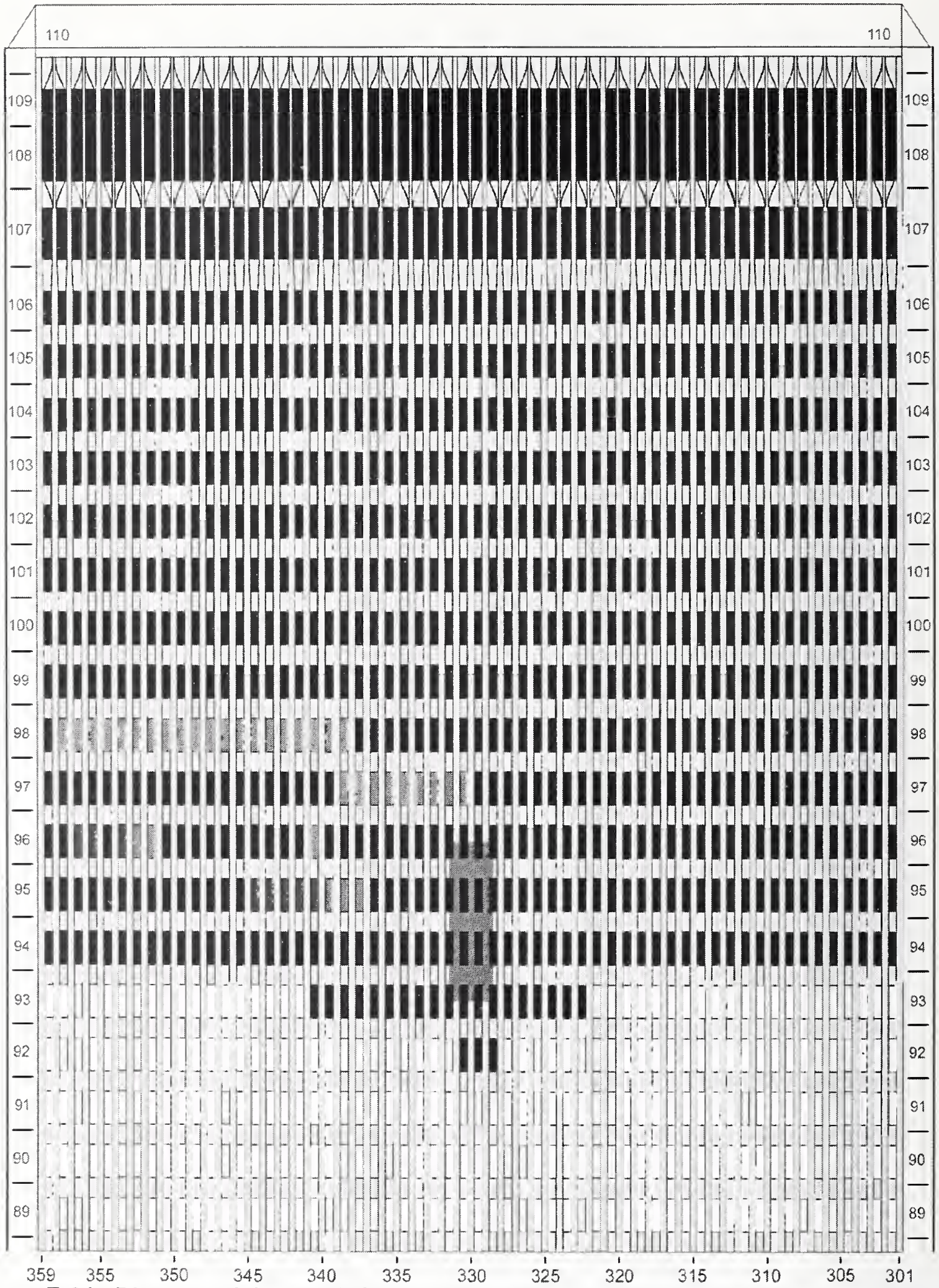

Figure E-31. Diagram of the south face of WTC 1 for floors 89 to 110 at 9:46 a.m. showing the condition of windows and locations of fires. 


$$
\text { WTC 1, South Face } \quad 9: 46 \text { a.m. }
$$

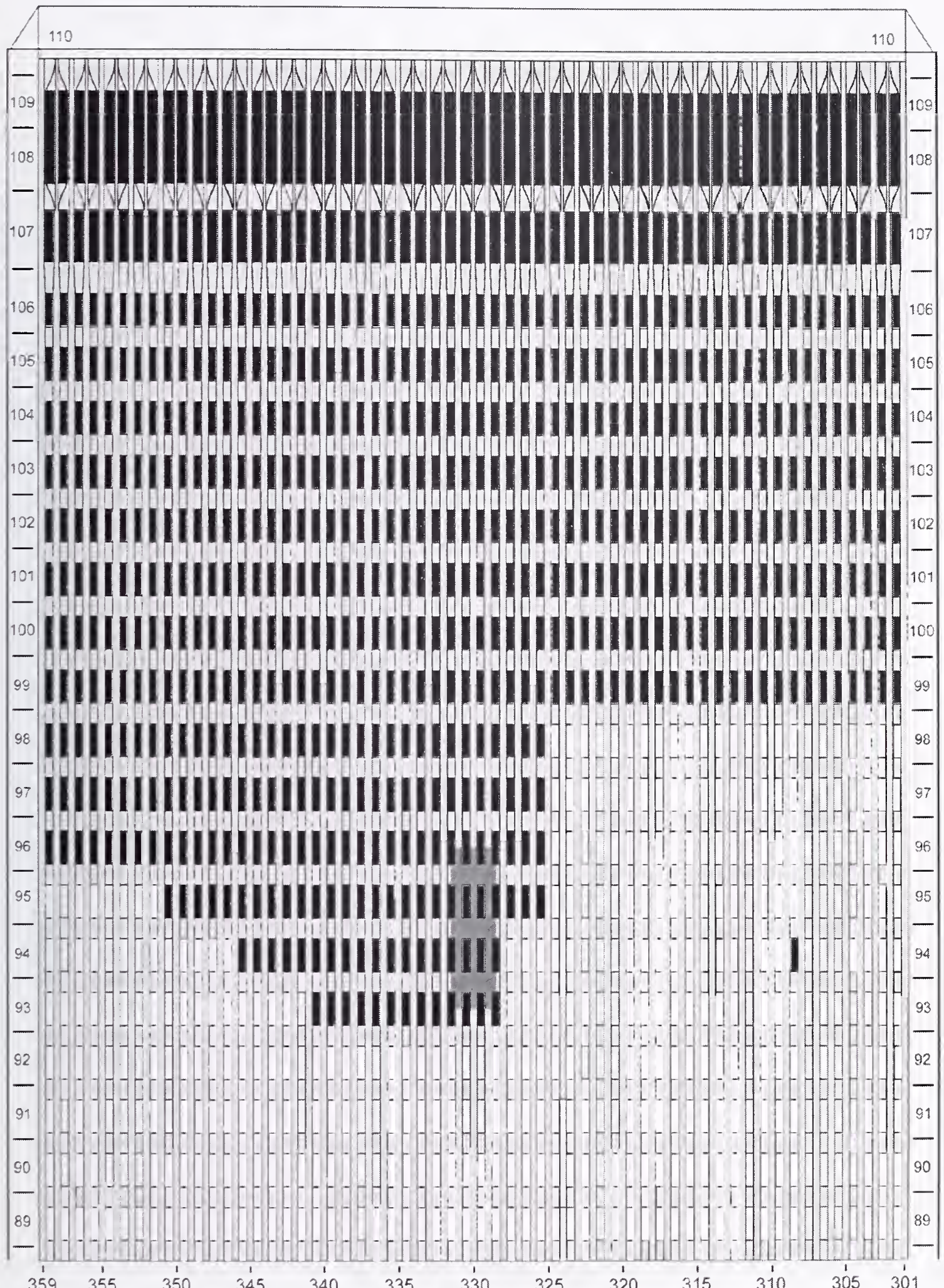

Figure E-32. Diagram of the south face of WTC 1 for floors 89 to 110 at 9:46 a.m. showing windows where smoke was observed and those that were hidden from view. 


$$
\text { WTC 1, South Face } \quad 9: 52 \text { a.m. }
$$



Figure E-33. Diagram of the south face of WTC 1 for floors 89 to 110 at 9:52 a.m. showing the condition of windows and locations of fires. 


$$
\text { WTC 1, South Face } \quad 9: 52 \text { a.m. }
$$

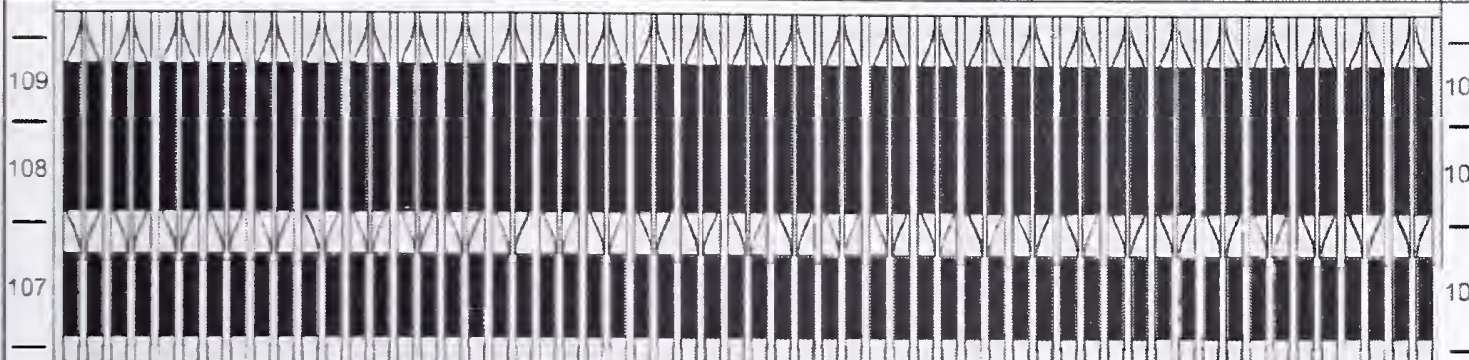

|

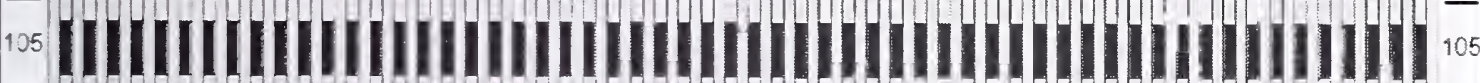

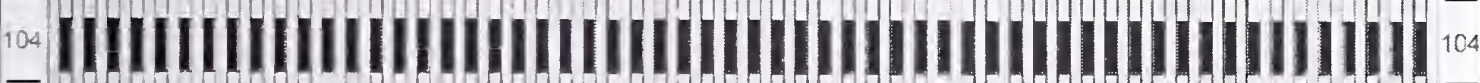

|

|

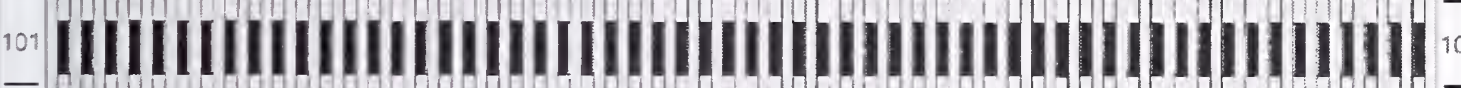

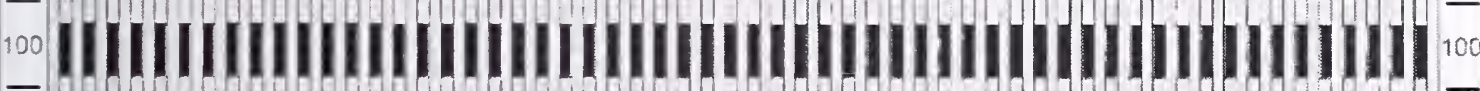

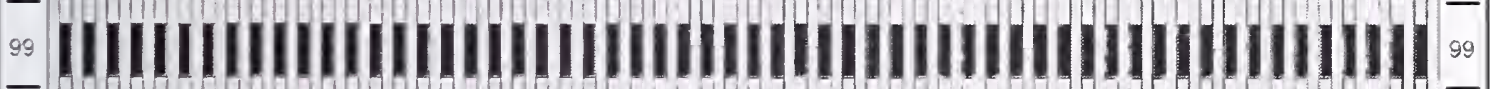

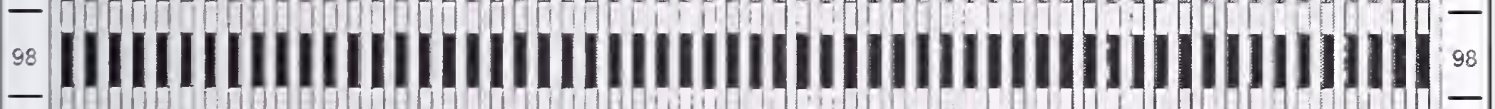

- |

-

-

IIIIIIIIIIIIIIIIIIIIIIIIIIIIIIIIIIIIIIIIIIII

III IIIIIIIIIIIIIIIIIIIIIIIIIIIIII

IIII IIIIII II

92
91
90
89

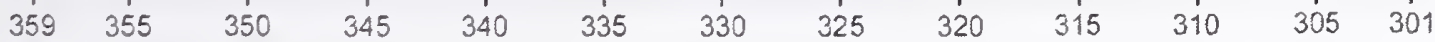

Figure E-34. Diagram of the south face of WTC 1 for floors 89 to 110 at 9:52 a.m. showing windows where smoke was observed and those that were hidden from view. 


$$
\text { WTC 1, South Face } \quad 9: 58 \text { a.m. }
$$

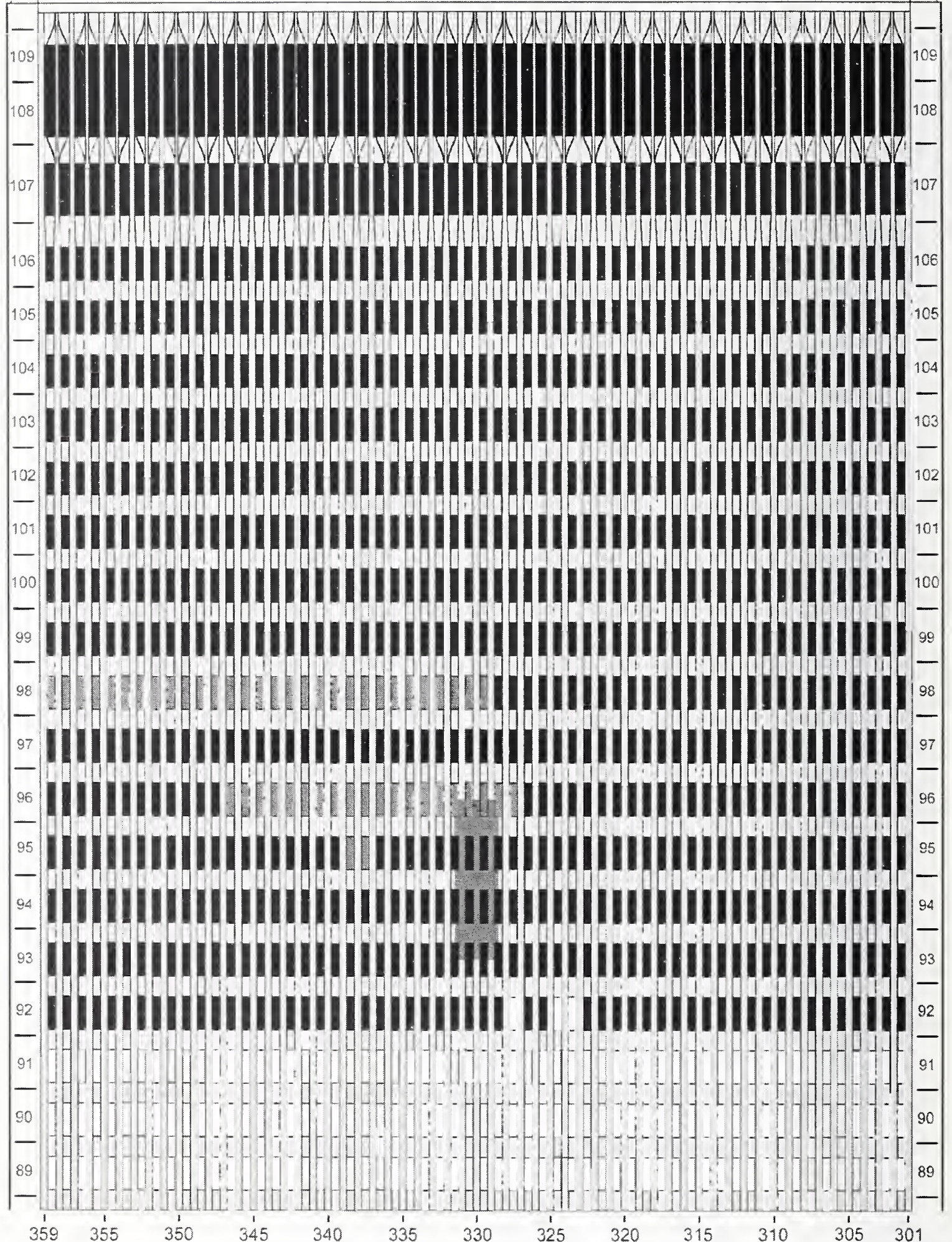

Figure E-35. Diagram of the south face of WTC 1 for floors 89 to 110 at 9:58 a.m. showing the condition of windows and locations of fires. 


$$
\text { WTC 1, South Face } \quad 9: 58 \text { a.m. }
$$

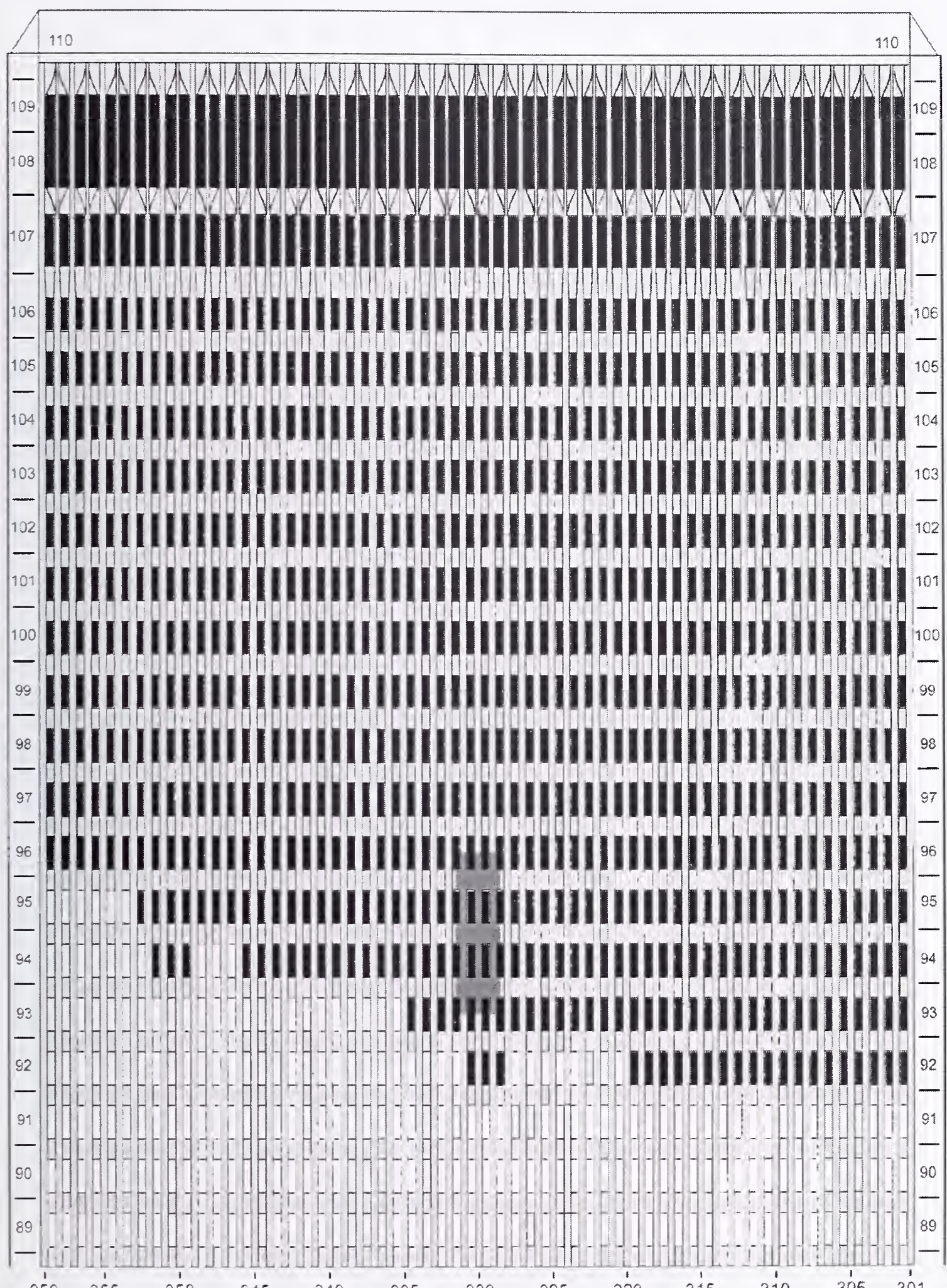

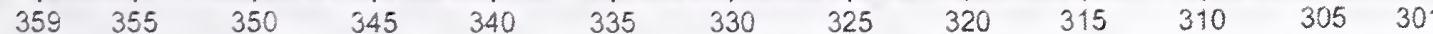

Figure E-36. Diagram of the south face of WTC 1 for floors 89 to 110 at 9:58 a.m. showing windows where smoke was observed and those that were hidden from view. 
WTC 1, South Face 10:00 a.m.

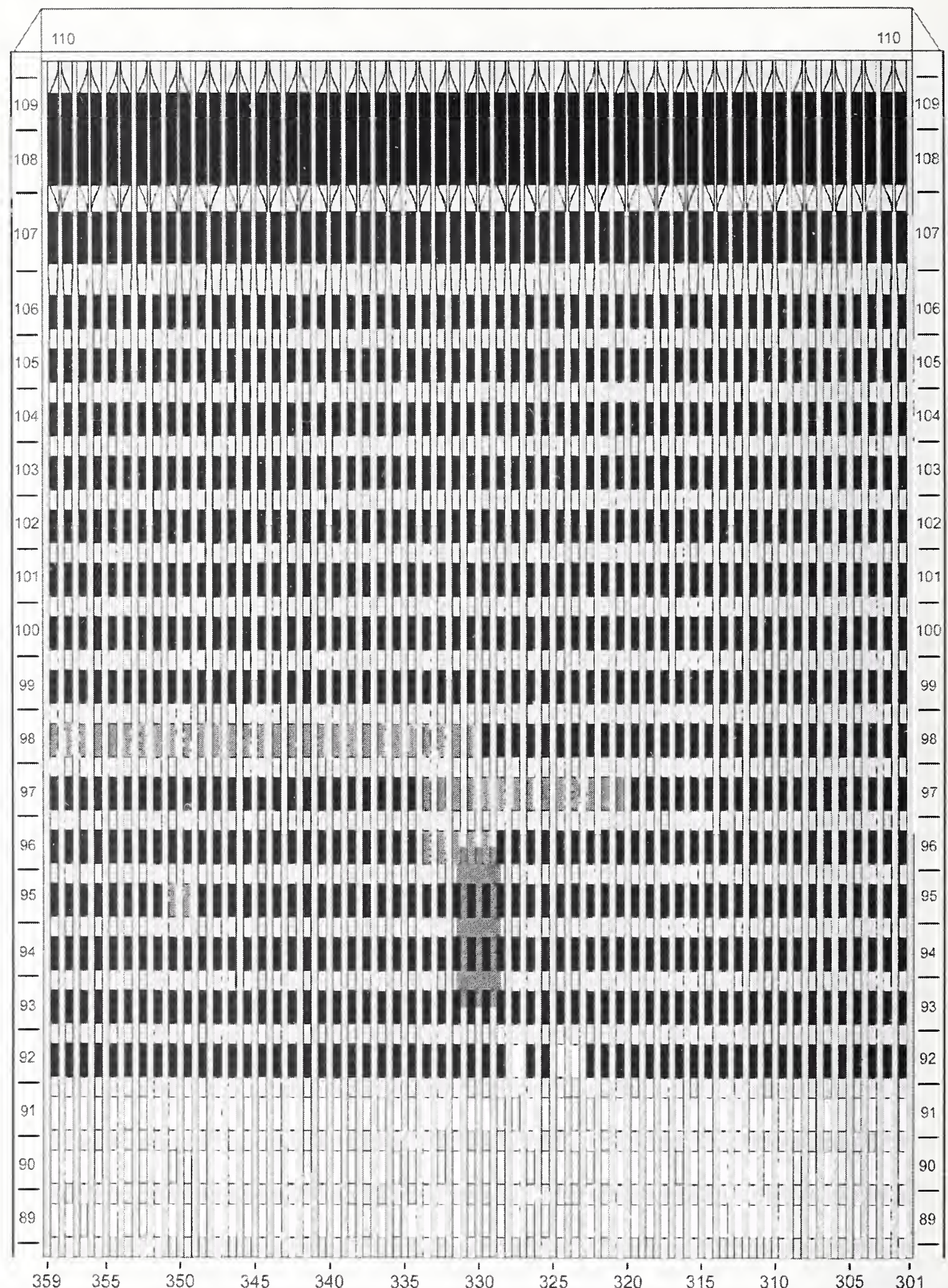

Figure E-37. Diagram of the south face of WTC 1 for floors 89 to 110 at 10:00 a.m. showing the condition of windows and locations of fires. 


$$
\text { WTC 1, South Face 10:00 a.m. }
$$

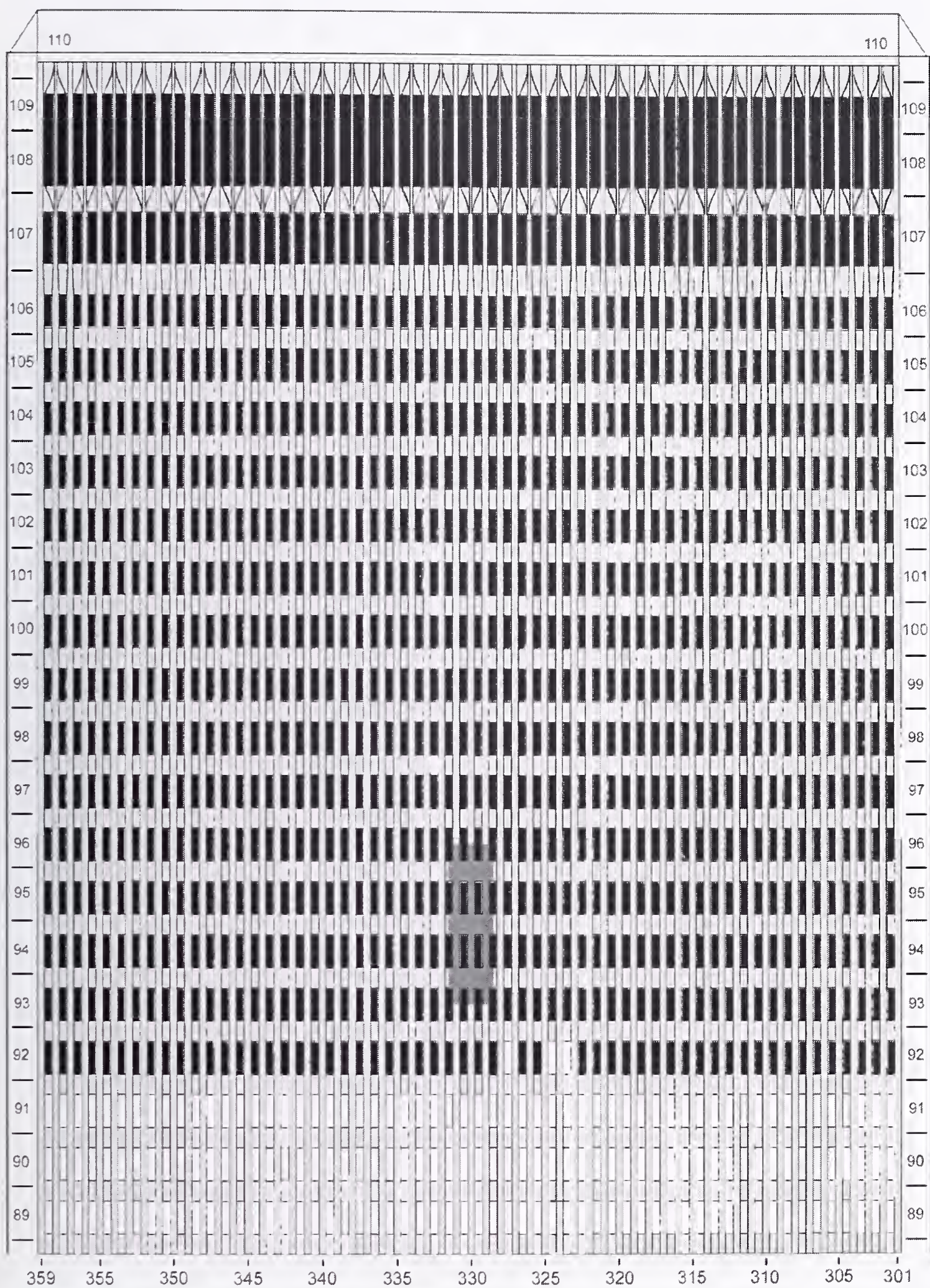

Figure E-38. Diagram of the south face of WTC 1 for floors 89 to 110 at 10:00 a.m. showing windows where smoke was observed and those that were hidden from view. 
WTC 1, South Face 10:10 a.m.

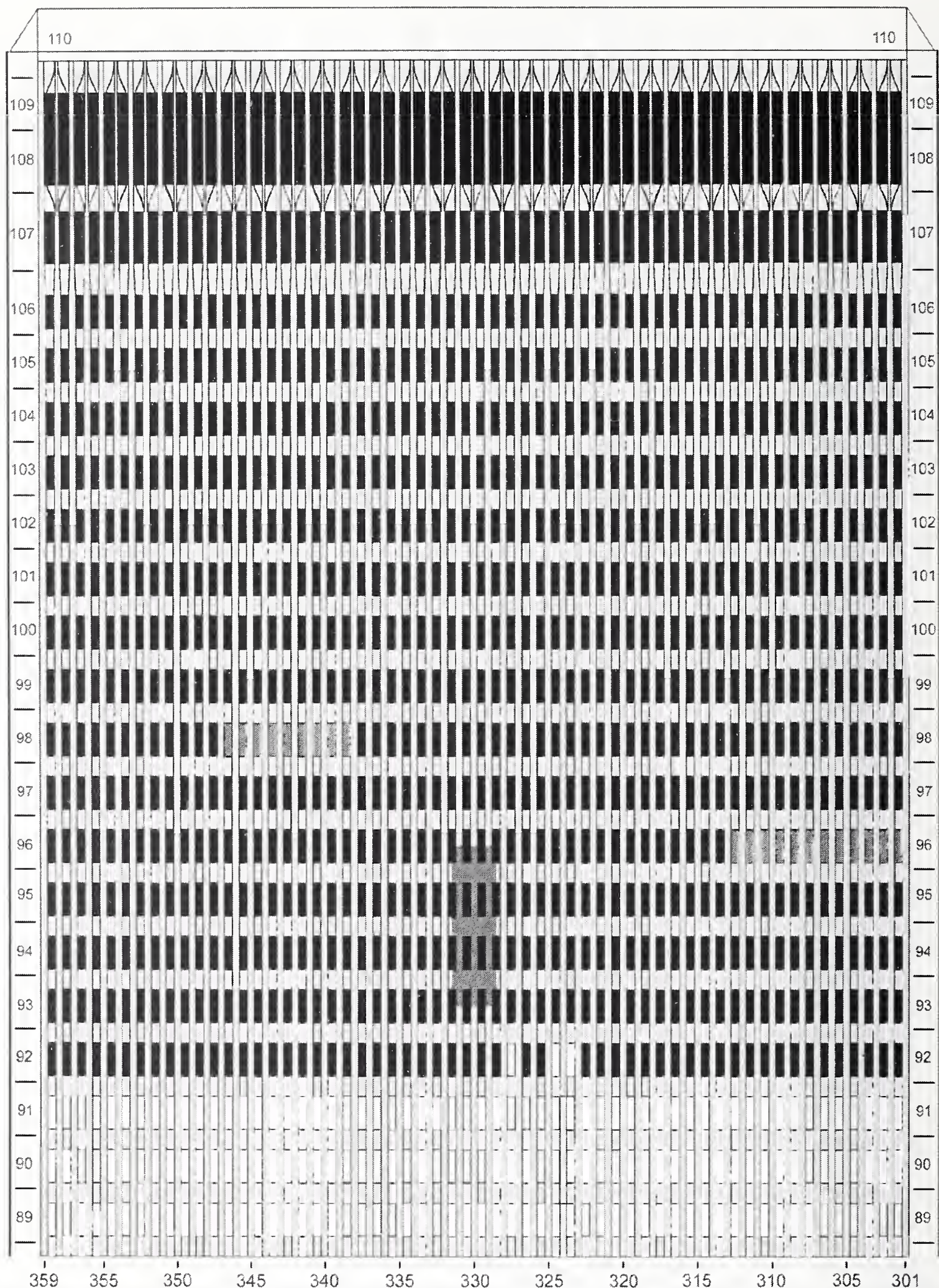

Figure E-39. Diagram of the south face of WTC 1 for floors 89 to 110 at 10:10 a.m. showing the condition of windows and locations of fires. 


$$
\text { WTC 1, South Face } \quad 10: 10 \text { a.m. }
$$

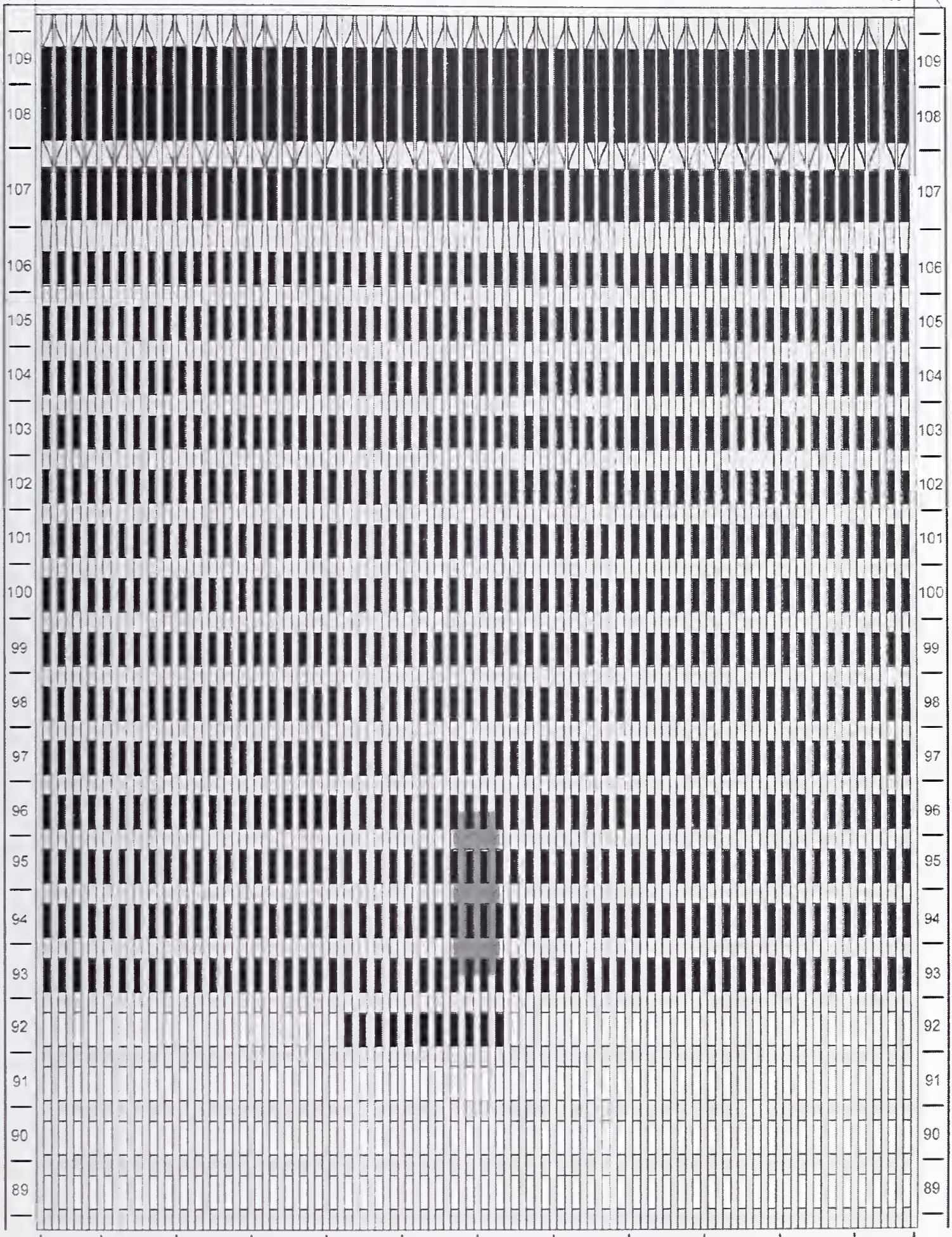

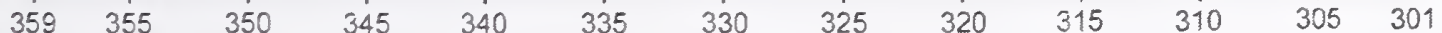

Figure E-40. Diagram of the south face of WTC 1 for floors 89 to 110 at 10:10 a.m. showing windows where smoke was observed and those that were hidden from view. 


$$
\text { WTC 1, South Face } \quad \text { 10:12 a.m. }
$$

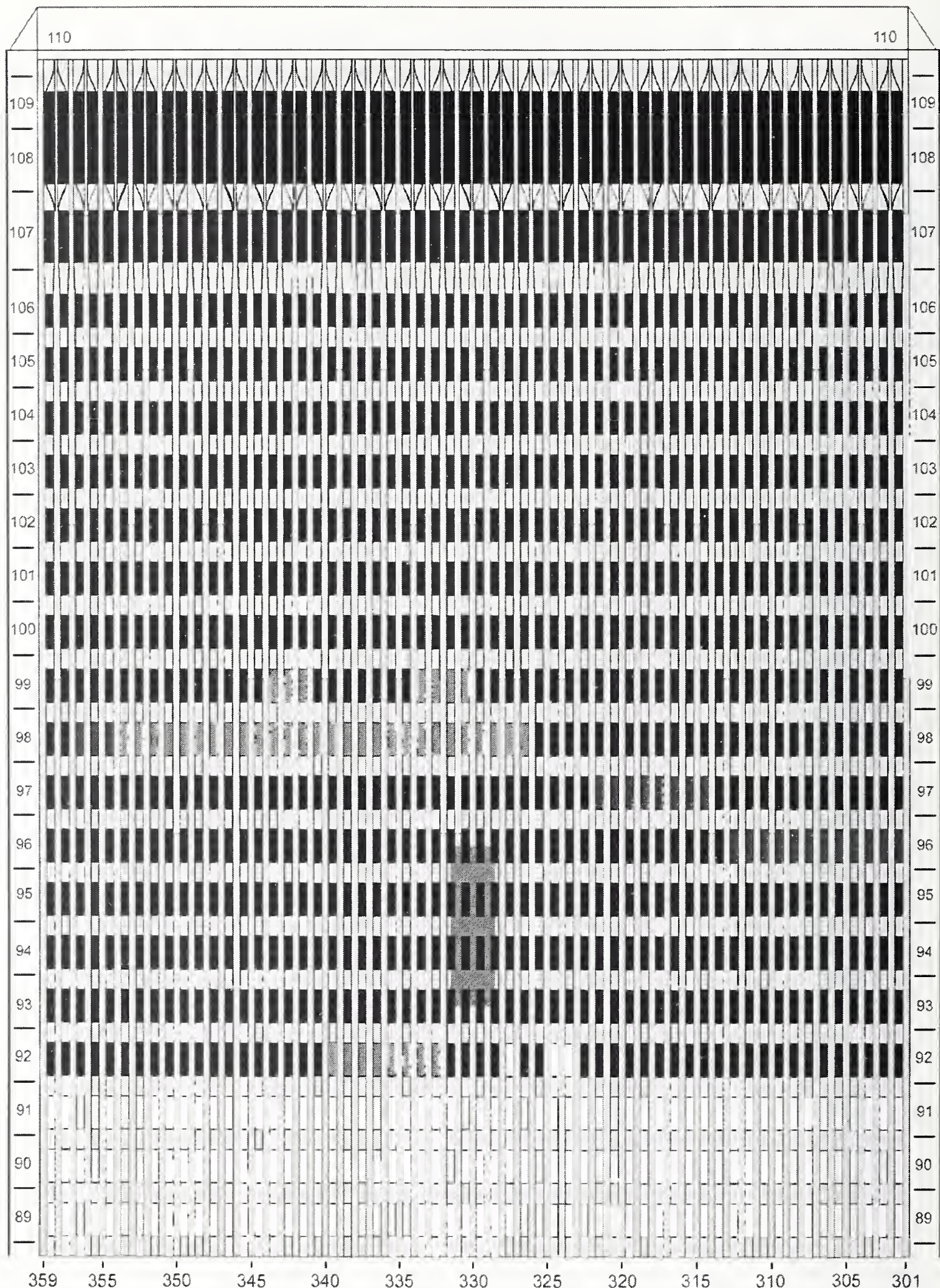

Figure E-41. Diagram of the south face of WTC 1 for floors 89 to 110 at 10:12 a.m. showing the condition of windows and locations of fires. 
WTC 1, South Face $\quad 10: 12$ a.m.

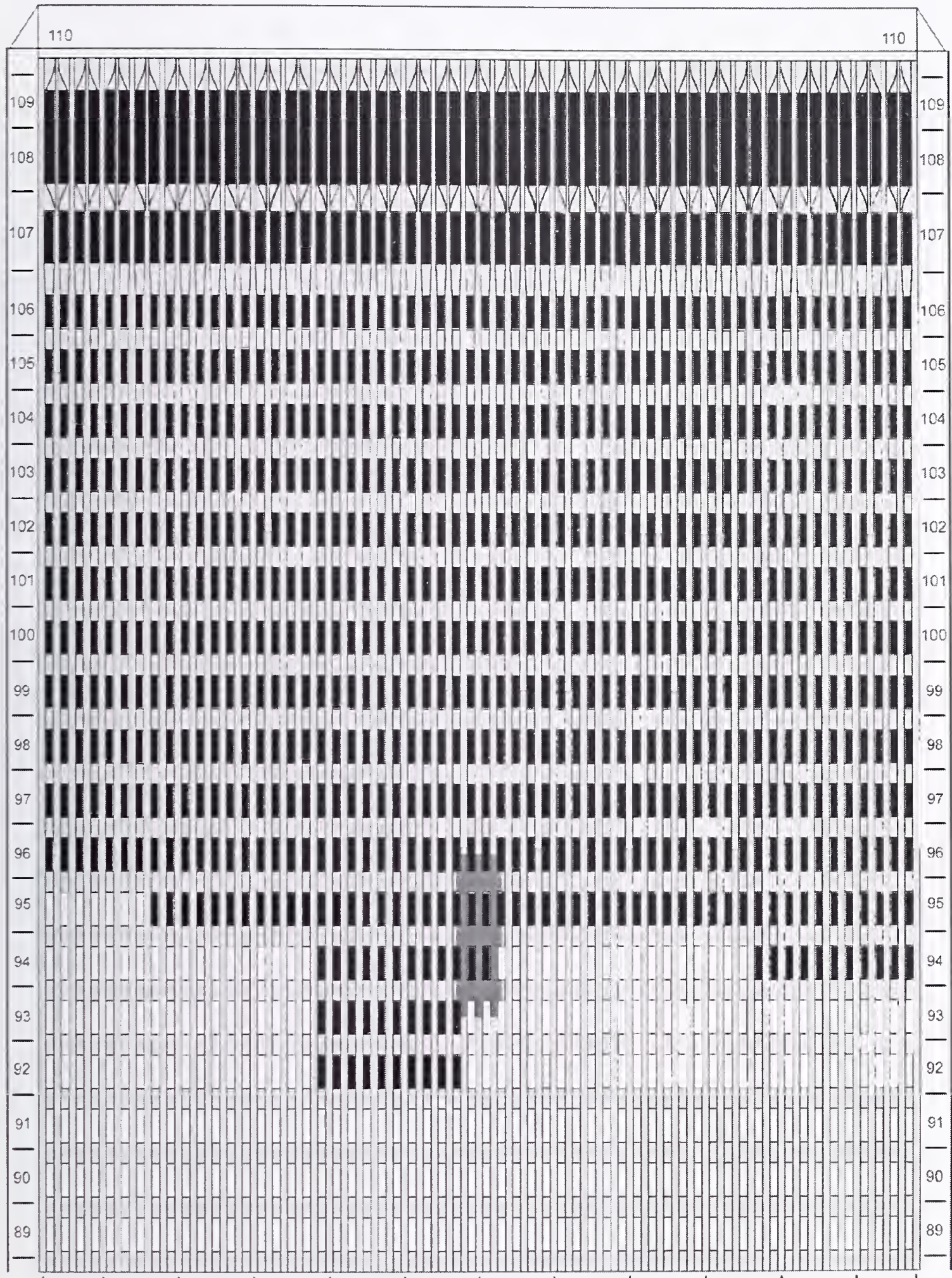

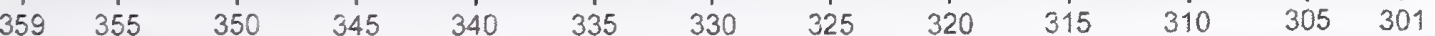

Figure E-42. Diagram of the south face of WTC 1 for floors 89 to 110 at 10:12 a.m. showing windows where smoke was observed and those that were hidden from view. 


$$
\text { WTC 1, South Face } \quad \text { 10:14 a.m. }
$$

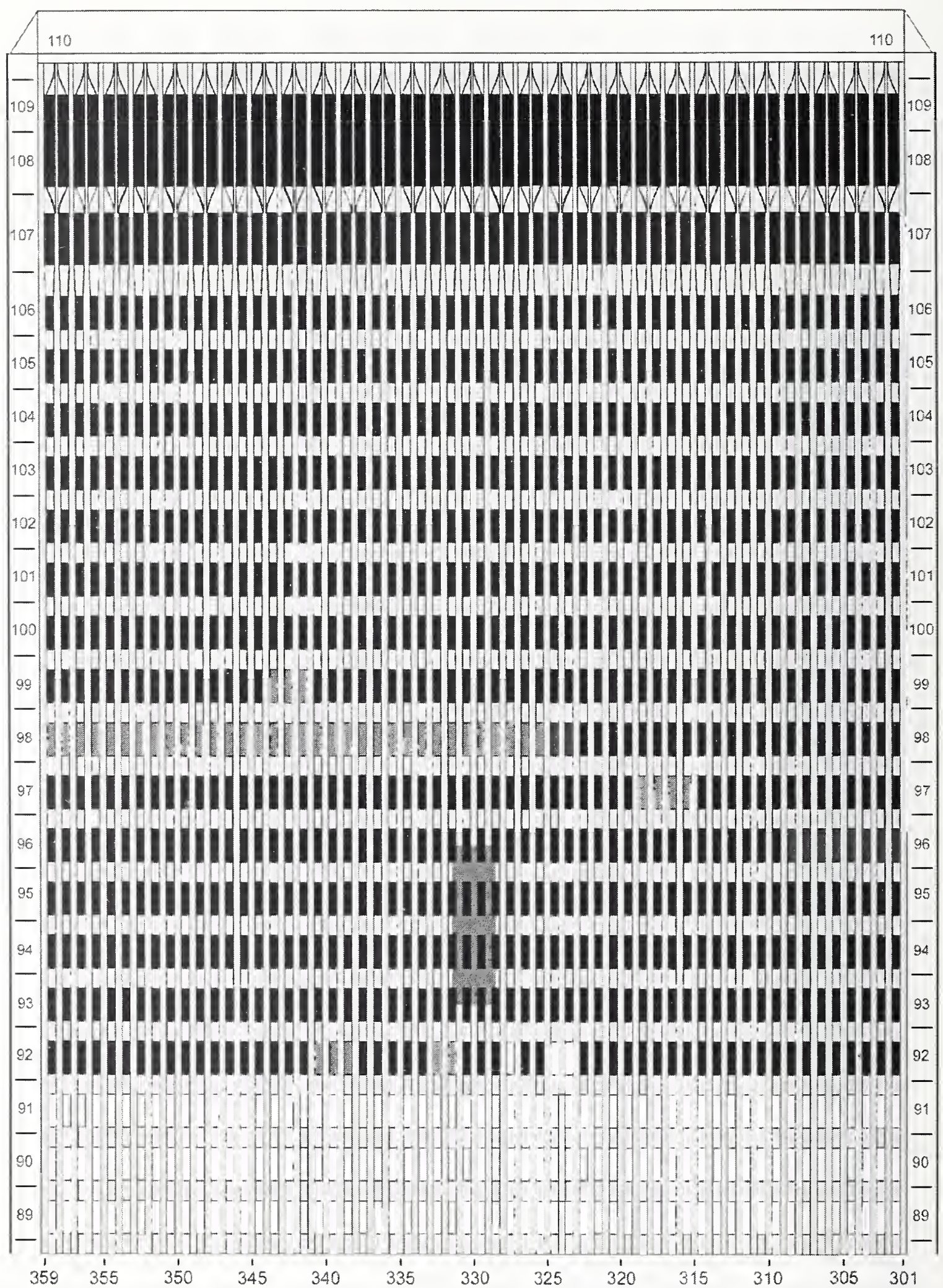

Figure E-43. Diagram of the south face of WTC 1 for floors 89 to 110 at 10:14 a.m. showing the condition of windows and locations of fires. 


$$
\text { WTC 1, South Face } \quad 10: 14 \text { a.m. }
$$

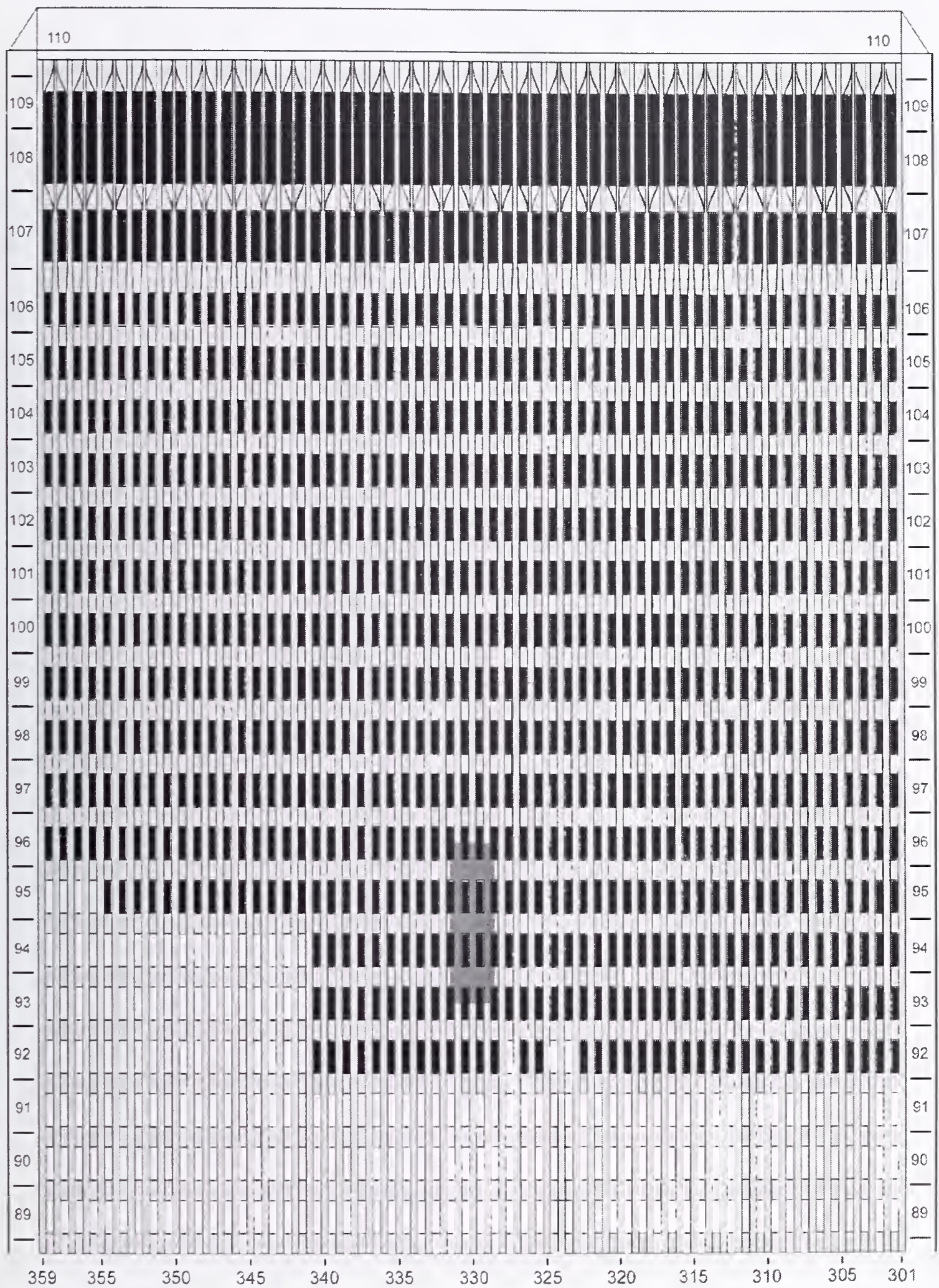

Figure E-44. Diagram of the south face of WTC 1 for floors 89 to 110 at 10:14 a.m. showing windows where smoke was observed and those that were hidden from view. 
WTC 1, South Face 10:18 a.m.

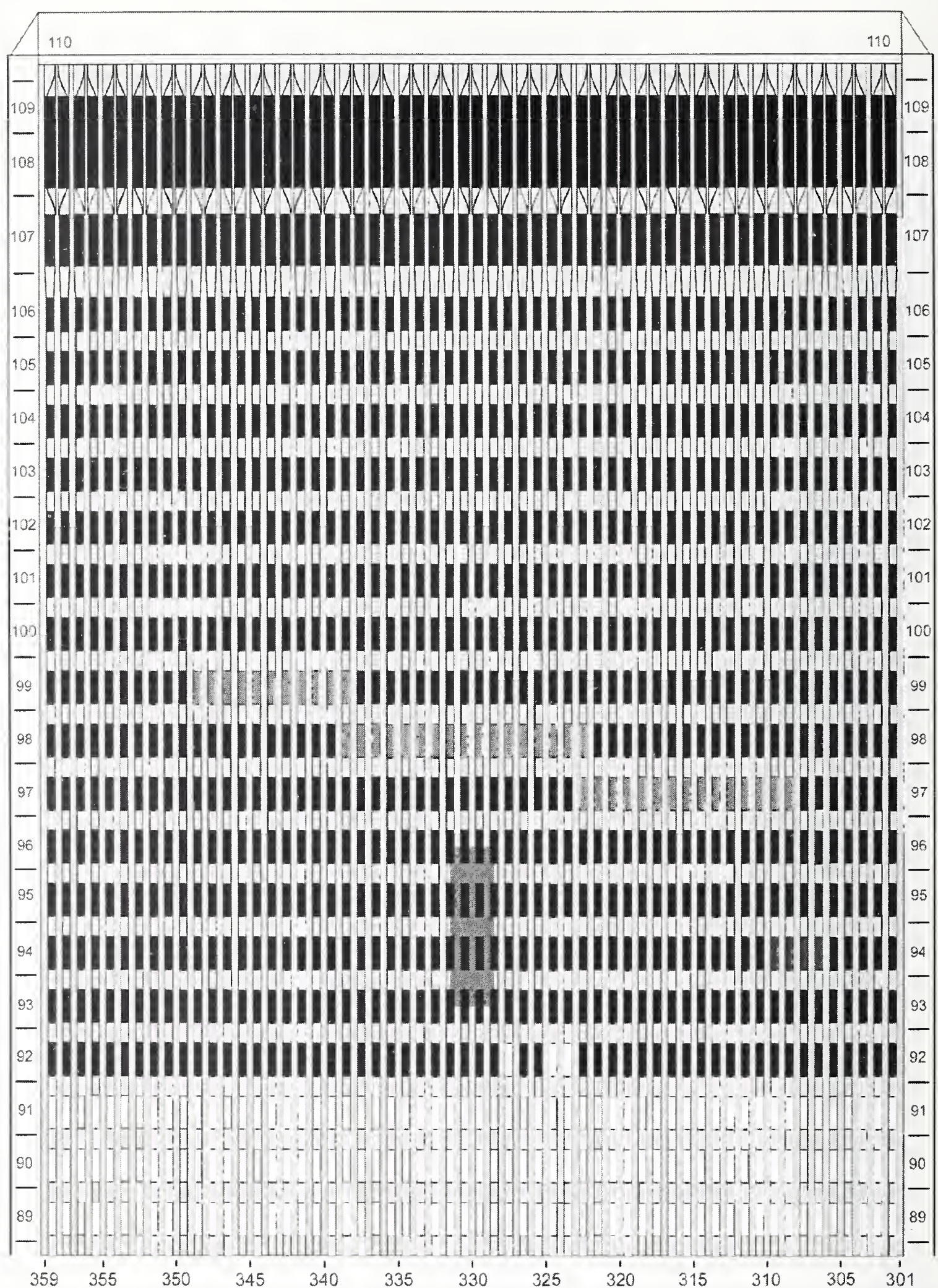

Figure E-45. Diagram of the south face of WTC 1 for floors 89 to 110 at 10:18 a.m. showing the condition of windows and locations of fires. 


$$
\text { WTC 1, South Face 10:18 a.m. }
$$

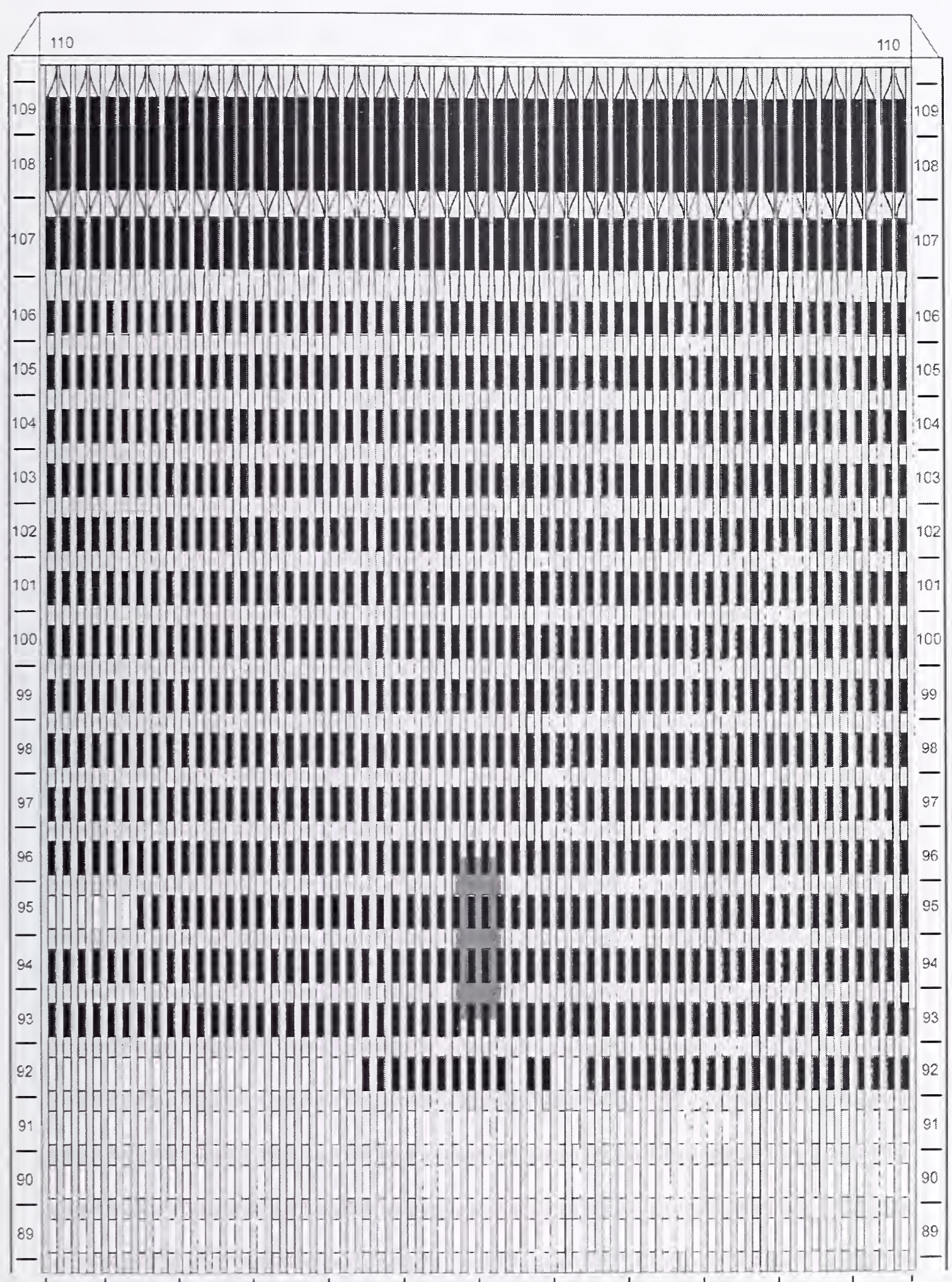

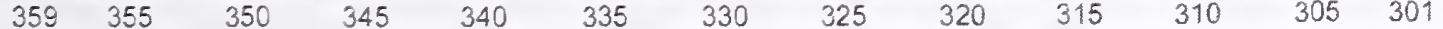

Figure E-46. Diagram of the south face of WTC 1 for floors 89 to 110 at 10:18 a.m. showing windows where smoke was observed and those that were hidden from view. 
WTC 1, South Face 10:22 a.m.

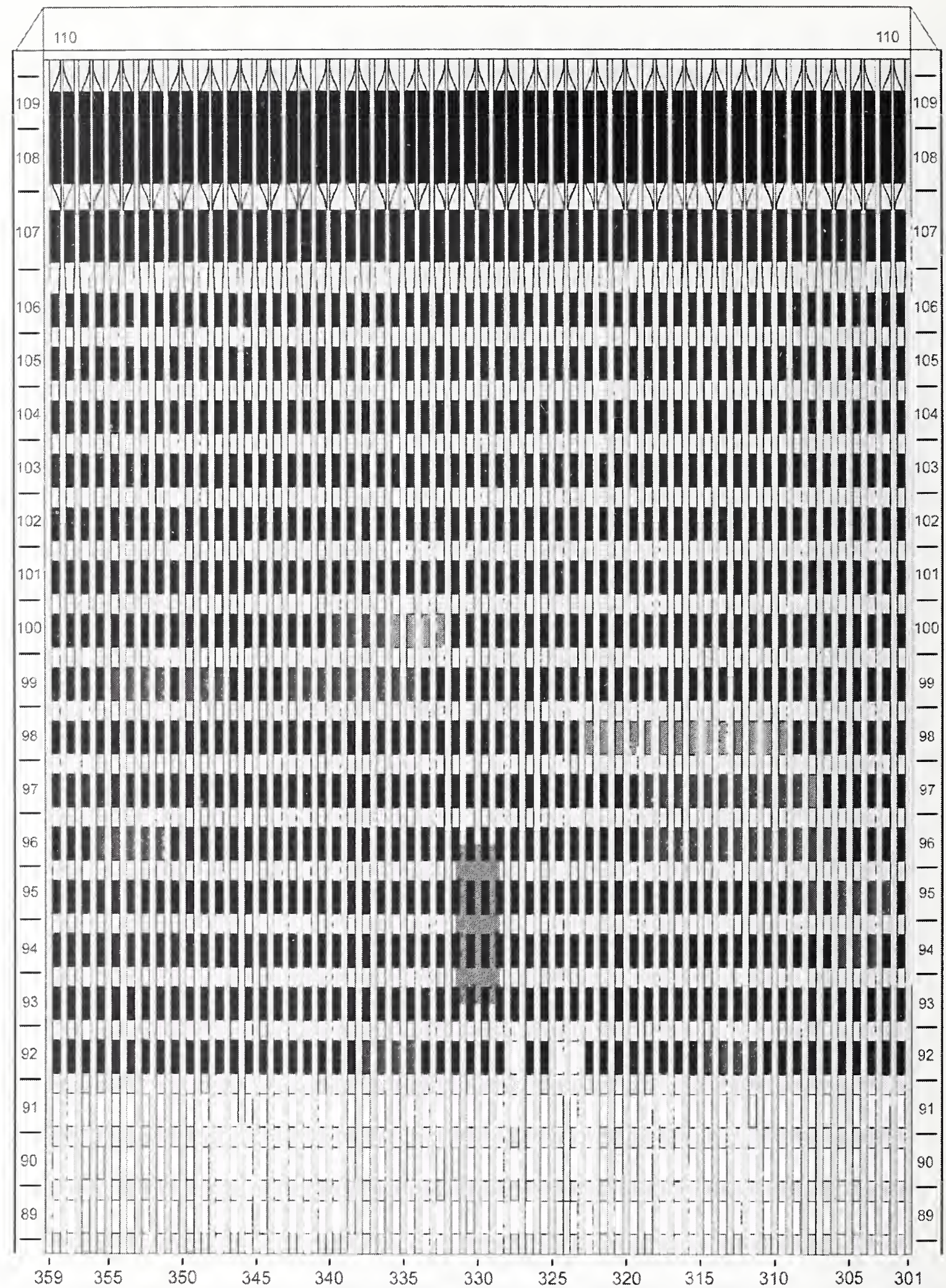

Figure E-47. Diagram of the south face of WTC 1 for floors 89 to 110 at 10:22 a.m. showing the condition of windows and locations of fires. 


\section{WTC 1, South Face 10:22 a.m.}

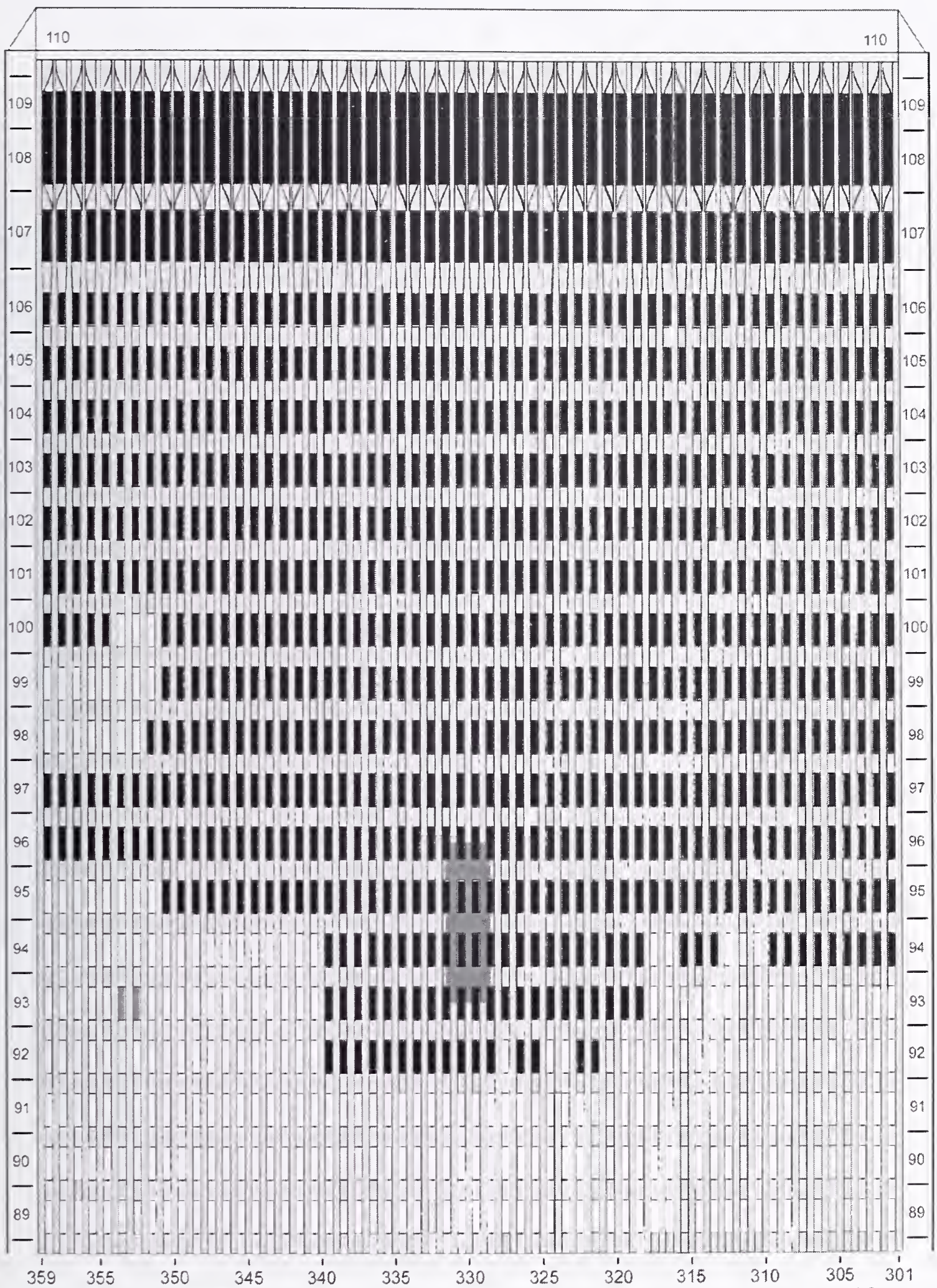

Figure E-48. Diagram of the south face of WTC 1 for floors 89 to 110 at 10:22 a.m. showing windows where smoke was observed and those that were hidden from view. 
WTC 1, South Face $\quad$ 10:28 a.m.

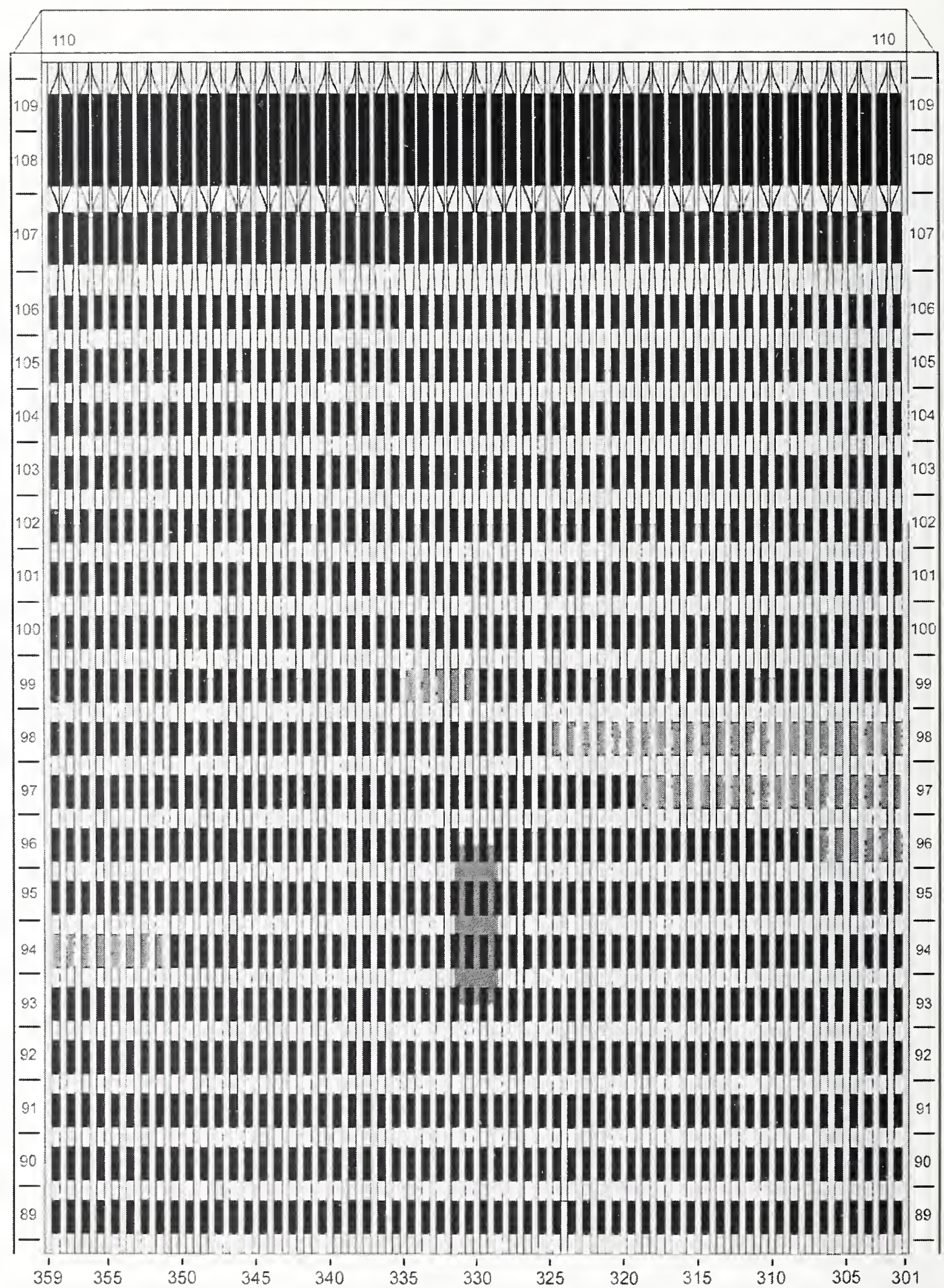

Figure E-49. Diagram of the south face of WTC 1 for floors 89 to 110 at 10:28 a.m. showing the condition of windows and locations of fires. 


$$
\text { WTC 1, South Face } \quad 10: 28 \text { a.m. }
$$

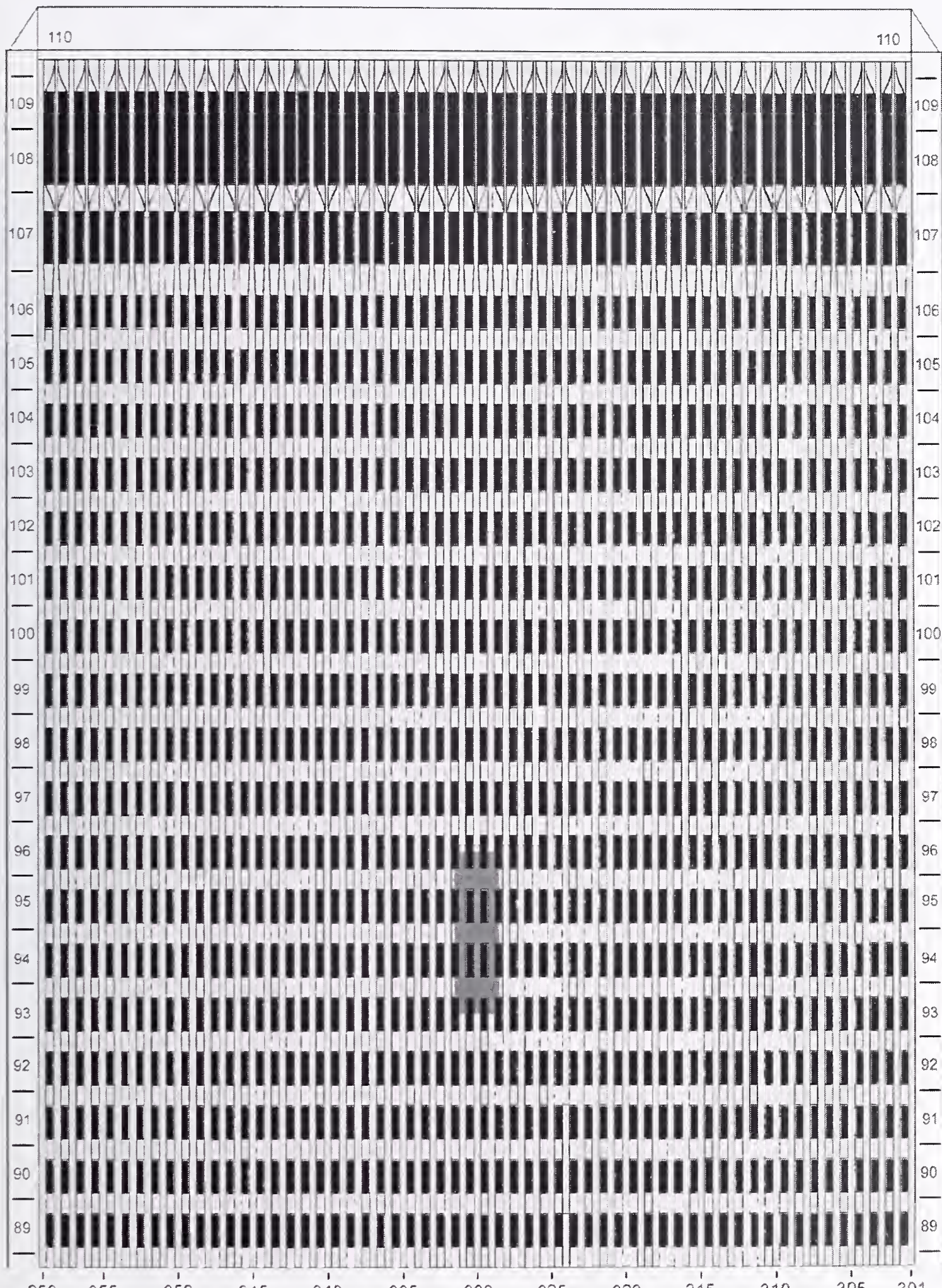

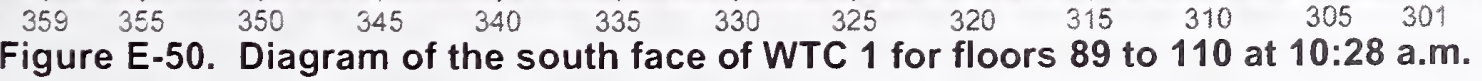
showing windows where smoke was observed and those that were hidden from view. 
This page intentionally left blank. 


\section{Appendix F \\ WTC 1 West FACE CoMBINEd FIRE AND WINDOW CONDITION AND WINDOW SMOKE CONDITION}

This appendix provides visual representations of the data contained in data sheets describing observations of fire, smoke, and window condition on a window-by-window basis as a function of time for the west face of WTC 1. The data for window condition and fire observations are combined into single representations, with the data for fire taking precedence. Observations are represented using the key provided in Figure 5-1 as follows: ]- window glass in place, - window open, - spot fire, I- fire visible inside, - external flaming, and - not visible. The data for smoke are shown in separate figures using the key from Figure 5-1 as follows: - no smoke visible, - light smoke, - heavy smoke, and - not visible. For a given time, the window condition and fire data are shown first. 


\section{WTC 1, West Face $\quad 8: 47$ a.m.}

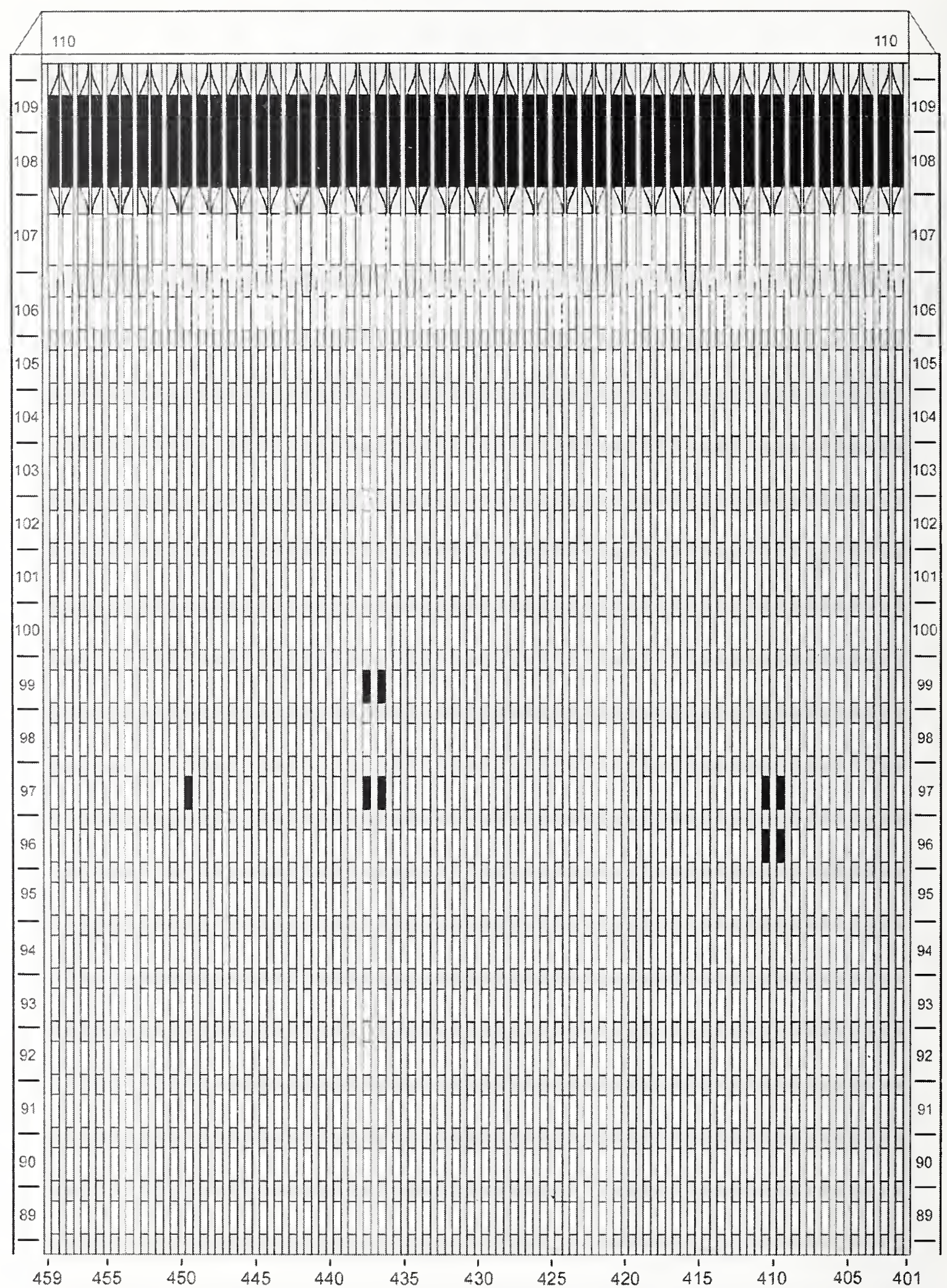

Figure F-1. Diagram of the west face of WTC 1 for floors 89 to 110 at 8:47 a.m. showing the condition of windows and locations of fires. 
WTC 1, West Face $\quad$ 8:47 a.m.

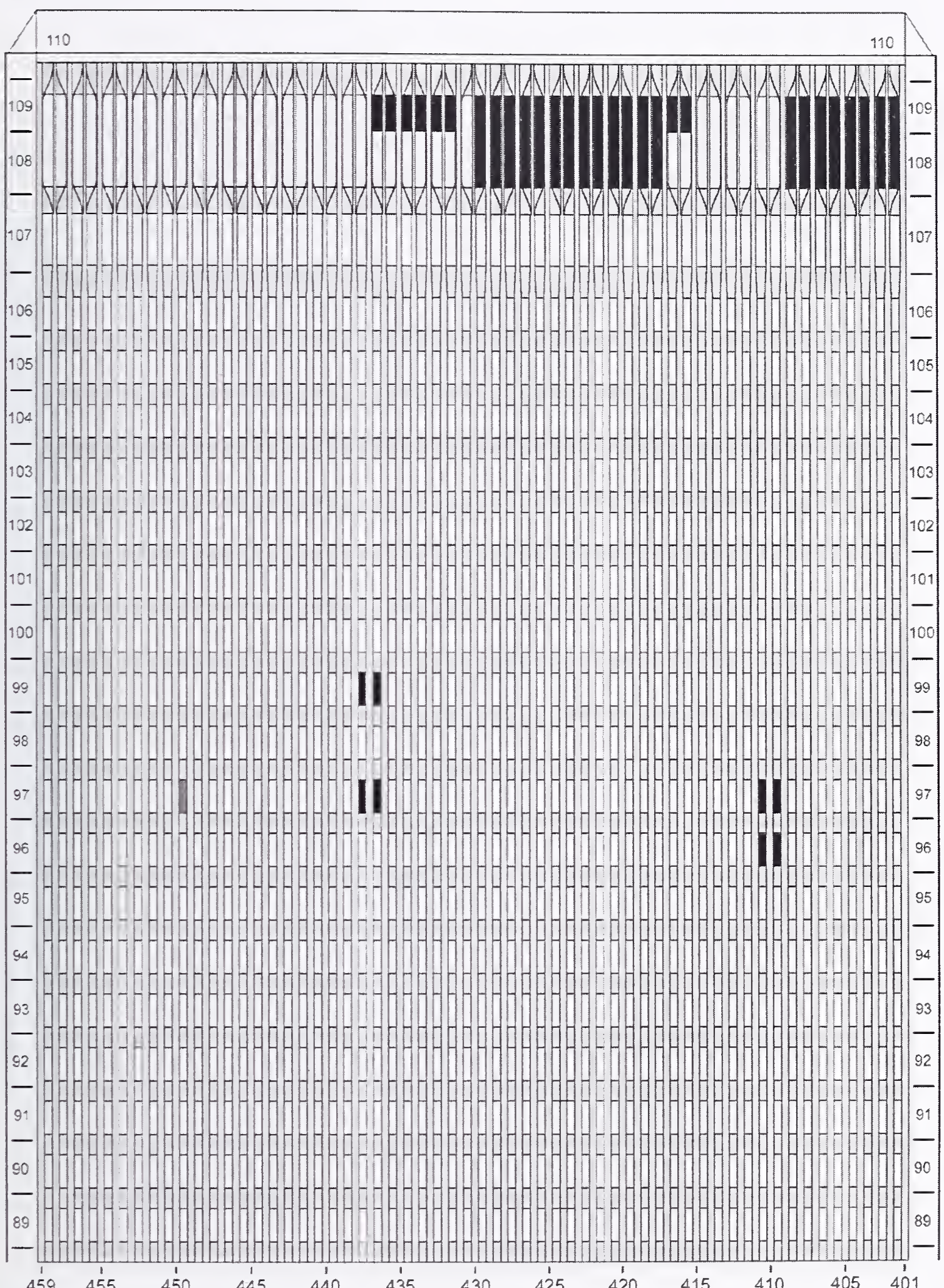

Figure F-2. Diagram of the west face of WTC 1 for floors 89 to 110 at $8: 47$ a.m. showing windows where smoke was observed and those that were hidden from view. 
WTC 1, West Face $\quad 8: 48$ a.m.

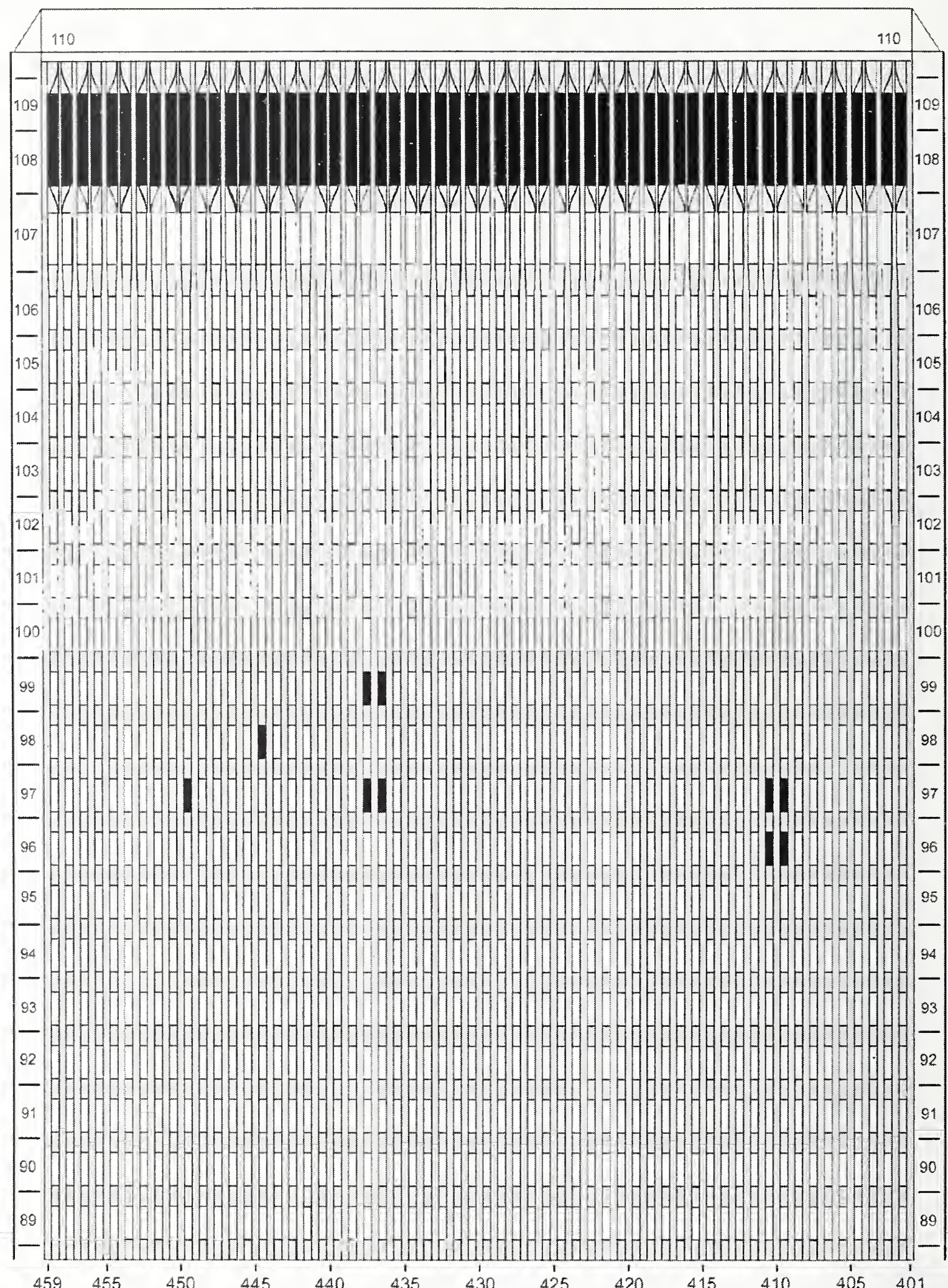

Figure F-3. Diagram of the west face of WTC 1 for floors 89 to 110 at 8:48 a.m. showing the condition of windows and locations of fires. 
WTC 1, West Face $\quad$ 8:48 a.m.

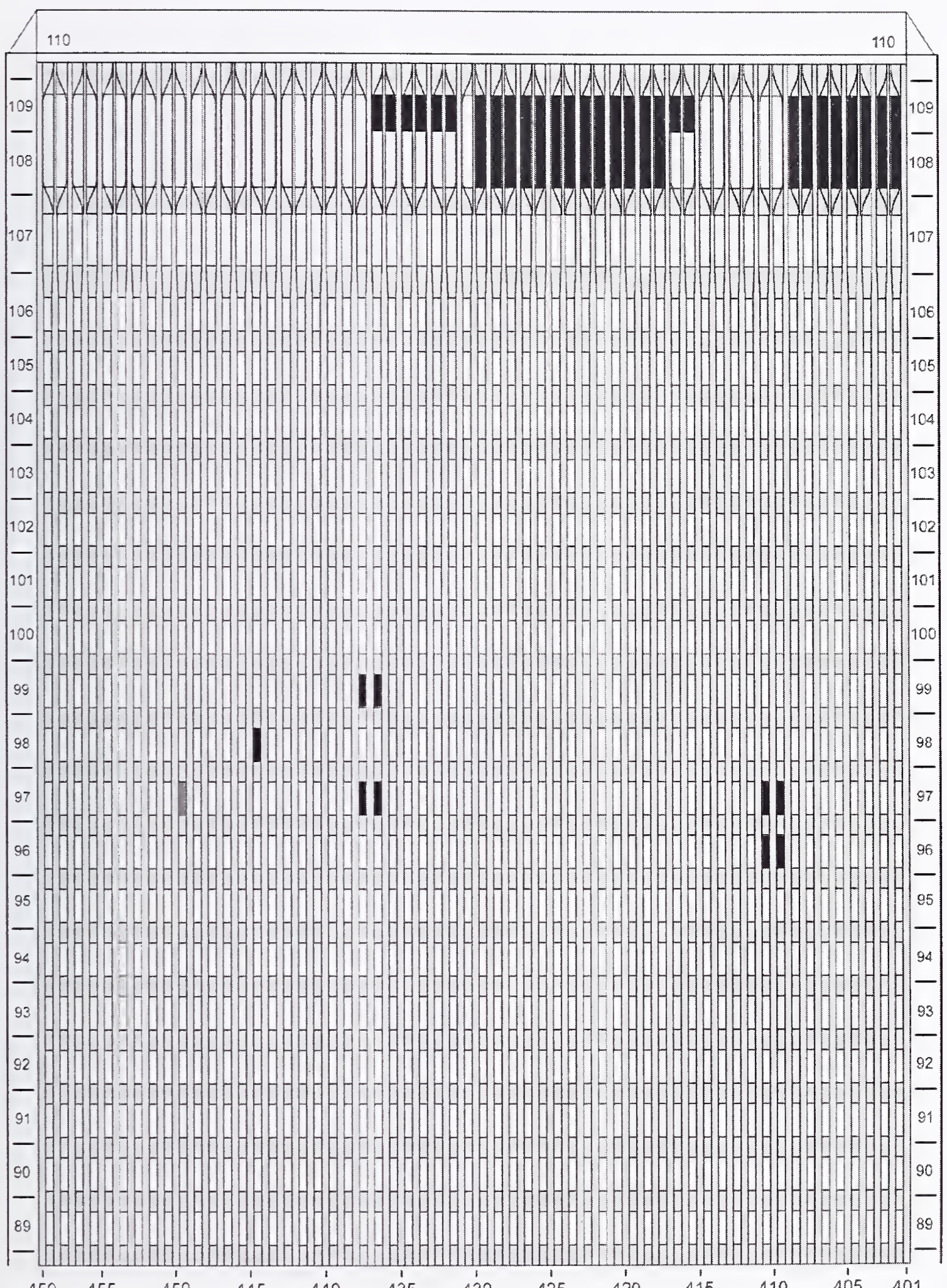

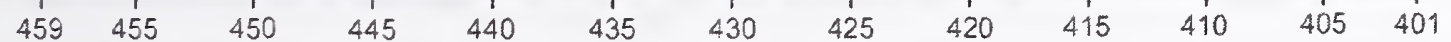

Figure F-4. Diagram of the west face of WTC 1 for floors 89 to 110 at $8: 48$ a.m. showing windows where smoke was observed and those that were hidden from view. 

WTC 1, West Face
8:50 a.m.

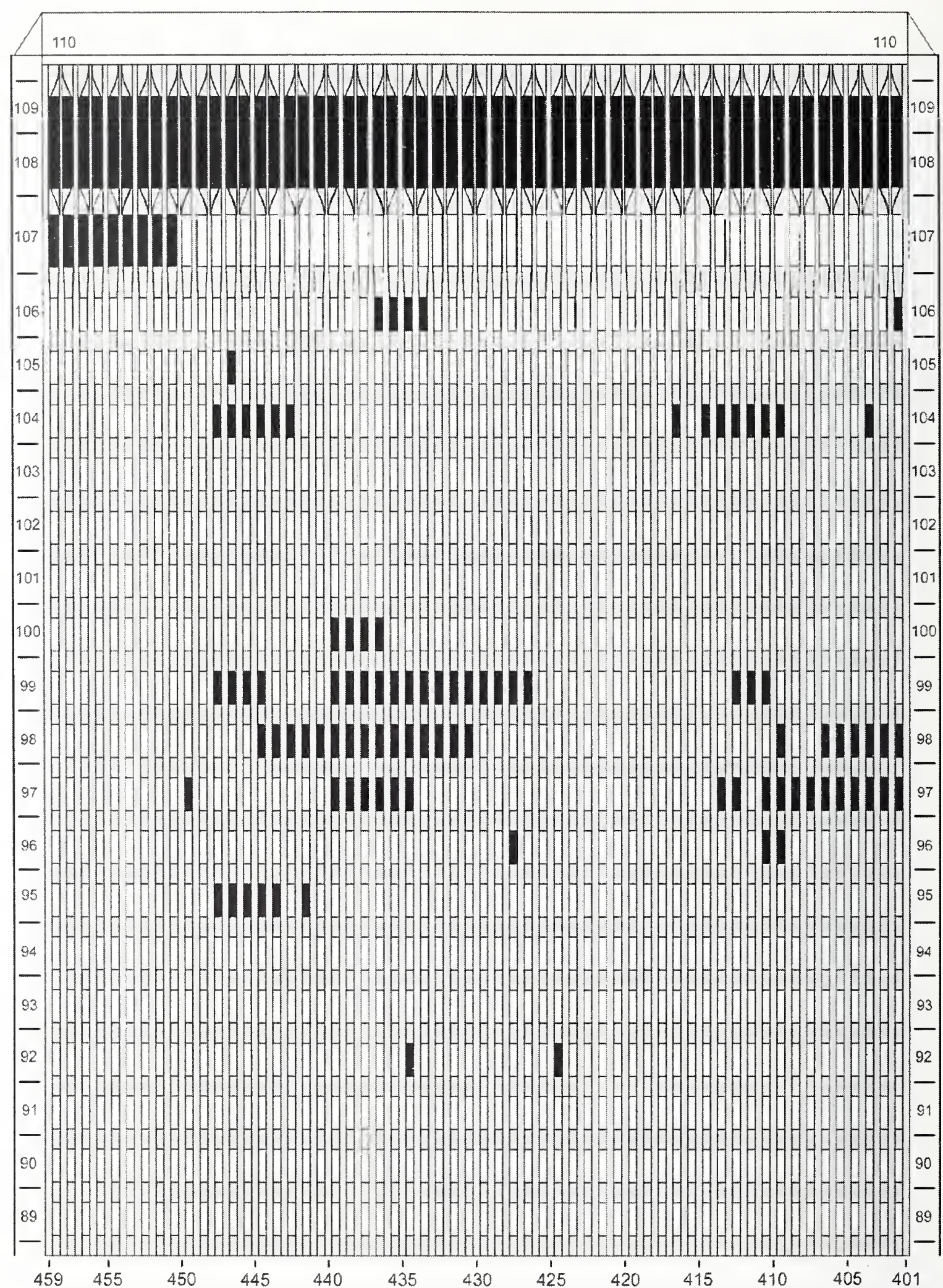

Figure F-5. Diagram of the west face of WTC 1 for floors 89 to 110 at 8:50 a.m. showing the condition of windows and locations of fires. 


$$
\text { WTC 1, West Face } \quad 8: 50 \text { a.m. }
$$

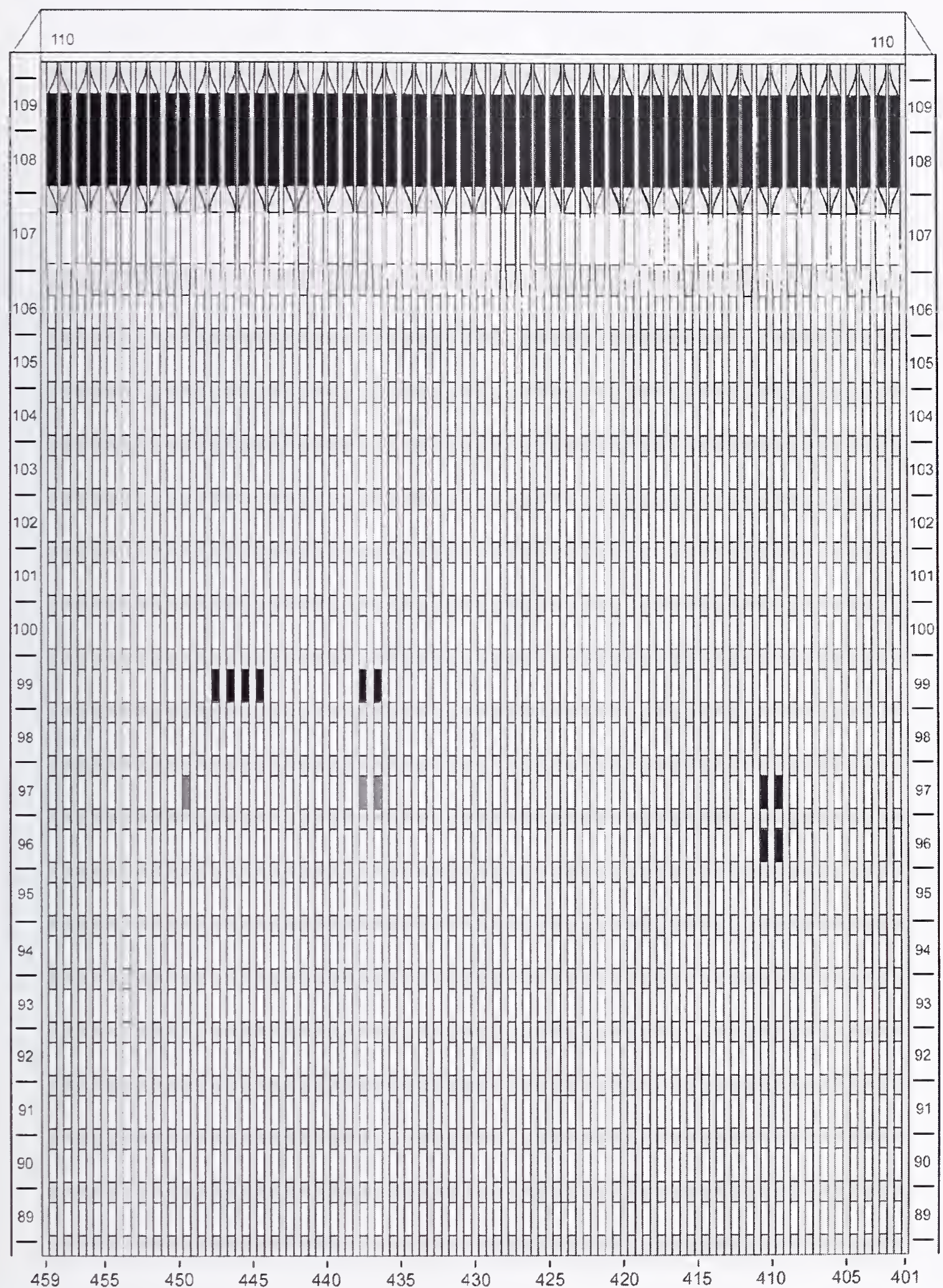

Figure F-6. Diagram of the west face of WTC 1 for floors 89 to 110 at $8: 50$ a.m. showing windows where smoke was observed and those that were hidden from view. 


$$
\text { WTC 1, West Face 8:52 a.m. }
$$

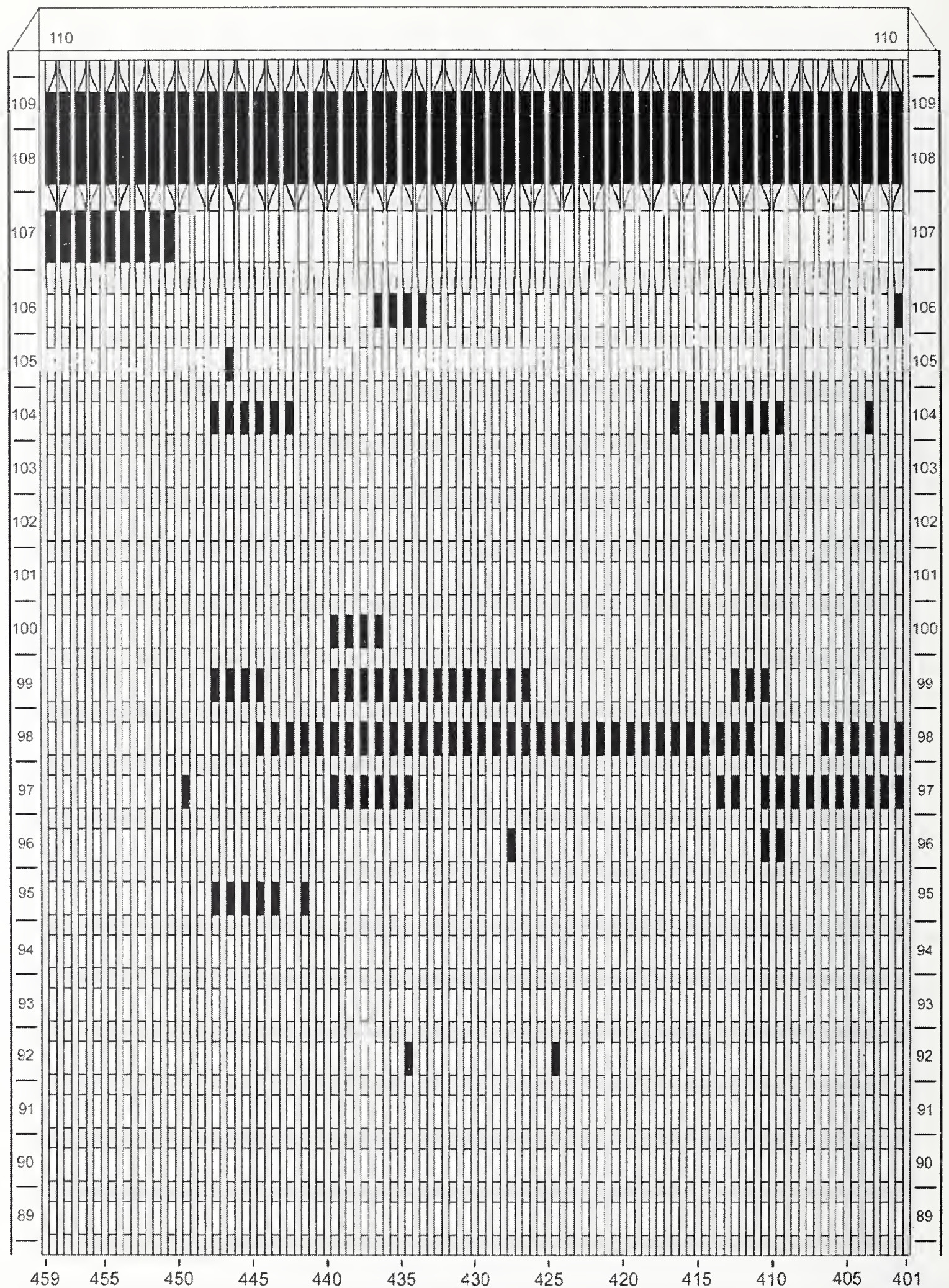

Figure F-7. Diagram of the west face of WTC 1 for floors 89 to 110 at 8:52 a.m. showing the condition of windows and locations of fires. 


$$
\text { WTC 1, West Face } \quad \text { 8:52 a.m. }
$$

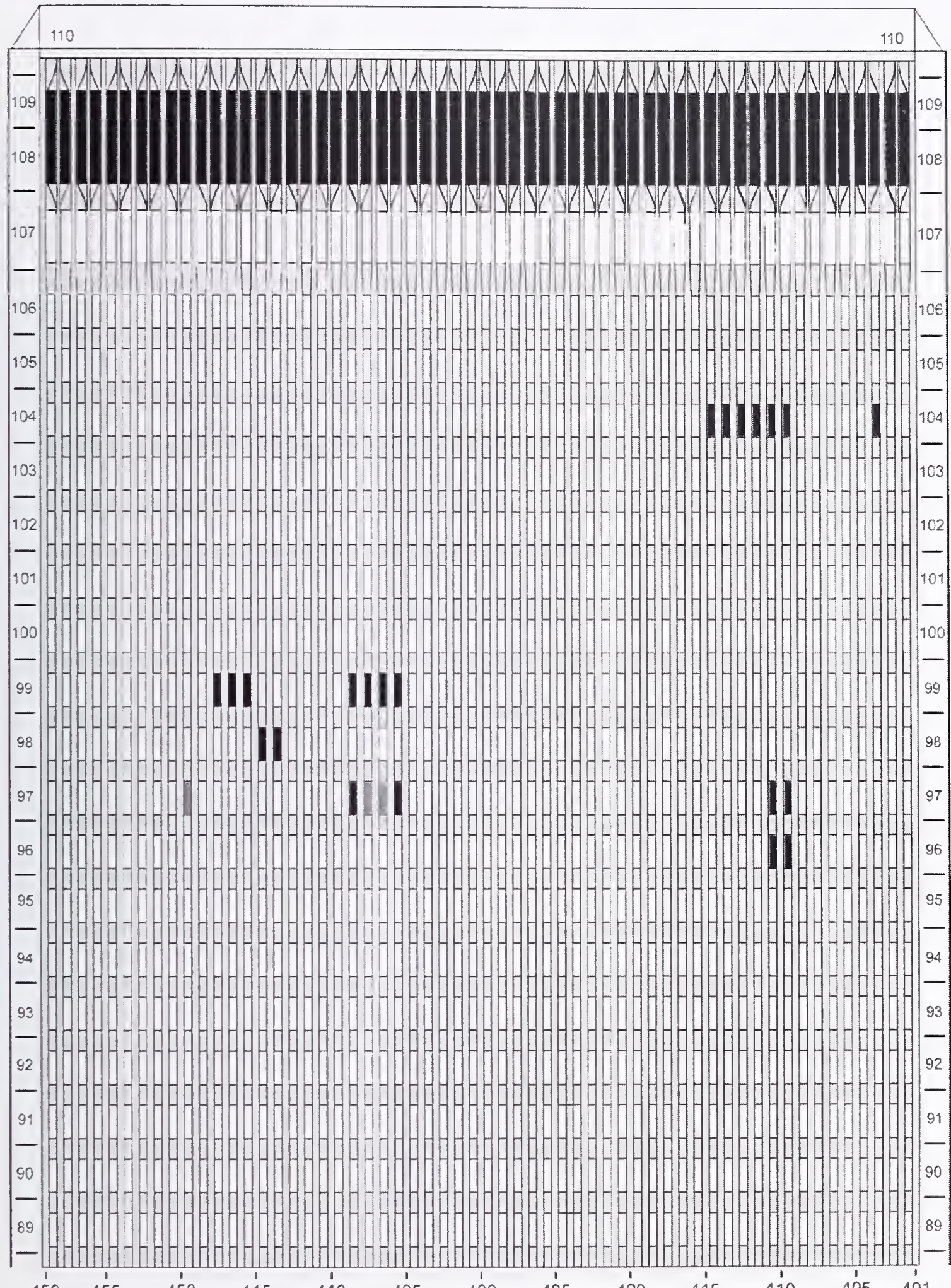

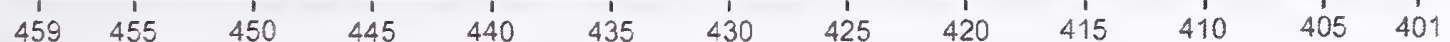

Figure F-8. Diagram of the west face of WTC 1 for floors 89 to 110 at $8: 52$ a.m. showing windows where smoke was observed and those that were hidden from view. 


\section{WTC 1, West Face $\quad$ 8:54 a.m.}

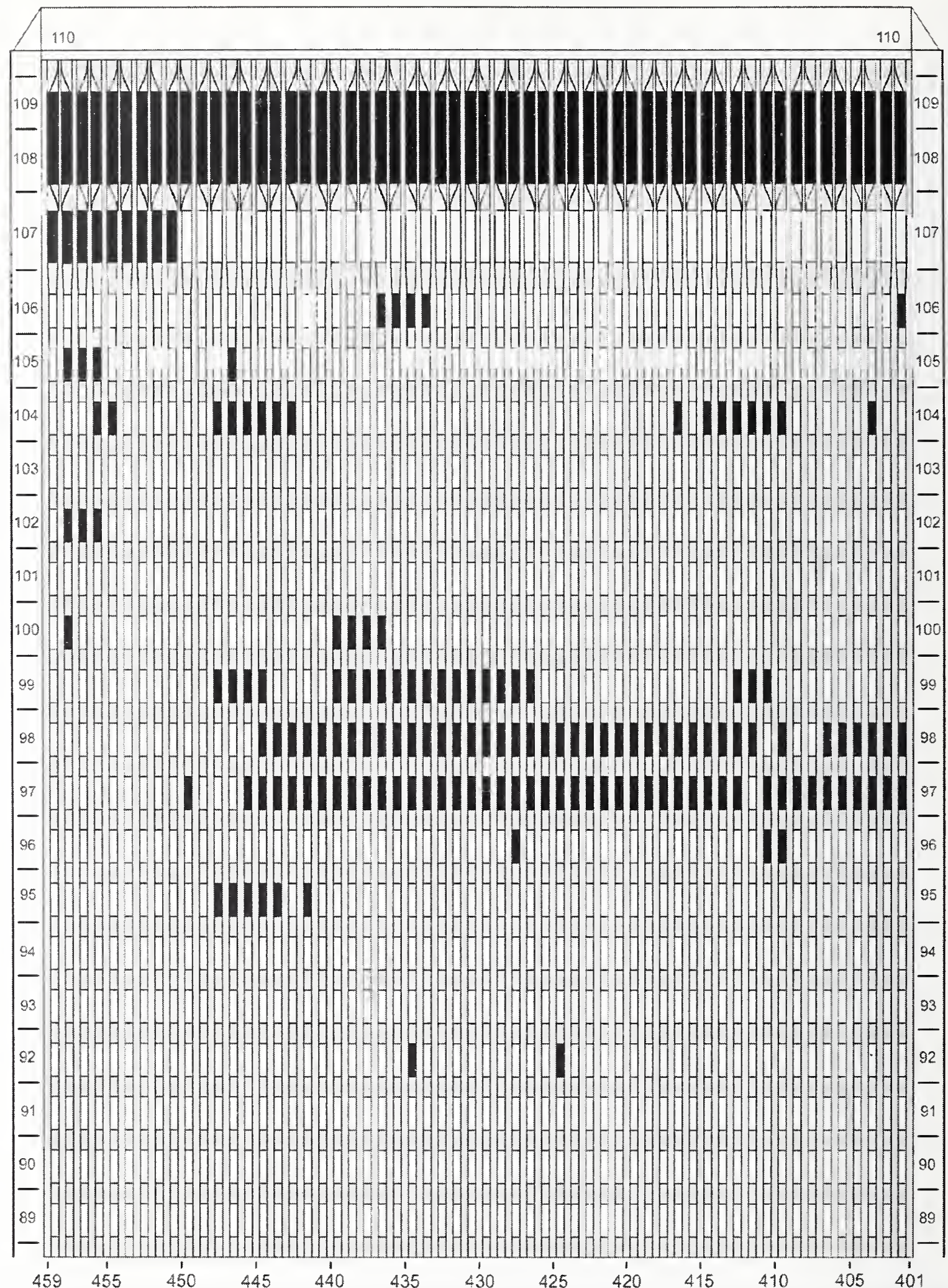

Figure F-9. Diagram of the west face of WTC 1 for floors 89 to 110 at 8:54 a.m. showing the condition of windows and locations of fires. 


$$
\text { WTC 1, West Face } \quad \text { 8:54 a.m. }
$$

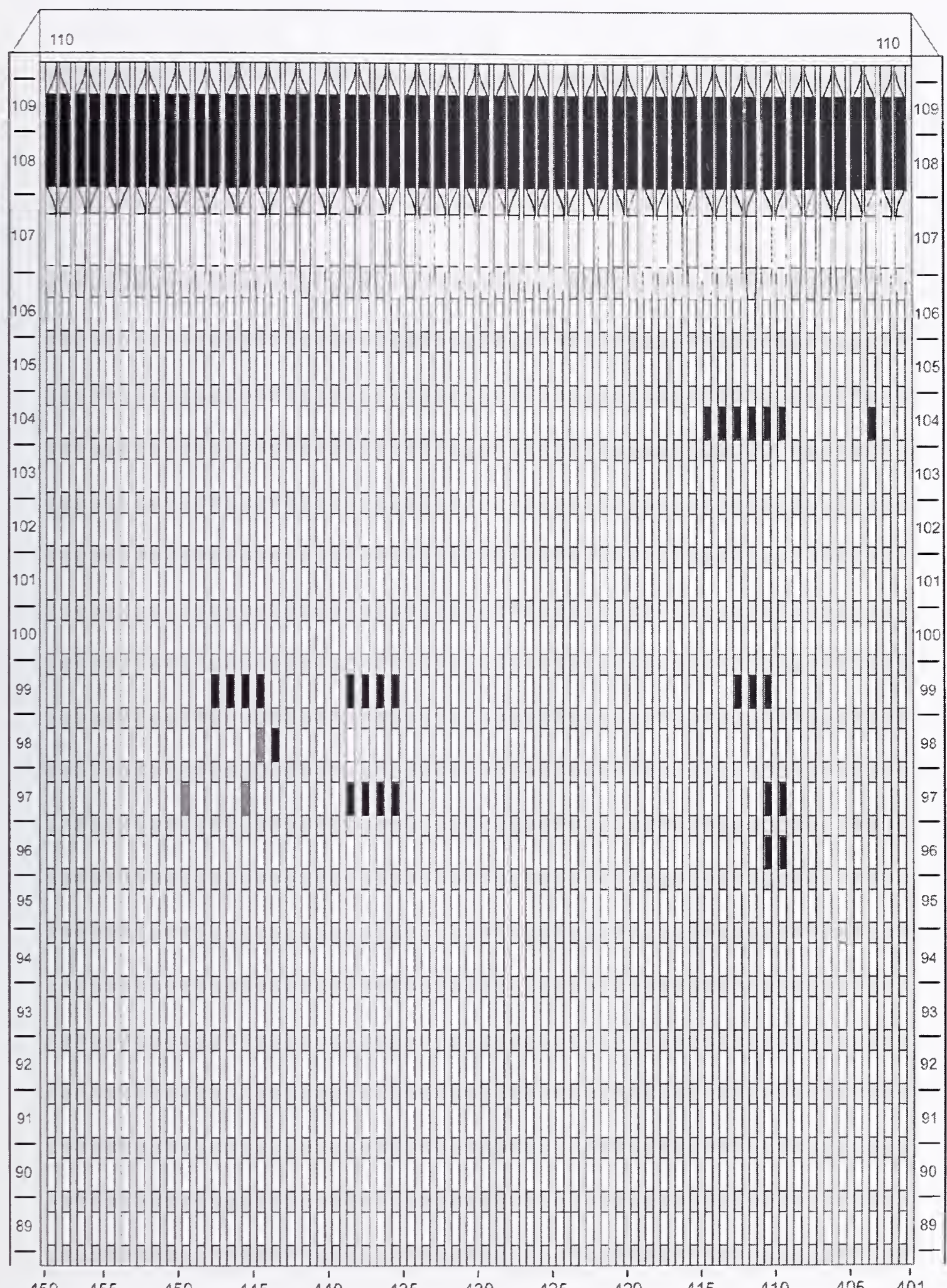

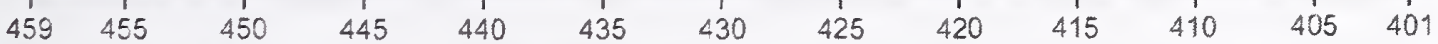

Figure F-10. Diagram of the west face of WTC 1 for floors 89 to 110 at $8: 54$ a.m. showing windows where smoke was observed and those that were hidden from view. 


\section{WTC 1, West Face $\quad 8: 56$ a.m.}

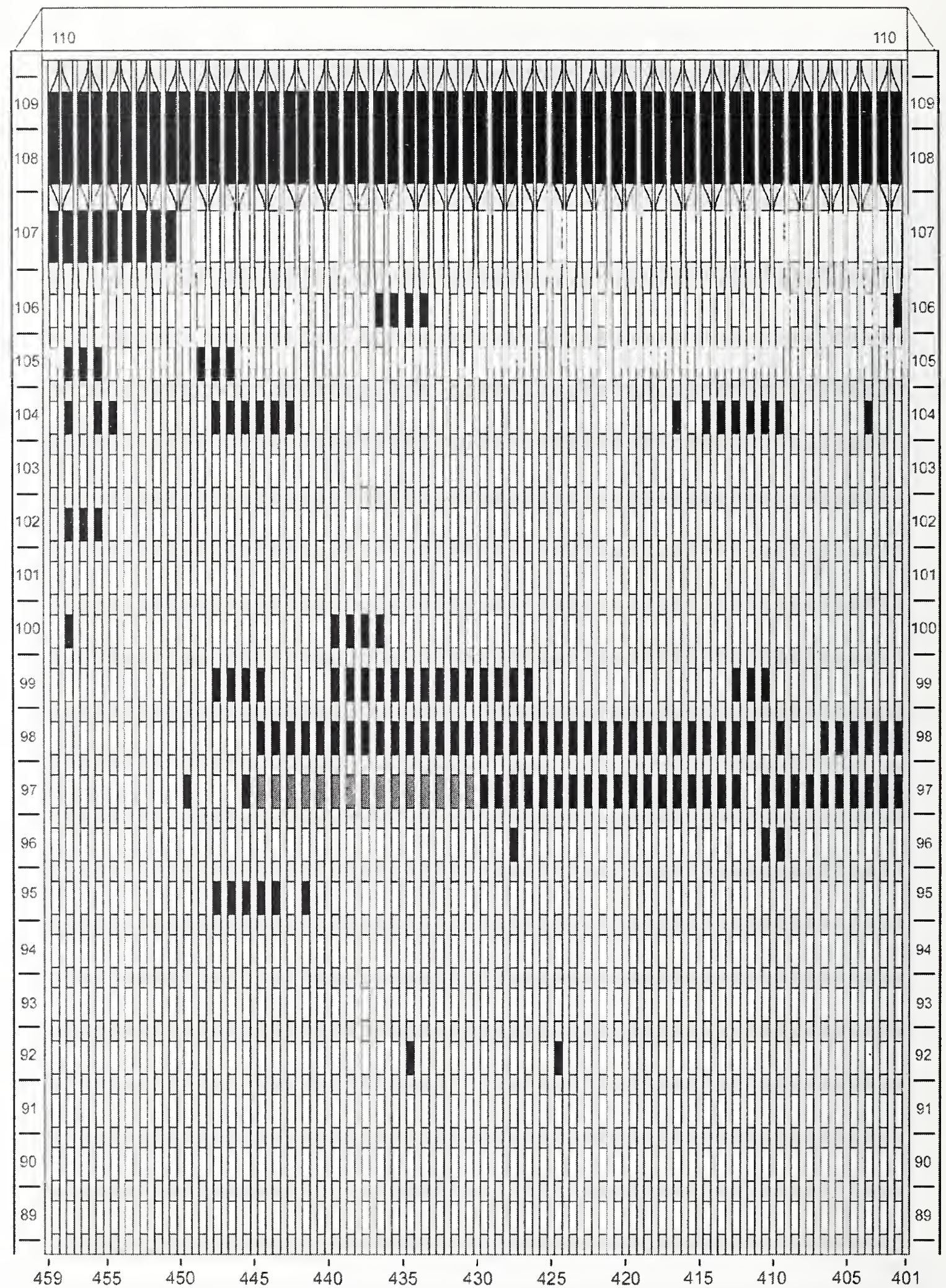

Figure F-11. Diagram of the west face of WTC 1 for floors 89 to 110 at 8:56 a.m showing the condition of windows and locations of fires. 
WTC 1, West Face $\quad$ 8:56 a.m.

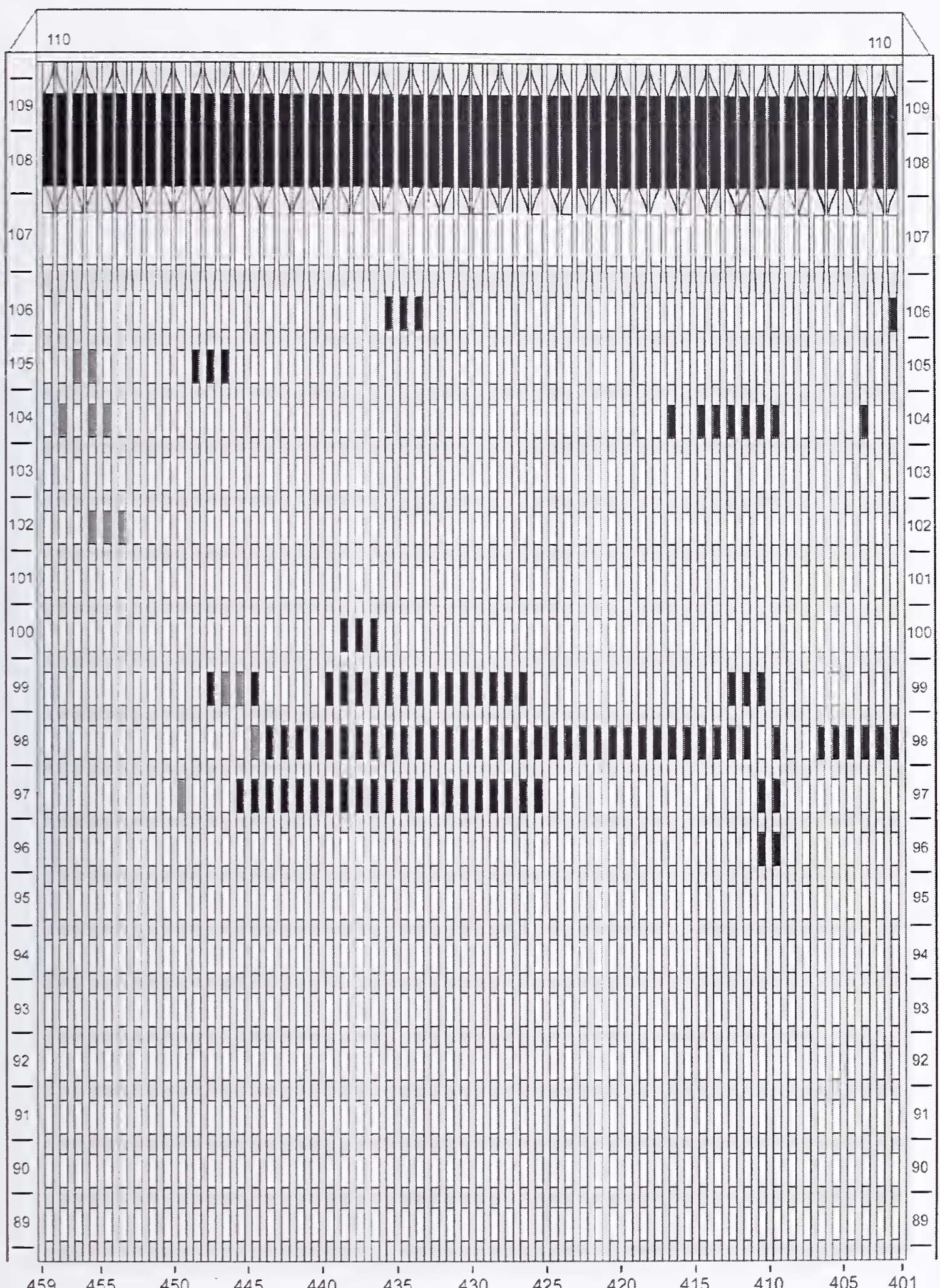

Figure F-12. Diagram of the west face of WTC 1 for floors 89 to 110 at $8: 56$ a.m. showing windows where smoke was observed and those that were hidden from view. 

WTC 1, West Face
8:58 a.m.

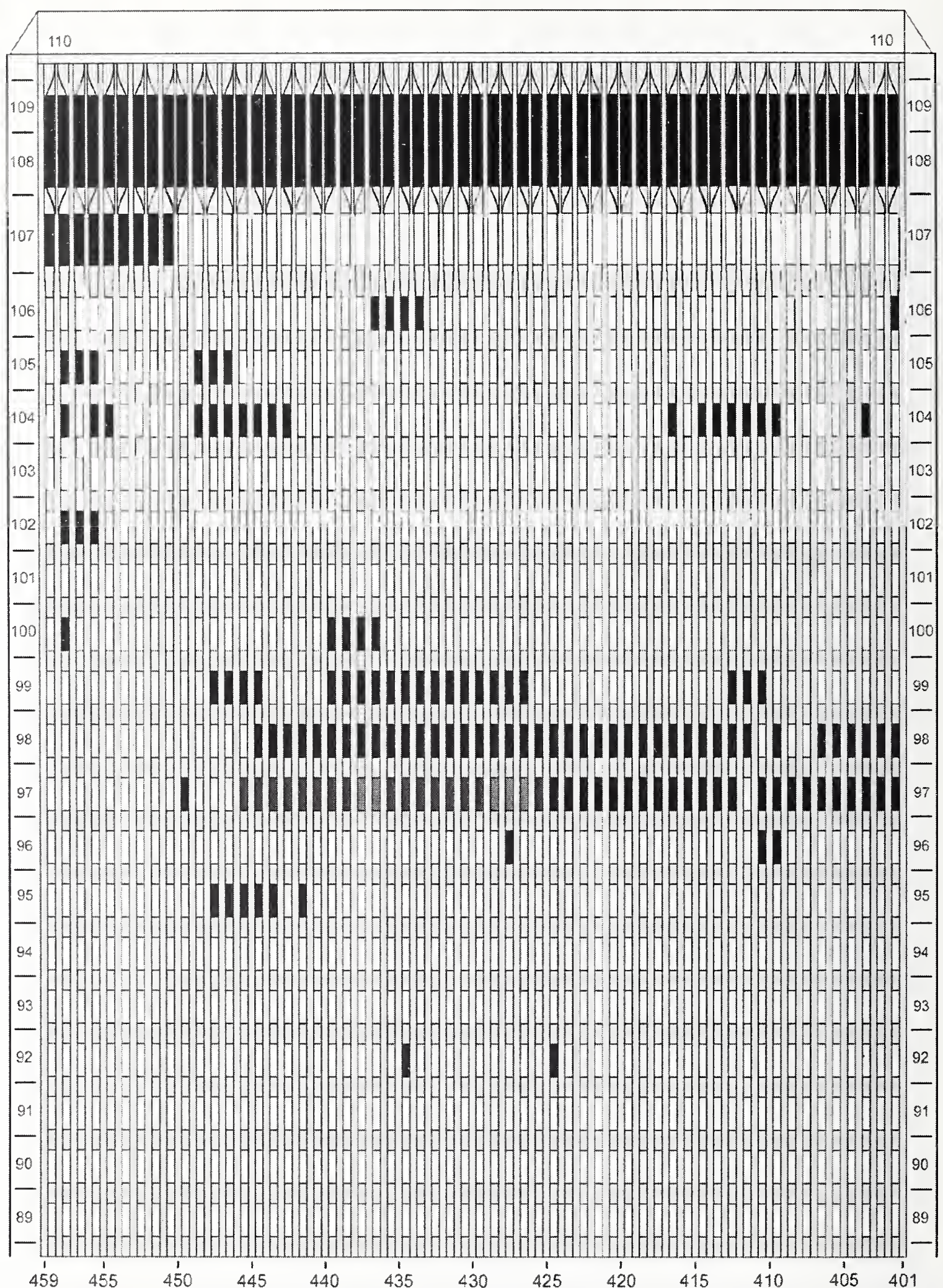

Figure F-13. Diagram of the west face of WTC 1 showing the condition of windows and locations of fires for floors 89 to 110 at 8:58 a.m. 


$$
\text { WTC 1, West Face } \quad 8: 58 \text { a.m. }
$$

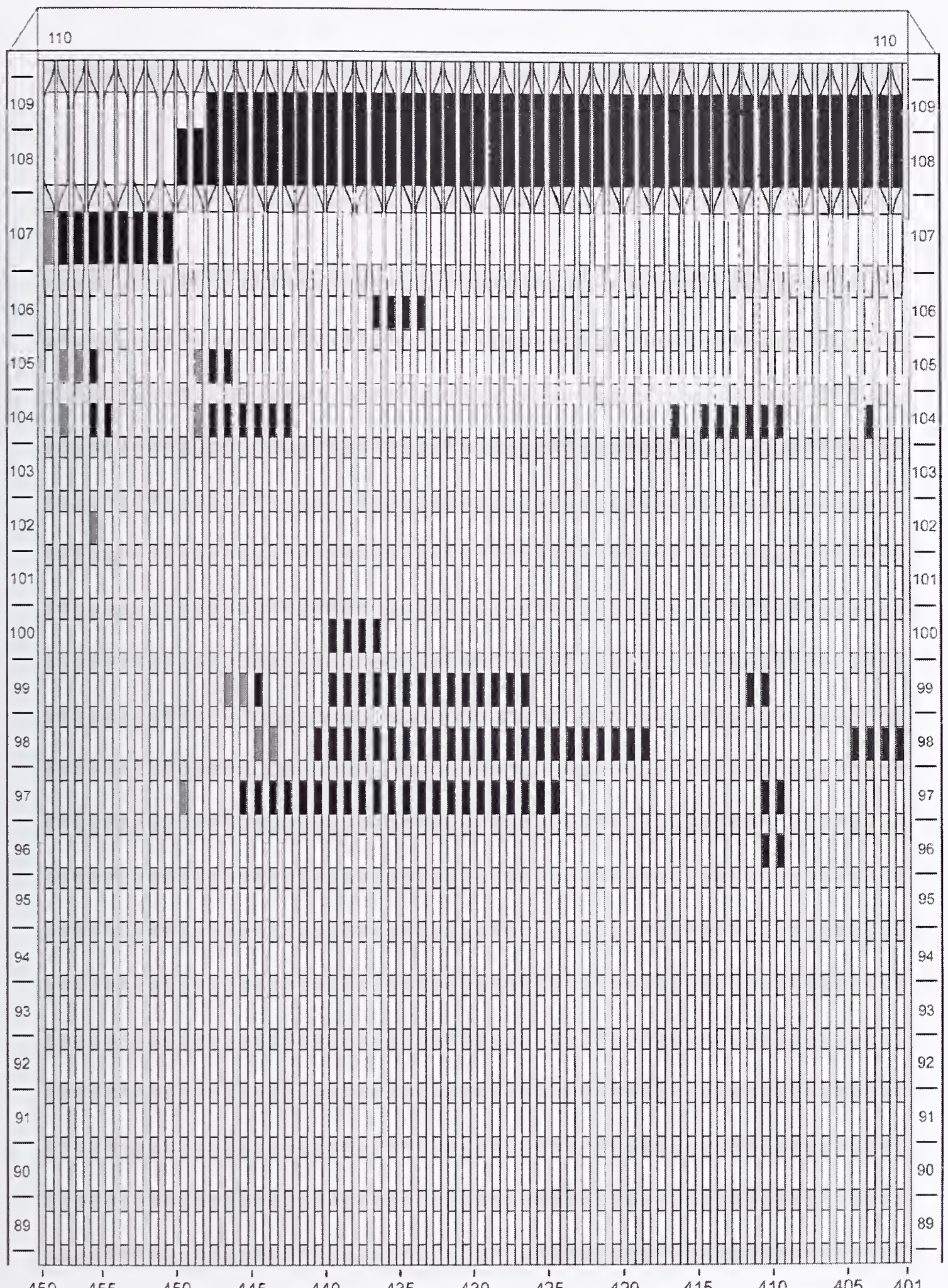

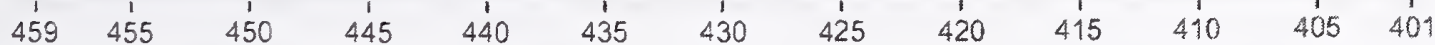

Figure F-14. Diagram of the west face of WTC 1 for floors 89 to 110 at $8: 58$ a.m. showing windows where smoke was observed and those that were hidden from view. 

WTC 1, West Face
9:00 a.m.



Figure F-15. Diagram of the west face of WTC 1 for floors 89 to 110 at 9:00 a.m. showing the condition of windows and locations of fires. 


$$
\text { WTC 1, West Face 9:00 a.m. }
$$

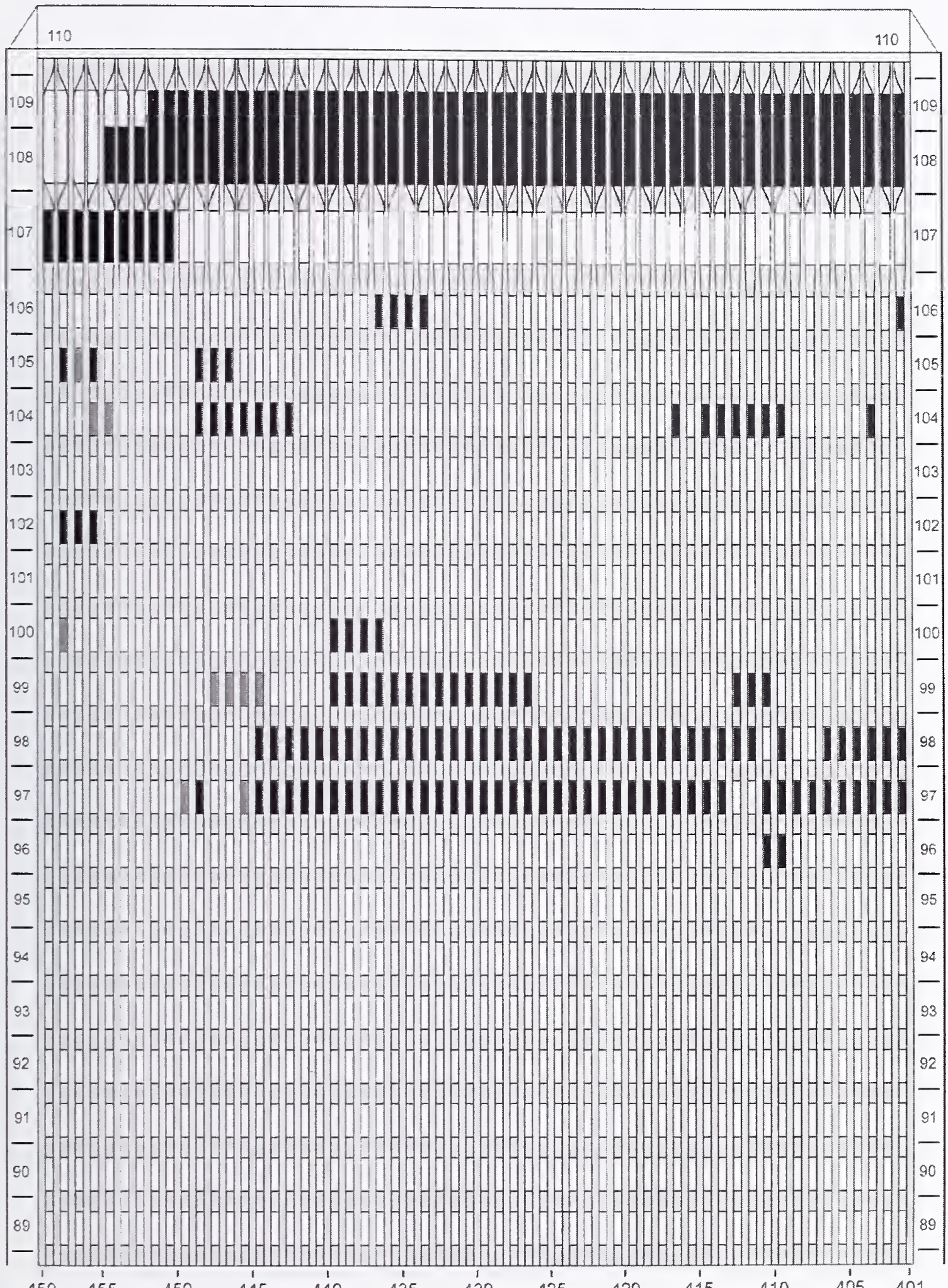

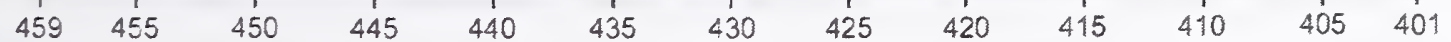

Figure F-16. Diagram of the west face of WTC 1 for floors 89 to 110 at 9:00 a.m. showing windows where smoke was observed and those that were hidden from view. 
WTC 1, West Face $\quad$ 9:02 a.m.

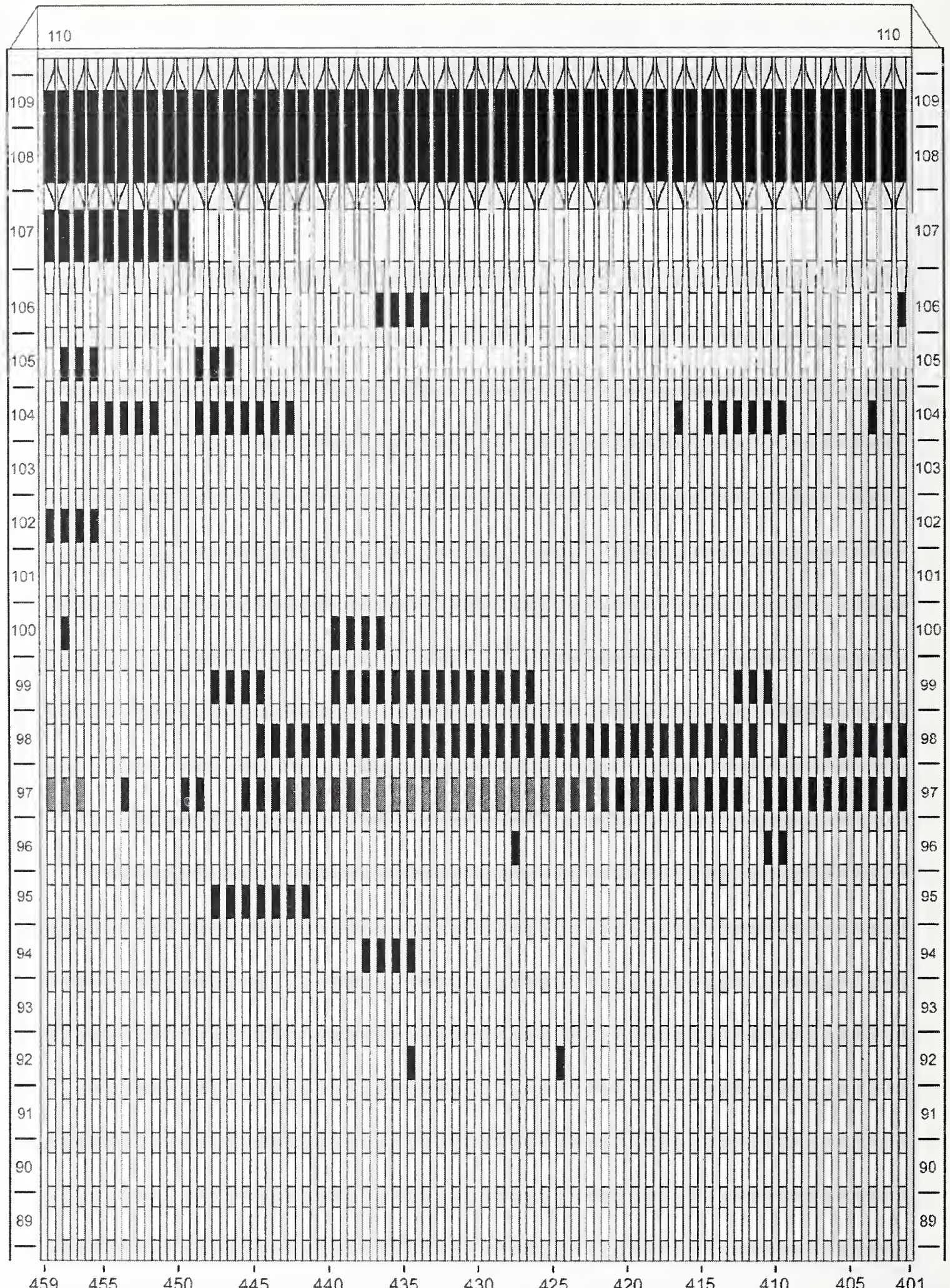

Figure F-17. Diagram of the west face of WTC 1 for floors 89 to 110 at 9:02 a.m. showing the condition of windows and locations of fires. 


$$
\text { WTC 1, West Face } \quad \text { 9:02 a.m. }
$$

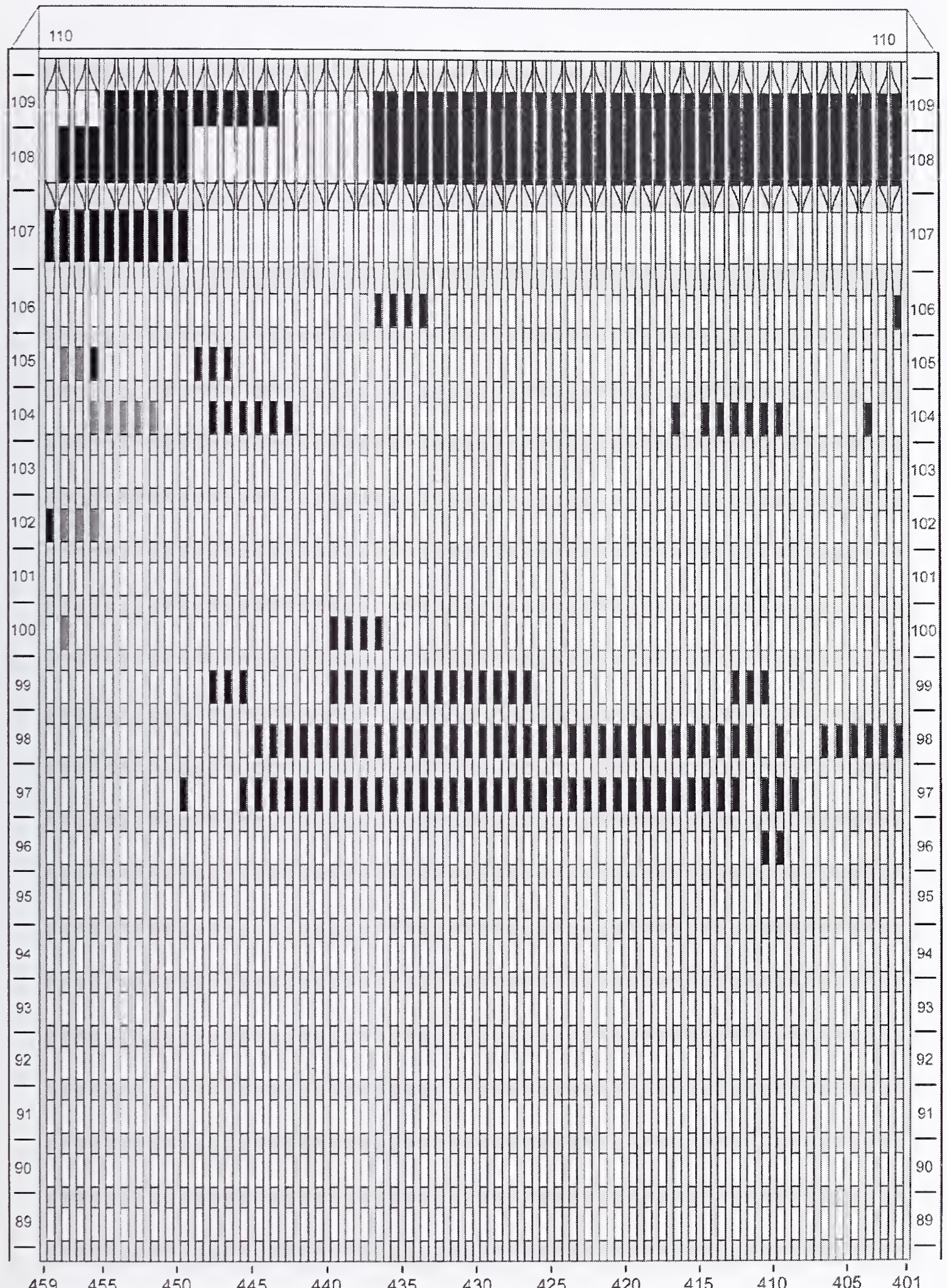

Figure F-18. Diagram of the west face of WTC 1 for floors 89 to 110 at 9:02 a.m. showing windows where smoke was observed and those that were hidden from view. 

WTC 1, West Face
9:04 a.m.

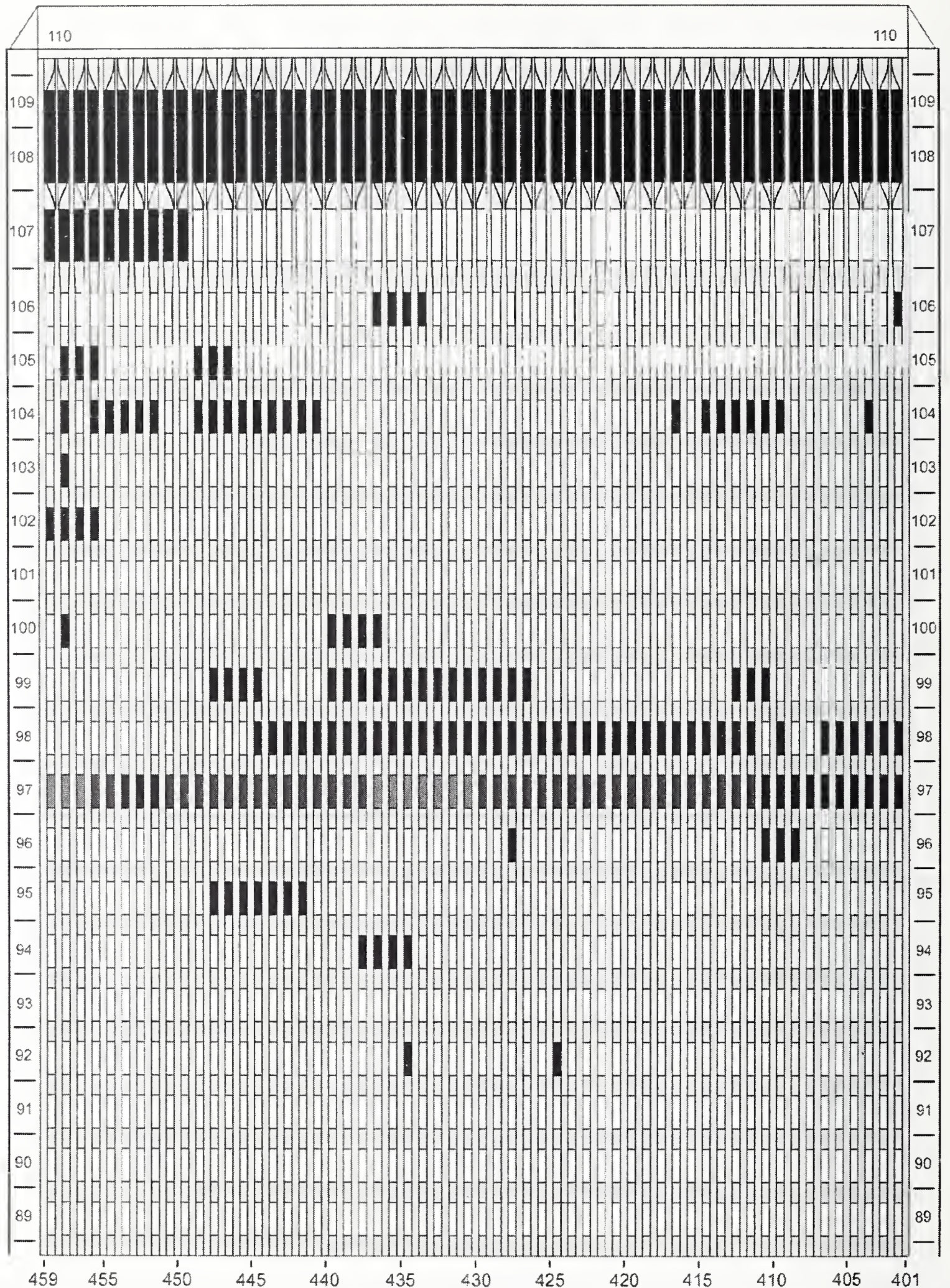

Figure F-19. Diagram of the west face of WTC 1 for floors 89 to 110 at 9:04 a.m. showing the condition of windows and locations of fires. 


$$
\text { WTC 1, West Face 9:04 a.m. }
$$

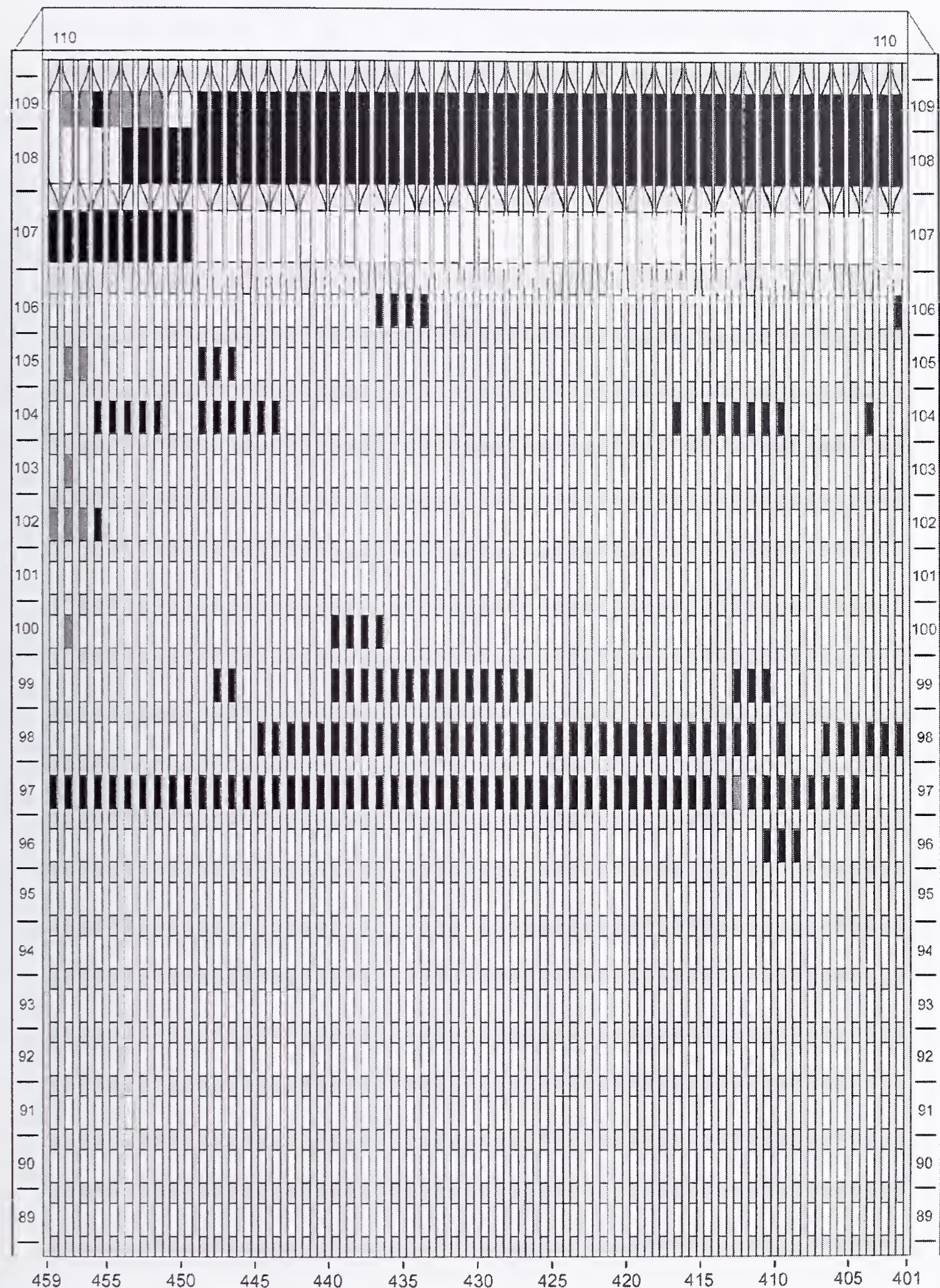

Figure F-20. Diagram of the west face of WTC 1 for floors 89 to 110 at 9:04 a.m. showing windows where smoke was observed and those that were hidden from view. 
WTC 1, West Face

9:06 a.m.

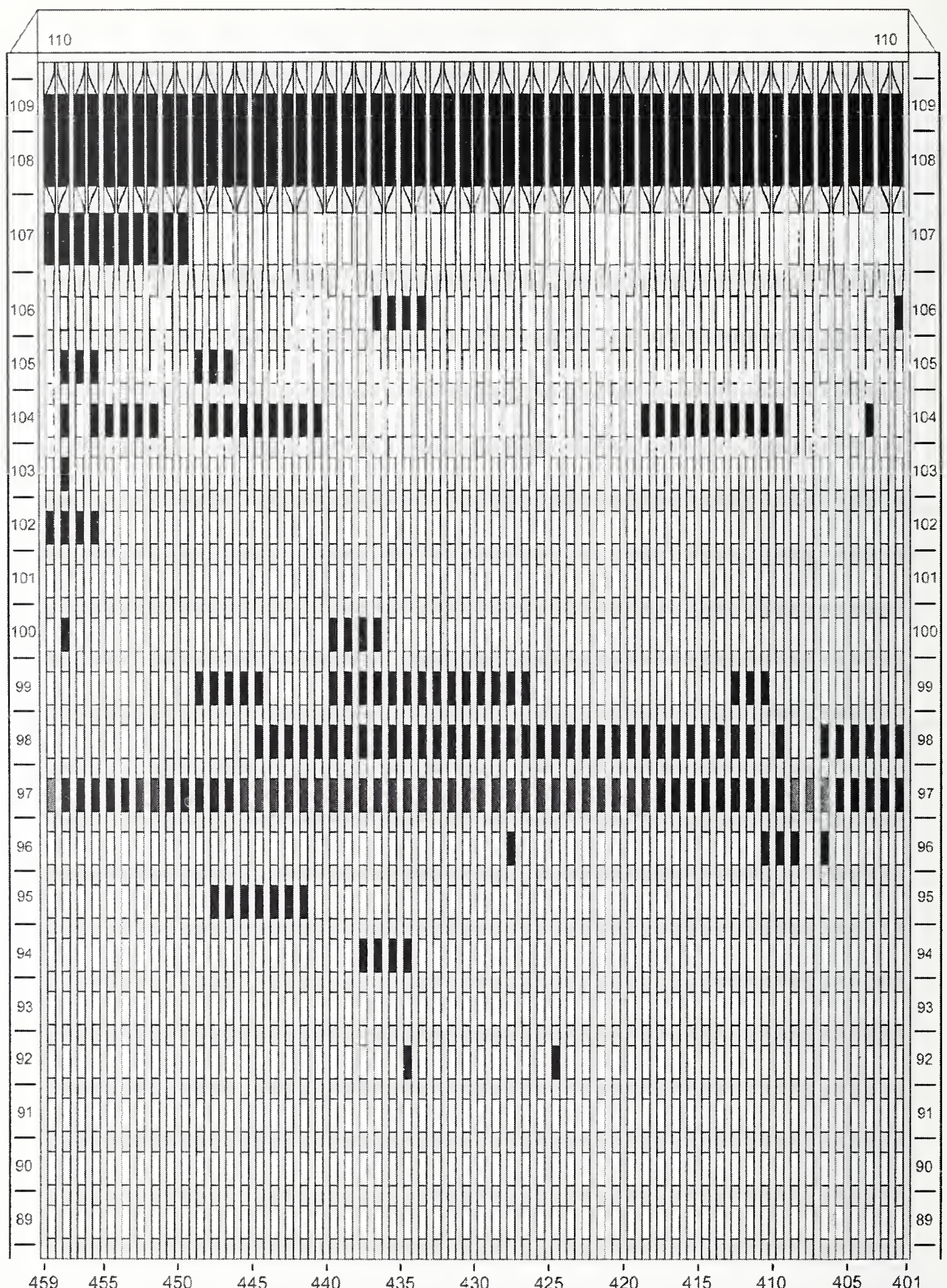

Figure F-21. Diagram of the west face of WTC 1 for floors 89 to 110 at 9:06 a.m. showing the condition of windows and locations of fires. 
WTC 1, West Face 9:06 a.m.

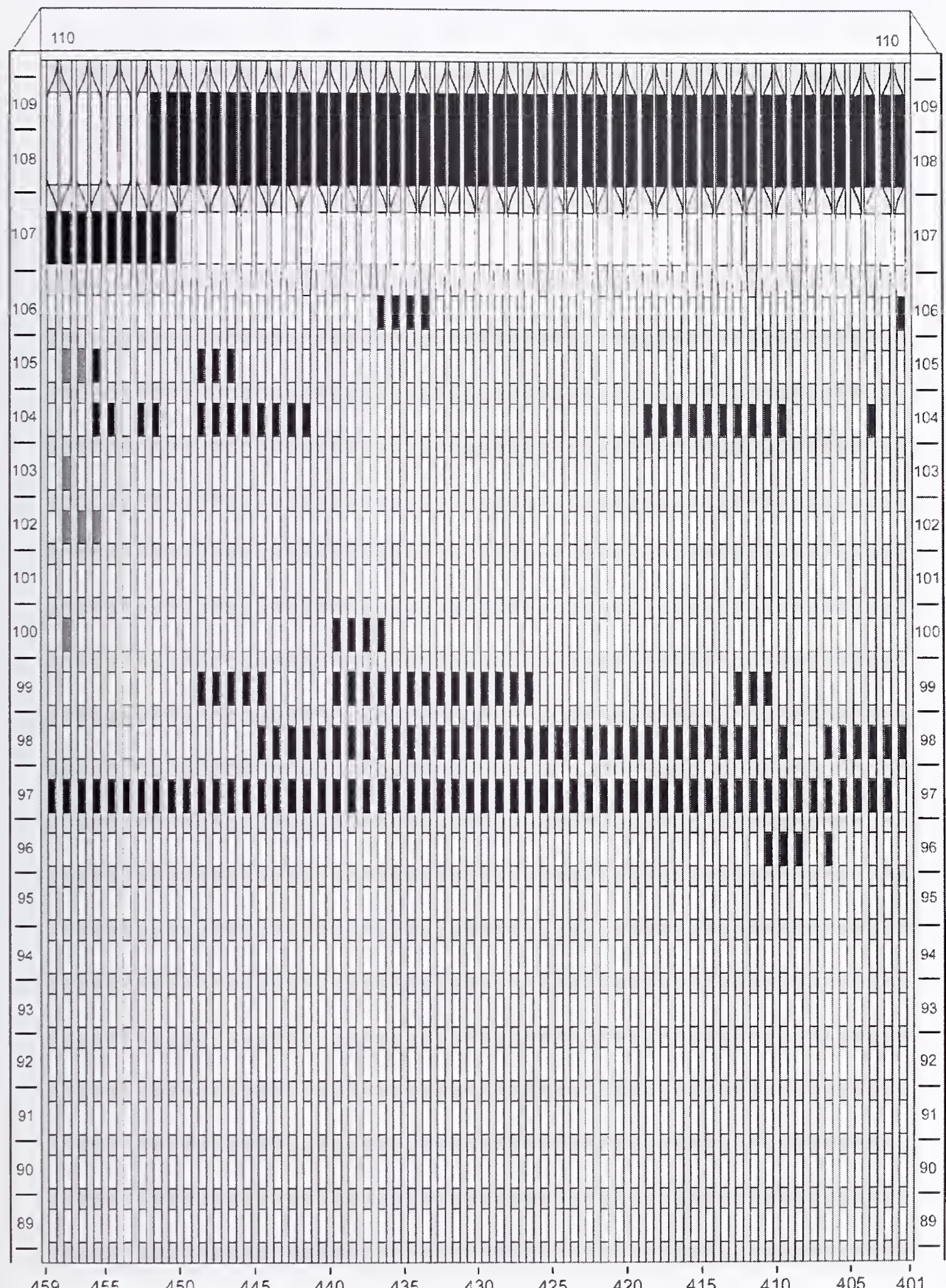

Figure F-22. Diagram of the west face of WTC 1 for floors 89 to 110 at 9:06 a.m. showing windows where smoke was observed and those that were hidden from view. 


\section{WTC 1, West Face 9:08 a.m.}

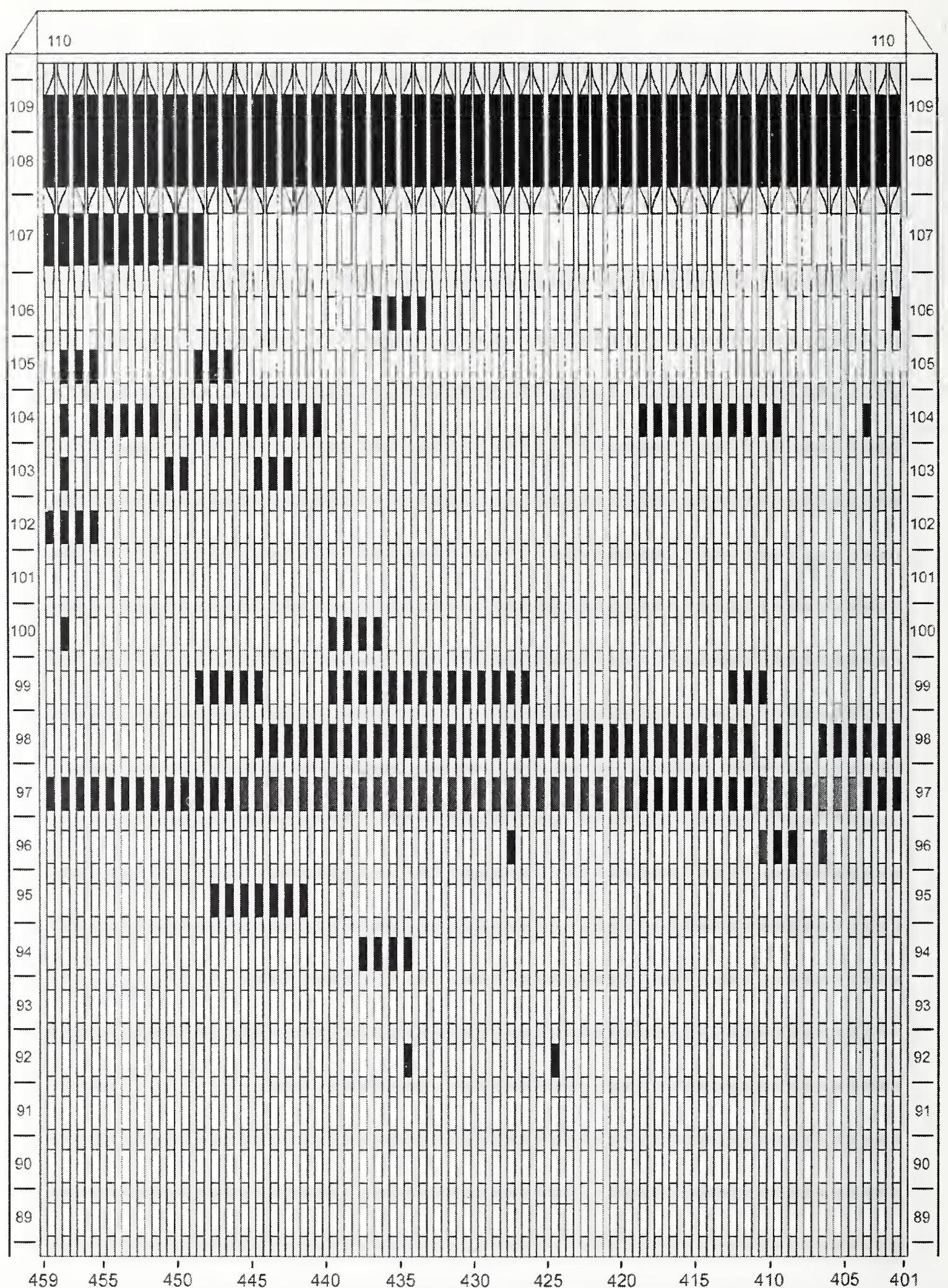

Figure F-23. Diagram of the west face of WTC 1 for floors 89 to 110 at 9:08 a.m. showing the condition of windows and locations of fires. 

WTC 1, West Face
9:08 a.m.



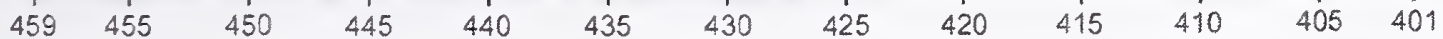

Figure F-24. Diagram of the west face of WTC 1 for floors 89 to 110 at 9:08 a.m. showing windows where smoke was observed and those that were hidden from view. 

WTC 1, West Face
9:10 a.m.



Figure F-25. Diagram of the west face of WTC 1 for floors 89 to 110 at $9: 10$ a.m. showing the condition of windows and locations of fires. 


$$
\text { WTC 1, West Face 9:10 a.m. }
$$

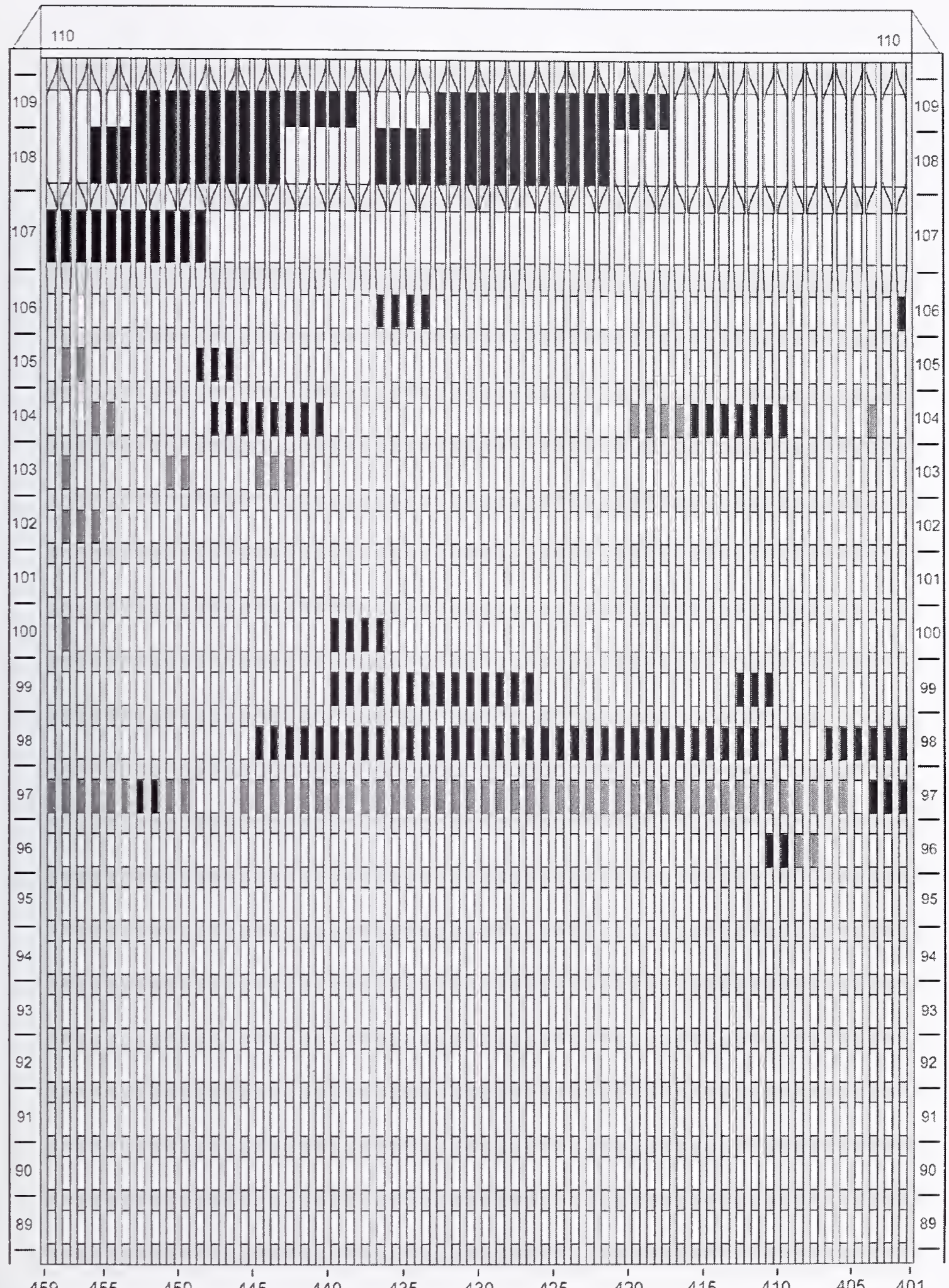

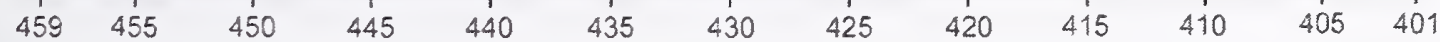

Figure F-26. Diagram of the west face of WTC 1 for floors 89 to 110 at $9: 10$ a.m. showing windows where smoke was observed and those that were hidden from view. 
WTC 1, West Face 9:14 a.m.

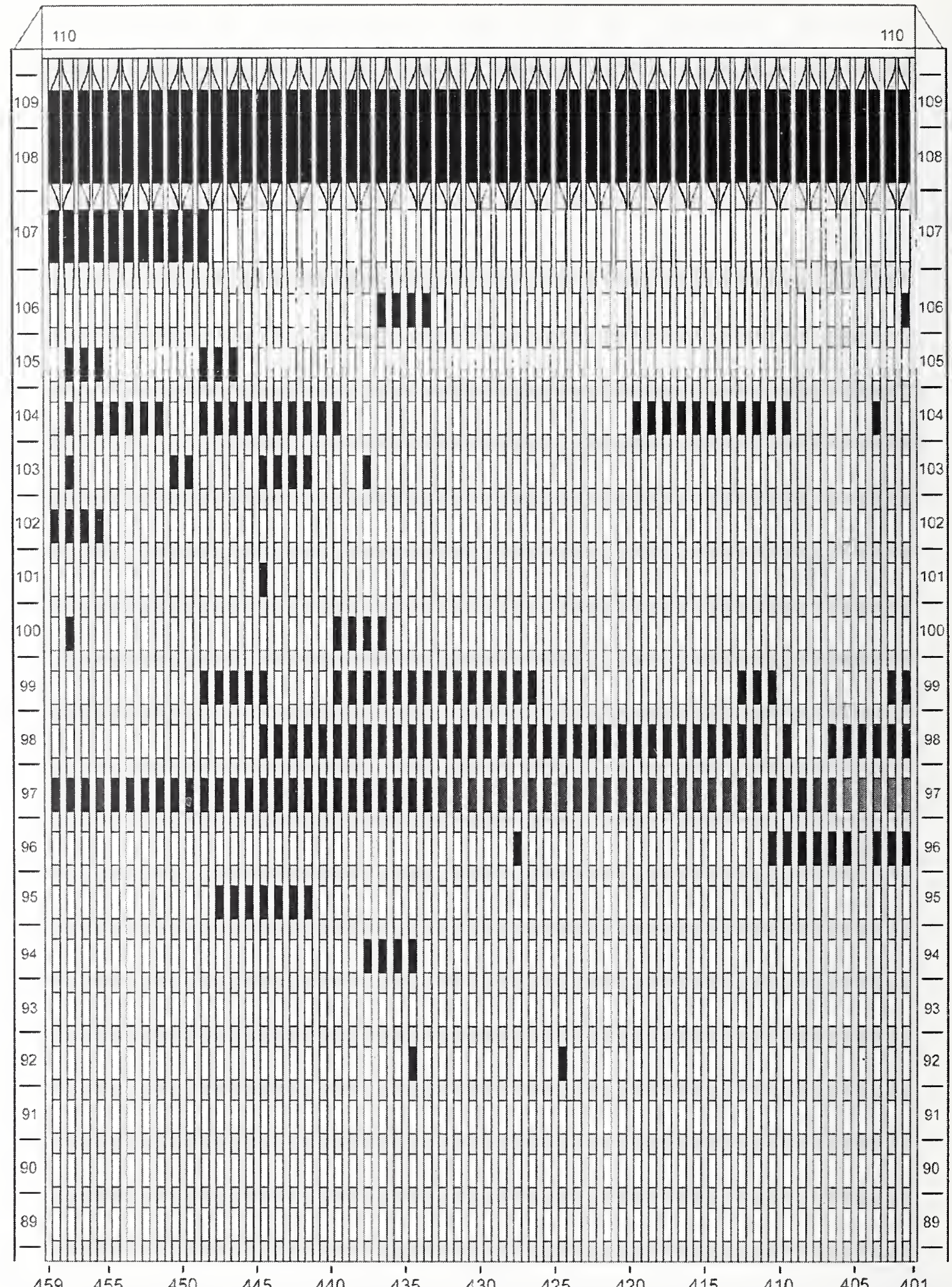

Figure F-27. Diagram of the west face of WTC 1 for floors 89 to 110 at 9:14 a.m. showing the condition of windows and locations of fires. 


$$
\text { WTC 1, West Face 9:14 a.m. }
$$



Figure F-28. Diagram of the west face of WTC 1 for floors 89 to 110 at $9: 14$ a.m. showing windows where smoke was observed and those that were hidden from view. 

WTC 1, West Face
9:20 a.m.

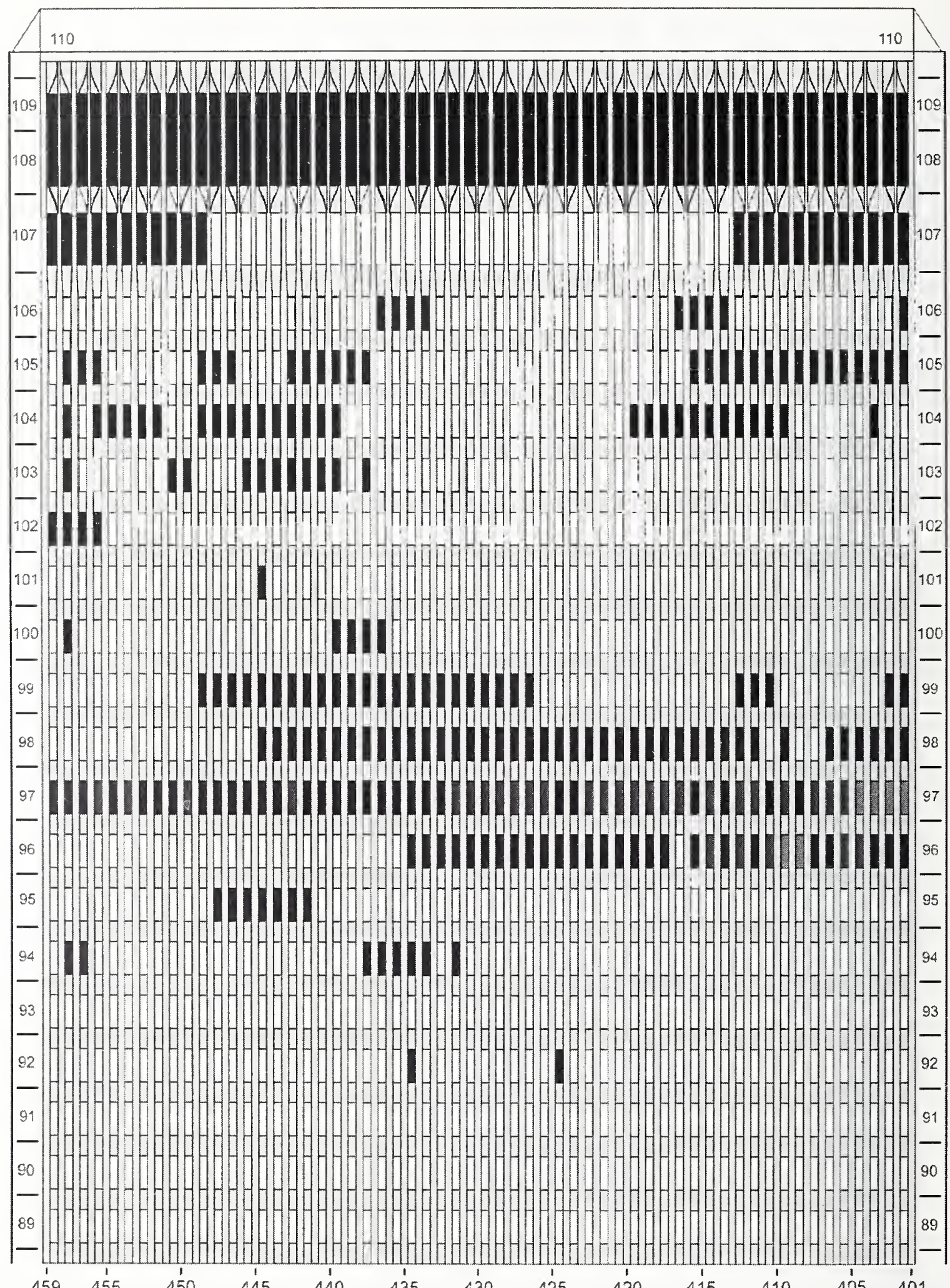

Figure F-29. Diagram of the west face of WTC 1 for floors 89 to 110 at 9:20 a.m. showing the condition of windows and locations of fires. 


$$
\text { WTC 1, West Face 9:20 a.m. }
$$

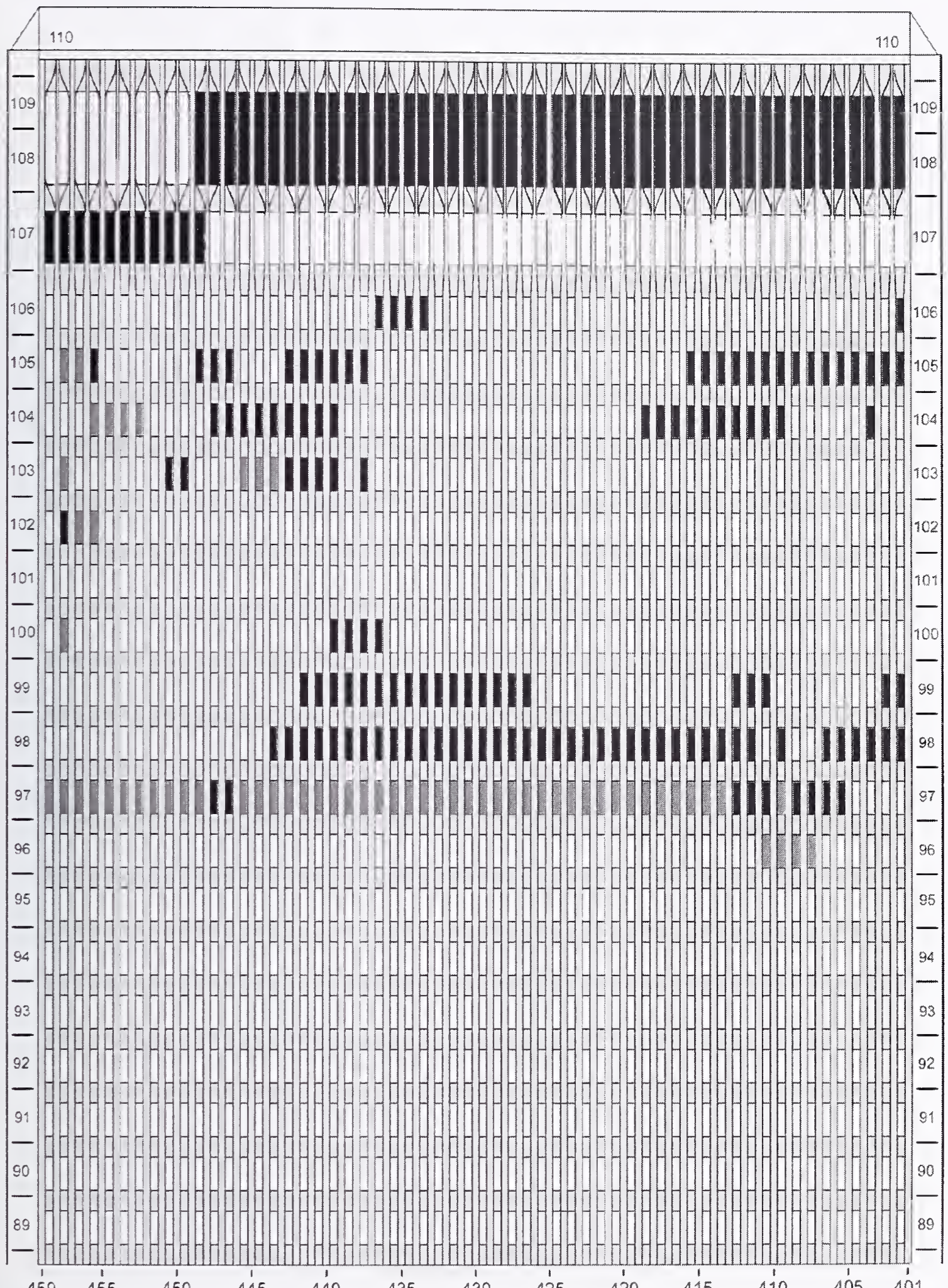

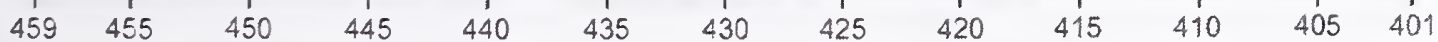

Figure F-30. Diagram of the west face of WTC 1 for floors 89 to 110 at 9:20 a.m. showing windows where smoke was observed and those that were hidden from view. 
WTC 1, West Face 9:24 a.m.

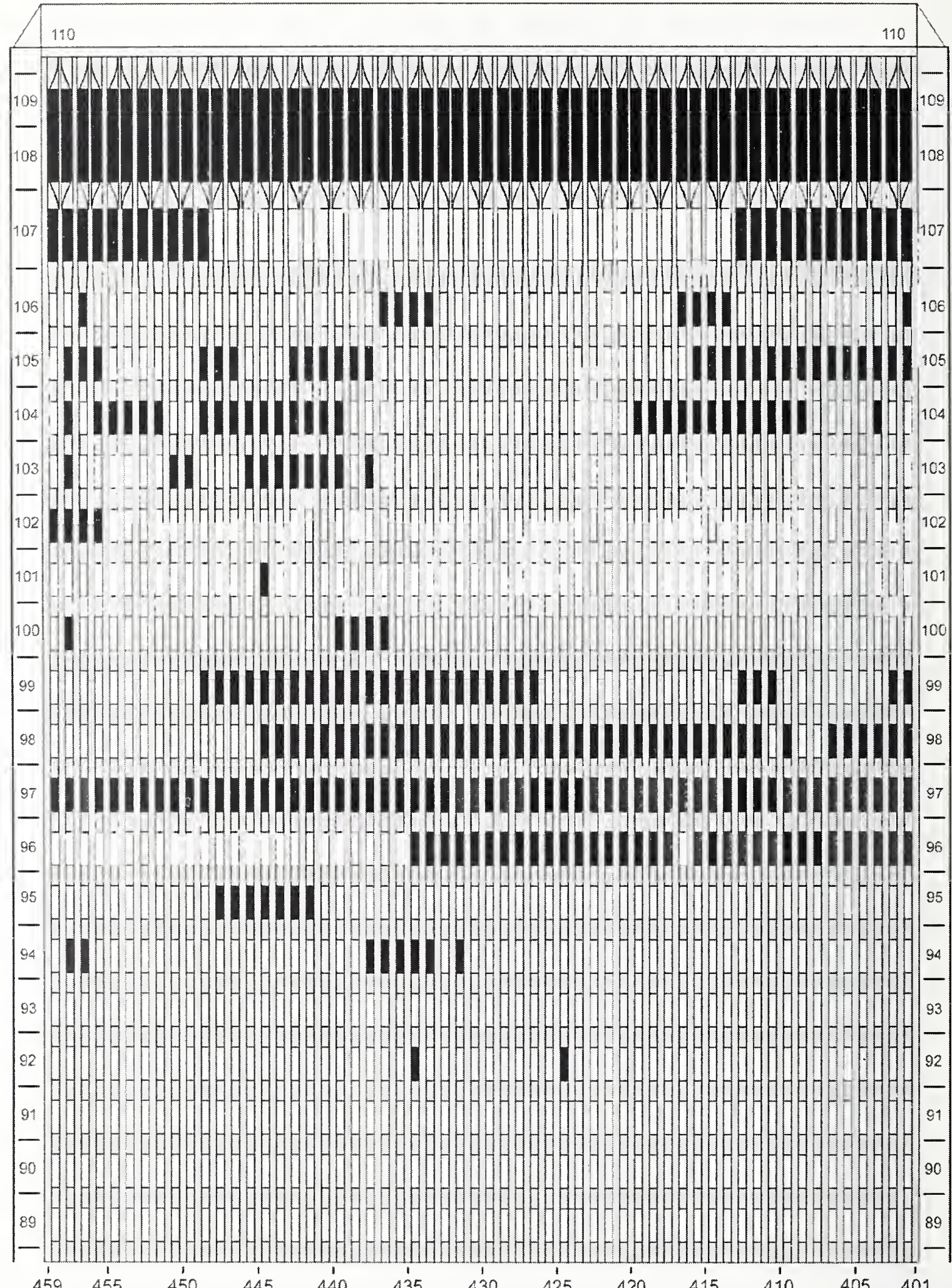

Figure F-31. Diagram of the west face of WTC 1 for floors 89 to 110 at 9:24 a.m. showing the condition of windows and locations of fires. 


$$
\text { WTC 1, West Face 9:24 a.m. }
$$

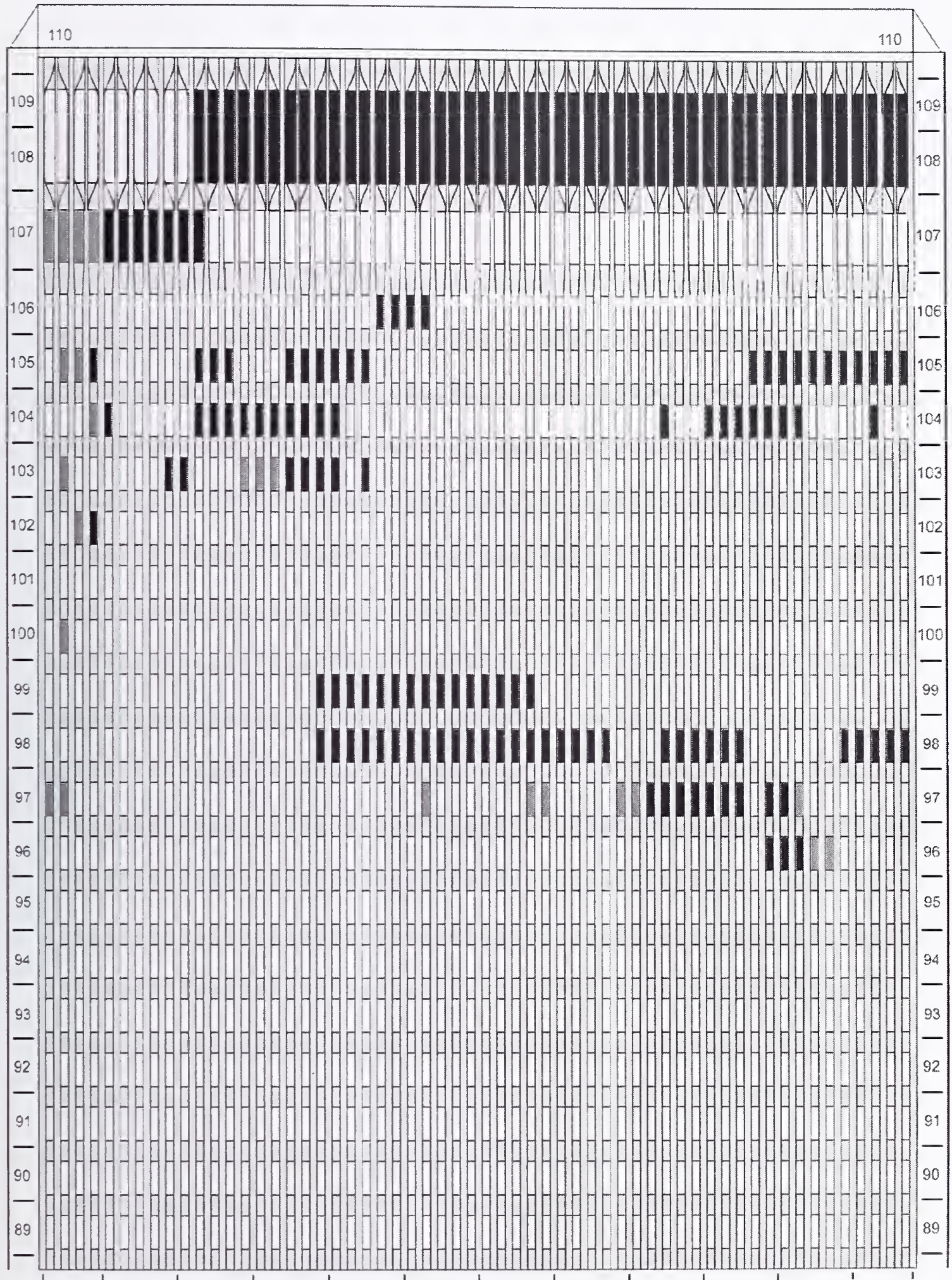

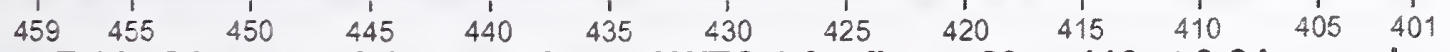

Figure F-32. Diagram of the west face of WTC 1 for floors 89 to 110 at 9:24 a.m. showing windows where smoke was observed and those that were hidden from view. 
WTC 1, West Face 9:30 a.m.



Figure F-33. Diagram of the west face of WTC 1 for floors 89 to 110 at 9:30 a.m. showing the condition of windows and locations of fires. 


$$
\text { WTC 1, West Face 9:30 a.m. }
$$

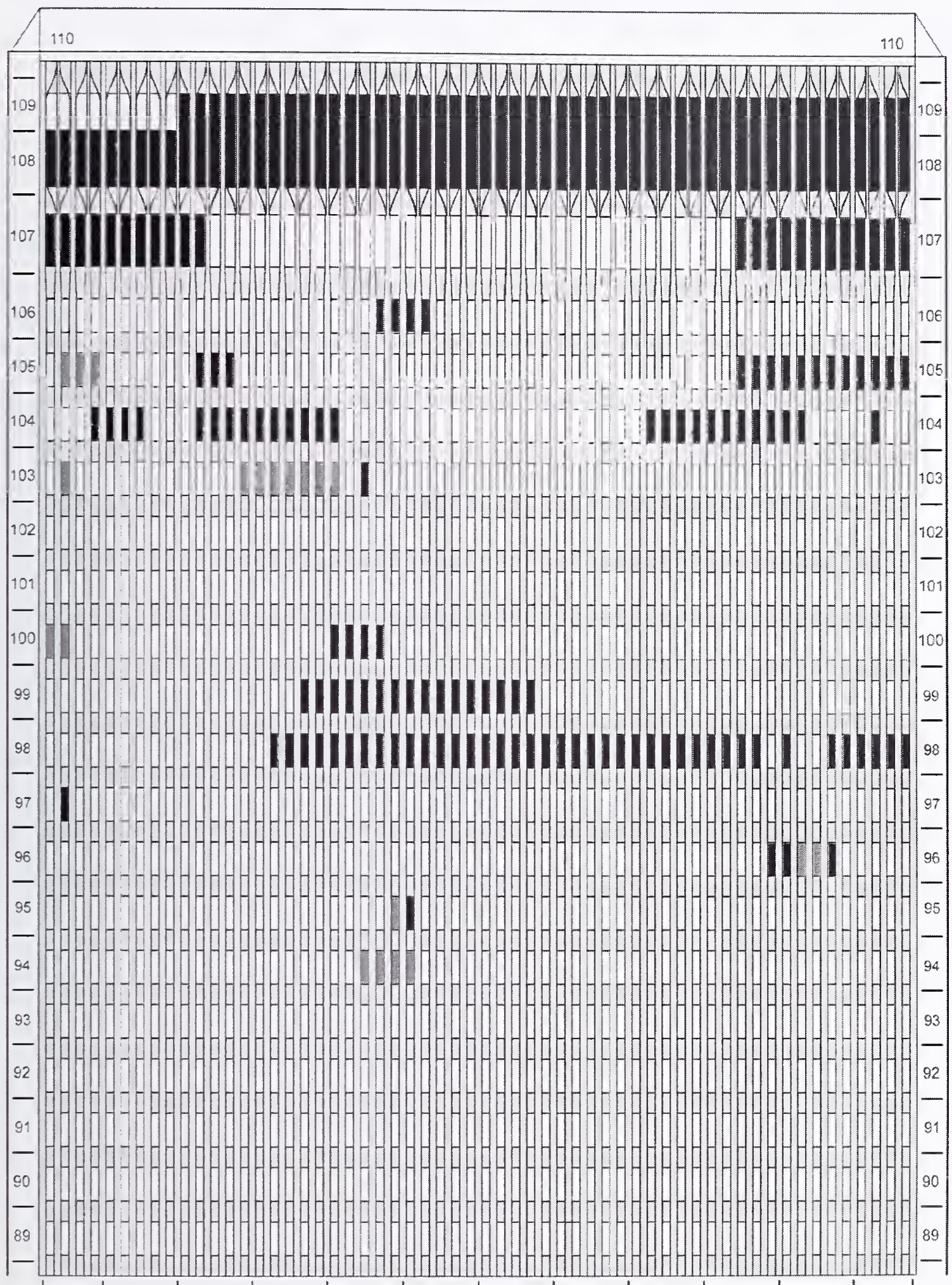

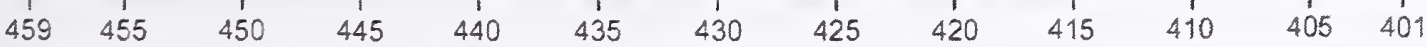

Figure F-34. Diagram of the west face of WTC 1 for floors 89 to 110 at 9:30 a.m. showing windows where smoke was observed and those that were hidden from view. 

WTC 1, West Face
9:36 a.m.

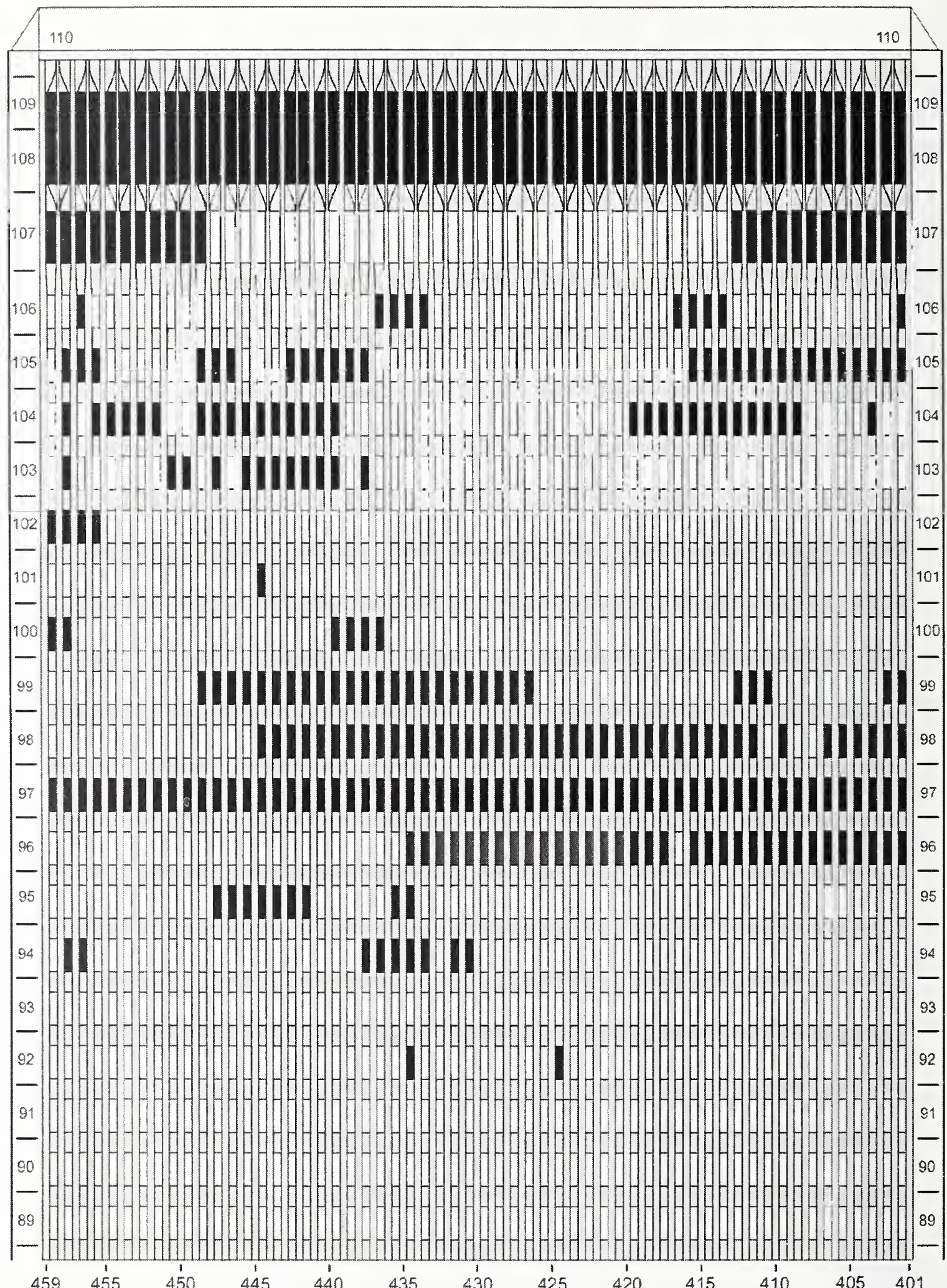

Figure F-35. Diagram of the west face of WTC 1 for floors 89 to 110 at 9:36 a.m. showing the condition of windows and locations of fires. 


$$
\text { WTC 1, West Face 9:36 a.m. }
$$



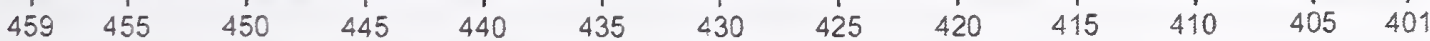

Figure F-36. Diagram of the west face of WTC 1 for floors 89 to 110 at 9:36 a.m. showing windows where smoke was observed and those that were hidden from view. 
WTC 1, West Face

9:40 a.m.

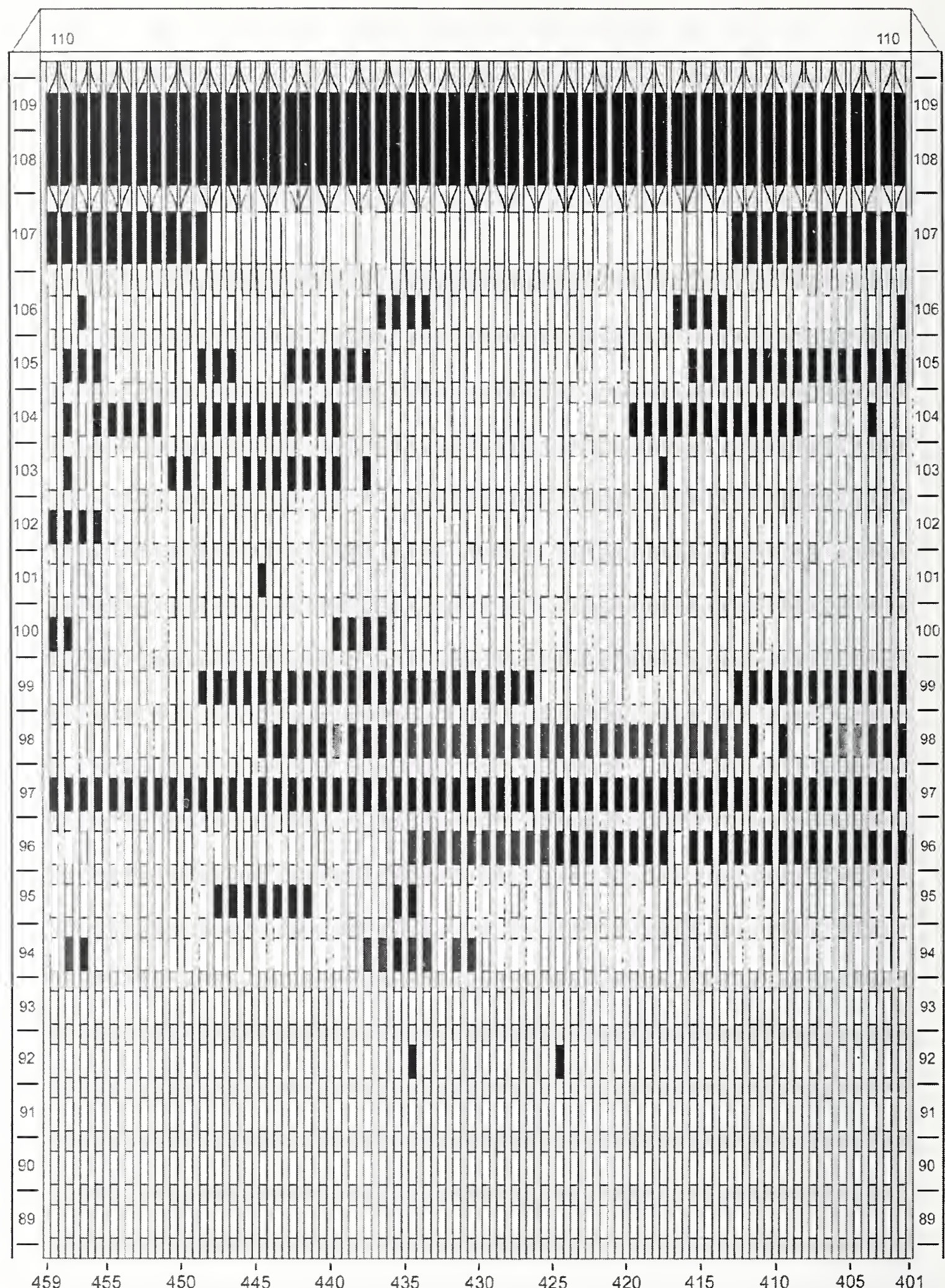

Figure F-37. Diagram of the west face of WTC 1 for floors 89 to 110 at 9:40 a.m. showing the condition of windows and locations of fires. 


$$
\text { WTC 1, West Face 9:40 a.m. }
$$

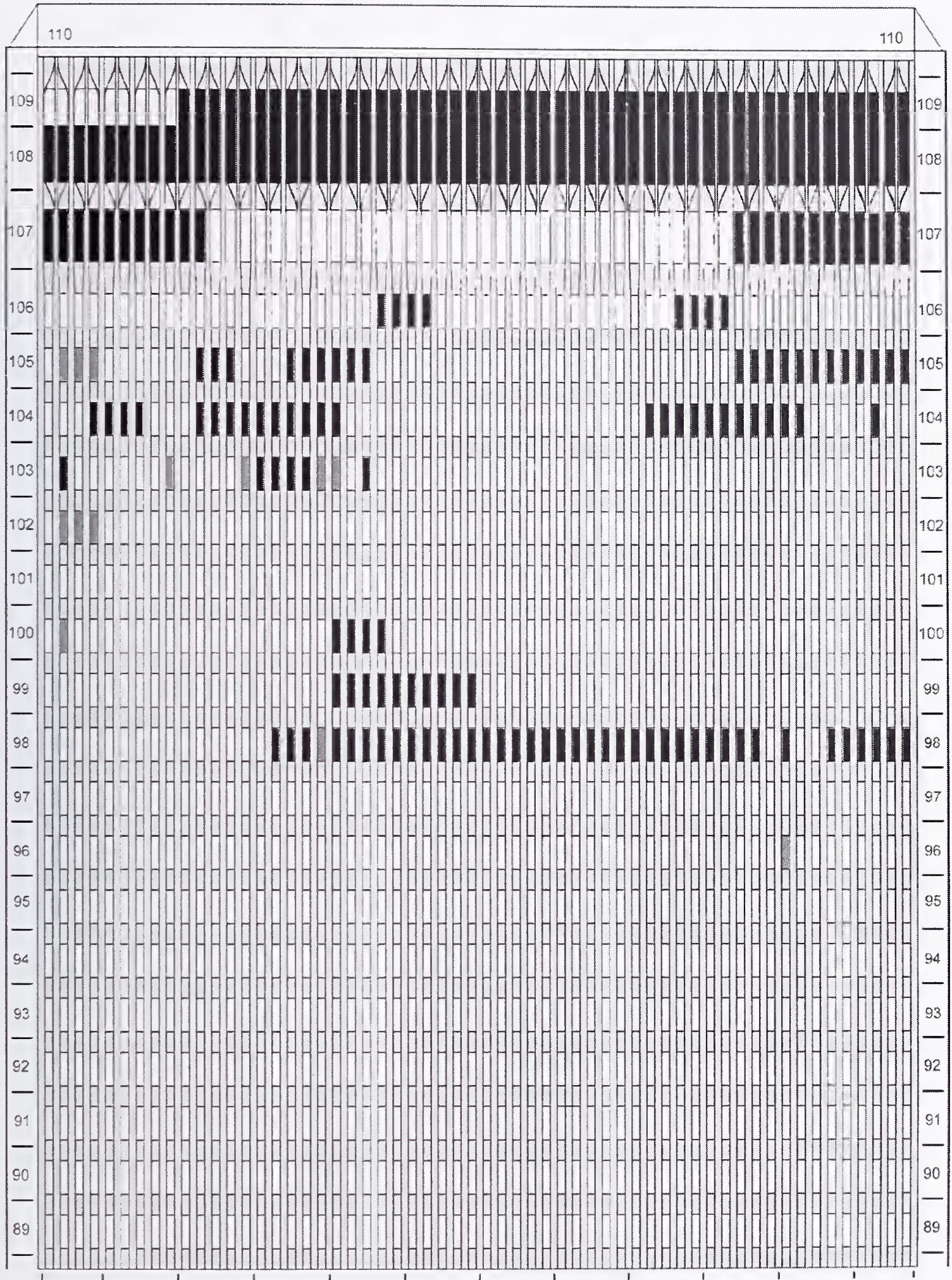

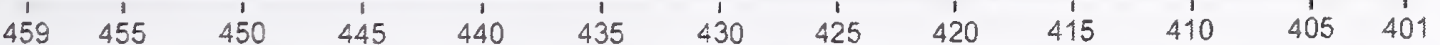

Figure F-38. Diagram of the west face of WTC 1 for floors 89 to 110 at 9:40 a.m. showing windows where smoke was observed and those that were hidden from view. 

WTC 1, West Face
9:42 a.m.

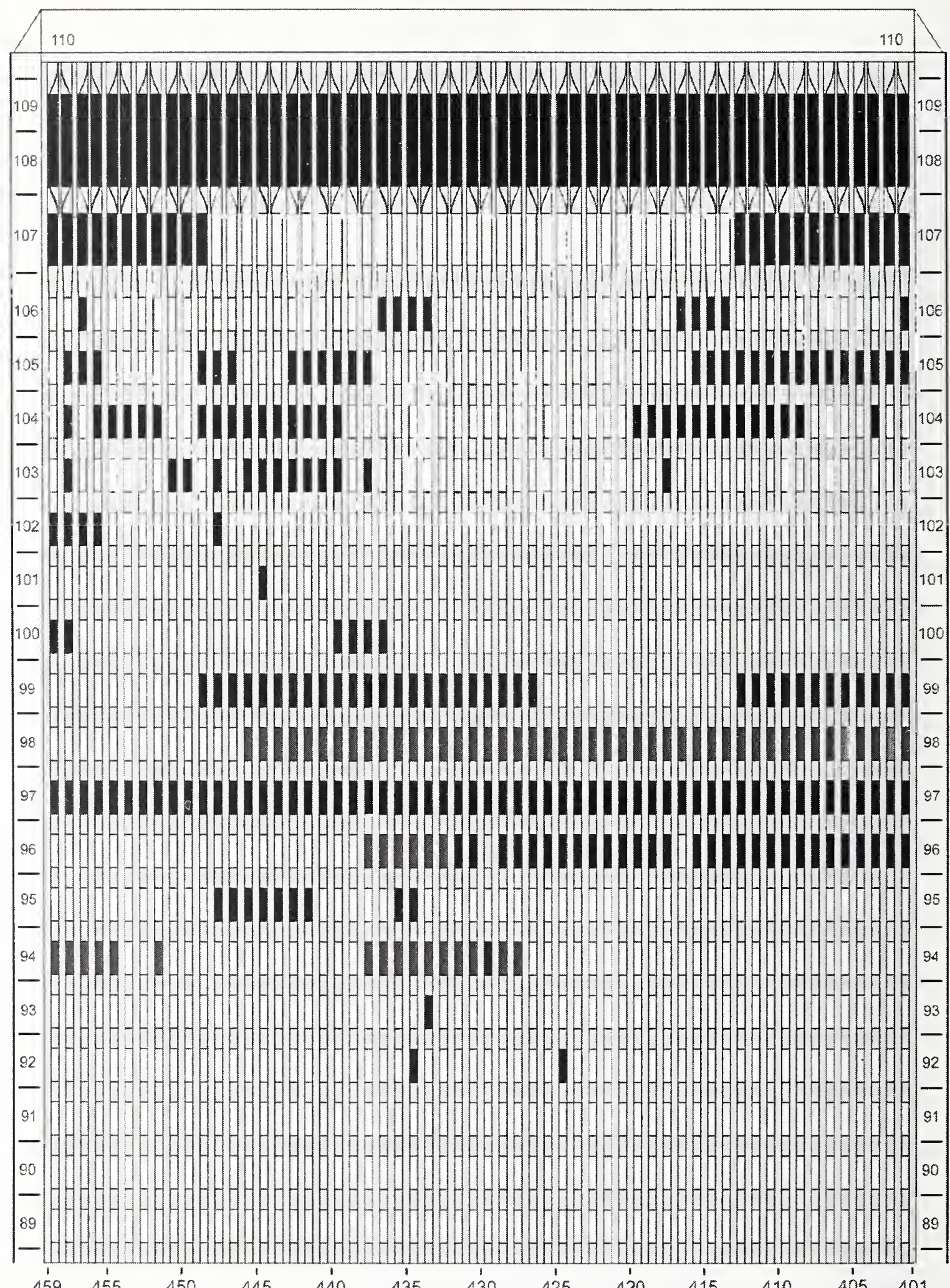

Figure F-39. Diagram of the west face of WTC 1 for floors 89 to 110 at 9:42 a.m. showing the condition of windows and locations of fires. 


$$
\text { WTC 1, West Face 9:42 a.m. }
$$

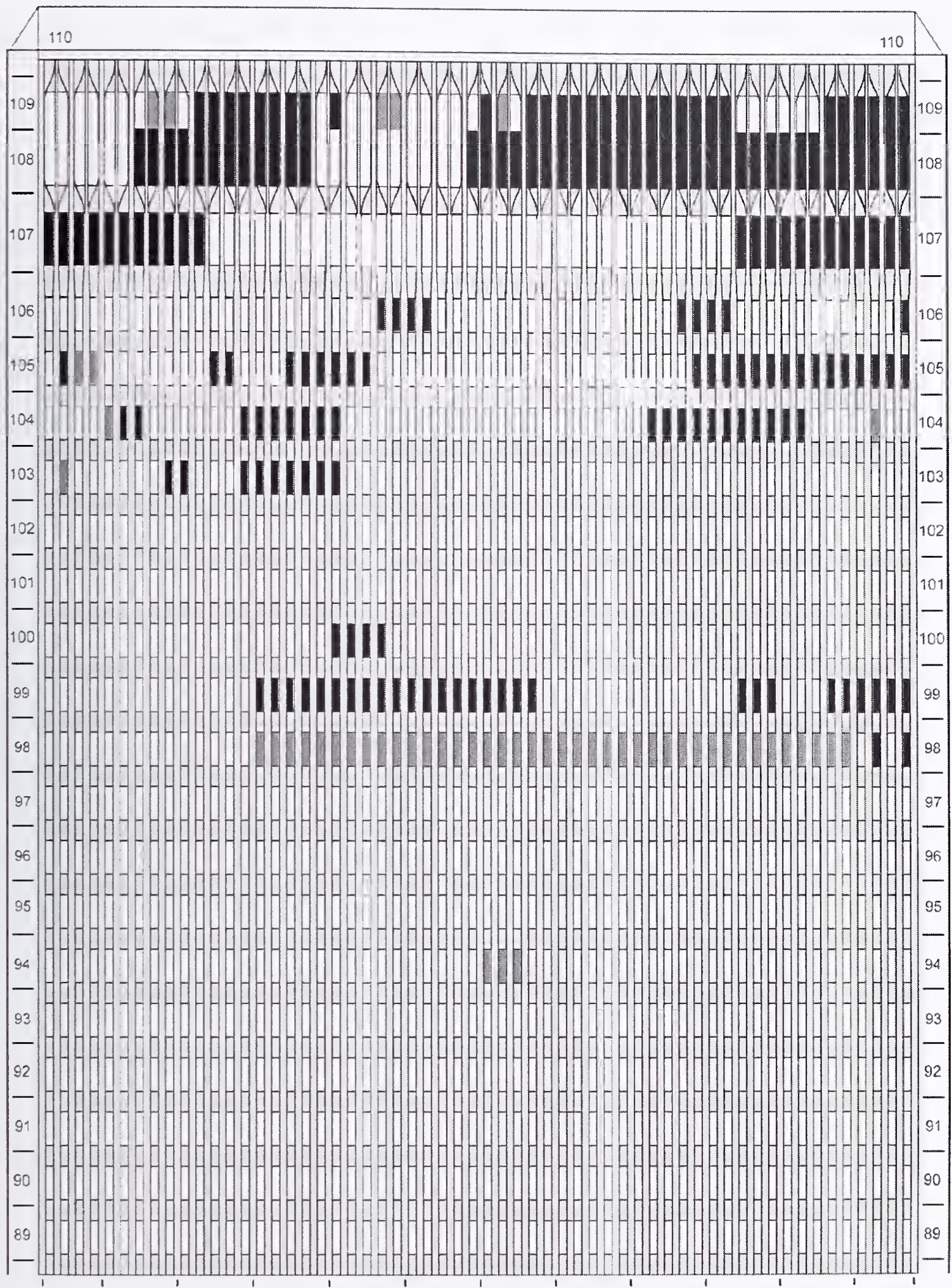

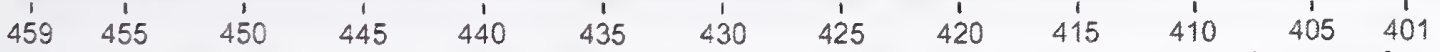

Figure F-40. Diagram of the west face of WTC 1 for floors 89 to 110 at 9:42 a.m. showing windows where smoke was observed and those that were hidden from view. 


\section{WTC 1, West Face 9:44 a.m.}

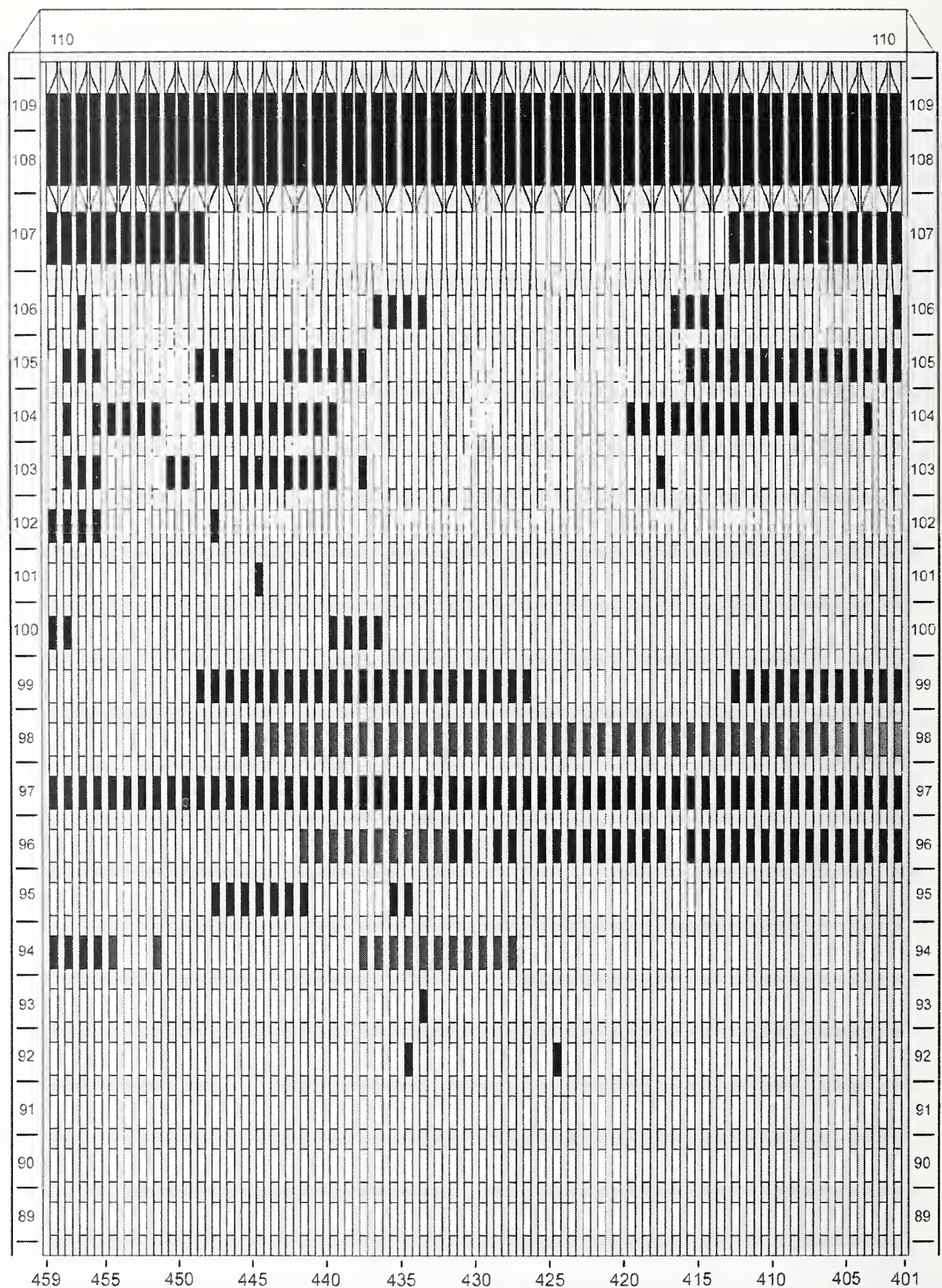

Figure F-41. Diagram of the west face of WTC 1 for floors 89 to 110 at 9:44 a.m. showing the condition of windows and locations of fires. 


$$
\text { WTC 1, West Face 9:44 a.m. }
$$

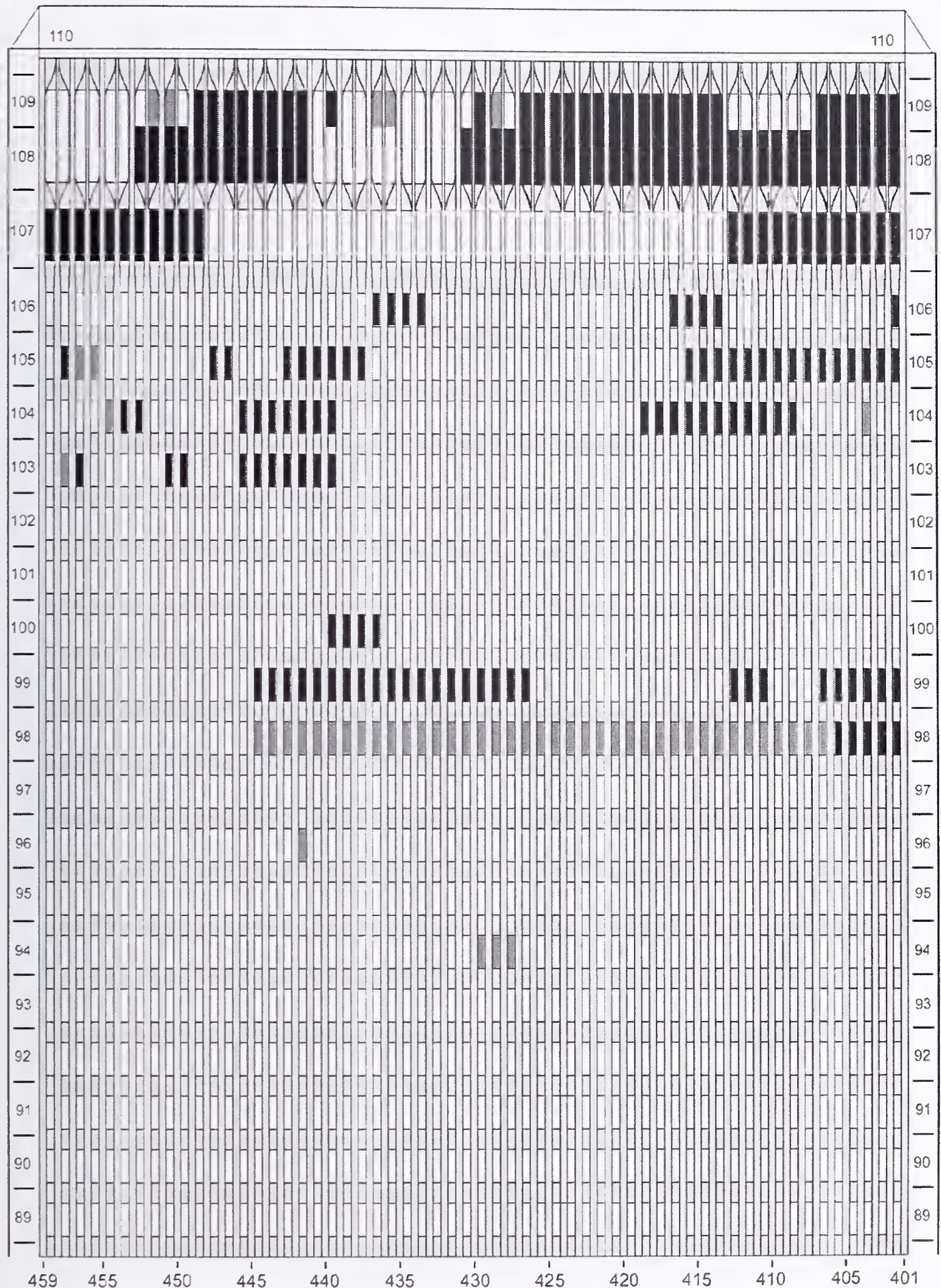

Figure F-42. Diagram of the west face of WTC 1 for floors 89 to 110 at 9:44 a.m. showing windows where smoke was observed and those that were hidden from view. 

WTC 1, West Face
9:48 a.m.

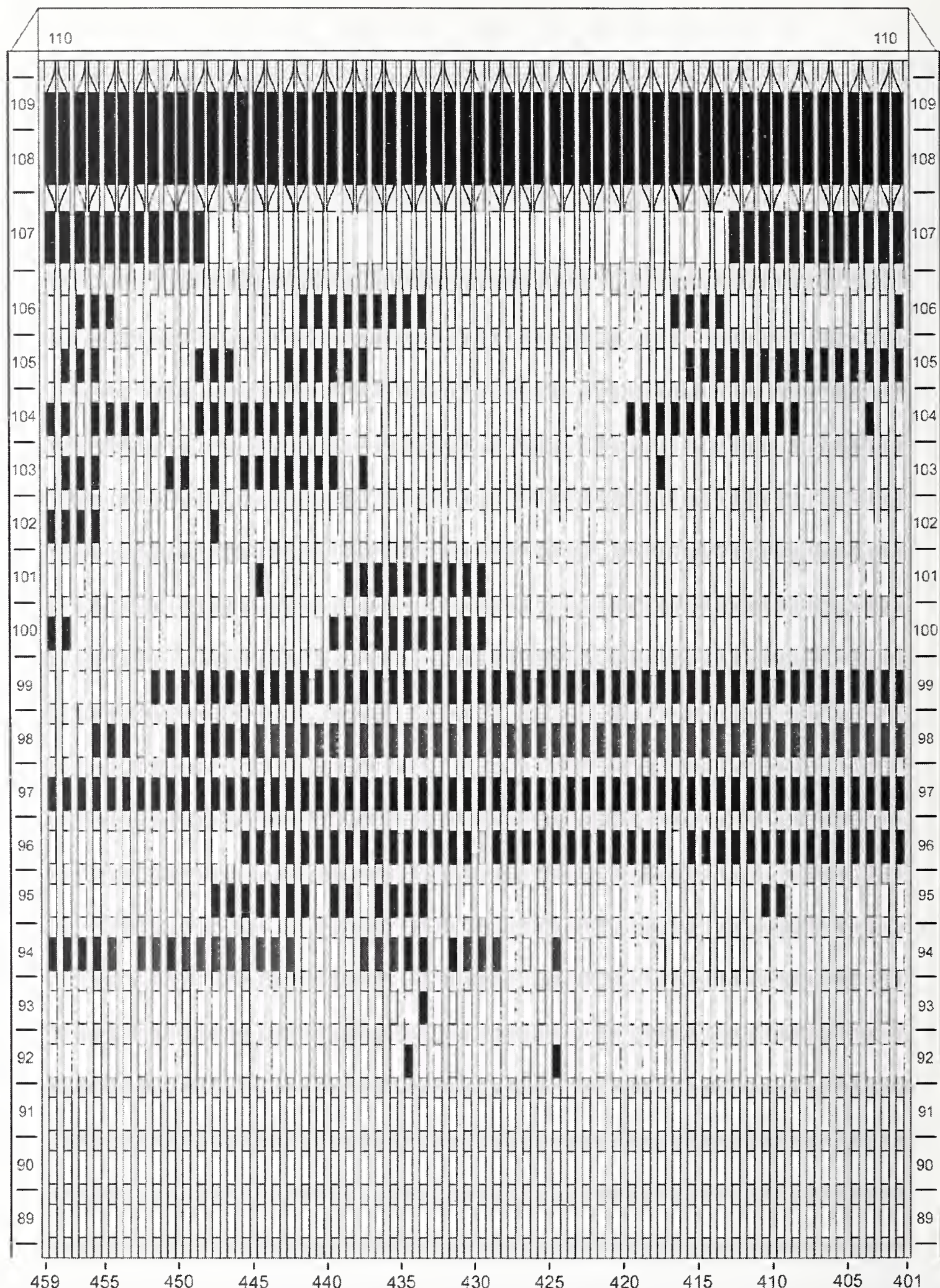

Figure F-43. Diagram of the west face of WTC 1 for floors 89 to 110 at 9:48 a.m. showing the condition of windows and locations of fires. 


$$
\text { WTC 1, West Face } \quad 9: 48 \text { a.m. }
$$

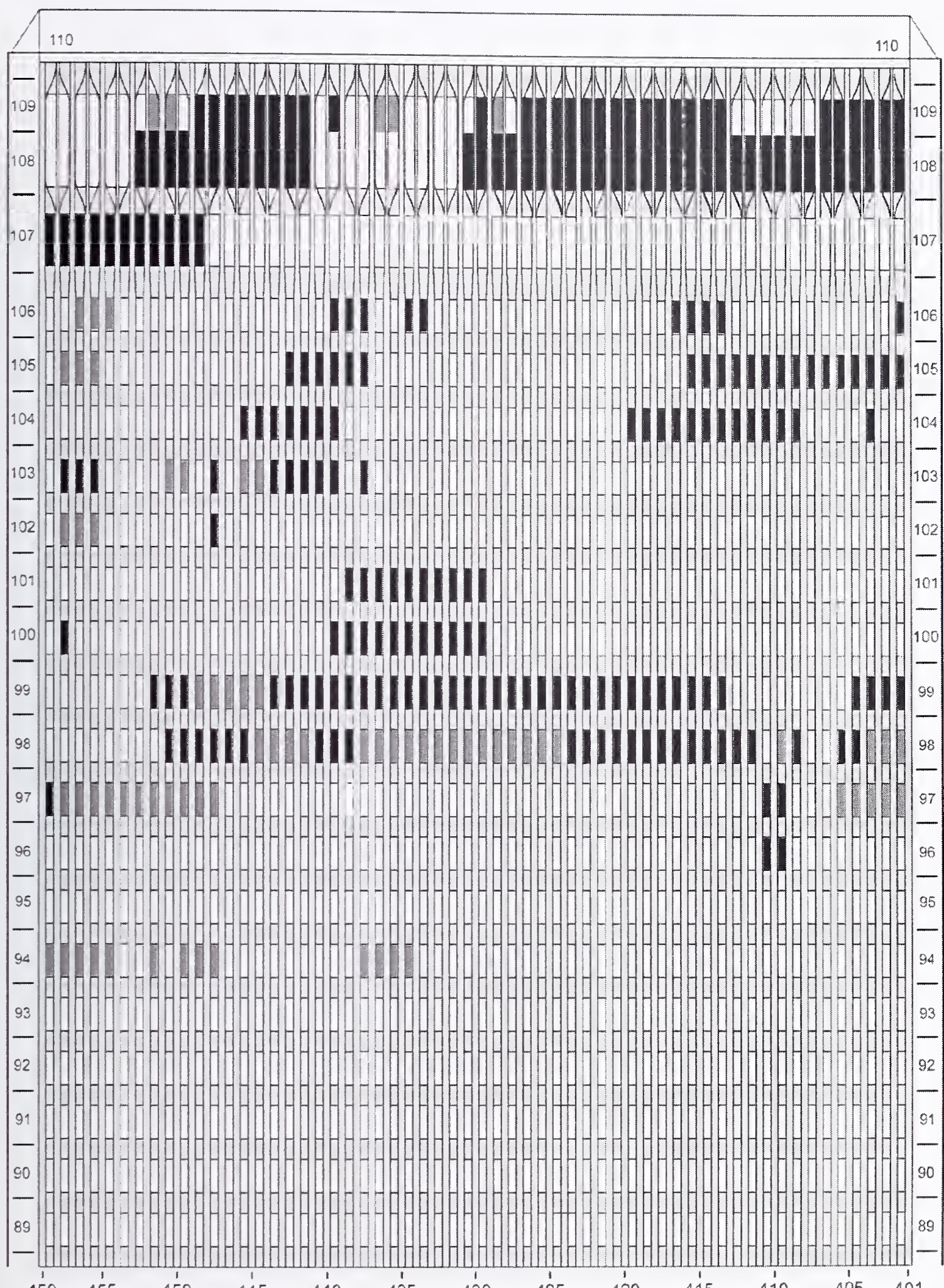

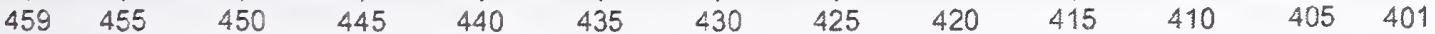

Figure F-44. Diagram of the west face of WTC 1 for floors 89 to 110 at 9:48 a.m. showing windows where smoke was observed and those that were hidden from view. 


$$
\text { WTC 1, West Face 9:52 a.m. }
$$

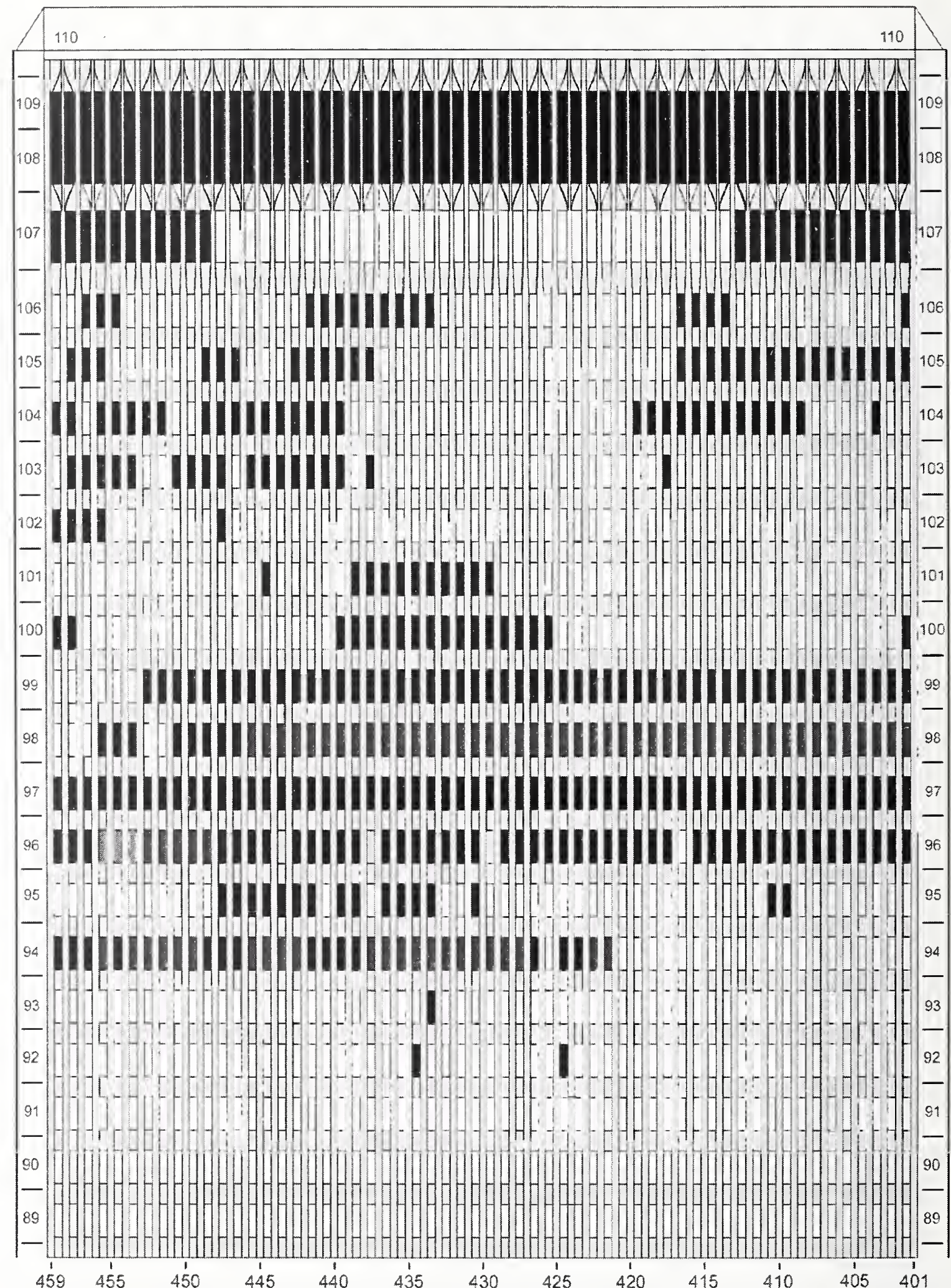

Figure F-45. Diagram of the west face of WTC 1 for floors 89 to 110 at 9:52 a.m. showing the condition of windows and locations of fires. 


$$
\text { WTC 1, West Face 9:52 a.m. }
$$

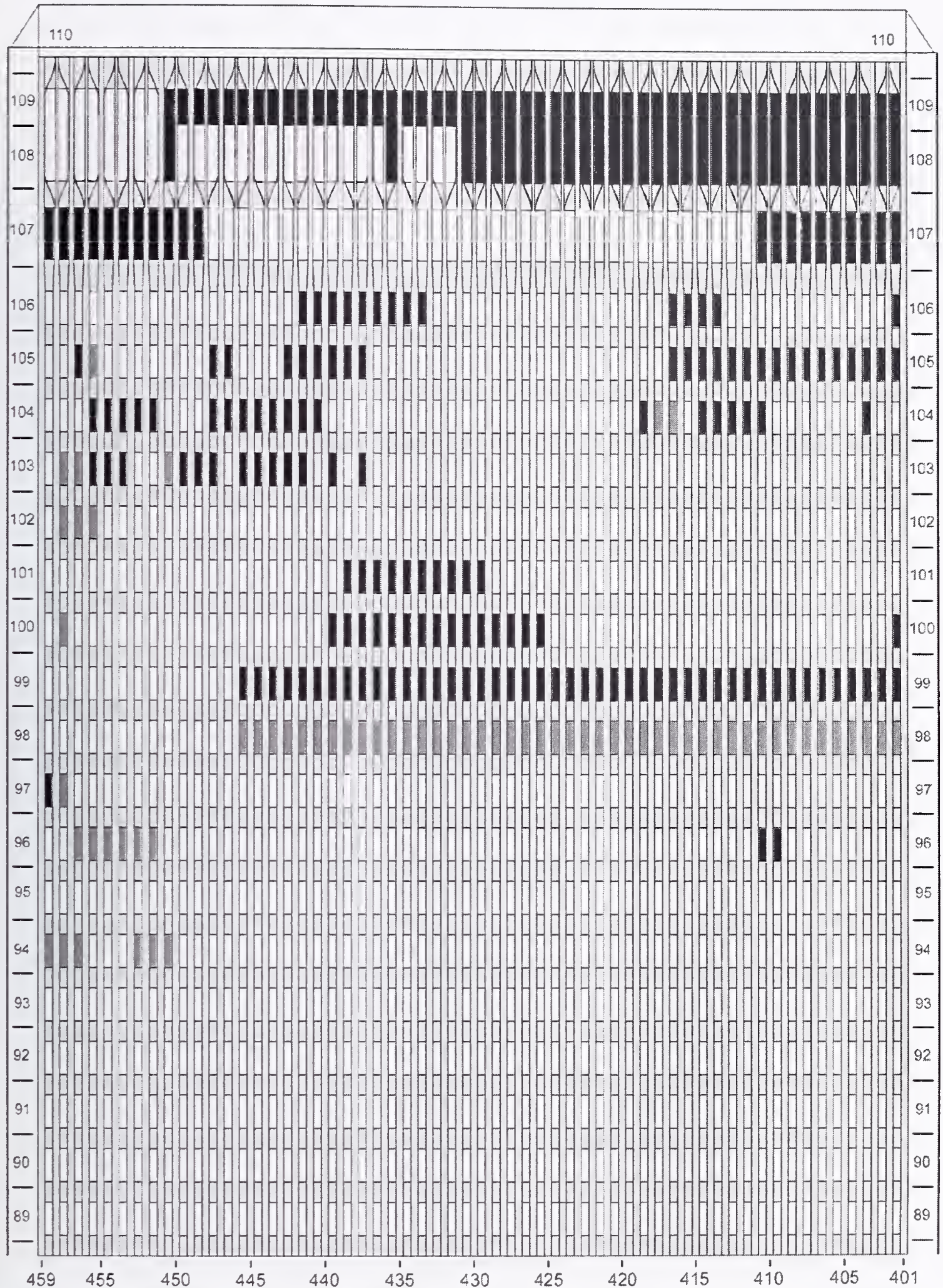

Figure F-46. Diagram of the west face of WTC 1 for floors 89 to 110 at 9:52 a.m. showing windows where smoke was observed and those that were hidden from view. 


$$
\text { WTC 1, West Face 9:54 a.m. }
$$

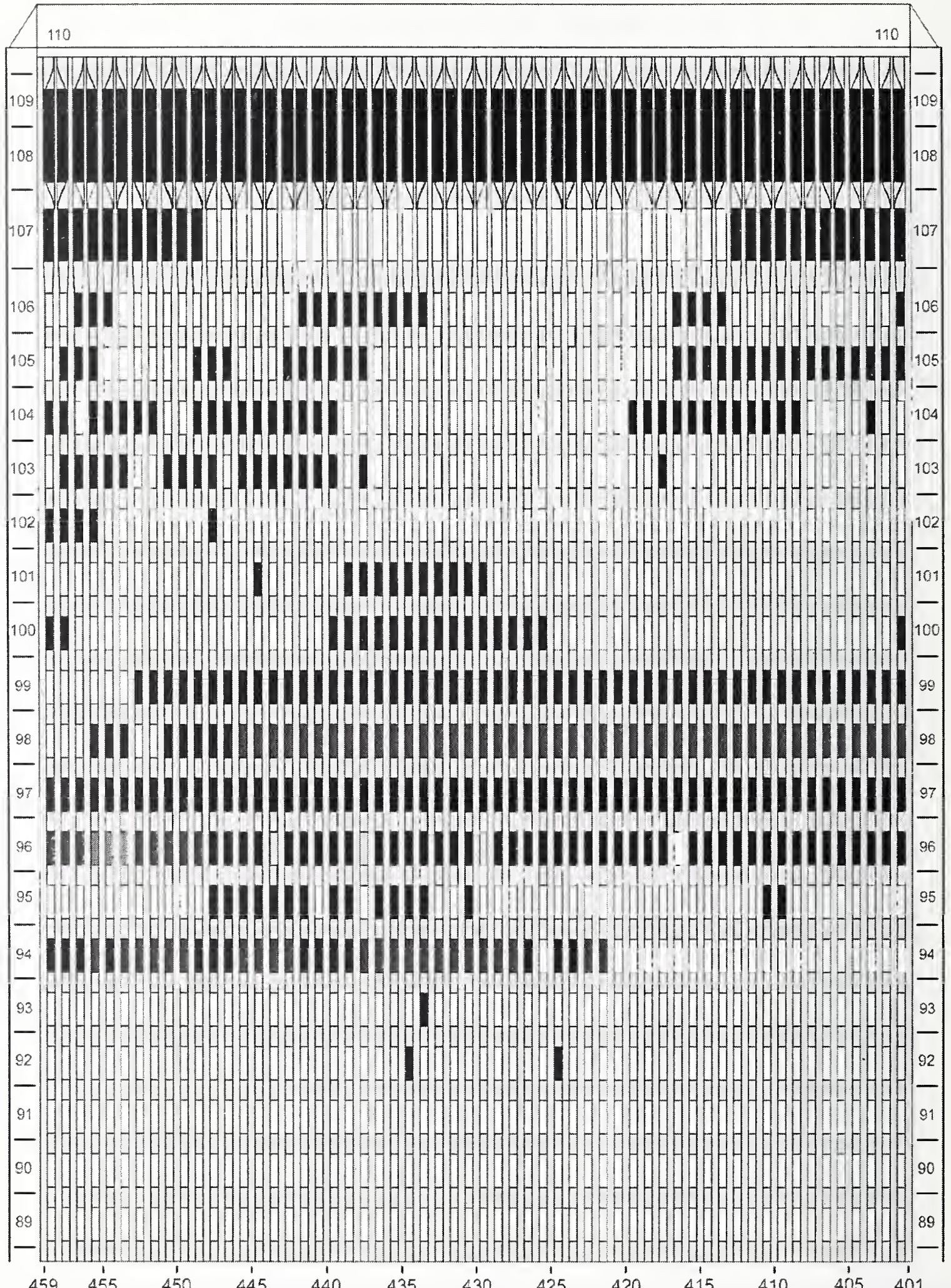

Figure F-47. Diagram of the west face of WTC 1 for floors 89 to 110 at 9:54 a.m. showing the condition of windows and locations of fires. 


$$
\text { WTC 1, West Face 9:54 a.m. }
$$

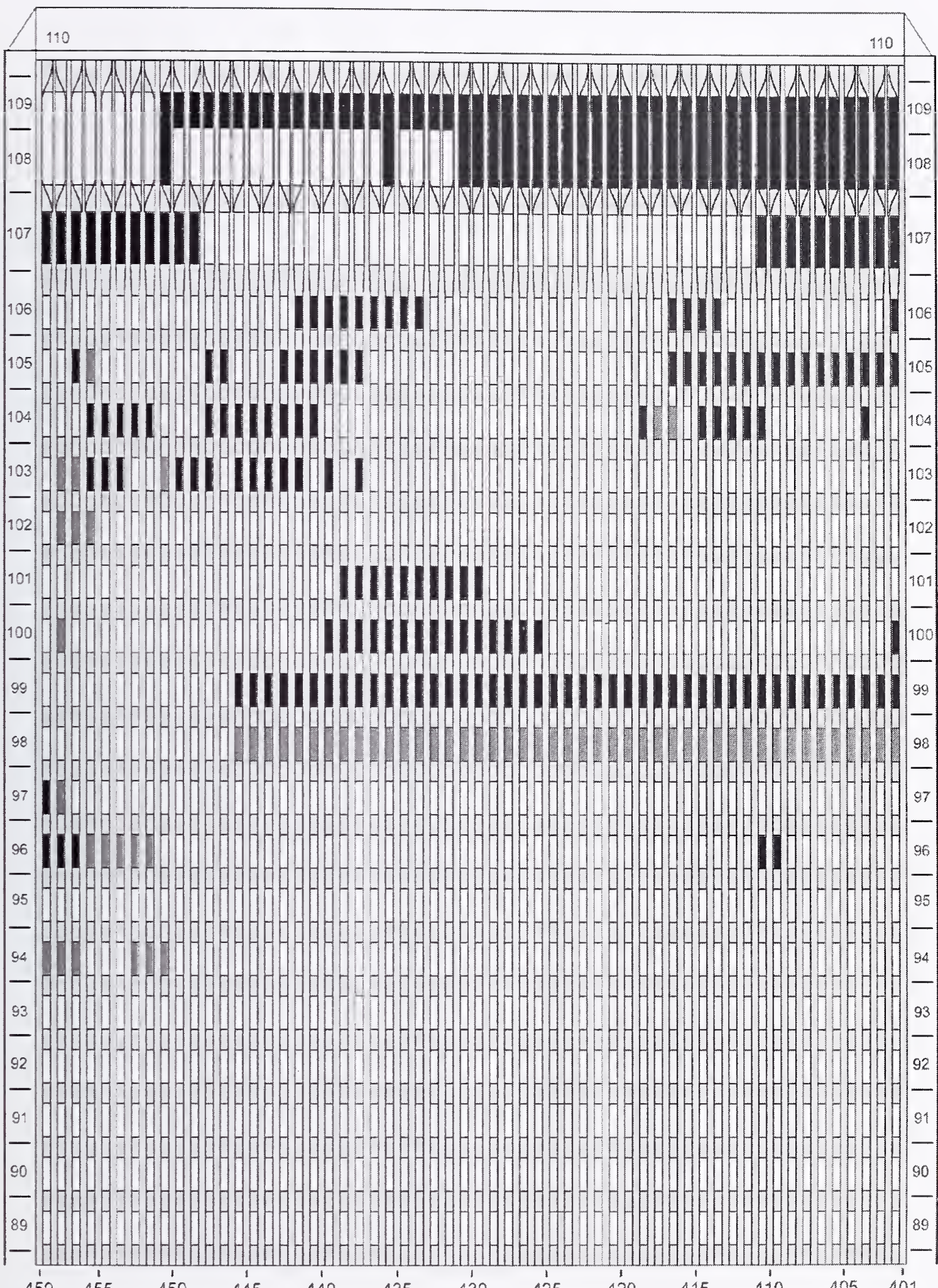

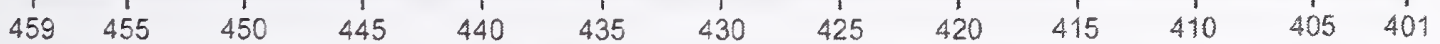

Figure F-48. Diagram of the west face of WTC 1 for floors 89 to 110 at 9:54 a.m. showing windows where smoke was observed and those that were hidden from view. 


$$
\text { WTC 1, West Face 9:58 a.m. }
$$

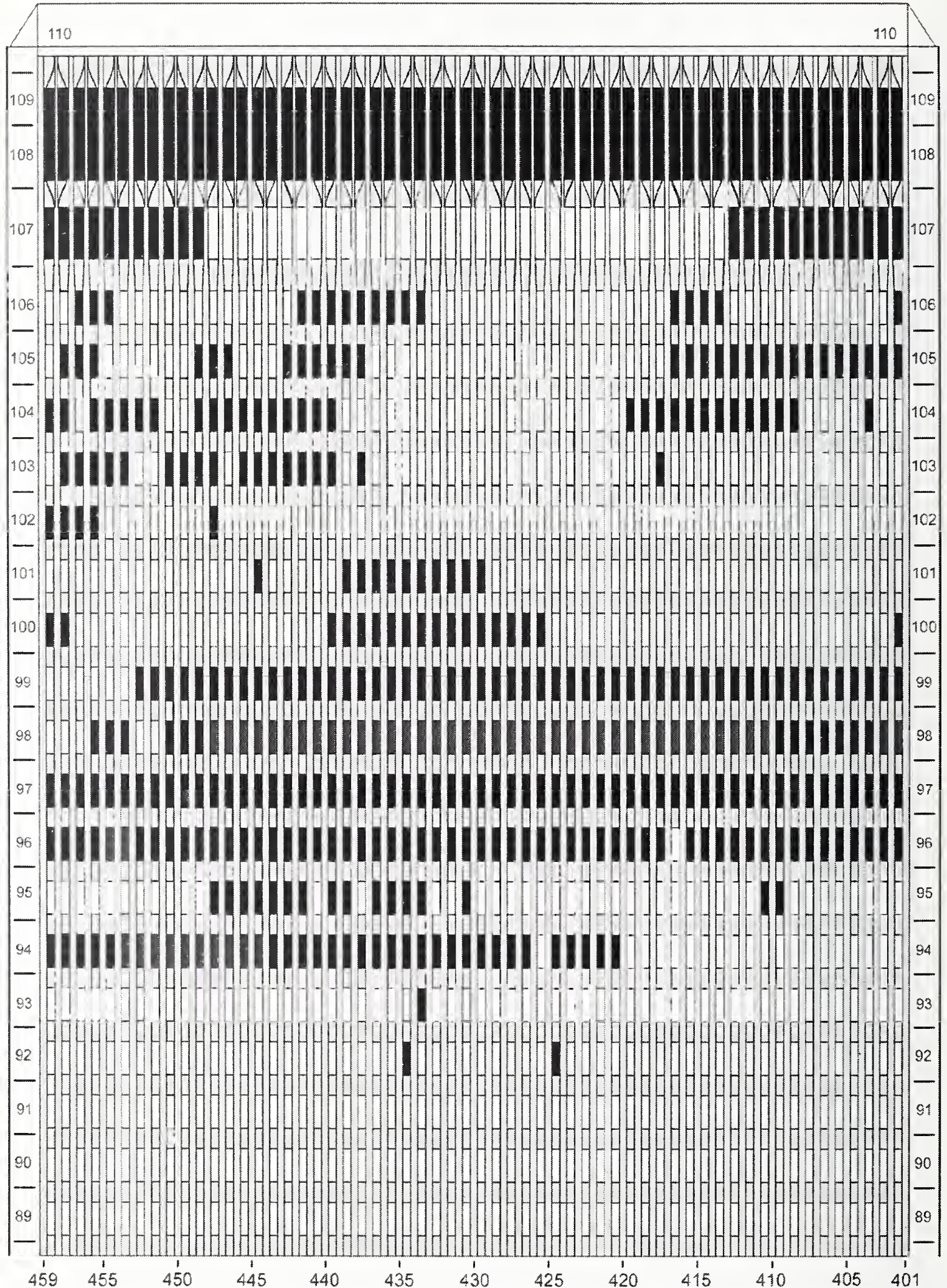

Figure F-49. Diagram of the west face of WTC 1 for floors 89 to 110 at 9:58 a.m. showing the condition of windows and locations of fires. 


$$
\text { WTC 1, West Face 9:58 a.m. }
$$

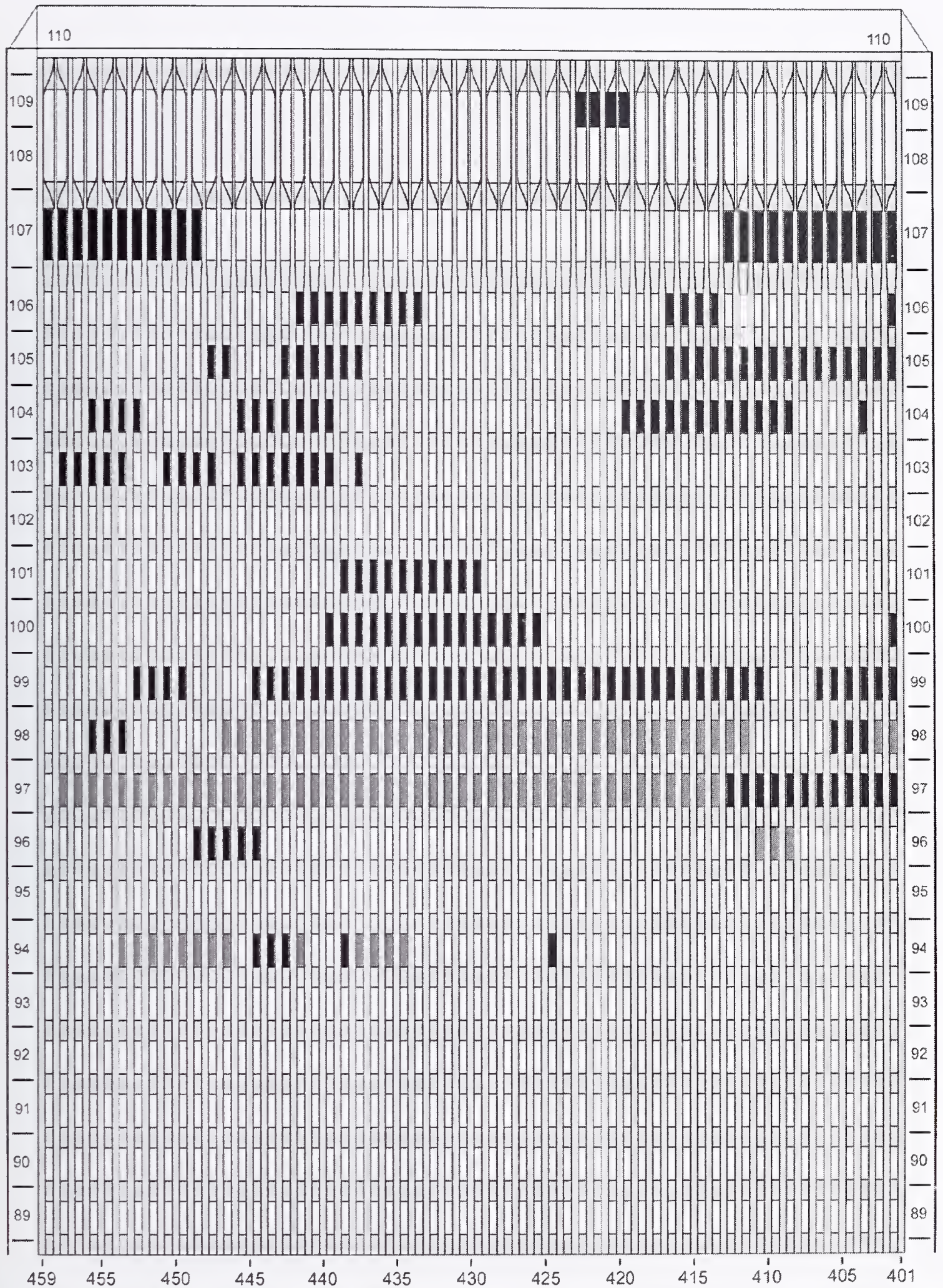

Figure F- 50. Diagram of the west face of WTC 1 for floors 89 to 110 at 9:58 a.m. showing windows where smoke was observed and those that were hidden from view. 
WTC 1, West Face 10:04 a.m.

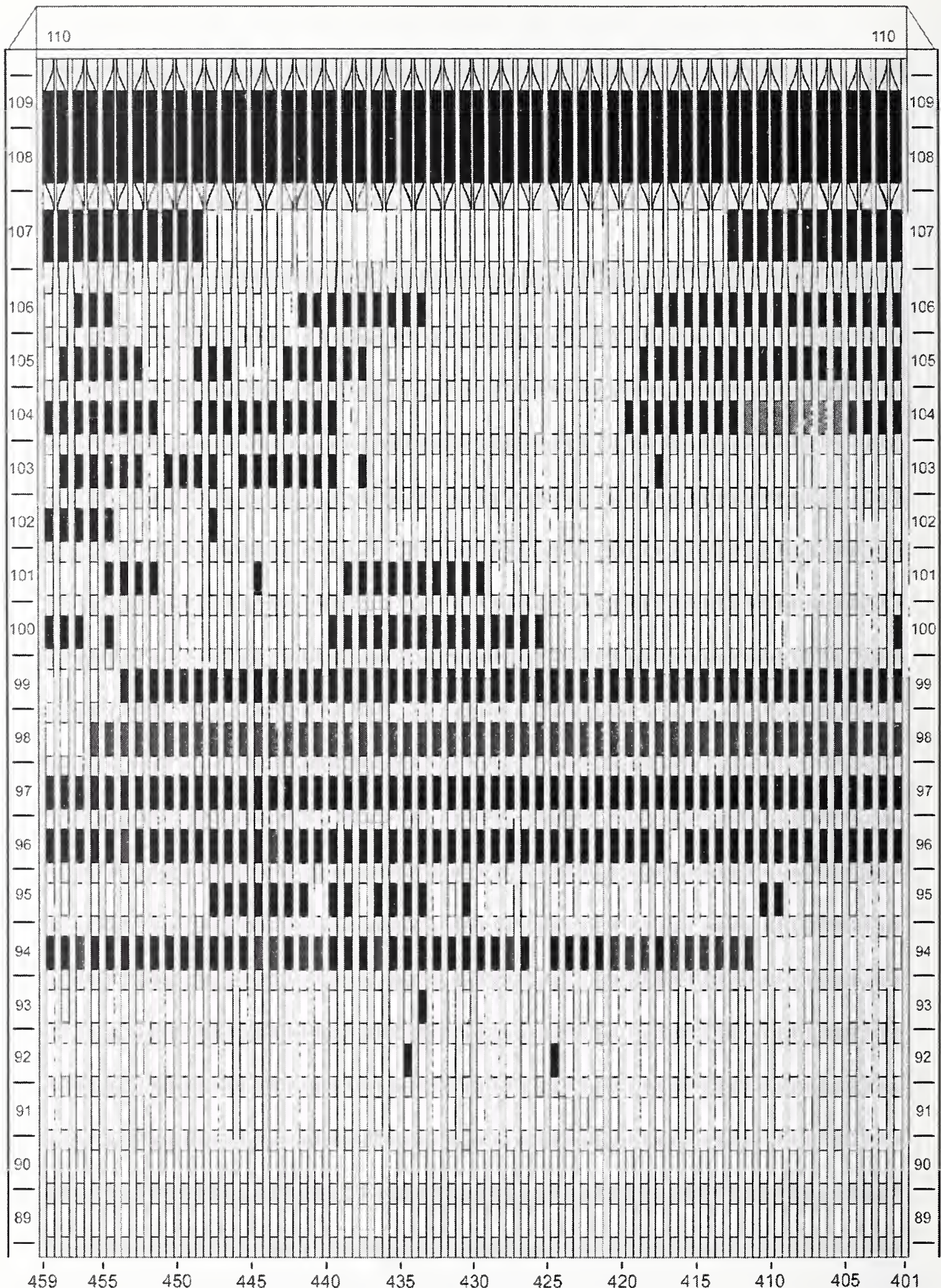

Figure F-51. Diagram of the west face of WTC 1 for floors 89 to 110 at 10:04 a.m. showing the condition of windows and locations of fires. 


$$
\text { WTC 1, West Face 10:04 a.m. }
$$

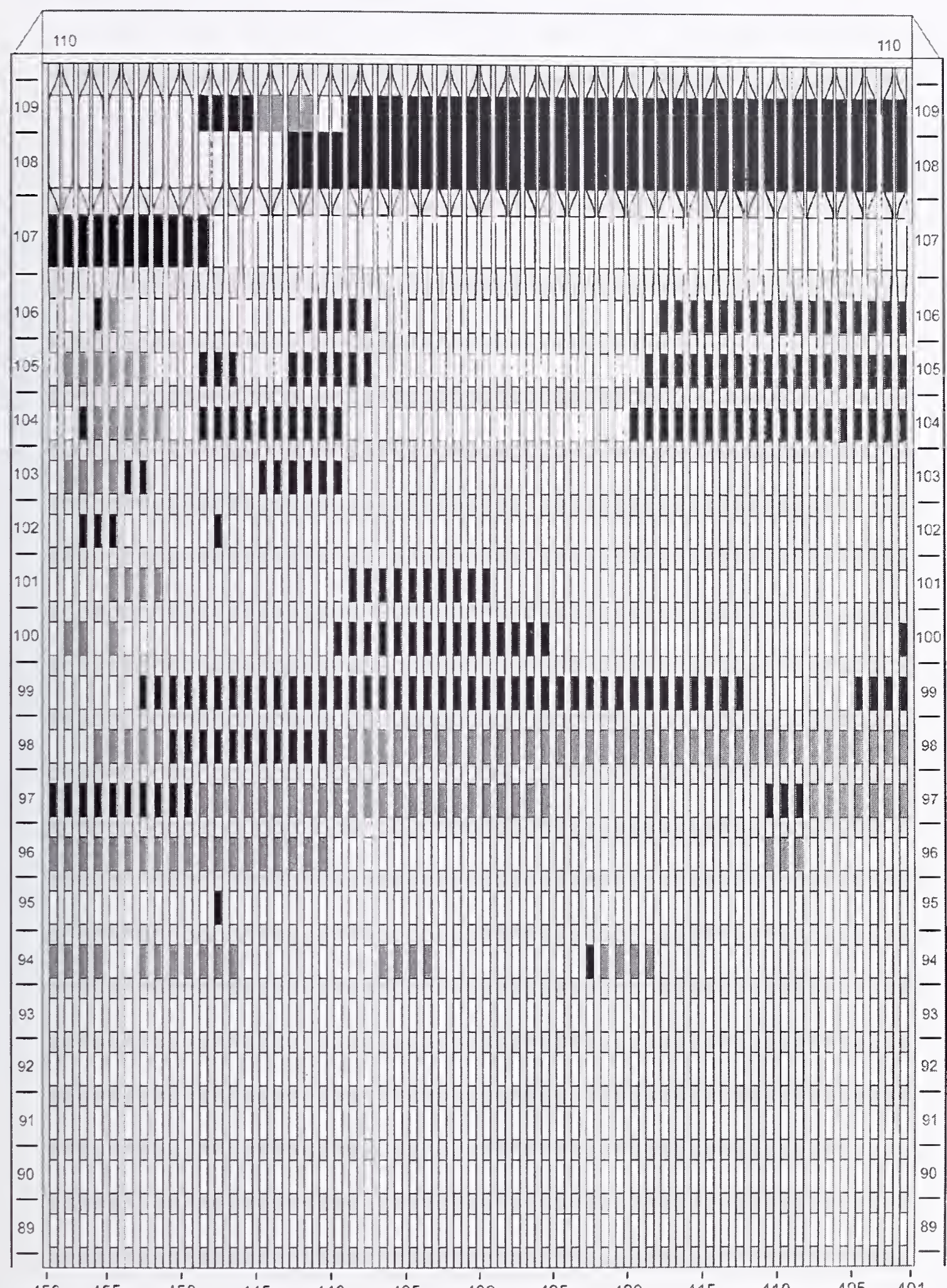

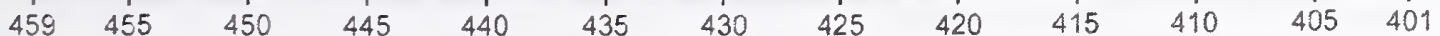

Figure F-52. Diagram of the west face of WTC 1 for floors 89 to 110 at 10:04 a.m. showing windows where smoke was observed and those that were hidden from view. 


$$
\text { WTC 1, West Face } \quad \text { 10:10 a.m. }
$$

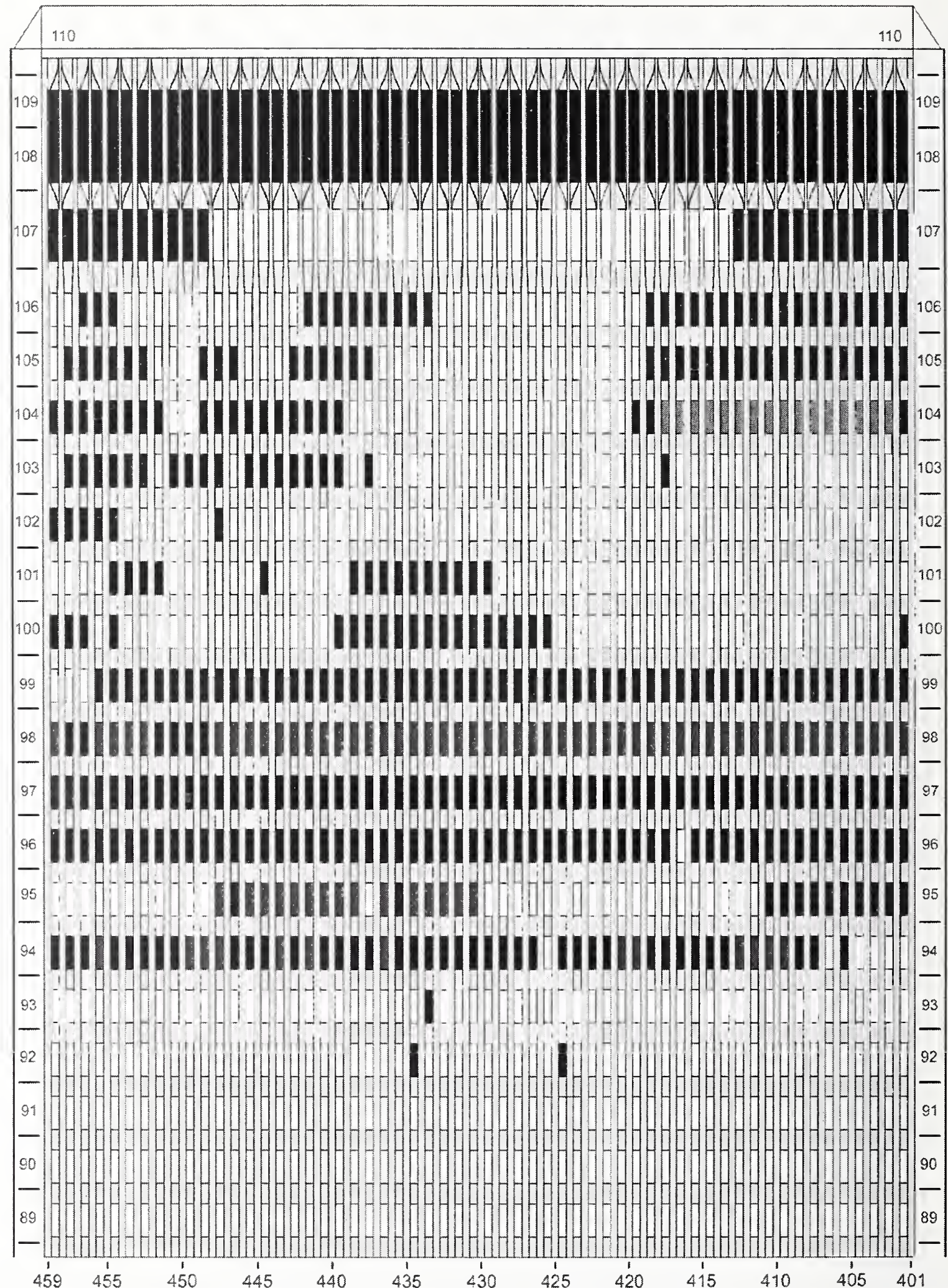

Figure F-53. Diagram of the west face of WTC 1 for floors 89 to 110 at 10:10 a.m. showing the condition of windows and locations of fires. 
WTC 1, West Face

10:10 a.m.

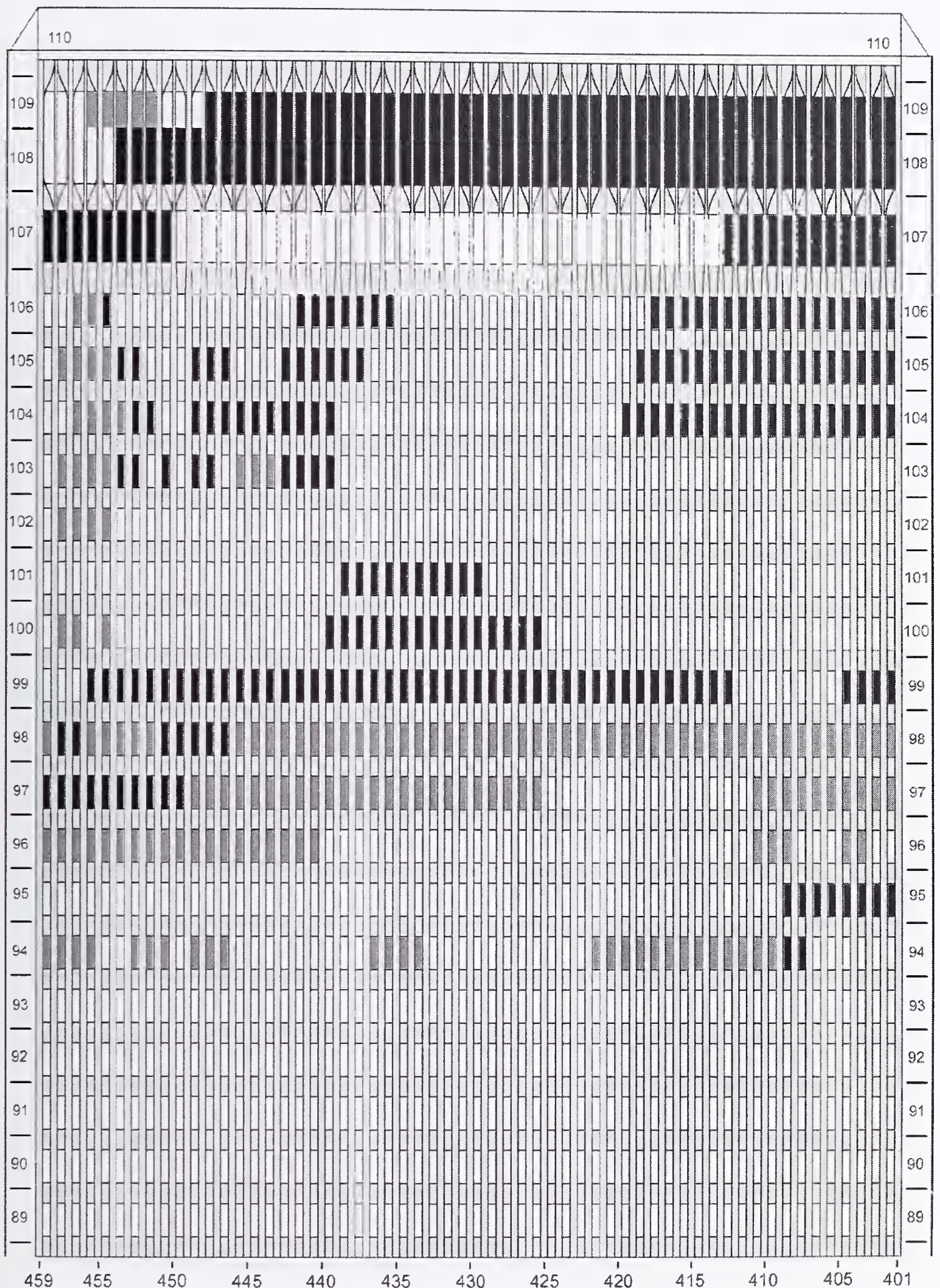

Figure F-54. Diagram of the west face of WTC 1 for floors 89 to 110 at 10:10 a.m. showing windows where smoke was observed and those that were hidden from view. 
WTC 1, West Face

10:16 a.m.

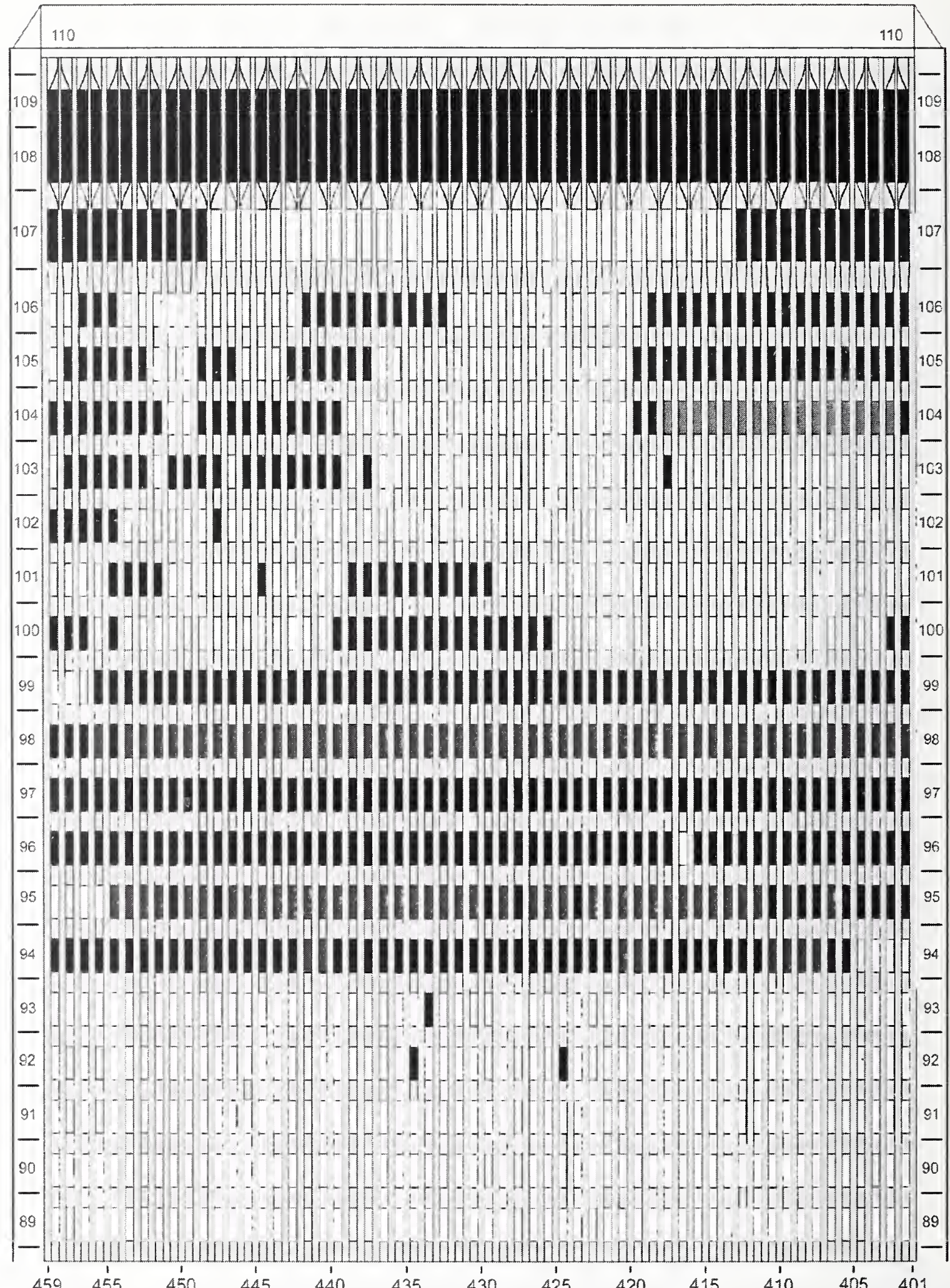

Figure F-55. Diagram of the west face of WTC 1 for floors 89 to 110 at 10:16 a.m. showing the condition of windows and locations of fires. 
WTC 1, West Face 10:16 a.m.

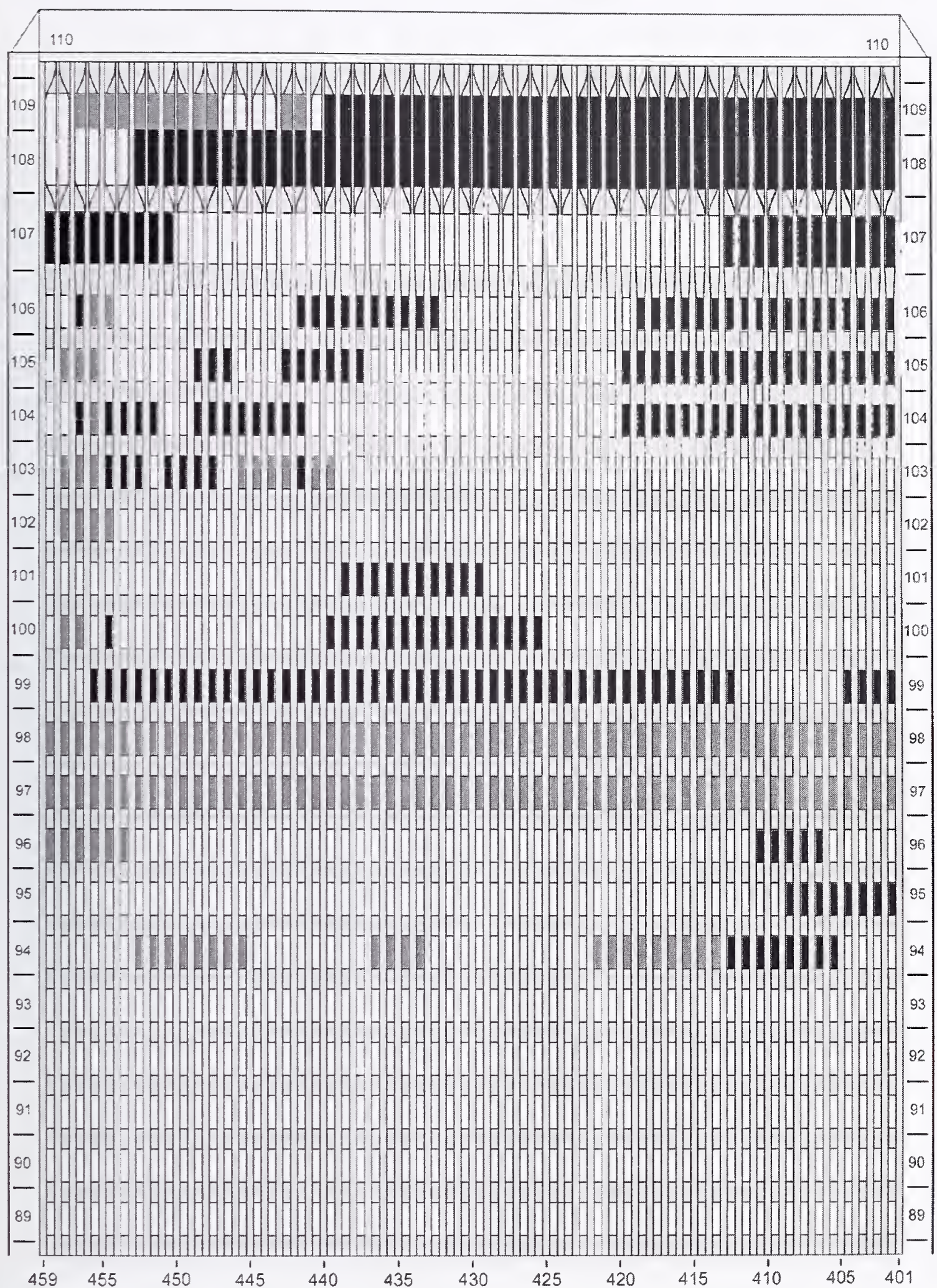

Figure F-56. Diagram of the west face of WTC 1 for floors 89 to 110 at 10:16 a.m. showing windows where smoke was observed and those that were hidden from view. 
WTC 1, West Face

10:18 a.m.

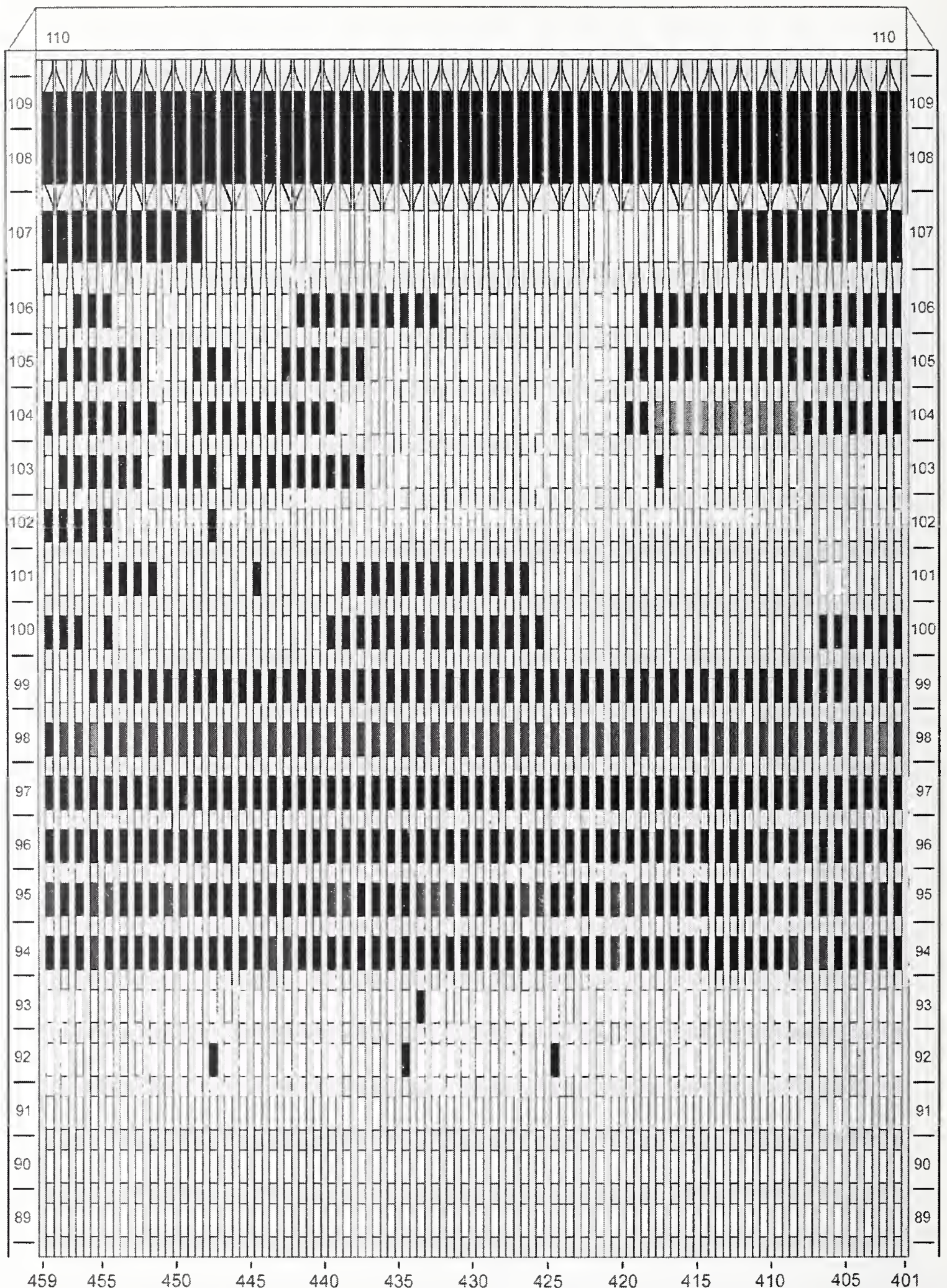

Figure F-57. Diagram of the west face of WTC 1 for floors 89 to 110 at 10:18 a.m. showing the condition of windows and locations of fires. 
WTC 1, West Face 10:18 a.m.

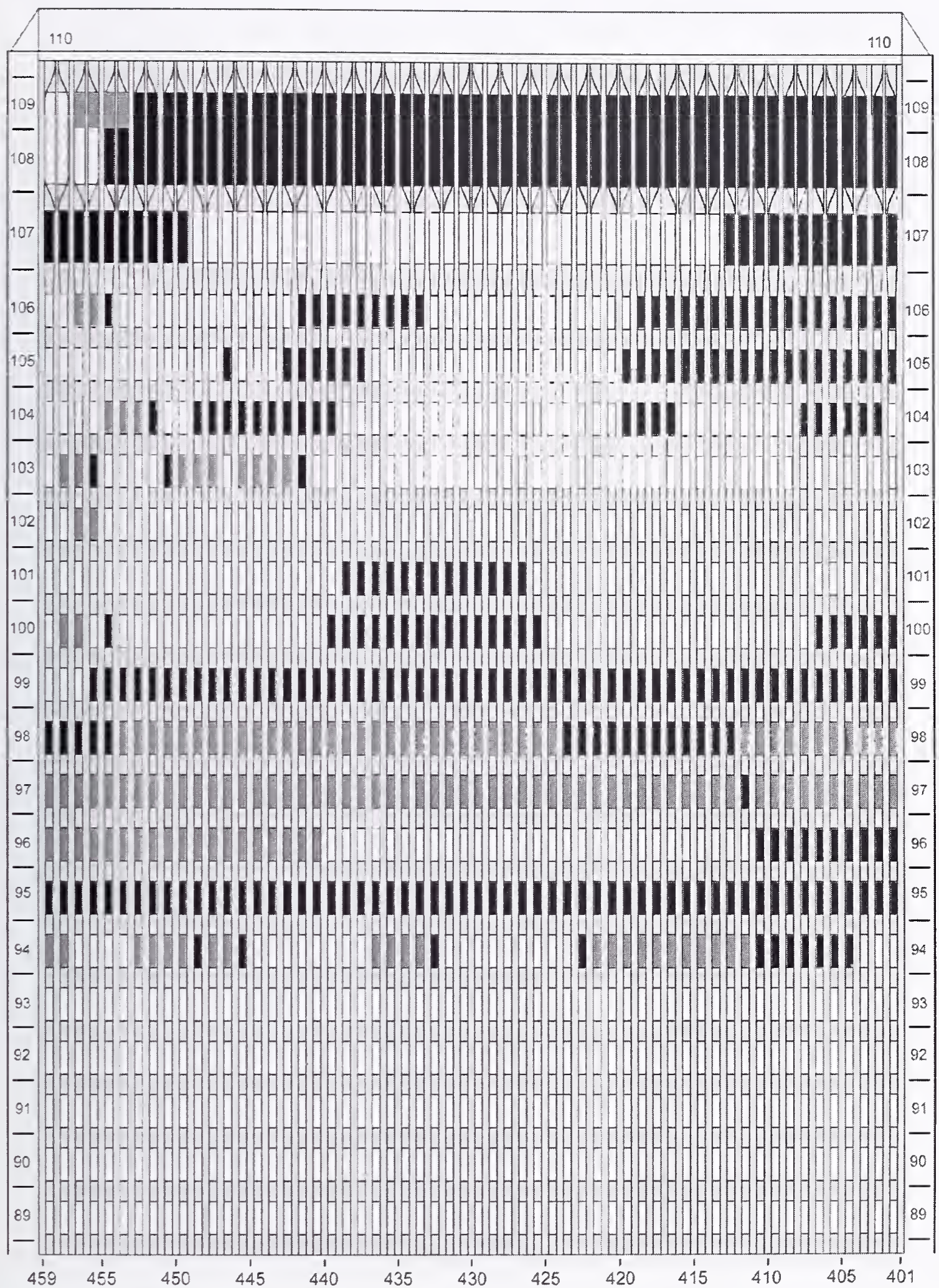

Figure F-58. Diagram of the west face of WTC 1 for floors 89 to 110 at $10: 18$ a.m. showing windows where smoke was observed and those that were hidden from view. 


\section{WTC 1, West Face 10:22 a.m.}

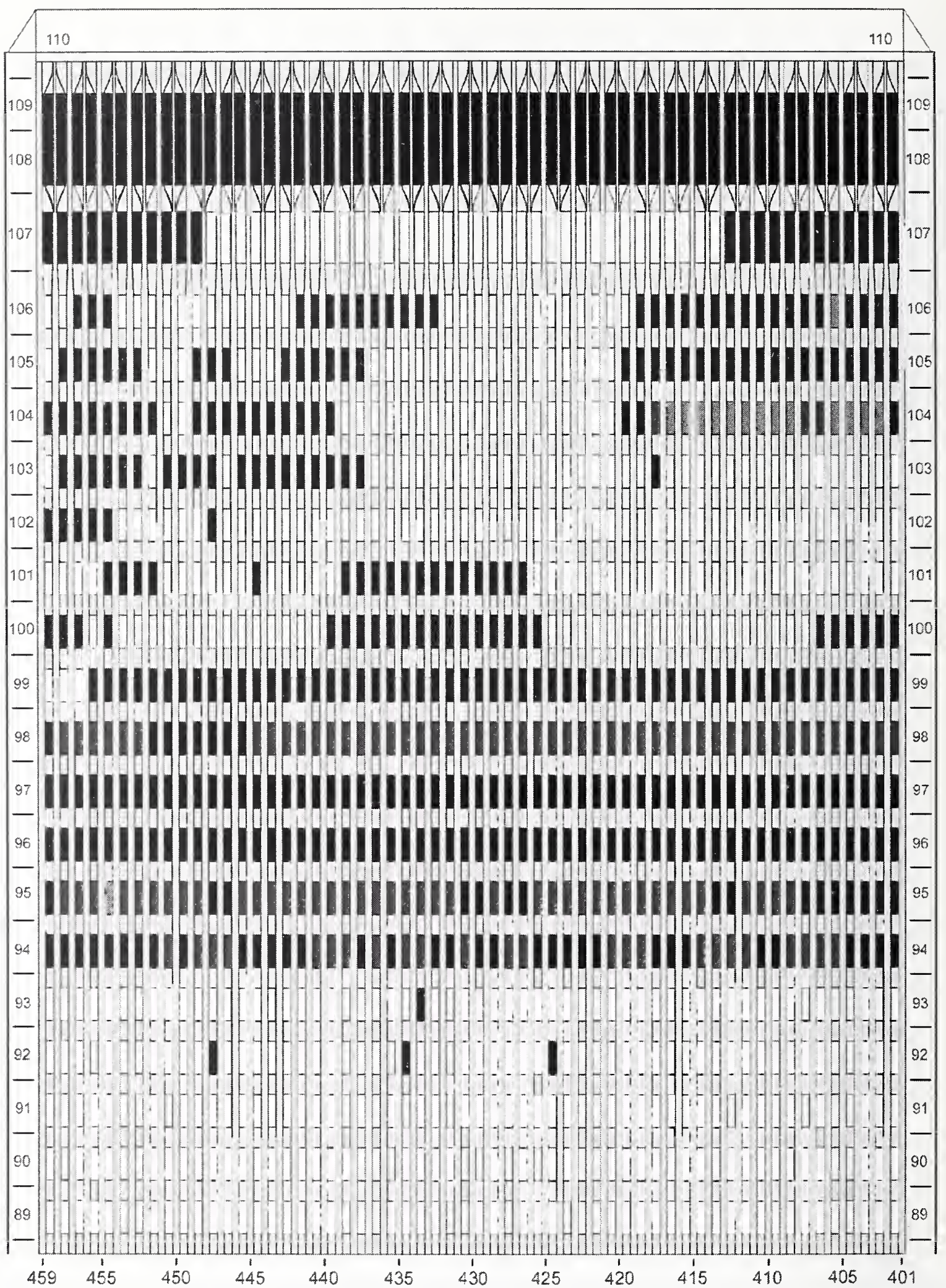

Figure F-59. Diagram of the west face of WTC 1 for floors 89 to 110 at 10:22 a.m. showing the condition of windows and locations of fires. 


$$
\text { WTC 1, West Face 10:22 a.m. }
$$

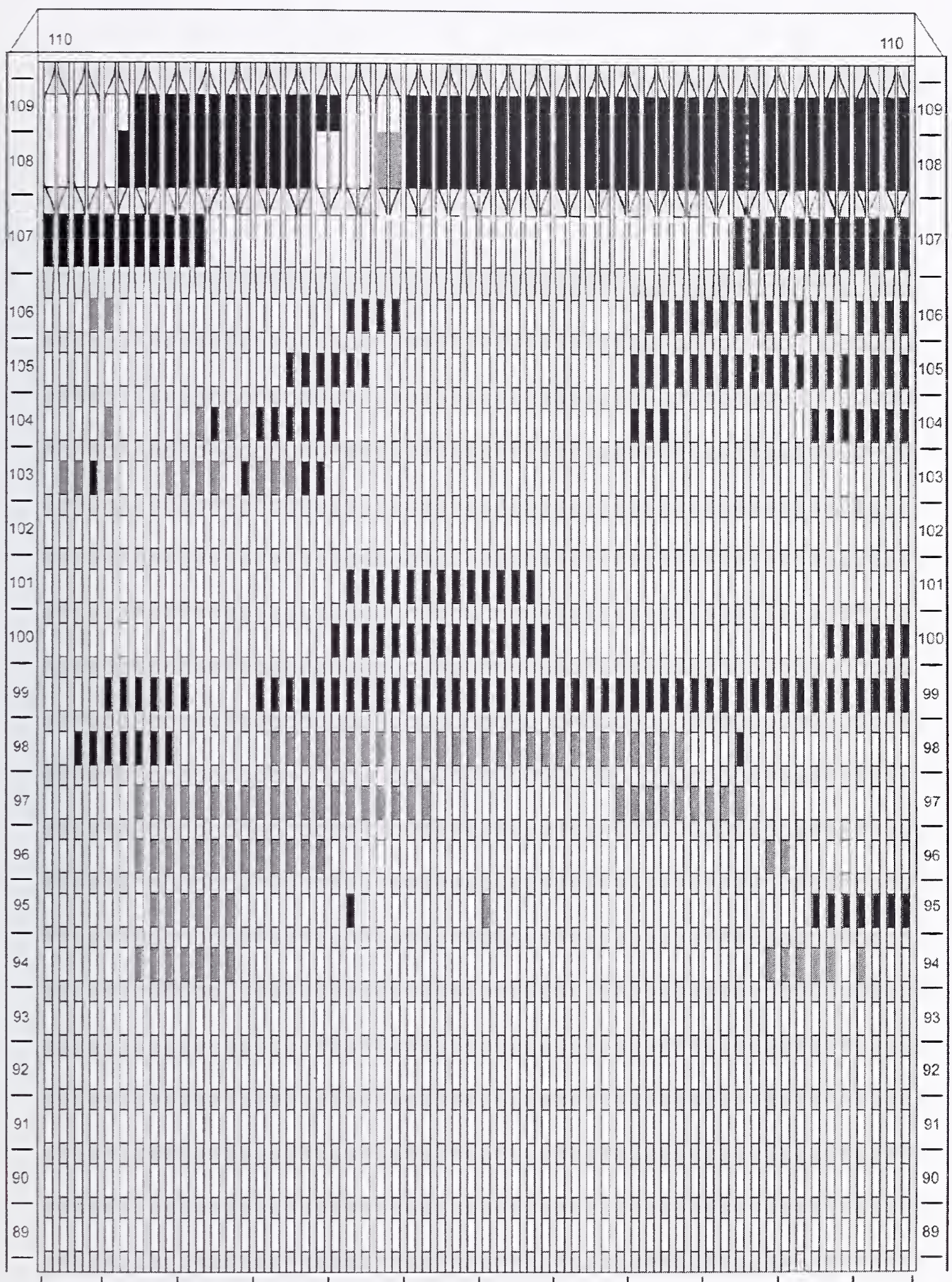

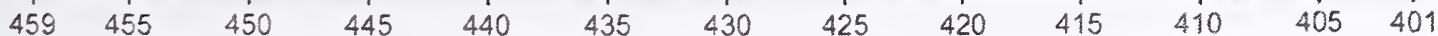

Figure F-60. Diagram of the west face of WTC 1 for floors 89 to 110 at 10:22 a.m. showing windows where smoke was observed and those that were hidden from view. 
WTC 1, West Face

10:28 a.m.

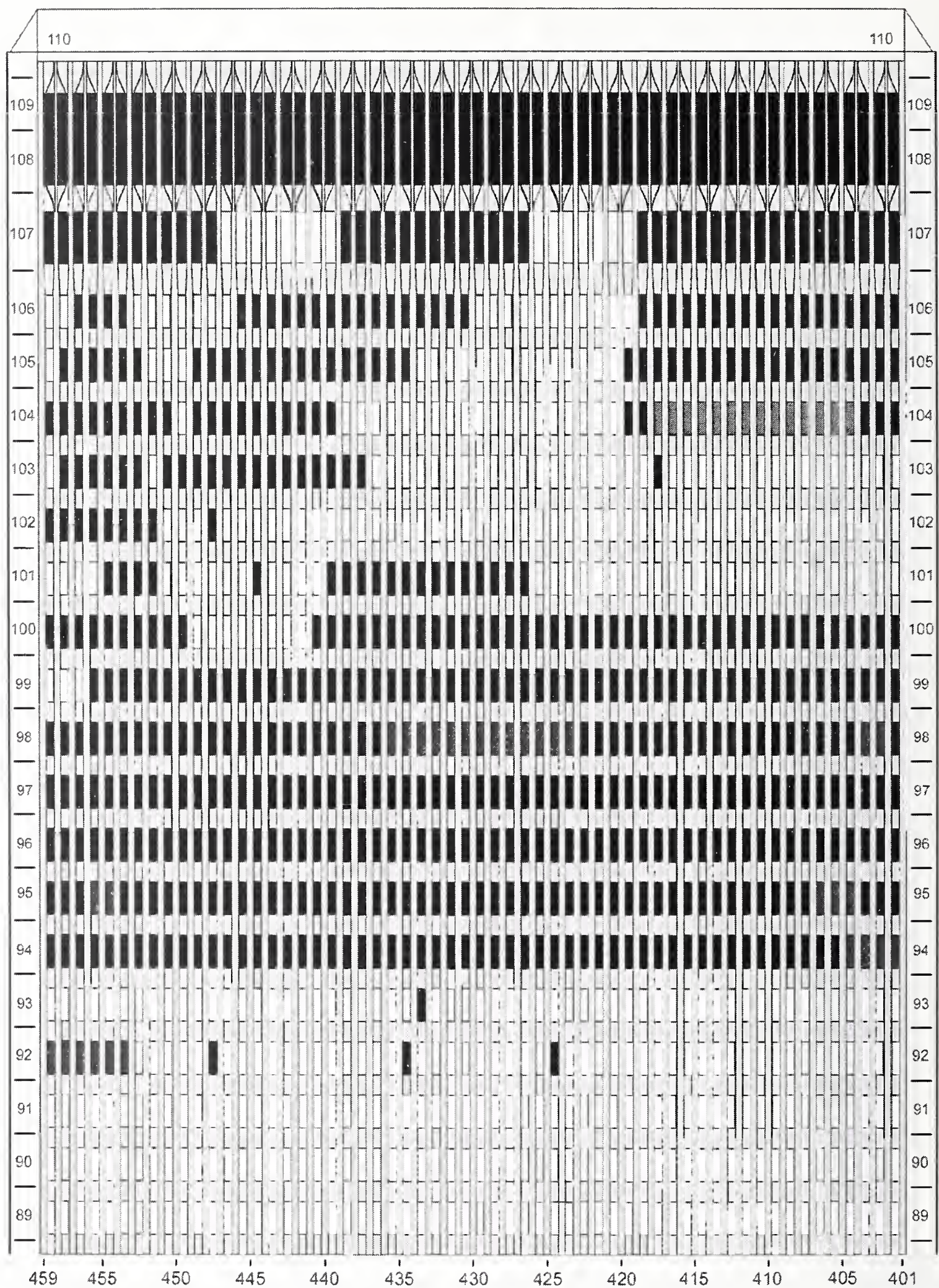

Figure F-61. Diagram of the west face of WTC 1 for floors 89 to 110 at 10:28 a.m. showing the condition of windows and locations of fires. 


$$
\text { WTC 1, West Face 10:28 a.m. }
$$

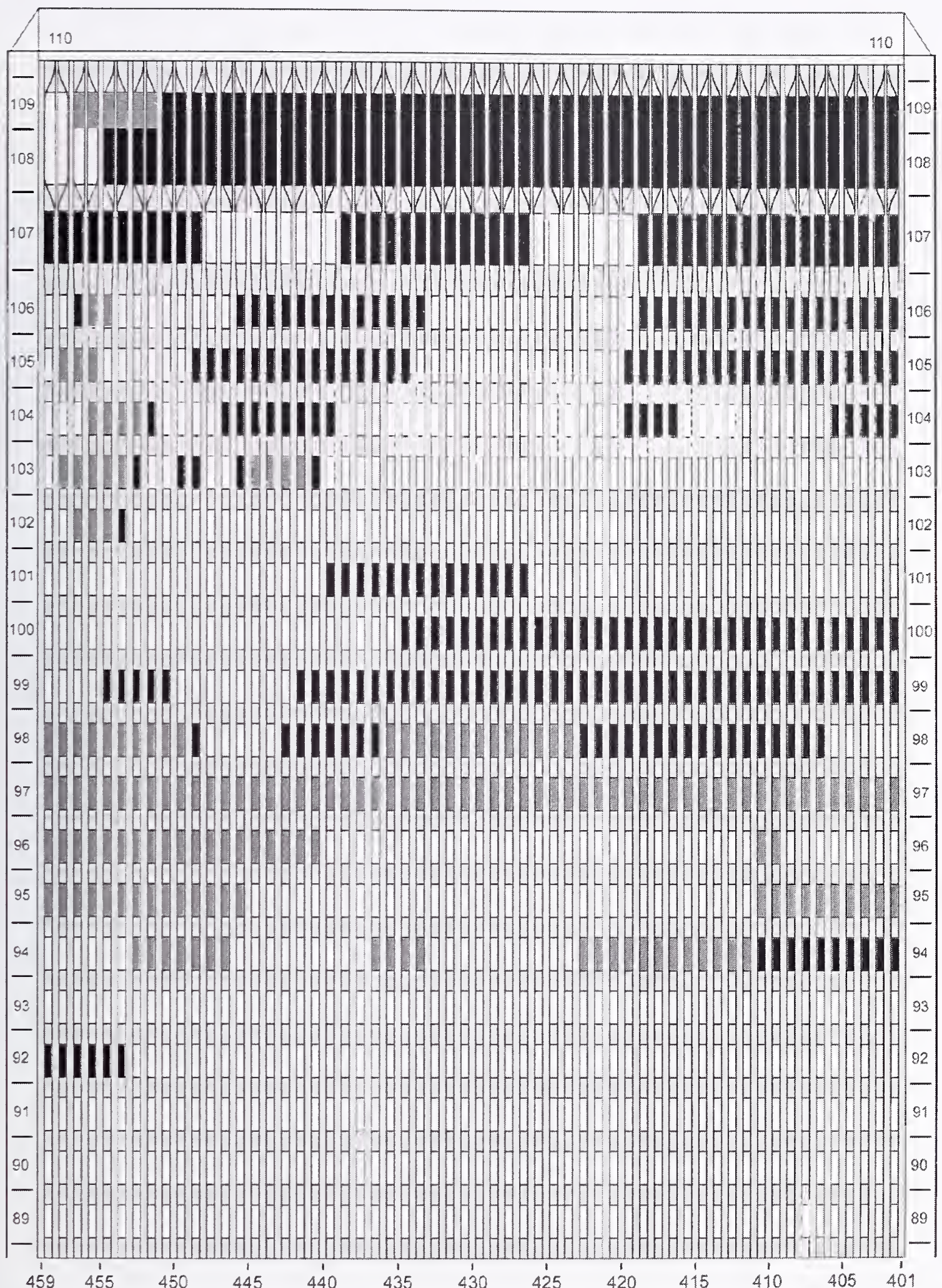

Figure F-62. Diagram of the west face of WTC 1 for floors 89 to 110 at 10:28 a.m. showing windows where smoke was observed and those that were hidden from view. 
This page intentionally left blank. 


\section{Appendix G \\ WTC 2 NORTH FACE COMBINED FIRE AND WINDOW CONDITION AND WINDOW SMOKE CONDITION}

This appendix provides visual representations of the data contained in data sheets describing observations of fire, smoke, and window condition on a window-by-window basis as a function of time for the north face of WTC 2. The data for window condition and fire observations are combined into single representations, with the data for fire taking precedence. Observations are represented using the key

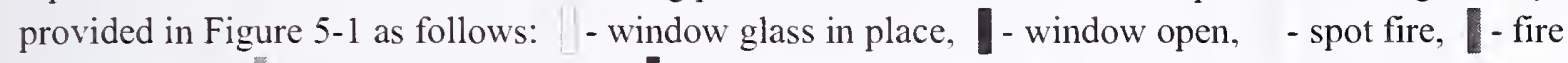
visible inside, [- external flaming, and - not visible. The data for smoke are shown in separate figures using the key from Figure 5-1 as follows: - no smoke visible, - light smoke, - heavy smoke, and - not visible. For a given time, the window condition and fire data are shown first. 
WTC 2, North Face

9:03 a.m.

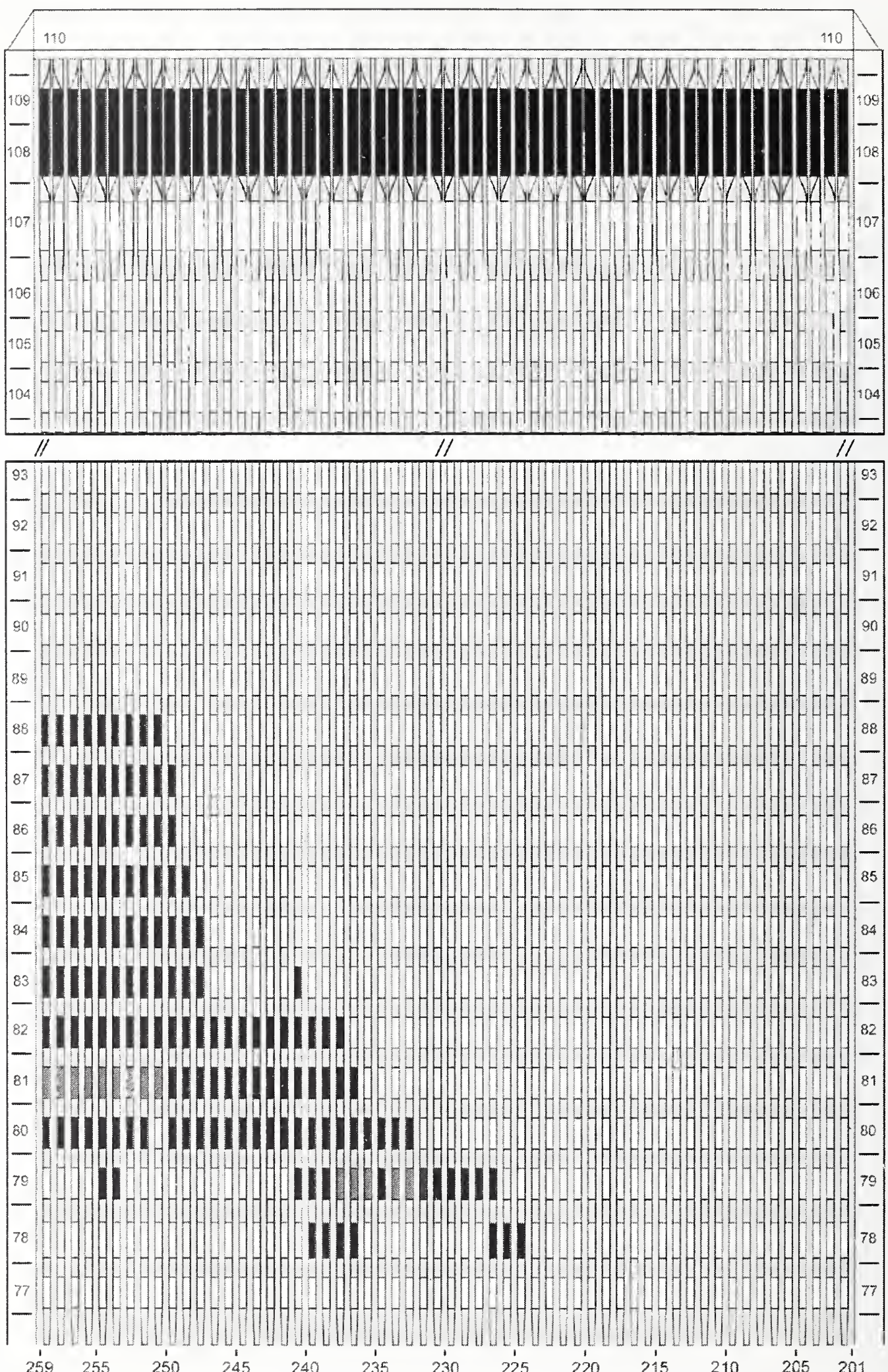

Figure G-1. Diagram of the north face of WTC 2 for floors 77 to 93 and floors 104 to 110 at 9:03 a.m. showing the condition of windows and locations of fires. 


$$
\text { WTC 2, North Face 9:03 a.m. }
$$

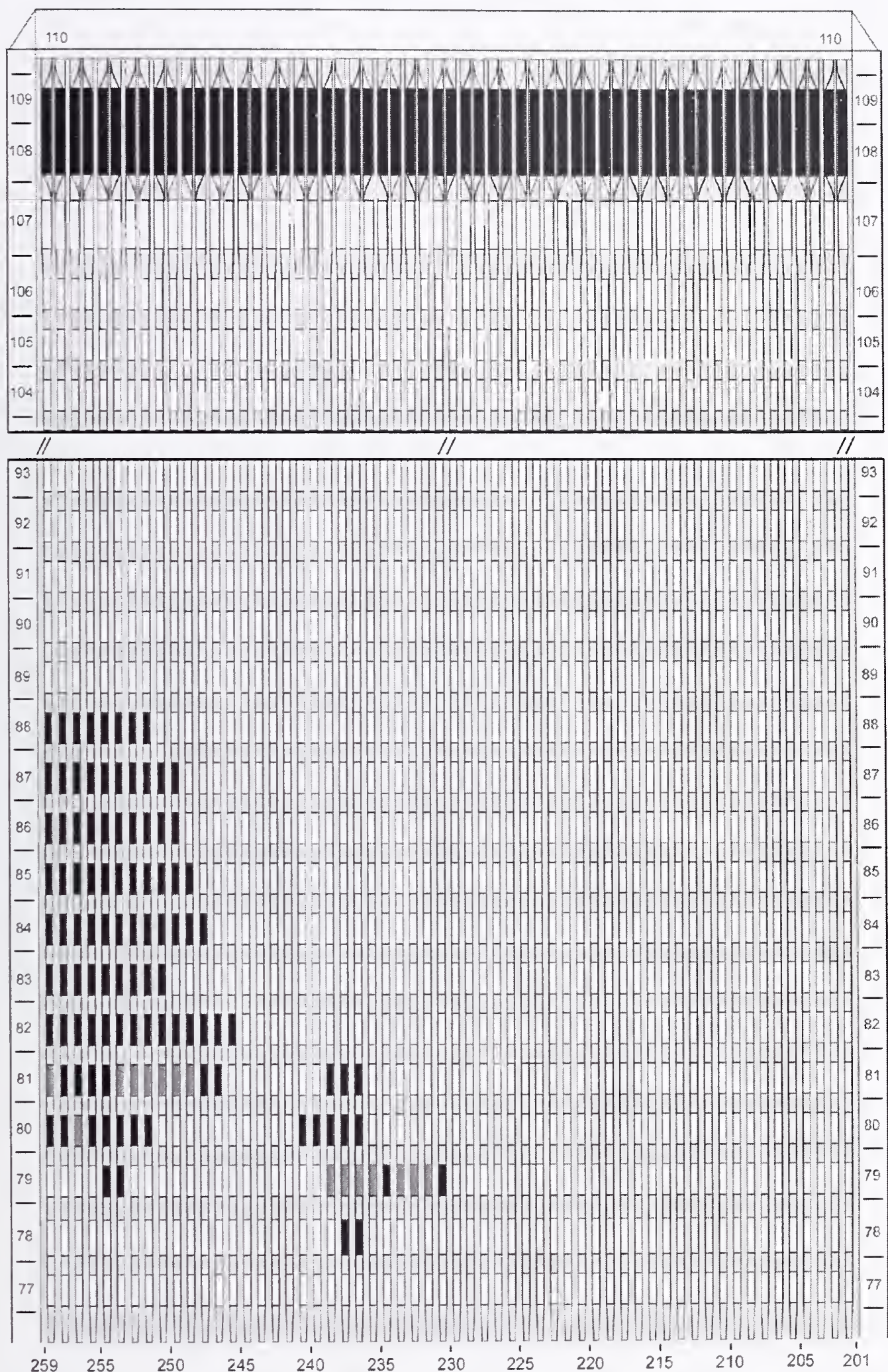

Figure G-2. Diagram of the north face of WTC 2 for floors 77 to 93 and floors 104 to 110 at 9:03 a.m. showing windows where smoke was observed and those hidden from view. 
WTC 2, North Face

9:04 a.m.

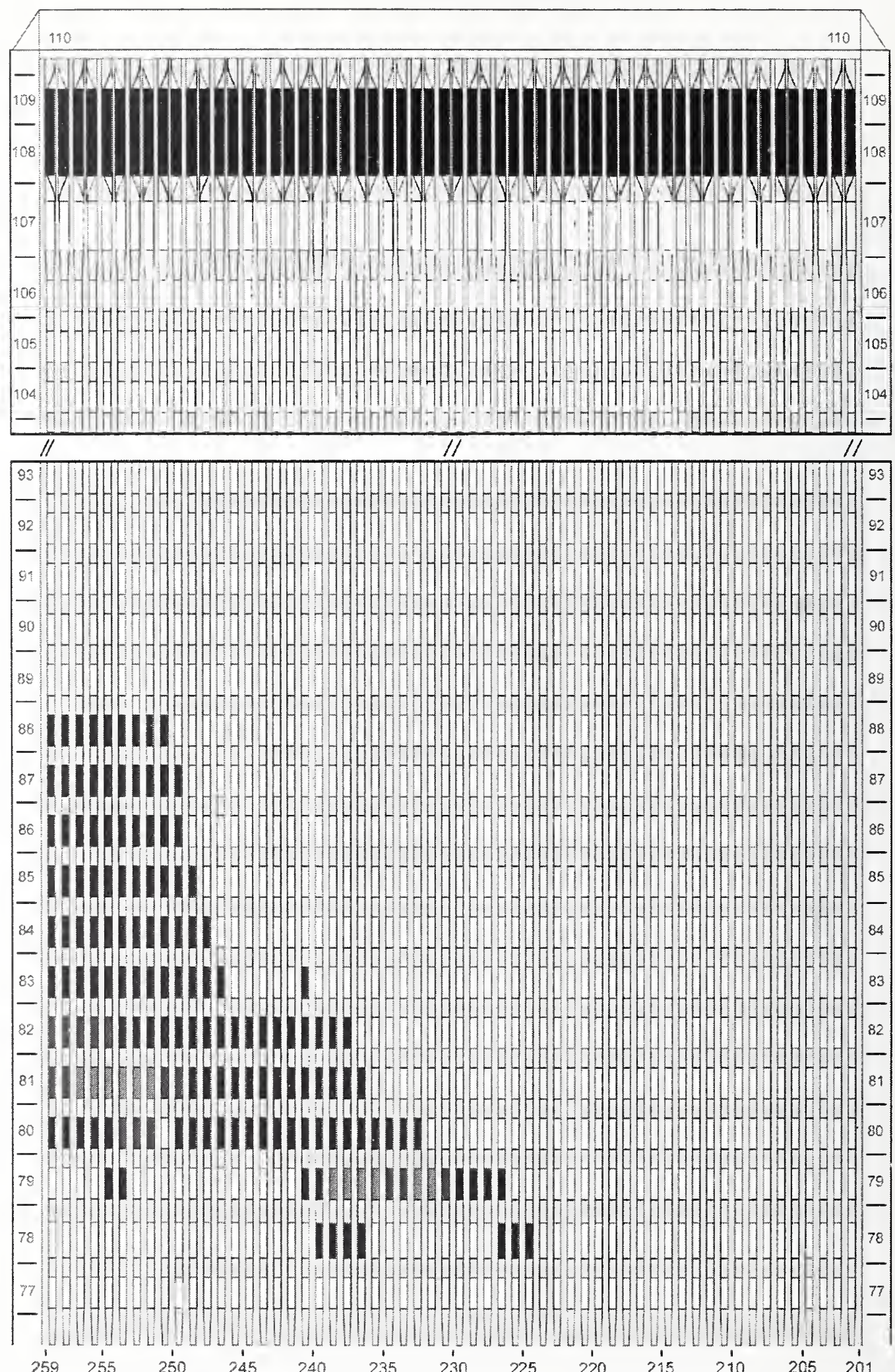

Figure G-3. Diagram of the north face of WTC 2 for floors 77 to 93 and floors 104 to 110 at 9:04 a.m. showing the condition of windows and locations of fires. 
WTC 2, North Face 9:04 a.m.

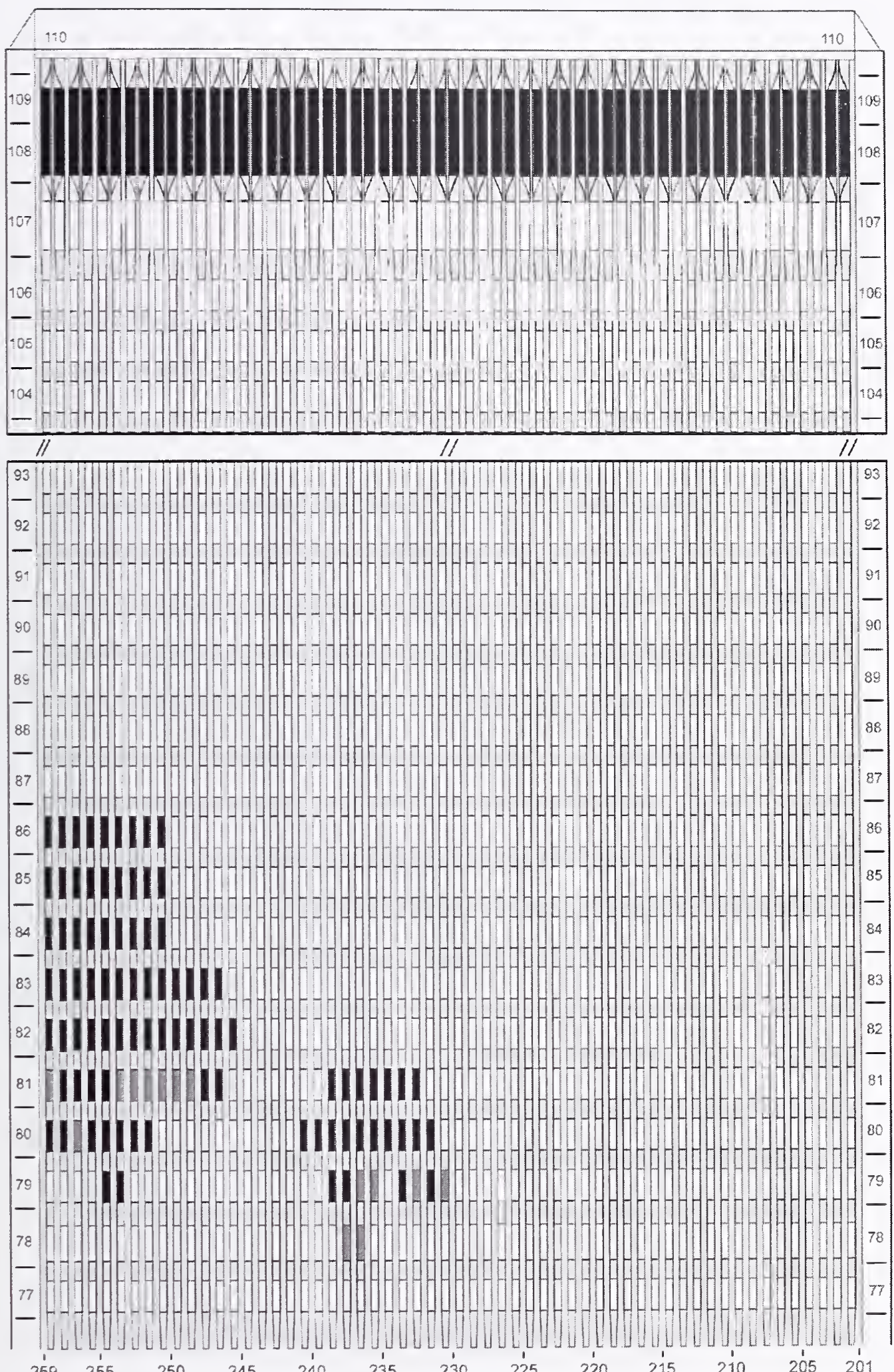

Figure G-4. Diagram of the north face of WTC 2 for floors 77 to 93 and floors 104 to 110 at 9:04 a.m. showing windows where smoke was observed and those hidden from view. 
WTC 2, North Face $\quad 9: 06$ a.m.

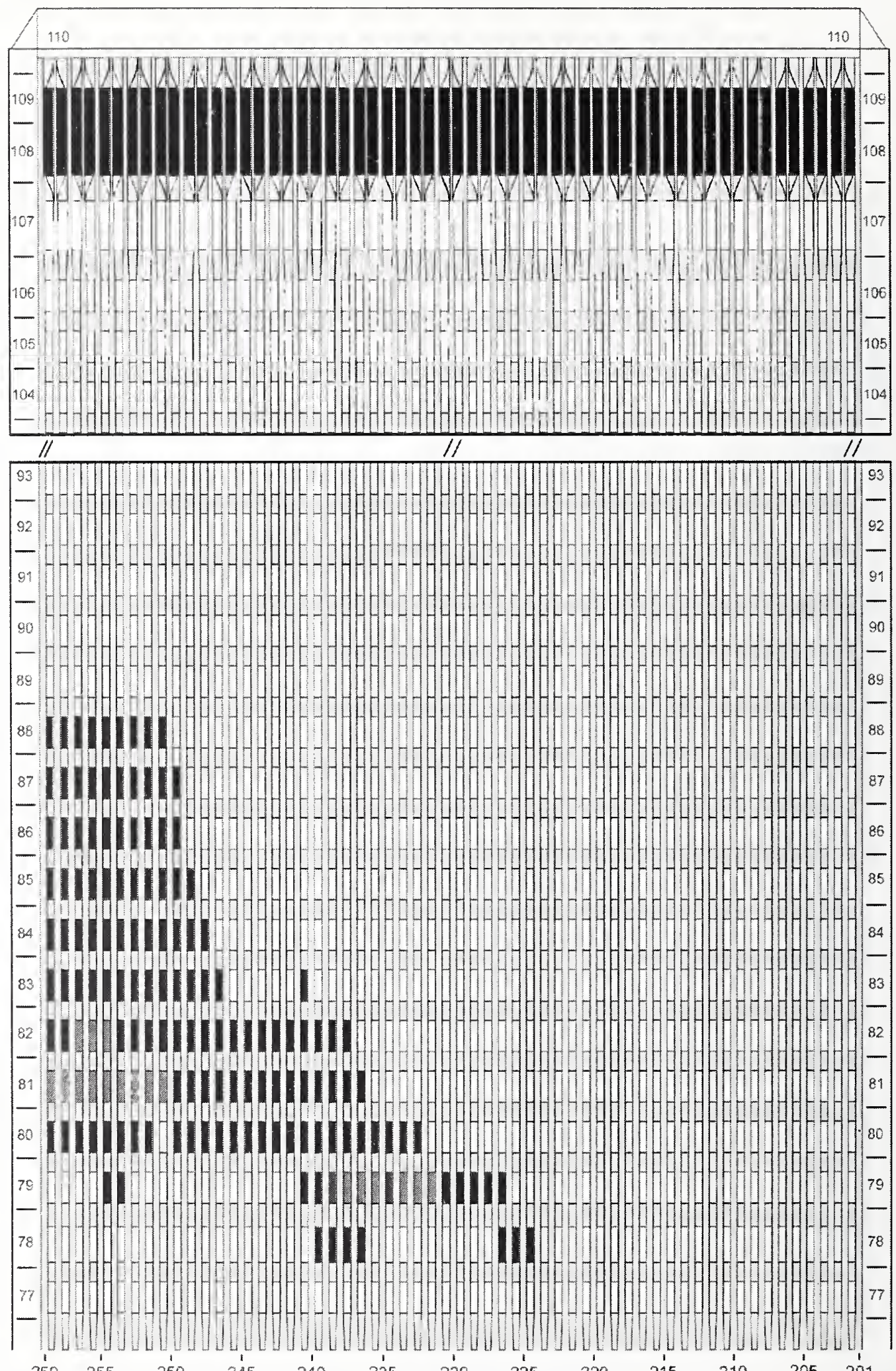

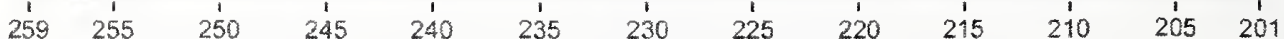

Figure G-5. Diagram of the north face of WTC 2 for floors 77 to 93 and floors 104 to 110 at 9:06 a.m. showing the condition of windows and locations of fires. 
WTC 2, North Face 9:06 a.m.

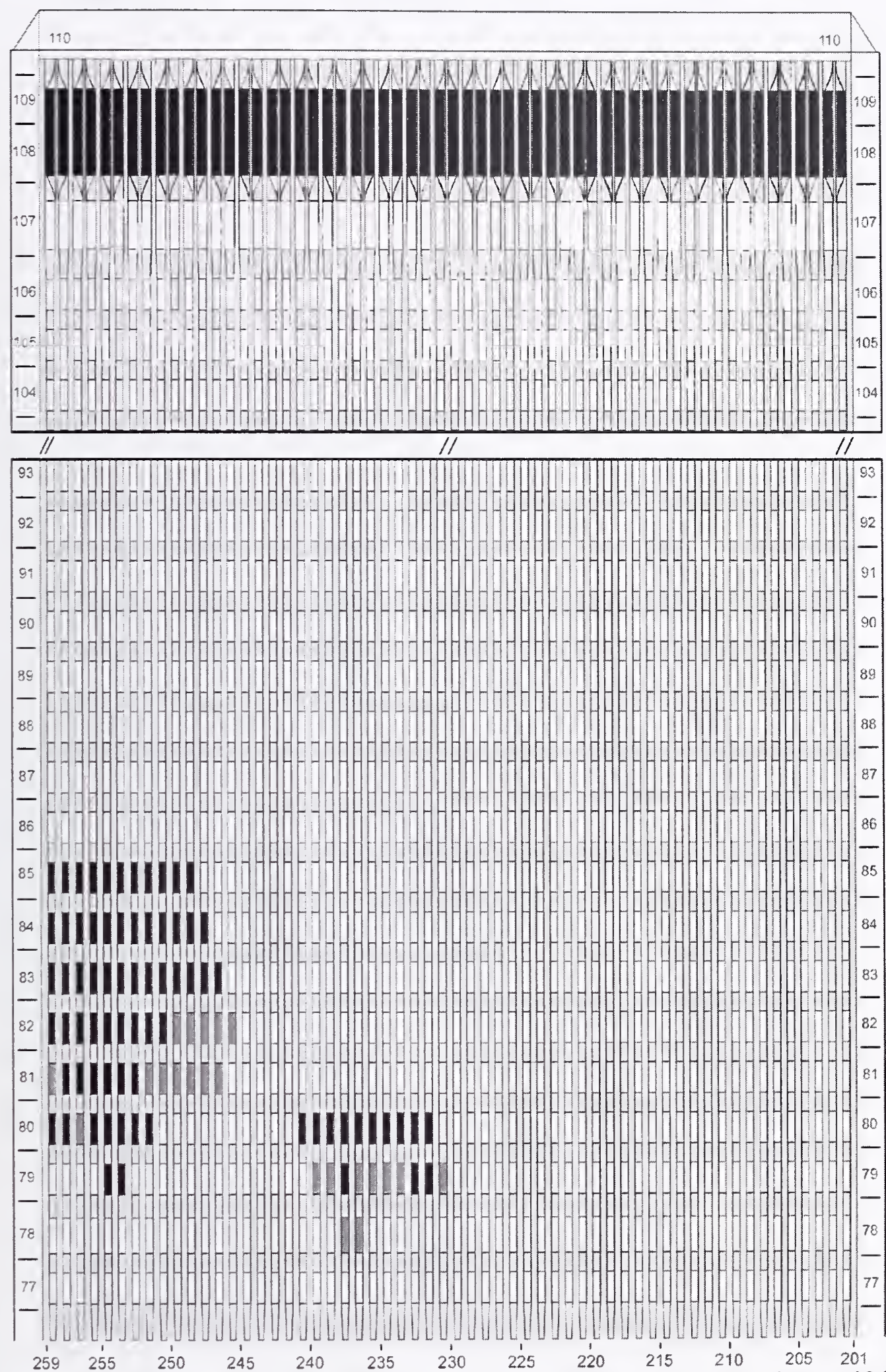

Figure G-6. Diagram of the north face of WTC 2 for floors 77 to 93 and floors 104 to 110 at 9:06 a.m. showing windows where smoke was observed and those hidden from view. 
WTC 2, North Face

9:08 a.m.

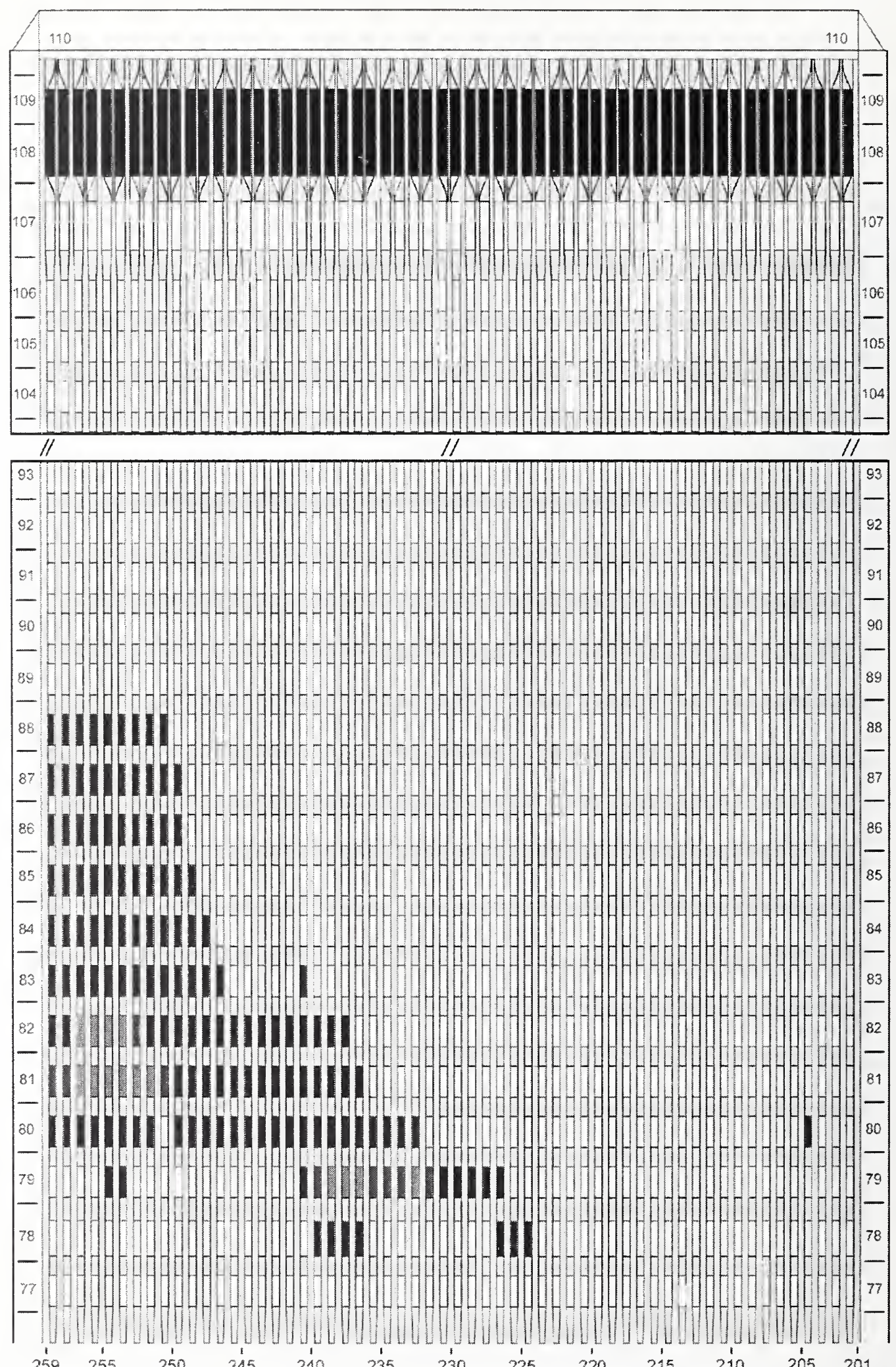

Figure G-7. Diagram of the north face of WTC 2 for floors 77 to 93 and floors 104 to 110 at 9:08 a.m. showing the condition of windows and locations of fires. 
WTC 2, North Face $\quad 9: 08$ a.m.

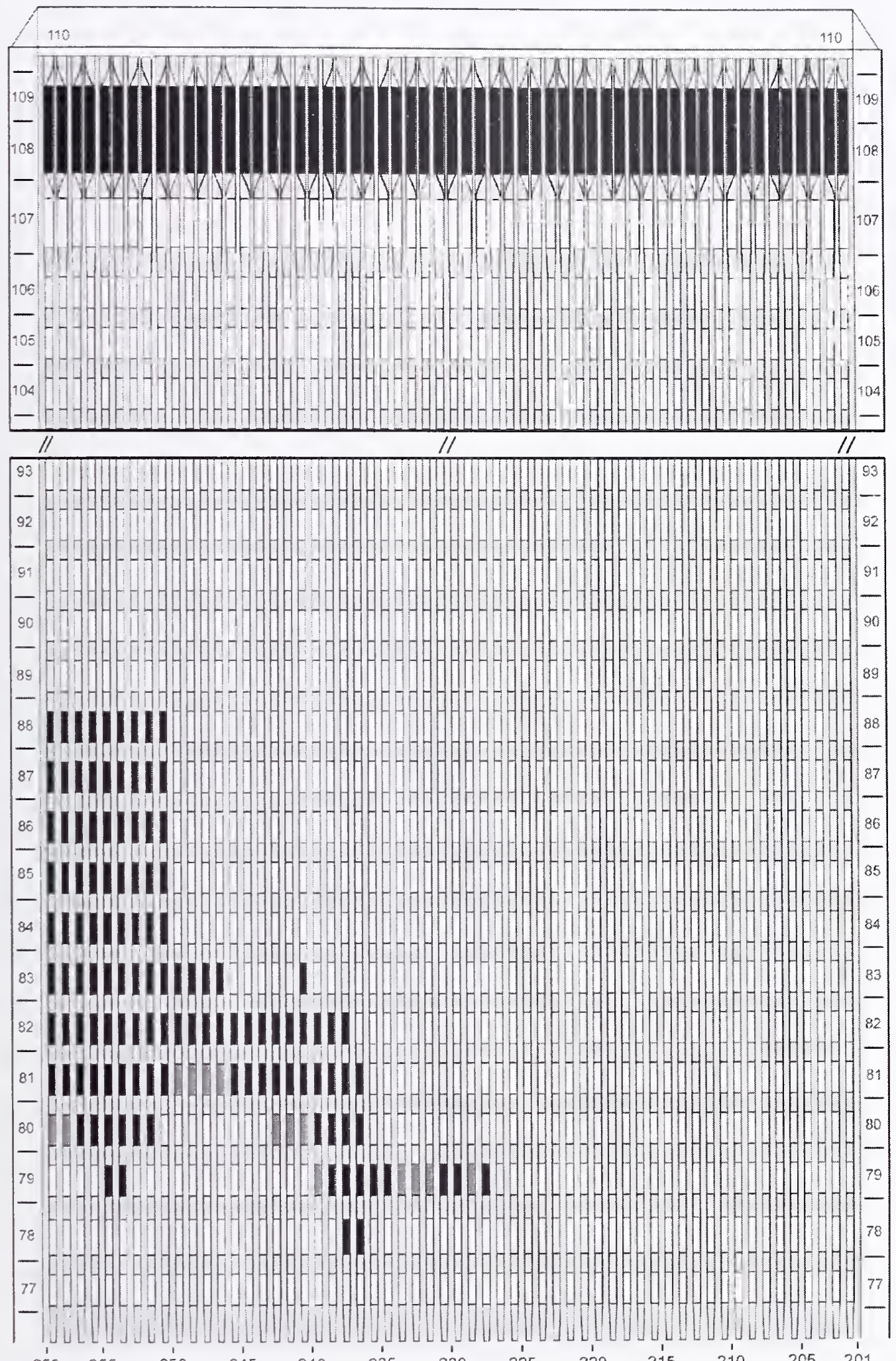

Figure G-8. Diagram of the north face of WTC 2 for floors 77 to 93 and floors 104 to 110 at 9:08 a.m. showing windows where smoke was observed and those hidden from view. 
WTC 2, North Face

9:10 a.m.

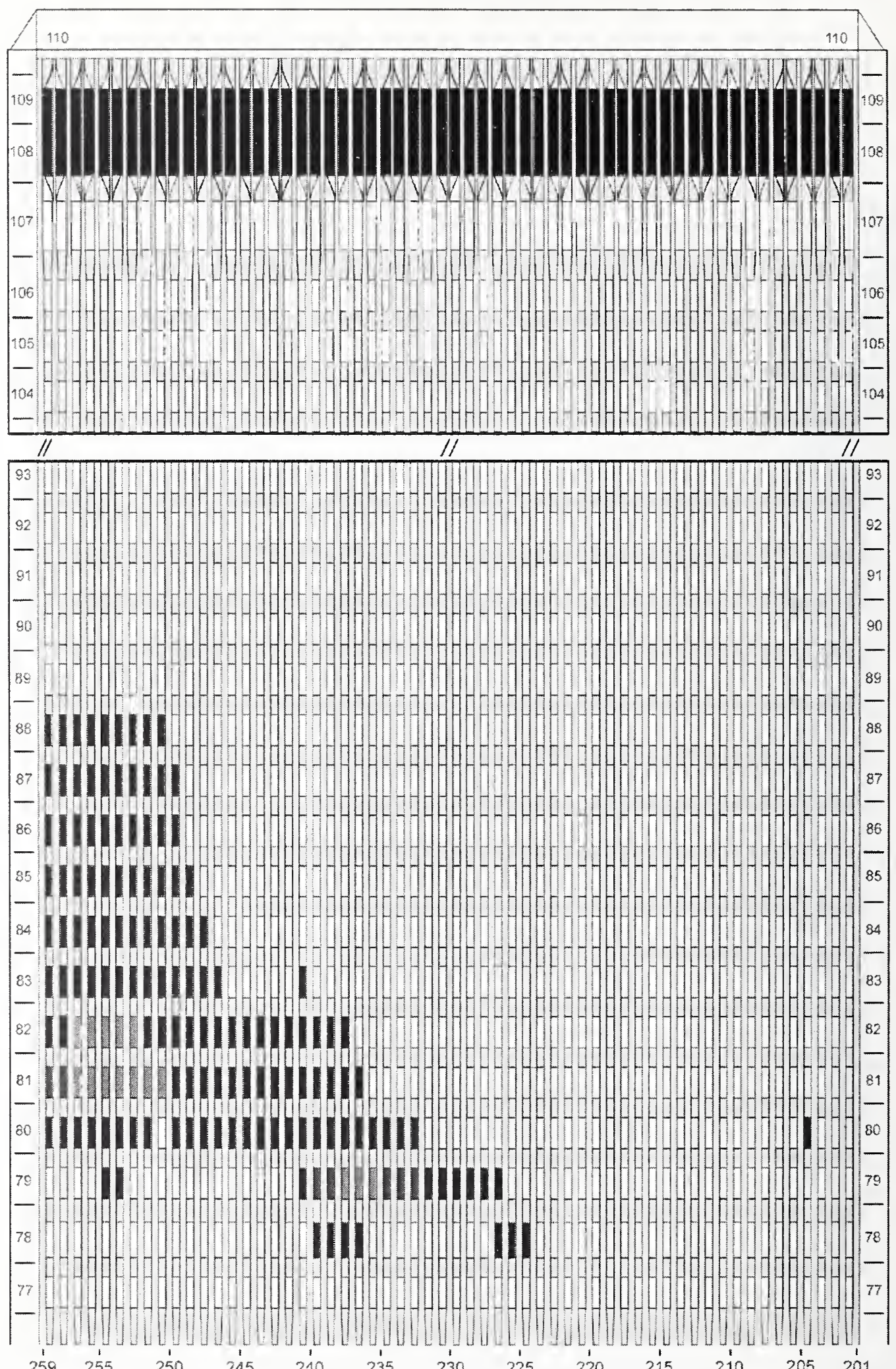

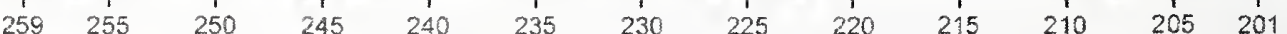

Figure G-9. Diagram of the north face of WTC 2 for floors 77 to 93 and floors 104 to 110 at 9:10 a.m. showing the condition of windows and locations of fires. 

WTC 2, North Face
9:10 a.m

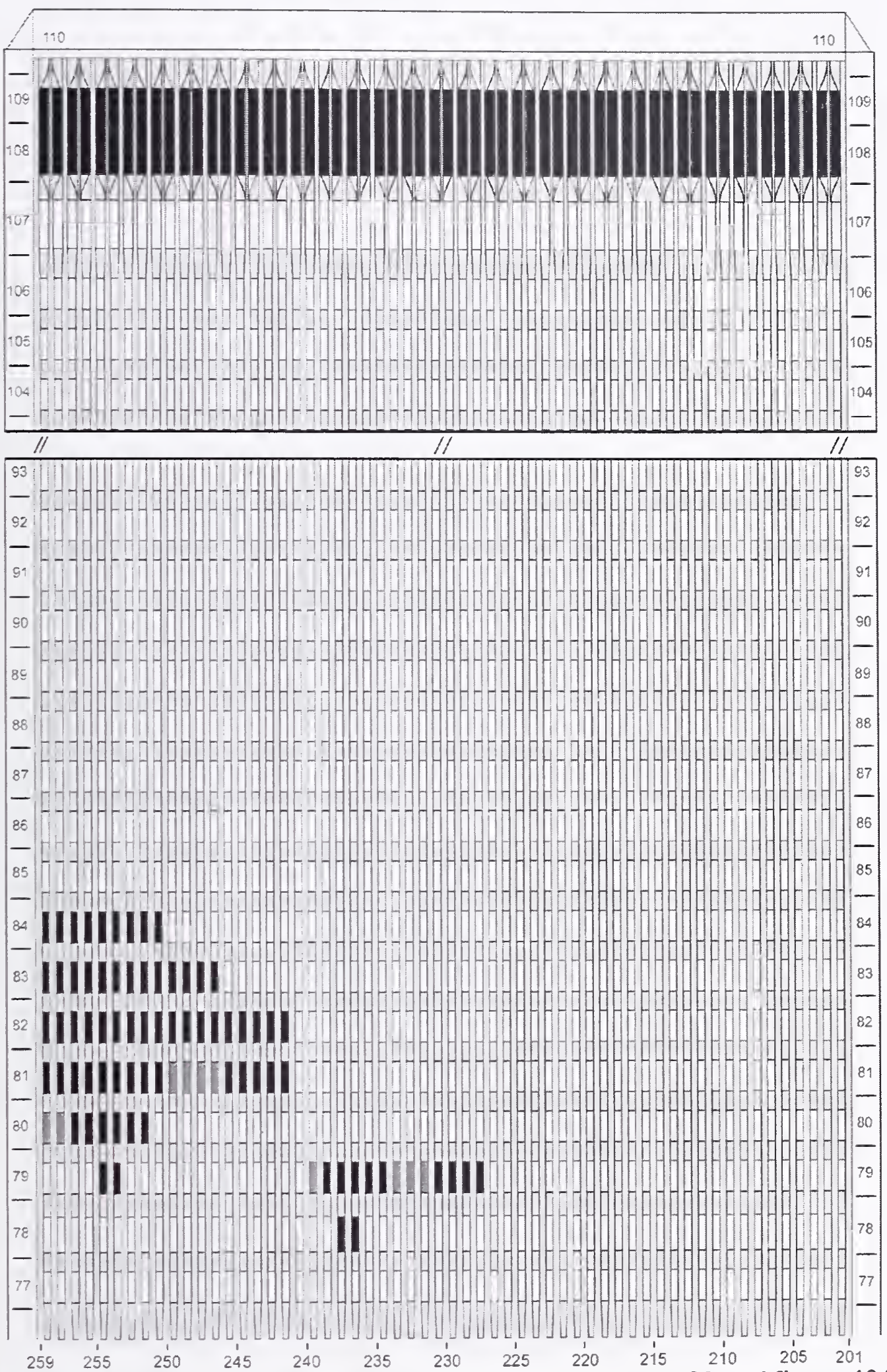

Figure G-10. Diagram of the north face of WTC 2 for floors 77 to 93 and floors 104 to 110 at 9:10 a.m. showing windows where smoke was observed and those hidden from view. 
WTC 2, North Face 9:12 a.m.

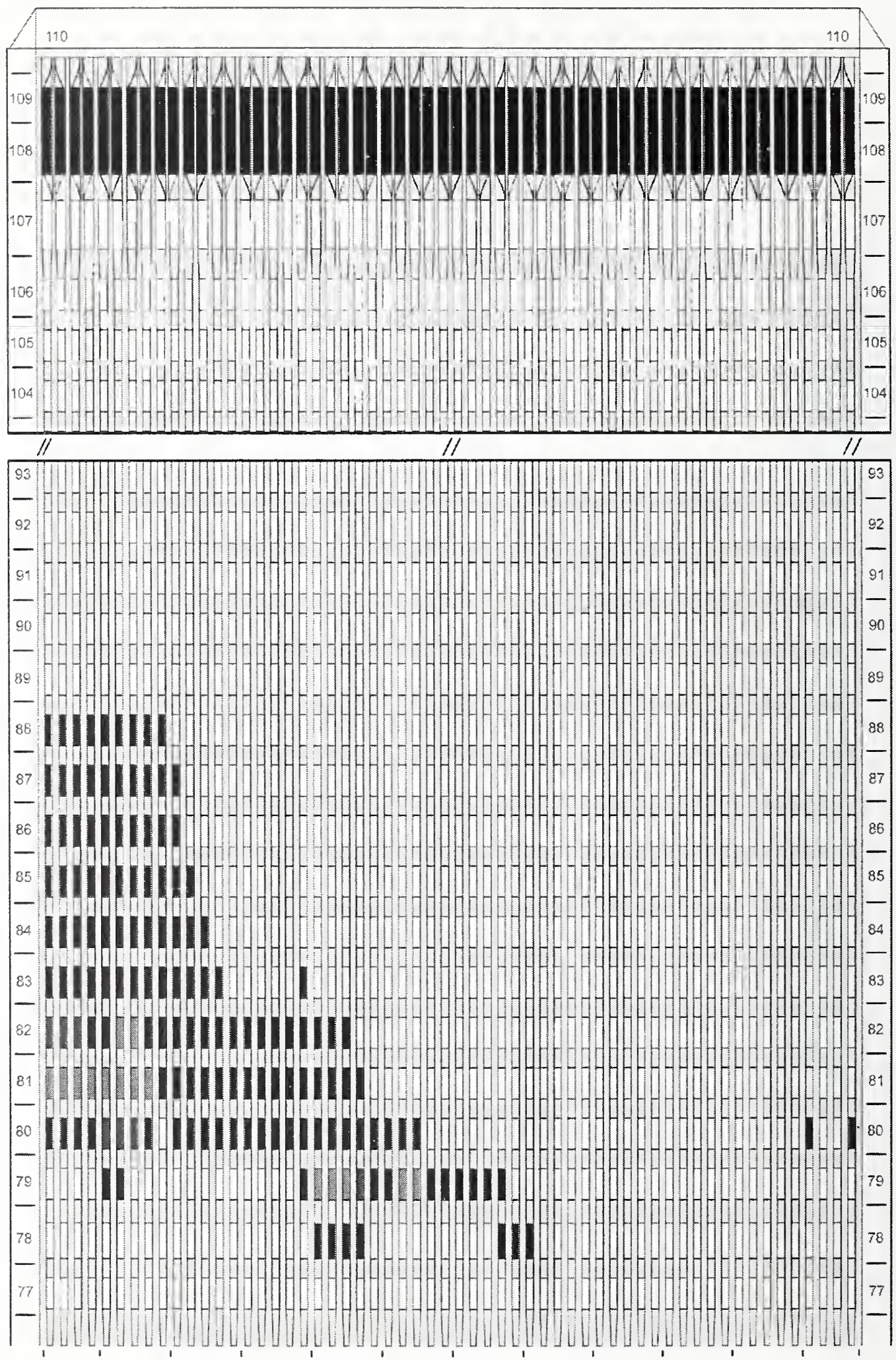

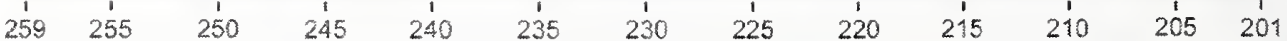

Figure G-11. Diagram of the north face of WTC 2 for floors 77 to 93 and floors 104 to 110 at 9:12 a.m. showing the condition of windows and locations of fires. 
WTC 2, North Face 9:12 a.m.

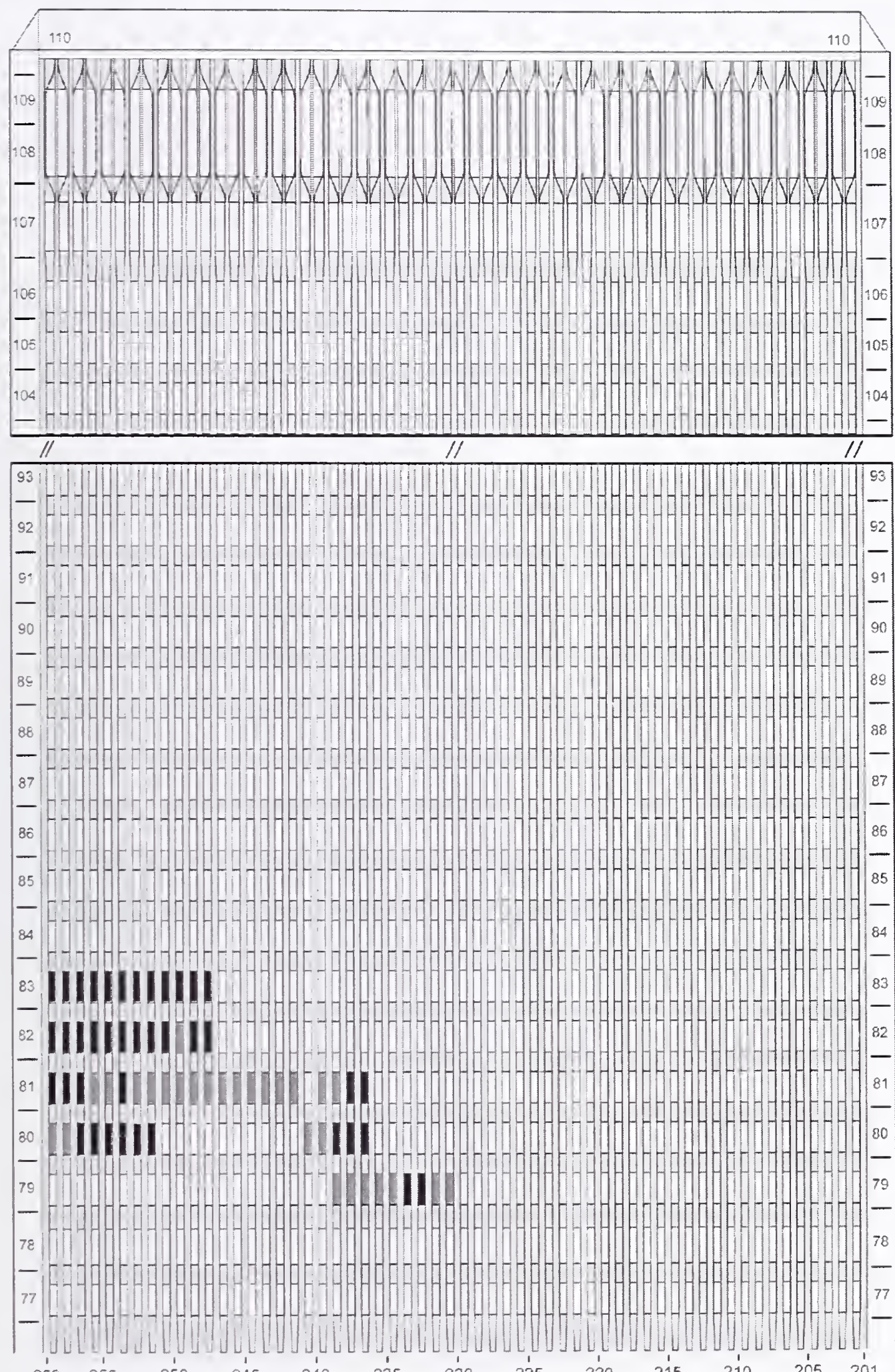

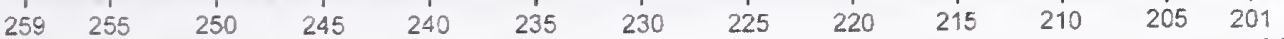

Figure G-12. Diagram of the north face of WTC 2 for floors 77 to 93 and floors 104 to 110 at 9:12 a.m. showing windows where smoke was observed and those hidden from view. 
WTC 2, North Face

9:14 a.m.

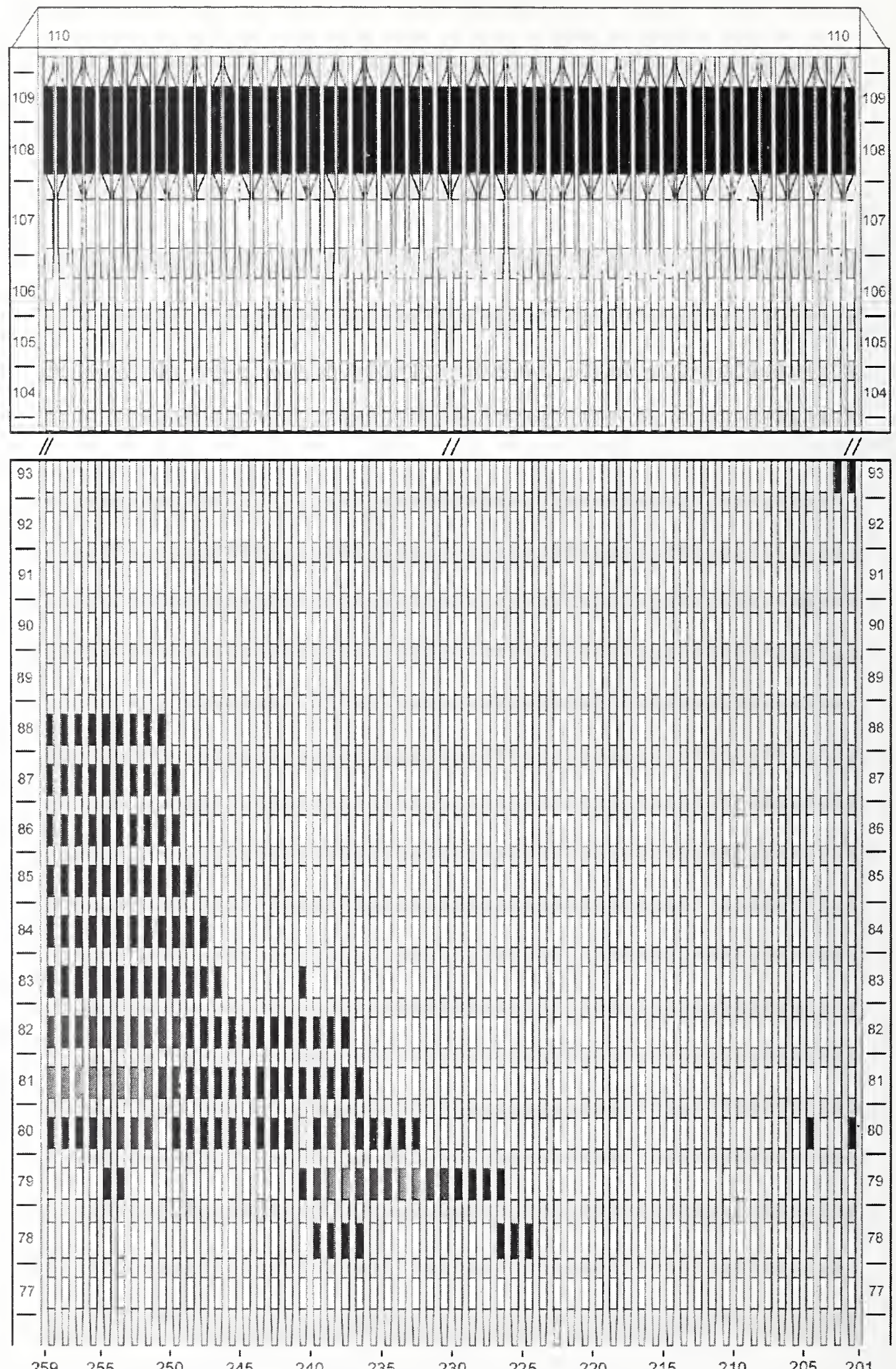

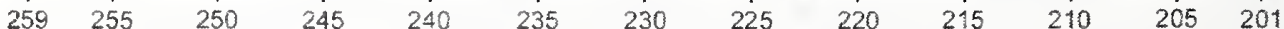

Figure G-13. Diagram of the north face of WTC 2 for floors 77 to 93 and floors 104 to 110 at 9:14 a.m. showing the condition of windows and locations of fires. 
WTC 2, North Face $\quad 9: 14$ a.m.

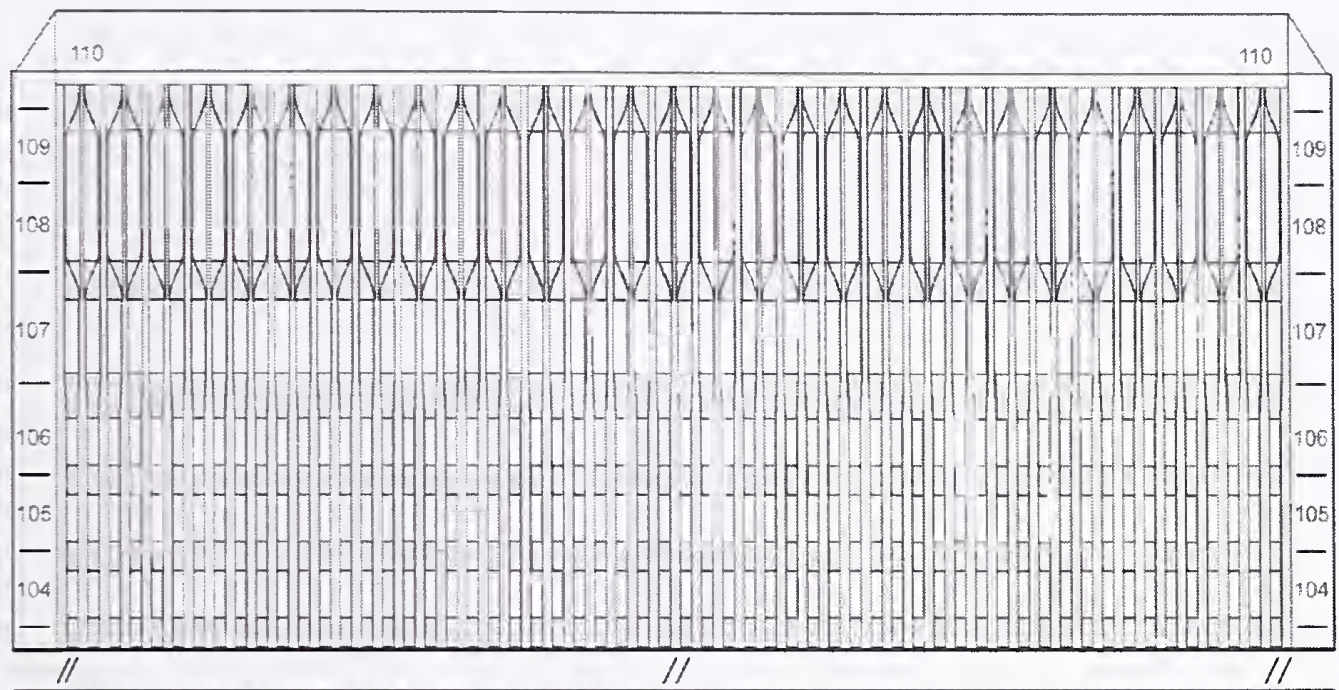

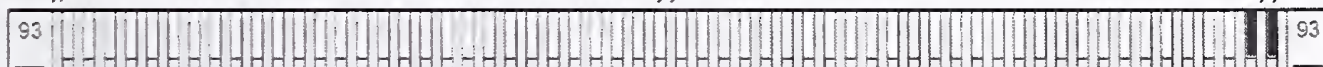

- D2-

$-$

- IIIIIIII

87 IIIIII

(26-- ||||||

85 IIIIIIIII

a IIIIIIIIIIIII

83 IIIIIIIIIIIII

32 IIIIIIIIIIII

81 IIIIIIIII

- [IIIIII [III

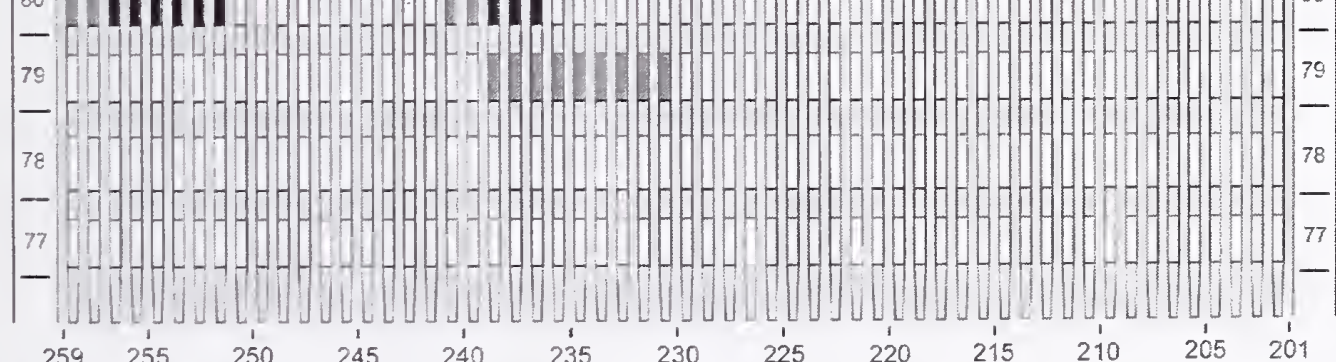

Figure G-14. Diagram of the north face of WTC 2 for floors 77 to 93 and floors 104 to 110 at 9:14 a.m. showing windows where smoke was observed and those hidden from view. 
WTC 2, North Face

9:16 a.m.

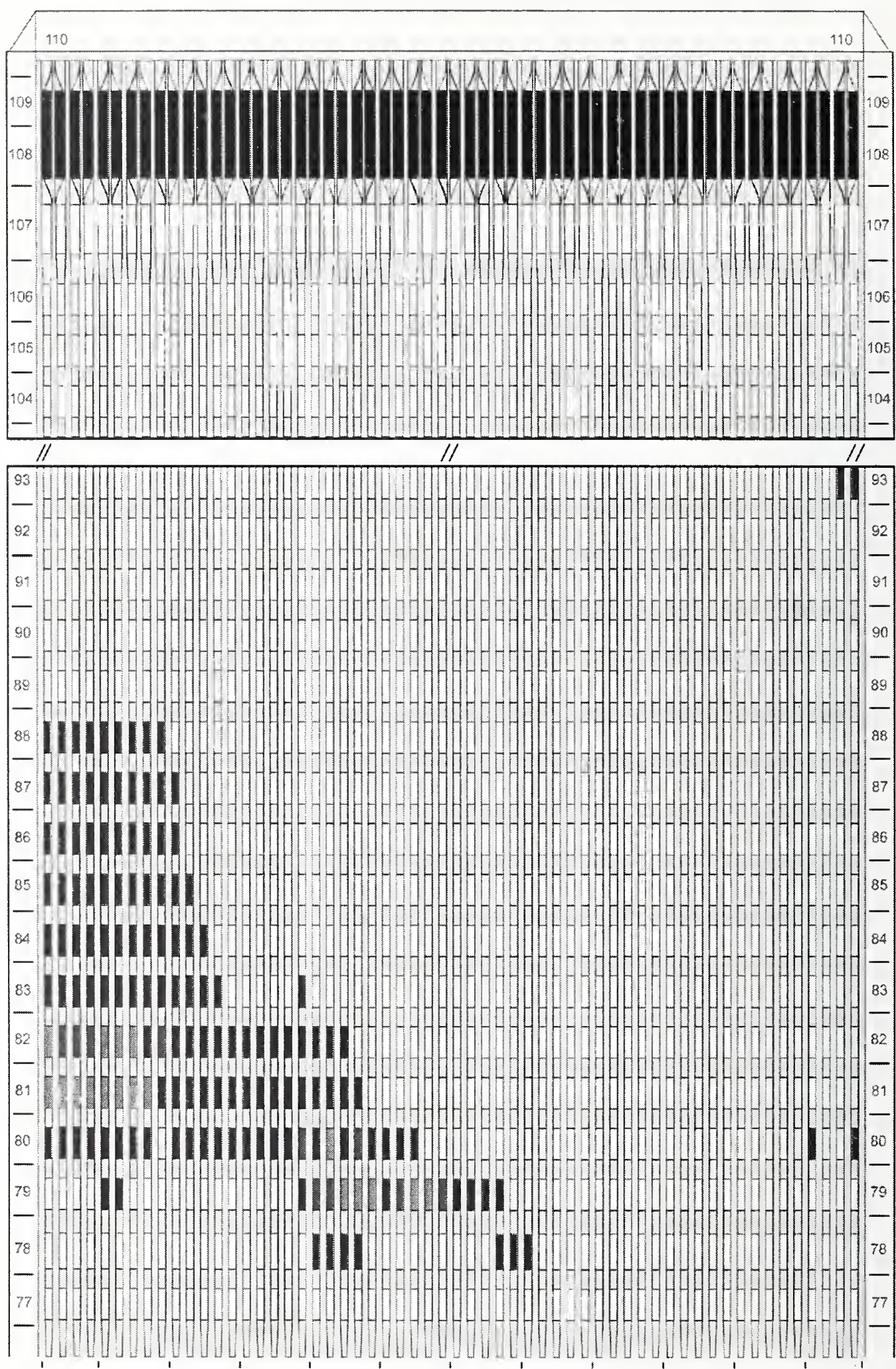

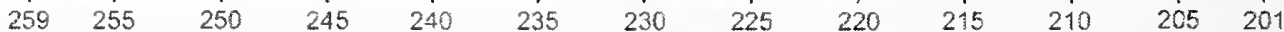

Figure G-15. Diagram of the north face of WTC 2 for floors 77 to 93 and floors 104 to 110 at 9:16 a.m. showing the condition of windows and locations of fires. 
WTC 2, North Face $\quad 9: 16$ a.m.

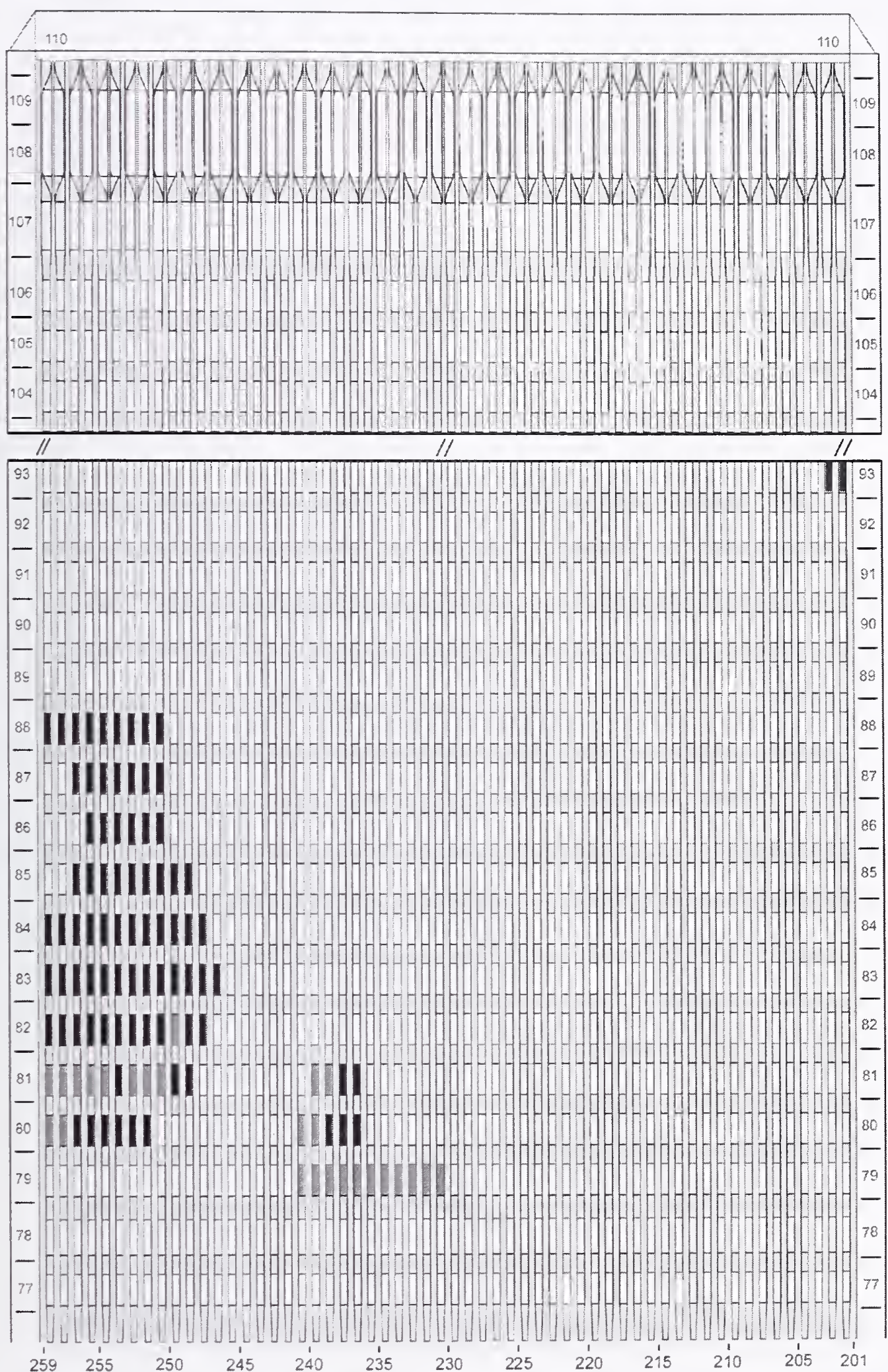

Figure G-16. Diagram of the north face of WTC 2 for floors 77 to 93 and floors 104 to 110 at 9:16 a.m. showing windows where smoke was observed and those hidden from view. 
WTC 2, North Face $\quad$ 9:18 a.m.

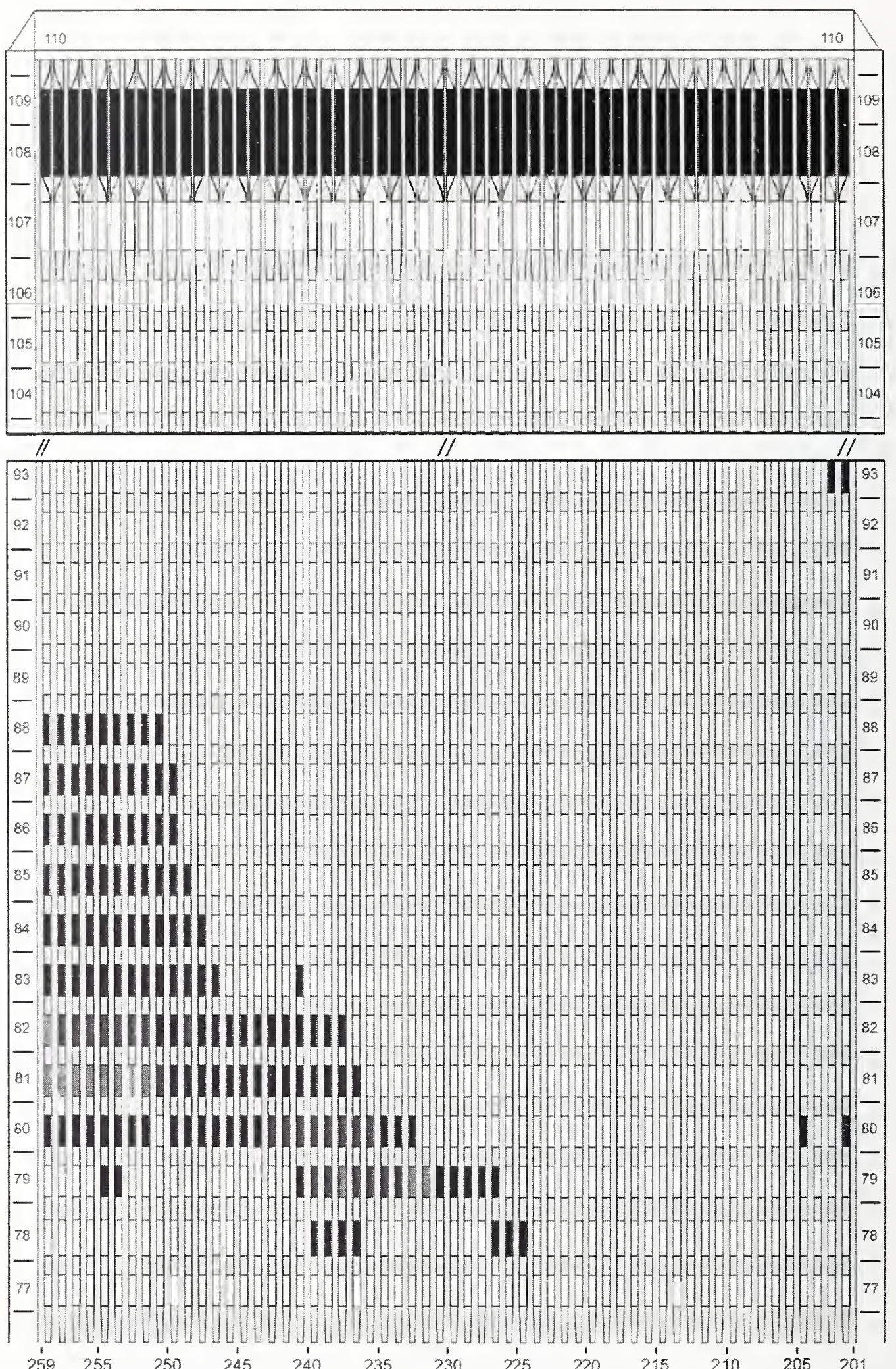

Figure G-17. Diagram of the north face of WTC 2 for floors 77 to 93 and floors 104 to 110 at 9:18 a.m. showing the condition of windows and locations of fires. 
WTC 2, North Face $\quad 9: 18$ a.m.

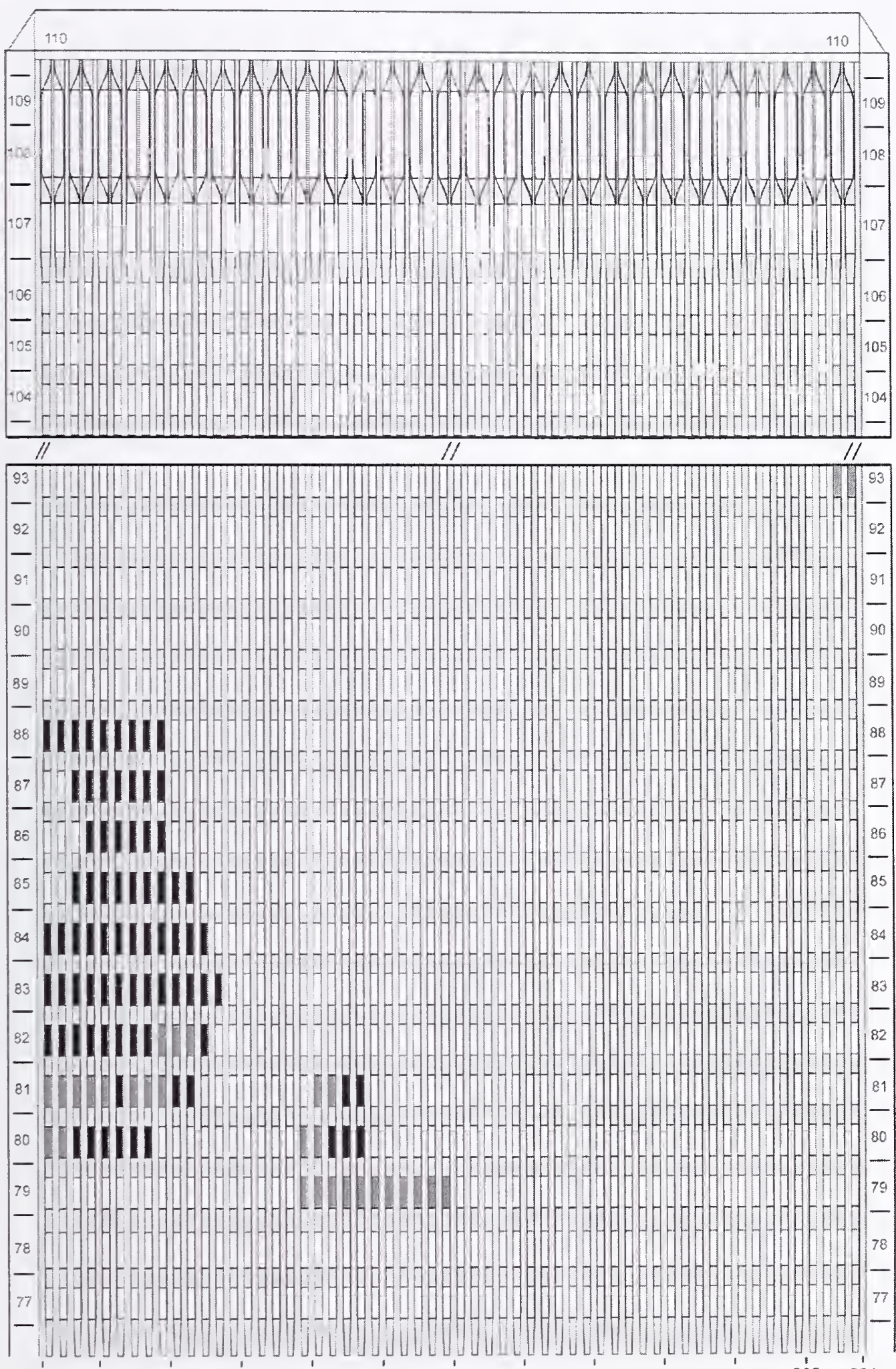

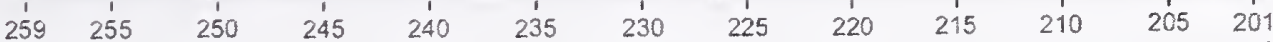

Figure G-18. Diagram of the north face of WTC 2 for floors 77 to 93 and floors 104 to 110 at 9:18 a.m. showing windows where smoke was observed and those hidden from view. 
WTC 2, North Face $\quad 9: 20$ a.m.

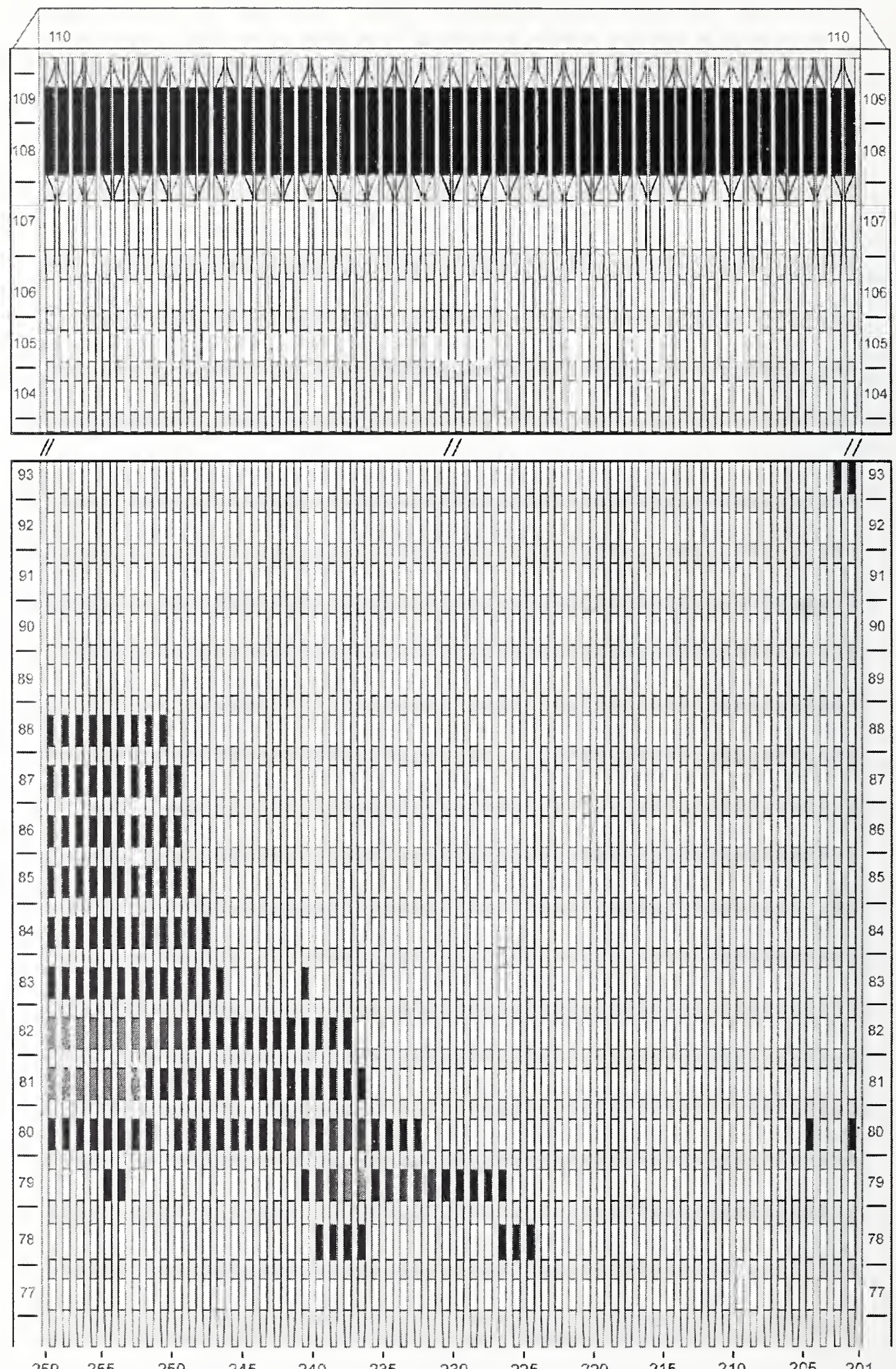

Figure G-19. Diagram of the north face of WTC 2 for floors 77 to 93 and floors 104 to 110 at 9:20 a.m. showing the condition of windows and locations of fires. 

WTC 2, North Face
9:20 a.m.

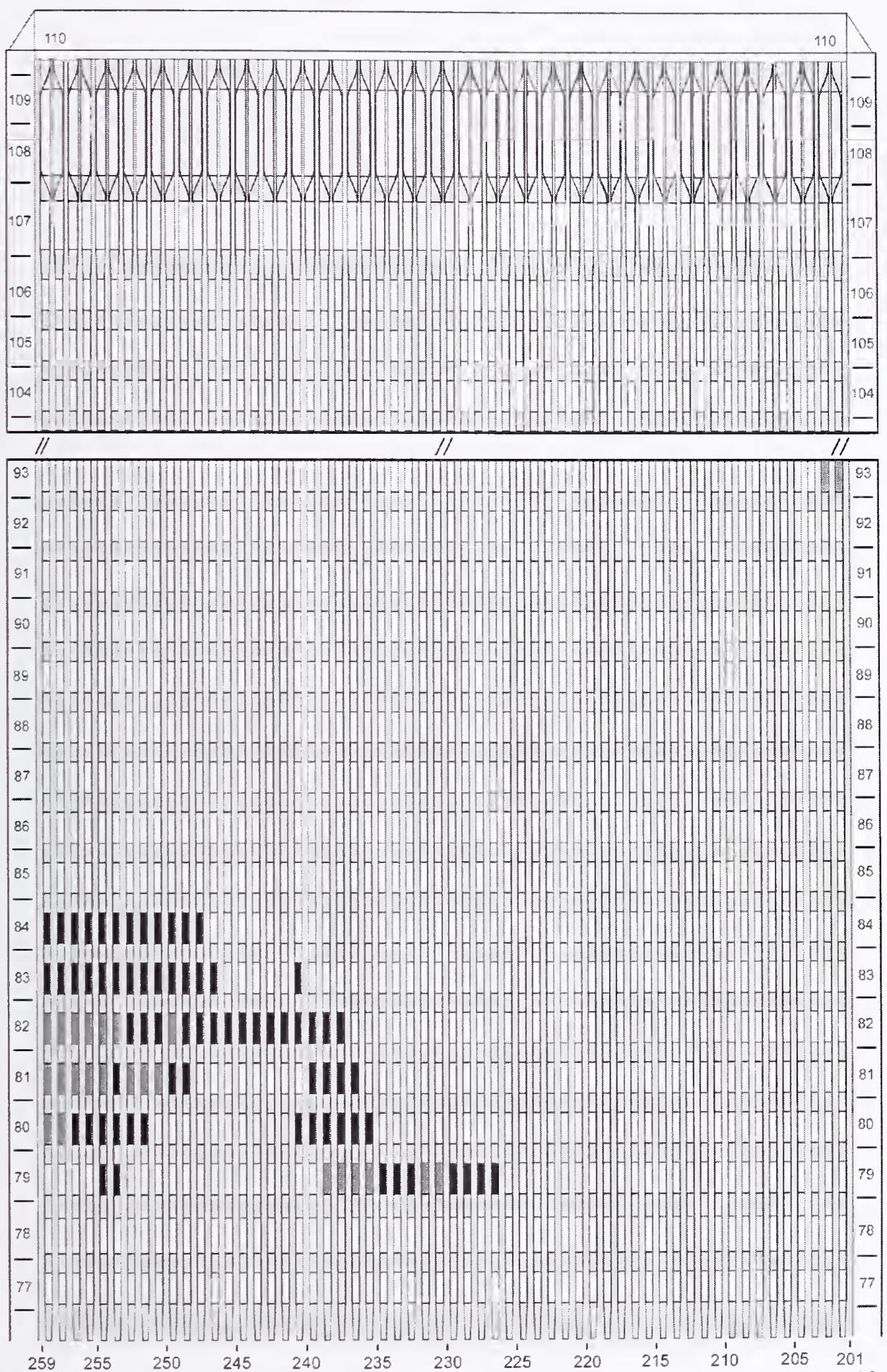

Figure G-20. Diagram of the north face of WTC 2 for floors 77 to 93 and floors 104 to 110 at 9:20 a.m. showing windows where smoke was observed and those hidden from view. 
WTC 2, North Face 9:22 a.m.

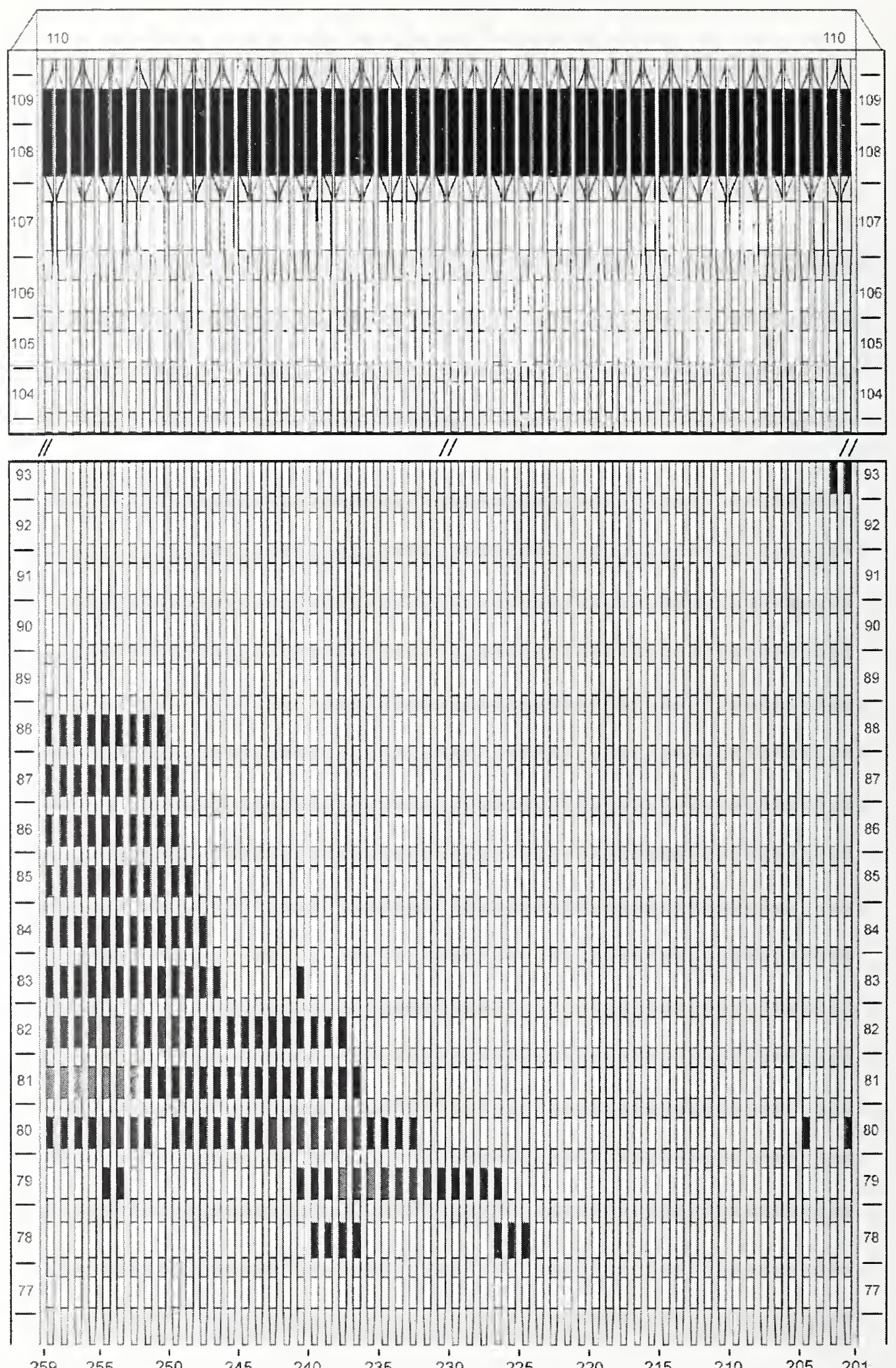

Figure G-21. Diagram of the north face of WTC 2 for floors 77 to 93 and floors 104 to 110 at 9:22 a.m. showing the condition of windows and locations of fires. 
WTC 2, North Face $\quad 9: 22$ a.m.
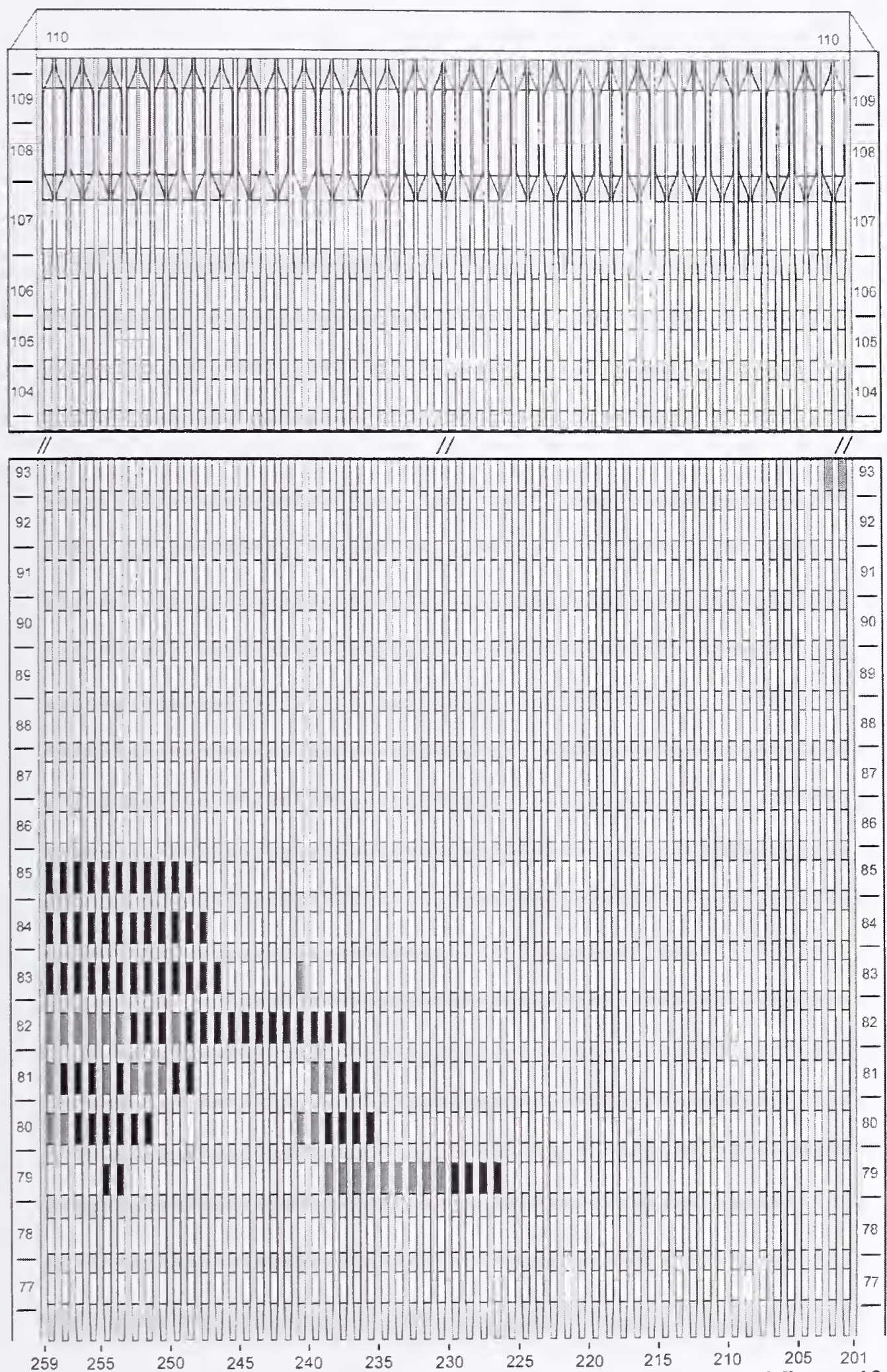

Figure G-22. Diagram of the north face of WTC 2 for floors 77 to 93 and floors 104 to 110 at 9:22 a.m. showing windows where smoke was observed and those hidden from view. 
WTC 2, North Face 9:24 a.m.



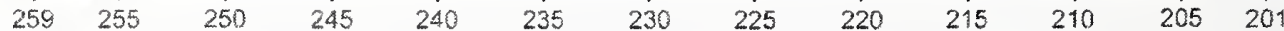

Figure G-23. Diagram of the north face of WTC 2 for floors 77 to 93 and floors 104 to 110 at 9:24 a.m. showing the condition of windows and locations of fires. 


$$
\text { WTC 2, North Face } \quad 9: 24 \text { a.m. }
$$

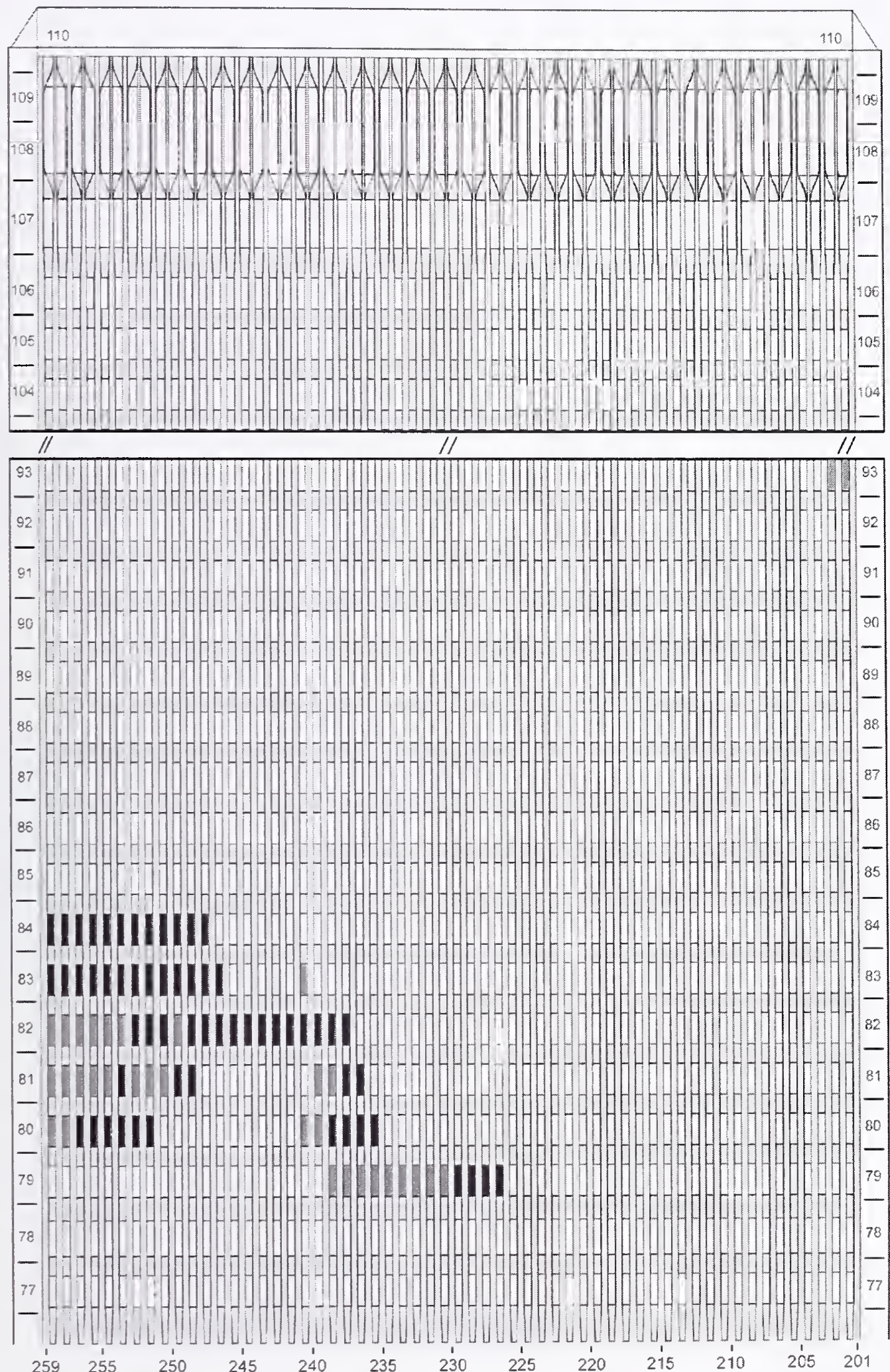

Figure G-24. Diagram of the north face of WTC 2 for floors 77 to 93 and floors 104 to 110 at 9:24 a.m. showing windows where smoke was observed and those hidden from view. 
WTC 2, North Face $\quad 9: 26$ a.m.

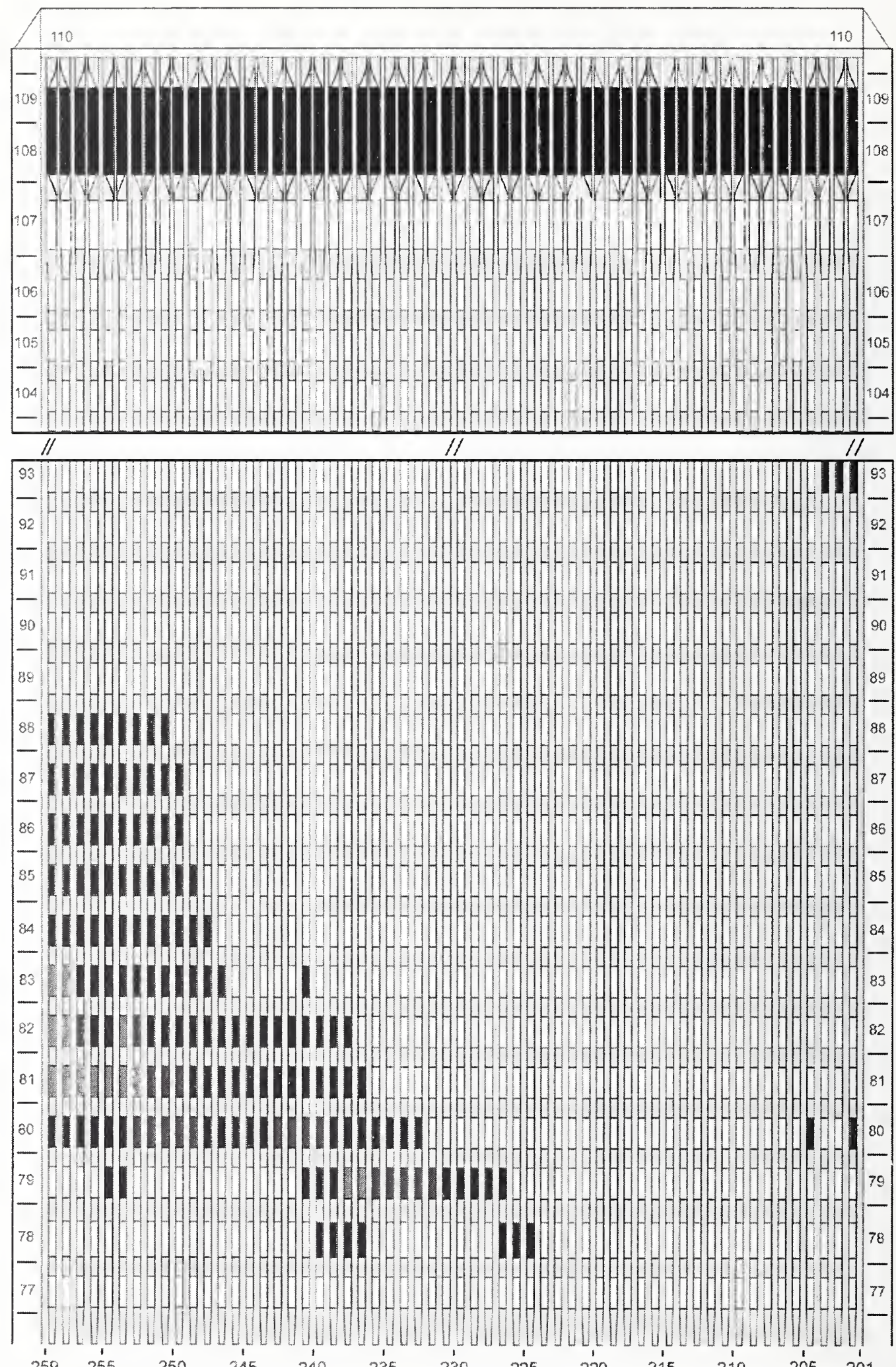

Figure G-25. Diagram of the north face of WTC 2 for floors 77 to 93 and floors 104 to 110 at 9:26 a.m. showing the condition of windows and locations of fires. 
WTC 2, North Face $\quad 9: 26$ a.m.

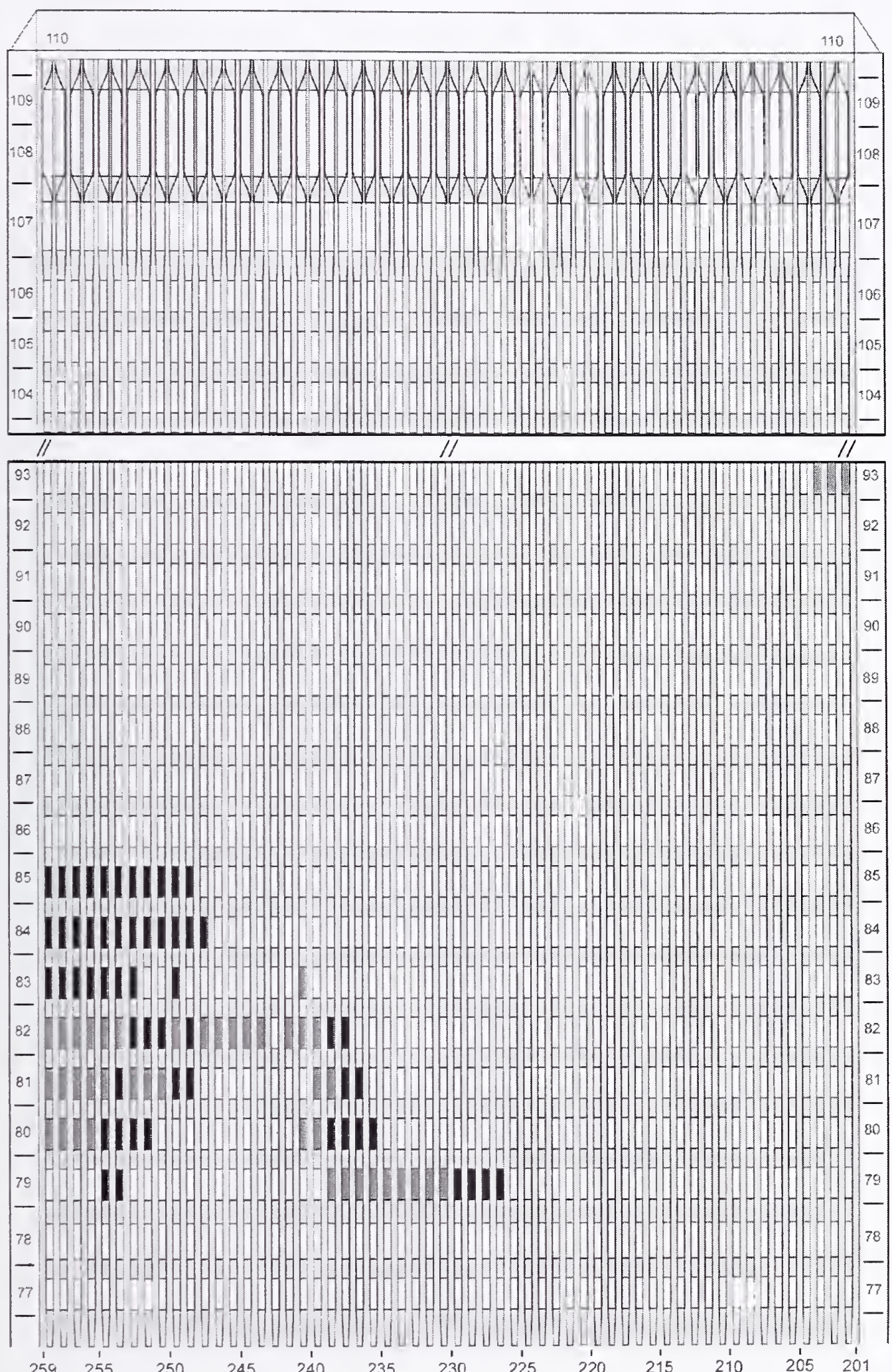

Figure G-26. Diagram of the north face of WTC 2 for floors 77 to 93 and floors 104 to 110 at 9:26 a.m. showing windows where smoke was observed and those hidden from view. 


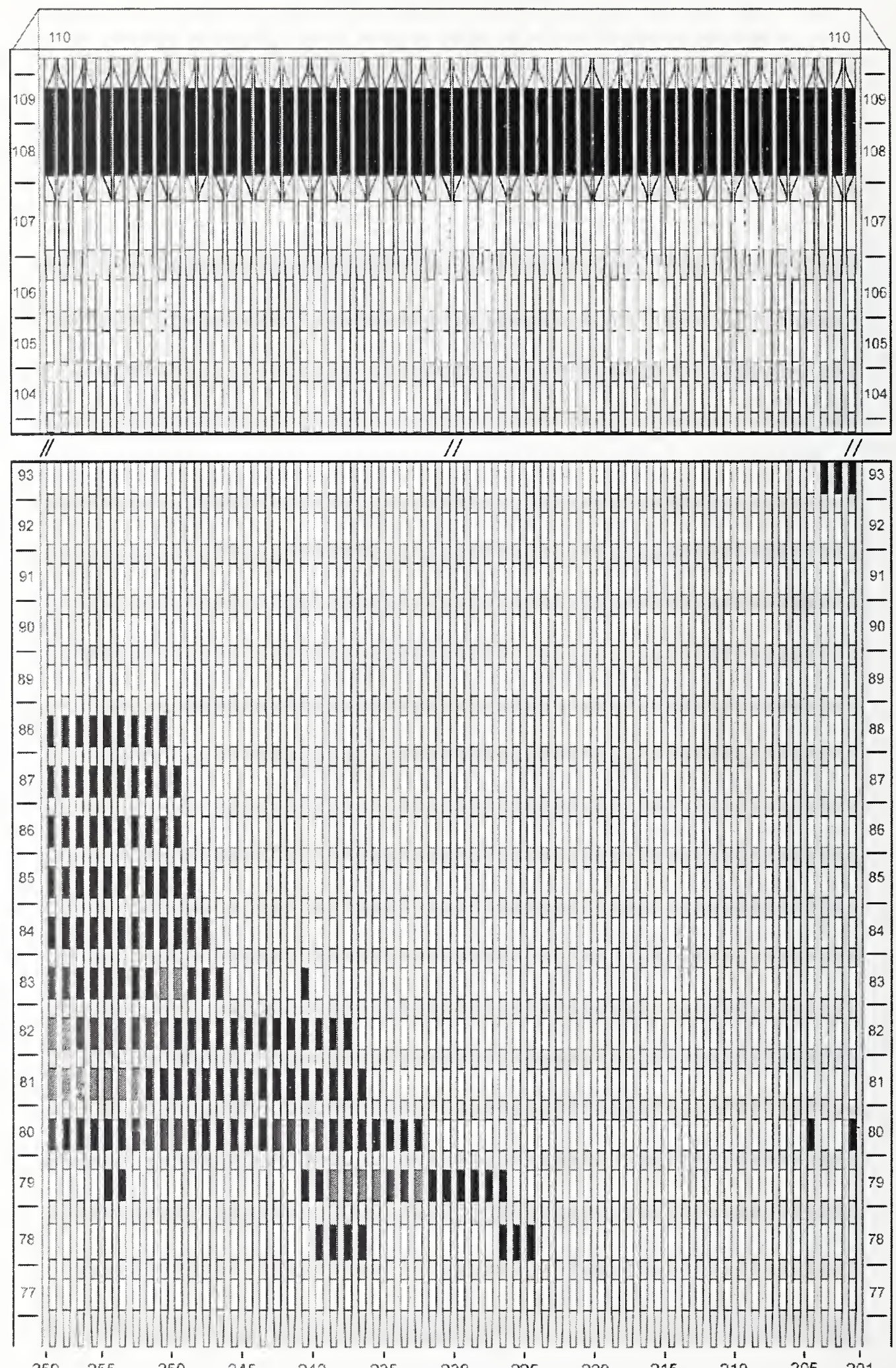

Figure G-27. Diagram of the north face of WTC 2 for floors 77 to 93 and floors 104 to 110 at 9:28 a.m. showing the condition of windows and locations of fires. 


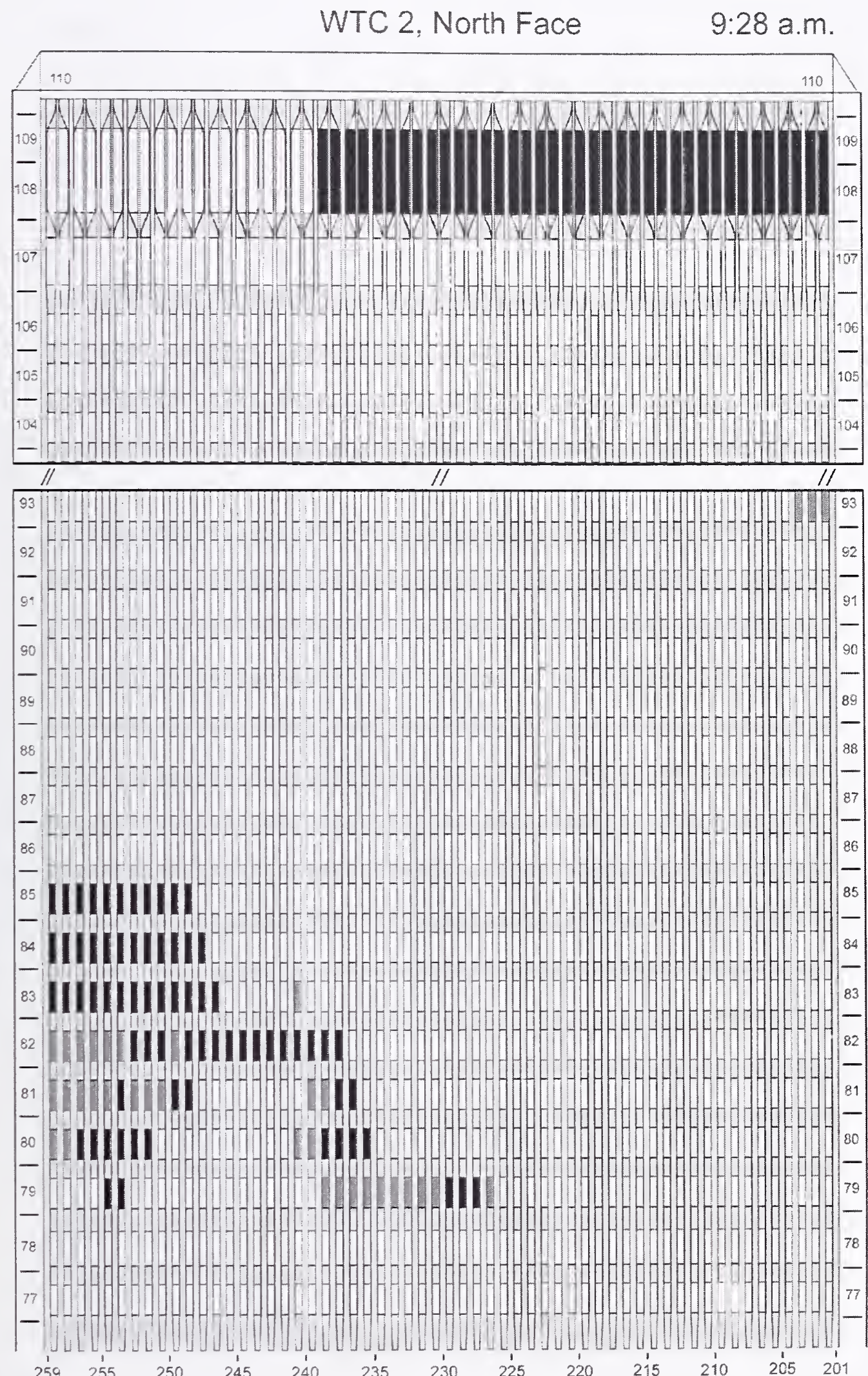

Figure G-28. Diagram of the north face of WTC 2 for floors 77 to 93 and floors 104 to 110 at 9:28 a.m. showing windows where smoke was observed and those hidden from view. 
WTC 2, North Face

9:30 a.m.

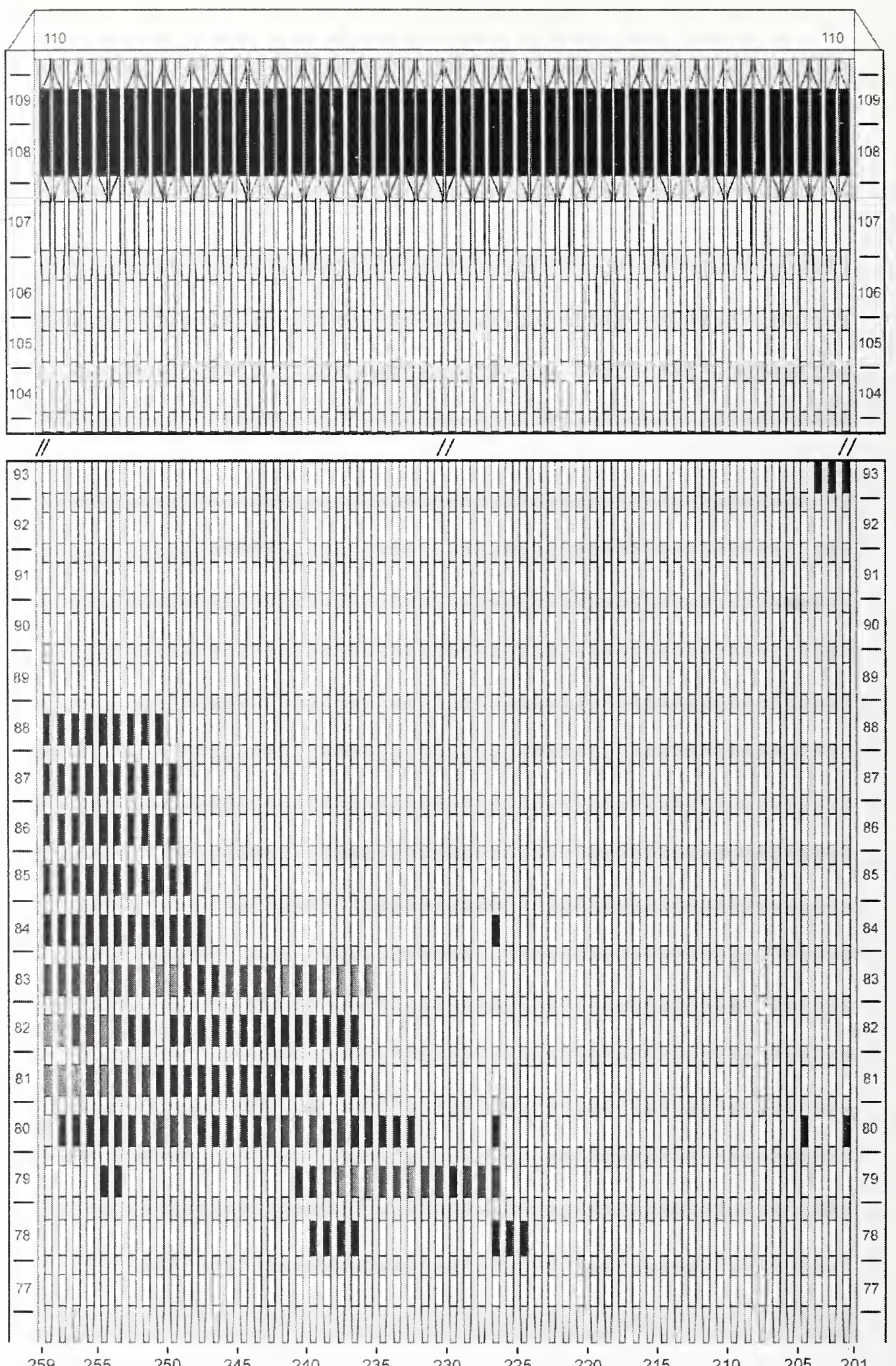

Figure G-29. Diagram of the north face of WTC 2 for floors 77 to 93 and floors 104 to 110 at 9:30 a.m. showing the condition of windows and locations of fires. 


$$
\text { WTC 2, North Face 9:30 a.m. }
$$
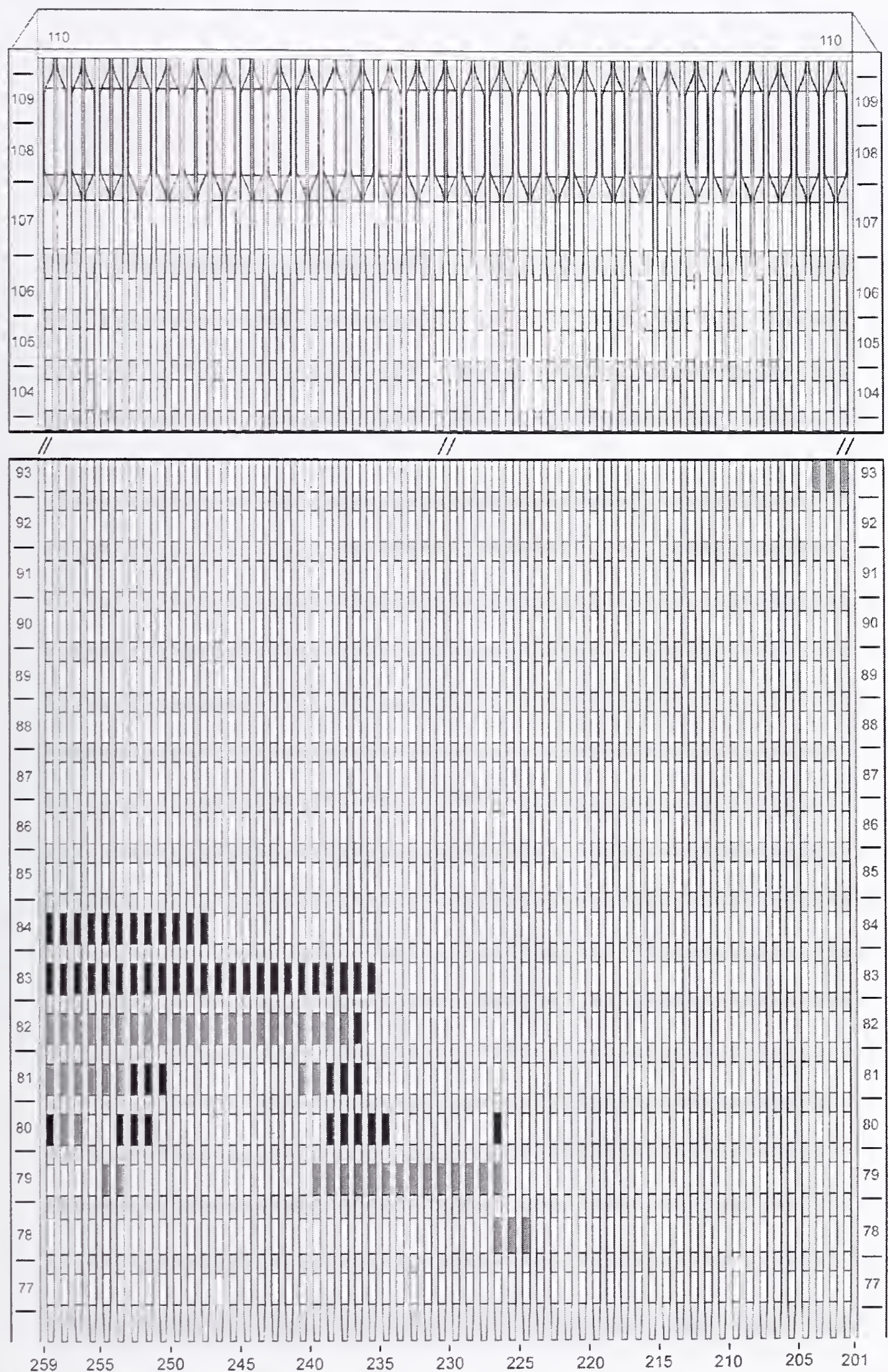

Figure G-30. Diagram of the north face of WTC 2 for floors 77 to 93 and floors 104 to 110 at 9:30 a.m. showing windows where smoke was observed and those hidden from view. 
WTC 2, North Face

9:32 a.m.

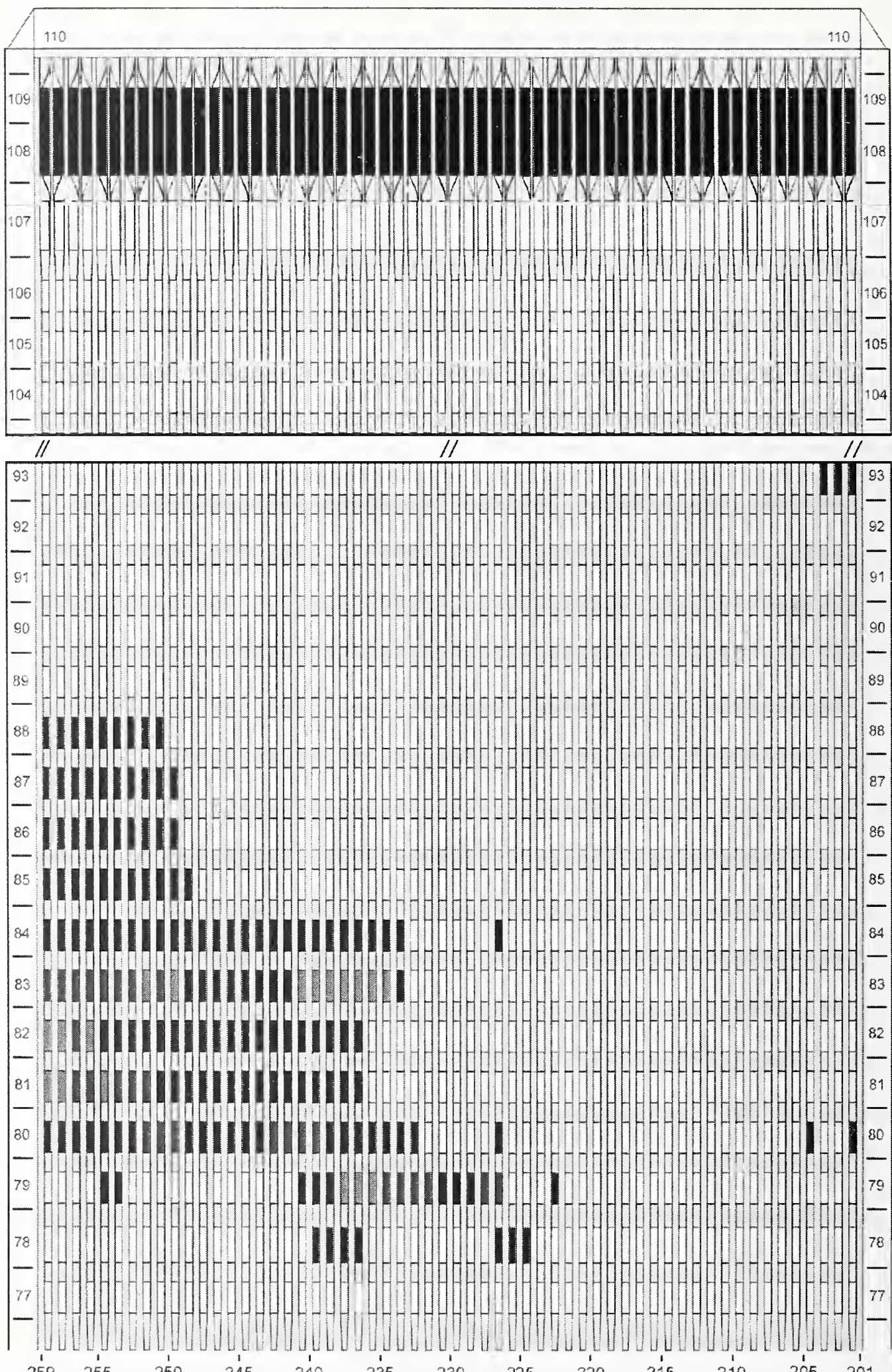

Figure G-31. Diagram of the north face of WTC 2 for floors 77 to 93 and floors 104 to 110 at 9:32 a.m. showing the condition of windows and locations of fires. 
WTC 2, North Face $\quad 9: 32$ a.m.

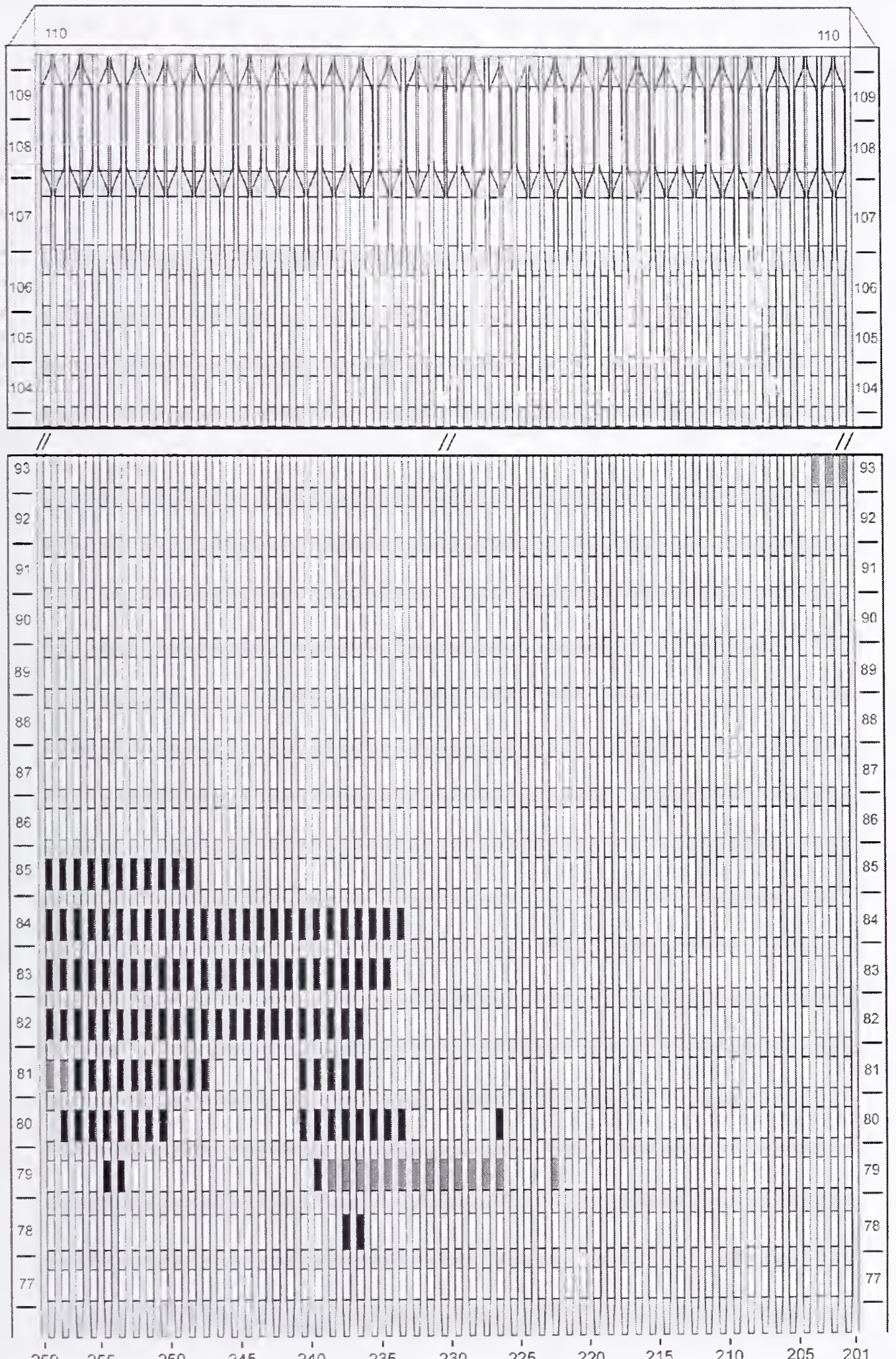

Figure G-32. Diagram of the north face of WTC 2 for floors 77 to 93 and floors 104 to 110 at 9:32 a.m. showing windows where smoke was observed and those hidden from view. 
WTC 2, North Face $9: 34$ a.m.

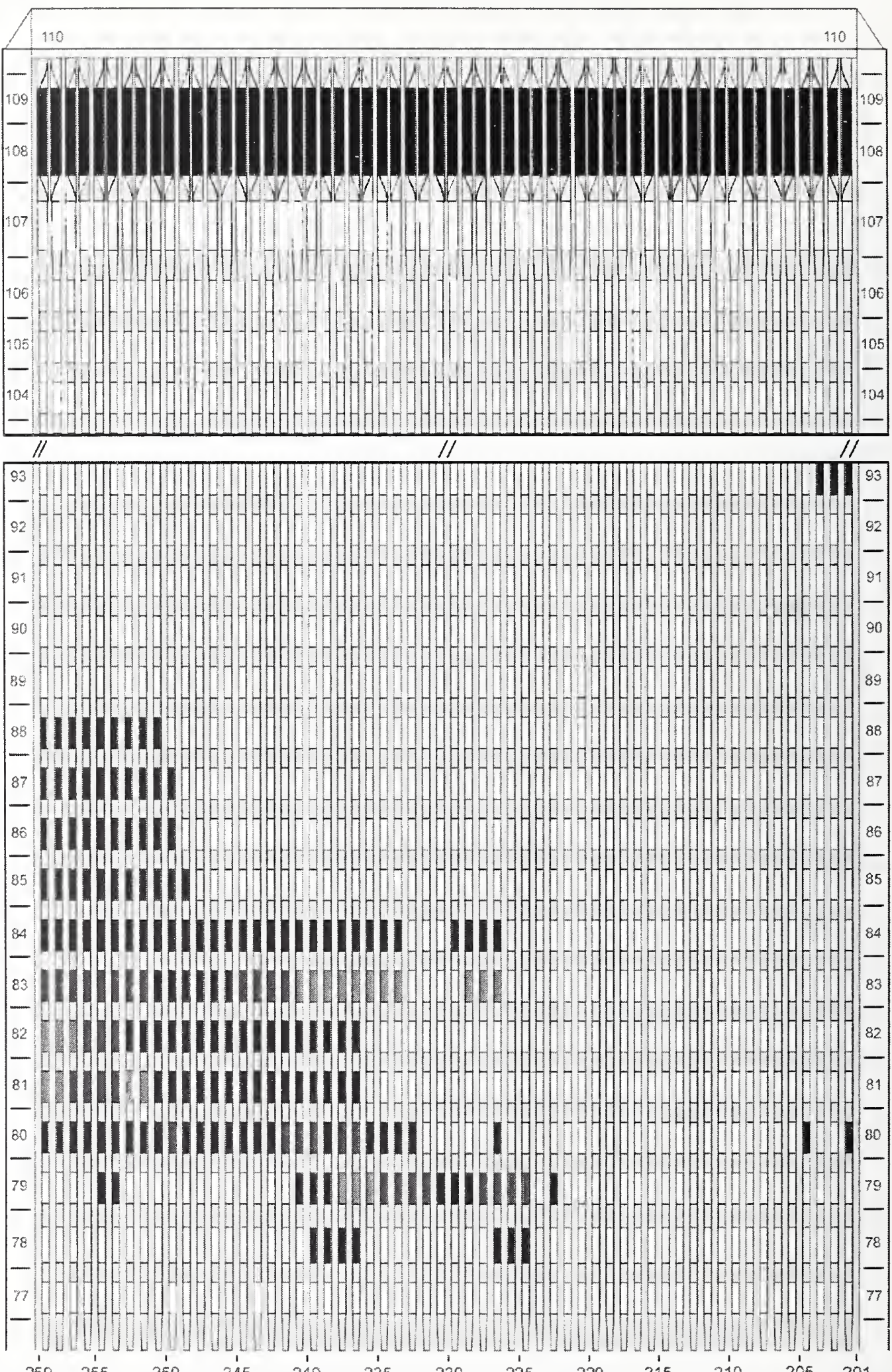

Figure G-33. Diagram of the north face of WTC 2 for floors 77 to 93 and floors 104 to 110 at 9:34 a.m. showing the condition of windows and locations of fires. 
WTC 2, North Face

9:34 a.m.

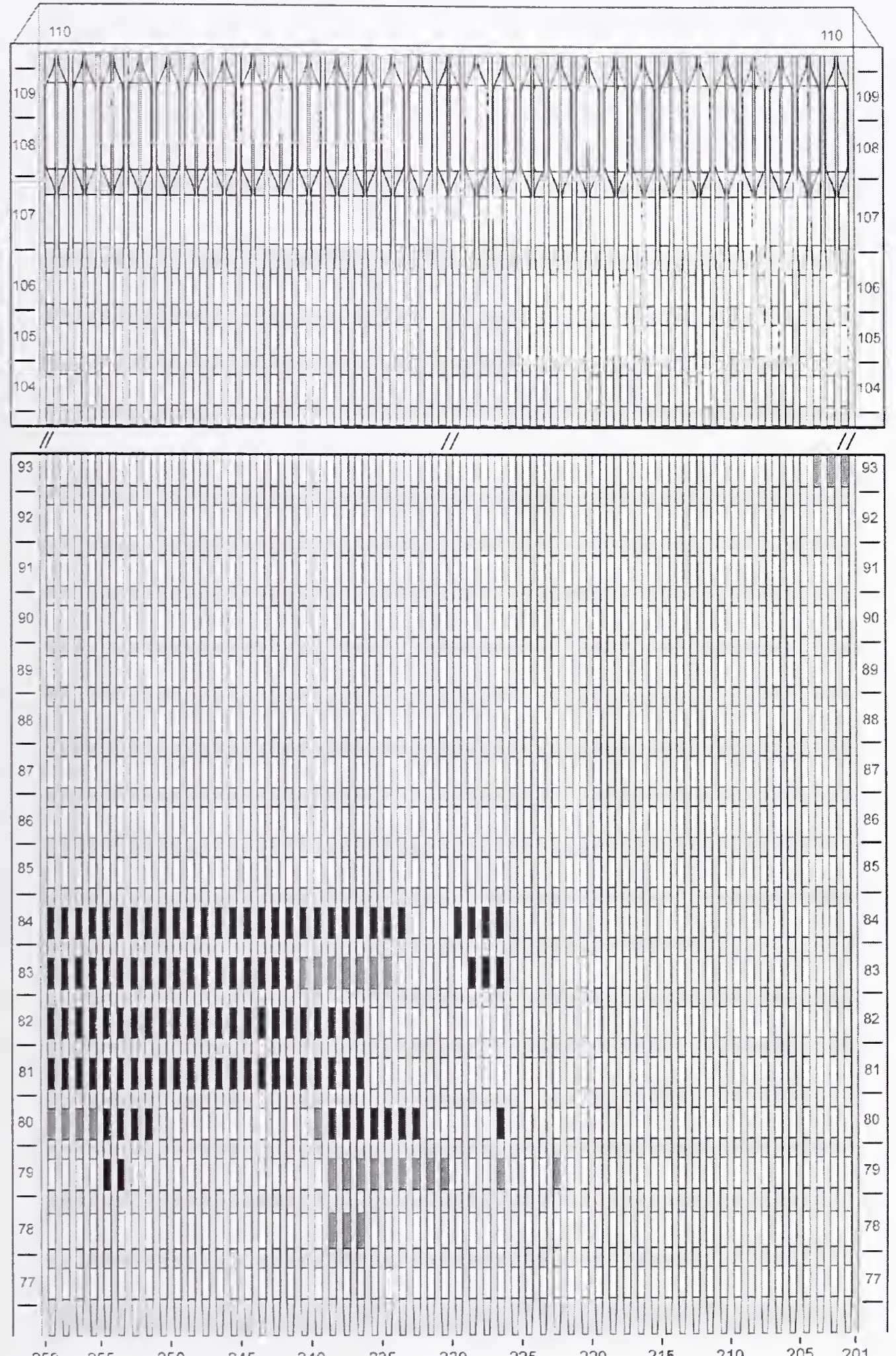

Figure G-34. Diagram of the north face of WTC 2 for floors 77 to $93^{25}$ and floors 104 to 110 at 9:34 a.m. showing windows where smoke was observed and those hidden from view. 
WTC 2, North Face

$9: 36$ a.m.

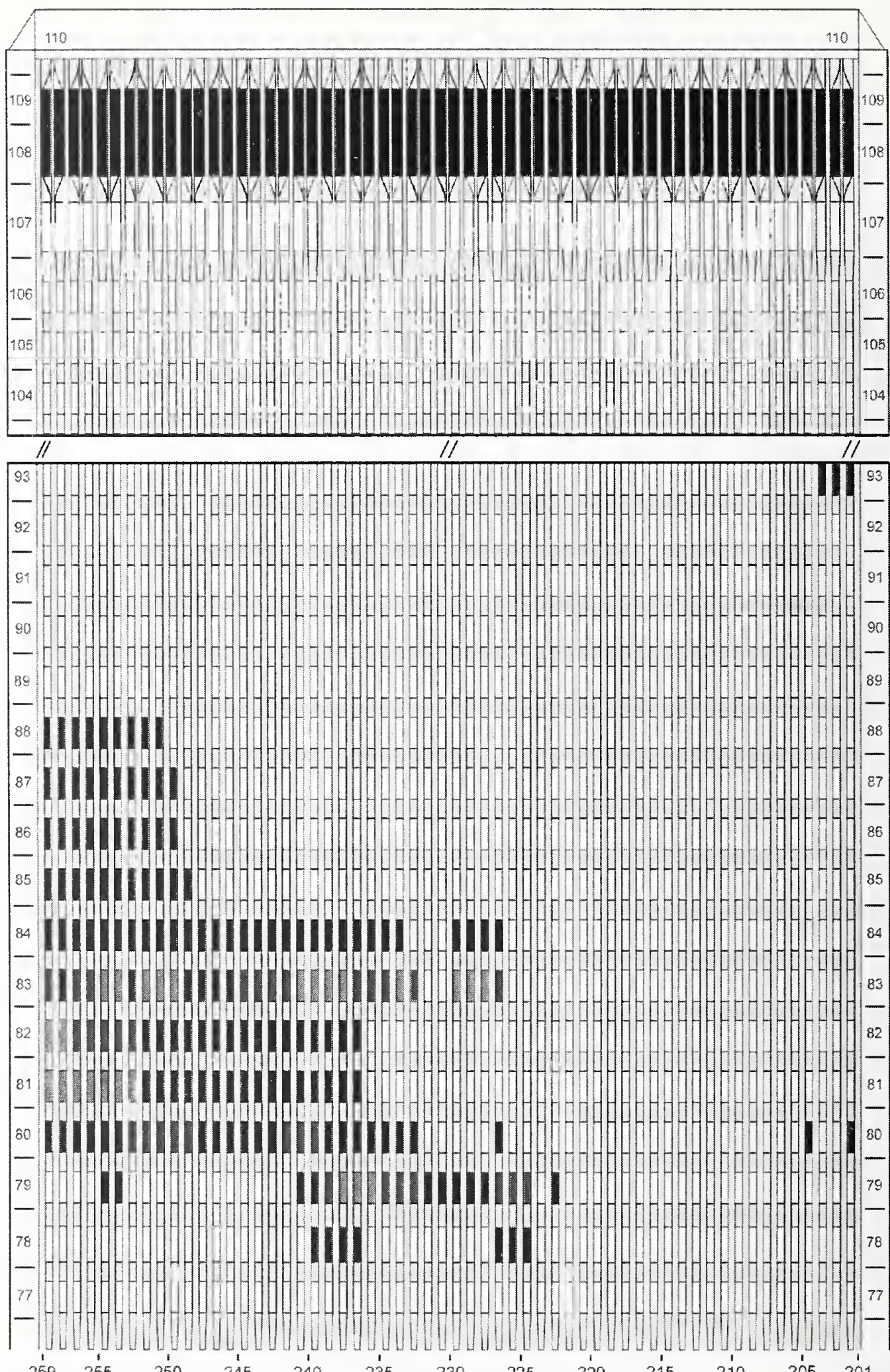

Figure G-35. Diagram of the north face of WTC 2 for floors 77 to 93 and floors 104 to 110 at 9:36 a.m. showing the condition of windows and locations of fires. 


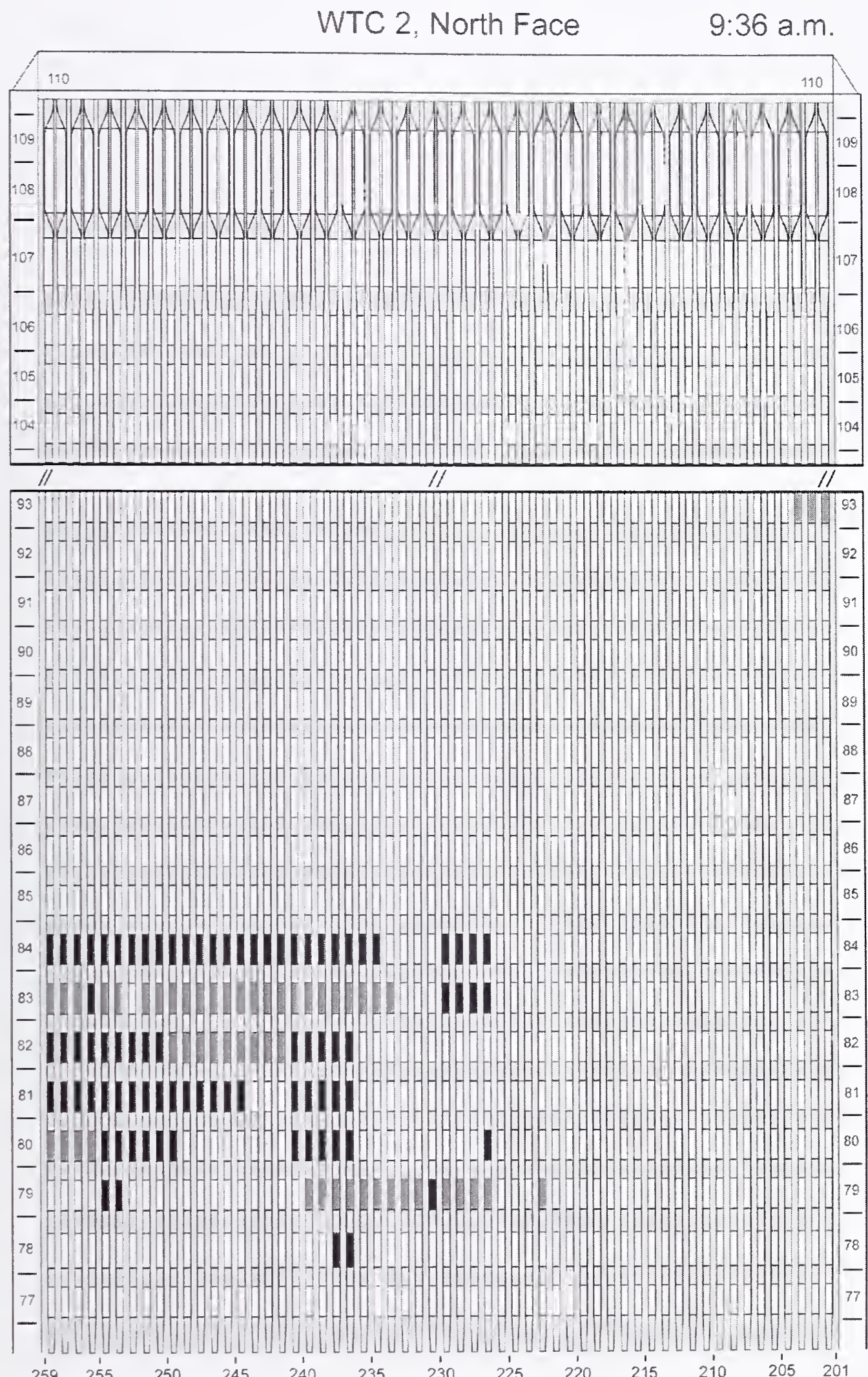

Figure G-36. Diagram of the north face of WTC 2 for floors 77 to 93 and floors 104 to 110 at 9:36 a.m. showing windows where smoke was observed and those hidden from view. 
WTC 2, North Face

$9: 38$ a.m.

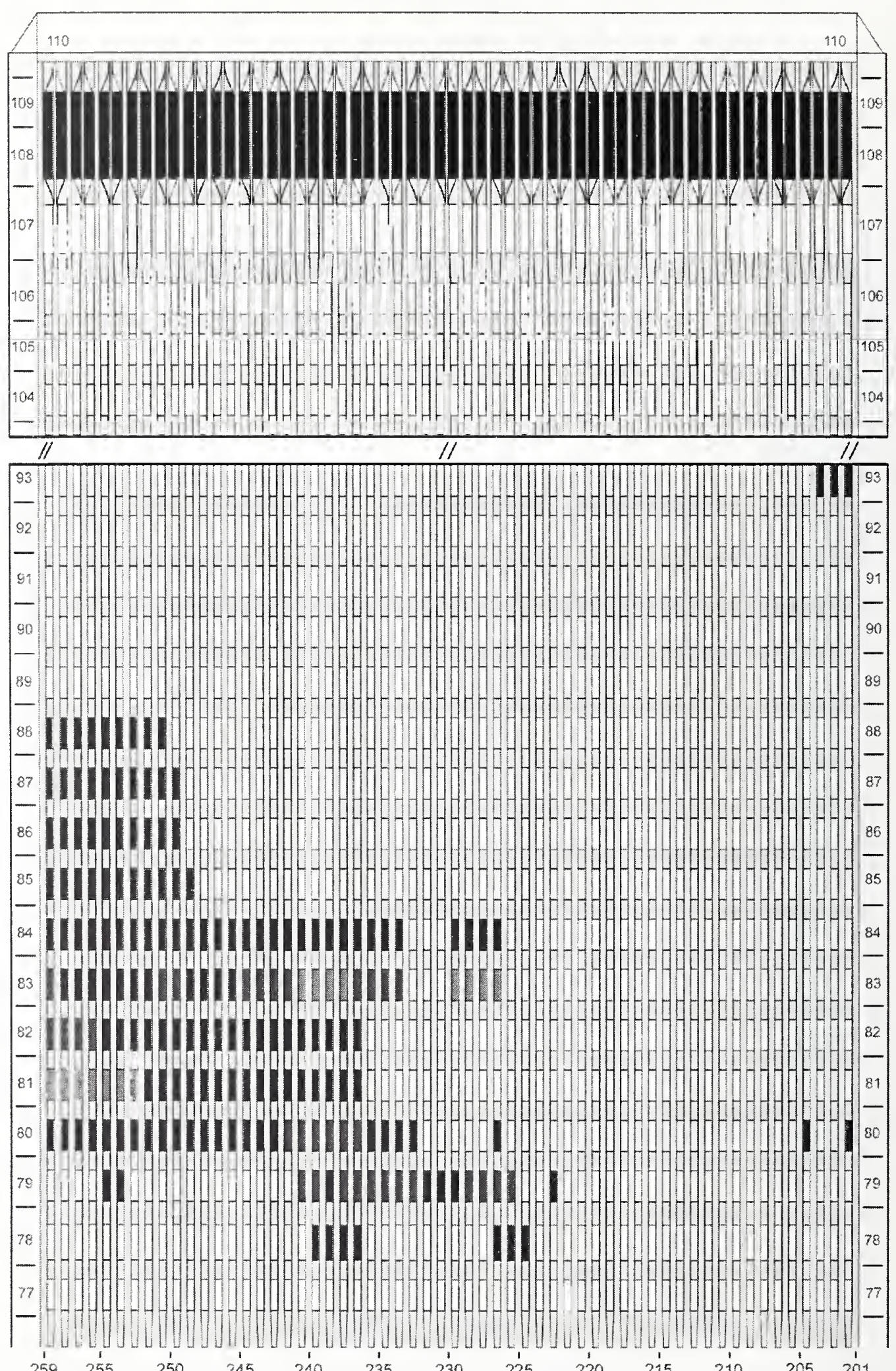

Figure G-37. Diagram of the north face of WTC 2 for floors 77 to 93 and floors 104 to 110 at 9:38 a.m. showing the condition of windows and locations of fires. 
WTC 2, North Face $\quad 9: 38$ a.m.

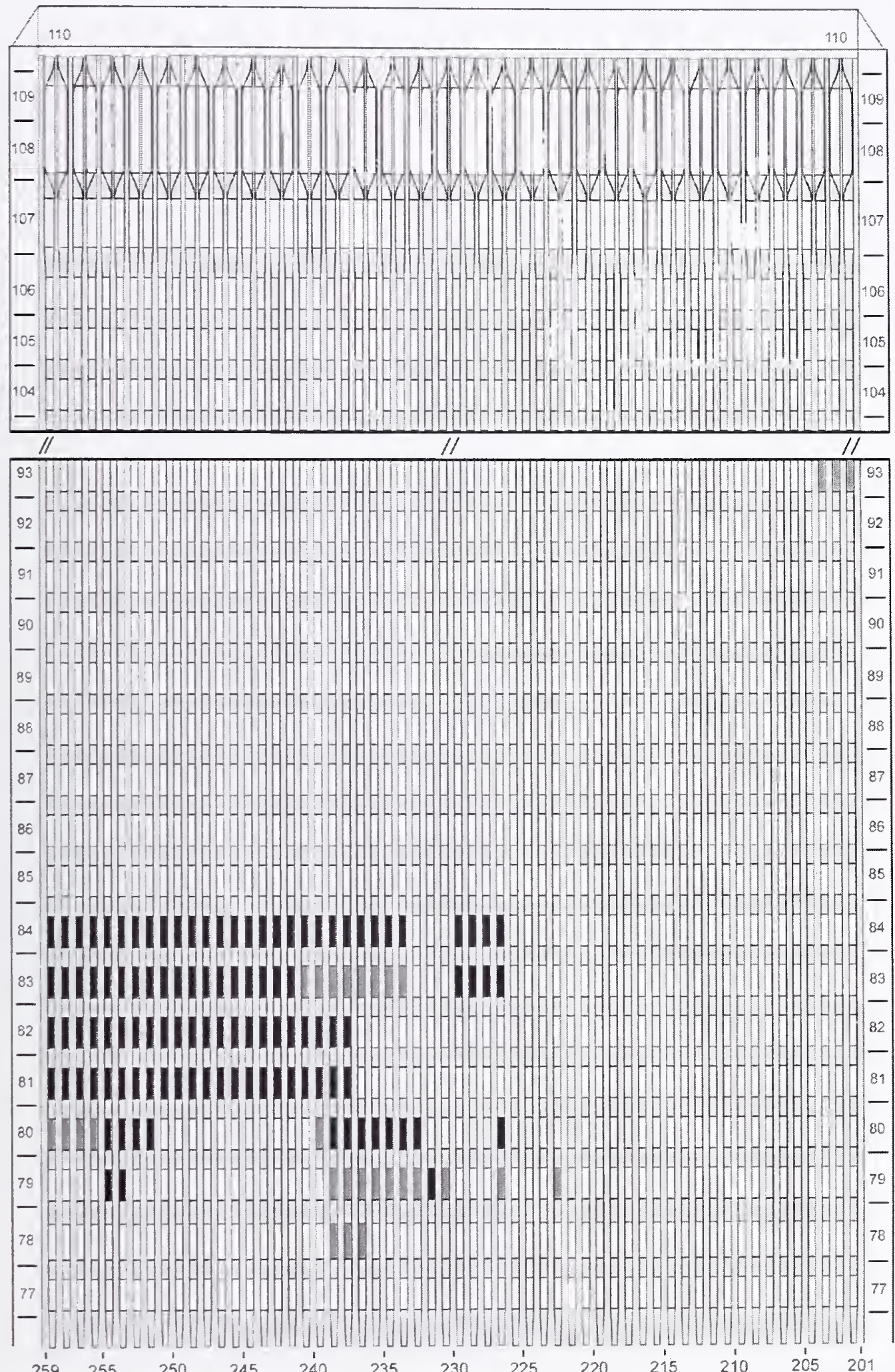

Figure G-38. Diagram of the north face of WTC 2 for floors 77 to 93 and floors 104 to 110 at 9:38 a.m. showing windows where smoke was observed and those hidden from view. 
WTC 2, North Face $\quad 9: 40$ a.m.

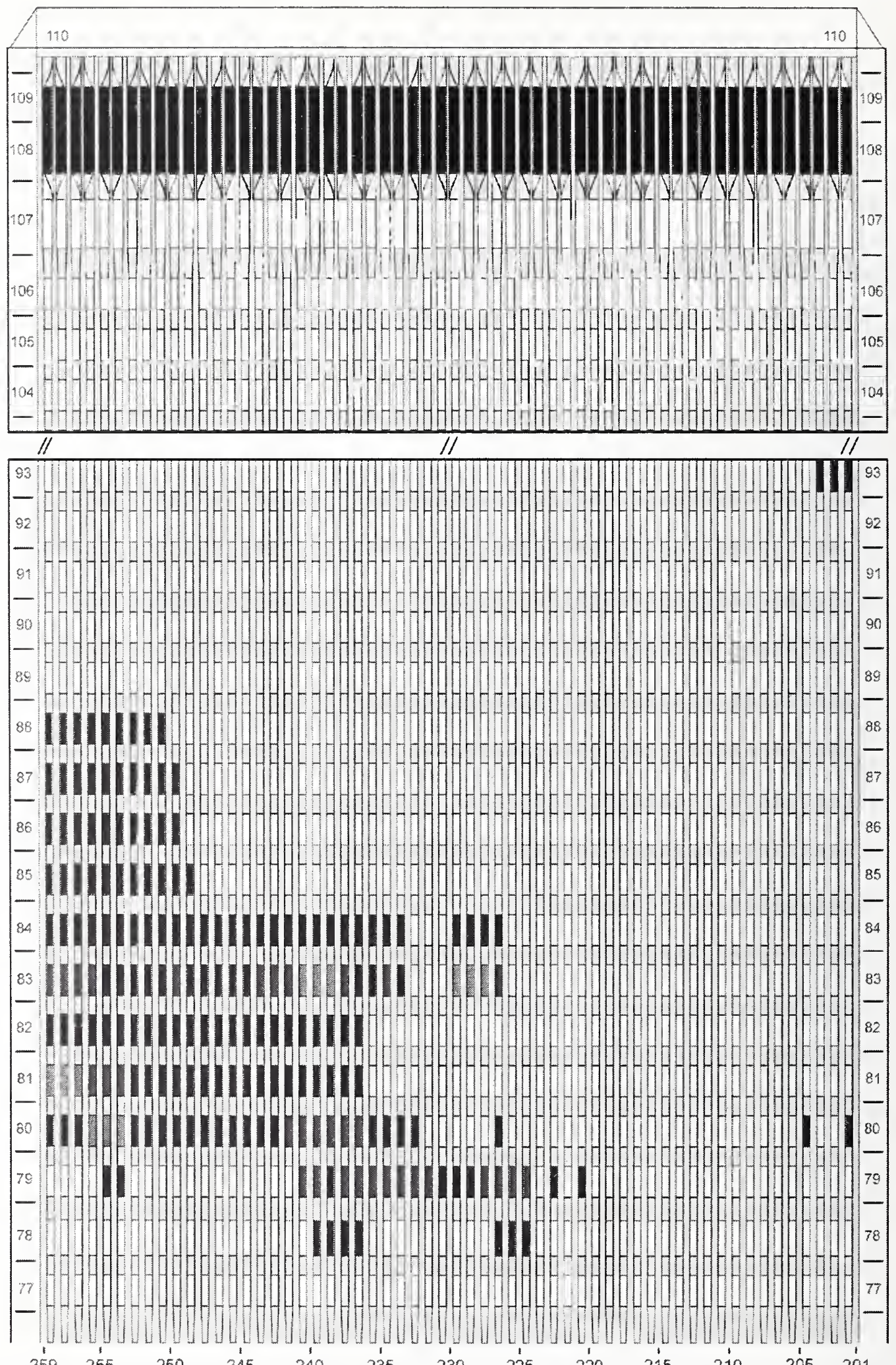

Figure G-39. Diagram of the north face of WTC 2 for floors 77 to 93 and floors 104 to 110 at 9:40 a.m. showing the condition of windows and locations of fires. 
WTC 2, North Face

$9: 40$ a.m.

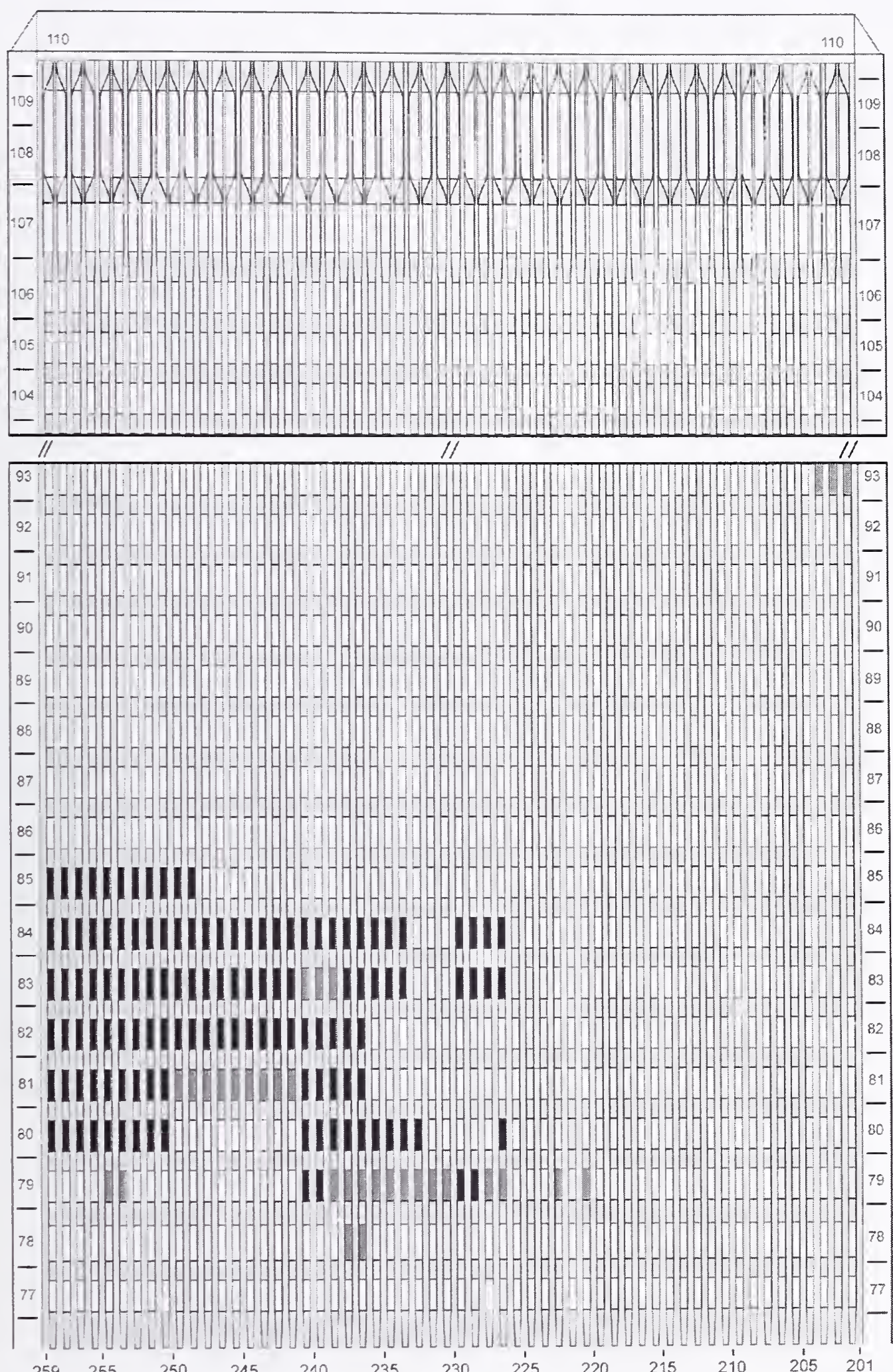

Figure G-40. Diagram of the north face of WTC 2 for floors 77 to 93 and floors 104 to 110 at 9:40 a.m. showing windows where smoke was observed and those hidden from view. 
WTC 2, North Face $\quad 9: 42$ a.m.

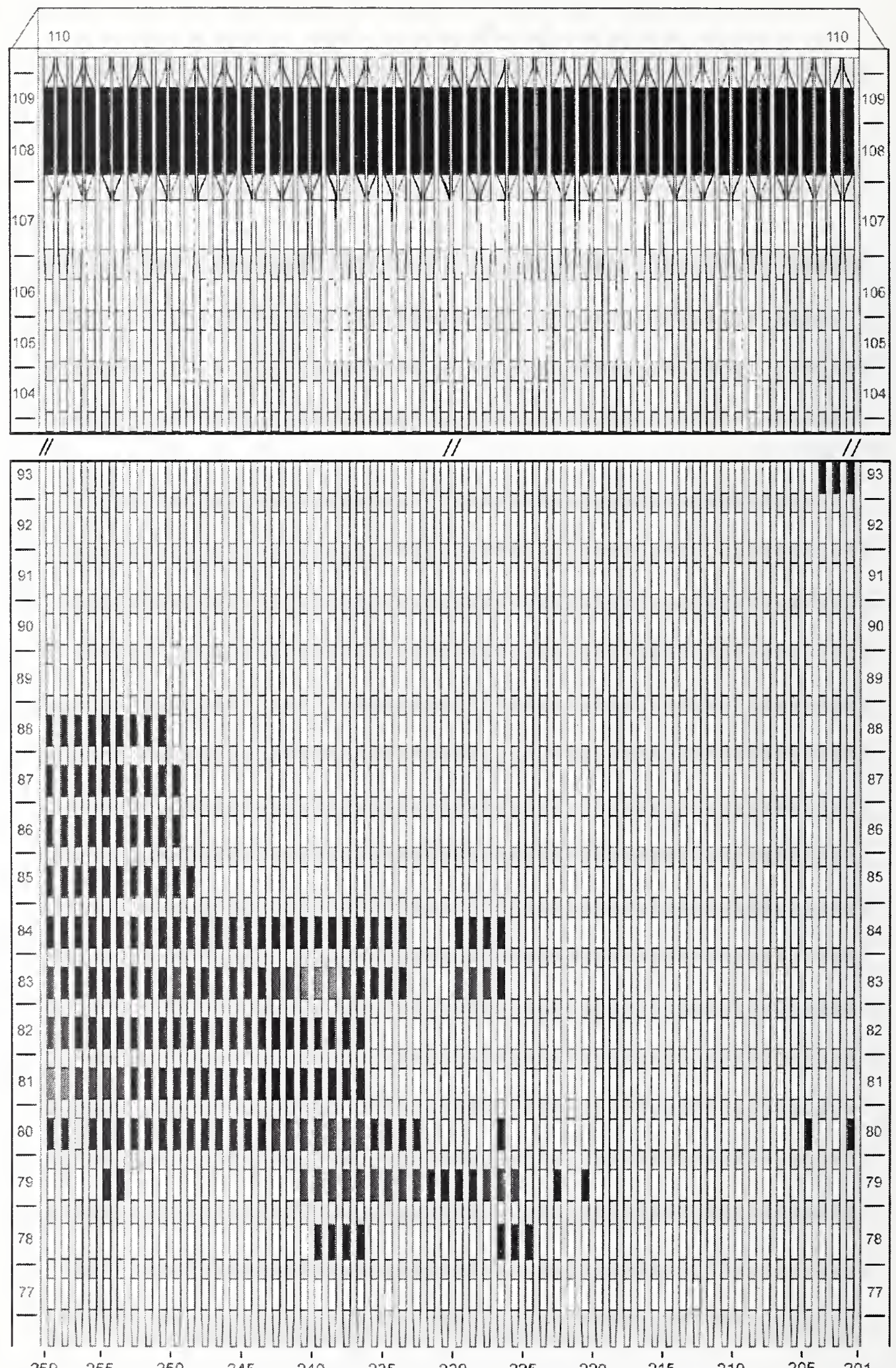

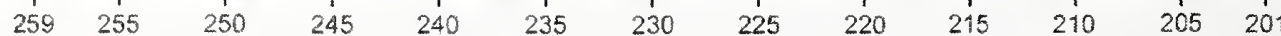

Figure G-41. Diagram of the north face of WTC 2 for floors 77 to 93 and floors 104 to 110 at 9:42 a.m. showing the condition of windows and locations of fires. 
WTC 2, North Face

9:42 a.m.

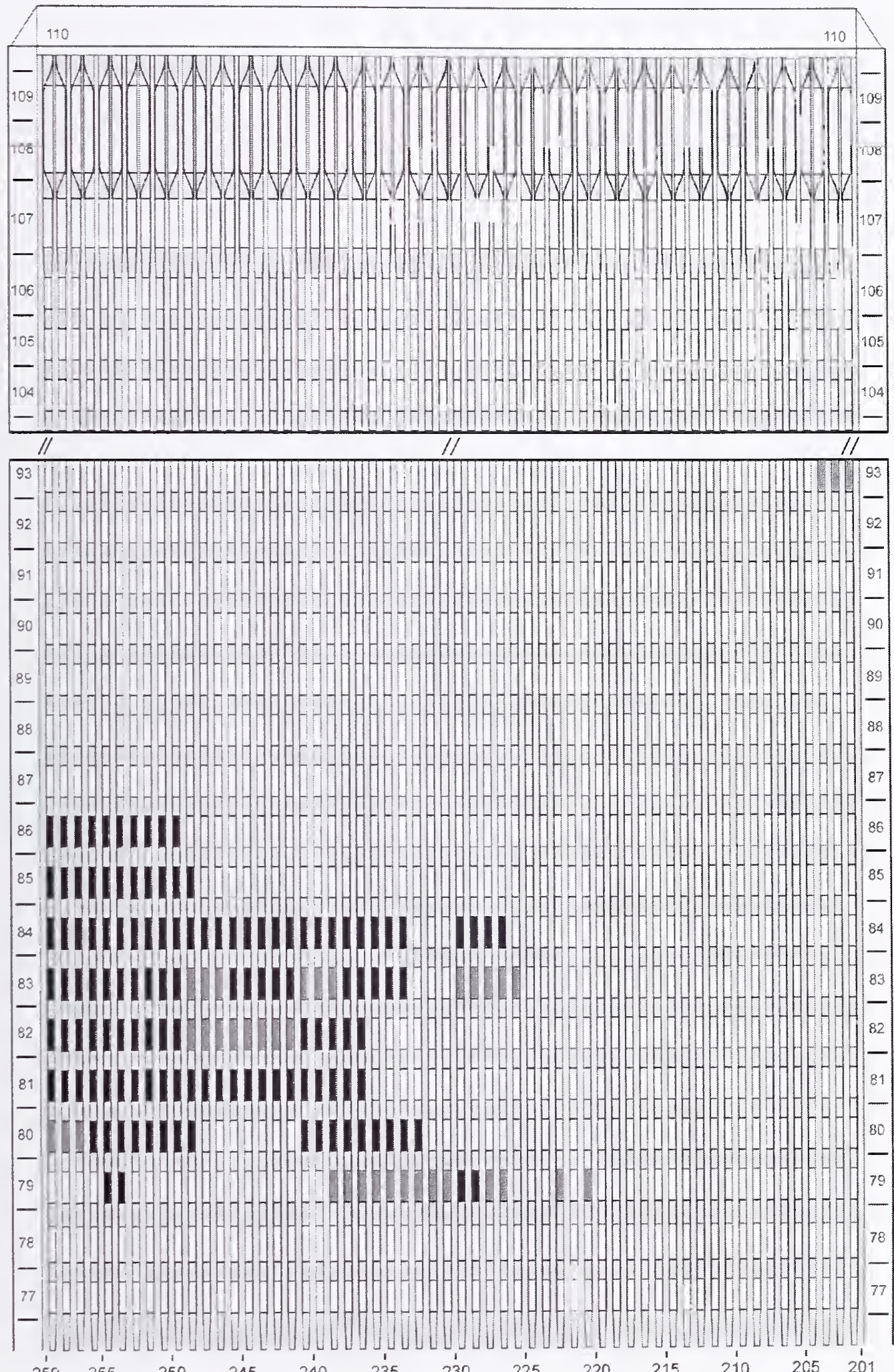

Figure G-42. Diagram of the north face of WTC 2 for floors 77 to 93 and floors 104 to 110 at 9:42 a.m. showing windows where smoke was observed and those hidden from view. 
WTC 2, North Face $\quad 9: 44$ a.m.

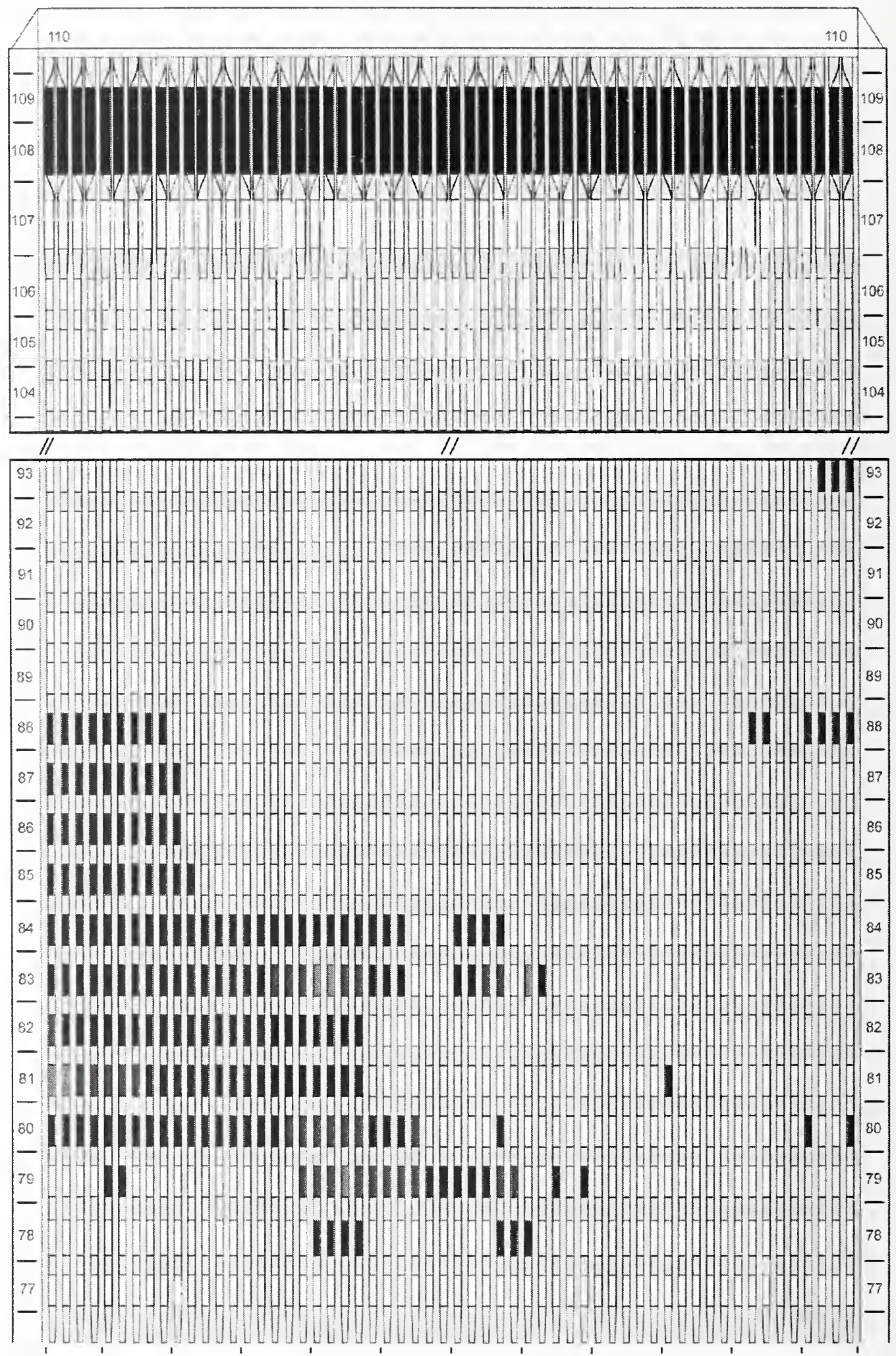

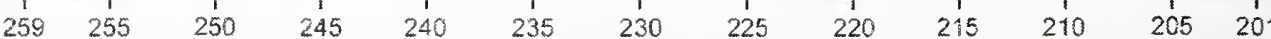

Figure G-43. Diagram of the north face of WTC 2 for floors 77 to 93 and floors 104 to 110 at 9:44 a.m. showing the condition of windows and locations of fires. 
WTC 2, North Face $\quad 9: 44$ a.m.

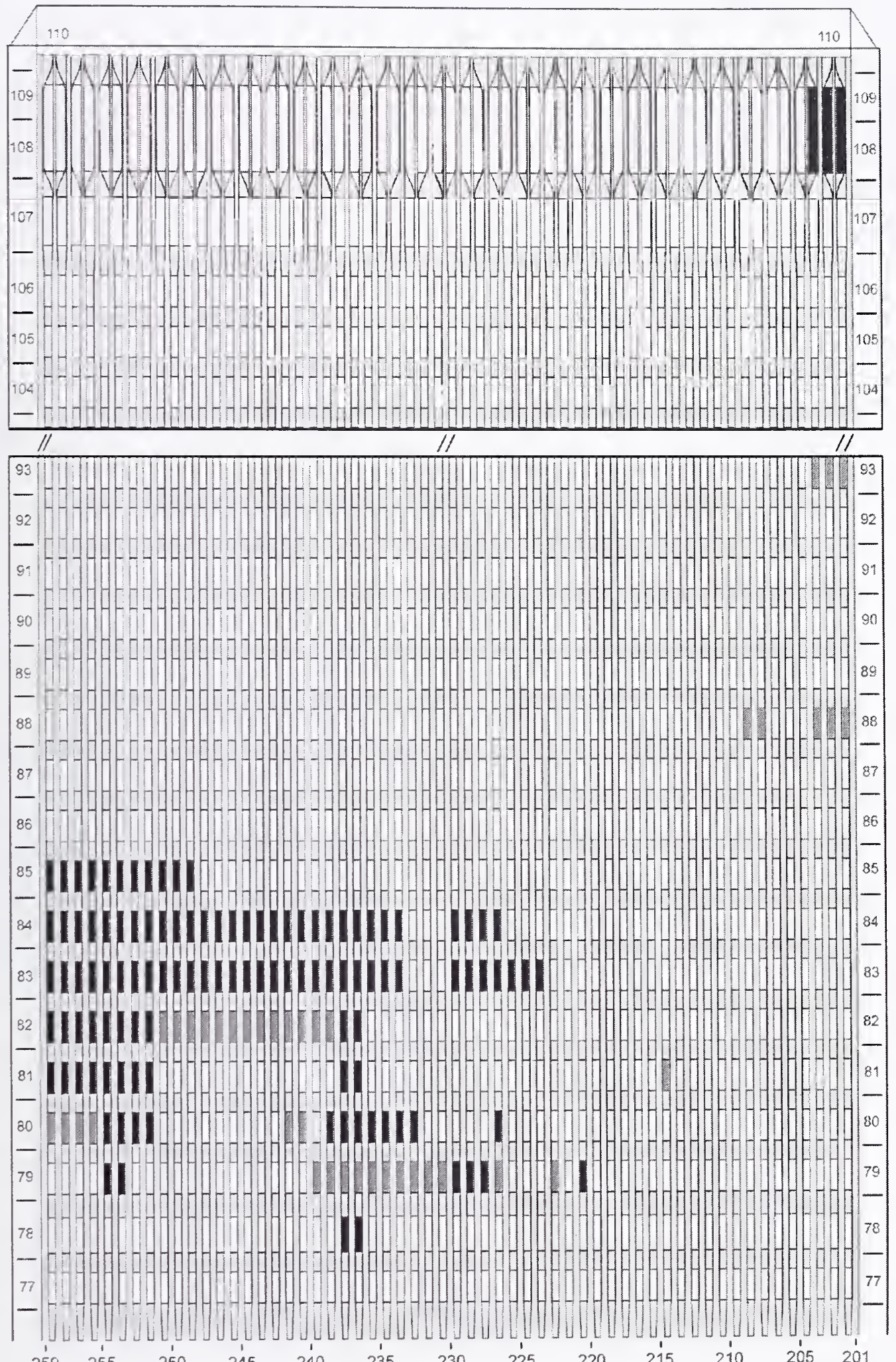

Figure G-44. Diagram of the north face of WTC 2 for floors 77 to 93 and floors 104 to 110 at 9:44 a.m. showing windows where smoke was observed and those hidden from view. 

WTC 2, North Face
9:46 a.m.
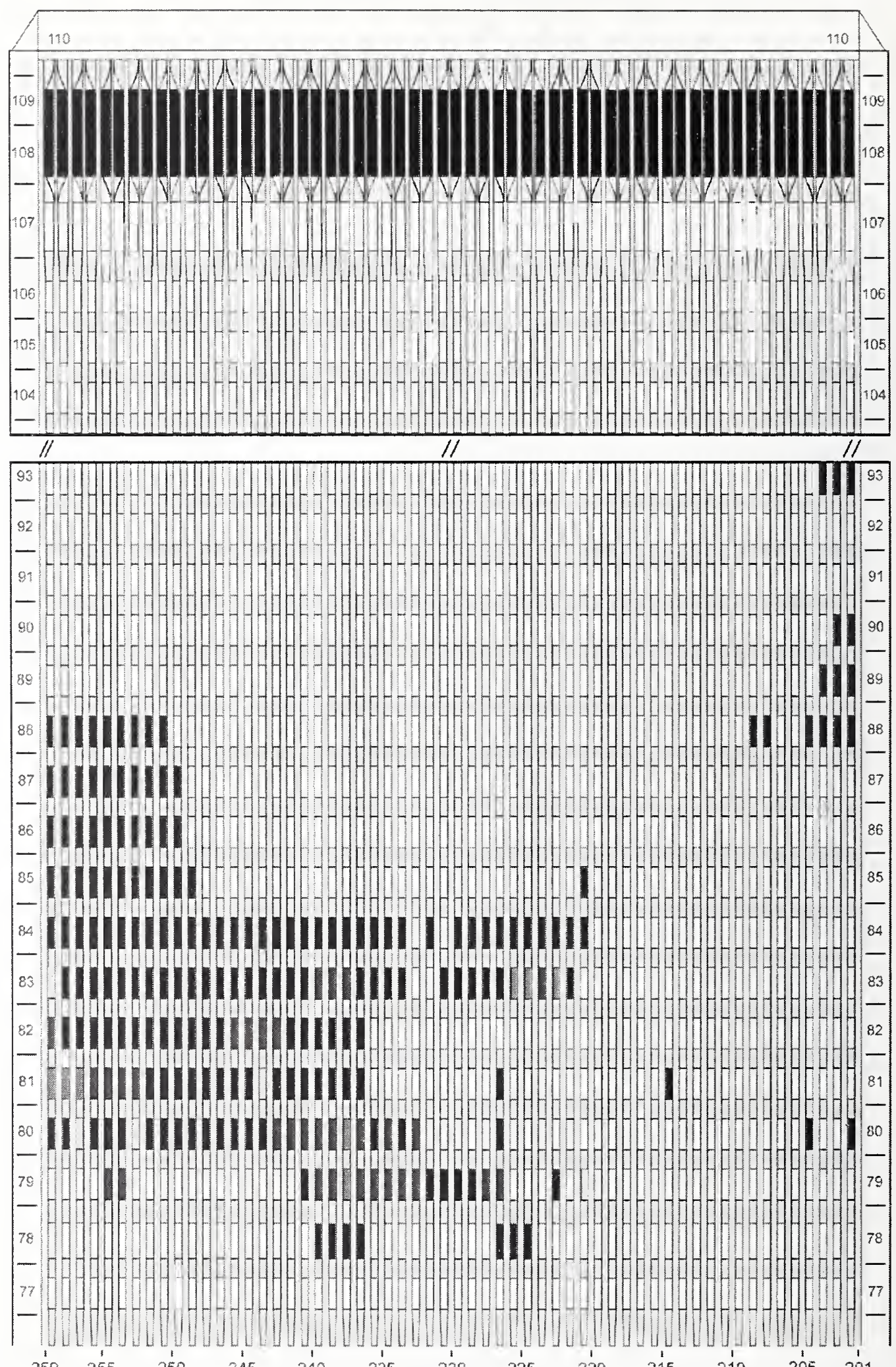

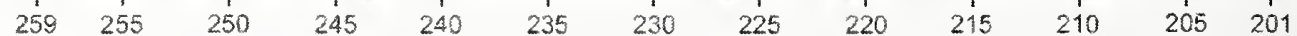

Figure G-45. Diagram of the north face of WTC 2 for floors 77 to 93 and floors 104 to 110 at 9:46 a.m. showing the condition of windows and locations of fires. 
WTC 2, North Face 9:46 a.m.

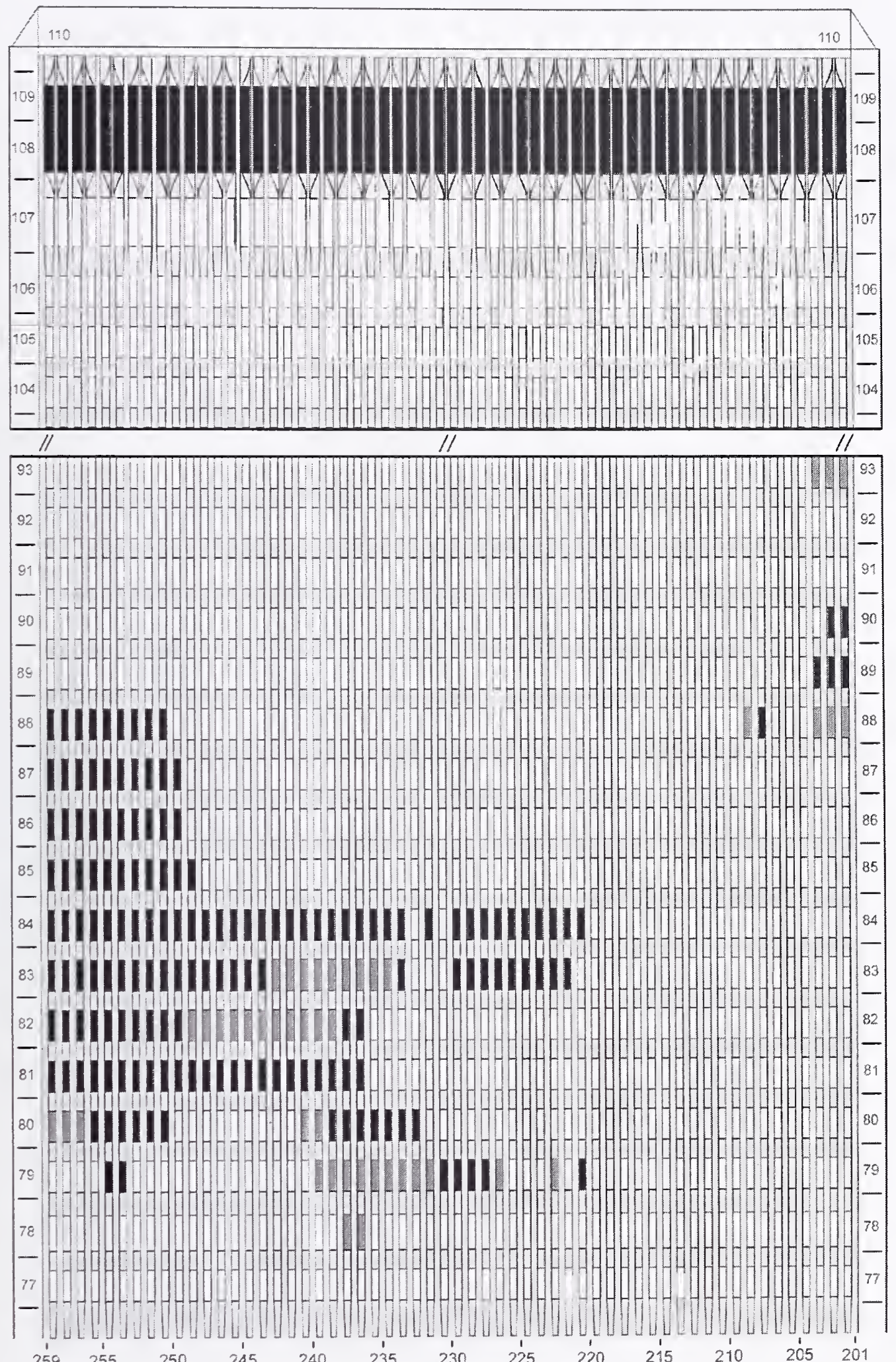

Figure G-46. Diagram of the north face of WTC 2 for floors 77 to 93 and floors 104 to 110 at 9:46 a.m. showing windows where smoke was observed and those hidden from view. 
WTC 2, North Face $\quad 9: 48$ a.m.



Figure G-47. Diagram of the north face of WTC 2 for floors 77 to 93 and floors 104 to 110 at 9:48 a.m. showing the condition of windows and locations of fires. 
WTC 2, North Face

9:48 a.m.

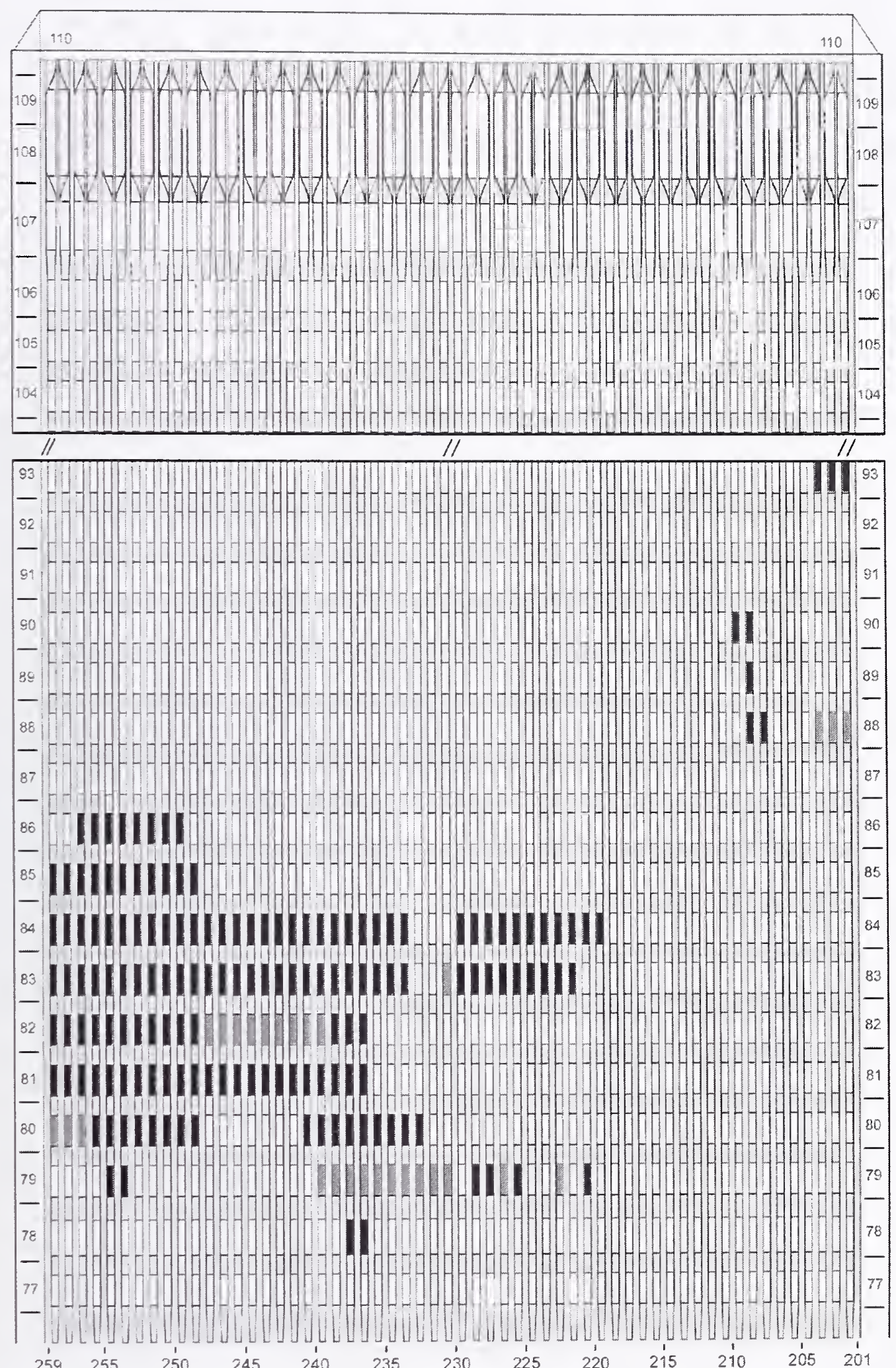

Figure G- 48. Diagram of the north face of WTC 2 for floors 77 to 93 and floors 104 to 110 at 9:48 a.m. showing windows where smoke was observed and those hidden from view. 
WTC 2, North Face $\quad 9: 50$ a.m.

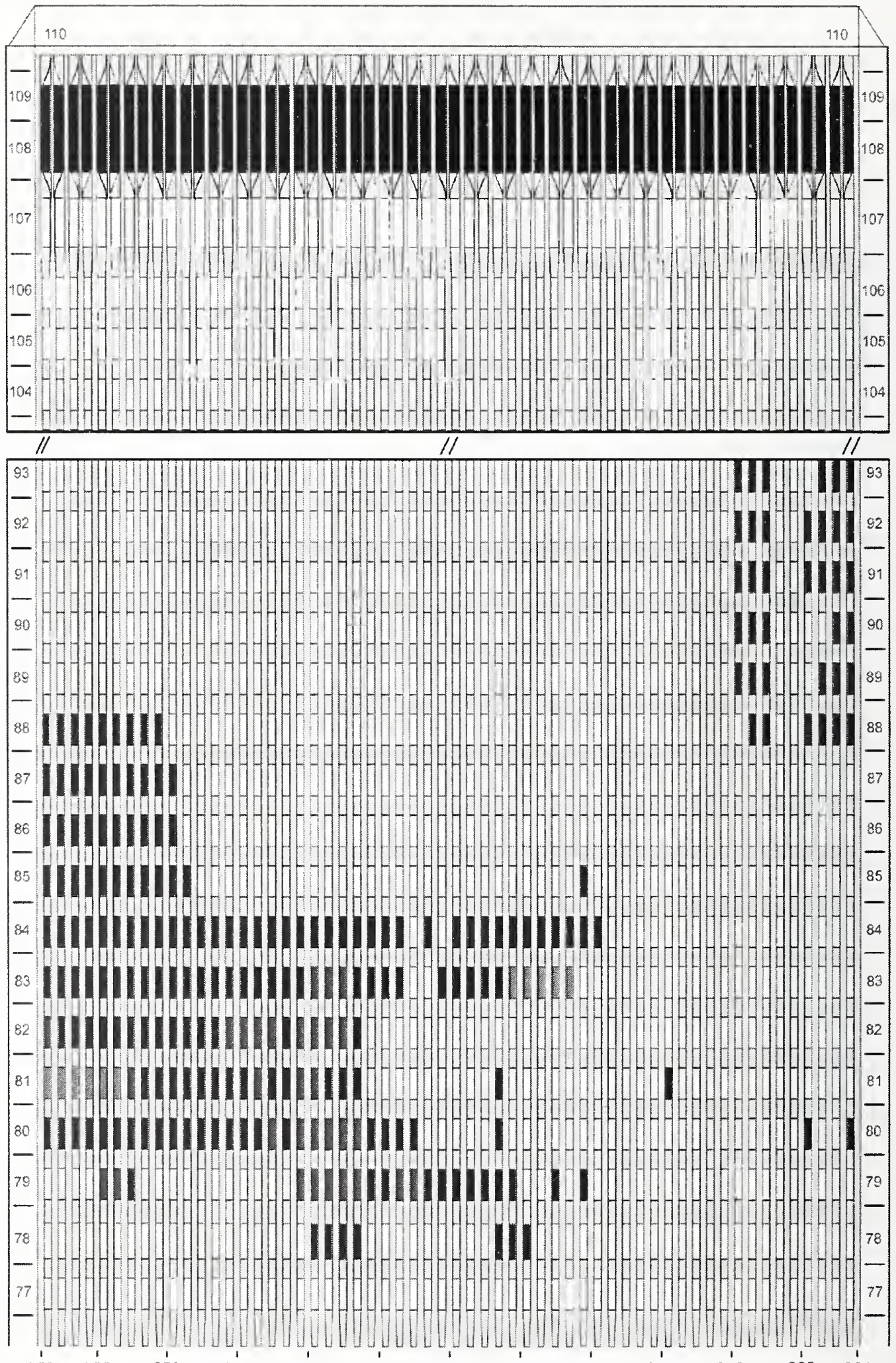

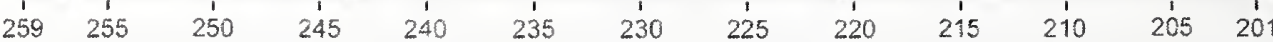

Figure G-49. Diagram of the north face of WTC 2 for floors 77 to 93 and floors 104 to 110 at 9:50 a.m. showing the condition of windows and locations of fires. 
WTC 2, North Face 9:50 a.m.

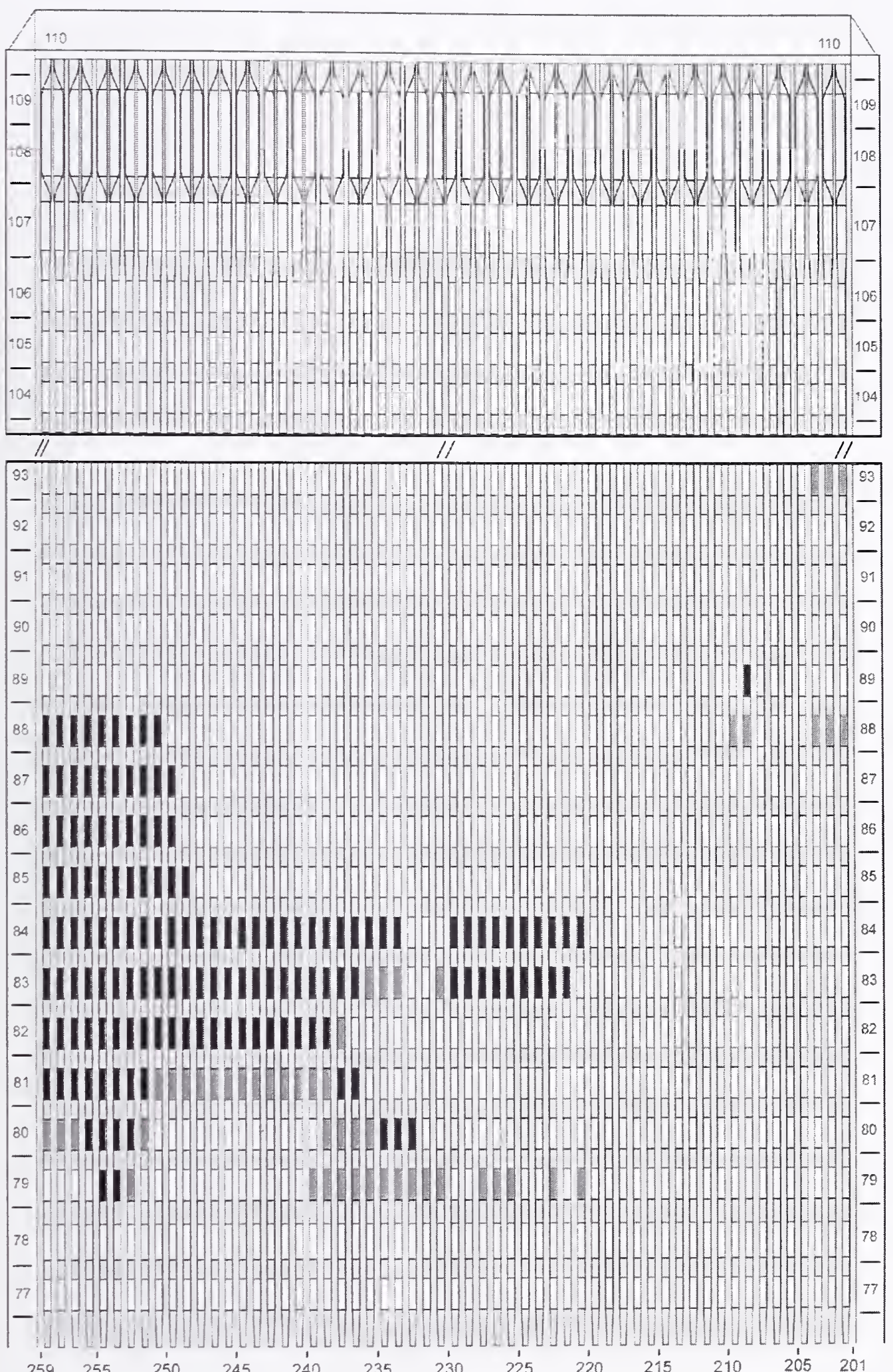

Figure G-50. Diagram of the north face of WTC 2 for floors 77 to 93 and floors 104 to 110 at 9:50 a.m. showing windows where smoke was observed and those hidden from view. 
WTC 2, North Face 9:52 a.m.

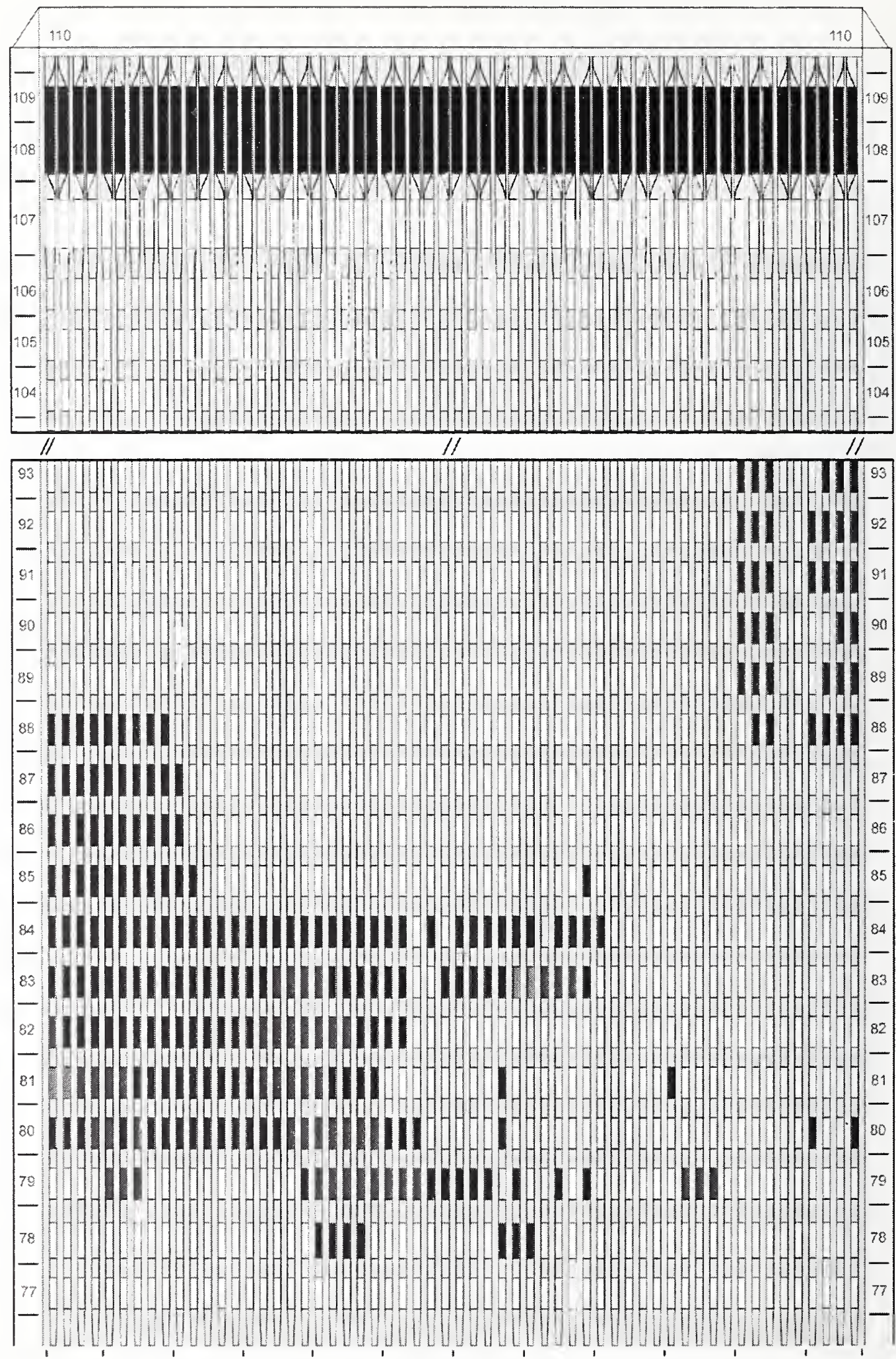

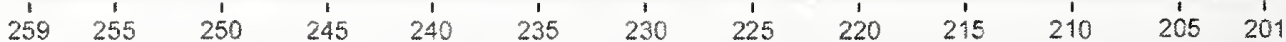

Figure G-51. Diagram of the north face of WTC 2 for floors 77 to 93 and floors 104 to 110 at 9:52 a.m. showing the condition of windows and locations of fires. 
WTC 2, North Face

$9: 52$ a.m.

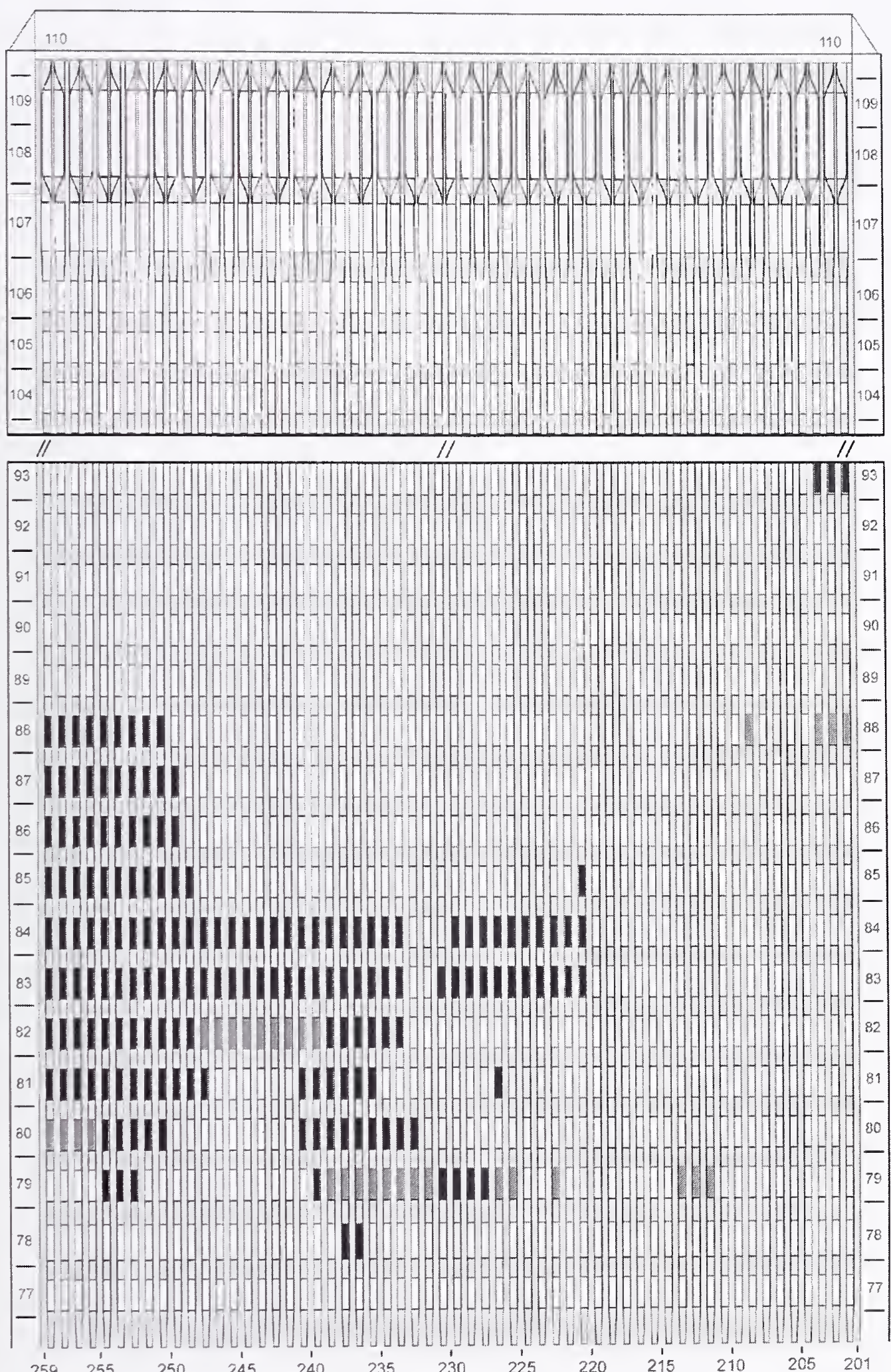

Figure G-52. Diagram of the north face of WTC 2 for floors 77 to 93 and floors 104 to 110 at 9:52 a.m. showing windows where smoke was observed and those hidden from view. 
WTC 2, North Face $\quad 9: 54$ a.m.

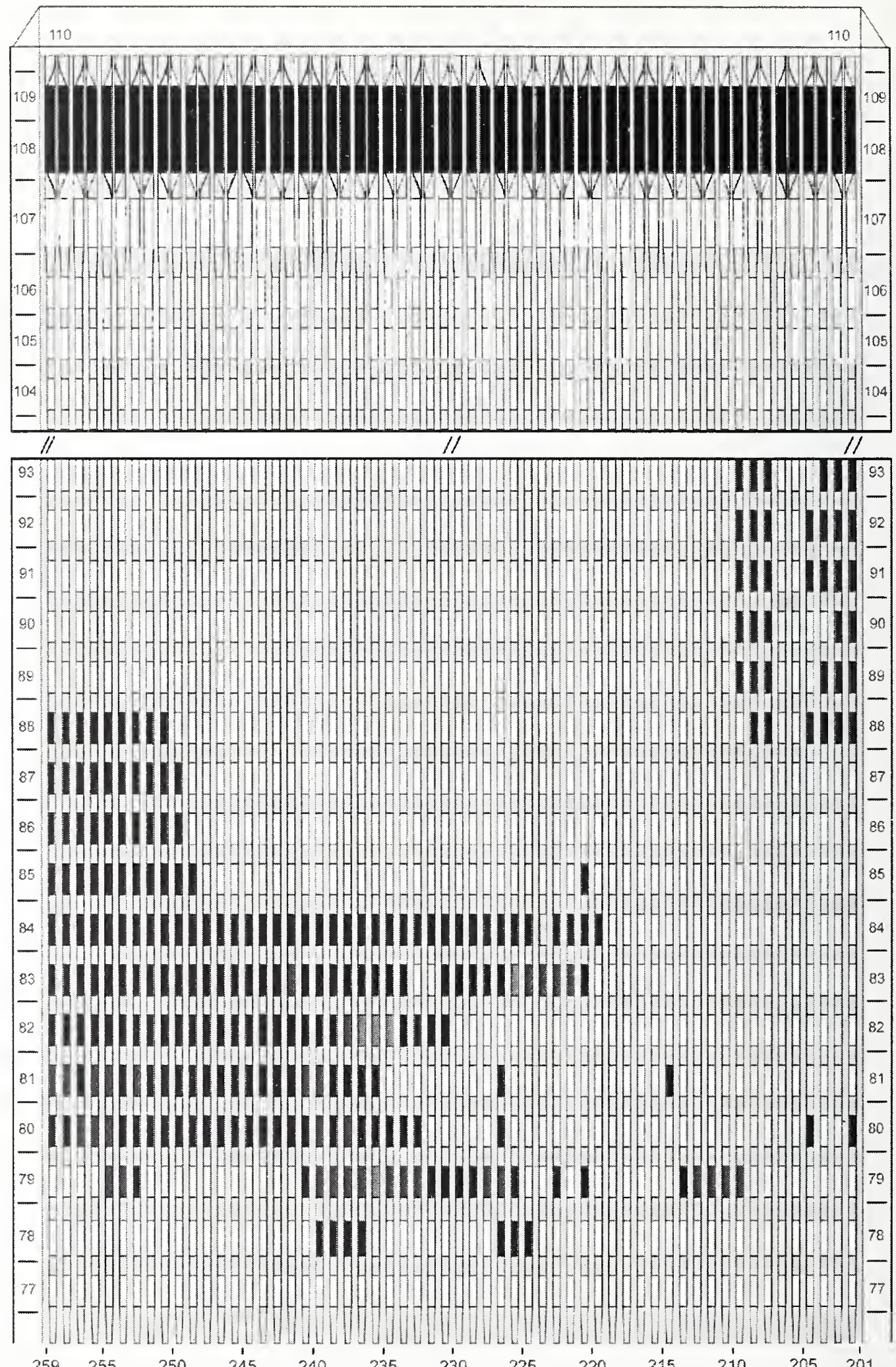

Figure G-53. Diagram of the north face of WTC 2 for floors 77 to 93 and floors 104 to 110 at 9:54 a.m. showing the condition of windows and locations of fires. 
WTC 2, North Face

$9: 54$ a.m.

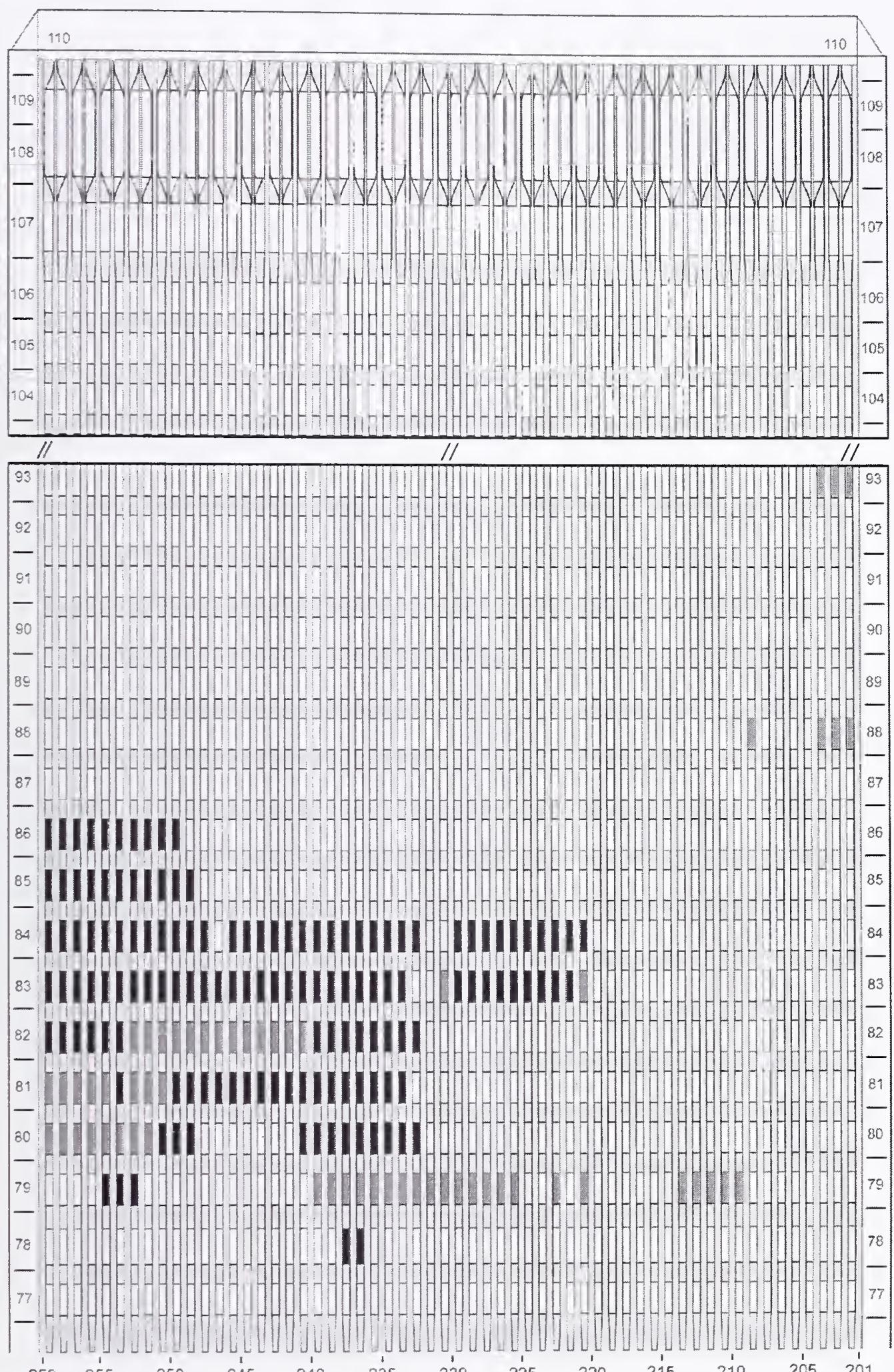

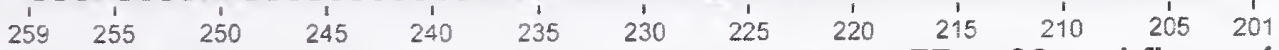

Figure G-54. Diagram of the north face of WTC 2 for floors 77 to 93 and floors 104 to 110 at 9:54 a.m. showing windows where smoke was observed and those hidden from view. 
WTC 2, North Face

9:56 a.m.

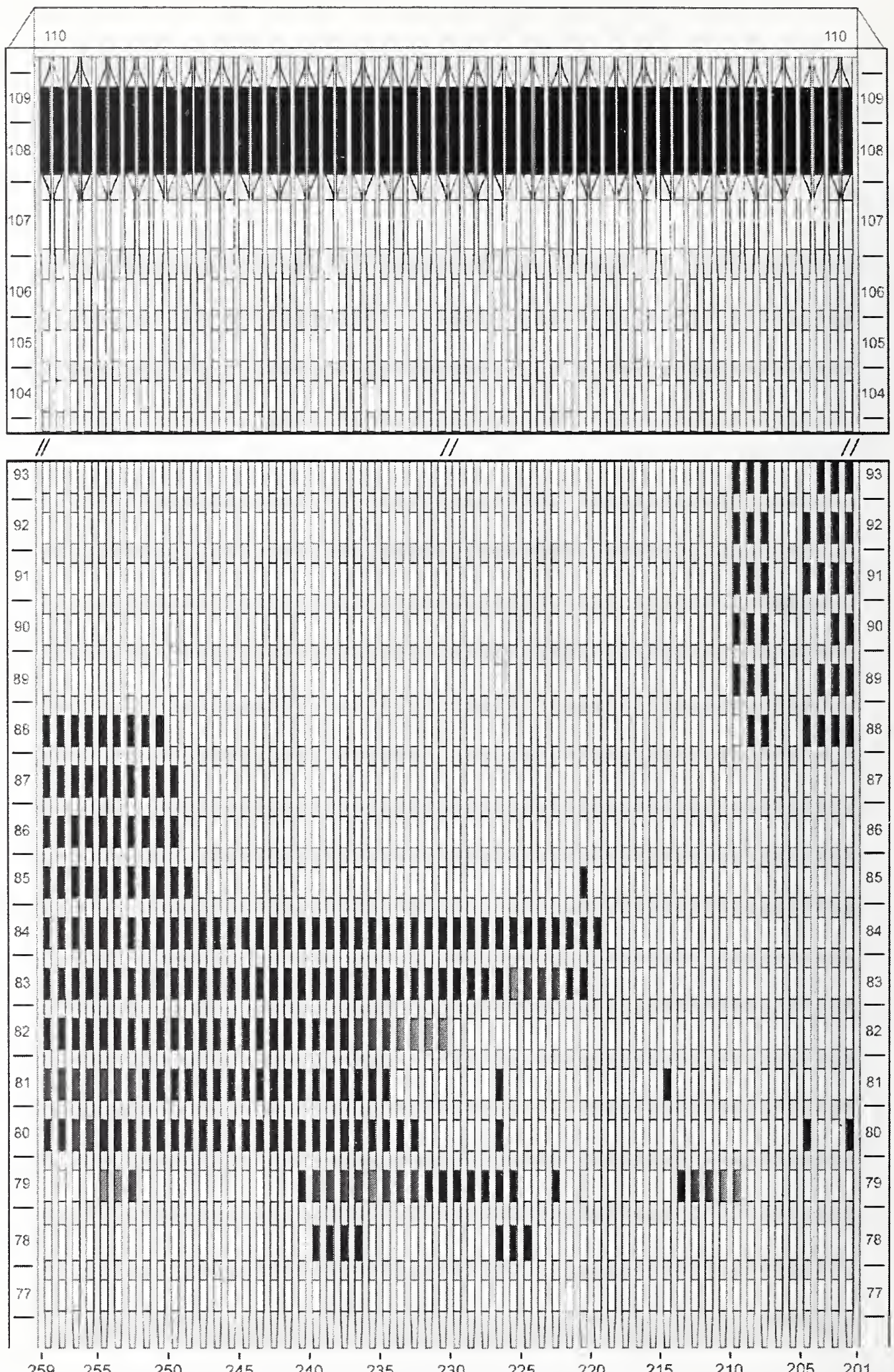

Figure G-55. Diagram of the north face of WTC 2 for floors 77 to 93 and floors 104 to 110 at 9:56 a.m. showing the condition of windows and locations of fires. 
WTC 2, North Face

9:56 a.m

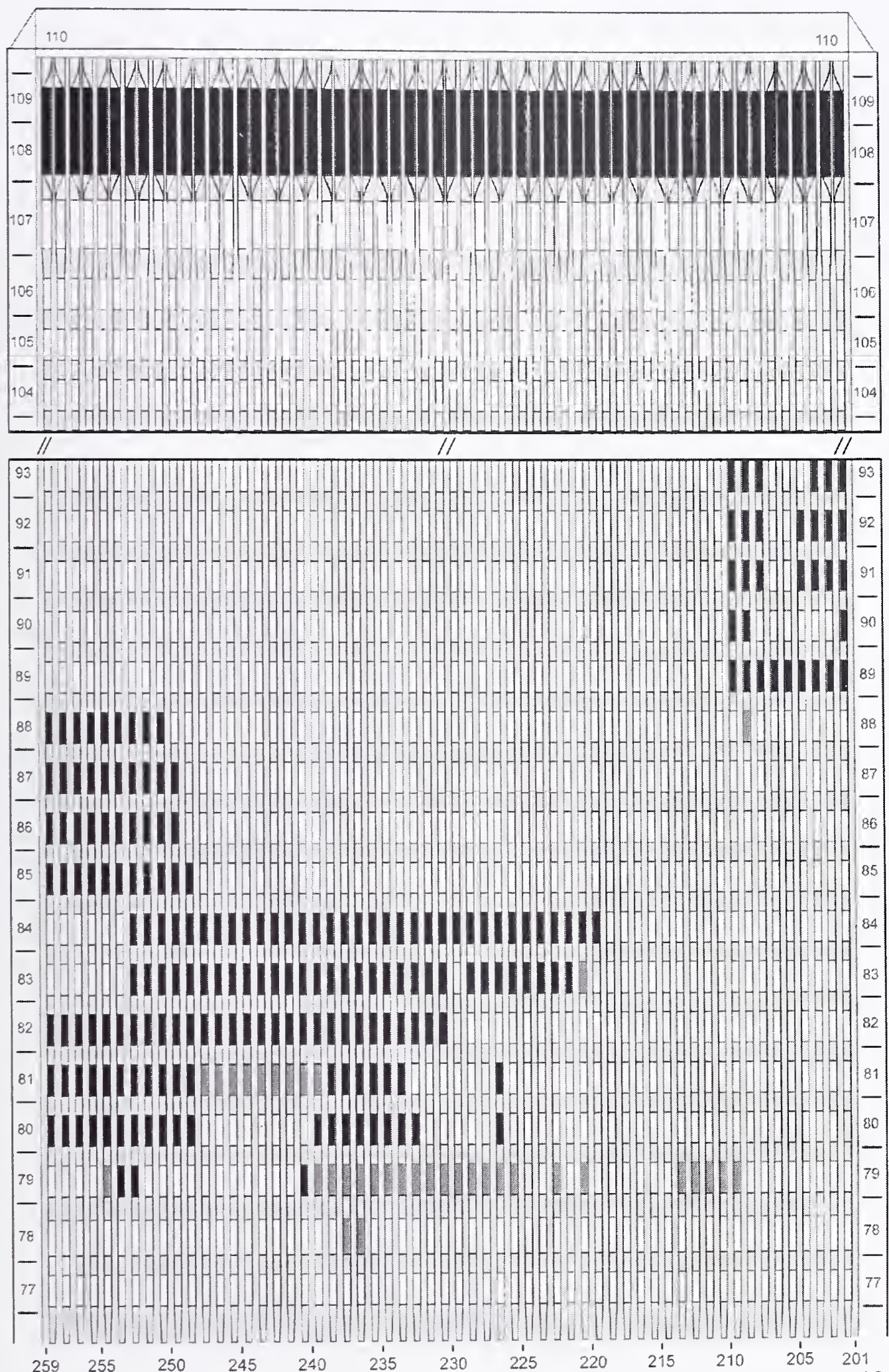

Figure G-56. Diagram of the north face of WTC 2 for floors 77 to 93 and floors 104 to 110 at 9:56 a.m. showing windows where smoke was observed and those hidden from view. 
WTC 2, North Face 9:58 a.m.

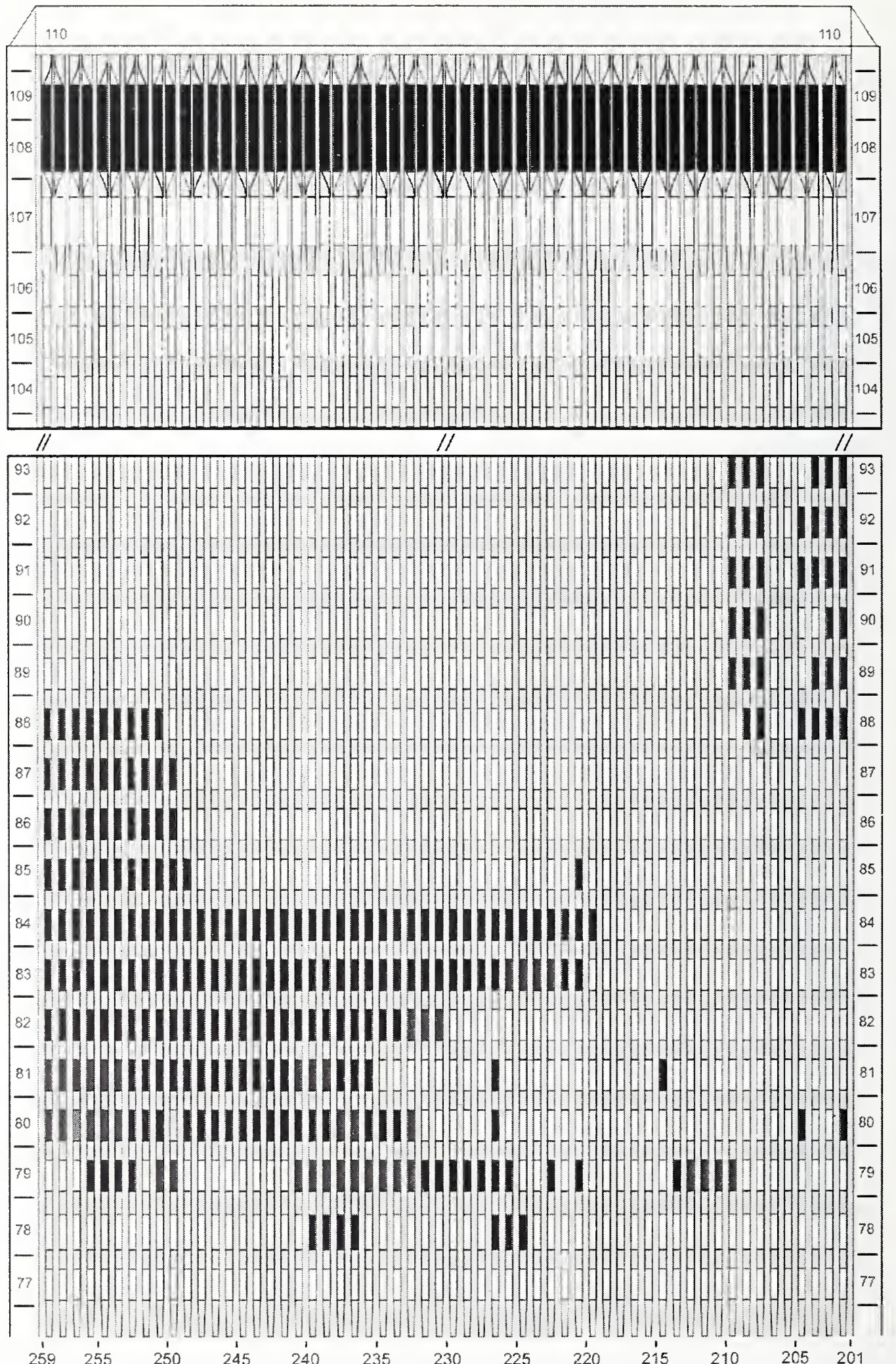

Figure G-57. Diagram of the north face of WTC 2 for floors 77 to 93 and floors 104 to 110 at 9:58 a.m. showing the condition of windows and locations of fires. 
WTC 2, North Face $\quad 9: 58$ a.m.

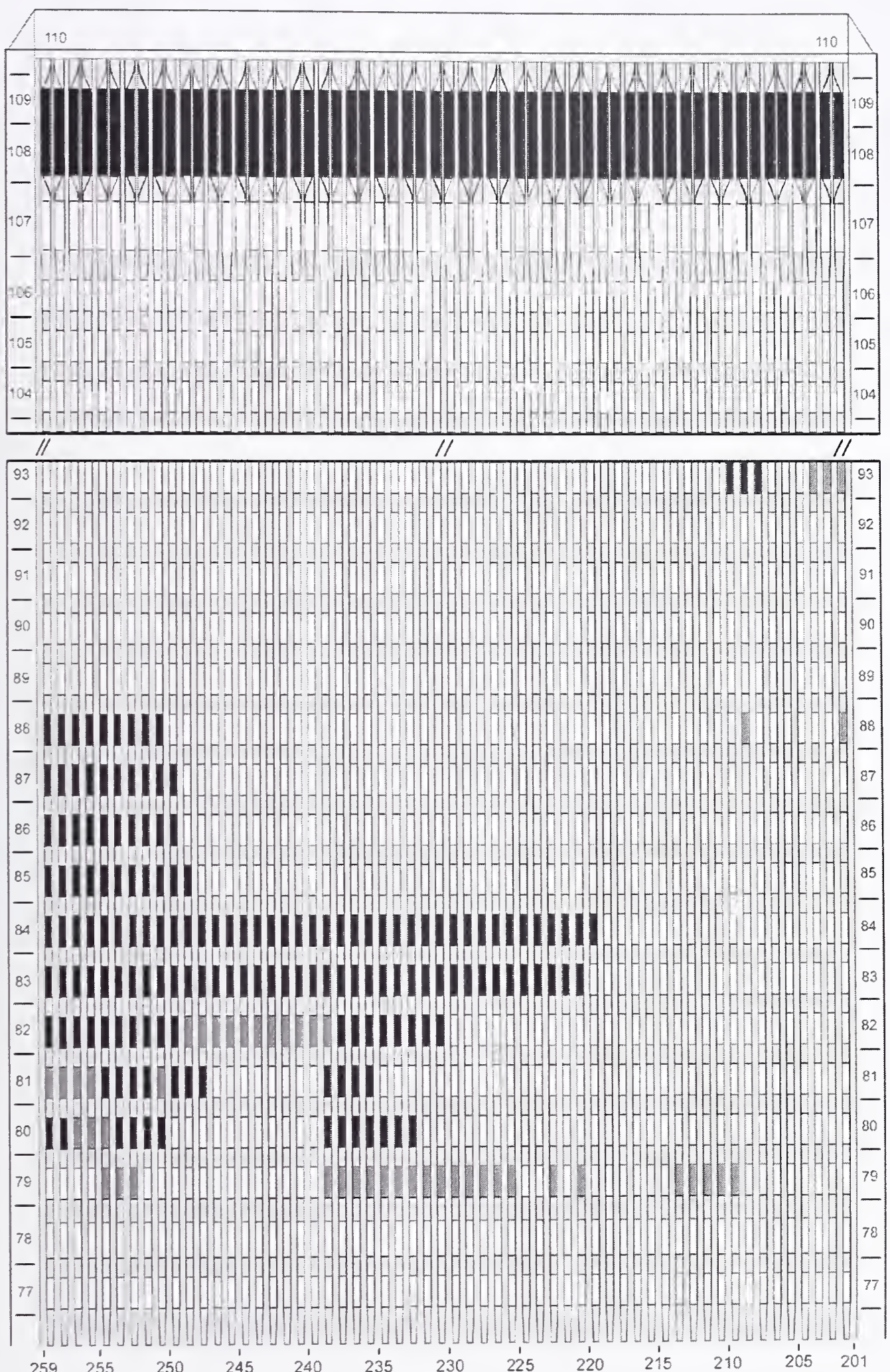

Figure G-58. Diagram of the north face of WTC 2 for floors 77 to 93 and floors 104 to 110 at 9:58 a.m. showing windows where smoke was observed and those hidden from view. 
This page intentionally left blank. 



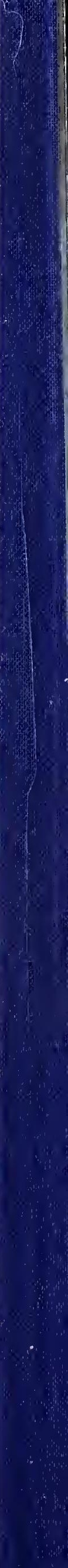

